\title{
NUGLEAR
}

AIR GLEANING

HANDBOOK

\section{DESIGN, CONSTRUCTION, AND TESTING OF HIGH-EFFICIENCY AIR CLEANING SYSTEMS FOR NUCLEAR APPLICATION}

C. A. Burchsted

J. E. Kahn

A. B. Fuller
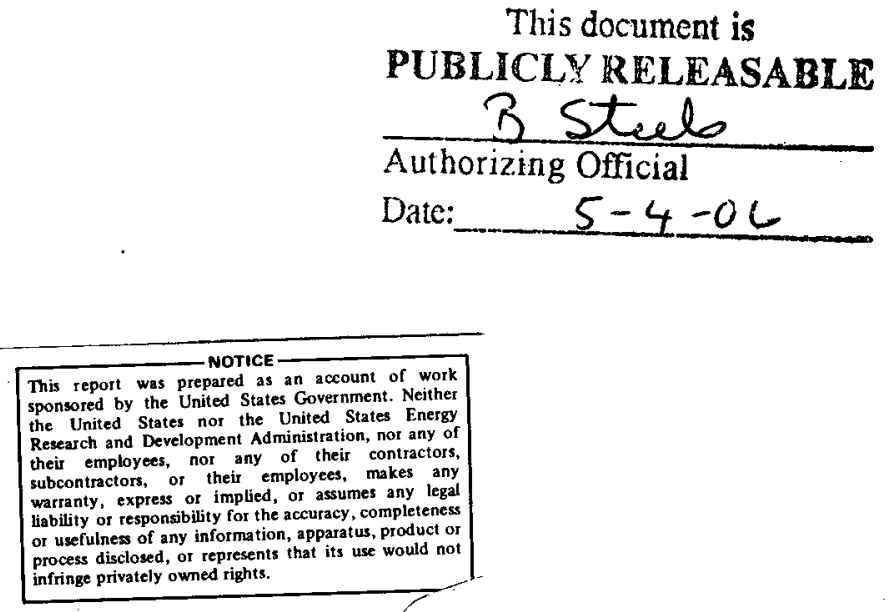

Contract No. W-7405-eng-26

OAK RIDGE NATIONAL LABORATORY

Oak Ridge, Tennessee 37830

OPERATED BY UNION CARBIDE CORPORATION

FOR THE ENERGY RESEARCH AND DEVELOPMENT ADMINISTRATION 


\section{DISCLAIMER}

This report was prepared as an account of work sponsored by an agency of the United States Government. Neither the United States Government nor any agency Thereof, nor any of their employees, makes any warranty, express or implied, or assumes any legal liability or responsibility for the accuracy, completeness, or usefulness of any information, apparatus, product, or process disclosed, or represents that its use would not infringe privately owned rights. Reference herein to any specific commercial product, process, or service by trade name, trademark, manufacturer, or otherwise does not necessarily constitute or imply its endorsement, recommendation, or favoring by the United States Government or any agency thereof. The views and opinions of authors expressed herein do not necessarily state or reflect those of the United States Government or any agency thereof. 


\section{DISCLAIMER}

Portions of this document may be illegible in electronic image products. Images are produced from the best available original document. 


\section{Foreword \\ to \\ Second Edition}

This handbook is a revision of ORNL/NSIC-65, Design, Construction, and Testing of High-Efficiency Air Filtration Systems for Nuclear Application, which was issued in January 1970. For simplification, the title has been shortened to Nuclear Air Cleaning $H a n d b o o k$, and the report has been issued under an ERDA number.

The new edition updates the information of the orginal volume, corrects some errors that appeared in it, and adds some new material, particularly in the areas of sand filters, deep-bed glass fiber filters, and requirements for plutonium and reprocessing plants. Although A. B. Fuller was unable to contribute directly to this edition, his earlier material on singlefilter installation and glove boxes has been largely retained, though rewritten and updated. With this issue, J. E. Kahn of the Union Carbide Corporation Nuclear Division's (UCCND) Engineering staff joins the writing team, contributing particularly in updating the material on glove boxes and writing the sections on sand filters and deep-bed glass fiber filters in Chap. 9. Others who have contributed to this edition include J. C. Little, UCCND Engineering, and a host of reviewers who provided technical evaluation of the draft. Particular thanks are due Dr. M. W. First of the Harvard University School of Public Health, and Mr. Humphrey Gilbert, consultant to the Energy Research and Development Administration (ERDA) and the Nuclear Regulatory Commission (NRC) and former safety engineer with the U.S. Atomic Energy Commission, for their detailed and thorough review of the complete draft. Others who reviewed the complete draft were J. F. Fish, chairman of ANSI Committee N45-8; J. C. Little, UCCND Engineering; J. C. Dempsey, ERDA Division of Nuclear Fuel Cycle and Production; A. B. Fuller, president of Fuller Engineering; and J. T. Collins of NRC. Thanks are also due to the members of ANSI Committee N45-8 who, perhaps unknowingly, supplied certain data and served as a sounding board for some of the concepts presented in the handbook. We wish to thank the miany vendors and ERDA contractors who supplied drawings and photographs used in the book. We also acknowledge the work of Oak Ridge National Laboratory's Technical Publications Department, particularly that of the Composition and Makeup groups, that of R. H. Powell who provided editorial assistance, and especially that of P. J. Patton who edited and coordinated publication of this handbook.

Reviewers who contributed in the technical review of particular sections of the handbook include

R. L. Alley, American Warming and Ventilating Company

J. E. Beavers, Union Carbide Corporation Nuclear Division

R. R. Bellamy, Nuclear Regulatory Commission

R. E. Blanco, Oak Ridge National Laboratory

P. J. Breman, Union Carbide Corporation Nuclear Division

C. L. Cheever, Argonne National Laboratory

J. C. Elder, Los Alamos Scientific Laboratory

A. G. Evans, Savannah River Laboratory

H. F. Farquhar, Lau Blower Company

S. S. Freeman, Mound Laboratory

R. T. Goulet, Cambridge Filter Corporation

R. K. Hilliard, Hanford Engineering Development Laboratory

D. J. Keigher, Los Alamos Scientific Laboratory

C. Lambert, Bechtel Power Corporation

F. D. Leckie, Nuclear Containment Systems, Inc.

H. A. Lee, Atlantic Richfield Hanford Company

J. Lipera, Lawrence Livermore Laboratory

R. A. Lorenz, Oak Ridge National Laboratory

W. Ng, Lawrence Livermore Laboratory 
W. C. Schimdt, Atlantic Richfield Hanford Company

F. R. Schwartz, Jr., North American Carbon Company

A. Shacter, U.S. Army, Aberdeen Proving Ground-EA
Sheet Metal and Air Conditioning Contractors' National Association.

A. A. Weintraub, Energy Research and Development Administration

R. E. Yoder, Rocky Flats Plant

D. P. Zippler, Savannah River Plant

C. A. Burchsted

Oak Ridge, Tennessee

March 31, 1976 


\section{Foreword}

\section{to}

\section{First Edition}

This handbook fills a large gap in the literature concerning air cleaning and filtration, the gap that encompasses design, construction, and testing of very high-efficiency air cleaning systems. The project was originally conceived by Mr. Humphrey Gilbert of the USAEC and was sponsored by the Division of Reactor Development and Technology of the USAEC. In preparing for the project we surveyed aircleaning. systems at atomic energy facilities and industrial installations throughout the United States and Canada. We visited AEC production reactors, commercial power reactors, laboratories, radiochemical plants, reactor fuel manufacturers, clean rooms, equipment manufacturers, and one chemical-biological warfare installation. The purposes of these visits were to review current practices in high efficiency air cleaning and to define the problems in operating, maintaining, and controlling contamination release from very high-efficiency aircleaning systems from experienced people who were dealing with such problems daily. The handbook reflects a consensus of our findings in these travels, in addition to information gleaned from the available literature.

The handbook is addressed primarily to designers and architect-engineers. We frequently observed a lack of communication and feedback from people with problems in the field to designers. Our intention is to bring to the attention of designers of future systems the kind of problems that an operator faces and what he, the designer, must do to preclude or alleviate them. We have purposely pointed out some poor practices in current design in addition to our recommendations in the hope that such practices will go no further. To give "do's" without "don'ts" may encourage some designers to offer a poor design because he mistakenly believes that "it worked before."

Those who have contributed to the handbook number literally in the hundreds and include those we consulted with and those who have given of their time in reviewing drafts or have supplied specific bits and pieces of information. We take this opportunity to thank the many friends we have made in the course of this project, particularly for their candidness in discussing problems and ways of solving those problems, and for their help in supplying photographs and information. In particular we want to thank Mr. Humphrey Gilbert and I. Craig Roberts of the USAEC for their guidance, W. B. Cottrell of ORNL for his help in getting the book published, T. F. Davis of the USAEC's Division of Technical Information for his assistance in indexing the material, J. H. Waggoner of ORNL for doing the illustrations, and Dr. M. W. First of Harvard University for his meticulous page-by-page review of the draft and suggestions for this final issue.

C. A. Burchsted

A. B. Fuller

Oak Ridge, Tennessee July 10, 1969 


\section{Preface}

This handbook is another step in the continuing effort of the Energy Research and Development Administration to ensure the safe operation of nuclear facilities. Gaseous effluents from these facilities are among the more difficult to control, and the AEC, now ERDA, has long carried on an intensive program aimed at their effective control. The record of this program is available in the proceedings of the biennial AEC Air Cleaning Conferences, the first of which was held in 1952 and the fourteenth to be held this year in Idaho. These proceedings and numerous technical reports issued on this topic describe research, development, and experience in specific facilities. In most cases they do not provide general or coordinated guidance for the designer.

The purpose of this handbook is to draw on the wealth of background data available, to digest and evaluate it, and to provide guidance to the engineer and technologist in the design of future facilities. The book is an update of the earlier ORNL/NSIC-65, issued through the Nuclear Safety Information Center at Oak Ridge National Laboratory, and has been prepared under the direction of the ERDA Division of Nuclear Fuel Cycle and Production. The previous edition has received worldwide recognition as the authoritative text in the field of nuclear air cleaning system design. We believe that publication of this new edition by ERDA is a significant contribution to the technical literature.

Frank P. Baranowski, Director Division of Nuclear Fuel Cycle and Production, Energy

Research and Development Administration 


\section{Contents}

FOREWORD TO SECOND EDITION ............................................................................... iii

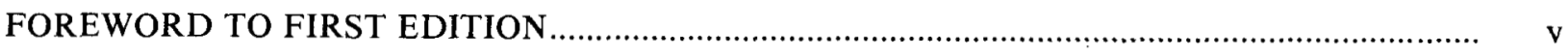

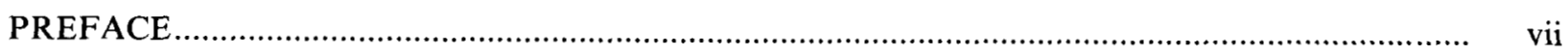

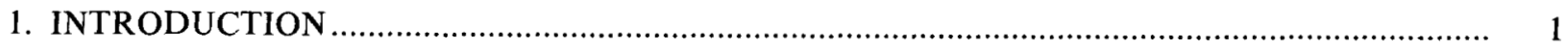

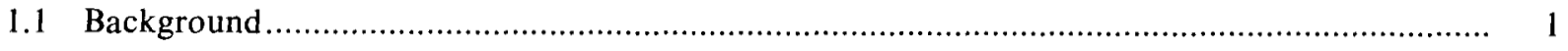

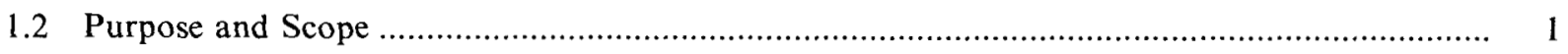

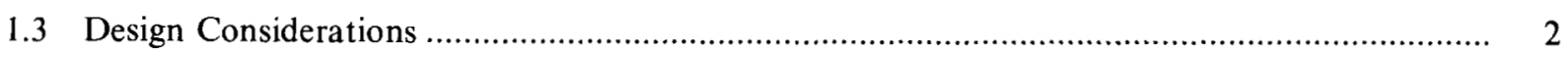

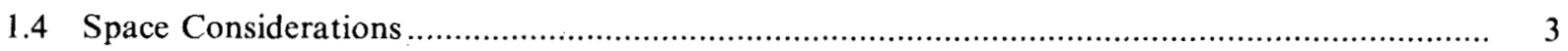

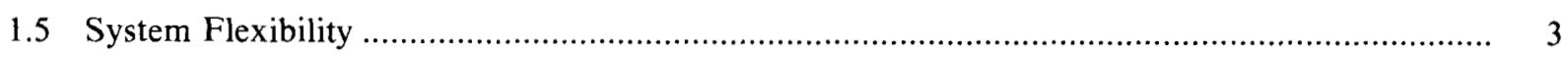

1.6 Coordination of Design and Construction..................................................................... 3

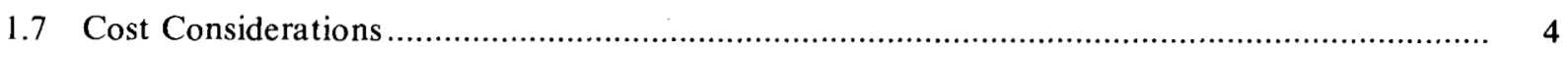

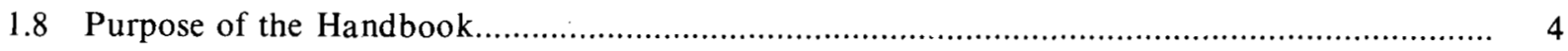

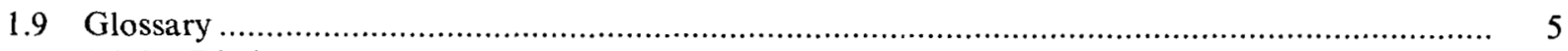

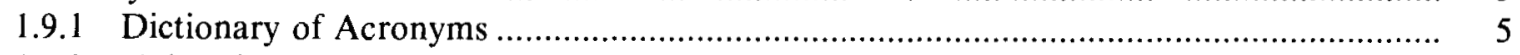

1.9.2 Units of Measure and Metric Equivalents Used in This Handbook .................................. 6

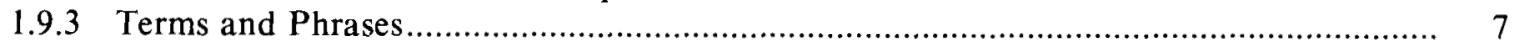

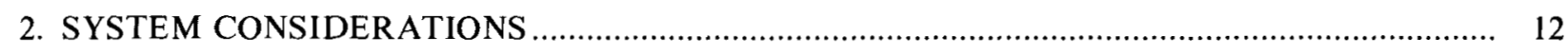

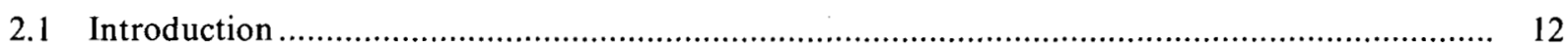

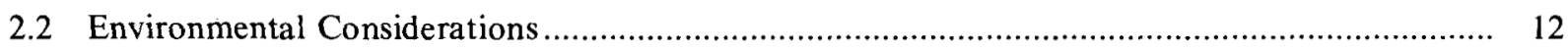

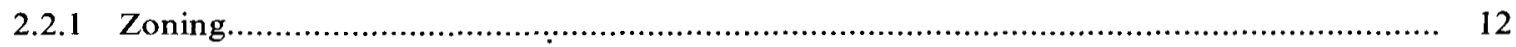

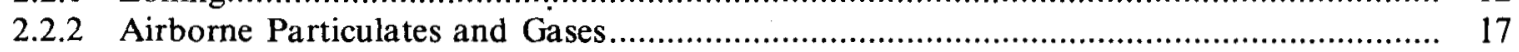

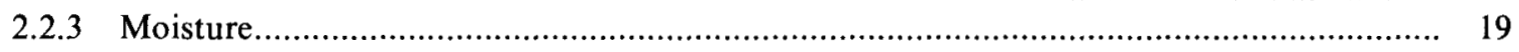

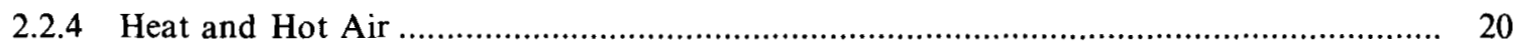

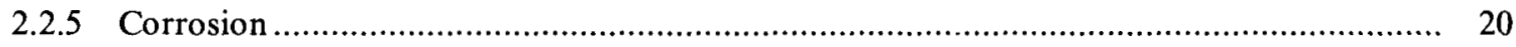

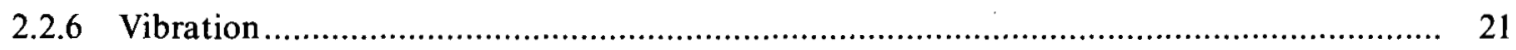

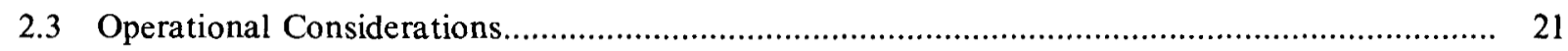

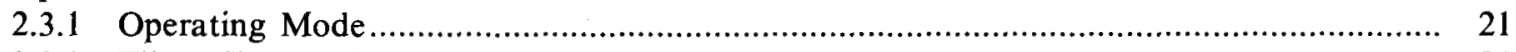

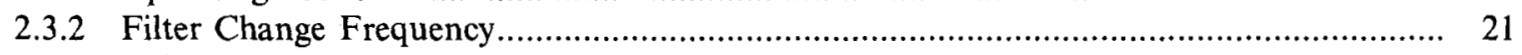

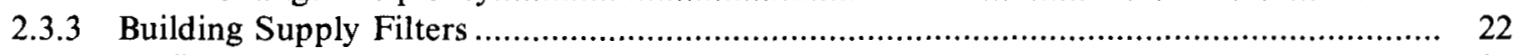

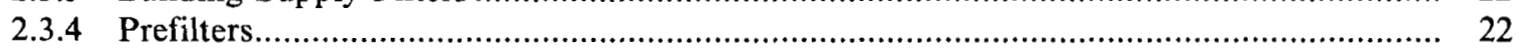

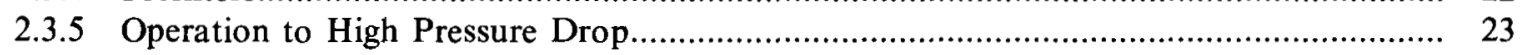

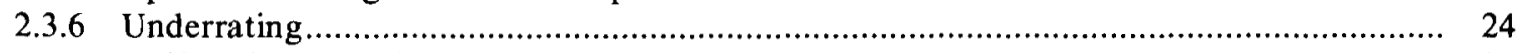

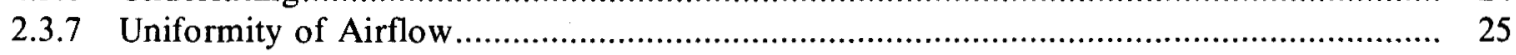

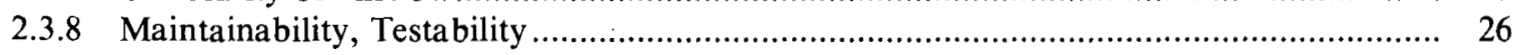




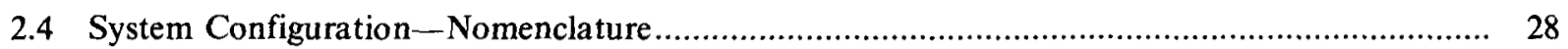

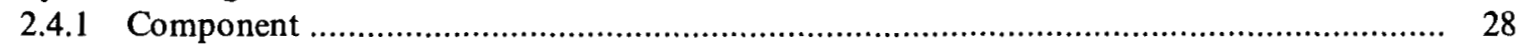

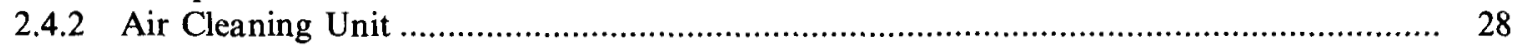

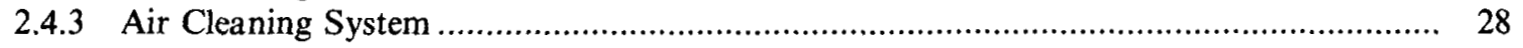

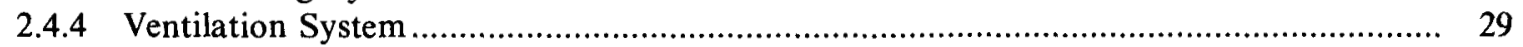

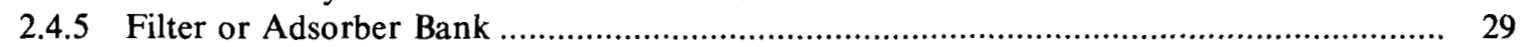

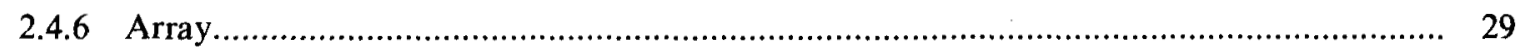

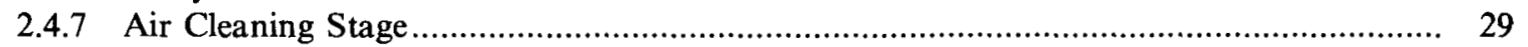

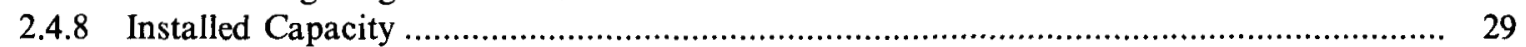

2.4.9 Single-Component Air Cleaning Unit ….................................................................. 29

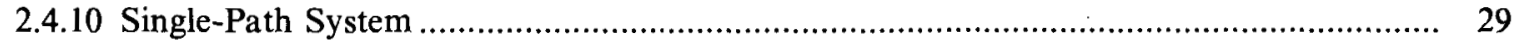

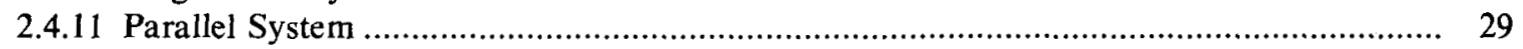

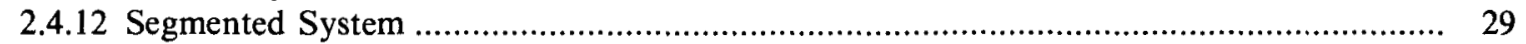

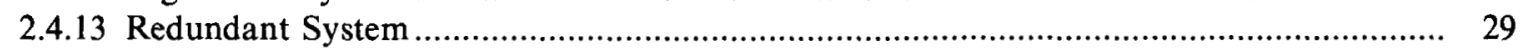

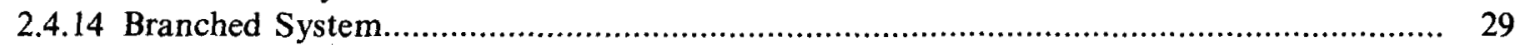

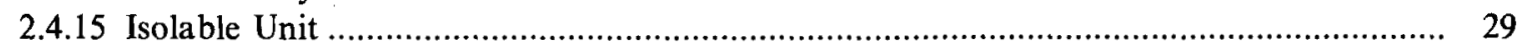

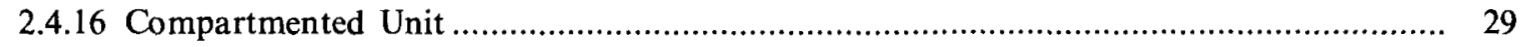

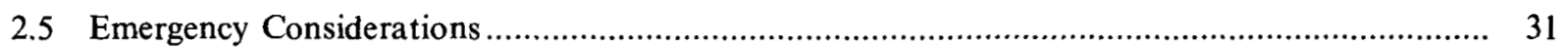

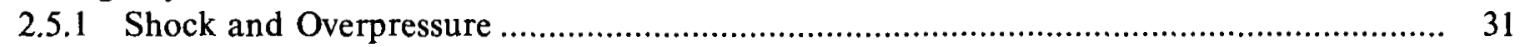

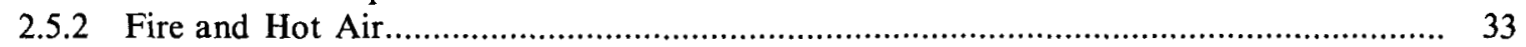

2.5.3 Power and Equipment Outage …............................................................................. 34

2.5.4 Air Cleaning System Layout Considerations .............................................................. 34

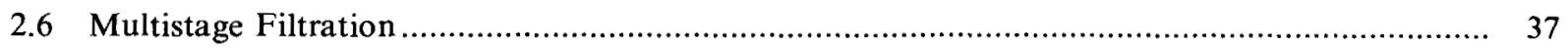

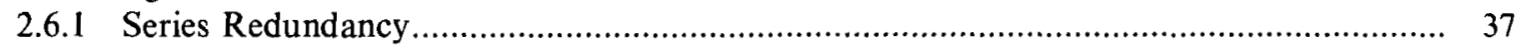

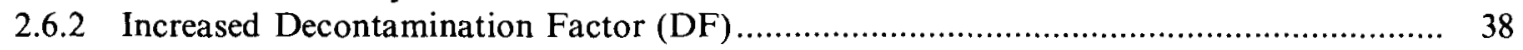

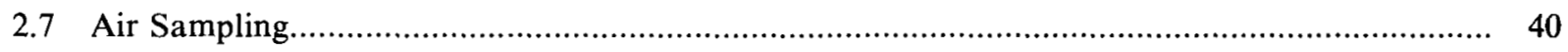

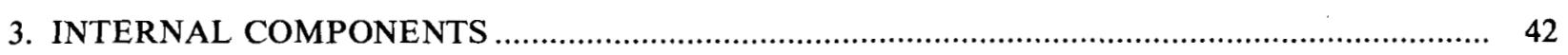

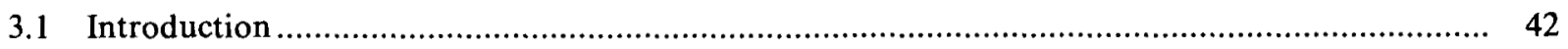

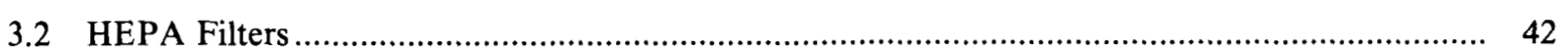

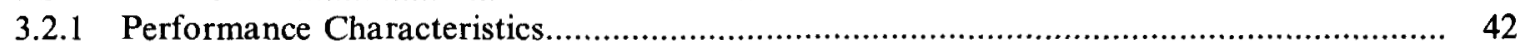

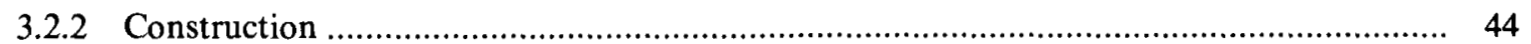

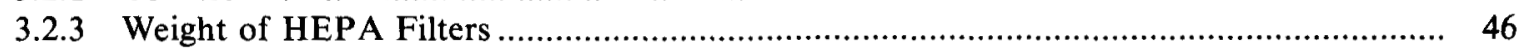

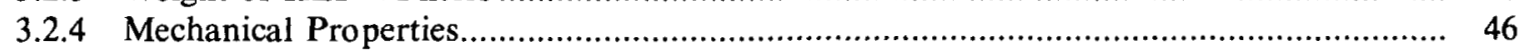

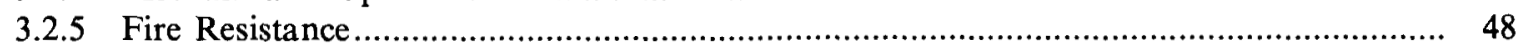

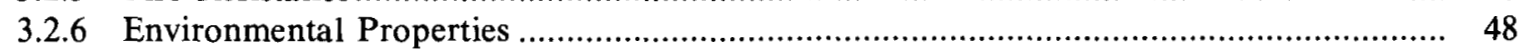

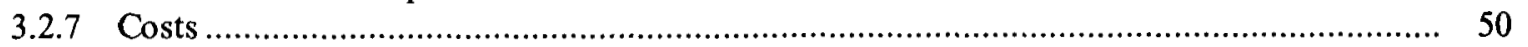

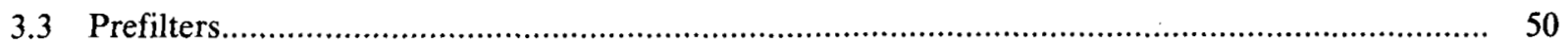

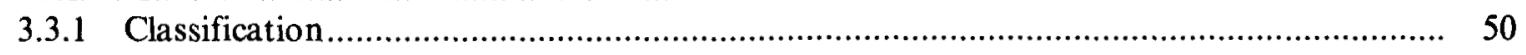

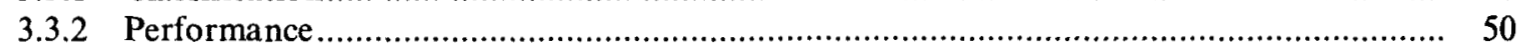

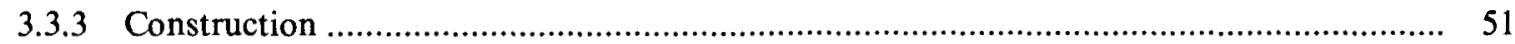

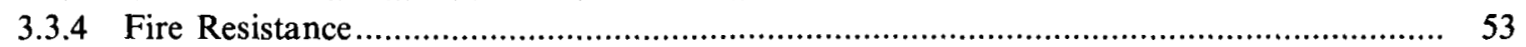

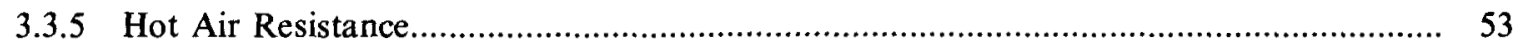

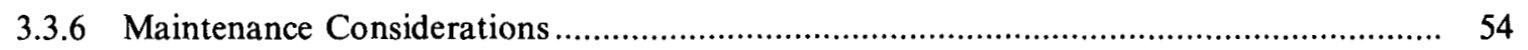

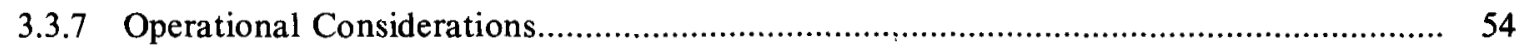

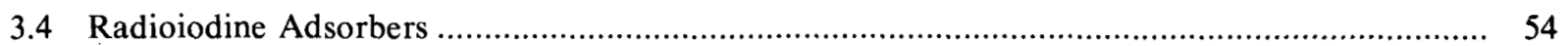

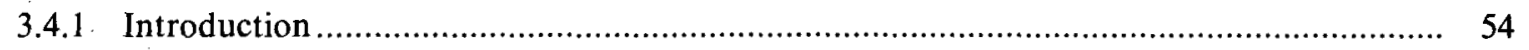

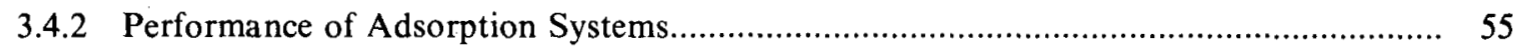

3.4.3 Adsorber Unit Design and Construction ................................................................. 58

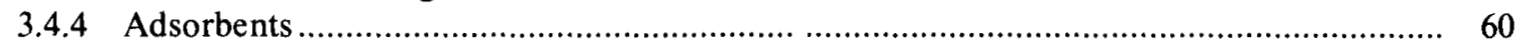


3.4.5 Inorganic Adsorbents.

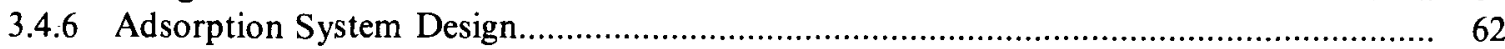

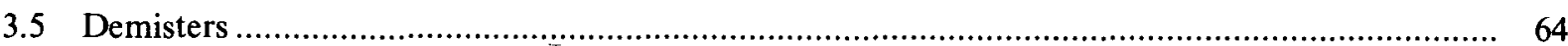

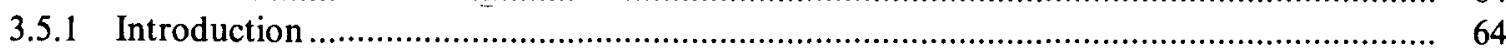

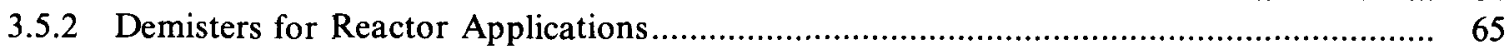

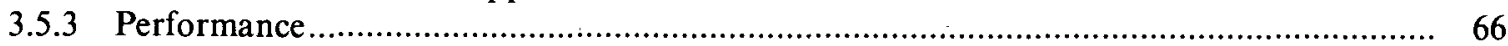

3.5.4 Normal Off-Gas Demisters for Radiochemical Service .............................................. 69

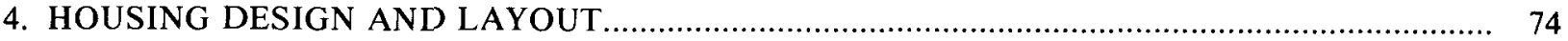

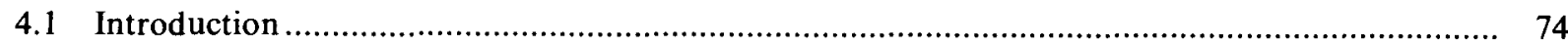

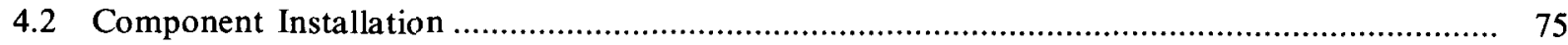

4.3 HEPA Filter, Adsorber Cell, and Demister Mounting Frames ............................................... 77

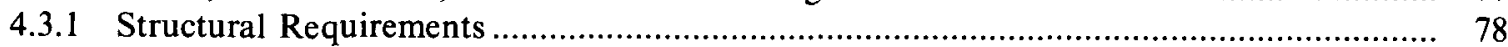

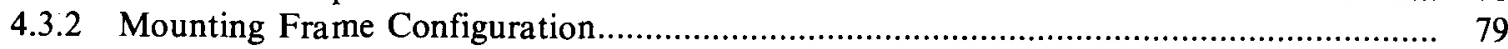

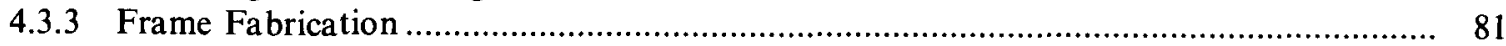

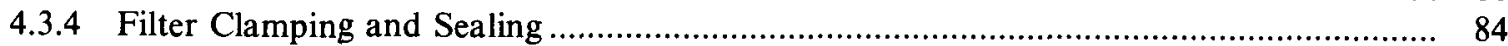

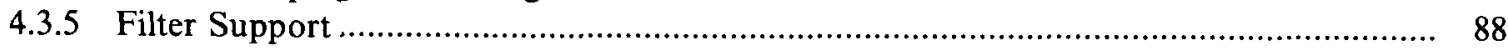

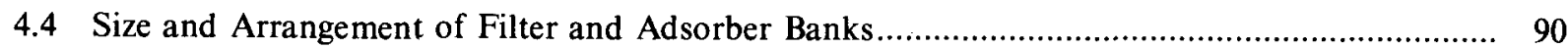

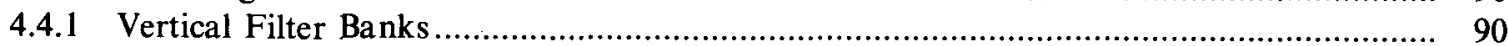

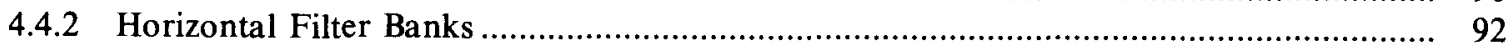

4.4.3 Location of Filters on Mounting Frame.................................................................. 92

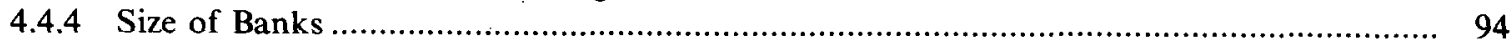

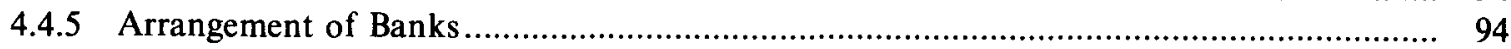

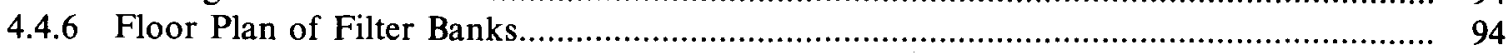

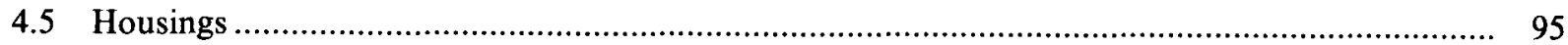

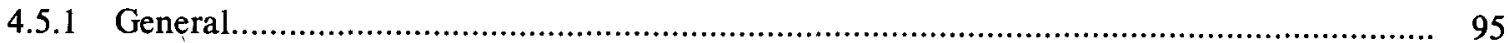

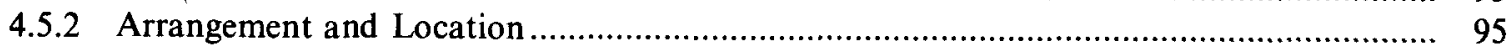

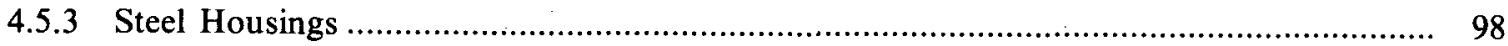

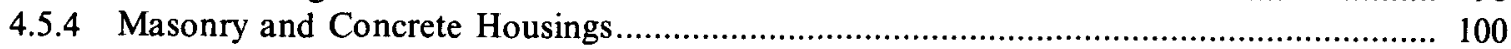

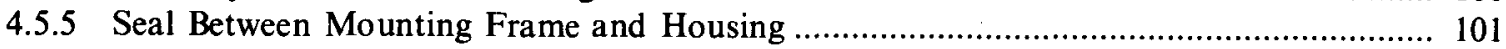

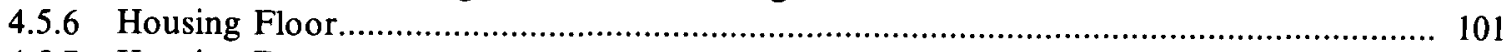

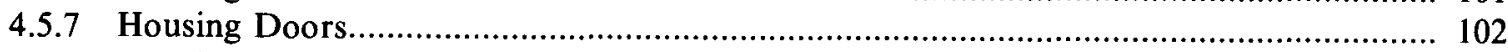

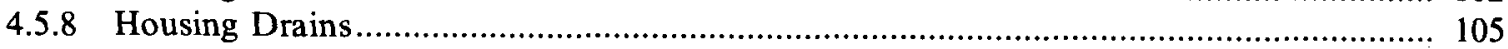

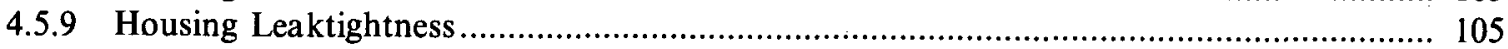

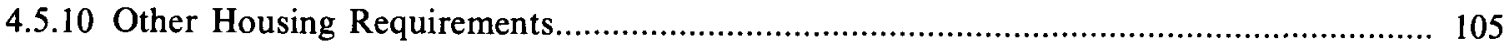

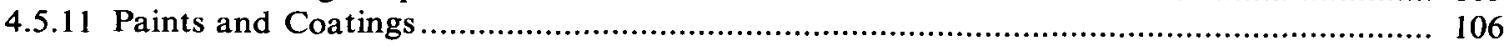

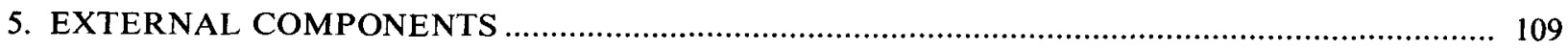

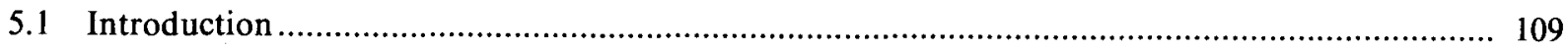

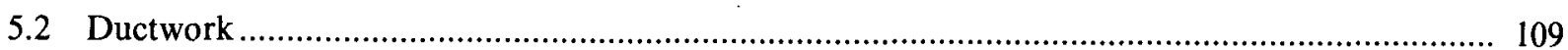

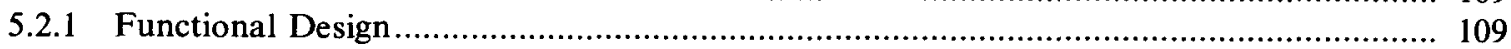

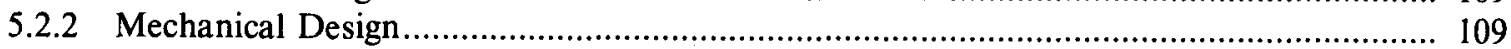

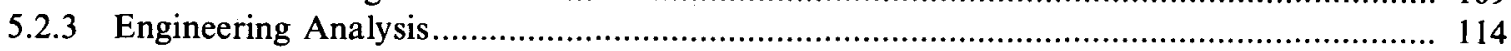

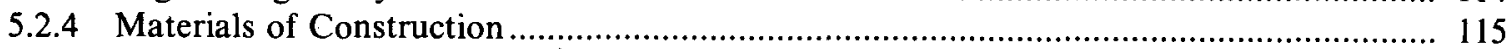

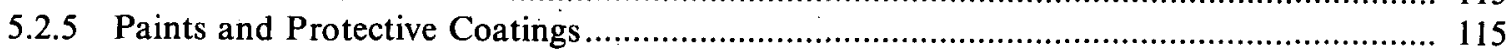

5.2.6 Hangers, Supports, and Anchors

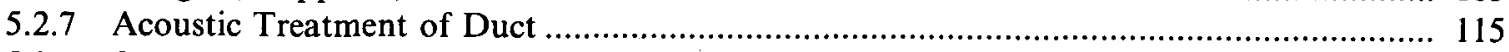

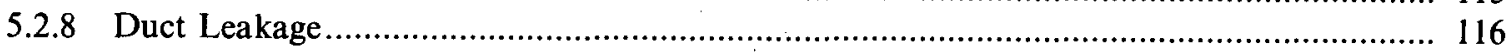




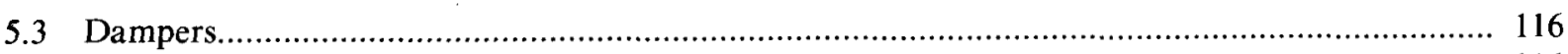

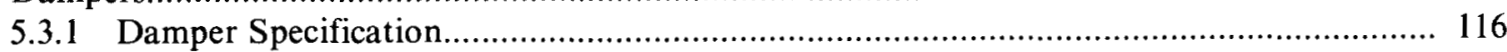

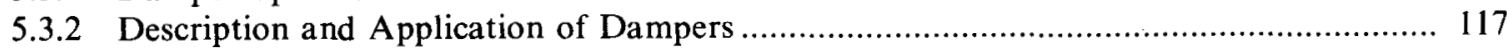

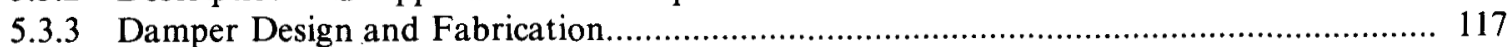

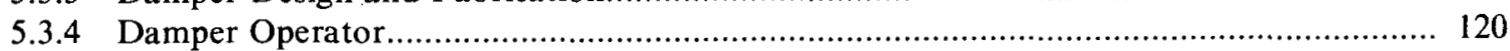

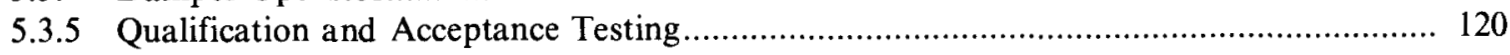

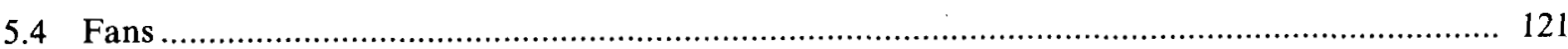

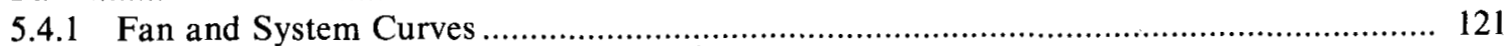

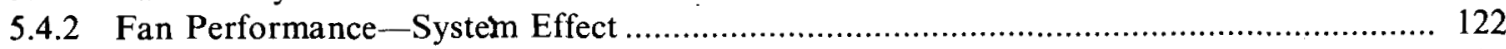

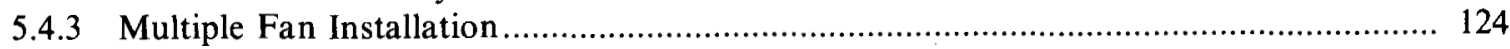

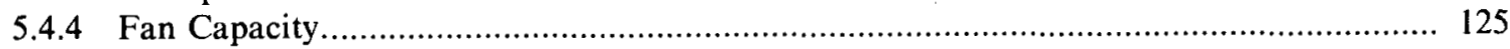

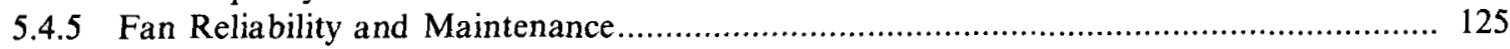

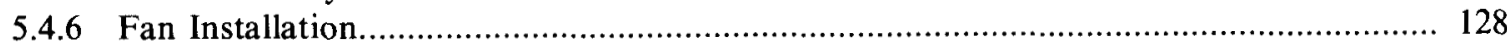

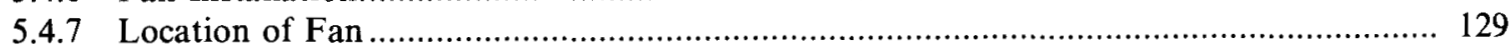

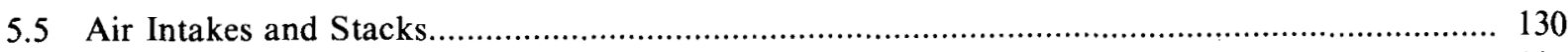

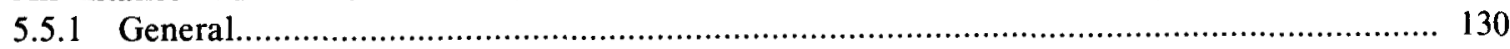

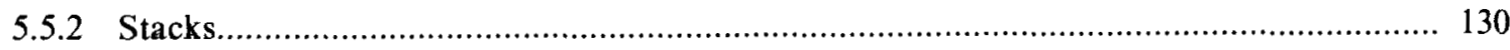

5.6 Ventilation System Control and Instrumentation .........................................................

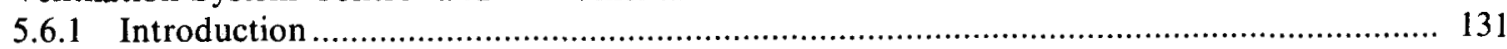

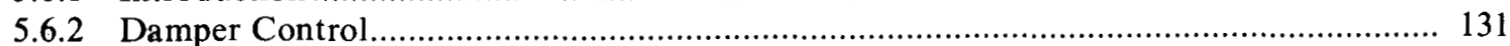

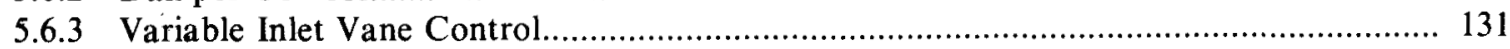

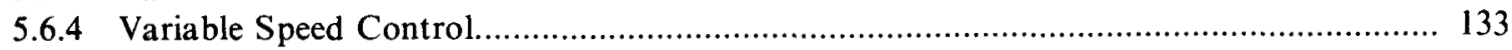

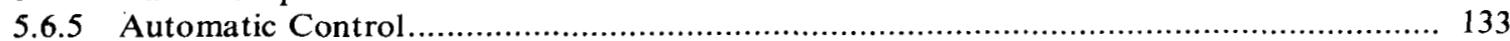

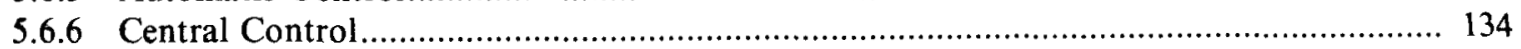

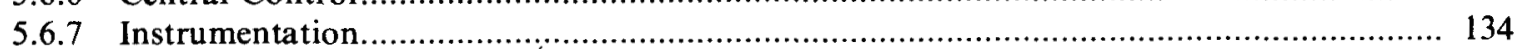

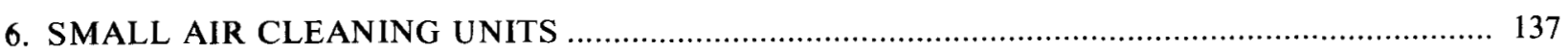

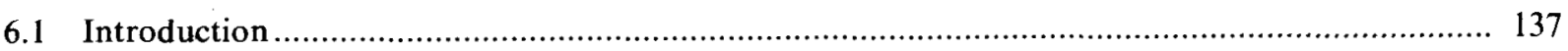

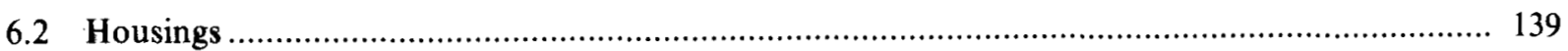

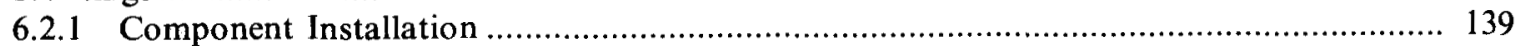

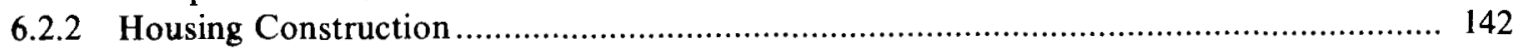

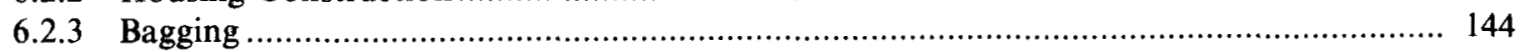

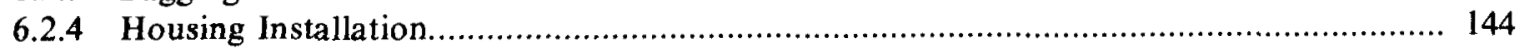

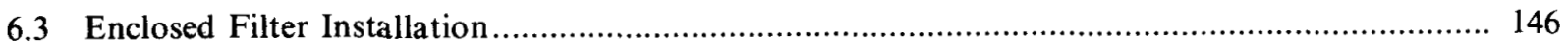

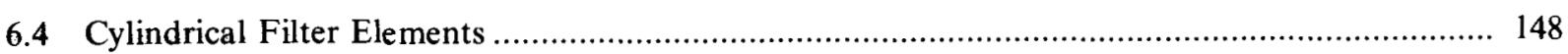

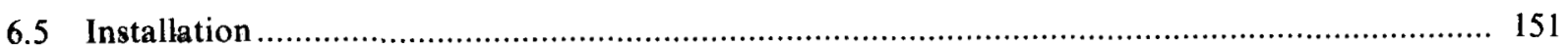

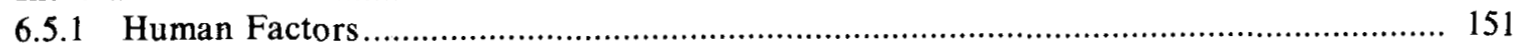

6.5.2 Fume Hood Filter Installations ........................................................................... 151

6.5.3 Emergency and Portable Air Cleaning Units ........................................................ 154

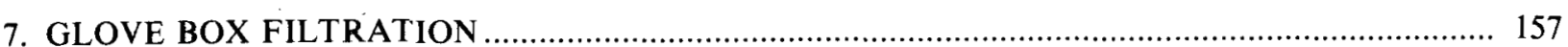

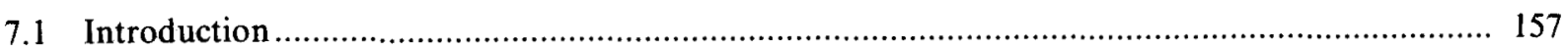

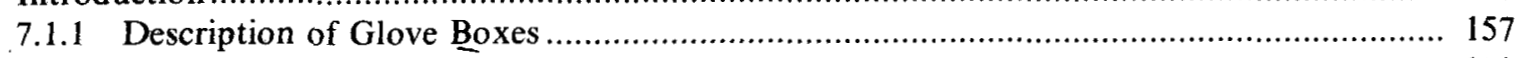

7.1.2 Importance of Glove Box Ventilation and Filtration................................................ 159

7.2 Design of Glove Box Ventilation Systems .................................................................. 159

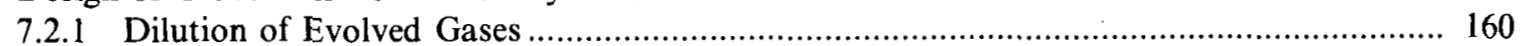

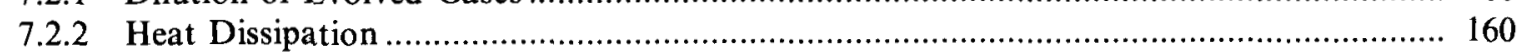

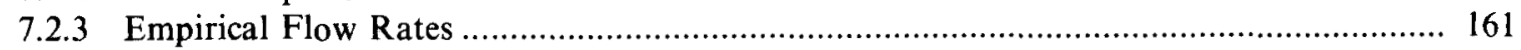

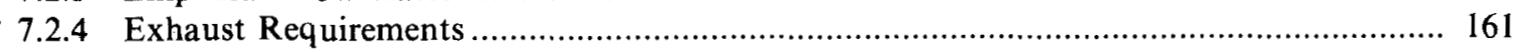




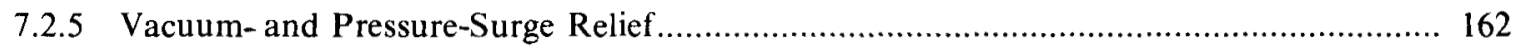

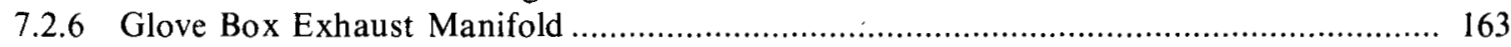

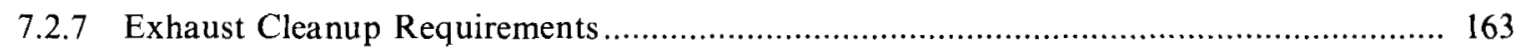

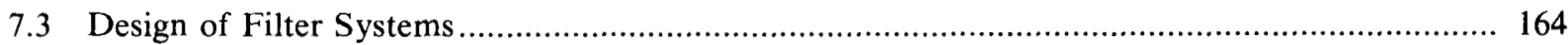

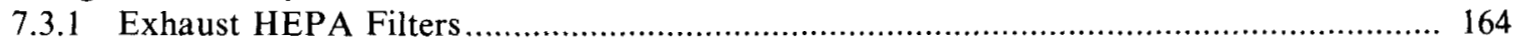

7.3.2 Exhaust Filter Located Inside the Glove Box....................................................... 166

7.3.3 Exhaust Filter Located Outside the Glove Box .................................................... 168

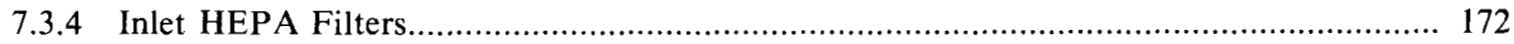

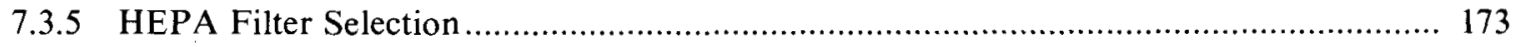

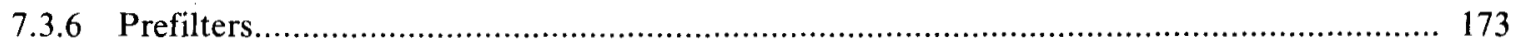

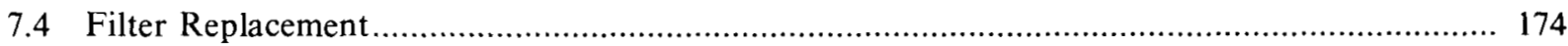

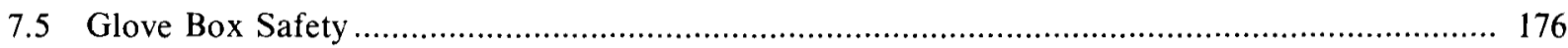

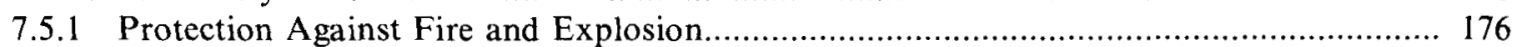

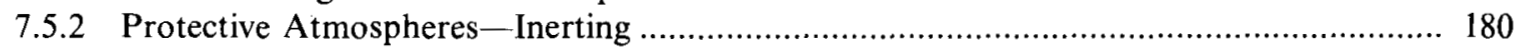

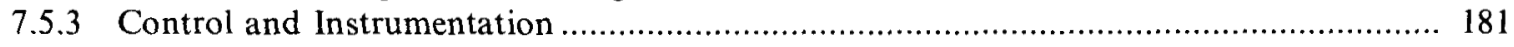

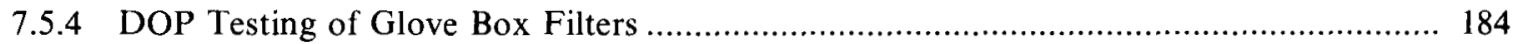

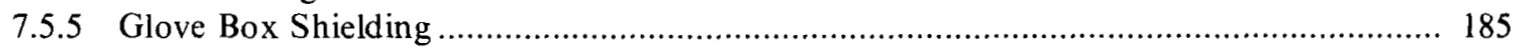

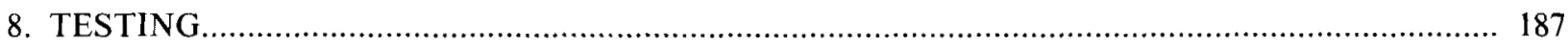

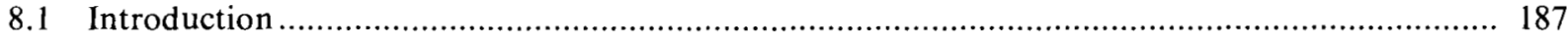

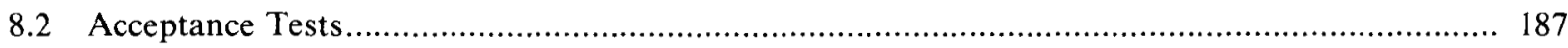

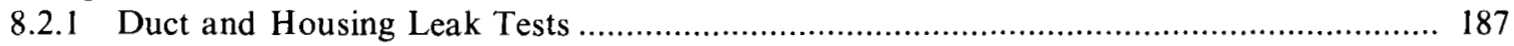

8.2.2 Mounting Frame Leak Tests............................................................................. 188

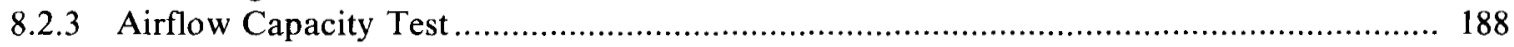

8.2.4 Airflow Distribution Test-Adsorber Residence Time ............................................ 188

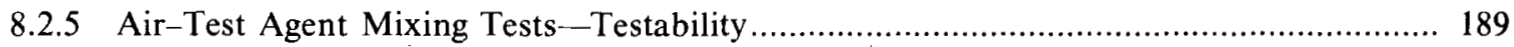

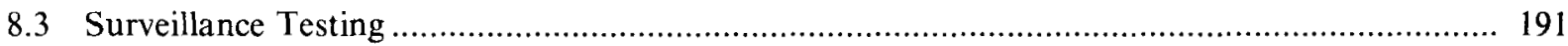

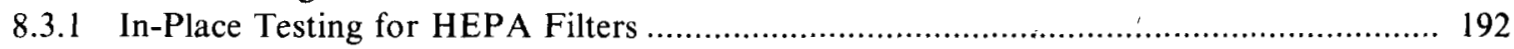

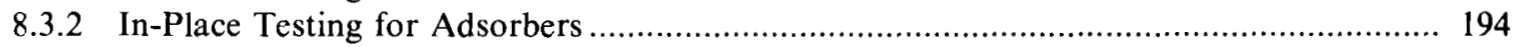

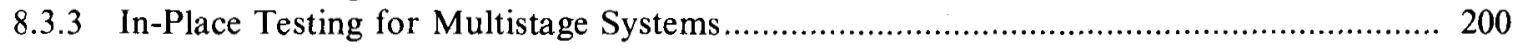

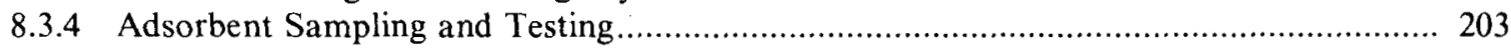

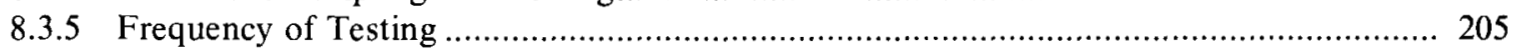

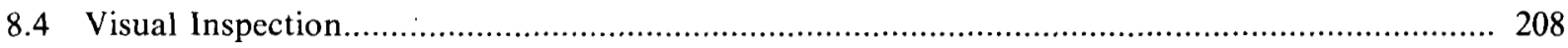

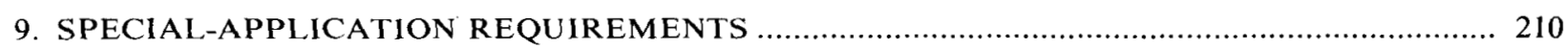

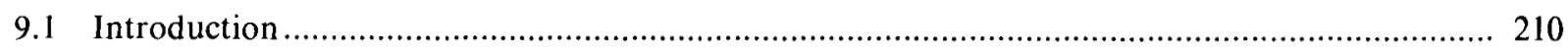

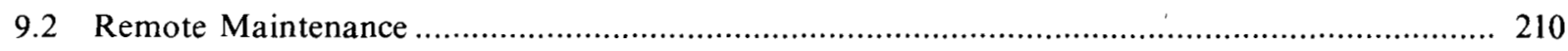

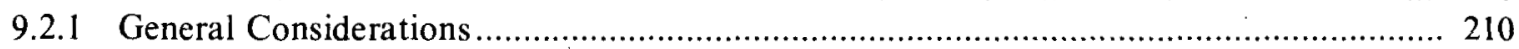

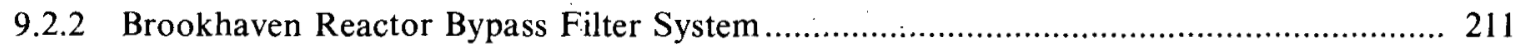

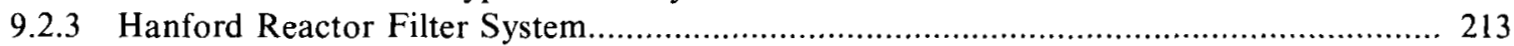

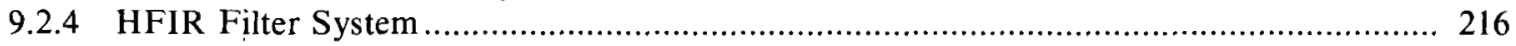

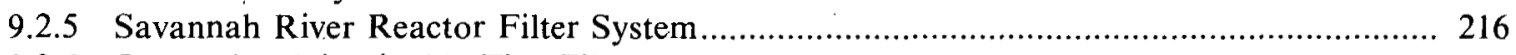

9.2.6 Remotely Maintainable Fish Filter System................................................... 218

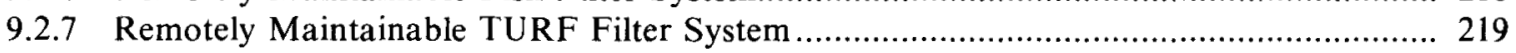

9.2.8 Remotely Maintainable HWESF Filter Assembly ............................................... 220

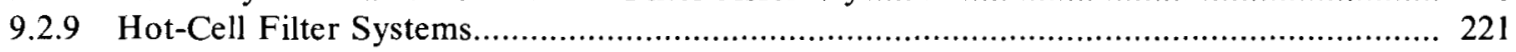

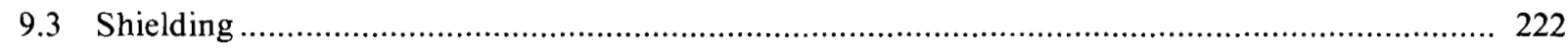




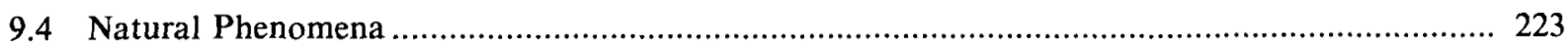

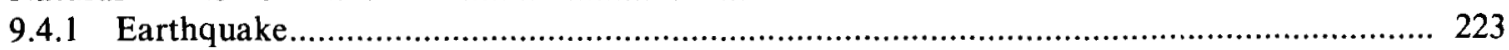

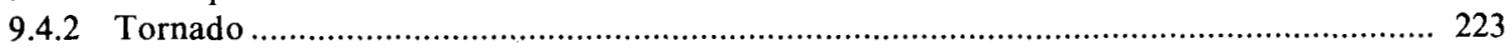

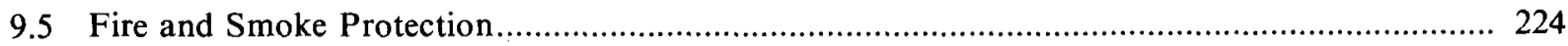

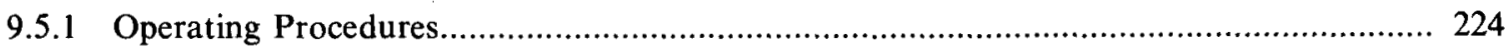

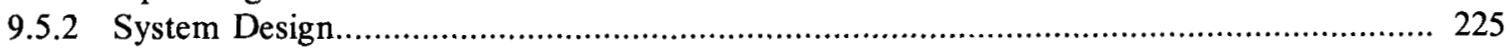

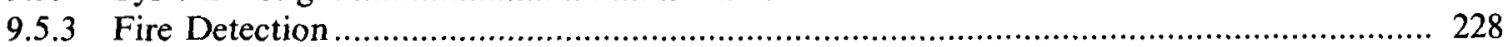

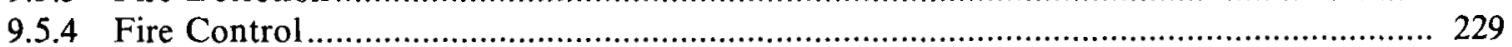

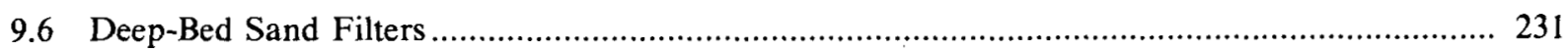

9.6.1 Design of Deep-Bed Sand Filters........................................................... 232

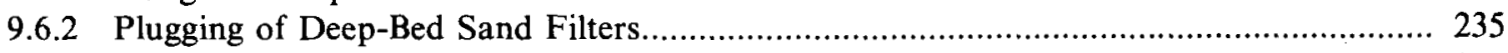

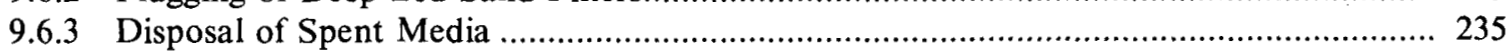

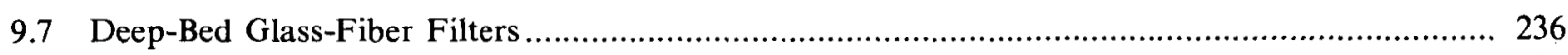

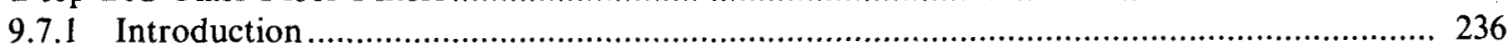

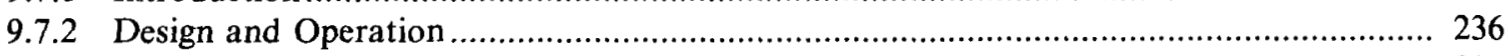

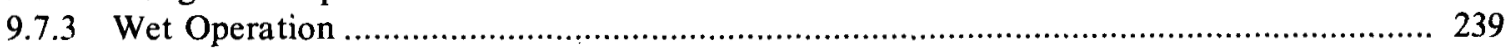

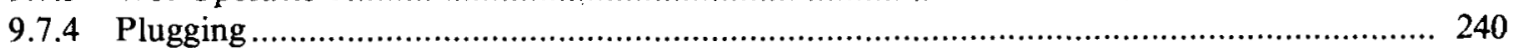

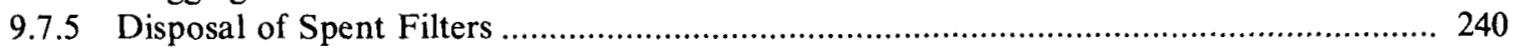

9.8 Reactor Engineered-Safety-Feature Air Cleaning Systems ....................................... 240

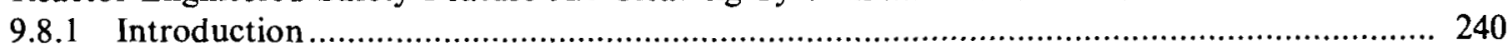

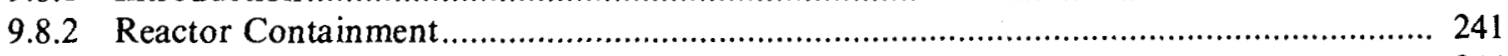

9.8.3 Light Water Reactors......................................................................... 244

9.8.4 High-Temperature Gas-Cooled Reactors ................................................... 245

9.8.5 Liquid-Metal Fast-Breeder Reactors......................................................... 245

9.8.6 Control Room Protection Air Cleaning Systems............................................... 246

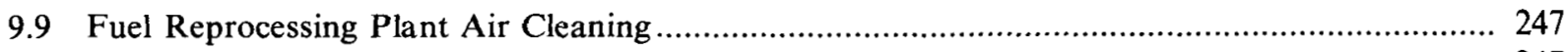

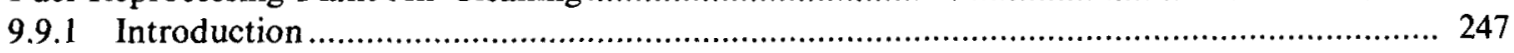

9.9.2 Light Water Reactor Spent Fuel Reprocessing ................................................... 248

9.9.3 LMFBR Spent Fuel Reprocessing ............................................................. 248

9.9.4 Near-Zero Release Concept .................................................................... 249

9.9.5 HTGR Spent Fuel Reprocessing Air Cleaning Systems .................................... 250

9.9.6 Air Cleaning System Costs, Fuel Reprocessing ........................................... 250

APPENDIX A Sample Air Cleaning Equipment Specifications........................................... 254

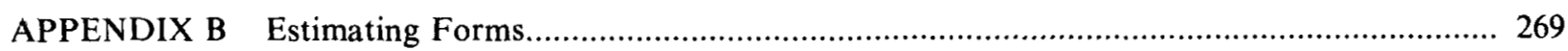

APPENDIX $C$ Care and Handling of HEPA Filters ...................................................... 271

APPENDIX D Seismic Design and Qualification of ESF Air Cleaning Systems.......................... 275

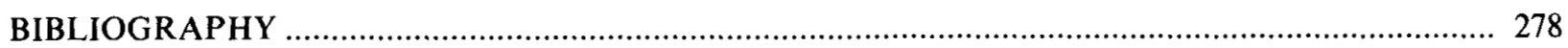

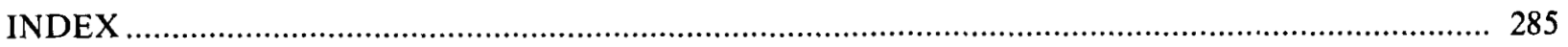




\section{Introduction}

\subsection{BACKGROUND}

A nuclear air cleaning system is provided to protect the public and plant operating personnel from airborne radioactive particles and gases which are, or could be, generated or released from operations conducted in a nuclear reactor, fuel fabrication or processing plant, radiochemical operation, laboratory, or other nuclear operation. Such a system is characterized by operation at very high contaminant collection levels, generally orders of magnitude greater than those exhibited by air cleaning systems employed in commercial, industrial, or pollution control applications. The component almost universally included in such systems is the high-efficiency particulate air (HEPA) filter. This type of filter may be supplemented by common air filters, bag filters, cyclones, scrubbers, or other devices used in more conventional applications but is nearly always employed in the nuclear air or gas cleaning system as the final barrier between a contained space (in which radioactive particulates could be generated) and the point of release to the atmosphere (i.e., the stack) or to an environmentally controlled space of the facility.

The prevention of even extremely low concentrations of airborne contamination is fundamental to the safe operation of a nuclear facility. ${ }^{1}$ It is also an important factor in the economic operation of such facilities. Although protection of the health and safety of the public and of plant personnel is the primary consideration, the high costs of decontamination and the possibility of shutdown of the facility in the event of an accidental airborne release of radioactive material are also important considerations.

Radioactive substances tend to deposit or "plate out" on ducts, components, and other exposed surfaces and, in time, become sources of persistent ionizing radiation. This deposition can severely complicate maintenance and operation of a facility unless eliminated close to the source. These problems are of particular concern in power reactors and fuel reprocessing facilities because of their potential for releasing large amounts of radioactive material in the event of a system malfunction or upset.

\subsection{PURPOSE AND SCOPE}

Much of the information pertinent to the design, construction, and testing of very-high-efficiency air and gas cleaning systems for nuclear applications is contained in limited-distribution topical reports, technical papers, and job specifications that are often not readily available to designers. Although there is a growing body of standards relating to the subject, the background information necessary for their effective interpretation is scattered. The purposes of this handbook are to summarize available information in a manner that is useful to the designer, to point out shortcomings in design and construction practice, and to provide guides and recommendations for the design of future systems. The handbook summarizes findings from the literature and air cleaning practices at laboratories, production facilities, power and research reactors, and radiochemical and fuel reprocessing facilities. The judgments and recommendations presented reflect the experience of users and conditions that exist in operating systems where airborne radioactive material is being successfully controlled on a day-to-day basis, often in situations where personnel have had to live with, or adapt to, serious deficiencies in design or construction.

This handbook is limited to the mechanical or hardware phase of design. Functional design-the sizing of a system or selection of components to meet the needs of a specific application-is beyond its scope. The design of ventilation systems, of which the air cleaning systems are a part, is also beyond the scope of the handbook except as the ventilation 
system design affects the operation and reliability of the air cleaning facilities, or, conversely, as the air cleaning facilities affect the operation and reliability of the ventilation system. The functional design of nuclear air cleaning systems is covered in Safety Monograph No. 17 of the International Atomic Energy Agency (IAEA), ${ }^{2}$ in various Regulatory Guides of the Nuclear Regulatory Commission (NRC), ${ }^{3-5}$ and in the ERDA Manual. ${ }^{6}$ The functional design of ventilating systems is covered in Industrial Ventilation,' the ASHRAE handbooks, ${ }^{8}$ ANSI Z9.2, ${ }^{y}$ and numerous textbooks. The handbook does not cover the theories of air filtration or gas adsorption; however, discussions of air filtration theory can be found in White's and Smith's High Efficiency Air Filtration ${ }^{10}$ and Davies' Air Filtration; ${ }^{11}$ gas adsorption theory of interest to the nuclear industry is covered best in the proceedings of the biennial AEC (now ERDA) Air Cleaning Conferences.

\subsection{DESIGN CONSIDERATIONS}

The design of nuclear air cleaning systems is complicated by the extremely high collection efficiencies required to meet the maximum permissible concentration (MPC) values that have been established for radioactive substances in air. ${ }^{1}$ In many conventional situations (i.e., commercial, industrial, and air pollution control), dust, chemical fumes, and other contaminants can be detected by the human senses before they reach concentrations that pose a serious immediate threat to health or safety. The situation is quite different in nuclear systems because of the complete insensitivity of man to the presence of radioactivity, even at levels that represent an immediate danger to life, and because of possible longterm effects of exposures even at low levels. The lowest threshold limit values (TLV) ${ }^{12}$ specified for most chemical contaminants in air are at least two orders of magnitude higher than the MPC of any radioactive material.

The common air filters used in conventional air cleaning applications are unable to decontaminate air to the levels required to meet these MPCs. Even the best of such filters exhibit number decontamination factors (DF) no greater than 6 to 7 for submicron particles (i.e., those having an aerodynamic diameter of less than $1 \mu \mathrm{m}$ ), and the DF of most filters is 2 or less for particles in this range. ${ }^{1,14}$ To meet the requisite MPCs for contaminants present as, or adsorbed on, particulate matter, the HEPA filter must be used. By definition, ${ }^{15}$ this type of filter must have a minimum number efficiency ${ }^{16}$ of $99.97 \%$ for $0.3-\mu \mathrm{m}$ particles; that is, a number decontamination factor of at least 3333 for all measurable particles, at any concentration, down to at least a $0.3-\mu \mathrm{m}$ aerodynamic diameter. Similarly, the iodine adsorption units used in nuclear air and gas cleaning service must also exhibit collection (i.e., decontamination) efficiencies substantially greater than adsorption units used in fume and odor control and most toxic or noxious gas control applications. For these components to function at their required performance levels, the manner in which they are installed, the connecting ductwork, and the ancillary components required to complete the air cleaning function must all meet standards of design and installation substantially higher than those which prevail in most nonnuclear situations. That such high standards can be met routinely and on a continuing basis is evidenced by the superlative safety record of most nuclear installations and by the control of releases to the atmosphere even under severe upset conditions.

If airborne radioactive material is released from the system, there is the possibility of seriously contaminating occupied spaces of the plant, as occurred in the St. Laurent fuel meltdown incident in France, or of contaminating the surrounding countryside, as occurred in the Windscale reactor incident in England several years ago. Even a minor incident, in terms of the actual weight or volume of radioactive material released, could shut down a costly facility for an extended period of time. The costs of decontamination can be thousands of times the losses due to such ordinary hazards as fire, explosions, or chemical spills, as illustrated below by the loss experience due to a small glove box accident at an ERDA laboratory. ${ }^{17}$

$\begin{array}{lr}\text { Casualty loss due to fire } & \$ 100 \\ \text { Casualty loss due to explosion } & \$ 500 \\ \text { Cost of cleanup and decontamination } & \$ 76,200\end{array}$

In addition, the deposition and "plate out" of radioactive particulate matter and gases in and on ductwork, housings (i.e., equipment casings), filters, and other air cleaning system components limits access, obstructs maintenance, and increases the cost of operation. The designer must appreciate these substantial differences between nuclear and conventionaletair cleaning systems. Concentrations of radiotoxic materials in the air cannot be maintained below statutory limits ${ }^{1}$ if the design or layout of the system, or selection or installation of components, is 
deficient. Some operations in the past have relied to some extent on dilution of airborne radioactive wastes with large volumes of air, followed by dispersal in the atmosphere. This practice is no longer acceptable in view of recent "as low as reasonably achievable" (ALARA) regulations, and heavy emphasis must be placed on positive removal of radioactive particulates, fumes, and gases by means of well-designed and-maintained filtration and adsorption systems.

\subsection{SPACE CONSIDERATIONS}

The location and space allocations for exhaust and air cleanup systems must receive close attention beginning with the early stages of building planning and layout and continuing through construction of the facility. Failure to provide adequate space for ductwork in early building layouts often results in the inability to achieve good aerodynamic design, in excessive velocity and pressure losses that can compromise system operation, and in dynamic conditions that can cause outleakage of contamination, even in ductwork that operates under negative pressure. Poor location of filter housings, fans, and dampers may limit their accessibility and thereby decrease ability to maintain the system. Also, since filters are collectors of radioactive (or potentially radioactive) dust, they can contain substantially greater concentrations of radioactive material than the air of the contained space served by the system. Changing filters in open attics or crowded spaces of the building increases risks at a time when risk is already higher than normal. Adequate access to and space surrounding housings and filter installations decreases this risk. Space allotted for access to housings and equipment must not be encroached upon forstorage, field shops, or other operational conveniences during the life of the facility.

\subsection{SYSTEM FLEXIBILITY}

A shortcoming often encountered in ventilation and air cleaning system designs is failure to anticipate the possibility of future system modification. Although lack of ventilation system flexibility may create no problems in nuclear reactors and other facilities that have a fixed function, in radiochemical operations and particularly in laboratories and experimental facilities where change is almost standard procedure, provision for future system modification at the time of original system design can pay for itself many times over. The rebuilding of radioactively contaminated ducts and air cleaning systems is costly and hazardous, at best, and can be even more costly and hazardous when some provision for flexibility has not been left in the original design. Because of the radioactivity problem, the costs of modifying or rebuilding a nuclear plant exhaust or air cleanup system may run five to ten times the cost of similar work carried out in a nonradioactive system. Provision for expansion of a system, including extra housing space, reserve fan and motor capacity, additional tie-on points, and sufficient mechanical joints in ductwork to permit reasonably easy dismantling, should be given serious consideration in initial planning.

Temporary systems may not justify the extra capital investment for providing flexibility. Nevertheless, the designer should keep in mind that temporary systems often become permanent or are adapted for other purposes. Short cuts in design that make the modification of even a temporary system difficult can often become very costly to the owner in the long run.

\subsection{COORDINATION OF DESIGN AND CONSTRUCTION}

The mechanical contractor cannot be expected to supply more than the minimum requirements shown in the drawings and specifications. He cannot be expected to build a system having the special features and requirements of a nuclear air cleaning system unless the design details and specifications clearly define them. It is the functional designer's responsibility to correctly interpret the owner's needs and to develop clear and accurate system criteria. It is the mechanical designer's responsibility, in turn, to interpret these criteria and translate the functional design requirements into detailed equipment and construction drawings and specifications that can be followed by workmen with no experience in this specialty. It is also the mechanical designer's responsibility to ensure that the system, as it will be built, will meet the owner's needs in terms of a safe, effective, reliable, maintainable, and economic system.

An example of poor design and construction coordination occurred in a power reactor containment purge system. The facility designer, when allocating space for ventilation and air cleaning 
equipment, allowed a nominal 144-in. width for a sixwide bank of $24-$ by 24 -in. HEPA filters. No verification of this dimension was made by the mechanical designer, and the drawings went to the constructor who proceeded to pour concrete. The difficulty that developed is that a six-wide bank of 24by 24-in. filters should be installed in a housing at least $151 \mathrm{in}$. wide (and preferably $158 \mathrm{in}$. wide) to provide room for a reliable filter mounting frame and to provide ease of filter changing. This type of error should have been recognized in the mechanical design stage. To make matters worse, no embedments were provided in the concrete to which the filter mounting frame could be seal-welded. This error resulted in a filter installation that is, at best, questionable.

It is also important for contractual relationships to be carefully defined and enforced. If the constructor is to be responsible for correct performance of the installed system, then test procedures, identification of the parties who will make and evaluate test results, and requirements for remedying errors and deficiencies must all be specified in the contract documents. It is not enough merely to require that the system meet some minimum dioctyl phthalate (DOP) test efficiency (usually $99.95 \%$ ). If the system is to meet its intended service requirements, technical requirements must be carefully followed during preparation, review, and contractual acceptance of drawings and specifications, as well as during performance of the work in the field.

\subsection{COST CONSIDERATIONS}

Shortcuts and compromises with good design practice result in unduly high operating costs throughout the life of the system, as well as reduced system reliability and performance. A common error in the planning and design of nuclear air cleaning systems is to place too much emphasis on first (i.e., capital) costs. Minimizing first costs often results in high operating and maintenance costs if desirable optional features are omitted or if sacrifices are made in the amount or quality of space provided for components, equipment, and ductwork. During the life of the system, operating and service costs usually far exceed the first cost of building the system. A survey by the Harvard Air Cleaning Laboratory showed that operation and maintenance accounted for more than $85 \%$ of the total cost of owning a nuclear air cleaning system, based on 20-year amortization. ${ }^{18}$
Errors are sometimes made in choosing between alternate methods of accomplishing a desired objective because of the failure to consider all aspects of cost. When estimating capital costs of air cleaning systems, for example, the costs of special filter housings, dampers, fire protection facilities, clothingchange facilities, and other unusual (as compared with conventional air cleaning practice) provisions are often overlooked and may result in substantial avoidable maintenance costs for the sake of a few dollars' savings in first costs. For example, higher efficiency prefilters may greatly extend the life of the downstream HEPA filters and perhaps have longer life themselves, thereby increasing the time between filter changes. This is important because replacement of highly contaminated HEPA filters may cost as much as $50 \mathrm{c}$ per $\mathrm{cfm}$ of installed capacity. ${ }^{19}$ Often, when estimating filter replacement costs, only the "do" phase of the operation is considered, with little or no heed given to the "make ready" and "put away" phases; yet these are generally the most time consuming and costly phases of a filter change in a nuclear air cleaning system. The time-consuming activities of clothing change, preinstallation inspections, health physics monitoring, and decontamination of the area and equipment after the change are often overlooked. Other factors overlooked are escalations of labor and materials cost and the ability to extend HEPA filter life by the selection of various combinations of prefilters, bank size, airflow rate, or other system parameters. Appendix B provides a form to assist the designer in estimating capital and operating costs and a form that breaks down a filter (or adsorber) change into at least its major elements.

\subsection{PURPOSE OF THE HANDBOOK}

The information given in this handbook will supplement the designer's previous knowledge and understanding of ventilation and air cleaning system design and construction by supplying background information on components and requirements for these very specialized applications. Hopefully through the use of this handbook, the experienced functional designer will be better able to evaluate an owner's requirements and to establish essential system criteria; the experienced mechanical designer will be better able to translate these criteria into effective system designs; and mechanical contractors will be provided with the knowledge needed to effectively carry out the intent of these designs to 
provide safe, reliable systems at reasonable cost. The previous issue of the handbook ${ }^{20}$ has provided background information for a growing family of national standards covering air and gas cleaning systems for nuclear applications; the new issue will hopefully assist designers and engineers in using and interpreting those standards. It is also hoped that the volume will provide a rationale for the engineer, the manager, and the designer to justify the more costly, but necessary, features that a nuclear air cleaning system demands.

\subsection{GLOSSARY}

\subsubsection{Dictionary of Acronyms and Initialisms}

ACGIH American Conference of Governmental Industrial Hygienists

ACI American Concrete Institute

AEC Atomic Energy Commission (see ERDA, NRC)

AFI Air Filter Institute

AgX Silver-exchanged zeolite

ALAP As low as practicable (obsolete term for ALARA)

AISC American Institute of Steel Construction

ALARA As low as reasonably achievable

AMCA Air Moving and Conditioning Association

AMD Aerodynamic mean diameter (of particles)

ANSI American National Standards Institute

ASHRAE American Society of Heating, Refrigerating and Air-Conditioning Engineers

ASME American Society of Mechanical Engineers

ASTM American Society for Testing and Materials

AWS American Welding Society

BET Brunauer, Emmett, and Teller (test for surface area of adsorbents)

BWR Boiling water reactor

CBR Chemical biological radiological (filter)

CFD Continuous fire detector
$\mathrm{CH}_{3} \mathrm{I} \quad$ Methyl iodide

CFR Code of Federal Regulations

CG Concentration guide

CRSI Concrete Reinforced Steel Institute

CWS Chemical Warfare Service

DBA Design basis accident

DBS Deep-bed sand (filter)

DBGF Deep-bed glass fiber (filter)

DF Decontamination factor

DOP Dioctyl phthalate

ERDA Energy Research and Development Administration

ES Equipment specification

ESF Engineered safety feature (system)

FRP Fiber-reinforced plastic

GMA Gas metal arc (welding)

GTA Gas tungsten arc (welding)

HEP Hazard equivalent plutonium

HEPA High-efficiency particulate air (filter)

HF Hydrogen fluoride

HTGR High-temperature gas-cooled reactor

HVAC Heating, ventilating, and air conditioning

HWESF Hanford Waste Encapsulation and Storage Facility

IAEA International Atomic Energy Agency

IES Institute of Environmental Sciences

KI Potassium iodide

Kr Krypton

LOCA Loss-of-coolant accident

LMFBR Liquid-metal fast breeder reactor

LWR

Light water reactor

MMD Mass median diameter (of particles)

MPC Maximum permissible concentration

MPL $_{d} \quad$ Maximum permissible loading, desorption

MPL $L_{t} \quad$ Maximum permissible loading, ignition 


\begin{tabular}{|c|c|c|c|}
\hline NBS & National Bureau of Standards & RH & Relative humidity \\
\hline NFPA & National Fire Protection Association & RSIC & Reactor Shielding Information Center \\
\hline NMD & Number mean diameter (of particles) & RTV & $\begin{array}{l}\text { Room temperature vulcanizing (sealant } \\
\text { or caulking compound) }\end{array}$ \\
\hline NRC & Nuclear Regulatory Commission & SAR & Safety analysis report \\
\hline NRL & Naval Research Laboratory & SGTS & Standby gas treatment system \\
\hline NSIC & Nuclear Safety Information Center & SMACNA & Sheet Metal and Air Condition \\
\hline OBE & Operating basis earthquake & SMACNA & $\begin{array}{l}\text { Sheet Metal and Aur Conditioning } \\
\text { Contractors' National Association }\end{array}$ \\
\hline ORNL & Oak Ridge National Laboratory & SRL & Savannah River Laboratory \\
\hline PL & Permissible leakage & SSE & Safe shutdown earthquake \\
\hline PSU & Permanent single unit (adsorber) & TEDA & Triethylene diamine \\
\hline PVA & Polyvinyl acetate & TFE & Tetrafluoroethylene (plastic) \\
\hline PWR & Pressurized water reactor & TLV & Threshold limit value \\
\hline QA & Quality assurance & TURF & Thorium-Uranium Recycle Facility \\
\hline QAS & Quality assurance station & UL & Underwriters' Laboratories \\
\hline $\mathbf{R G}$ & Regulatory Guide & $\mathrm{Xe}$ & Xenon \\
\hline
\end{tabular}

\subsection{Units of Measure and Metric Equivalents Used in This Handbook}

\begin{tabular}{|c|c|c|c|c|c|}
\hline $\mathrm{cfm}$ & cubic feet per minute & $x$ & 0.000472 & $=\mathrm{m}^{3} / \mathrm{sec}$ & cubic meters per second \\
\hline fpm & feet per minute & $x$ & 0.00508 & $=\mathrm{m} / \mathrm{sec}$ & meters per second \\
\hline $\mathrm{ft}$ & feet & $\times$ & 0.3048 & $=\mathrm{m}$ & meters \\
\hline $\mathbf{g}$ & grams & & & & \\
\hline gal & gallons & $\times$ & 3.78532 & $=$ liters & liters \\
\hline gpm & gallons per minute & $x$ & 0.06309 & $=$ liters $/ \mathrm{sec}$ & liters per second \\
\hline gr & grains & $x$ & 0.0648 & $=\mathrm{g}$ & grams \\
\hline $\begin{array}{l}\mathrm{gr} / \mathrm{ft}^{3} \\
\mathrm{hr}\end{array}$ & $\begin{array}{l}\text { grains per cubic foot } \\
\text { hours }\end{array}$ & $x$ & 2.288 & $=\mathrm{g} / \mathrm{m}^{3}$ & grams per cubic meter \\
\hline $\mathrm{Hz}$ & Hertz & & & & \\
\hline in. & inches & $x$ & 2.54 & $=\mathrm{cm}$ & centimeters \\
\hline in.wg & inches water gage & $x$ & 0.24836 & $=\mathbf{k P a}$ & kilopascals \\
\hline in..$^{3}$ & cubic inches & $x$ & 0.01639 & $=$ liters & liters \\
\hline $\mathbf{l b}$ & pounds & $x$ & 0.4536 & $=\mathbf{k g}$ & kilograms \\
\hline m & meter & & & & \\
\hline$\mu \mathrm{m}$ & micrometer & & & & \\
\hline $\mathrm{mCi}$ & millicuries & & & & \\
\hline$\mu \mathrm{Ci}$ & microcuries & & & & \\
\hline $\min$ & minutes & & & & \\
\hline $\mathrm{mR}$ & milliroentgen & & & & \\
\hline millirem & milliroentgen equivalent man & & & & \\
\hline millirad & millirad & & & & \\
\hline ppm & parts per million & & & & \\
\hline psf & pounds per square foot & $x$ & 4.883 & $=\mathrm{kg} / \mathrm{m}^{3}$ & kilograms per cubic meter \\
\hline psi & pounds per square inch & & 57.820 & $=\mathrm{g} / \mathrm{cm}^{2}$ & grams per square centimeter \\
\hline $\mathrm{rad}$ & (unit of) radiation & & & & \\
\hline rem & roentgen equivalent man & & & & \\
\hline scfm & standard cubic feet & & & & \\
\hline & per minute (see $\mathrm{cfm}$ ) & & & & \\
\hline $\sec$ & seconds & & & & \\
\hline tonne & 1000 kilograms & & & & \\
\hline
\end{tabular}




\subsubsection{Terms and Phrases}

absolute filter. Obsolete term for HEPA filter.

acceptance test. A test made upon completion of fabrication, installation, repair, or modification of a system, unit, component or part to verify to the user or owner that the item meets specified requirements.

activation analysis. A method for identifying and quantitatively measuring chemical elements in a sample. Atoms in the sample are first made radioactive by bombardment with neutrons, charged particles, or other nuclear radiation; they then give off characteristic nuclear radiation by which they can be identified and their relative abundance can be determined.

adsorber. A device for removing gases or vapors from air by means of preferential physical condensation and retention of molecules on a solid surface. Adsorbers used in nuclear applications are often impregnated with chemicals to increase their activity for organic radioactive iodine compounds.

adsorber cell. A modular replaceable adsorber element.

AEC filter. A HEPA filter with fiberglass medium. Obsolete term for HEPA filter.

aerosol. A dispersion of very small particles and/or droplets in air.

air cleanup system. A system provided to decontaminate the air in, or exhausted from, a contained space following a system upset or prior to personnel access to the contained space.

air-generated DOP. See DOP.

ALAP. As low as practicable. Obsolete term for ALARA.

ALARA. As low as reasonably achievable. The design philosophy used to determine the need for, or extent of, air cleaning and off-gas facilities, based on their cost effectiveness in reducing adverse impacts with respect to offsite and onsite dose criteria. Formerly known as ALAP.

bag in, bag out. A method of introducing and removing items from a contaminated enclosure that prevents the spread of contamination or opening of the contaminated space to the atmosphere through the use of plastic bagging material.

case, casing. The frame or cell sides of a modular filter element. clean-air device. A clean bench, clean work station, downflow module, or other equipment designed to control air cleanness (particle count) in a localized working area and incorporating, as a minimum, a HEPA filter and a fan.

clean-air system. An air cleaning system designed to maintain a defined level of air cleanness, usually in terms of a permissible number of particles in a given size range, within an enclosed working area.

clean room. An occupied room designed to maintain a defined level of air cleanness under operating conditions. Inlet air is cleaned by HEPA filters.

coating. Paint or other protective surface treatment applied by brushing, spraying, or dipping (does not include metallic plates).

contained space (contained volume). A building, building space, room, cell, glove box, or other enclosed volume in which air supply and exhaust are controlled.

containment (containment vessel or building). A gastight enclosure around a nuclear reactor or other nuclear facility designed to prevent fission products from escaping to the atmosphere.

contaminated exhaust system. An air cleaning system that is designed to remove harmful or potentially harmful particulates, mists, or gases from the air exhausted from contained space.

contamination. Any unwanted material in the air, in process fluids, or on surfaces. For the purposes of this handbook, contamination is usually assumed to be radioactive.

contamination zone. An isolable area which is, or which could become, contaminated and which is designed to facilitate decontamination.

controlled area. An area to which access is restricted.

cover gas. An inert gas, under pressure, provided in a contained space or process equipment item to prevent inleakage of air.

criticality. The state of sustaining a chain reaction, as in a nuclear reactor. When fissionable materials are handled or processed, they must be kept in a subcritical geometry, configuration, or mass to avoid accidental criticality.

critical system, unit, or item. One that is essential for adequate or safe operation, failure of which would cause loss of function.

CWS filter. Chemical Warfare Service filter-a term used for a HEPA filter with cellulose-asbestos 
medium, kraft paper separators, and untreated plywood casing. Obsolete term for HEPA filter.

decay heat. The heat produced by radioactive materials as nuclides spontaneously transform into other nuclides or into different energy states. Each decay process has a definite half-life.

decontamination. The removal of unwanted substances from personnel, rooms, building surfaces, equipment, etc.

decontamination factor. A measure of air cleaning effectiveness; the ratio of the concentration of a contaminant in the untreated air or gas to the concentration in the treated air or gas.

demister. The preferred generic term for devices used to remove entrained moisture from air (see Thesaurus of Engineering and Scientific Terms). Also a trademark of Otto H. York Company.

design basis accident (DBA). The most serious accident that can be hypothesized from an adverse combination of equipment malfunction, operating errors, and other unforeseen causes.

design pressure. The pressure that is used for the structural design of a unit, component, or system, and which includes allowance for forces encountered under system upset conditions.

double filtration. An arrangement of two filters in series with the second providing backup protection against leakage or failure of the first. Also a series arrangement intended to increase the total filtration efficiency.

DOP aerosol. A dispersion of dioctyl phthalate (DOP) droplets in air. Monodisperse DOP is generated by controlled vaporization and condensation of liquid dioctyl phthalate to give a cloud of droplets with diameters of approximately $0.3 \mu \mathrm{m}$. Polydisperse DOP is generated by blowing compressed air through liquid dioctyl phthalate and exhausting through special nozzles under controlled conditions to produce a cloud of droplets with a light-scattering mean diameter of approximately $0.7 \mu \mathrm{m}$.

dose. The amount of ionizing radiation energy absorbed per unit mass of irradiated material at a specific location. In the human body it is measured in rems; in inanimate bodies it is measured in rads.

double containment. An arrangement of double barriers in which the second barrier provides backup protection against leakage through or failure of the first.

dry-type filter. A filter having a medium that is not coated with an oil or adhesive to improve its retention of large particles.

enclosed filter. A filter that is completely enclosed on all sides and both faces except for reduced end connections or nipples for direct connection into a duct system. Enclosed filters are installed individually because there is a separate run of duct to each filter unit.

engineered safety feature (ESF). A unit or system that is provided to directly mitigate the consequences of a DBA.

extended-medium filter. A filter having a pleated medium or a medium in the form of bags, socks, or other shape to increase the surface area relative to the frontal area of the filter.

face guard. A screen, usually made from 4-mesh galvanized hardware cloth, permanently affixed to the face of a filter unit to protect it against damage caused by mishandling.

face shield. A screen or protective grille placed over a filter unit after it is installed to protect it from damage that might be caused from operations carried on in the vicinity of the filter.

fail safe. A design to give equipment the capability to fail without producing an unsafe condition.

filter. A device having a porous or fibrous medium for removing suspended particles from air or gas that is passed through the medium.

filter bank. A parallel arrangement of filters on a common mounting frame enclosed within a single housing.

final filter. The last filter unit in a set of filters arranged in series.

functional design. The establishment of airflow rates, airflow capacities, types of components to be employed, general system layout, operational objectives and criteria, decontamination factors and rates, space allocations, and other overall features of a system.

gas chromatograph. An analytical instrument used for quantitative analysis of extremely small quantities of organic compounds whose operation is based upon the absorption and partitioning of a 
gaseous phase within a column of granular material:

gas residence time. The calculated time that a contaminant or test agent theoretically remains in contact with an adsorbent, based on active volume of adsorbent and air or gas velocity through the adsorber bed.

glove box. A sealed enclosure in which all handling of items inside the box is carried out through long rubber or neoprene gloves sealed to ports in the walls of the enclosure. The operator places his hands and forearms in the gloves from the room side of the box so that he is physically separated from the glove box environment but is able to manipulate items inside the box with relative freedom while viewing the operation through a window.

HEPA filter. High-efficiency particulate air filter-also obsoletely known as AEC, CWS, superinterception, absolute, and superhighefficiency filter. A throwaway extended-pleatedmedium dry-type filter with (1) a rigid casing enclosing the full depth of the pleats, (2) a minimum particle removal efficiency of $99.97 \%$ for thermally generated monodisperse DOP smoke particles with a diameter of $0.3 \mu \mathrm{m}$, and (3) a maximum pressure drop of $1.0 \mathrm{in.wg}$ when clean and operated at its rated airflow capacity.

hot. Highly radioactive.

hot cell. A heavily shielded enclosure in which radioactive materials can be handled remotely with manipulators and viewed through shielding windows to limit danger to operating personnel.

in-box. Refers to an item within a glove box that can be handled or manipulated only by means of the box gloves or tools within the box.

in-cell. Refers to an item located within a cell or enclosure that can be handled or manipulated only by means of manipulators and/or a crane and other tools within the cell.

in-duct filter. Refers to a single-filter arrangement in which the filter unit is clamped between two sections of duct or taped into a space between two sections of duct.

in-place test. Penetration test of filter units or charcoal adsorbers made after they are installed.

inches of water. A unit of pressure or pressure differential ( 1 in.wg $=0.036$ psi). ionizing radiation. Any radiation (alpha, beta, or gamma) that directly or indirectly displaces electrons from the outer domains of atoms.

isotope. One of several forms or nuclides of the same chemical element that have the same number of protons in the nucleus and therefore have the same chemical properties, but have differing numbers of neutrons and differing nuclear properties.

kidney system. An air cleaning system that recirculates the air of a contained space.

leaktightness. The condition of a system, unit, or component where leakage through its pressure boundary is less than a specified maximum value at a specified pressure differential across the pressure boundary.

maximum permissible dose. The dose of ionizing radiation which competent authorities have established as the maximum that can be absorbed without risk to human health.

mechanical design. Detailed design of a system which results in exact layouts, equipment specifications, shop drawings, installation details and drawings, sizing and layout of ducts, housings and equipment, and other details necessary to achieve the objectives and meet criteria established in functional design.

medium (plural, media). The filtering material in a filter.

mounting frame. The structure to which a filter unit is clamped and sealed.

normal off-gas. The normal gaseous discharge from a process or process equipment item.

nuclear reactor. An apparatus in which a chain reaction of fissionable material is initiated and controlled.

off-gas. The gaseous effluent from a process or operation.

off-line system. One that is not operating or is normally held in standby.

on-line system. One that is operating or is normally in operation, as opposed to an off-line system.

open-face filter. A filter with no restrictions over the ends or faces of the unit, as opposed to the enclosed filter with reduced-size end connections.

operating pressure. The desired pressure corresponding to any single condition of operation. 
overpressure. Pressure in excess of the design or operating pressure.

particle, particulate. A minute piece of solid matter having measurable dimensions. Also a radioactive particle (alpha, beta) which can liberate ionizing radiation or (neutron) which can initiate a nuclear transformation.

penetration. The measure of the quantity of a test agent that leaks through or around an air cleaning device when the device is tested with an agent of known characteristics under specified conditions; penetration is expressed as a percentage of the concentration of the test agent in the space upstream of the air cleaning device.

poison. Any material that tends to decrease the effectiveness of an adsorbent by occupying adsorption sites on the surface of the adsorbent or by reacting with the impregnants in the adsorbent.

prefilter. A filter unit installed ahead of another filter unit to protect the second unit from high dust concentrations or other environmental conditions. The prefilter usually has a lower efficiency for the finest particles present in the aerosol than the filter it protects (see roughing filter).

production test. A test made on each item or a sample of items or product from a production run to verify that the item meets specification requirements.

PSU adsorber. An adsorber that is permanently installed in a system and that can be emptied of and refilled with adsorbent without removing it from the system.

qualification test. A test made on a product or equipment item when it is proposed as a candidate to meet certain service requirements, which will verify to the user or owner that the item can meet his requirements (see production test).

rad. Radiation absorbed dose, the basic unit of ionizing radiation. One rad is equal to the absorption of 100 ergs of radiation energy per gram of matter.

radiation. The propagation of energy through matter or space in the form of electromagnetic waves or fast-moving particles (alpha and beta particles, neutrons, etc.). Gamma rays are electromagnetic radiation in which the energy is propagated in "packets" called photons. radioactivity. The spontaneous decay or disintegration of an unstable atomic nucleus accompanied by the emission of radiation.

redundant unit or system. An additional and independent unit or system which is capable of achieving the objectives of the basic system and is brought on-line in the event of failure of the basic system.

rem. Roentgen equivalent man. The unit of absorbed radiation dose in rads multiplied by the relative biological effectiveness of the radiation.

roughing filter. A prefilter with high efficiency for large particles and fibers but low efficiency for small particles; usually of the panel type.

scrubber. A device in which the gas stream is brought into contact with a liquid so that undesirable components in the gas stream are removed by reacting with or dissolving in the liquid.

separators. Corrugated paper or foil (usually aluminum alloy or plastic) used to separate the folds of a pleated filter medium and to provide air channels between them.

service environment. The aggregate of conditions (temperature, pressure, humidity, radioactivity, chemical contaminants, etc.) that surround or flow through a system, unit, or component while serving the conditions of design.

shielding. A mass of absorbing material placed around a radioactive source to reduce ionizing radiation to levels not hazardous to personnel.

shock overpressure. The pressure intensity over and above atmospheric or operating pressure produced by a shock wave from an explosion, a suddenly closed damper, or other event.

specific radioactivity. Radioactivity per unit weight of a material.

spill. Accidental release of radioactive or other contaminating materials.

split system. A filter system consisting of two or more trains operating in parallel; one or more of the trains may be on standby.

standby system. One held in reserve.

surveillance test. A test made periodically to establish the current condition of a system, unit, component, or part. 
system upset. An accident, system malfunction, or transient condition.

test program. A formalized schedule of tests which specifies the sequence of tests, the procedures to be employed, and the acceptance criteria.

train. A set of components arranged in series. In a filter system this may be as simple as a damper, HEPA filter, fan, and damper or as complex as a damper, condenser, moisture separator, heater, prefilter, HEPA filter, charcoal adsorber, another charcoal adsorber, HEPA filter, fan, and damper.

treatment. The process of removing all or a part of one or more chemical components, particulate components, or radionuclides from an off-gas stream.

\section{REFERENCES FOR CHAP. 1}

1. "Standards for Protection Against Radiation," Code of Federal Regulations, Title 10, Part 20 (10 CFR 20).

2. Techniques for Controlling Air Pollution from the Operation of Nuclear Facilities, International Atomic Energy Agency, Vienna, Safety Series No. 17, 1966.

3. Regulatory Guide 1.52, Design, Testing and Maintenance Criteria for Atmosphere Cleanup System Air-Filtration and Adsorption Units of Light-Water-Cooled Nuclear Power Plants, U.S. Atomic Energy Commission, Washington, D.C., 1973.

4. Regulatory Guide 3.12, General Design Guide for Ventilation Systems of Plutonium Processing and Fuel Fabrication Plants, U.S. Atomic Energy Commission, Washington, D.C., 1973.

5. Regulatory Guide 1.32, General Design Guide for Ventilation Systems for Fuel Reprocessing Plants, U.S. Atomic Energy Commission, Washington, D.C., 1972.

6. ERDA Manual, Appendix 6301, "General Design Criteria."

7. Industrial Ventilation, American Conference of Governmental Industrial Hygienists, 13th ed., Lansing, Mich., 1974.

8. ASHRAE Handbook-Systems, ASHRAE Handbook-Applications, American Society of Heating, Refrigerating and Air-Conditioning Engineers, New York.
9. ANSI Z9.2, The Design and Operation of Local Exhaust Systems, American National Standards Institute, New York, 1971. 10. P. A. F. White and S. E. Smith, eds., High Efficiency Air Filtration, Butterworth and Co., London and Washington, D.C., 1964.

11. C. N. Davies, Air Filtration, Academic Press, London and New York, 1973.

12. TLVS-Threshold Limit Values for Chemical Substances and Physical Agents in the Workroom Environment, American Conference of Governmental Industrial Hygienists, Cincinnati, Ohio (issued annually).

13. Number decontamination factor, DF, is the ratio of the number concentration of particles in the unfiltered air to the number concentration in the filtered air. Where no subscript is appended, decontamination factor in this handbook means number decontamination factor, as opposed to decontamination factor based on mass of particulate $\left(\mathrm{DF}_{m}\right)$, intensity of radioactivity $\left(D F_{r}\right)$, or volume concentration $\left(D F_{v}\right)$.

14. E. J. Bauer et al,, "The Use of Particle Counts For Filter Evaluation," ASHRAE Journal, American Society of Heating, Refrigerating and Air-Conditioning Engineers, October 1973,pp. 53-59.

15. IES CS-1, Standard for HEPA Filters, Institute of Environmental Sciences, Mt. Prospect, Ill., current issue. (Formerly American Association for Contamination Control.)

16. The efficiency of a HEPA filter is based on its absolute particulate collection effectiveness; that is, the efficiency is relative to the number of particles present (see Sect. 3.2). The efficiency of common air filters, on the other hand, is based on the mass concentration of particulate matter (see Table 2.4) or on the staining effect of particulates on a reference surface, which is, of course, very subjective.

17. "Hazardous Solvent Use Causes Explosion In Glove Box" in Serious Accidents, U.S. Atomic Energy Commission, Issue No. 261, Feb. 25, 1966.

18. M. W. First and L. Silverman, "Cost and Effectiveness of Air-Cleaning Systems," Nucl. Saf. 4(1), 61-66 (September 1962).

19. This cost includes $\$ 120$ for the new filter, $\$ 150$ or more for handling and testing, and as much as $\$ 240$ per filter for disposal and retrievable storage.

20. C. A. Burchsted and A. B. Fuller, Design, Construction, and Testing of High-Efficiency Air Filtration Systems for Nuclear Application, USAEC Report ORNL/NSIC-65, Oak Ridge National Laboratory, January 1970. 


\section{System Considerations}

\subsection{INTRODUCTION}

A nuclear air cleaning installation is one of an assemblage of interrelated and interactive parts that include a ventilation system, the contained space served by that system (i.e., glove box, hot cell, room, or building), and the processes carried out there. The design of an air cleaning installation has a direct bearing on the performance and operating costs of the ventilation system of which it is a part, and equally, the design of the ventilation system directly affects the performance and costs of the air cleaning facility. This chapter discusses, in general terms, some of the factors that must be considered when designing nuclear air cleaning facilities.

\subsection{ENYIRONMENTAL CONSIDERATIONS}

The complexity of the air cleaning system needed to provide satisfactory working conditions for personnel and to prevent the release of radioactive or toxic substances to the atmosphere depends on the nature of the contaminants to be removed (e.g., radioactivity, toxicity, corrosivity, particle size and size distribution, particle shape, and viscidity); on heat, moisture, and other conditions of the environment to be controlled; on the probability of an upset or accident; and on the extent of hazard in the event of such upset.

\subsubsection{Zoning}

Workroom ventilation rates are based primarily on cooling requirements, the potential combustion hazard, and the potential inhalation hazard of substances present in, or which could be released to, the workroom. Concentrations of radioactive gases and aerosols in the air of occupied and occasionally occupied areas should not exceed the concentration guides (CG) established for occupationally exposed persons under normal or abnormal operating conditions, and releases to the atmosphere must not exceed the permissible limits for nonoccupationally exposed persons. ${ }^{1,2}$ Because radioactive gases and aerosols might be released accidentally in the event of an equipment failure, a spill, or system upset, the ventilation and air cleaning facilities must be designed to maintain airborne radioactive material within prescribed limits, even following the worst conceivable accident that could occur in the plant. ${ }^{2}$ Control is made more difficult by the "as low as reasonably achievable" (ALARA) requirements which, at least for light water reactors, restrict gaseous and airborne particulate effluents to levels such that continuous exposure of persons in unrestricted spaces of the plant and its environs will not exceed the design objective annual dose limits set forth in Appendix I of 10 CFR 50. ${ }^{3}$ These guides limit annual dose to the whole body to 5 millirems, annual dose to the skin to 15 millirems, and annual radioiodine exposure to the thyroid to 15 millirems. In addition, the calculated air doses due to gamma and beta radiation should not exceed 10 and 20 millirads respectively. These limits are design objectives and can be modified when rationalized by a costbenefit analysis.

Radioactive materials may be grouped as shown in Tables 2.1 and 2.2 with respect to relative inhalation hazard. Quantities of radioactive materials greater than those indicated in Table 2.2 must be handled within a special containment, such as a hot cell or glove box, which often has ventilation and/or exhaust facilities independent of those serving the building space in which the special containment is located. The current CGs for radioactive substances in air are specified in 10 CFR $20{ }^{1}$ A building or facility can be divided into confinement zones with respect to the hazard classes shown in Table 2.2 and based on the criteria shown in Table 2.3. The limits given in Table 2.3 are guides and should not be considered as absolute. By introducing such indexes of potential hazard and limitations on the quantities of materials that can be handled, it is possible to establish a basis for ventilation and air cleaning 
Table 2.1. Hazard classification of radioisotopes

\begin{tabular}{|c|c|c|c|}
\hline $\begin{array}{l}\text { Hazard } \\
\text { class }\end{array}$ & Hazard & $\begin{array}{c}\text { CG, air } \\
\text { (Ci/liter) }\end{array}$ & $\begin{array}{l}\text { Amount of radioactive } \\
\text { materials permitted } \\
\qquad(\mu \mathrm{Ci})\end{array}$ \\
\hline 1 & Very high & $\leqslant 10^{-13}$ & 0.1 \\
\hline 2 & High & $10^{-13}$ to $10^{-11}$ & 1.0 \\
\hline 3 & Moderate & $10^{-11}$ to $10^{-9}$ & 10.0 \\
\hline 4 & Negligible & $\leqslant 10^{-9}$ & 100.0 \\
\hline
\end{tabular}

${ }^{a}$ Amount of radioactive material that can be handled without special protection for personnel. requirements in various parts of a building or plant. Figure 2.1 illustrates a typical zoning plan for a nuclear facility showing permitted occupancies, pressure differentials between zones required for proper ventilation and contaminant control, and zone assignments. Not all of the zones listed in Table 2.3 would be required in all buildings, and an entire building could quite possibly be designated as a single zone. Zones are defined, with respect to function and permitted occupancy, as follows:

Table 2.2. Classification of isotopes according to relative radiotoxicity based on inhalation hazard ${ }^{a}$ amounts (Ci) equivalent to $1 \mathrm{~g}$ of Pu-239 (HEP)

Italicized isotopes are fissile and require special consideration for nuclear safety

Class 1

(Very high radiotoxicity)

HEP $\leqslant 0.07$

Class 2

(High radiotoxicity)

$\mathrm{HEP}=0.86$ to 17

Class 3

(Moderate radiotoxicity)

$\mathrm{HEP}=22$ to 220
Class 4

(Slight radiotoxicity)

HEP $>430$
$\mathrm{Sr}-90+\mathrm{Y}-90$, Po-210, Po-210 + Bi-210, Ra-226, Th-228, U-232, $\mathrm{Np}-236, \mathrm{Pu}-238, \mathrm{Pu}-239, \mathrm{Pu}-240, \mathrm{Pu}-241, \mathrm{Am}-24 \mathrm{I}, \mathrm{Am}-242^{m}$, $\mathrm{Cm}-242, \mathrm{Am}-243, \mathrm{Cm}-243, \mathrm{Cm}-244, \mathrm{Cm}-245, \mathrm{Cm}-246$, $C m-247, B k-249, C f-249$ Cf-250, Cf-251, Cf-252

$\mathrm{Na}-22, \mathrm{P}-32, \mathrm{Ca}-45, \mathrm{Sc}-46, \mathrm{~V}-48, \mathrm{Fe}-59$, Co-58, Co-60, Ni-63, Zn-65, Rb-86, Sr-89, Y-91, Zr-95 + Nb-95, Ru-103, Ru-106 + Rh-106, Ag-105, Ag-110, Cd-109 + Ag-109, Cd-115, In-114, Sn-113, Sb-122, Sb-124, Sb-125, I-131, Cs-134, Cs-137 + Ba-137, Ba-140 + La-140, Ce-144 + Pr-144, Pm-147, Sm-151, Eu-152, Eu-154, Tm-170, Hf-181, Ta-182, Ir-192, Hg-203, Tl-204, Bi-210, At-211, U-233, ${ }^{c}$ Th-234 + Pa-234, Np-237, Pu-242

$\mathrm{Be}-7, \mathrm{Na}-24, \mathrm{~S}-35, \mathrm{~K}-42, \mathrm{Ca}-47, \mathrm{Sc}-47, \mathrm{Sc}-48, \mathrm{Mn}-52, \mathrm{Mn}-54$ Fe-55, Mn-56, Cu-64, Ga-72, As-74, As-76, As-77, Se-75, Br-82, Sr-85, Y-90, Nb-95, Mo-99, Pd-103 + Rd-103, Rh-105, Pd-109, Ag-111, Cd-115, Sb-122, Te-127, Ba-131, La-140, $\mathrm{Ce}-141, \mathrm{Pr}-142, \mathrm{Pr}-143, \mathrm{Nd}-147$, Ho-166, Sm-153, Ho-170, Lu-177, W-181, W-185, W-187, Re-183, Re-186, Os-191, Ir-190, Ir-192, Ir-194, Pt-191, Pt-193, Au-196, Au-198, Au-199, Hg-197, Tl-200, Tl-201, Tl-202, Ac-227, pure U-233, $\mathrm{U}-234$

$\mathrm{H}-3, \mathrm{C}-14, \mathrm{~F}-18, \mathrm{Cl}-36, \mathrm{~A}-37, \mathrm{Cr}-51$, Ni-59, Ge-71, Kr-85, Tc-98, Tc-99, Ru-97, Rh-103, Te-129, I-129, 1-132, Xe-133, $\mathrm{Pb}-203, U-235, \mathrm{U}-236$, natural thorium, $\mathrm{U}-238$, natural uranium

${ }^{a}$ These values are based on inhalation and immersion (for inert gases) hazard only. Other factors that must be considered are criticality, chemical toxicity and reactivity, and pyrophoricity.

${ }^{b} \mathrm{HEP}=$ hazard equivalent plutonium.

$c \leqslant 500 \mathrm{ppm} \mathrm{U}-232$.

Note:

$\mathrm{HEP}=\left(2.16 \times 10^{9}\right)(\mathrm{CG} \times \mathrm{A})$

where

$\mathrm{CG}=\mu \mathrm{Ci} / \mathrm{cm}^{3}$ for $40-\mathrm{hr}$ week;

$\mathrm{A}=\mathrm{g} / \mathrm{Ci}$ or 0.1 , whichever is greater.

Sample calculation-Determine curie HEP for Am-24I.

CG 40 -hr week for Am-241 $=6 \times 10^{-12} \mu \mathrm{Ci} / \mathrm{cm}^{3}$

lnverse specific activity $(\mathrm{g} / \mathrm{Ci})=0.311$.

$\therefore$ HEP $=2.16 \times 10^{9} \times 6 \times 10^{-12} \times 0.311$

$$
=4.03 \times 10^{-3}
$$

Therefore, $4.03 \times 10^{-3} \mathrm{Ci}$ of $\mathrm{Am}-241$ has the same hazard equivalent potential as $1 \mathrm{~g}$ of $\mathrm{Pu}-239$.

Source: Procedures and Practices for Radiation Protection, Health Physics Manual, Oak Ridge National Laboratory, Oak Ridge, Tenn. 
Table 2.3. Zoning of facilities based on radiotoxicity of materials handled

\begin{tabular}{lllll}
\hline & \multicolumn{4}{c}{ Quantity of material handled vs radiotoxicity } \\
\cline { 2 - 5 } $\begin{array}{l}\text { Radiotoxicity } \\
\text { of isotopes }\end{array}$ & Zone I & Zone II & Zone III & Zone IV \\
\hline Very high & & & & \\
High & $>10 \mathrm{mCi}$ & $10 \mu \mathrm{Ci}-10 \mathrm{mCi}$ & $0.1 \mu \mathrm{Ci}-10 \mu \mathrm{Ci}$ & $0-0.1 \mu \mathrm{Ci}$ \\
Moderate & $>100 \mathrm{mCi}$ & $100 \mu \mathrm{Ci}-100 \mathrm{mCi}$ & $1.0 \mu \mathrm{Ci}-100 \mu \mathrm{Ci}$ & $0-1.0 \mu \mathrm{Ci}$ \\
Slight & $>1 \mathrm{Ci}$ & $1 \mathrm{mCi}-1 \mathrm{Ci}$ & $10 \mu \mathrm{Ci}-1 \mathrm{mCi}$ & $0-10 \mu \mathrm{Ci}$ \\
& $>10 \mathrm{Ci}$ & $10 \mathrm{mCi}-10 \mathrm{Ci}$ & $100 \mu \mathrm{Ci}-10 \mathrm{mCi}$ & $0-100 \mu \mathrm{Ci}$ \\
\hline
\end{tabular}

${ }^{a}$ There is an upper limit to the quantity of transuranium elements which should be approved for glove box operations. As a general rule, for those isotopes having a gram HEP index number below $10^{-4}$, the limiting quantity should be $100 \mathrm{mg}$. (For example, $100 \mathrm{mg}$ of $\mathrm{Cm}-244$ generates the same hazard equivalent potential as $4.3 \mathrm{~kg}$ of $\mathrm{Pu}-239$.) Any operation involving more than $100 \mathrm{mg}$ of such isotopes should be conducted at facilities with more absolute containment features than are offered by glove boxes alone. This number may require further reduction due to penetrating radiation. One gram of $\mathrm{Cf}-252$, for example, generates a dose rate of $2400 \mathrm{rems} / \mathrm{hr}$ at a distance of $1 \mathrm{~m}$ in air.

Source: Procedures and Practices for Radiation Protection, Health Physics Manual, Oak Ridge National Laboratory, Oak Ridge, Tenn.

ORNL-DWG 75-17171R

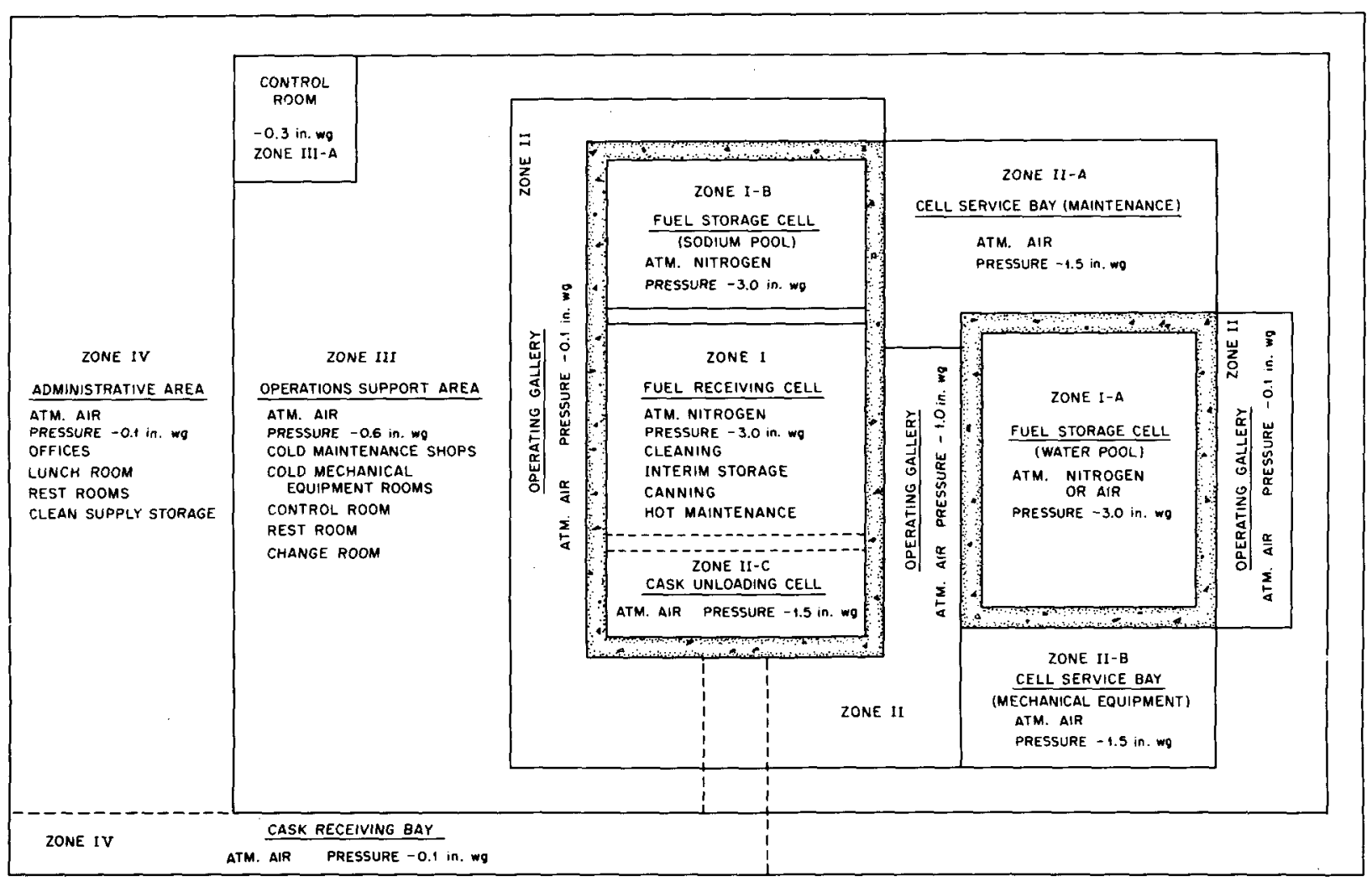

Fig. 2.1. Typical zoning plan for nuclear facility, showing type occupancy and operations permitted and static pressure that must be maintained in each zone to prevent backflow of air to areas of less contamination. 
Zone I: The interior of a hot cell, glove box, or other containment for handling highly radioactive material. Containment features must prevent the spread of radioactive material within and release from the building under both normal operating and upset conditions up to and including the design basis accident (DBA) for the facility. Complete isolation (physical separation) from neighboring facilities, laboratories, shop areas, and operating areas necessary. Entry forbidden until area is cleaned up to Zone II classification. Air-exhaust system independent of those serving surrounding areas required. High efficiency filter, preferably HEPA type, required in air inlet; two independently testable stages of HEPA filters required in exhaust. Entry only with full-body protective clothing and with respirators or full-face gas masks, as specified by health physicist.

Zone II: Glove box operating area, hot cell service or maintenance area, or other building space where high levels of radiation could be present. Particularly hazardous operations conducted in chemical fume hoods or glove boxes. Sufficient air supply to produce inward airflow into fume hoods or glove box ports (with glove removed) of at least 100 lin fpm, and may be $200 \mathrm{fpm}$ for particularly hazardous operations or if hot plates, burners, or aspirators are operated within such containment. Air locks or personnel clothing-change facility recommended at entry of zone. Continous monitoring of airborne radioactive material required. Personnel should wear at least laboratory coats and possibly shoe covers in glove box operating areas, and full protective clothing in service areas. Respirators or full-face gas masks should be available in the event of an operational upset. Restricted access areas are generally considered to be Zone II hazard classification.

Zone III: Hot cell operating areas, general chemical laboratories, maintenance, and other general working areas which are usually "cold" but which are subject to low levels of radiation in the air. Chemical fume hoods required for operations which could produce greater than CG for radioactive material or TLV for toxic or noxious material. Operating personnel should wear lab coats or equivalent special clothing, with respiratory gear available for emergencies. Routine airborne radiation monitoring required.

Zone IV: Office and "cold" shop areas. No specific protective clothing requirements. Radiation monitoring may be required at exit points. Bench-top operations permitted in laboratories, but chemical fume hoods should be considered where airborne concentrations could exceed levels stipulated in Table 2.1.

Multizoned buildings are usually ventilated so that airflow is from the less contaminated zone to the more contaminated zone. Recirculation within a zone, with the air circulated through a high-efficiency air cleaning system before discharge back to the zone, might be permitted, but recirculation from a zone of higher contamination back to a zone of lesser contamination is prohibited. The inside of exhaust and recirculating ductwork is considered to be of the same hazard classification as the zone it serves. Airflow must be sufficient to provide the necessary degree of contaminant dilution and cooling and to maintain sufficient pressure differentials between zones where there can be no backflow of air to spaces of lower contamination, even under upset conditions. A pressure differential $(\Delta p)$ of at least 0.1 in.wg between building zones is recommended, and substantially higher differentials ( 0.3 to 1.0 in.wg) are often specified between Zone II and Zone I spaces. The following criteria are specified at one of the ERDA national laboratories for the design and operation of radiochemical and laboratory facilities and for the buildings that contain them.,

Hot cells, caves, and canyons

1. Vacuum equal to or greater than 1 in.wg relative to surrounding spaces shall be maintained at all times to ensure a positive flow of air into the containment.

2. Containment exhaust shall be at least $10 \%$ of cell volume per minute to minimize possible explosion hazards due to the presence of volatile solvents and to ensure that, in the event 
of cell pressurization due to an explosion, the containment will be returned to normal operating pressure (1 in.wg) in a minimum of time.

3. Maximum permissible leak rate shall be $1 \%$ of cell volume per minute for unlined cells, and $0.1 \%$ of cell volume per minute for lined and sealed cells at a $\Delta p$ of 2 in.wg to ensure that the escape of radioactive material will be minimized in the event of cell pressurization; maximum permissible leak rate of ductwork is $0.1 \%$ of duct volume per minute at $\Delta p$ equal to 1.5 times the static pressure of ductwork.

4. Seals and doors shall withstand a $\triangle p$ of at least 10 in.wg to ensure integrity of closures and penetrations under all operating and design basis upset conditions.

5. The containment structure shall withstand the DBA for that facility without structural damage or loss of function.

6. Operating procedures shall be designed to limit quantities of flammable and smoke-producing materials and solvents within limits that can be accommodated by the ventilation system without endangering functionability of the air cleaning facility.

\section{Glove boxes}

1. Vacuum shall be at least 0.3 in.wg between the glove box and surrounding room.

2. Exhaust rate is not specified but must be adequate for heat load and dilution requirements of operations conducted in the glove box.

3. Airflow shall be sufficient to provide at least 5 scfm to the glove box and to maintain an inward velocity of at least $100 \mathrm{lin}$ fpm through one open glove port in every five glove boxes in the system to ensure adequate inflow to prevent the escape of contamination in the event of glove failure.

4. Individual glove boxes shall be isolated or isolable (under upset conditions) to prevent spread of fire from one box to another.

\section{Chemical fume hoods}

1. Vacuum shall be at least 0.1 in.wg between the laboratory in which the fume hood is installed and the corridor from which the laboratory is entered.
2. Exhaust rate of the fume hood shall be sufficient to maintain sufficient airflow face velocity into the hood to prevent eduction of fumes from the hood to the room, even when the operator walks rapidly back and forth in front of, and close to, the hood face. A face velocity of at least 100 lin fpm is recommended for operations with radioactive materials; $150 \mathrm{fpm}$ is desirable.

3. Each hood in the laboratory should be isolable by means of dampers to prevent backflow through a hood when it is not in service.

4. Each hood used for handling radioactive materials should have a HEPA filter in its exhaust duct, located close to the duct entrance. All hoods should, where practicable, exhaust to a common stack.

5. Hoods should operate on a once-through mode with no recirculation to the room. (This requirement may be reexamined in light of current energy conservation objectives; if a recirculatory system is considered, the air cleaning system must be such that there is no possibility of releasing radioactive or toxic particulates, fumes, or gases to the room, even under the worst foreseeable operating or accident conditions.)

\section{Secondary containment structure or building}

1. The building (structure) shall be designed to prevent the dispersal of airborne contamination to the environment in the event of an accident in a hot cell, glove box, fume hood, or building space.

2. Under emergency conditions the building shall be capable of being maintained at a vacuum of at least 0.3 in.wg relative to the atmosphere. For increased reliability and simplicity, some buildings are held at this pressure under normal operating conditions; if this is not practicable, the ventilation system must be capable of reducing building static pressure to $0.3 \mathrm{in.wg}$ in $20 \mathrm{sec}$ or less. All building air shall be exhausted through at least one stage of HEPA filters. During an emergency, the differential between Zone I spaces (glove boxes, hot cells) and other building spaces must also be maintained.

3. Airflow within the building must be from areas of less contamination to areas of higher (or potentially higher) contamination. 
4. Recirculation of air within the same zone or room is permitted, but recirculation from the central exhaust system is prohibited.

\section{Air handling system}

1. Ventilation (recirculating or exhaust) and offgas systems shall be backed up by redundant air cleaning facilities (including filters and fans) to maintain containment in the event of fan breakdown, filter failure, power outage, or other operational upset. Airflow shall always be from the less hazardous to the more hazardous area under both normal and upset conditions.

2. Air exhausted from occupied or occasionally occupied areas shall be passed through prefilters and at least one stage of HEPA filters. Contaminated and potentially contaminated air exhausted from a hot cell, cave, canyon, glove box, or other primary containment structure or vessel shall be passed through at least two individually testable stages of HEPA filters in series, plus prefilters, adsorbers, scrubbers, or other air cleaning facilities as required by the particular application. Air that is normally clean but has the potential of becoming contaminated in the event of an operational upset (e.g., exhaust from a Zone II operating area) or during service operations when the zone is opened to a zone of higher contamination (e.g., a hot cell service area) and air from a potentially mildly contaminated space (e.g., Zone III area) require only one stage of HEPA filters in the exhaust.

3. Corrodents or moisture in the exhaust capable of damaging or unduly loading the HEPA filters (or other components, such as adsorbers) shall be removed or neutralized before they can reach components that can be affected.

4. HEPA filters and adsorbers (where required) shall be tested in place at a prescribed frequency (usually twice per year). HEPA filter stages shall exhibit a DF of no less than $3333(99.97 \%$ efficiency) as determined by an in-place test (see Chap. 8); because of the sensitivity of the test, some facilities now specify a minimum DF equivalent to the predelivery test efficiency of the filters (as determined by the manufacturer or ERDA Quality Assurance Station); that is, a DF of 10,000 for the in-place test may be specified where filters having a predelivery test efficiency of $99.99 \%$ (which is common today) are used in the system.
Concentration limits for radioactive substances in air are specified in $10 \mathrm{CFR} 20$. $^{1}$ Threshold limit values (TLV) of toxic and noxious substances, including irritant and nuisance substances, are specified in Title 29 of the Code of Federal Regulations (Labor) but are more conveniently tabulated by the American Conference of Governmental Industrial Hygienists in the annual issue of TLVs. ${ }^{6}$ The latter gives a procedure for determining TLVs for mixed toxicants and also gives limit values for heat stress, nonionizing radiation, and noise.

\subsubsection{Airborne Particulates and Gases}

Although process-generated dust and particulate matter are the primary reason for installing exhaust or air cleanup filters, a major portion of the dust and particulate matter collected in those filters actually consists of atmospheric dust brought into the building with the supply air or by infiltration and of "people-generated" particulates (e.g., lint, skin, and hair). These particulates contribute to degradation of the filters and sometimes become radioactive when exposed to certain operating environments (e.g., by adsorption of radioactive vapors or gases or by agglomeration with already radioactive particles). Because particles in the size range of 0.05 to $5 \mu \mathrm{m}$ tend to be retained by the lungs when inhaled, they are of primary concern in operations that involve radioactive material ${ }^{7}$ they are also recognized as health hazards of nonradioactive air pollution. ${ }^{8}$ As Table 2.4 shows, over $99 \%$ of the actual number of particles present in atmospheric air falls in this size range.

Reports of dust concentration in air are generally based on the mass of particulate matter present. As Table 2.4 shows, mass accounts for only a negligible portion of the total number of particles in the air. This is important in filter selection because it indicates that some filters that have high efficiency based on weight may be inefficient on a true count basis. That is, they are efficient for large particles but inefficient for small $(>0.75 \mu \mathrm{m})$ particles. This is true of most common air filters used as prefilters. The HEPA filter, on the other hand, is highly efficient for all particle sizes, down to and including the smallest shown in Table 2.4 . The $99.97 \%$ minimum efficiency claimed for these filters is actually for the most penetrating size particles, those in the range from 0.07 to $0.3 \mu \mathrm{m}$. Dust concentrations vary widely from place to place and, for the same location, from season to season and from time to time during the same day. Concentrations in the atmosphere may vary from as low as 0.01 grain per $1000 \mathrm{ft}^{3}$ in rural areas to more 
Table 2.4. Distribution of particles in typical urban air sample

\begin{tabular}{ccccc}
\hline $\begin{array}{c}\text { Mean } \\
\text { particle size } \\
(\mu \mathrm{m})\end{array}$ & $\begin{array}{c}\text { Particle size } \\
\text { range } \\
(\mu \mathrm{m})\end{array}$ & $\begin{array}{c}\text { Approximate } \\
\text { particle count } \\
\text { per cubic foot of air }\end{array}$ & $\begin{array}{c}\text { Percent } \\
\text { by } \\
\text { weight }\end{array}$ & $\begin{array}{c}\text { Percent } \\
\text { by } \\
\text { count }\end{array}$ \\
\hline 20.0 & $50-10$ & $12.5 \times 10^{3}$ & 28 & $1 \times 10^{-11}$ \\
7.5 & $10-5$ & $10 \times 10^{4}$ & 63 & $8 \times 10^{-111}$ \\
2.5 & $5-1$ & $12.5 \times 10^{6}$ & 6 & $1 \times 10^{-7}$ \\
0.75 & $1-0.5$ & $10 \times 10^{7}$ & 2 & $8 \times 10^{-7}$ \\
0.25 & $0.5-0.1$ & $12.5 \times 10^{9}$ & 1 & $1 \times 10^{-4}$ \\
0.05 & $0.1-0.001$ & $12.5 \times 10^{15}$ & $<1$ & 99.9999 \\
\hline
\end{tabular}

Source: From the Frank Chart, American Air Filter Co., Louisville, Ky.

than 10 grains per $1000 \mathrm{ft}^{3}$ in heavily industrialized areas. Dust-producing operations may generate concentrations as great as several thousand grains per $1000 \mathrm{ft}^{3}$ at the work place. Because the weight percent determinations on which these concentrations are based account for only a small fraction of the number of particles present, the true count of particles smaller than $5 \mu \mathrm{m}$ may number in the billions per $1000 \mathrm{ft}^{3}$. Atmospheric dust concentrations are usually lowest during the summer months (June 1 to August 1)-as much as $30 \%$ lower at that time than during the remainder of the year. ${ }^{y}$ Filter selection, particularly prefilter selection and building supply filter selection, must take into consideration the atmospheric dust concentrations that can be encountered at the particular site at any time of the year.

Figure 2.2 shows the distribution of particles (by weight percent) in atmospheric air as a function of particle shape. Variations in particle shape, mean particle size, particle size range, and concentration affect filter life, maintenance costs, and operational effectiveness. The size range of various types of particles, the technical nomenclature of various types of aerosols, and the applicability of various types of air cleaning devices as a function of particle size are shown in Fig. 2.3. A major source of the lint often found on filters is derived from the abrasion of clothing as people move about. In addition, a person at rest gives off more than 2.5 million particles (skin, hair, etc.) and moisture droplets per minute, in the size range of $0.3 \mu \mathrm{m}$ to $1 \mu \mathrm{m} .{ }^{10}$ Process-generated aerosols fall into two general size ranges. Those produced by machining, grinding, polishing, and other mechanical operations are generally large, probably from $1 \mu \mathrm{m}$ to several hundred micrometers, according to the nature of the process, and can be removed effectively by common air filters or other conventional air cleaning techniques. The other class includes those produced by evaporation/condensa-

\begin{tabular}{|c|c|c|c|c|}
\hline \multirow{2}{*}{ Description } & \multirow{2}{*}{ Appeorance } & \multirow{2}{*}{ Kinds } & \multicolumn{2}{|c|}{$\begin{array}{c}\text { Percent Present } \\
\text { by Weight }\end{array}$} \\
\hline & & & Ronge & Averoge \\
\hline Spherical & & $\begin{array}{l}\text { Smokes } \\
\text { Pollens } \\
\text { Fly osh }\end{array}$ & $0-20$ & 10 \\
\hline $\begin{array}{l}\text { Irregular } \\
\text { cubic }\end{array}$ & & $\begin{array}{l}\text { Mineral s } \\
\text { Cindor }\end{array}$ & $10-90$ & 40 \\
\hline Flakes & & $\begin{array}{l}\text { Minerols } \\
\text { Epidermis }\end{array}$ & $0-10$ & 5 \\
\hline Fibrous & & $\begin{array}{l}\text { Lint } \\
\text { Plant } \\
\text { fibers }\end{array}$ & 3-35 & 10 \\
\hline $\begin{array}{l}\text { Conden sation } \\
\text { flocs }\end{array}$ & 19 & $\begin{array}{l}\text { Carbon } \\
\text { Smokes } \\
\text { Fumes }\end{array}$ & $0-40$ & 15 \\
\hline
\end{tabular}

Fig. 2.2. Distribution of airborne particulates in the atmosphere, by particle shape. From K. T. Whitby; see ref. 9 .

tion and other chemical operations, which generate droplets and solid particles that are often in the submicron size range. These aerosols are more difficult to separate from air or gases, and recourse must be made to collectors such as HEPA filters. Other process-generated contaminants include radioactive halogen and noble gases. Because of their chemical inertness, limited reactivity with available sorbents, and great difficulty of separation, the noble gases (xenon and krypton) were treated in the past by simple holdup, to give time for radioactive decay of the shorter half-life elements, and dilution before discharge to the atmosphere. Newer practice, particularly in view of ALARA requirements, is to separate the noble gases by cryogenic fractionation, charcoal adsorption, or fluorocarbon absorption, and store them until a significant degree of radioactive decay can take place. The halogen gases, essentially elemental iodine and certain organic iodides, constitute the largest fraction of the gaseous effluents and are captured by adsorption on activated carbon or certain synthetic zeolites. 


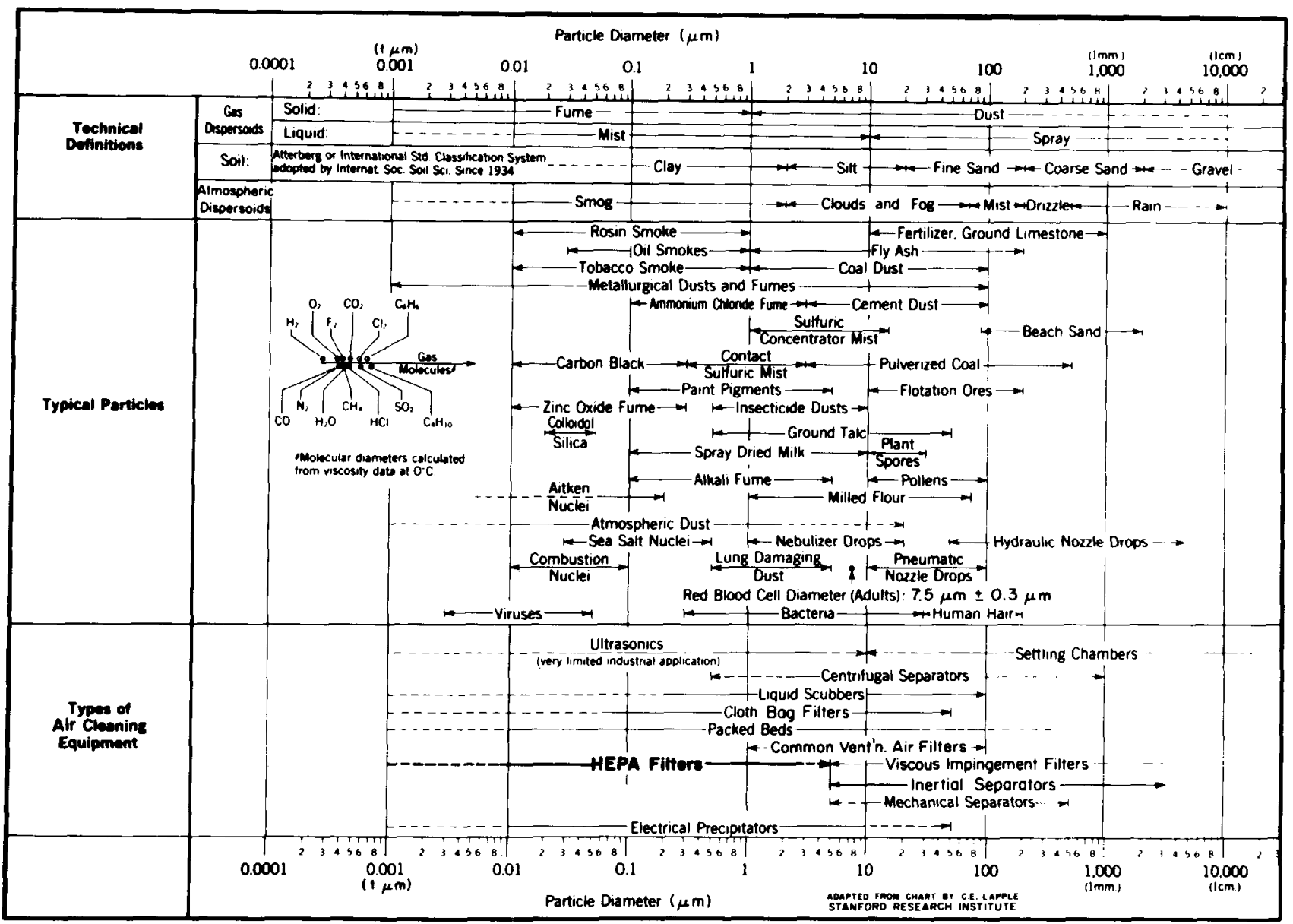

Fig. 2.3. Characteristics of atmospheric and process-generated particulates, fumes, and mists and effective ranges of typical air cleaning equipment. Solid line indicates range of normal application. Courtesy C. E. Lapple, Stanford Research Institute.

\subsubsection{Moisture}

Sensible moisture in the air is a hazard to prefilters, HEPA filters, and adsorbents. Where heavy concentrations of water mist or steam can be expected, under either normal or upset conditions, heaters, demisters, or other means of reducing entrained moisture to tolerable levels must be provided upstream of the filters to prevent plugging, deterioration, and reduced performance. Condensation from saturated air or gas streams and carry-over from air washers and scrubbers are common sources of sensible moisture. When fire-protection sprinklers are provided in operating areas or ducts, moisture can be drawn into the filters if they are activated in the event of a fire. In nuclear reactors, large volumes of steam and moisture would be expected in the very unlikely event of a major loss-of-coolant accident or heat-exchanger failure; this moisture can impair the performance and integrity of HEPA filters and adsorbers unless removed before reaching those components.

Condensation is particularly troublesome when filters are installed in underground pits, in housings located outdoors, or in unheated spaces of buildings. Even when air entering ducts is above the dew point, duct walls, dampers, or filters may be cold enough to cause condensation on their surfaces. Condensation can also take place in standby systems, particularly when groundwater can evaporate into the filter housing to condense on the walls, mounting frames, or filters; salts that leach from wood filter casings can rapidly deteriorate aluminum separators. In one instance, the separators of a bank of HEPA filters were nearly destroyed by this action in a three-month period. Periodic ventilation of standby filters, on a 
monthly or even weekly basis, is recommended to prevent such occurrences.

\subsubsection{Heat and Hot Air}

Continuous operation at high temperature $\left(>200^{\circ} \mathrm{F}\right)$ may be detrimental to both HEPA filters and activated-carbon-filled adsorbers. At high temperatures, the shear strength of adhesives used in the manufacture of HEPA filters and some prefilters diminishes, thereby limiting the safe pressure drop to which they can be subjected. The limiting temperature varies with the specific adhesive used and should be checked with the filter manufacturer where operation at elevated temperatures is considered. ${ }^{11}$ Limiting temperatures for HEPA filters are given in Chap. 3. For continued operation at temperatures in excess of $200^{\circ} \mathrm{F}$, a specially constructed HEPA filter, in which the filter core is sealed into the steel case by means of a compressed mat of glass fibers, is generally employed; however, tests show that the efficiency of these filters, when operating at high temperatures (about $800^{\circ} \mathrm{F}$ ), decreases substantially below the specified value of 99.97\% minimum number efficiency for submicron particles, even though the filters purportedly operated within that specification value when tested at room temperature before and after the high temperature runs. ${ }^{12}$ Apparently the steel case sides expand away from the filter core, thus permitting some degree of bypassing at high temperature. Most commercially available prefilters are not resistant to heat, and special constructions must be specified if continuous operating temperatures exceed $200^{\circ} \mathrm{F}$.

Ceramic-fiber filters having efficiencies as high as $80 \%$ for $0.3-\mu \mathrm{m}$ particles are suitable for service at temperatures up to $2000^{\circ} \mathrm{F}$. As with other types of HEPA filters, however, DOP efficiency tests have been made only at room temperature, and the actual efficiency at high temperature is unknown. Ceramicfiber filters are very expensive, extremely fragile, and must be handled and installed with great care.

The limiting temperature of adsorbents for capturing radioactive iodine and iodine compounds is related to the desorption temperature of the adsorbed compound and of the impregnants with which the material has been treated to enhance its adsorption of organic radioiodides. For triethylene diamine (TEDA) impregnated activated carbon, this temperature may be as low as 300 to $350^{\circ} \mathrm{F}$.

When temperatures higher than the operating limits of air cleaning system components must be accommodated, heat sinks, dilution with cooler air, or some other means of cooling must be provided to reduce temperatures to levels that those components can tolerate. Consideration must also be given to thermal expansion and heat resistance of ducts, dampers, filter housings, component mounting frames and clamping devices, and fans. Consideration must also be given to flammability of dust collected in the ducts and on the filters.

\subsubsection{Corrosion}

Many radiochemical operations generate acid or caustic fumes that can damage or destroy filters, other system components, and materials of construction. High levels of system performance and reliability cannot be ensured when filters are exposed, even occasionally, to corrosive fumes unless corrosionresistant HEPA filters, with specially treated media and separators and wood cases, and stainless steel ducts, housings, and mounting frames are employed. A new hydrogen fluoride resistant HEPA filter medium has been developed $;{ }^{13}$ however, the material has not yet been produced commercially. Although stainless steel filter cases have sometimes been employed for corrosion resistance, this is false economy because the life of standard case materials is nearly always greater than the life of the filter core. Stainless steel should not be specified for HEPA filter separators because it makes the filter core impossible to fabricate; separatorless filters, corrosion-resistant asbestos separators, or even plastic-coated aluminum separators are recommended.

Stainless steel is recommended for ductwork and housings when corrosion can be expected. Even this material may be insufficient in some cases, and coated (e.g., vinyl, epoxy) stainless steel or fiberreinforced plastics may be necessary (corrosionresistant coatings are covered by ANSI N512) $;^{14}$ plastics must be used with caution because they will soften and may collapse if exposed to high temperatures, as might be encountered during a fire in the workroom.

Scrubbers or air washers may be employed to pretreat the air or gas before it enters the final filters, but consideration must also be given to moisture carry-over if the scrubbers or airwashers are not designed and operated properly. Demisters should be provided ahead of the filters. Corrosion is always a danger but is not always obvious. In activatedcarbon-filled adsorbers, for example, even trace amounts of $\mathrm{NO}_{2}$ or $\mathrm{SO}_{2}$ will concentrate in the 
adsorbent over a period of time; in the presence of moisture, they can form nitric or sulfuric acids that are capable of corroding metal parts of the adsorber. In one instance, this sequence of events required replacement of several hundred carbon-steel-cased adsorber cells with stainless steel units, at a very substantial cost. Aluminum and carbon steel are subject to corrosion when in contact with moistureladen carbon. For this reason, stainless steel is always recommended for adsorber cells and for adsorber-cell mounting frames.

\subsubsection{Vibration}

Vibration and pulsation can be produced in an air or gas cleaning installation by turbulence generated in poorly designed ducts, transitions, dampers, and fan inlets and by improperly installed or balanced fans and motors. Apart from discomfort to personnel, excessive vibration or pulsation can result in eventual mechanical damage to system components when vibrational forces become high or when accelerative forces (e.g., from an earthquake or tornado) coincide with the resonant frequencies of those components. Weld cracks in ducts, housings, and component mounting frames may be produced by even low-level local vibration if sustained, and vibrations or pulsations that produce no apparent short-term effects may cause serious damage after long duration.

Vibration produces noise that can range from the unpleasant to the intolerable. An important factor in the prevention of excessive vibration and noise is planning at the stage of initial building layout and space allocation to ensure that adequate space is provided for good aerodynamic design of ductwork and fan connections. Spatial conflicts with the process and with piping, electrical, and architectural requirements should also be resolved during early design so that the compromises that are so often made during construction, which lead to poor duct layout and resultant noise and vibration, can be avoided. Ducts should be sized to avoid excessive velocities while maintaining the necessary transport velocities to prevent the settling out of particulate matter during operation. Fan vibration can be minimized through the use of vibration isolators and inertial mountings, although the use of these must be balanced against seismic design requirements where necessary (some designers prefer hard-mounting of fans where continued operation during and after an earthquake must be considered). Flexible connec- tions between the fan and ductwork are often employed, but these must be designed to resist the high static pressures often incurred in this class of system, particularly in those parts of the system which are under negative pressure. Finally, the ductwork system must be balanced after installation, not only to ensure the desired airflows and resistances, but to "tune out" any objectionable noise or vibration that may have been inadvertently introduced during construction.

\subsection{OPERATIONAL CONSIDERATIONS}

\subsubsection{Operating Mode}

According to operational requirements, an air cleaning system may be operated full-time, part-time, or simply held in standby for emergency service. If processes in the building are operated only one or two shifts a day, the designer may have a choice between continuous operation and operation only during those shifts. He must evaluate the effects of daily starts and stops on the performance and life of filters and other components vs the higher power and maintenance costs that may be incurred by continuous operation. Experience has shown that, all factors considered, continuous operation of air cleaning facilities, perhaps at reduced flow during weekends and holidays, is generally the most satisfactory mode of operation for buildings in which radioactive operations are conducted. Unless ducts, filter housings, damper frames, and fan housings (i.e., the pressure boundary) are extremely leaktight, outleakage of contaminated dust into occupied spaces of the building may occur during shutdown periods.

Many facilities require standby exhaust or air cleanup systems that are operated only in the event of an emergency or redundant air cleaning facilities that are brought into operation when a parallel online facility is shut down because of failure or for maintenance. When designing stand by systems, the engineer must keep in mind the possibility of corrosion and filter and adsorber deterioration even when the system is not in use.

\subsubsection{Filter Change Frequency}

The principal costs in operating a high-efficiency air cleaning system are power (i.e., for fans), replacement filters and adsorbers, and labor. The principal factor that affects these costs is the frequency of 
changing filters (adsorbers). Replacement filters and adsorbers and labor costs may make up as much as $70 \%$ of the total cost of owning a system (including capital costs) over a 20 -year period. Power accounted for only $15 \%$ of the total owning costs in a study made by the Harvard Air Cleaning Laboratory. ${ }^{15}$ Measures such as the use of high-efficiency building supply-air filters, the use of prefilters ahead of HEPA filters, operation of the system below its rated airflow capacity, and operation of HEPA filters until they have reached high airflow resistance before replacement all tend to decrease filter change frequency and thereby reduce costs. These factors are discussed in the following sections.

\subsubsection{Building Supply-Air Filters}

Atmospheric dust brought into the building with ventilation air constitutes a substantial fraction of the dirt load in the building and the dust load in the exhaust air cleaning system. Removal of this dust before it gets inside the building has the double advantage of protecting the exhaust filters from premature dust loading and of reducing janitorial and building maintenance costs. When operations within a building do not generate heavy concentrations of smoke, dust, or lint, it may be possible, by providing medium-efficiency (50 to 65\% ASHRAE efficiency) ${ }^{16}$ building supply-air filters, to substantially reduce the dust loading in the exhaust system, thereby shifting much of the burden of what would otherwise be a change of "hot" (radioactive) prefilters in the exhaust system to a more economical change of "cold" supply-air filters. The labor costs involved in replacing "cold" filters is a small fraction of those for replacing "hot" filters.

Noticeable reductions in janitorial costs have been observed in several ERDA installations after changing to higher efficiency building supply-air filters. There is also a trend toward using better building supply-air filters in commercial buildings; one operator of a commercial office building reported that the time interval between major cleaning and repainting had doubled after replacing his original panel-type furnace filters with $60 \%$ ASHRAEefficiency filters. ${ }^{17}$

Louvers or moisture separators or both must be provided at the air inlet to protect the supply filters from the weather. Rain, sleet, snow, and ice can damage or plug building supply-air filters, resulting not only in increased operating costs but upset of pressure conditions within the building and possible impairment of the more critical exhaust air cleaning system. Heaters are desirable in the building supply system, even in warm climates. Icing has caused severe damage to building supply-air filters at a number of ERDA installations, even in the South. Screens should be provided over supply-air inlets located at ground or roof level to protect inlet filters and demisters from grass clippings, leaves, dirt, and windblown trash. If possible, inlets should be located well above grade or adjacent roofs so they are not burdened by such materials, preferably at the secondfloor level (or equivalent height above an adjacent roof) or higher.

\subsubsection{Prefilters}

HEPA filters are intended primarily for removal of submicron particles and should not be used as coarsedust collectors. They have relatively low dust-holding capacity, particularly for large particles and lint, and may plug rapidly when exposed to high concentrations of such material or smoke; lint may tend to bridge the pleats of the filter, even further reducing its capacity. The HEPA filter is also the most critical particulate-removal element in the air cleaning system from the standpoint of preserving containment, and its failure will result in a failure of system function. Prefilters, installed either locally at the entrances to intake ducts, in the central exhaust filter house, or both, extend the life of HEPA filters and provide at least a measure of protection against damage. Local duct-entrance filters also minimize dust accumulation in ducts and reduce an otherwise potential fire hazard. A typical increase in HEPA filter life through the use of prefilters is illustrated in Fig. 2.4. The increase for a specific application is, of course, dependent on the quality of the prefilter selected and the nature and concentration of dusts and particulate matter in the system.

Generally, prefilters should be provided when the potential dust concentration in the air leading to the air cleaning system exceeds 10 grains per $1000 \mathrm{ft}^{3}$ and should be considered if the dust concentration exceeds 1 grain per $1000 \mathrm{ft}^{3}$. The use of prefilters is recommended in engineered safety feature (ESF) systems for nuclear reactors. ${ }^{18}$ The decision to install prefilters should be based on providing the best operational balance between HEPA filter life, with its attendant decrease in HEPA filter change frequency, and procurement and maintenance costs for the prefilters. 

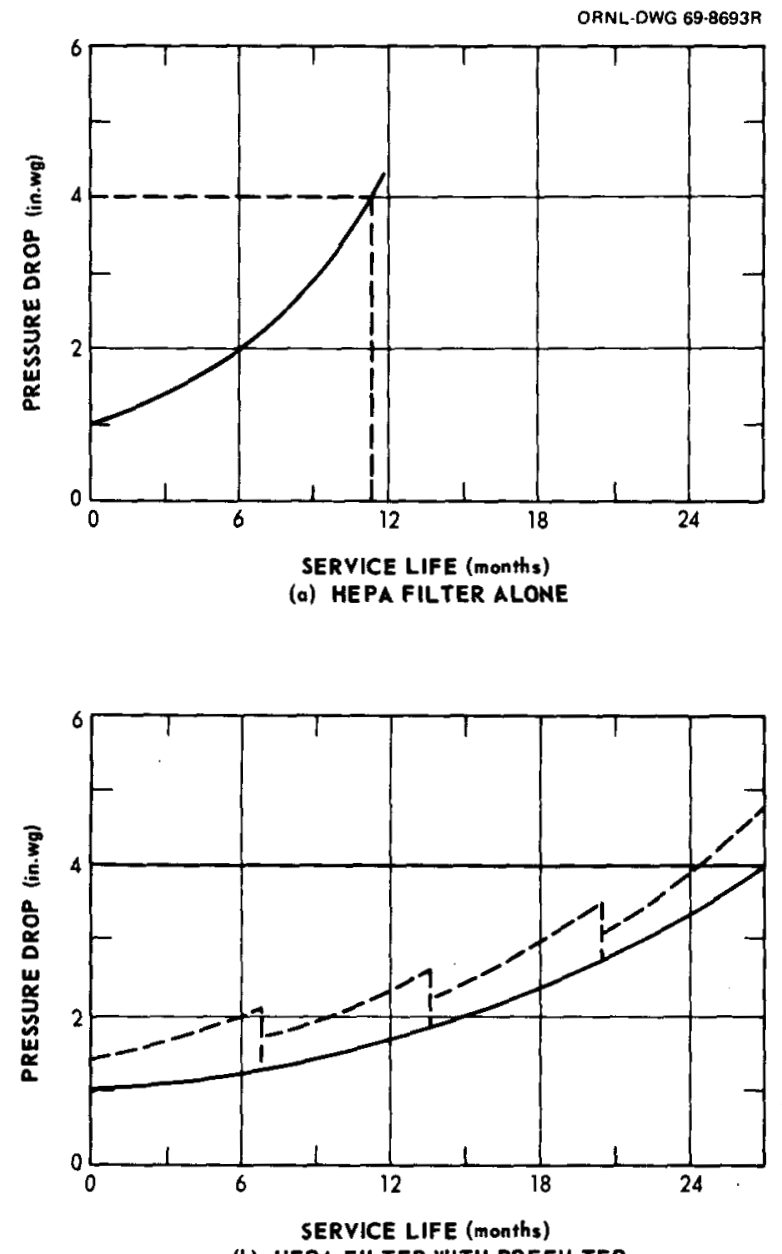

(b) HEPA FILTER WITH PREFILTER

Fig. 2.4. Comparison of HEPA filter life with and without prefilter. HEPA filter replaced at 4 in.wg pressure drop and prefilter replaced when pressure drop across it reaches twice the clean-filter pressure drop. From P.A.F. White and S. E. Smith, eds., High Efficiency Air Filtration, Butterworth \& Co., London, 1964.

Duct-entrance prefilters can be changed without entering or interrupting the central air cleaning facility, can minimize dust buildup in the ducts, and can provide a measure of protection against duct corrosion, accidental high-moisture loadings, and flaming trash or sparks that may be produced by a fire in the working space. On the other hand, a system that has a number of local prefilter installations may cost from two to three times as much as one in which the same prefilter capacity is installed in a central housing. ${ }^{15}$

Prefilters in a central air cleaning system shoúld not be attached directly to or installed back-to-back to HEPA filters; they should be installed on a separate mounting frame located at least 4 to $5 \mathrm{ft}$ upstream of the HEPA filters. This installation requires more building space and higher investment costs (particularly when building space is at a premium), but it is justified by increased safety and greater system reliability. Adequate space between prefilters and HEPA filters is needed for access and maintenance and to minimize the propagation of fire by sparks or direct flame impingement. If the possibility of fire is a serious consideration, a removable screen, fine enough to stop sparks ( 10 to 20 mesh), may be installed on the downst ream side of the prefilters.

\subsubsection{Operation to High Pressure Drop}

Most HEPA filter manufacturers' literature suggests the replacement of HEPA filters when the resistance due to dust loading has reached 2 in.wg. However, HEPA filters, by specification, are capable of withstanding a pressure drop, when clean, of at least $10 \mathrm{in} . w g$ without structural damage or reduction of efficiency ${ }^{19}$ (see Chap. 3). Replacement at a pressure drop of only 2 in.wg, when other factors such as radioactivity and fan capacity do not have to be considered, is underutilization of the filter. At many ERDA facilities, HEPA filters are operated routinely to pressure drops as high as 4 to 5 in.wg. Figure 2.5 shows the effect of such operation on filter life and maintenance costs.

The advantages of operating to high pressure drop must be weighed against first cost (higher-staticpressure fans, larger motors, and heavier ductwork), higher power costs, and less efficient fan operation. The installed fan and motor must have sufficient capacity to deliver the design airflow at the maximum differential pressure to which the system will operate, with the filters at maximum dirty-filter pressure drop prior to change. Consideration must therefore be given not only to the increased installed capacity required to operate to the higher pressure drop, but also to the fact that the fan operates at a penalty for much of the time to provide the required airflow over the wide span of pressure drop between installation and replacement of filters.

The cost of ductwork, on the other hand, may not be significantly affected by operation to a high pressure drop because there is a minimum sheetmetal thickness (18 gage recommended for shop operations, 16 gage for field operations) for effective welding, regardless of pressure requirements (see Chap. 5). The cost of fans and motors is a function of 
ORNL.OWG 69-8694R

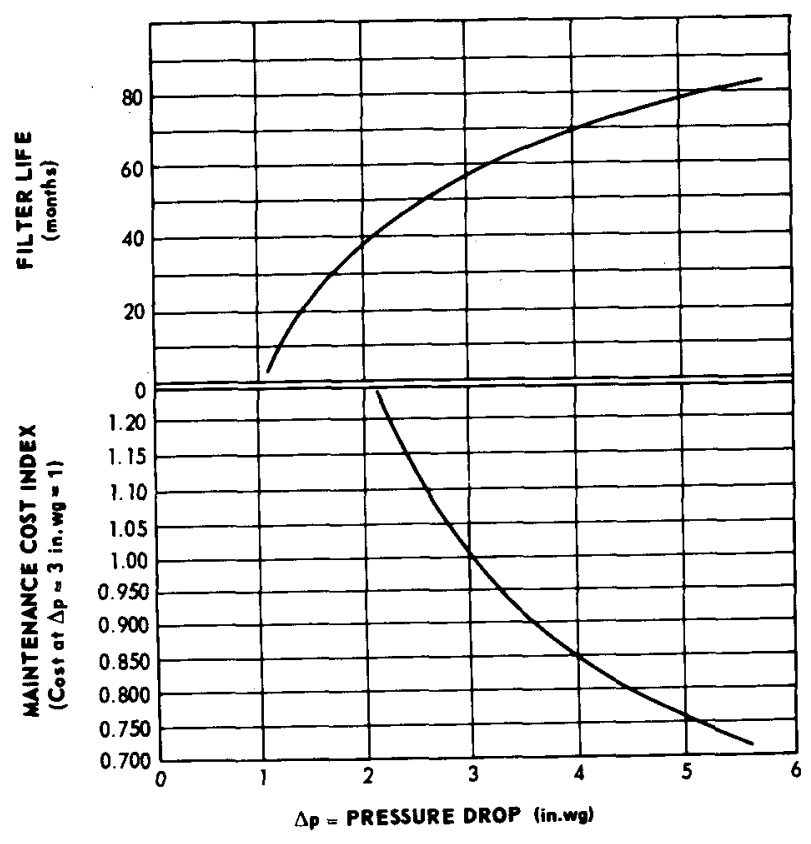

Fig. 2.5. Effect of operating HEPA filters to high pressure drop on filter life and maintenance cost (including replacement filters and labor). From W. V. Thompson, High Efficiency Particulate Filter History and Activities as of August, 1964, 117-B, $C, D R, F, H, K E$, and $K W$ Buildings, USAEC Report RL-REA1000, Hanford Atomic Products Operation, April 12, 1965.

the maximum total pressure that must be developed. Fan horsepower can be estimated from the equation ${ }^{20}$

$$
\mathrm{hp}_{f}=\frac{Q \Delta p}{6356 E_{f}},
$$

where

$$
\begin{aligned}
\mathrm{hp}_{f}= & \text { fan horsepower, } \\
Q= & \text { system airflow, cfm, } \\
\Delta p= & \text { maximum pressure drop across air cleaning } \\
& \text { system, in.wg, at time of filter replacement, } \\
E_{f}= & \text { fractional efficiency of fan ( } 0.60 \text { usually } \\
& \text { assumed for estimating). }
\end{aligned}
$$

Motor horsepower can be estimated from the equation ${ }^{20}$

$$
\mathrm{hp}_{m}=\frac{\mathrm{hp}_{f}}{E_{m}},
$$

where

$$
\begin{aligned}
\mathrm{hp}_{m}= & \text { motor horsepower, } \\
\mathrm{hp}_{f}= & \text { fan horsepower, } \\
E_{m}= & \text { fractional motor efficiency }(0.90 \text { usually } \\
& \text { assumed for estimating for } 20 \text {-hp motors and } \\
& \text { larger }) .
\end{aligned}
$$

Annual power costs can be estimated from the equation ${ }^{20}$

$$
C=\frac{Q \Delta p h r}{8520 \overline{E_{f}^{\prime}} E_{m}^{\prime}},
$$

where

$$
\begin{aligned}
& C=\text { annual power cost, dollars, } \\
& h=\text { hours of operation per year, } \\
& r=\text { cost of power, cents } / \mathrm{kWhr},
\end{aligned}
$$

$E_{f}^{\prime}$ and $E_{m}^{\prime}=$ efficiency of fan and motor, respectively, over the period of operation from filter installation to replacement; these will be less than the design efficiencies.

Although investment and power costs will be lower for the system operated to 2 in.wg pressure drop, the total annual cost of owning the system, including materials and labor costs for filter replacement, may be less for the system in which HEPA filters are replaced at pressure drops on the order of 4 to 5 in.wg. Total savings for the facility as a whole may be even greater when the reduced interruption of building operations due to the reduced frequency of filter change is taken into consideration.

Some prefilters can be operated to higher pressure drops than recommended by their manufacturers. In Great Britain prefilters are commonly operated to dirty-filter pressure drops of 3 to 4 in.wg. ${ }^{21}$ This results in less frequent prefilter change than if the prefilters are changed at a pressure drop of only two or three times the clean-filter pressure drop (usually 0.1 to 0.5 in.wg) recommended by most manufacturers. Care must be taken in the selection of prefilters. Because of the many types, efficiencies, configurations, and constructions available, the designer must specifically investigate the safe overpressure allowance for the particular model under consideration. Figure 2.6 shows clearly the results of overpressuring prefilters. In this case the problem of filter blowout was overcome by working with the manufacturer to reinforce the filter itself. Some benefit could also have been obtained by installing a screen or expanded metal grille on the downstream face of the prefilters against which the filter cores could bear; in any event, screens or grilles would have prevented damage to the HEPA filters when pieces of prefilter struck them.

\subsubsection{Underrating}

The service of all internal components, except moisture separators, can be extended and system 


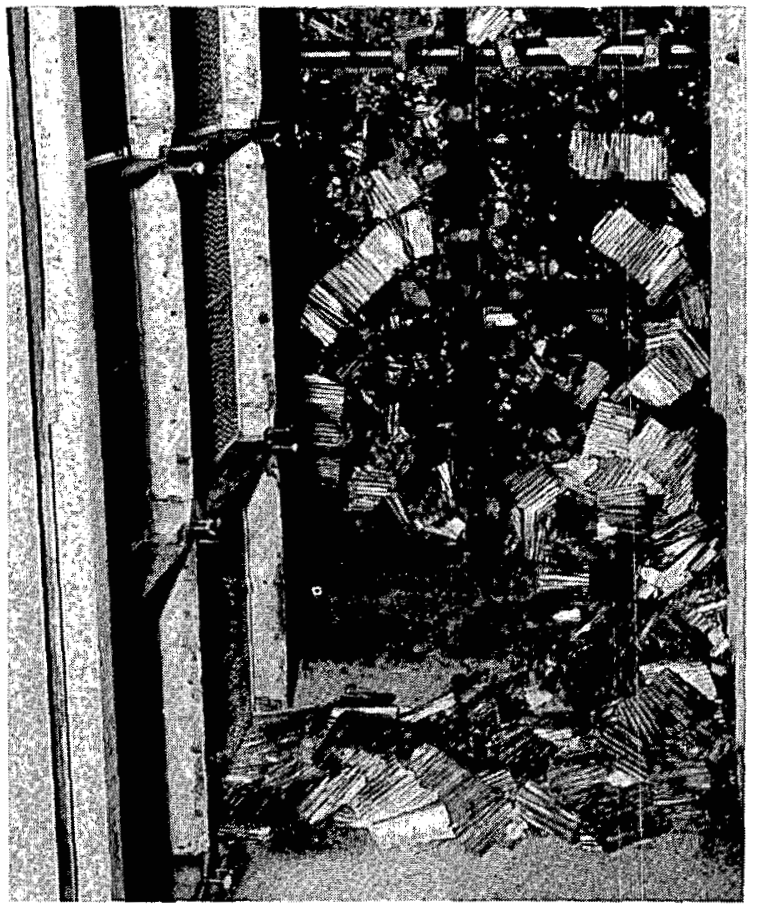

Fig. 2.6. Result of coverpressuring prefilters. Note damage to HEPA filters in, rear. Courtesy Union Carbide Corporation, Nuclear Division, 1 Y:12 Plant.

pressure drop for a given level of dust:loading can be reduced tby underrating - that is, by roversizing the system and installing more filter and adsotber capacity than is required, based on nominal airflow rating of those components, to meet system design airflow needs. Figure 2.7 shows that the increase in filter life obtainable by underrating is roughly proportional to the square root of the degree of underrating. A study by the Harvard Air Cleaning Laboratory suggests that the economic limit of underrating is about $20 \%$ (i.e., system design:airflow equal to $80 \%$ of installed airflow capacity): ${ }^{15}$

Overrating. The operation of a system at airflows greater than the installed airflow capacity of the system should be avoided, particularly in systems with radioiodine adsorbers whose performance is dependent on residence time of air within the adsorbent bed. When airflow rates exceed rated airflow capacity of HEPA;filters, filter life decreases more rapidly than the equivalent increase in iflow rate, as can be seen from the $120 \%$ curve in Fig. 2.7. As noted above, the residence time of contaminantladen air in adsorber units is inversely related to airflow rate; overrating of these units decreases their ability to trap gaseous contaminants and thereby degrades their function.

\subsubsection{Uniformity of Airflow}

In large air cleaning systems, because of the stratification of airflow due to poor transitions between ducts and housings or between housings and fans or because of poorly designed housings, filters or adsorbers at the center of a bank may receive higher airflow than those on the periphery of the bank. This not only results in nonuniform dirt loading of filters but may result in excessive penetration of those HEPA filters closer to the air intake if the degree of a airflow nonuniformity is great. Figure 2.8 shows that the penetration of HEPA filters by very small particles is directly velocity-dependent and increases significantly at very high airflow rates. Conversely, the penetration of HEPA filters by particles larger than $\mathbb{I}, \mu \mathrm{m}$ may increase at very low flow rates due to the reduction in effectiveness of the impaction mechanism on which trapping of those particles depends. If some filters are operating at very high airflow and some at very low airflow, as could happen in:a poorly designed housing and filter bank, it is possible that significant penetration could occur even though the filters are in good condition. Low flow rates improve the efficiency of radioiodine adsorbers, but high flow rates decrease efficiency, as discussed in the previous section. Therefore, significant nonuniformity of airflow through a bank of adsorber cells can reduce the overall efficiency for trapping radioactive gases of interest. Figure 4.28 shows a well-designed duet-to-housing transition

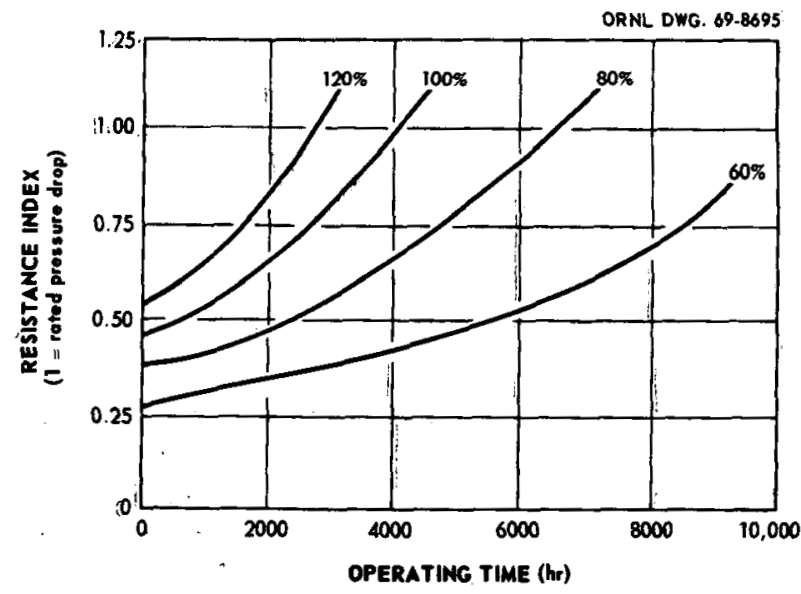

Fig. 2.7. Effect of underrating on service life of extendedmedium filters, based on percentage of manufacturer's rated filter airflow capacity. From P. M. Engle and C. J. Bauder, "Characteristics and Application of High Performance Dry Filters," ASHRAE Journal, American Society of Heating, Refrigerating and Air-Conditioning Engineers, May 1964, pp. 72-75. 


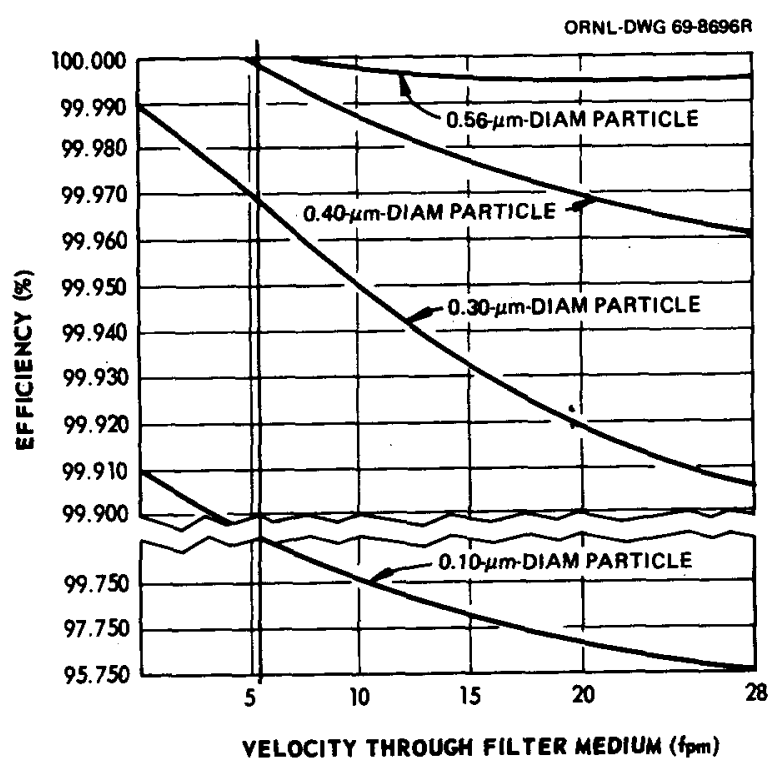

Fig. 2.8. Penetration of HEPA filter medium by submicron particles as function of flow rate through medium. Normal flow rate, at manufacturer's rated filter capacity, is approximately $5 \mathrm{fpm}$ (vertical line). From MSA Ultra-Aire Filters, Bulletin No. 1505-20, Mine Safety Appliances Co.

that will produce satisfactory airflow distribution through the banks of filters and adsorbers.

\subsubsection{Maintainability, Testability}

Maintenance and testing are two operational factors whose cost can be minimized by good initial design and layout of ventilation and air cleaning facilities. Inadequate attention to maintenance and testing requirements at the initial phase of the project can result in operating costs much higher than they should be. Two elements that largely influence the costs of these functions are the accessibility of components requiring periodic test and service and frequency of filter and adsorber replacement. In systems that involve the handling of radioactively contaminated filters and adsorbers, the frequency of changing these components and the time to accomplish the change can be especially critical, because the total integrated radiation dose a workman can be permitted to receive in each calendar period is limited. When all personnel have received their maximum permissible dose for the period, the supervisor faces the prospect of having no one available to carry out a needed filter change or a scheduled test. Maintenance and testing of radioactively contaminated systems is much more costly than the same operations in nonradioactive systems because of the time required for personnel to change into and out of protective clothing; to decontaminate and clean up the area, tools, and equipment after the operation; to dispose of contaminated filters; and to bathe and be monitored by health physicists. There is also the extra attention that must be given to filter or adsorber cell installation (as compared with common air filters, for example). If the system does not meet test requirements after the change, the work must be repeated. There is also a need for health physics monitoring before, during, and after the operation. The fact that personnel have to work in clumsy protective clothing, including respirator or full-face gas mask, also adds to the time required. Regardless of these inherently high time and money costs, proper maintenance and testing are primary factors in ensuring the reliability of the air cleaning system, and they cannot be done properly unless the physical facilities have been properly designed and built.

Frequency of Maintenance and Testing. All measures that reduce the frequency of filter (HEPA and prefilter) and adsorber replacement also reduce system costs and downtime. Several of the factors discussed earlier - the use of good building supply-air filters and prefilters, operation of HEPA filters to high pressure drop, and underrating-serve to extend component life and reduce the frequency and cost of service. Exhaust system HEPA filter and adsorber installations must be tested after each component change so that any extension of service life also directly reduces testing costs.

Accessibility. When laying out ventilation and air cleaning facilities the designer must consider the location of fans, dampers, instruments, and filter housings and working space adjacent to them; working space and spacing of banks within manentry housings; height and array of filter and adsorber banks; and routes to be used for moving new and used filters and adsorbers between storage, installation, and disposal areas. Failure to provide adequate space in and around housings and mechanical equipment (fans, dampers, etc.) results in high maintenance and testing costs, inhibits proper care and attention, creates hazards, and increases the chance for the accidental spread of contamination during service or testing operations. Recommendations for the arrangement and space requirements for air cleaning components are given in Chaps. 4, 6, and 7. Even greater space requirements are needed for remotely maintainable systems. 
Ease of Maintenance. Simplicity of maintenance and testing is a primary factor in minimizing the time personnel must remain inside a contaminated housing and restricted areas of a building during a filter or adsorber change or test and is, therefore, an important factor in reducing both personnel exposures and costs. Simplicity of maintenance and testing is achievable through the following means:

1. A housing layout that minimizes reaching, stooping, and the use of ladders or temporary scaffolding for gaining access to filter or adsorber cells. Some reaching and stooping are unavoidable in man-entry housings, but it should not be necessary for workmen to go through physical contortions or climb ladders to femove and replace filters in single-filter installations. Similarly, in bank systems it should not be necessary for workmen to climb ladders or temporary scaffolding to gain access to the upper tiers of filters or adsorbers.

2. Adequate finger space $(1 \mathrm{in}$. minimum is desirable) between filter elements and provision of handles on heavy components such as adsorber cells.

3. Cradles or benches built into the component mounting frame for aligning and supporting filters (adsorbers) prior to clamping to facesealed mounting frames (see Sect. 4.3.5).

4. Simple filter and adsorber clamping devices. A properly designed bolt-and-nut clamping system has proven most satisfactory in the past, although numerous methods of minimizing or eliminating loose parts are currently being investigated. Toggle clamps, over-center latches, and other devices are easily manipulated and require no tools; however, they often tend to jam, become difficult to operate, or lose their ability to properly clamp the filter or adsorber cell after extended exposure to the hostile environment of a contaminated air cleaning system. Such devices should be used only after due consideration of the difficulties that would be involved in replacing them in a contaminated system (see Sect. 4.3.4).

5. Elimination of ledges and sharp corners that a workman might stumble over or might snag or tear his protective clothing on.

6. Adequate lighting in and adjacent to the filter house and adjacent to other items that require periodic service, inspection, or testing.
7. Provision for communication between personnel inside and outside the filter house (e.g., communication ports or portable talker system).

8. Floor drains in housings and adjacent work spaces to facilitate easy removal of water spilled or applied during decontamination of the area after a filter or adsorber change. Drains must be designed so that no air can bypass filters or adsorbers.

9. Availability of electrical, water, and compressed air connections nearby, but in no case inside, the filter house.

10. Materials-handling facilities, including dollies for moving new and used filters and adsorbers, hoists or other means of handling the heavy adsorber cells in systems containing those components, and elevators or ramps for moving loaded dollies up and down within the building.

11. Location of filter housings inside the building. It is undesirable for personnel to (a) conduct a filter change or test out of doors where wind or rain may cause a spread of contamination, (b) cross a roof to gain access to a filter house, or (c) wait for good weather to carry out a scheduled filter or adsorber change or test.

12. Rigid, double-pin-hinged doors on man-entry housings large enough for personnel to pass through without excessive stooping or twisting. It should not be necessary to remove several dozen nuts from a hatch to gain entry to a manentry or single-filter housing. Not only is this too time consuming, but nuts tend to cross-thread or gall to the extent that it is often necessary to cut off the bolt to open a hatch; or the nuts get dropped and lost and are often not replaced, thus compromising the seal of the hatch. Sliding doors are not suitable because they will jam with any distortion of the housing wall (see Sect. 4.5.7) and are difficult to seal.

13. Nearby decontamination and clothing-change facilities (including showers).

14. Well-planned and rehearsed maintenance and testing procedures.

15. Adequate space for materials and test equipment and access (through preplanned doors or panels) to both sides of filter and adsorber banks.

16. Preplanned test-agent injection ports and sampling ports (see Chap. 8). 
Construction. The design for maintainability requires careful attention to details of construction, including tolerances, surface finishes, and the location of adjacent equipment and service lines. Ducts and housings should have a minimum of interior ledges, protrusions, and crevices that can collect dust or moisture or that can impede personnel or create a hazard in the performance of their work. Provision of relatively high efficiency (40 to 65\% ASHRAE) prefilters at duct inlets will minimize the accumulation of dust and contamination in the ducts. If these are not provided, easily opened ports and hatches for inspection and cleaning must be provided at strategic and accessible locations in the duct. Duct runs should have enough mechanical joints to permit easy erection and dismantling. Otherwise, replacement of radioactively contaminated ducts can be an expensive and hazardous job.

Housings, ductwork, and component mounting frames must be able to withstand anticipated system pressures and shock loadings without distortion, fatigue, or yielding that permits inleakage or bypassing of the filters or adsorbers. Ability to meet an air pressure test of $\pm 12 \mathrm{in}$. $\mathrm{wg}$ for $\mathrm{I} \mathrm{hr}$ without distortion or excessive leakage (see Table 5.6) is often specified.

Interior surfaces and finishes warrant special attention. Regardless of the formulation when coatings are used, a primary factor in long and dependable service life is proper preparation of the surface to be coated. Coating (or paint) manufacturer's instructions must be followed exactly. All coated or painted metal surfaces that will be exposed to contaminated air and gases should be grit-blasted to white or near-white metal. The first primer coat should be applied as soon as possible, preferably within $4 \mathrm{hr}$, after completion of grit-blasting. Coatings should be thoroughly cured and ducts and housings completely purged of paint and solvent fumes before installation of adsorber cells or filling of permanent fill-in-place adsorbers.

\subsection{SYSTEM CONFIGURATION - NOMENCLATURE}

The design, manufacture, installation, testing, operation, and service of nuclear air cleaning facilities involve the efforts of many people of different backgrounds. There has been a tendency on the part of some users to employ different terms for essentially the same items when used in different applications. The result is that similarities of basically similar systems or items are obscured, and experience gained in one situation is not transferred to another. Use of common terminology helps to alleviate this lack of communication. To promote consistency and common understanding, the terms defined in this section are suggested for describing the configuration and arrangement of such systems and their parts.

\subsubsection{Component}

A component is a filter, adsorber cell, fan, damper, or other basic element of an air cleaning system which cannot be disassembled without nullifying the capability of performing its designed task. Components are often designated by the function they perform (e.g., filter, demister). Items purchased by manufacturer's name and catalog number are generally components. Internal components are normally enclosed within the system or a housing, including such items as filters, demisters, adsorber cells, and heaters. Major internal components are discussed in Chap. 3. External components include those that form part of the pressure boundary of the system and those (such as instruments) that are installed externally to the pressure boundary of the system. Major external components, such as ducts, dampers, fans, and instruments, are discussed in Chap. 5. Housings are discussed in Chaps. 4 and 6.

\subsubsection{Air Cleaning Unit}

An air cleaning unit is an assembly of components which comprises a single subdivision of a complete air cleaning system, including all components necessary to perform the air cleaning function of that subdivision. Such a unit includes a single-filter housing and its internal components. In a parallel or branched system, the unit includes all components in a single branch of the system. This conforms with the terminology of ANSI N509.

\subsubsection{Air Cleaning System}

An air cleaning system is an assembly of one or more air cleaning units (see Sect. 2.4.2) plus all external components needed to convey air or gases. from one or more intake points, through the air cleaning units, to one or more points of discharge. The system may be either recirculating, in which the cleaned air is returned to the space from which the contaminated air was drawn or to a space subject to greater contamination, or once-through, in which the cleaned air is discharged directly to the atmosphere, for an exhaust system, or to a building space in the case of a supply system. 


\subsubsection{Ventilation System}

The ventilation system includes the total facilities required to supply air to, circulate air with $\mathbf{n}$, and remove air from a building or building syace by natural or by mechanical means. The ventilation system may or may not include air cleaning, airconditioning, or heating facilities.

\subsubsection{Filter or Adsorber Bank}

A filter or adsorber bank is an assembly of two or more internal components of the same kind, in parallel, within the same housing.

\subsubsection{Array}

An array is the arrangement of internal components in a bank, expressed as the number of components across the width of a bank times the number high (e.g., a 4 by 3 array of HEPA filters).

\subsubsection{Air Cleaning Stage}

An air cleaning stage is a single component or a bank of identical components in an air cleaning unit or an air cleaning system. A system that has one bank of components (e.g., HEPA filters) in each of three air cleaning units, arranged in parallel, is a single stage system. A multistage unit or system has two or more stages in tandem.

\subsubsection{Installed Capacity}

Installed capacity is the manufacturer's rated airflow capacity of a bank or stage of the same kind of components, expressed as the number of components (in the bank or stage, respectively) times the rated airflow of the individual components.

\subsubsection{Single-Component Air Cleaning Unit}

A single-component air cleaning unit is one in which there is only one component (HEPA filter, prefilter, etc.) per stage, as opposed to a bank installation in which there are two or more components per stage.

\subsubsection{Single-Path System}

A single-path system is one in which the total installed capacity of the air cleaning system is installed in a single air cleaning unit (see Sect. 2.4.2).

\subsubsection{Parallel System}

A parallel system has two or more air cleaning units in parallel (see Fig. 2.9).

\subsubsection{Segmented System}

A segmented system is a parallel configuration in which the installed capacity necessary to meet system design airflow requirements has been subdivided into two or more parallel air cleaning units (see Fig. 2.9).

\subsubsection{Redundant System}

A redundant system is a parallel system (Sect. 2.4.11) that contains one or more independently operable air cleaning units (Sect. 2.4.2) which provides excess system design airflow capacity, either in multiples of the system design airflow capacity or in integral fractions of that capacity (see Fig. 2.9).

\subsubsection{Branched System}

A branched system is a parallel configuration having a common entrant duct or inlet, a common discharge duct, or both (see Fig. 2.9).

\subsubsection{Isolable Unit}

An isolable unit is an air cleaning unit which, by means of dampers, backflow preventers (dampers), fan location, or system layout, can be isolated from other units that comprise the system and which can be operated simultaneously with, or alternatively to, the other units that comprise the system.

\subsubsection{Compartmented Unit}

A compartmented unit is an air cleaning unit in which stages of components are installed in individual compartments in series, as illustrated in Fig. 2.10 . 


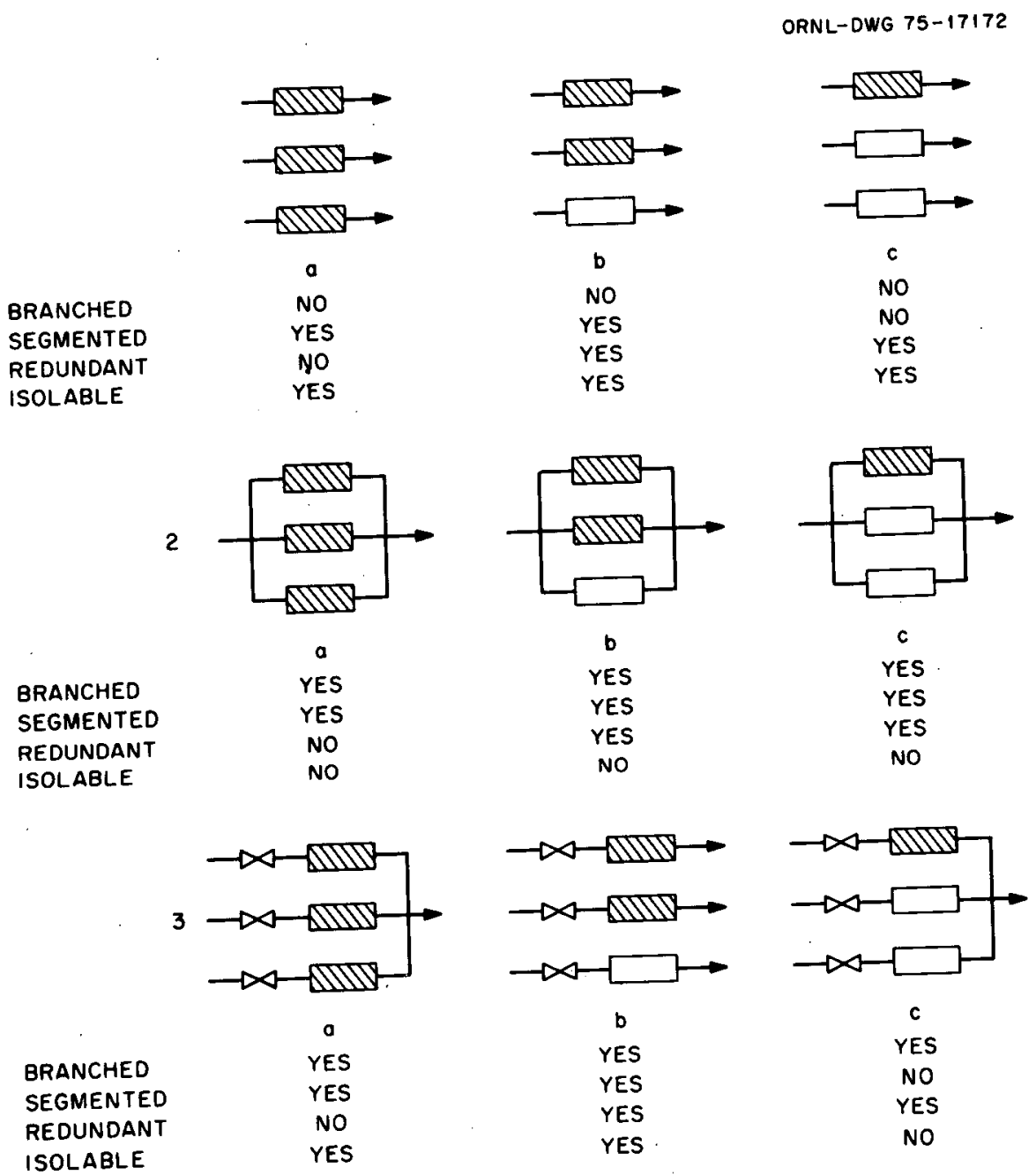

Fig. 2.9 Properties of some parallel air cleaning system configurations.

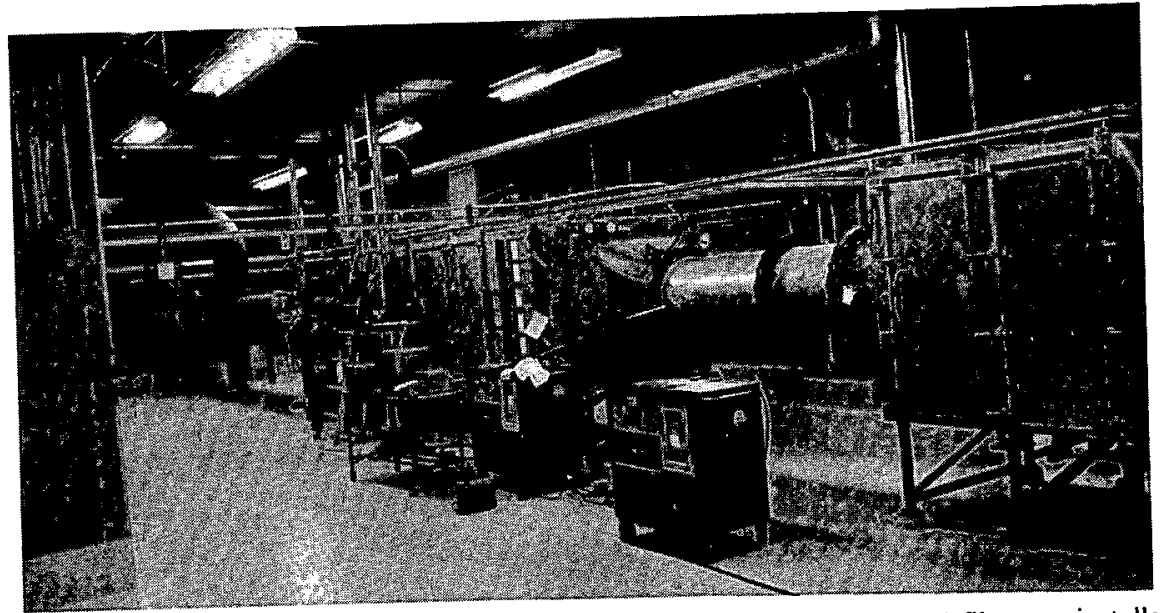

compartmented air cleaning system. Demisters, prefilters, and first-stage HEPA filters are installed in the compartment Fig. 2.10. Series-compartmented air cleaning system. Demisters, prefliters, compartment on the right. on the left; adsorber cells and second-stage HEPA filters are installed in the compartment on the right. 


\subsection{EMERGENCY CONSIDERATIONS}

The ventilation and air cleaning systems of a building in which radioactive materials are handled or processed are integral parts of the building's containment. In some cases, these systems may be shut down in the event of an operational upset, power outage, accident, fire, or other emergency; in other cases they must remain operational to maintain the airflows and pressure differentials between building spaces and between the building and the atmosphere, which are needed to maintain containment. In some cases, airborne radioactive material may not be a problem until an emergency occurs. In all of these cases, a particular danger is damage to or failure of the final HEPA filters (and adsorbers in those facilities where radiolytic gases could be released) that constitute the final barrier between the contained space (hot cell, glove box, room, or building) and the atmosphere or adjacent building spaces. Even if the system can be shut down in the event of an emergency, protection of the final filters is essential to prevent the escape of contaminated air to the atmosphere or to occupied spaces of the building.

Some damage to or degradation of the ventilation system, the final filters, or the adsorbers is probably not completely avoidable in the event of a serious incident. Consideration must be given to (1) the possible effects of operational upsets, power outages, accidents, fires, and other emergencies on the ventilation and air cleaning systems, including damage to the filters and adsorbers from shock, overpressure, heat, fire, and high sensible-moisture loading; (2) the design and arrangement of ducts and air cleaning components to alleviate these conditions; (3) means of switching to a redundant air cleaning unit, fan, or alternate power supply; and (4) methods of controlling the exhaust system during failure conditions. To provide the necessary protection to the publiciand to plant personnel, air cleaning and ventilation system components on which containment leakagecontrol depends must remain essentially intact and serviceable under the upset conditions. These components must be capable of withstanding the differential pressures, heat, moisture, and stress of the most serious accident predicted for the facility, with minimum damage and loss of integrity, and they must remain operable long enough to satisfy system objectives.

\subsubsection{Shock and Overpressure}

Mechanical shock in an air cleaning system can be produced by explosion in an operating area of the building, by an earthquake, or by rapid compression or decompression of the air inside a system caused by sudden opening or closing of a damper or housing doors. When pressure transients last for periods measurable in seconds, static pressure is primarily responsible for any destructive effect. For shocks that have a duration of only a few milliseconds with nearly instantaneous pressure rise, as occurs in most chemical explosions, destructiveness is primarily a function of the momentum of the shock wave. Shocks produced by an earthquake or inadvertent opening or closing of a damper usually fall somewhere between these two extremes. Protection of the final filters and adsorbers against failure from shock can be accomplished by isolating them to prevent the transmission of forces to them and by increasing the shock resistance of ducts, housings, mounting frames, and equipment supports. The shock resistance of HEPA filters can be enhanced by face guards (see Sect. $3.2 .4),{ }^{22}$ and similar treatment may sometimes improve the shock resistance of prefilters. Most prefilters used today, however, probably have low shock and overpressure resistance, and a screen installed between them and the HEPA filters is recommended to prevent the condition shown in Fig. 2.6. Adsorbers, both unit-tray and permanent singleunit (PSU) types (see Sect. 3.4), are generally of a robust construction that should be relatively unaffected by shock loadings if properly installed. Filter and adsorber mounting frames and housings designed in accordance with recommendations in Chap. 4 will probably have adequate shock resistance for most applications. The difference in the ability of the two fan installations, shown in Fig. 2.11, to withstand a substantial degree of shock is readily apparent.

Protection of the primary air cleaning components can be achieved by providing sharp turns, heavy perforated plates, or cushion chambers in the ductwork to "snub" shock forces and by using fastacting isolation dampers. Although turning vanes, dampers, moisture separators, and prefilters may be damaged by a shock wave, they may also serve to attenuate its force to some degree and thereby provide a measure of protection to the HEPA filters downstream. Damage to dampers, however, can result in inability to control flows or isolate branch lines. Sand filters are employed in some ERDA facilities for protection of the final filters and to prevent loss of containment in the event of explosion, earthquake, tornado, fire, or shock. As discussed in Chap. 9, sand filters are large deep beds of graded sand and gravel, installed in underground concrete enclosures. In some cases they are employed as final 
filters. Because their submicron particle-collection efficiency probably does not exceed about $99.7 \%$ and because some leakage may occur in the event of an explosion or other shock, they should be backed up by HEPA filters. On the other hand, airflow is upward through the bed, and leakage should be only momentary because of the great mass of sand and gravel comprising the filter and the disposition of the disturbed sand to fall back to heal the breach. This large mass of sand and gravel also provides a substantial heat sink in the event of fire in a ventilated space. The disadvantages of sand filters are very high first cost and high pressure drop. $^{23}$

Explosion in an operating area of a building is probably the most likely type of shock-generating incident that one can expect in radiochemical,
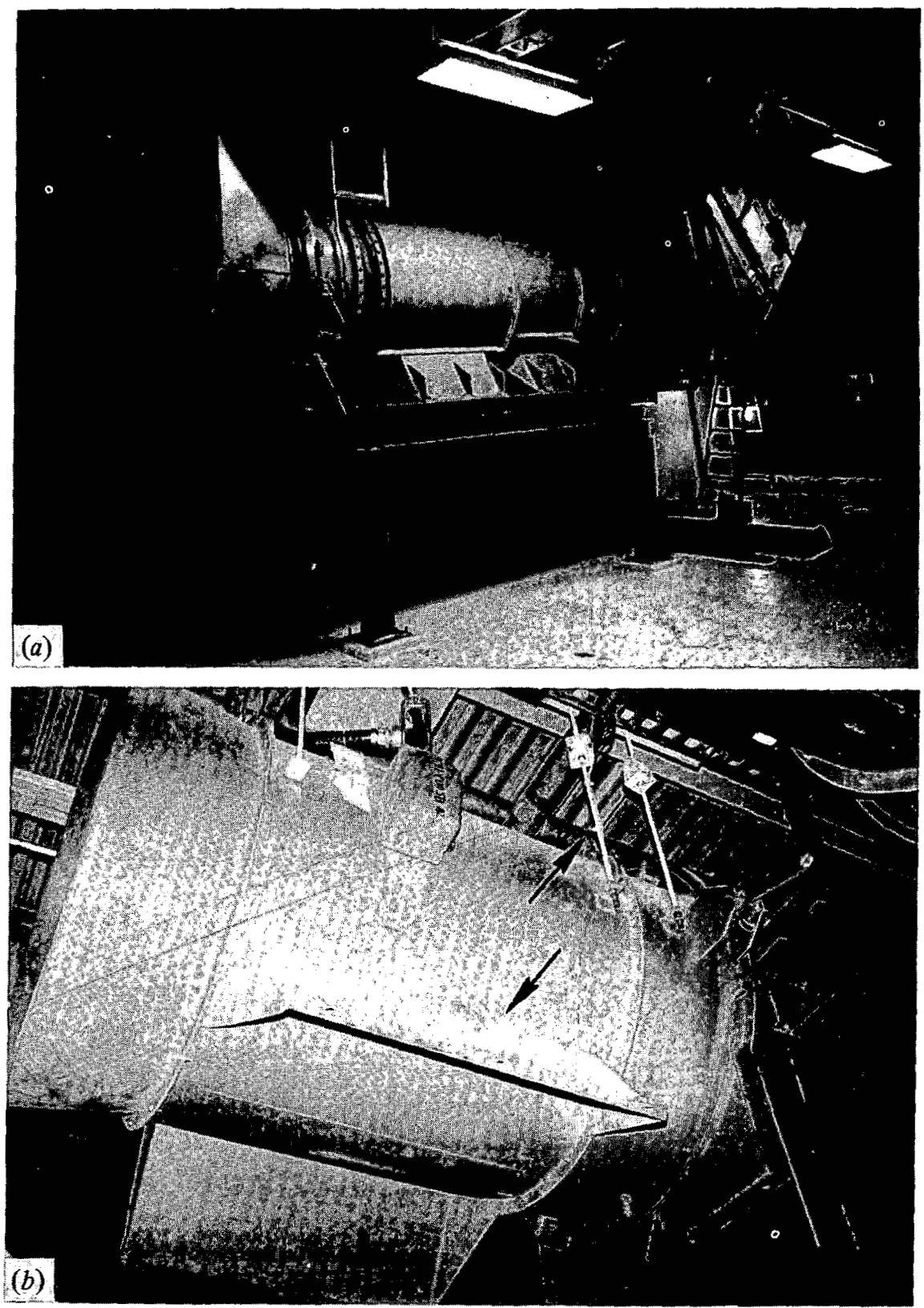

Fig. 2.11. Methods employed for installing axial-centrifugal fans in different nuclear reactor ESF air cleaning systems. (a) Shockresistant base-mounted fan; $(b)$ hanger-rod supported fan. Note anchor plates provided by fan manufacturer, but not used. 
laboratory, and experimental facilities. A chemical explosion is no more than a rapidly burning fire and therefore, in a confined space, can be arrested if a suppressant can be introduced quickly enough. Fluorocarbon gases such as halon-1301 (bromotrifluoromethane) have been found effective for this purpose in many situations. A concentration of 15 to $20 \%$ halon-1301 is required to suppress an explosion, after which a concentration of only 3 to $4 \%$ is needed to prevent reignition. In operation, an extremely sensitive pressure-sensor signals an explosive-actuated release valve on the halon cylinder installed in the contained space. Tests have confirmed that an incipient explosion can be suppressed in less than $60 \mathrm{msec}$ with a maximum pressure rise in the contained space (a glove box in the test) of 2 psi. ${ }^{24,25}$ Halon-1301 is particularly attractive for glove box and hot cell applications except where pyrophoric metal dusts or chips are being handled.

\subsubsection{Fire and Hot Air}

Fire in activated-carbon adsorption systems has received considerable attention in the last few years, but the possibility of fire in other components of the air cleaning system, such as ducts and filters, has been largely overlooked, except by the national laboratories and other ERDA prime contractors. ${ }^{26}$ Adequate provision for the detection and control of duct and filter fires has been the exception rather than the rule. Fires in glove boxes, hot cells, laboratories, and other nuclear containment facilities pose special difficulties to air cleaning and ventilation systems because of the need to contain airborne radioactive material during the emergency. The release of contaminated smoke through a ruptured HEPA filter or other breach of the containment building may have more serious consequences than any potential casualty losses from the fire itself.

Where it is possible to completely isolate and seal off the contained space within a building in which a fire occurs, or the building itself, including the ventilation system, consideration of effects of the fire on the filters and other air cleaning components may not be so important. In most facilities, however, it is essential to maintain some degree of airflow through the exhaust system in order to maintain the pressure differentials necessary to prevent backflow of contamination to occupied spaces of the building. An exhaust system also provides a vent for relief of pressure and hot air and a means for removing radioactive and potentially radioactive smoke before the air is released to the atmosphere. In these cases, the effect of the fire on the air cleaning system and its components becomes an important consideration. In many cases the final filters and fan, or a redundant set of filters and fan, must be operable during and following an emergency. If the building is zoned to control airflow from areas of less hazard to areas of greater hazard, pressure differentials between zones must be preserved to prevent pressurization of the contained space in which the fire occurs and to prevent backflow of contamination. Even when the ventilating system can be shut down in the event of a fire, protection of the final filters is important to provide for cleanup of the contaminated air in the building after the fire has been extinguished.

Five hazards to the final air cleaning unit which arise from fire situations are (1) heat and hot air that can (a) damage filters, (b) ignite dust accumulated in ducts or filters, and (c) distort metal parts to the point that filters are bypassed or fans and dampers are made inoperable; (2) sparks and burning trash that can ignite dust and melt holes in the filter media;(3) smoke that can plug demisters, prefilters, and HEPA filters to the point that system airflow is seriously reduced and/or the filters are ruptured due to the increased resistance; (4) overpressure due to air expansion which, when coupled with smoke plugging, can lead to rupture of the filters; and (5) droplets of spray from sprinklers or particles of other types of fire extinguishing agents, which can perforate filter medium, plug filters, or lead to reduction of their structural properties.

The first line of defense against duct and filter fires is the development and enforcement of safe operating practices in the contained and operating spaces served by those ducts and filters. This means eliminating one or more of the basic fire elements-fuel, ignition source, or oxygen. It includes control over the kinds and quantities of combustible liquids and gases in contained spaces; control of hot plates, burners, furnaces, and other sources of heat or flame; and sometimes, for totally contained spaces such as glove boxes or hot cells, inerting of the box or cell environment with nitrogen, argon, $\mathrm{CO}_{2}$, or other gas. Safe operating practices also include (1) the development and rehearsal of preplanned fire- and damage-control procedures in the contained and occupied spaces of the building and (2) means for rapidly detecting and suppressing a fire. To date there has been no case of loss of containment due to fire in any ERDA facility equipped with a properly designed sprinkler system. ${ }^{27}$ 
Conventional fire protection practices, such as the provision of fire dampers in ducts where they pass through a fire wall or floor, are sometimes ignored or discounted in the design of nuclear ventilation and air cleaning systems on the basis that they are not needed. Such established fire protection practices should not be omitted without careful consideration of the consequences. In most cases, customary fire protective design practices should be followed in the design of air-conditioning and ventilation facilities for "cold" (Zone IV) regions of the building.

\subsubsection{Power and Equipment Outage}

The design for emergency must plan for the probable occurrence of power and equipment (particularly fan) failures. Such failures, if not properly planned for, can result in a contamination hazard to the public or operating personnel, particularly in buildings with zone ventilation where airflow must be maintained to preserve pressure gradients between zones and to prevent backflow of contaminated air to occupied spaces. Possible emergency measures include redundant fans, redundant fan motors (perhaps served from independent power sources), and alternate power supplies (e.g., steam turbine or emergency diesel-electric generator). Where continuous airflow must be maintained, facilities for rapid automatic switching to an alternate fan, power supply, or emergency source, or to a standby air cleaning unit, are essential. However, if brief interruptions of flow can be tolerated, manual switching may be permissible at less expense. In any event, visible and audible alarms should be provided, both locally and at a central control station, to signal the operator when a malfunction has occurred. In addition, indicator lights to show the operational status of fans and controls in the system should be provided in the central control room.

\subsubsection{Air Cleaning System Layout Considerations}

The layout and location of air cleaning facilities can have a direct bearing on the system's capability of effecting control under upset conditions and of limiting the adverse consequences of such an upset.

Compartmentation and Segmentation. A higher degree of control is required in the event of a fire, explosion, equipment outage, or other system upset if the air cleaning system is segmented or if the individual air cleaning units are compartmented. Segmentation permits isolation of a damaged unit and minimizes the chance that the entire system will become inoperable at the same time. Series compartmentation (see Fig. 2.10) is employed in some potentially high-risk applications to permit further isolation of the less critical air-pretreatment facilities (demisters, prefilters) from the more critical final HEPA filters and adsorbers. Series parallel arrangement of a central exhaust filter system that handles high-specific-activity alpha-emitting materials is shown in Fig. 2.12. In the event of fire or equipment damage in any one housing of this system, in the prefilters, or in the HEPA filters, the housing can be isolated and the remainder of the system kept in service. Also, any one of the housings can be isolated for testing or filter change (under normal operating conditions) without interruption of work being conducted in the building. NRC Regulatory Guide 1.52 recommends that the installed capacity of any one air cleaning unit be no greater than $30,000 \mathrm{cfm}$ to permit more effective control in the event of an emergency and to permit more reliable surveillance testing of the HEPA filter and adsorber stages of the unit. ${ }^{18}$

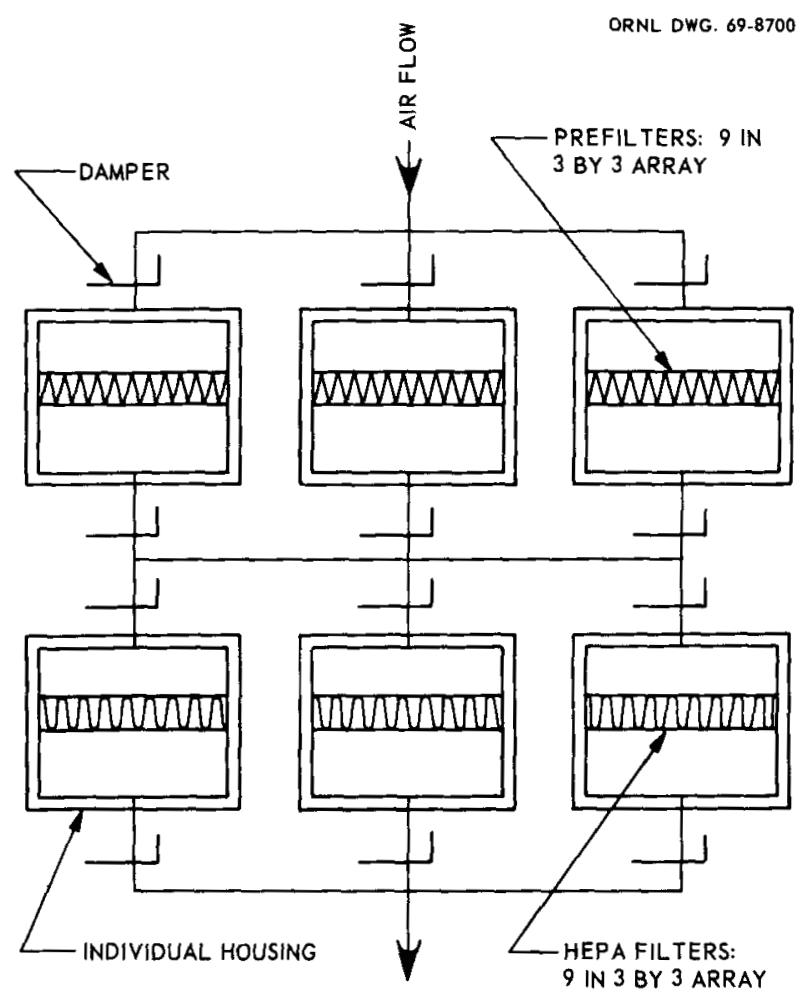

Fig. 2.12. Series-parallel arrangement of central exhaust filter system of a high-hazard radiochemical laboratory. Note dampers that permit isolation of any housing without stopping exhaust airflow. 
Redundancy. Redundant air cleaning facilities are often required in potentially high-risk operations, such as reactors and radiochemical plants, to ensure continuous ventilation in the event of failure of an online air cleaning unit. In the case of reactor postaccident cleanup systems, redundant air cleaning units are required even though the system is normally in a standby condition. Figure 2.13 shows the segmented, redundant, normal off-gas and buildingexhaust air cleaning systems of an experimental water-cooled reactor with vented containment. Of the two units of each system which are normally online, one is capable of meeting exhaust requirements when the building supply fans are shut down in the event of an emergency. High-quality isolation dampers are essential in redundant systems not only to protect the off-line units when not in service but to prevent bypassing of the air cleaning system through a damaged off-line air cleaning unit.

Location of Air Cleaning Facilities. The location of filters, fans, and other air cleaning components can play a major part in minimizing component damage and spread of contamination in the event of a fire, system upset, or other emergency. A common but undesirable practice has been to install such items in random locations in attics or unused building spaces. Figure 2.14 illustrates a type of filter installation in which a wood-cased filter was simply clamped between two flanged duct transitions in an open attic

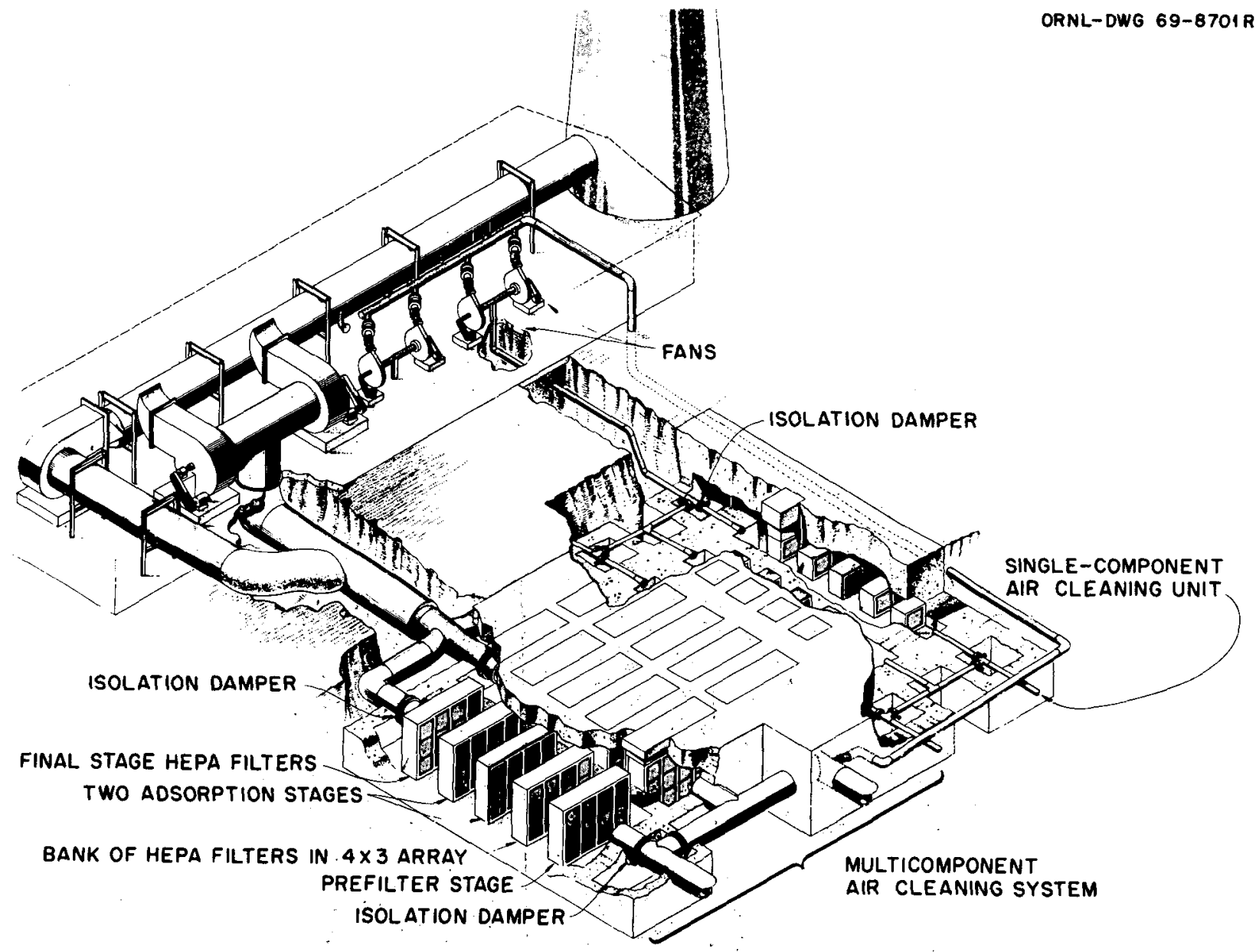

Fig. 2.13. Air cleaning facilities of an experimental reactor, illustrating application of the suggested nomenclature for nuclear air cleaning systems. Two parallel air cleaning systems are shown, one a multicomponent installation, the other a single-component installation. The components of the multicomponent system are installed in banks arranged in a 4 by 3 array; the system has an installed capacity of 36,000 $\mathrm{cfm}$, with $12,000 \mathrm{cfm}$ installed capacity in each of the three air cleaning units. In each of the systems, two units are capable of meeting normal system airflow needs, and one is capable of meeting airflow requirements under upset conditions; all units can be isolated by means of the isolation dampers. The systems are segmented, redundant, isolable, and branched. 
space. There is no floor or catwalk adjacent to the filter, with the danger that service personnel risk falling through the ceiling to the room below, and access is limited by the adjacent hangers and ducts. Furthermore, because the location is in an open attic space, dropping a used filter during a filter change, or

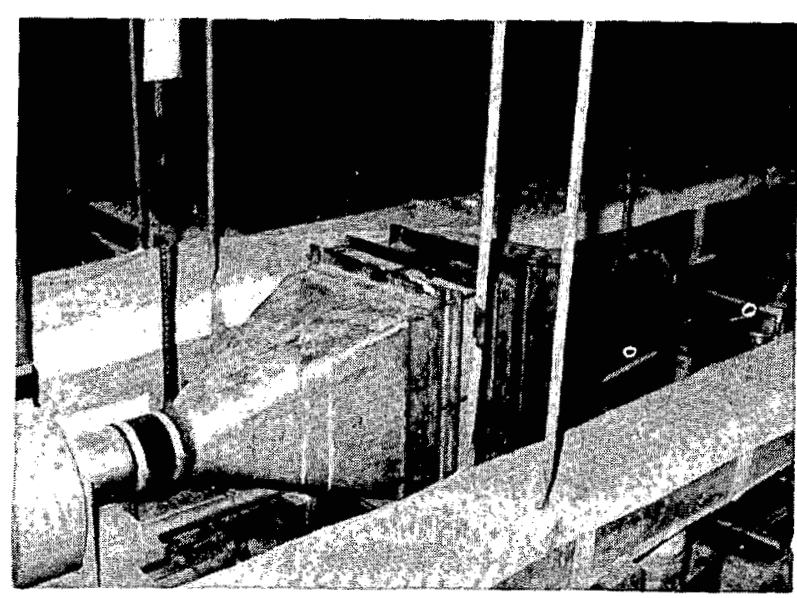

Fig. 2.14. An illustration of poor filter installation practice. Note unprotected wood-cased filter simply clamped between two transitions; difficult access for service; location in open attic; lack of floor or catwalk. breach of the wood filter case in the event of a fire, would result in the spread of contamination throughout the entire attic, which would be difficult, if not impossible, to clean up. In-duct installations of this type, in which the wood filter case is part of the pressure boundary, do not conform with NFPA $90 \mathrm{~A}^{28,29}$

Figure 2.15 illustrates another example of poor filter installation and location. The location of the light troffer indicates that the air cleaning unit (which is provided for control room ventilation in a nuclear reactor) is located about $20 \mathrm{ft}$ off the floor, and access is seriously impeded by hangers, cable trays, piping, and other equipment. This unit is a wood-cased chemical, biological, radiological (CBR) filter which, like the filter installation shown in Fig. 2.14, does not comply with NFPA 90A. Again, this unit is located in an open and normally occupied building space where a serious spread of contamination could result if the filter were dropped during service or breached in an accident or fire. Furthermore, fire external to the filter could also breach the filter case and permit contamination to spread from the room to other portions of the building. Figure 2.10 illustrates better practice by showing an air cleaning facility installed in a large room that can be isolated as a

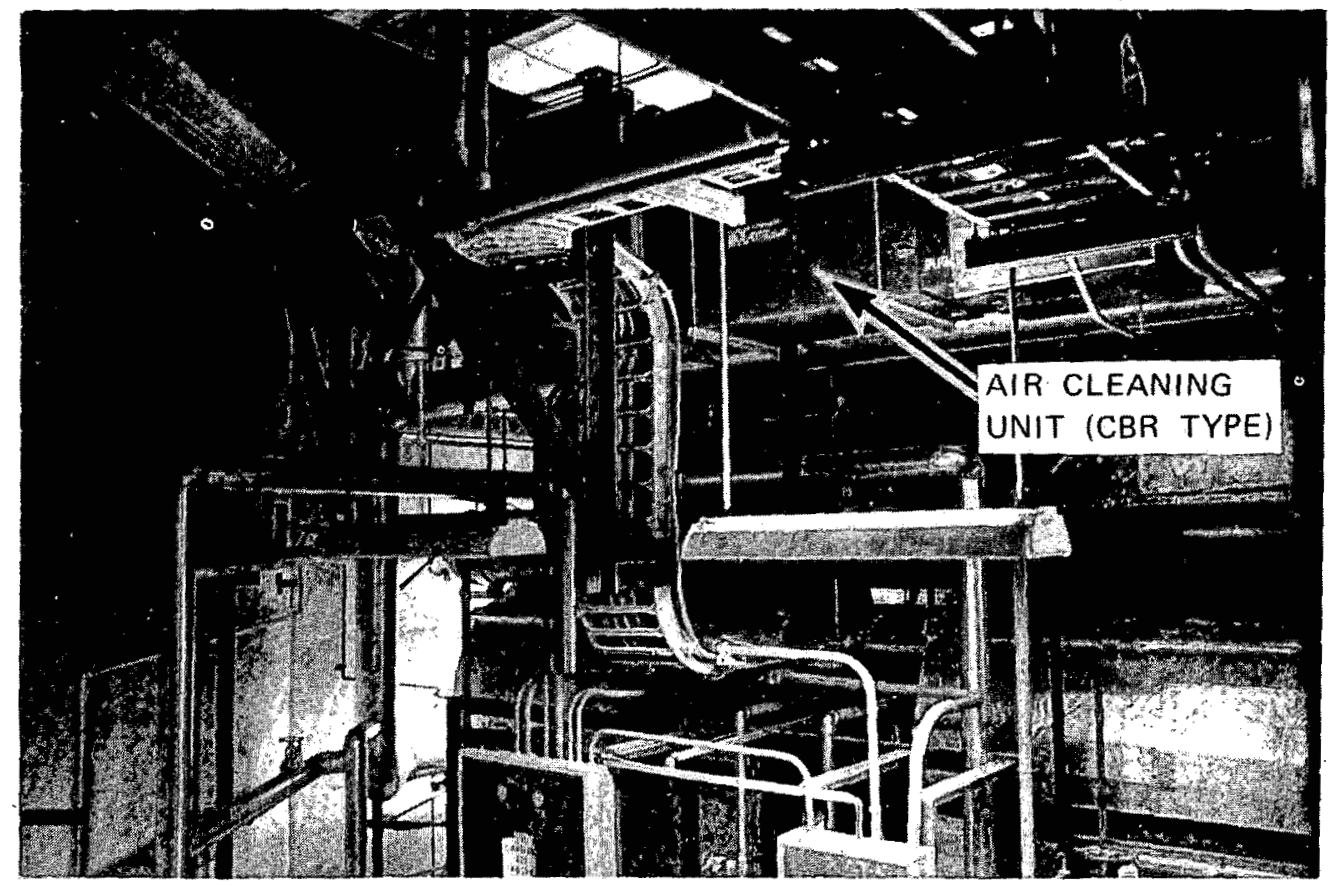

Fig. 2.15 An illustration of poor filter installation practice. Arrow points to wood-cased CBR filter. Light troffer indicates that height above floor is about $20 \mathrm{ft}$, requiring that ladders be brought in to gain access to the unit. Note cable tray, hangers, piping, and other obstructions. 
radiation zone in the event of an emergency or spill without risking contamination of adjacent facilities.

Another common practice has been to install ducts and filter housings on the roof of a building which are accessible only over the roof. In the event a used filter is dropped during maintenance, there is a potential for contamination spread not only to a surface (the roof), which would be difficult to decontaminate, but to the atmosphere as well. For all systems, but especially for potentially high-hazard systems, it is recommended that all air cleaning components, including ductwork, be located inside a building space to provide a secondary containment against breach of the pressure boundary. Preferably, such building spaces should be heated to minimize condensation in the ducts during the winter months, and they should be easily accessible for inspection and service. Housings should be located in rooms that can be isolated during service or an emergency and that have walls and floors that can be easily decontaminated in the event of a spill. As a minimum precaution, the general area surrounding the housing should be one that can be cordoned off as a contamination zone. Off-the-shelf bag-out housings of the type shown in Fig. 2.16 are being used increasingly for single-filter installations. Although the bag-in bag-out provisions of these housings offer a measure of protection against spills during service operations, the plastic bags employed can be easily torn by the sharp corners of steel-cased filter elements and adsorber cells. It is recommended, therefore, that these caissons be installed in isolable rooms or controlled building spaces, at least in those cases where intermediate to high-level radioactive material is, or could be, present in the duct. Additional information on caissons and bag-in bag-out filter installations is given in Chap. 6.

\subsection{MULTISTAGE FILTRATION}

Although a single stage of HEPA filters is sufficient to meet most decontamination requirements, two, three, or even more stages may be required to meet the stringent requirements of facilities in which plutonium and other transuranic materials are handled. Multistage HEPA filtration is also employed to increase system reliability through series redundancy.

\subsubsection{Series Redundancy}

Installations such as the ERDA national laboratories and production facilities which have lived with radiation on a day-to-day basis for many years have found it necessary to employ series

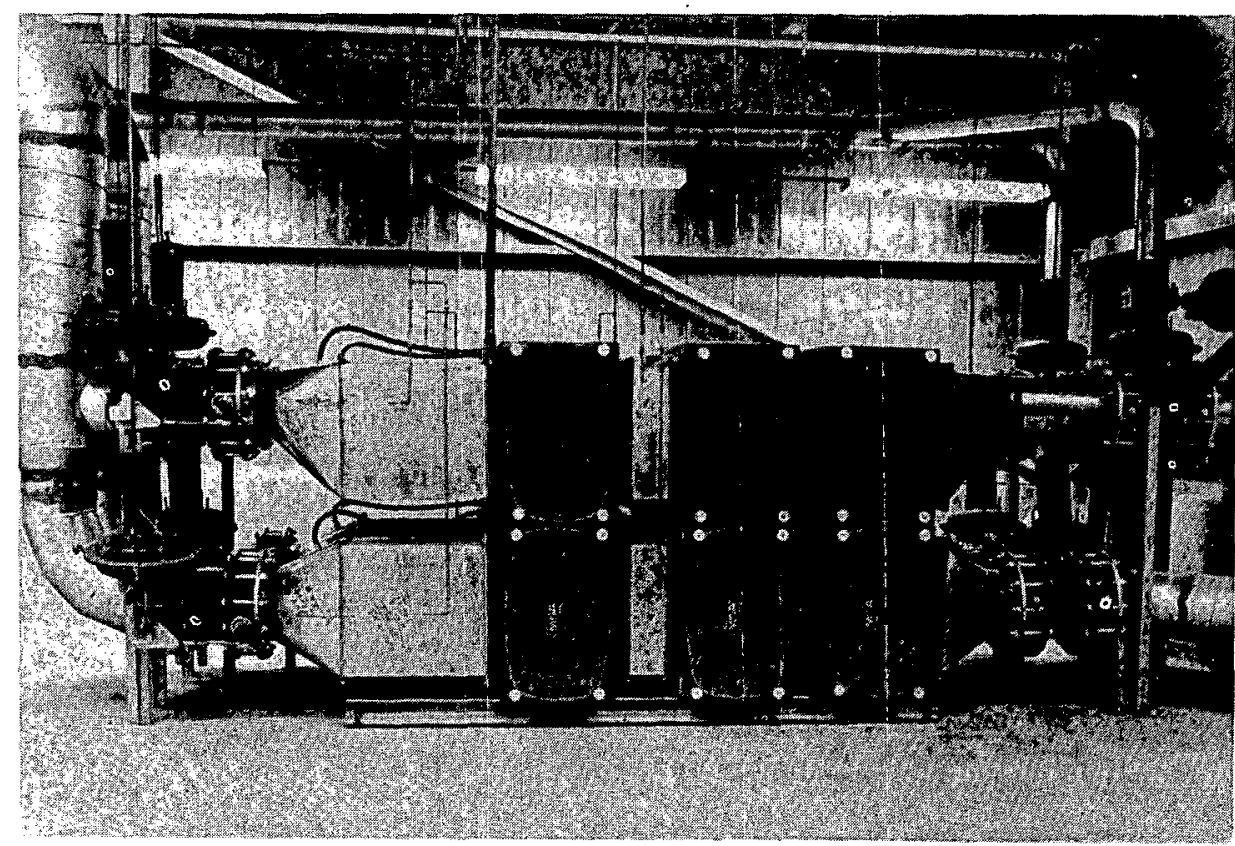

Fig. 2.16. Exhaust air cleaning system of radiopharmaceutical company. System is 1000 -cfm capacity with isolable redundant branch. Each branch contains one HEPA filter and two type I (pleated bed) adsorber cells in series. Filters and adsorber cells are installed in bag-out housings. Courtesy MSA Research Corp. 
redundancy of HEPA filters in exhaust and air cleanup facilities for Zone I, and often Zone II, containments. The purpose is to increase the reliability of the system by providing backup filters in the event of damage, deterioration, or failure of the first-stage filters. As noted in Sect. 2.2.1 (item 2 under air handling systems), each stage of filters must be individually testable if credit for redundancy is to be claimed. That is, if the stages are not individually testable, the combination of two or more stages must be considered as only a single stage from the standpoint of reliability. On the other hand, each untestable stage contributes to the overall filtration efficiency of the combination, although not to an extent equivalent to the nominal stage efficiency of $99.97 \%(\mathrm{DF}=3333)$; a maximum efficiency of $99.8 \%(\mathrm{DF}=500)$ has been allowed in the past for untestable second- and third-stage filters, with full credit for the first stage. ${ }^{30}$

Redundant stages should be well spaced, the first often being a duct-entrance filter in a room, glove box, or hot cell, and the second being the final filters of a central exhaust system. In some systems, as for example the ESF air cleaning units of nuclear power plants (shown in Figs. 2.10,2.13, and elsewhere in this handbook), the series-redundant filter banks are installed within the same housing. In any event, redundant stages should be spaced sufficiently far apart to allow for effective in-place testing and inspection of both faces of the filters; they should not be installed back-to-back to one another or to other components of the system such as prefilters or adsorber cells (see Figs. 4.23 and 9.2).

\subsubsection{Increased Decontamination Factor (DF)}

The particle sizes of plutonium and plutonia aerosols generated in chemical operations employed in nuclear fuel fabrication and reprocessing fall within the range of the size of maximum penetration (SMP) for HEPA filters, 0.07 to $0.3 \mu \mathrm{m}$ lightscattering mean diameter (LMD); although $0.3 \mu \mathrm{m}$ LMD is considered the SMP for dust and other unitdensity particles, the SMP for high-density particles, such as plutonium, is substantially higher; the aerodynamic mean diameter of plutonium particles formed by condensation is thought to lie between 0.4 and $0.7 \mu \mathrm{m} .{ }^{30}$ A HEPA filter, by definition, ${ }^{31}$ has a minimum filtration efficiency of $99.97 \%(\mathrm{DF}=3333$ ) for $0.3-\mu \mathrm{m}$ particles (although most of the HEPA filters currently being validated by the ERDA Quality Assurance Stations exhibit DFs on the order of
$10^{4}$ ). Current NRC Regulatory Guides recommend a total plant DF of at least $10^{11}$ for plutonium in gaseous effluents. Although some decontamination is effected by plant operations, the greatest portion must come from the HEPA filters, which means that two, three, or even more stages of filters may be necessary.

Theory predicts that the primary mechanisms in the arrestance of particles by a HEPA filter are diffusion and inertia; the effectiveness of these mechanisms varies with particle size, airflow velocity through the medium, and, to a lesser extent, particle density as shown in Fig. 2.17. Direct interception, or impaction, is a secondary mechanism that is independent of these parameters. As evident from Fig. 2.17, these mechanisms combine to produce a statistical average DF, not an absolute value for a given particle size. For this reason, the effect of adding stages of HEPA filters is multiplicative and does not produce a screening effect that theoretically results in an absolute minimum DF for any given particle size. (In practice, however, some screening of particles substantially larger than the SMP can be expected.) In theory, therefore, the DF of a multistage HEPA filter installation would be $\mathrm{DF}_{f}^{n}$, where $\mathrm{DF}_{f}$ is the definition DF of the HEPA filter (3333) and $n$ is the number of stages. Recent work by Ettinger et al. ${ }^{32}$ at the Los Alamos Scientific Laboratory suggests that this theory is essentially true; DFs of $10^{4}$ for stages one and two and of somewhat less than $5 \times 10^{3}$ for the third stage of a three-stage system, with an average DF of $5 \times 10^{3}$ for each of the three stages, were determined..$^{30}$ These results were obtained in a smallscale test system (about $25 \mathrm{cfm}$ ) in which conditions were idealized by eliminating gasket leakage and employing filter units that exhibited a test efficiency (according to ERDA Quality Assurance Station testing) of greater than $99.99 \%$.

Earlier less definitive tests and experience had indicated substantially lower values of DF in the second and third stages, and conservatism suggests that values lower than those obtained in the Los Alamos tests should be used in practice. Conservatism also suggests that a value no higher than $D_{F}$ (3333) be used for the first stage and probably somewhat less to allow for filter degradation under service conditions. Although DF improves with dust loading of the filter, aging and exposure to moisture and corrodents may decrease the ability of the filter to maintain the higher DF under system upset conditions. For purposes of estimating the capability of a 

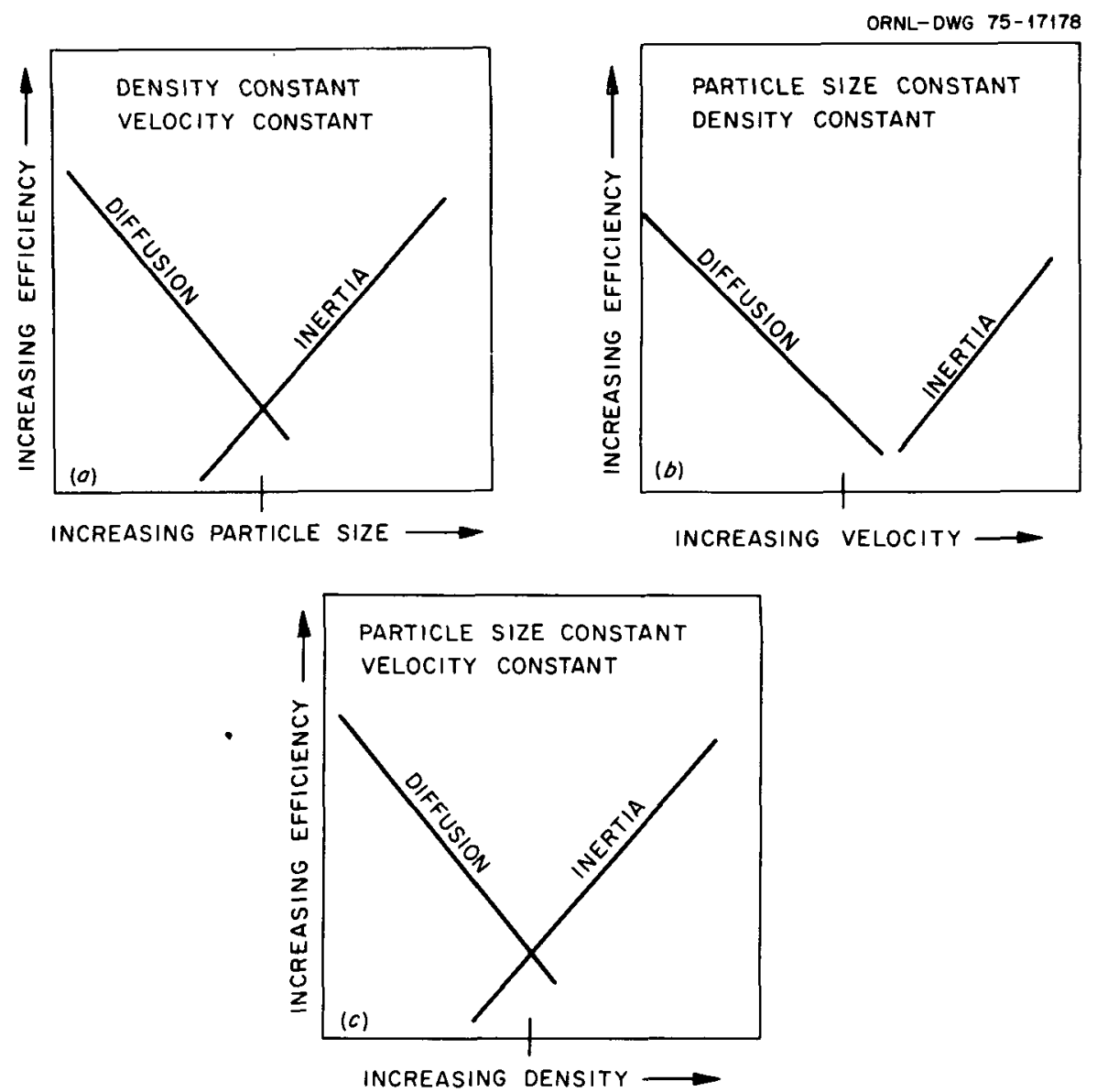

Fig. 2.17. General effect of principal mechanisms that affect the arresting efficiency of the HEPA filter. (a) Effectiveness of diffusion mechanism decreases with increasing particle size; effectiveness of inertial mechanism increases, at a given velocity. (b) Effectiveness of diffusion decreases with increasing velocity; effectiveness of inertial effects increase, for a given particle size. (c) For a given particle size and velocity, increased density decreases effectiveness of diffusion and increases effectiveness of inertial effect.

multistage HEPA filter installation under normal operating conditions, a DF of $\left(3 \times 10^{3}\right)^{n}$ can be safely used with systems that adhere to the design, construction, testability, and maintainability principles of this handbook or of ANSI N509. ${ }^{33}$

For purposes of assigning safety credit in facility safety analysis reports, however, consideration must be given to possible degradation of the filters under upset conditions. This requires that substantially more conservative DFs be used, particularly for the first-stage HEPA filters that would probably receive the brunt of any adverse effects due to a system upset. The concensus of a meeting held in the Albuquerque AEC Operations Office in 1971 to review filtration requirements for plants in which plutonium is handled ${ }^{34}$ was that a safety credit of $2 \times 10^{3}$ should be allowed for each stage of a system in which each stage is individually testable for the normal operating situation. It was recommended that lower values (not stipulated) be used for the accident condition and for the system in which stages were not individually testable. The value of $2 \times 10^{3}$ is considered low for normal operation in view of the Los Alamos work and the quality of filters now being received at the ERDA Quality Assurance Stations (most exhibit a penetration $<0.01 \%$ or an efficiency $>99.99 \%$ ). In view of the fact that no thorough hazard analysis exists to predict what might happen in an accident, a lower DF of $5 \times 10^{2}$ for the first stage and ( 1 to $2 \times$ $10^{3}$ ) for each of the remaining stages appears defensible for the accident condition, ${ }^{28}$ assuming that each stage is individually testable. If stages are not individually testable, the recommendations of Sect. 2.6.1 apply; that is, the additional stages should also be given a safety credit of no more than $5 \times 10^{2}$. 


\subsection{AIR SAMPLING}

Air samples are often taken from the stack or other locations downstream of the filters to monitor the amount of radioactive material being released to the atmosphere. If the sampling system is not properly designed, underestimation of released radioactive material may result. The element often at fault is the sampling line itself. If the sampling line is too long or too small in diameter (relative to flow velocity in the line), it may act as a diffusion tube to remove small particles or as an inertial separator to capture large particles before they can reach the counting and recording requipment. Sharp-angle bends, valves, and other flow restrictions must be minimized to avoid losses due to inertia, impaction, and impingement. Horizontal runs must be minimized to avoid gravitational settling. Conduit diameter must be large enough and consistent with flow velocity to minimize diffusion losses and turbulence that can cause migration of particles to the conduit walls, where they may be captured (turbulence in sampling lines can take place at a Reynolds number of 1200 or lower). ${ }^{35}$ The optimum sampling line diameter, considering both line losses and practical limitations on line size, can be found from the equation ${ }^{36}$

$$
d=\frac{Q}{150}
$$

where

$$
\begin{aligned}
& d=\text { diameter of sampling conduit, } \mathrm{cm} \\
& Q=\text { sampling rate, } \mathrm{cm}^{3} / \mathrm{sec} .
\end{aligned}
$$

Sampling nozzles should be sized for isokinetic inlet velocity. Sampling lines should be vertical, insofar as practicable, and should be as short as possible between collector nozzle and counting instruments (some stack sampling instruments are located on the stack at the same level as the sampling point). Sample lines should be stainless steel or copper; they must be clean and smooth on the inside and should be detachable to permit occasional field cleaning. Oil and moisture on the inner surfaces of the sample lines will trap particles and give false readings. Cleaning by procedures that meet the requirements of ASTM $\mathrm{A} 380^{37}$ is recommended.

\section{REFERENCES FOR CHAP. 2}

1. "Standards for Protection Against Radiation," Code of Federal Regulations, Title 10, Part 20 (10 CFR 20).

2. "Reactor Site Criteria," Code of Federal Regulations, Title 10, Part 100 (10 CFR 100).
3. "Licensing of Production and Utilization Facilities," Code of Federal Regulations, Title 10, Part 50 (10 CFR 50).

4. "Design Criteria for Radiochemical Plants and Laboratories," Procedures and Practices for Radiation Protection-Health Physics Manual, Oak Ridge National Laboratory, Appendix A-11, Jan. 8, 1974.

5. Numerical values can be relaxed or increased, depending on the requirements for the operating conditions and the DBA for that facility.

6. TLVs-Threshold Limit Values for Chemical Substances and Physical Agents in the Workroom Environment, American Conference of Governmental Industrial Hygienists, Cincinnati, Uhio (issued annually).

7. "Keport of International Atomic Energy Agency (IAEA) Symposium on Airborne Radioactivity," Nucleonics Week, 8(9), 7 (July 20, 1966).

४. R. E. Harrington, "Fine Particulates-The Misunderstood Air Pollutant,"J. Air Pollut.Control Assoc. 24(10), 927-29 (October 1974)

9. K. T. Whitby et al., "The ASHAE Air-Borne Dust Survey," Heat. Piping Air Cond. 185-192 (November 1957).

10. P. R. Austin, "Personnel Emissions in Laminar-Flow Clean Rooms," Contam. Control, 5(7), 29 (July 1966).

11. C. A. Burchsted, "Environmental Properties and Installation Requirements for HEPA Filters," Proc. Symp. Treat. Airhorne Radioact. Wastes, International Atomic Energy Agency, Vienna, 1968.

12. M. W. First, "Performance of Absolute Filters at lemperatures from Ambient to $1000^{\circ} \mathrm{F}$, " Proc. 12 th AEC Air Clean. Conf., USAEC Report CONF-720823, January 1973.

13. W. L. Belvin et al., Final Report, Development of FluorideResistant HEPA Filter Medium, ERDA Report TID-26649, August 1975.

14. ANS1 N512, Protective Coatings (Paints) for the Nuclear Industry, American National Standards Institute, New York, 1975.

15. M. W. First and L. Silverman, "Cost and Effectiveness of Air Cleaning Systems," Nucl. Saf. 4(1), 61-66 (September 1962).

16. ASHRAE 52-68, Method of Testing Air Cleaning Devices Used in General Ventilation for Removing Particulate Matter, American Society of Heating, Refrigerating and AirConditioning Engineers, New York, current issue.

17. News item, Air Conditioning. Heating and Refrigeration New's, July 30, 1967, p. 28.

18. Kegulatory Guide 1.52, Design. Testing, and Maintenance Criteria for Aimosphere Cleanup System Air-Filtration and Adsorption Units of Light-Water-Cooled Nuclear Power Plants, U.S. Atomic Energy Commission, Washington, D.C., 1973.

19. Military Specification MIL-F-51068, Filter, Particulate, High-Efficiency, Fire Resistant, current issue.

20. From engineering data furnished by the American Air Filter Co., Inc., Louisville, Ky.

21. P. A. F. White and S. E. Smith, eds., High Efficiency Air Filtration, Butterworth \& Co., Ltd., London, 1964.

22. A face guard is a hardware cloth or expanded metal screen installed on the face of a filter to protect the fragile filter medium from contact with fingers or extraneous items during handling and installation.

23. G. A. Schurr et al., "Deep Bed Filter Performance Tests," Proc. 12th AEC Air Clean. Conf., USAEC Report CONF-720823, January 1973.

24. NFPA 12A, "Standard on Halogenated Fire Extinguishing Agent Systems-Halon 1301," and NFPA 12B, "Standard on 
Halogenated Fire Extinguishing Agent Systems--Halon 1211," National Fire Codes, vol. I, National Fire Protection Association, current issue.

25. A. H. Hill, Savannah River Laboratory, personal communication to C. A. Burchsted.

26. See proceedings of the biennial AEC air cleaning conferences, 1966 to 1974.

27. H. Gilbert, Safety Engineer, U.S. Atomic Energy Commission, and D. J. Keigher, Fire Protection Engineer, Los Alamos Scientific Laboratory, personal communications to $\mathrm{C}$. A Burchsted.

28. NFPA 90A, "Air Conditioning and Ventilating Systems," National Fire Codes, National Fire Protection Association, vol.9, 1975.

29. R. E. Stevens, National Fire Protection Association, personal communication to C. A. Burchsted.

30. J. C. Elder, Aerosol Studies Section, Los Alamos Scientific Laboratory, letter to C. A. Burchsted, Nov. 25, 1975.

31. AACC CS-1, Standard for HEPA Filters, obtainable from the Institute of Environmental Sciences, Mt. Prospect, Ill., current issue.
32. H. J. Ettinger et al., "Performance of Multiple HEPA Filters Against Plutonium Aerosols, January 1 Through June 30. 1974," Proc'. 13th AEC Air Clean. Conf., USAEC Report CONF740807, March 1975.

33. ANSI N509, Nuclear Power Plant Air Cleaning Units and Components, American National Standards Institute, 1976.

34. HEPA Filtration Guidelines, unpublished minutes of a meeting held at the Albuquerque AEC Operations Office, Dec. $7-8,1971$.

35. J. W. Thomas, USAEC Health and Safety Laboratory, personal communication to C. A. Burchsted.

36. J. W. Thomas, "Particle Loss in Sampling Conduits," Proc. Annu. Conf. Int. At. Energl Agency, Vienna, 1967.

37. ASTM A380, Recommended Practice for Cleaning and Descaling Stainless Steel Parts, Equipment, and Sistems, American Society for Testing and Materials, Philadelphia, current issue. 


\section{Internal Components}

\subsection{INTRODUCTION}

This chapter covers the major air cleaning components used in nuclear ventilation and air cleanup systems, including HEPA filters, prefilters for HEPA filters, gas adsorbers, and demisters. It reviews the characteristics, construction, and application of these components including limitations and problems in their use. Other types of air and gas cleaning equipment are beyond the scope of this handbook.

\subsection{HEPA FILTERS}

HEPA filters [also called absolute, superinterception, very-high-efficiency, extreme efficiency, AEC (Atomic Energy Commission), and CWS (Chemical Warfare Service) filters] have proven to be the most effective, reliable, and economical devices for removing low concentrations of submicron particles at extremely higin ( $\geqslant 99.97 \%$ ) collection efficiency. By definition,' a HEPA filter is a "throwaway, extended-medium, dry-type" filter having (1) a minimum particle removal efficiency of no less than $99.97 \%$ for $0.3-\mu \mathrm{m}$ particles, (2) a maximum resistance, when clean, of $1.0 \mathrm{in} . \mathrm{wg}$ when operated at rated airflow capacity, and (3) a rigid casing extending the full depth of the medium (Fig. 3.1).

\subsubsection{Performance Characteristics}

Efficiency and Resistance. The Institute of Environmental Sciences (IES) standard for HEPA filters, CS-1, ' lists three classes of HEPA filters with respect to performance: type $A$, which are tested for overall penetration (i.e., 100 minus percent efficiency) at rated flow only; type $\mathrm{B}$, which are tested for overall penetration at rated flow andalso at $20 \%$ of rated flow, with the filter encapsulated to disclose casing leaks; and type $\mathrm{C}$, which are scanned filters. Scanning is a special leak test usually employed for filters used in clean rooms and clean benches but is generally not used for nuclear applications. Type A filters are recommended primarily for use in recirculating air cleanup systems. Type B filters are efficiency-tested at both $100 \%$ and $20 \%$ of rated flow to disclose pinhole leaks that may not show up in the $100 \%$ flow test. Type B filters are recommended for most nuclear applications, particularly in oncethrough systems. The ERDA Quality Assurance Stations that test HEPA filters will make either the single-flow or two-flow test, as specified by the user.

The measured particle-collection efficiency ${ }^{2}$ of most HEPA filters that are individually tested by

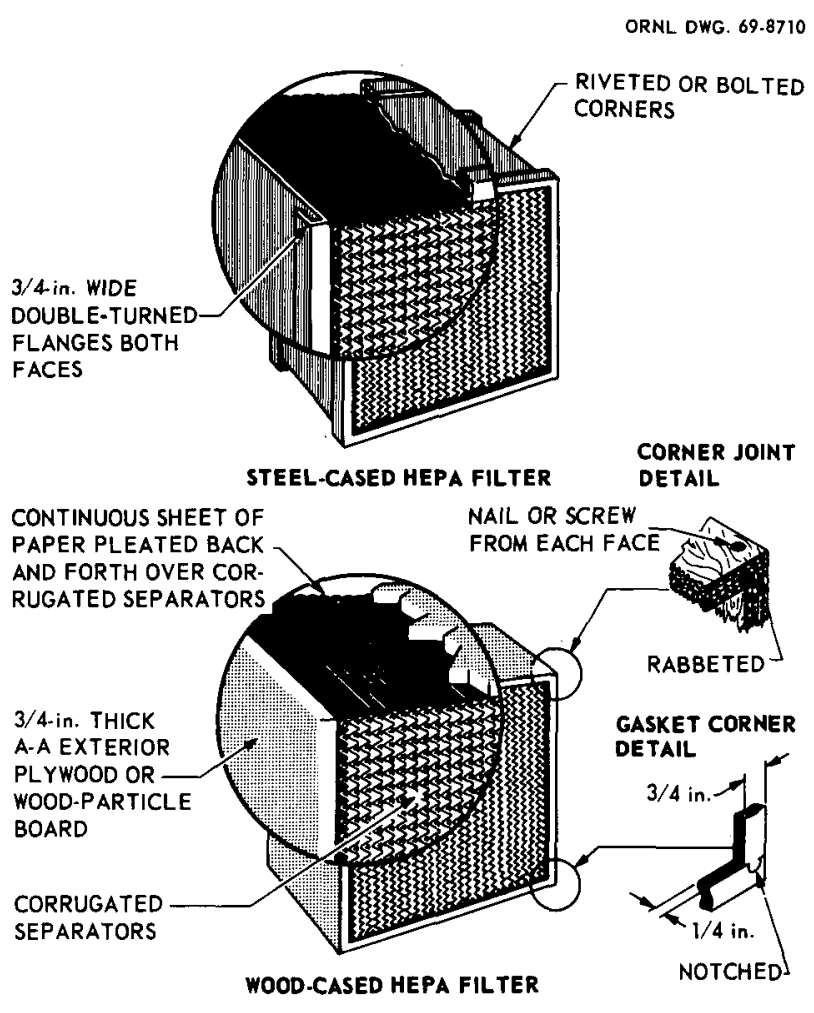

Fig. 3.1. Construction of open-face HEPA filter. 
these stations is currently close to $99.99 \%$. The value actually reported is penetration (100 minus percent efficiency). Penetration, resistance, and airflow capacity are determined at the same time and are marked on the case of each filter unit.

The penetration and airflow resistance of each HEPA filter unit are determined by the manufacturer before it is shipped from the factory, using procedures that were developed by the U.S. Army, U.S. Navy, and AEC (now ERDA). Most ERDA facilities require a confirmation test by one of the quality assurance stations (at either Oak Ridge, Tennessee; Richland, Washington; or the Rocky Flats Plant, (iolden, Colorado) as a condition of acceptance. This confirmation test is an important quality control factor and is recommended to nongovernment organizations. The service is available to industrial and utility organizations, from ERDA, at cost plus 15\% ( $\$ 13.50$ for a 1000-cfm filter in 1975, with a minimum charge of $\$ 135.00$ ). ${ }^{3}$ Efficiency tests are made with a monodisperse, thermally generated DOP aerosol having a count-median droplet diameter of $0.3 \pm 0.03 \mu \mathrm{m}$ in equipment of the type shown in Fig. 3.2.

Size and Airflow Capacity. The dimensions shown in Table 3.1 have been standardized for HEPA filters used in nuclear service. Other sizes can be obtained but should be considered as specials. Although the $24 \times 24 \times 5 \%$ in. size is included, it is structurally weak and should be avoided. The airflow capacities shown are nominal for the specified maximum resistance of 1 in.wg. Although some manufacturers claim substantially higher airflows at this resistance, the values shown in Table 3.1 should be used for design purposes. The use of higher design airflow could create future logistic problems for the user and could result in lower-than-expected system performance if filters of different manufacture should be substituted at a future date.

Resistance. The standard resistance of a HEPA filter is 1 in.wg maximum when new and operated at rated airflow capacity. Resistance increases with dust ${ }^{4}$ loading.

Dust-Holding Capacity. Dust-holding capacity of a filter in an actual situation is a function of the type, shape, size, density, "stickiness," and concentration of dust particles to which it is exposed. For HEPA filters it is often not an important factor since they will be protected by prefilters, particularly in highdust-concentration applications. The HEPA filter will hold a considerable quantity of fine granular dust, but fibrous dust and lint tend to bridge the space

Table 3.1. Standard HEPA filters

\begin{tabular}{|c|c|c|}
\hline $\begin{array}{l}\text {. Face } \\
\text { dimensions } \\
\text { (in.) }\end{array}$ & $\begin{array}{l}\text { Depth, } \\
\text { less gaskets } \\
\text { (in.) }\end{array}$ & $\begin{array}{l}\text { Design airflow capacity } \\
\text { at clean-filter resistance } \\
\text { of } 1.0 \mathrm{in} . \mathrm{wg}(\mathrm{scfm})\end{array}$ \\
\hline $24 \times 24$ & $11 \frac{1}{2}$ & 1000 \\
\hline $24 \times 24$ & $5 \%$ & 500 \\
\hline $12 \times 12$ & $5 \%$ & 125 \\
\hline $8 \times 8$ & $5 \%$ & 50 \\
\hline $8 \times 8$ & $3 \frac{1}{1} 16$ & 25 \\
\hline
\end{tabular}

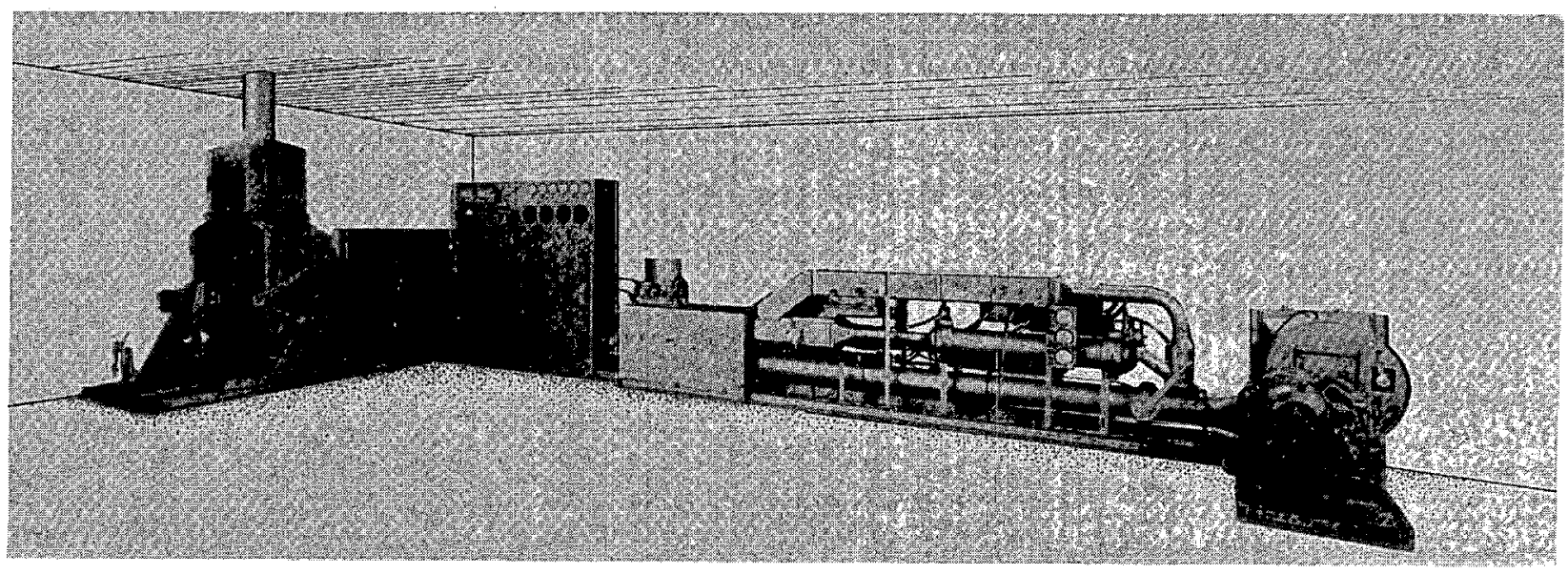

Fig. 3.2. Q-107 penetrometer used for efficiency testing of 1000-cfm HEPA filters by the manufacturer and ERDA Quality Assurance Stations. Courtesy Air Techniques, Inc. 
between pleats, thus decreasing the filter's dustholding capacity. Fibrous and flake-like particles comprise a substantial portion of atmospheric and "people-generated" dust. Dust-holding capacity has a bearing on filter life, however, and for design purposes is generally considered to be $4 \mathrm{lb}$ per 1000 $\mathrm{cfm}$ of rated capacity. This is a conservative value for granular dusts. Actual life under service conditions can be determined only from simulation tests under comparable operating conditions or from experience.

\subsubsection{Construction}

Figure 3.1 shows typical construction of woodcased and steel-cased open-face HEPA filters. The core (i.e., filter pack) is generally made by pleating a continuous web of fiberglass paper (the medium) back and forth over corrugated separators that add strength to the core and form air passages between the pleats. The core is sealed into a full-depth wood or steel casing (frame) with an elastomeric adhesive or polyurethane sealant. An alternative core construction of one manufacturer is made by wet-forming the medium into corrugations and pleats as it comes off the papermaking machine, thereby providing the necessary air passages and core structure without the use of separators. As of March 1976, this is the only domestic separatorless HEPA filter construction that is capable of meeting the specified minimum overpressure resistance of 10 in.wg. In fact, this filter has been shown to be capable of withstanding over twice this pressure load without damage.

Medium (Filter Paper). HEPA filter papers are composed of very fine (submicron diameter) fibers in a matrix of larger diameter ( 1 to $4 \mu \mathrm{m}$ ) fibers. An organic binder is added to hold the fibers during the papermaking process and to give added paper tensile strength. All fire-resistant papers are made from glass fibers, sometimes with a small percentage of asbestos added to improve resistance to hydrogen fluoride. Tensile strength of the paper is sharply reduced (about $50 \%$ ) at temperatures above $400^{\circ} \mathrm{F}$ due to burnout of the binder. ${ }^{5}$ Minimum properties for HEPA filter papers are given in Appendix A (sample specification ACES-1) and in Military Specification MIL-F-51079. ${ }^{6}$ Combustible papers, made from a mixture of cellulose and asbestos fibers, are no longer made. A special corrosion-resistant medium, developed for improved resistance to hydrogen fluoride, has been demonstrated but is not yet commercially available? This paper is fragile, and filters made from it must be handled with care to avoid damage.
Separators. Corrugated separators are interleaved with the pleats of the filter medium to space the pleats, thus forming airways, and to add strength to the assembled filter core. Separators of fire-resistant filters (Construction Class 1 , IES CS-1) ${ }^{1}$ are made from very hard (H-19 temper) 0.0015-in.-thick corrugated aluminum foil or from corrugated waterand corrosion-resistant asbestos paper. Kraft paper and other combustible materials, often used for separators of clean room and clean bench HEPA filters, are not permissible for nuclear grade filters. Plastics have been used for corrosion resistance by some manufacturers but are generally not suitable, because the corrugations often flatten at only moderately high temperatures $\left(90\right.$ to $110^{\circ} \mathrm{F}$ ), thus permitting the filter core to collapse and subsequently blow out if exposed to even design airflow. Minimum properties for separator materials, including corrosion resistance, are given in Appendix A (sample specification ACES-1).

Sealant. The sealant traditionally used to seal the filter core into the case is a heat- and moistureresistant elastomeric adhesive. Some manufacturers use a chemically expanded self-extinguishing urethane foam. Filters that will be operated continuously at high temperature (above $400^{\circ} \mathrm{F}$ ) are sealed with compressed glass-fiber matting or ref ractory adhesives. The sealant must (1) be moisture-and corrosion-resistant, (2) not deteriorate excessively under exposure to radiation or lose its resilience under alternating exposure to heat and cold or to dry or humid air, (3) not crack or delaminate from the frame at high temperature $\left(5 \mathrm{~min}\right.$ at up to $\left.750^{\circ} \mathrm{F}\right)$, and (4) maintain a reliable seal between the filter core and casing under continuous service at design operating conditions. The high-temperature sealants do not meet all of these criteria, and their use, except in very special circumstances, is not recommended. Glassfiber matting may not maintain a reliable seal at high temperature, silicone sealants delaminate quickly at only 10 or $20^{\circ} \mathrm{F}$ above their rated temperatures, and refractory sealants are extremely brittle after heating.

Case (Frame or "Cell Sides"). The usual. case materials are fire-retardant exterior-grade plywood or wood-particle board and cadmium-plated or chromized carbon steel. Thicknesses of $3 / 4$ in. for wood and No. 14 U.S. gage for steel are required for rigidity and to resist the compressive loads imposed when the filter is clamped to a mounting frame (axial compressive loads of $400 \mathrm{psi}$, or higher, may be encountered in service). Grade A-A plywood has previously been specified to avoid manufacturing

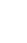


errors, but the current shortage of plywood sometimes makes necessary the use of wood-particle board or lesser grades of plywood; wood-particle board is usually used today. When a lesser grade of plywood is permitted, the ply adjacent to the filter core must be sound (grade A, if possible) and must be coated completely with sealant to prevent lateral air leakage through the case. Exterior grade is specified for both plywood and wood-particle board to provide adequate moisture resistance. A minimum density of $45 \mathrm{lb} / \mathrm{ft}^{3}$ and impermeable coating of both faces is specified for wood-particle board to ensure adequate impermeability and strength. Panels (four are required) for steel filter cases are cadmium-plated after all shearing and forming operations are complete. Because of shortages and restrictions on the use of cadmium, chromized steel is more widely used today. Chromized steel has been shown to have better corrosion resistance than either cadmium- or zincplated (galvanized) steel, ${ }^{8}$ and cost is comparable. Chromized steel is a titanium-stabilized carbon steel with a diffusion-bonded chromium coating on each face. Steel cases should be used where continuous wetting or high humidity [ $>90 \%$ relative humidity $(\mathrm{RH})]$ at high temperatures $\left(>90^{\circ} \mathrm{F}\right)$ can be expected. Wood-particle board, in particular, swells considerably when exposed to excessive moisture.

Gaskets. Gaskets are critical items. Tests have shown that excessive variation in gasket thickness, poorly formed gasket corners, and improperly glued gaskets result in air leakage that exceeds the acceptance level of the filter. ${ }^{y}$ If the gasket material is too hard, excessive bolt loading may be required to properly seal the filter to the mounting frame, thus resulting in possible filter damage. If the gasket material is too soft, excessive compression-set may take place, thus resulting in air leakage as clamping bolts and casing materials relax or expand under service conditions. Flat $(5 / 8$ to $3 / 4$-in. wide by $1 / 4$-in. thick) gaskets made from ASTM D1056 grade SCE 43 closed-cell neoprene sponge with cut surfaces on both faces are recommended. Gaskets are usually made in strips $(3 / 4$ by $1 / 4$ in.) with notched or dovetailed corners as shown in Fig. 3.1. Gaskets must be carefully aligned so they do not extend beyond the edges of the case flanges.

Because silicone mold-release compounds used in the manufacture of gasket material may prevent the adhesive from making a good bond with the neoprene, cut surfaces should be specified.

Gasket Adhesives. Fire-retardant rubber-base adhesives are used for gluing gaskets to the case.
Since the gasket is completely constrained once the filter has been installed, the chief requirement on these adhesives is that they hold the gasket firmly until the filter is installed.

Configuration. Rectangular and cylindrical HEPA filters are available in both open-face and enclosed configurations. Figure 3.1 shows open-face construction: The enclosed filter (Fig. 3.3) is similar except that the case is longer and closed, with nipples for attachment of the filter to the ducts. The casing of the enclosed filter is part of the system pressure boundary. Currently available enclosed filter units cannot meet the fire- and hot-air resistance requirements of UL-586, ${ }^{10}$ nor do wood-cased enclosed filter units meet the rquirements of NFPA $90 \mathrm{~A} .{ }^{11}$ Steel-cased enclosed filters, because of their bolted or riveted corners, generally leak under service conditions; therefore, acceptance tests of steel-cased units should specify encapsulation to ensure freedom from corner leaks. Enclosed filters generally have higher airflow resistance than open-face filters because of the restriction caused by the nipples. Cylindrical filters often seem to be an ideal solution to some design problems. However, because experience with them has often been poor and because they cannot be qualified for fire resistance under UL-586, they are generally not recommended.

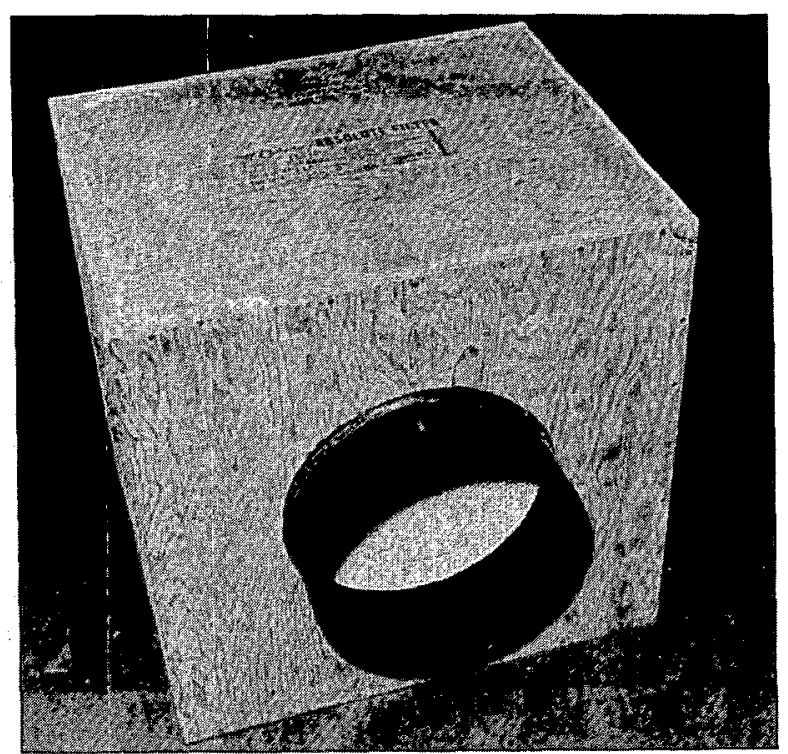

Fig. 3.3. A 1000-cfm wood-cased enclosed HEPA filter. Courtesy Cambridge Filter Co. 


\subsubsection{Weight of HEPA Filters}

The weight of filter elements is an important factor in design and maintenance. Table 3.2 gives the cleanfilter weight of open-face and enclosed rectangular models. Dirty-filter weights, for design purposes, are approximately $4 \mathrm{lb}$ more per $1000 \mathrm{cfm}$ of rated capacity.

\subsubsection{Mechanical Properties}

Other factors (heat, moisture) being equal, woodcased filters are preferred to steel-cased filters because of their greater rigidity, superior vibrationdamping characteristics, greater corrosion resistance, and greater corner strength. "Common practice in nuclear plant exhaust systems is to compress filter gaskets by $80 \%$ or more. This amount of gasket compression requires a clamping force of at least 18 $\mathrm{lb}$ per square inch of gasket surface, or a total load of $1250 \mathrm{lb}$ or more on the frame of a $1000-\mathrm{cfm}$ filter unit. Because its section modulus is nearly 20 times that of a steel case, the wood case is better able to withstand such high compressive, axial loads on the case panels. The wood case, with properly constructed (rabbeted) corners, also has about twice the corner strength of a steel case of the same size and is therefore better able to withstand racking or skewing when subjected to a force couple. Racking, which frequently occurs during handling, shipping, and installation, can damage either the filter medium or the seal between the core and case, or both. Face guards, consisting of hardware cloth or expanded-metal screens fastened to each face of the filter, increase the resistance to racking or skewing.
Resistance to shock pressures is important in a HEPA filter because it is often the final barrier between the contaminated space and the atmosphere. The shock overpressure resistance of open-face rectangular filters, based on tests by the U.S. Navy, ${ }^{12}$ is given in Table 3.3 and Fig. 3.4. The recommended values are the maximum shock overpressure that the filters should withstand without visible damage or loss in filtration efficiency when exposed to a shock of approximately $50 \mathrm{msec}$ duration. Filters with face guards on both faces have about $40 \%$ greater shock resistance than those without. Dirt-loaded filters in the Navy tests exhibited about 15\% less shock resistance than clean filters. At overpressures of 0.5 to 1.0 psi greater than the failure value, the filter medium burst on the downstream side of the pleats; at overpressure of 2 psi greater than the failure value, extensive damage to the core occurred; and at $5 \mathrm{psi}$, blowout of the entire filter core occurred. ${ }^{12}$ The greater shock resistance of filters with face guards is significant. The AEC, and more recently ERDA and NRC, has long advocated face guards on HEPA filters to minimize damage to the fragile core during handling and installation. The additional factor of higher shock overpressure resistance gives added weight to this recommendation. The shock overpressure resistance of enclosed filters is probably less than that of open-face filters, because the shock loading will be concentrated at the center of the core. However, no tests have been made to verify this phenomenon.

Another important property of HEPA filters is their ability to withstand continuous overpressure. By

Table 3.2. Weight of unused HEPA filters

\begin{tabular}{|c|c|c|c|}
\hline \multirow{2}{*}{$\begin{array}{l}\text { Filter } \\
\text { size } \\
\text { (in.) }\end{array}$} & \multirow{2}{*}{$\begin{array}{l}\text { Nominal airflow } \\
\text { capacity } \\
\text { (cfm) }\end{array}$} & \multicolumn{2}{|c|}{$\begin{array}{l}\text { Approximate weight (lb) } \\
\text { of filters with - }\end{array}$} \\
\hline & & Wood case & Steel case \\
\hline \multicolumn{4}{|c|}{ Open-face } \\
\hline $8 \times 8 \times 3 \frac{1}{1} 16$ & 25 & 2 & 3 \\
\hline $8 \times 8 \times 5 / 8$ & 50 & 3.6 & 5.8 \\
\hline $12 \times 12 \times 5 \%$ & 125 & 4.8 & 7.3 \\
\hline $24 \times 24 \times 5 \%$ & 500 & 17 & 22 \\
\hline $24 \times 24 \times 11^{1 / 2}$ & 1000 & 32 & 40 \\
\hline \multicolumn{4}{|c|}{ Enclosed } \\
\hline $8 \times 8$ cross section & 25 & 5 & 9 \\
\hline $8 \times 8$ cross section & 50 & 7 & 10.5 \\
\hline $12 \times 12$ cross section & 125 & 17 & 20 \\
\hline $24 \times 24$ cross section & 500 & 64 & 72 \\
\hline $24 \times 24$ cross section & 1000 & 78 & 95 \\
\hline
\end{tabular}


Table 3.3. Shock overpressure resistance of open-face HEPA filters

\begin{tabular}{|c|c|c|c|c|}
\hline \multirow{3}{*}{\multicolumn{2}{|c|}{$\begin{array}{c}\text { Filter } \\
\text { dimensions (in.) }\end{array}$}} & \multicolumn{3}{|c|}{ Overpressure (psig) } \\
\hline & & \multirow{3}{*}{$\begin{array}{c}\text { Overpressure } \\
\text { at } \\
\text { failure }^{a}\end{array}$} & \multicolumn{2}{|c|}{$\begin{array}{c}\text { Recommended } \\
\text { design limit for used filters }\end{array}$} \\
\hline & & & \multirow{2}{*}{$\begin{array}{l}\text { With face } \\
\text { guards }\end{array}$} & \multirow{2}{*}{$\begin{array}{l}\text { Without fac } \\
\text { guards }\end{array}$} \\
\hline Face & Depth & & & \\
\hline $8 \times 8$ & $31 / 16$ & 3.7 & $b$ & 2.0 \\
\hline $8 \times 8$ & $57 / 8$ & 4.5 & $b$ & 2.5 \\
\hline $12 \times 12$ & $5^{7} / 8$ & 3.6 & $b$ & 2.0 \\
\hline $24 \times 24$ & $5^{7} / 8$ & 2.2 & 1.7 & 1.2 \\
\hline $24 \times 24$ & $11^{1 / 2}$ & 3.2 & 2.7 & 1.8 \\
\hline
\end{tabular}

${ }^{a}$ Clean filter with 4 - by 4 -mesh face guards on both faces.

${ }^{b}$ Face guards not available.

Source: W. L. Anderson and T. Anderson, "Effect of Shock Overpressure on High Efficiency Filter Units," Proc. 9th AEC Air Clean. Conf., USAEC Report CONF-660904, 1966.

specification, new HEPA filters must have sufficient structural strength to withstand a continuously applied overpressure of 10 in.wg, or higher, for at least $15 \mathrm{~min}$ without visible damage or loss of efficiency. For used filters, a value of 8 in.wg is recommended for design or planning purposes. In addition, the filter should be able to withstand the considerably higher, but short duration, overpressures that might be encountered in a tornado or when a damper inadvertently slams shut in the duct system. Although the design basis tornado specifies an overpressure of 3 psi for a period of $3 \mathrm{sec},{ }^{13}$ it is unlikely that the HEPA filters would be subject to such a condition because of the attenuating effects of the stack, ductwork, and fans. Tests at Los Alamos showed that an $8 \times 8 \times 3 \% 16$ in. HEPA filter could withstand a $9-\sec$ pressure pulse during which the maximum pressure of $3 \mathrm{psi}$ was held for $3 \mathrm{sec}$ without visible damage or reduction in efficiency. ${ }^{14} \mathrm{Com}-$ parison of these results with the data of Table 3.3 indicates that the $24 \times 24 \times 11^{\frac{1}{2}} / 2$ in. filter can probably withstand a 9-sec pressure pulse of $2.5 \mathrm{psi}$, which is probably substantially worse than what the filter would experience in the event of a tornado.

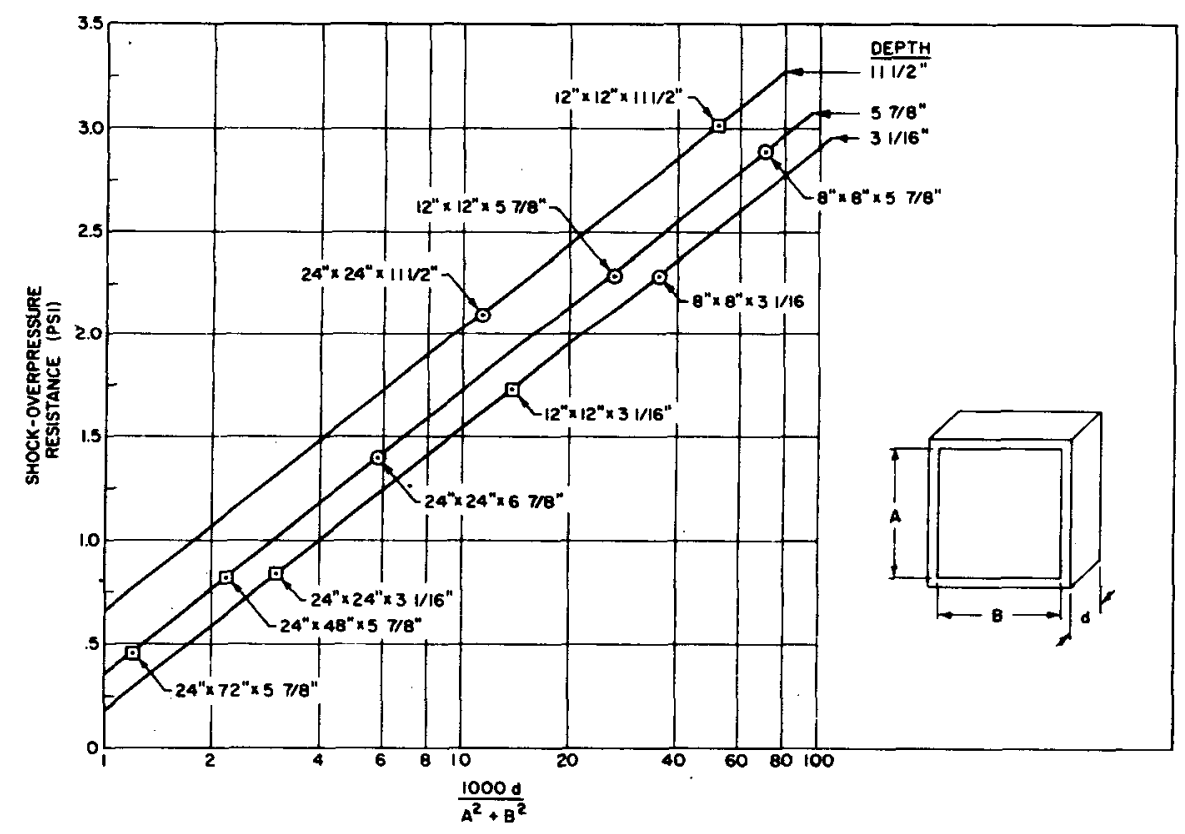

Fig. 3.4. Shock-overpressure resistance of clean HEPA filters (separator type) as a function of size. From C. A. Burchsted, "Environmental Properties and Installation Requirements of HEPA Filters," Proc. Symp. Treat. Airborne Radioaci. Wastes, International Atomic Energy Agency, Vienna, 1968. 


\subsubsection{Fire Resistance}

Standard IES CS- $1{ }^{15}$ lists three grades of filter with respect to fire resistance: (1) fire resistant (made from fire-resistant or fire-retardant materials throughout), (2) semicombustible (having a fireresistant medium but combustible separators and case), and (3) combustible (having flammable medium, separators, and case). Although grade 3 (combustible) filters are no longer available, they were previously used in the collection of process effluents that could be recovered by incineration of the filters and in applications where corrosive fumes would destroy fiberglass filter medium. Only fireresistant (grade 1) filters are permitted in nuclear exhaust applications.

Fire-resistant HEPA filters are qualified and labeled by Underwriters' Laboratories. ${ }^{10}$ The qualification test requires that the filter withstand (1) 700 to $750^{\circ} \mathrm{F}$ air for $5 \mathrm{~min}$ at rated airflow capacity with no significant reduction of filtration efficiency and (2) a spot-flame test in which a Bunsen burner flame is played on the filter core with no afterburning when the flame is removed. For sizes not specifically covered by UL -586 , the buyer should specify filters of the same materials of construction as filters that have been qualified. Both wood- and steel-cased filters in the rectangular open-face configuration can be made to meet the requirements of UL-586; enclosed and cylindrical filters are qualified by the UL-586 test.

\subsubsection{Environmental Properties}

Hot Air Resistance. 'Tables 3.4 and 3.5 give limiting continuous service temperatures for woodand steel-cased HEPA filters, based on sealants commonly used for sealing the core into the case. Filters with both types of case are designed to withstand temperatures to $750^{\circ} \mathrm{F}$ for 5 to $10 \mathrm{~min}$. Continuous operation at high temperature is limited primarily by the sealant. At temperatures well below the char or "checkering" point, elastomeric sealants lose their shear strength (from about 850 psi at room temperature to as low as 15 psi at $300^{\circ} \mathrm{F}$ ). The buyer should determine the manufacturer's limiting continuous service temperature if continuous operation at high temperature is necessary (see discussion of sealants in Sect. 3.2.2).

Moisture and Corrosion Resistance. The HEPA filter has limited resistance to corrosion. The separators, which must retain their shape to prevent collapse of the filter core, are the elements most subject to attack. Of the common separator materials, treated asbestos has the best corrosion resistance but has almost no moisture resistance unless specially treated. Aluminum has excellent moisture resistance and adequate corrosion resistance to the low concentrations of acids and caustics encountered in most applications. Plastic separators (polystyrene, polyvinyl chloride) are generally not suitable for nuclear applications because of their poor heat resistance; more importantly, the corrugations of plastic separators tend to flatten out when exposed to warm $\left(90\right.$ to $\left.120^{\circ} \mathrm{F}\right)$ air, thus resulting in a decrease of filter core strength. Stainless steel separators are unavailable because of filter manufacturing problems introduced by this material. If treated asbestos is specified, it must be qualified for exposure to the corrodents expected in service, because, if the coating is destroyed, the separator will collapse if exposed to moisture. A standard qualification test for moisture and corrosion-resistant separators has been developed. ${ }^{16}$

Wood casings are more resistant to chemical attack than cadmium-plated steel casings. However, wood is not suitable for extended continuous operation (seven days or more) in very humid ( 95 to $100 \% \mathrm{RH}$ ) environments at temperatures higher than $130^{\circ} \mathrm{F}$, particularly when periods of operation at service temperature will alternate with periods of shutdown at room temperature. Under these conditions, moisture condensed on the casing surface may infiltrate the wood, softening and releasing fibers beneath the sealant, which may cause a failure of the seal or even complete release of the filter core. Woodcased filters should not be used in very dry $(<1 \% \mathrm{RH})$ environments. When steel cases are required, either chromized steel, "muffler grade" stainless steel (e.g, Type 409), or cadmium-plated carbon steel is recommended. Galvanized or zinc-plated carbon steel is not recommended. Austenitic stainless steels have been used for filter cases but are very costly and not warranted because of their throwaway nature. Reclamation of stainless steel cases, a process attempted by one ERDA contractor, proved impracticable because of the high cost of decontaminating the reclaimed cases. Wood-cased filters are not recommended for use in extremely dry air or inert gas (1 or $2 \% \mathrm{RH}$ or less) because the wood will deteriorate.

The glass-fiber filter medium has fair resistance to corrosion and deterioration by water. However, glass, and therefore most fiberglass media, have poor resistance to hydrogen fluoride (HF). An HFresistant medium has been developed by the Herty 
Table 3.4. Recommended limiting service temperatures for steel-framed fire-resistant HEPA filter units sealed with elastomeric adhesives

\begin{tabular}{lccccc}
\hline & \multicolumn{5}{c}{ Temperature to which filter was exposed $\left({ }^{\circ} \mathrm{F}\right)$} \\
\cline { 2 - 6 } \multicolumn{1}{c}{$\begin{array}{c}\text { Sealant } \\
\text { used }\end{array}$} & $\begin{array}{c}\text { Up to } \\
10 \mathrm{~min}^{a}\end{array}$ & $\begin{array}{c}\text { Up to } \\
2 \mathrm{hr}\end{array}$ & $\begin{array}{c}\text { Up to } \\
48 \mathrm{hr}\end{array}$ & $\begin{array}{c}\text { Up to } \\
10 \text { days }\end{array}$ & Indefinitely \\
\hline $\mathrm{HT}^{\prime}-30-\mathrm{FR}^{b}$ & 750 & 350 & 325 & 300 & 260 \\
$\mathrm{Z}^{b} 73^{c}$ & 750 & 325 & 300 & 275 & 200 \\
EC-2155 $^{\text {Polyurethane foam }}$ & 750 & 250 & 220 & 200 & 200 \\
\hline
\end{tabular}

${ }^{a}$ Some reduction in efficiency may occur after $5 \mathrm{~min}$ of exposure.

${ }^{b}$ Goodyear.

${ }^{c}$ Pittsburgh Plate Glass.

${ }^{d}$ Minnesota Mining and Manufacturing (3M).

Table 3.5. Recommended limiting service temperatures for wood-framed fire-resistant HEPA filter units ${ }^{a}$

\begin{tabular}{lccccc}
\hline & \multicolumn{5}{c}{ Temperature to which filter was exposed $\left({ }^{\circ} \mathrm{F}\right)$} \\
\cline { 2 - 6 } $\begin{array}{c}\text { Frame } \\
\text { material }\end{array}$ & $\begin{array}{c}\text { Up to } \\
10 \mathrm{~min}\end{array}$ & $\begin{array}{c}\text { Up to } \\
2 \mathrm{hr}\end{array}$ & $\begin{array}{c}\text { Up to } \\
48 \mathrm{hr}\end{array}$ & $\begin{array}{c}\text { Up to } \\
10 \text { days }^{b}\end{array}$ & Indefinitely $^{b}$ \\
\hline $\begin{array}{l}3 / 4 \text {-in. thick } \\
\text { plywood } \\
\begin{array}{l}3 / 4-\text { in. wood- } \\
\text { particle board }\end{array}\end{array}$ & 750 & 300 & 275 & 200 & 180 \\
\hline
\end{tabular}

${ }^{a}$ Subject to sealant limitations given in Table 3.4.

${ }^{b}$ Maximum temperature of $120^{\circ} \mathrm{F}$ where relative humidity is $75 \%$ or higher.

${ }^{c}$ Exterior grade fire-retardant treated.

${ }^{d}$ Minimum density $=45 \mathrm{lb} / \mathrm{ft}^{3}$.

Foundation for ERDA for use in radiochemical and fuel reprocessing operations where HF resistance is required. ${ }^{5}$ In in-service tests at ERDA's Rocky Flats Plant, wood-cased filters with corrosion-resistant asbestos separators and HF-resistant media withstood operation in one of the most severe plant environments (containing high, but unspecified, concentrations of $\mathrm{HF}$ and $\mathrm{HNO}_{3}$ ) for 24 weeks with no observable breakdown of media, separators, or cases; whereas standard fire-resistant HEPA filters with fiberglass media last considerably less than 100 days on the average and are described as having "mushy" media on withdrawal. ${ }^{\top \overline{7}}$ The. $\mathrm{HF}$-resistant medium is not yet commercially a vailable.

Radiation Resistance. Although most applications for HEPA filters do not involve exposure to high levels of radiation, postaccident cleanup systems and some fuel reprocessing applications will involve such exposure. Exposure of HEPA filters to high levels of radiation (integrated beta or gamma dose of $3.5 \times 10^{7}$ rads or greater) can result in significant reduction in the moisture resistance (and therefore the wet strength) of fiberglass media. This must be taken into consideration when establishing the worst conditions that the filters can be exposed to under normal or abnormal system operation. In tests at Savannah River, ${ }^{18}$ HEPA filters of various commercial makes and constructions were irradiated to an integrated gamma dose of from $1 \times 10^{7}$ to $7 \times 10^{7}$ rads. They were then subjected to a steam-air flow $\left(90^{\circ} \mathrm{C}\right)$ of slightly more than three times the rated airflow capacity of the filters (a steam-air flow substantially greater than that expected under postaccident conditions in the Savannah River reactors). The tests showed a substantial increase in the tendency of the filters to plug with moisture (ref. 19, Fig. 11) with increasing irradiation and with increasing service life before exposure to radiation. The tests verified the need to 
provide excellent protection from water in systems where exposure to spray or to condensing steam is possible, particularly when this may be coupled with high levels of radiation.

Resistance to Plugging. Because HEPA filters are excellent collectors of very small particles, they are likely to plug when subjected to high loadings of sensible moisture, dust, or smoke. Dust plugging can be reduced by the use of effective prefilters, and moisture plugging can be minimized by the use of demisters. Demisters must be effective against very small droplets (at least down to 1 to $10 \mu \mathrm{m}$ ) because tests $^{19}$ have shown that exposure of HEPA filter media to radiation and to air pollutants such as soot and nitrous oxides may significantly reduce water repellency and, therefore, wet strength. As of March 1976, there is still no satisfactory device to protect the HEPA filter against the extremely high concentrations of particulate matter produced by a fire. Prefilters help to remove smoke but they may also plug rapidly and contribute to reduced airflow in the system; when resistance of the prefilter reaches an excessive value, the prefilter ruptures and the HEPA filter downstream is then exposed to the full concentration of smoke, which leads to its eventual plugging and probable rupture. Because rupture of the HEPA filter constitutes a breach of containment, some practical means of suppressing smoke before it reaches the filter system is required. Studies of smoke plugging are being made by ERDA, and means of alleviating the problem will be recommended as the studies progress. ${ }^{20}$

\subsubsection{Costs}

Table 3.6 gives relative costs of various open-face HEPA filter constructions. Commercial costs of 1000-cfm HEPA filters, at the time this handbook is published, are about $\$ 80$ to $\$ 120$, depending on discounts and quantity purchased.

Table 3.6. Relative costs of various HEPA filter constructions

\begin{tabular}{|c|c|c|c|c|}
\hline \multirow[b]{2}{*}{$\begin{array}{c}\text { Casing } \\
\text { material }\end{array}$} & \multicolumn{4}{|c|}{ Separator material } \\
\hline & $\begin{array}{l}\text { Untreated } \\
\text { asbestos }\end{array}$ & $\begin{array}{c}\text { Aluminum } \\
\text { foil }\end{array}$ & $\begin{array}{c}\text { Treated } \\
- \text { asbestos }^{a}\end{array}$ & Plastic \\
\hline Wood & 1.00 & 1.02 & 1.08 & 1.71 \\
\hline Carbon steel $^{b}$ & 1.13 & 1.16 & 1.22 & 1.89 \\
\hline Stainless steel & 1.96 & 1.99 & 2.04 & 2.69 \\
\hline
\end{tabular}

${ }^{a}$ Qualified for moisture and corrosion resistance in accordance with procedures established by ERDA.

${ }^{b}$ Cadmium-plated or chromized.

\subsection{PREFILTERS}

\subsubsection{Classification}

Air filters used as prefilters and as building supplyair filters in nuclear facilities are classified as shown in Table 3.7. The classification is based on arrestance (weight percent) and dust-spot (stain) efficiency as determined by ASHRAE 52-68. ${ }^{21}$ ASHRAE 52-68 requires both a dust-spot (i.e., stain) efficiency test made with atmospheric dust and a synthetic-dust weight arrestance test. The standard emphasizes the correct use of, and distinction between, the two terms; and to avoid confusion, it requires that both values be tested and reported. Table 3.7 shows typical values for the two tests.

By comparison, a HEPA filter has an ASHRAE atmospheric dust-stain efficiency of $100 \%$. Because the atmospheric dust-stain test is based on the staining capacity of the dust that penetrates the filter, compared to the staining capacity of the entering dust, it is not a true measure of particle-removal efficiency for any given particle size range. Table 3.8 gives a more meaningful comparison.

\subsubsection{Performance}

The performance of air filters is defined by particleremoval efficiency, resistance to airflow (i.e., pressure drop), airflow capacity, and dust-holding capacity. To understand manufacturers' rated efficiencies, it is important for the buyer to know what test method was used; whether the reported efficiency refers to weight, stain, or particle count; what test dust was used; and whether clean-filter efficiency or average efficiency over the projected life of the filter is reported. Three test methods were formerly used for rating group I, II, and III filters: the Air Filter Institute (AFI) weight method, ${ }^{22}$ the AFI method, ${ }^{23}$ and the National Bureau of Standards (NBS) dustspot method. ${ }^{24}$ Although these tests have been replaced by ASHRAE 52-68, ${ }^{21}$ they are still used occasionally. In general, the results obtained with the older tests are comparable to those of the respective parts of ASHRAE 52-68.

ASHRAE 52-68 has two basic efficiency tests--a synthetic-dust weight arrestance or weight test, and an atmospheric dust-spot efficiency test. The weight test determines the percent of a synthetic dust that is collected by the filter during an accelerated test. As Table 2.4 shows, more than $97 \%$ of the particles in a typical air sample are larger than $1 \mu \mathrm{m}$ on a weight basis, whereas on a count basis over $99.99 \%$ are 
Table 3.7. Classification of common air filters

\begin{tabular}{lllcl}
\hline Group & Efficiency & \multicolumn{1}{c}{ Filter type } & $\begin{array}{c}\text { Stain test } \\
\text { efficiency (\%) }\end{array}$ & $\begin{array}{c}\text { Arrestance } \\
(\%)\end{array}$ \\
\hline I & Low & Viscous impingement, panel type & $<20^{a}$ & $40-80^{a}$ \\
II & Moderate & Extended medium, dry type & $20-60^{a}$ & $80-96^{a}$ \\
III & High & Extended medium, dry type & $60-98^{b}$ & $96-99^{a}$ \\
HEPA & Extreme & Extended medium, dry type & $100^{c}$ & $100^{a}$ \\
\hline
\end{tabular}

"Test using synthetic dust.

"Stain test using atmospheric dust.

'ASHRAE/52-68, American Society of Heating, Refrigerating and Air-Conditioning Engineers.

Table 3.8. Comparison of air filters by percent removal efficiency for various particle sizes

\begin{tabular}{|c|c|c|c|c|c|}
\hline \multirow{2}{*}{ Group } & \multirow{2}{*}{ Efficiency } & \multicolumn{4}{|c|}{$\begin{array}{c}\text { Removal efficiency (\%) for } \\
\text { particle size of - }\end{array}$} \\
\hline & & $0.3 \mu \mathrm{m}$ & $1.0 \mu \mathrm{m}$ & $5.0 \mu \mathrm{m}$ & $10.0 \mu \mathrm{m}$ \\
\hline 1 & Low & $0-2$ & $10-30$ & $40-70$ & $90-98$ \\
\hline II & Moderate & $10-40$ & $40-70$ & $85-95$ & $98-99$ \\
\hline III & High & $45-85$ & $75-99$ & $99-99.9$ & 99.9 \\
\hline HEPA & Extreme & $99.97 \mathrm{~min}$ & 99.99 & 100 & 100 \\
\hline
\end{tabular}

smaller. Therefore, a filter with a high weight efficiency may be inefficient for removal of small particles.

The dust-spot tests are made by comparing the opacities of stains made on filter papers by air samples withdrawn from the test duct upstream and downstream of the filter. Because particles in the submicron range are chiefly responsible for staining the samples, even though they represent only a fraction of the total weight of dust charged to the filters, the test essentially measures the efficiency of the filter for small particles. However, because the atmospheric dust-spot test uses ambient air as the challenge, results depend on the nature of the atmospheric dust in the location where the tests are made.

The results of the various tests are not comparable, and an efficient filter by one test may be inefficient by another. The user should examine filter efficiency data very carefully to ensure that the precise meaning of the reported data is clear. Efficiency tests for group I, II, and III filters are made on prototypes only, and the results are extrapolated to the various filter sizes of similar design made by the same manufacturer. A predelivery test of each filter unit would be too costly and is not recommended. Table 3.9 gives the comparative performance of group I, II, and III filters (average over the life of the filter to the manufacturer's recommended maximum pressure drop).

The values for dust-holding capacity are based on tests with a synthetic dust. Because actual dustholding capacity varies with the nature and composition of the dust, the dust-holding capacity under service conditions cannot be accurately predicted on the basis of manufacturers' catalog data.

\subsubsection{Construction}

Panel Filters. Group I panel filters (viscous impingement filters) are shallow, tray-like assemblies of coarse fibers (glass, wool, vegetable, or plastic) or crimped metal mesh enclosed in a steel or cardboard casing. The medium is coated with a tacky oil or adhesive to improve retention of trapped particles. Throwaway, replaceable-medium, and cleanablemedium types of panel filters are available. The latter have metal mesh and are generally not used in contaminated exhaust service because of the difficulties and high labor costs associated with cleaning. Figure 3.5 shows typical throwaway and replaceable-medium types. Panel filters have fairly high dust-holding capacity, high airflow capacity with low resistance, and high removal efficiency for large particles. They are particularly effective against fibrous dust and heavy concentrations of visible particles but are useless for protection against submicron particulates. Except when large particles are present from a production operation such as grinding, panel filters are of limited value as prefilters for nuclear exhaust applications because of their limited effectiveness against small particles ( $5 \mu \mathrm{m}$ and less) and because they are rapidly plugged by lint and other fibrous materials. Panel filters of the type shown in Fig. 3.5 have low initial cost and low operating cost, but they may be more expensive than 
Table 3.9. Airflow capacity, resistance, and dust-holding capacity of air filters

\begin{tabular}{llllll}
\hline Group & Efficiency & $\begin{array}{c}\text { Airflow } \\
\text { capacity } \\
\text { (cfm per square foot } \\
\text { of frontal area) }\end{array}$ & $\begin{array}{l}\text { Clean } \\
\text { filter }\end{array}$ & $\begin{array}{c}\text { Used } \\
\text { filter }\end{array}$ & $\begin{array}{c}\text { Resistance } \\
\text { Dust-holding } \\
\text { capacity } \\
\text { (g per 1000 cfm of } \\
\text { airflow capacity) }\end{array}$ \\
\hline I & Low & $300-500$ & $0.05-0.1$ & $0.3-0.5$ & $50-1000$ \\
II & Moderate & $250-750$ & $0.1-0.5$ & $0.5-1.0$ & $100-500$ \\
III & High & $250-750$ & $0.20-0.5$ & $0.6-1.4$ & $50-200$ \\
\hline
\end{tabular}

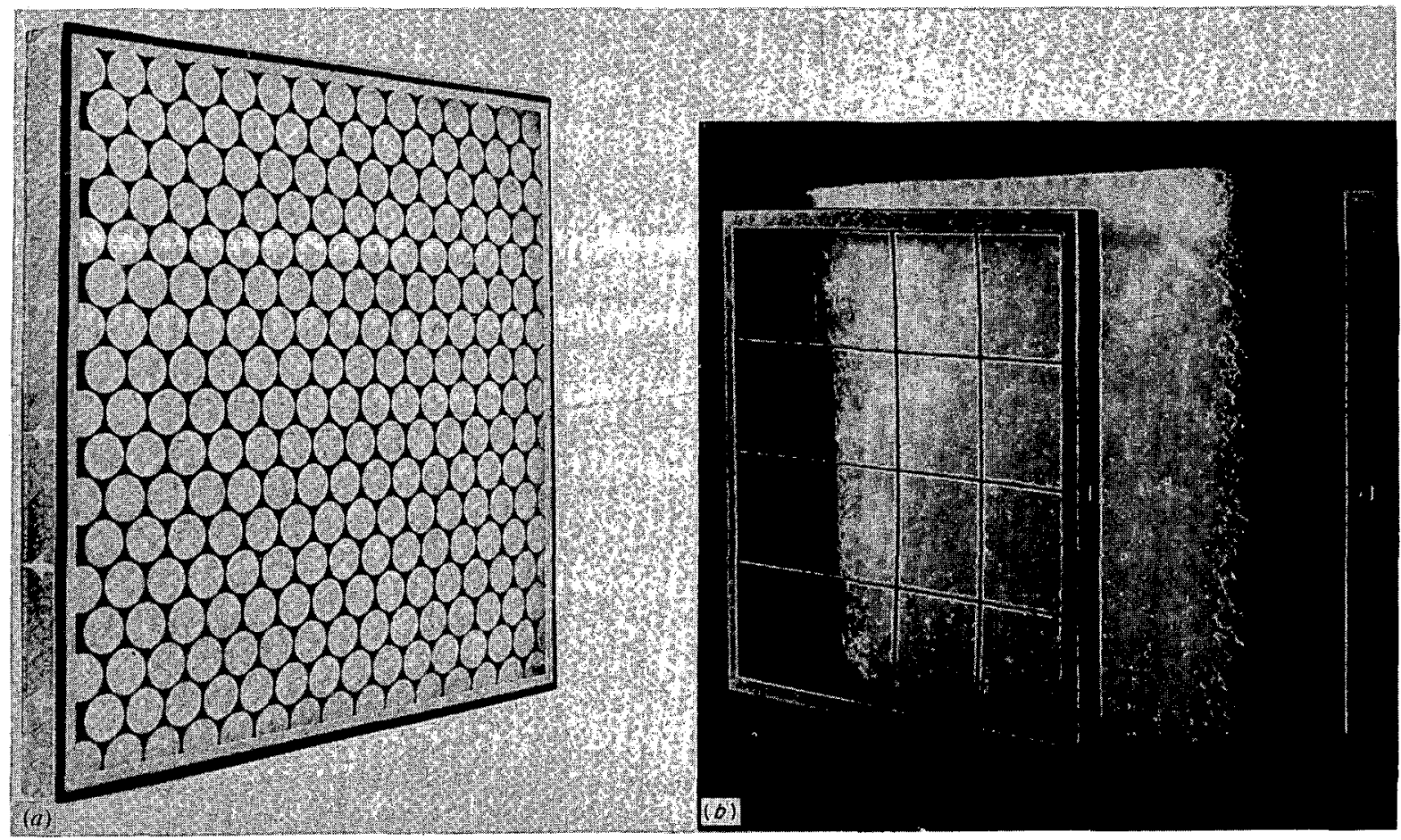

Fig. 3.5. Viscous-impingement panel-type group I air filters. (a) Throwaway paper-cased furnace filter; (b) replaceable-medium type.

an initially more costly group II or III filter that gives better protection to the HEPA filters.

Group II and III Filters. Group II (moderate efficiency) and group III (high efficiency) filters are extended-medium dry-type units. That is, the medium is pleated or formed as bags or "socks" to give a large surface area with minimum frontal area, and the medium is not coated with an oil or adhesive. Throwaway cartridge (Fig. 3.6), replaceable-medium (Figs. 3.7 and 3.8), and cleanable-medium types are available. The particle-removal efficiency of group II filters is moderate to poor for submicron particles but often approaches $100 \%$ for particles larger than $5 \mu \mathrm{m}$. In most cases the pressure drop of extended-medium filters varies directly with efficiency. Group II filters are recommended for high lint and fiber loading applications. The large area of the medium relative to frontal area permits the use of extended-medium filters at duct velocities equal to or higher than those permissible with panel filters. Group III filters are used when higher efficiency for smaller particles is desired, but their dust-holding capacity may be low relative to the lower efficiency group II filter. 


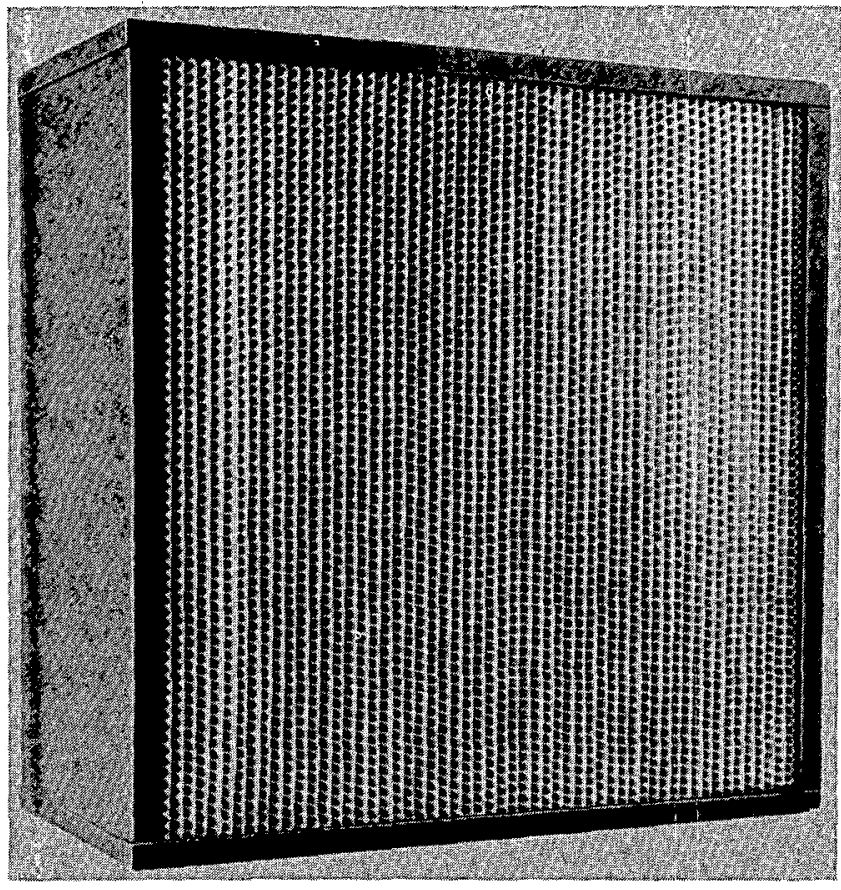

Fig. 3.6. Extended-medium dry-type throwaway, group III (high-efficiency) air filter with fiberglass medium, aluminum separators, and mineral-board case.

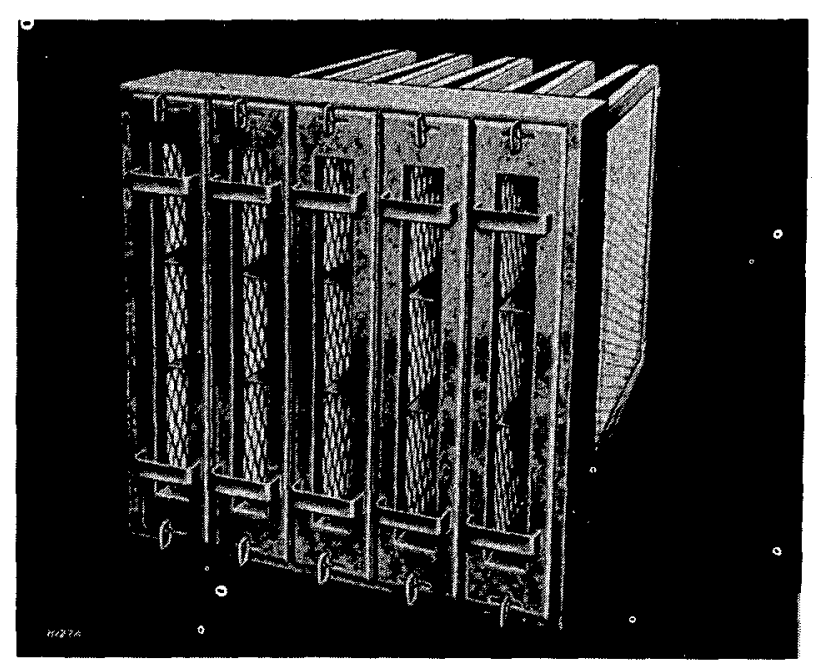

Fig. 3.7. Replaceable-medium, group III (high-efficiency ventilation-type) air filter with wire medium supports. Note individually removable core segments. Unit is $24 \times 24 \times \sim 30 \mathrm{in}$. deep. Courtesy American Air Filter Co.

\subsubsection{Fire Resistance}

Underwriters' Laboratories classifies common ventilation air filters in two categories with respect to

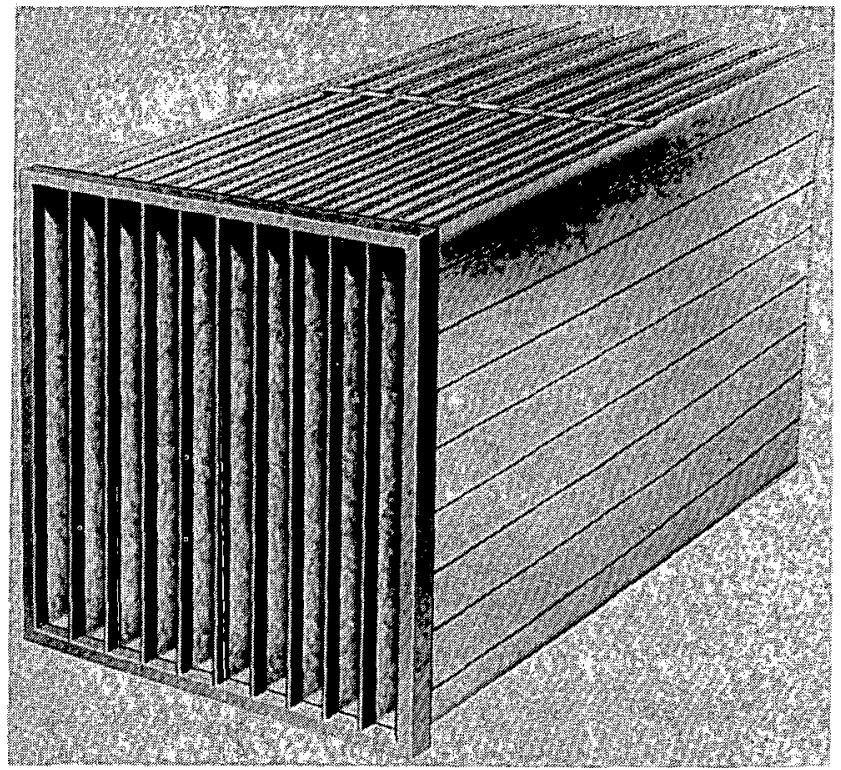

Fig. 3.8. Bag-type, replaceable-medium, group III (highefficiency) ventilation-type air filter. Courtesy Cambridge Filter Corp.

fire resistance. ${ }^{25}$ When clean, UL class 1 filters do not contribute fuel when attacked by flame and emit only negligible amounts of smoke. UL class 2 filters may contain some combustible material but must not contribute significantly to a fire. Because dust collected on either a UL class 1 or class 2 filter may burn vigorously and create a fire that is difficult to extinguish, the use of UL-rated filters should not lead to an unwarranted sense of security on the part of the user. Filters that meet the UL requirements are listed in the current UL Building Materials List. ${ }^{26}$ NFPA $90 \mathrm{~A}^{11}$ makes the use of UL-rated filters mandatory.

\subsubsection{Hot Air Resistance}

Most types of common air filters are suitable for continuous operation at temperatures no higher than 150 to $250^{\circ} \mathrm{F}$. Certain types with glass-fiber media in steel or mineral board casings may be used at temperatures as high as $400^{\circ} \mathrm{F}$. With any hightemperature filter, the user should take a conservative view of performance claims, particularly for efficiency at operating temperature. The user should examine his application closely to determine if the filters will be exposed to continuous high temperature or if the high-temperature exposure will be intermittent or under emergency conditions. 


\subsubsection{Maintenance Considerations}

Air filters may be classified by the method of medium renewal, that is, throwaway cartridge, replaceable medium, or cleanable medium. The first choice for nuclear exhaust applications is the throwaway cartridge. The filter shown in Fig. 3.6 is typical of this class. Replaceable-medium filters offer an advantage over throwaways in that the bulk of material to be disposed of is smaller. This may reduce handling and disposal costs (radioactively contaminated filters are usually buried at NRCauthorized burial grounds), but reentrainment of contaminated dust is possible as the medium is removed from the holding frame and crumpled up to be stuffed into a bag for disposal. Replaceablemedium designs, therefore, are not recommended for nuclear exhaust applications. Cleanable filters are unsatisfactory because they require a long downtime of the facility, and they require decontamination facilities adjacent to the filter house. Cleaning of filters transfers radioactive material from the filter to the contaminated liquid waste system, which may pose a more difficult problem than the disposal of dry throwaway units.

Corrosion and Moisture. The choice of filter type may be limited by corrodents and moisture in the air stream in which it is to operate. Many filter media will not withstand acid or caustic fumes; fiberglass will withstand exposure to most reagents except hydrofluoric acid or gaseous hydrogen fluoride. Aluminum parts (e.g., separators) may deteriorate in sea air or when caustic substances are deposited on them. Plastics have poor heat resistance and generally will not meet UL requirements. Heavy concentrations of water droplets or condensate may plug or deteriorate filters and result in frequent filter replacement. In general, common air filters that have equivalent construction to the HEPA filters used in the system will have equivalent moisture and corrosion resistance. Plugging as a result of moisture will not be quite so severe for class III filters and will probably be considerably less for class $I I$ and class I filters. Radiation resistance of common air filters has not been investigated.

Change Frequency. Pressure drop (resistance) is the primary factor in prefilter replacement. Replacement at the pressure drop suggested by the manufacturer is recommended. Panel filters will plug rapidly under heavy lint loads, whereas some lint, by breaking up the uniformity of the dust deposit, may be beneficial to extended-medium filters. Extended-medium filters will plug rapidly in heavy concentrations of soot or smoke. Operation at airflow levels below the manufacturer's rated capacity extends filter life and reduces filter change frequency (see discussion of underrating, Sect. 2.3.6). On the other hand, when airflow exceeds the manufacturer's recommendations by more than about 15 to $20 \%$, dust-loading rate (and consequently filter replacement costs) begins to increase exponentially with arithmetic increases in airflow.

\subsubsection{Operational Considerations}

The decision to use prefilters must be determined for each application on the basis of total air cleaning system costs and the consequences of exposing the HEPA filters to the environment without protection. In some cases, prefilters may double or triple the life of HEPA filters; in other cases, the increase may be insignificant. In general, HEPA filters should be protected from (1) particles larger than 1 or $2 \mu \mathrm{m}$ in diameter, (2) lint, and (3) dust concentrations greater than 10 grains per $1000 \mathrm{ft}^{3}$. Resistance (and corresponding power costs), system installation costs, and filter element replacement costs generally increase with increasing prefilter efficiency. Table 3.10 shows the relative price ranges of filters usually used for prefiltering.

Table 3.10. Price indexes of common air filters per 1000-cfm capacity

\begin{tabular}{llll}
\hline Group & Efficiency & \multicolumn{1}{c}{ Type } & Price index \\
\hline 1 & Low & Panel, viscous impingement & 1 \\
II & Moderate & Extended medium & $2-10$ \\
III & High & Extended medium & $30-70$ \\
- & HEPA & HEPA & $80-100$ \\
\hline
\end{tabular}

\subsection{RADIOIODINE ADSORBERS}

\subsubsection{Introduction}

The gas (i.e., adsorbate) of primary interest in nuclear air and gas cleaning systems is radioiodine. Although concentrations will be extremely low (probably no more than $1 \mu \mathrm{Ci} / \mathrm{ml}$, or about $8 \mu \mathrm{g} / \mathrm{m}^{3}$, in the primary containment air of a reactor following a DBA), the high affinity of radioiodine for the thyroid makes it a major contributor to potential radiation doses to the public and plant personnel. Radioiodine may be in the form of elemental iodine $\left(\mathrm{I}_{2}\right)$ or organic iodine compounds, principally methyl iodide $\left(\mathrm{CH}_{3} \mathrm{I}\right)$. 
Adsorption on activated carbon is the most frequently used method for removing radioiodine from air and gas streams in large ventilation and air cleanup systems. Inorganic adsorbents, such as silver-exchanged zeolite, are sometimes used in small systems where high temperatures or the presence of contaminants, such as nitrogen oxides, which react with carbon make the use of activated carbon unadvisable. The high cost of inorganic adsorbents often makes their use unattractive for large systems.

Activated carbon is produced by first making a charcoal from coconut, nut shell, wood, bituminous coal, or petroleum sludge. This charcoal is then activated by controlled heating in a steam atmosphere to drive off organic matter and generate large internal surfaces on which adsorption can take place. The internal area of activated carbon ranges from 700 to $1800 \mathrm{~m}^{2} / \mathrm{g}$, based on the nitrogen gas isotherm by the BET method. The activated carbon may then be impregnated with chemicals, at this time principally $\mathrm{I}_{2}, \mathrm{KI}$, and triethylene diamine (TEDA), to enhance its ability to adsorb organic radioiodine compounds. Definitions of terms relating to adsorbents and activated carbon are given in ASTM D2652. ${ }^{27}$

\subsubsection{Performance of Adsorption Systems}

Important properties of a nuclear adsorption system are efficiency for trapping radioiodine, breakthrough capacity, ability to retain sorbed radioiodine, airflow capacity and velocity, resistance to airflow, and resistance to ignition. The remainder of this section discusses important performance considerations. The discussion is based primarily on activated carbon because it is the most widely used material. However, much of the material applies equally to zeolite and other inorganic adsorbents.

Efficiency. The efficiency of a nuclear grade adsorbent is the measure of its ability to remove (i.e., sorb) from a flowing air or gas stream an adsorbate of interest (i.e., $\mathrm{I}_{2}, \mathrm{CH}_{3} \mathrm{I}$ ). The efficiency of activated carbon is a function of (1) the degree of activation, ash and moisture content, and impurities present in the carbon; (2) the type and quantity of impregnant; (3) granule size (efficiency varies inversely with average granule size); (4) gas residence time (i.e., the contact time between the gas and the carbon in the adsorber bed - at constant airflow velocity, efficiency increases with increasing gas residence time); (5) airflow velocity through the adsorber bed (at constant gas residence time, efficiency increases with increasing airflow velocity); and (6) temperature and relative humidity of the air or gas stream (for activated carbons, efficiency tends to decrease with increasing temperature and relative humidity; for silver zeolite, efficiency increases with increasing temperature). Special high-purity low-ash grades of activated carbon are generally used for trapping radioiodine. Efficiency is determined by laboratory testing using radioactively traced iodine and methyl iodide.

Minimum efficiencies for elemental iodine and methyl iodide at low $\left(70^{\circ} \mathrm{F}\right)$ and elevated $\left(180^{\circ} \mathrm{F}\right)$ temperatures and low $(70 \%)$ and high $(>95 \%)$ relative humidity are specified in RDT M 16-1 ${ }^{28}$ and ANSI $\mathrm{N} 509 .{ }^{29}$ Trapping of elemental radioiodine involves physical adsorption only, and the efficiency of nearly any good grade of activated carbon, impregnated or not, will be at least $99 \%(D F=100)$ under any combination of temperature and humidity that would be encountered in a nuclear air cleaning system. Trapping of organic radioiodine compounds, on the other hand, requires an impregnated carbon and involves physical adsorption, chemical reaction, and/or isotopic exchange. Efficiency for those compounds is dependent on temperature and relative humidity of the air or gas stream, and performance of the adsorbent must be qualified under a range of operating conditions as specified by RDT M 16-1. ${ }^{28}$ The tests of RDT M 16-1 bracket the range of conditions that are likely to be encountered in most nuclear applications.

In addition to efficiency tests, qualification of a nuclear grade adsorbent requires a $4-\mathrm{hr}, 180^{\circ} \mathrm{C}$ radioiodine desorption test and a number of physical properties tests that characterize the adsorbent from the standpoint of particle size, resistance to attrition, ignition temperature, and impregnation. Efficiency tests are very costly and are made only at the time a grade of product is qualified. If there is any significant change in base material, impregnation, or manufacture, however, the material must be requalified and a new grade designation assigned. Subsequent production lots of material are evaluated on the basis of (1) physical properties tests, the results of which must conform, within reasonable tolerances, to the values established at the time of qualification and (2) a $4-\mathrm{hr} 180^{\circ} \mathrm{C}$ radioiodine desorption test. The radioiodine desorption test has been found very sensitive for differentiating between candidate products and for detecting changes or errors in manufacture. Substantial differences have been observed between ostensibly equivalent 
products (e.g., penetrations of four $\mathrm{KI}-\mathrm{I}_{2}$ impregnated activated carbons, claimed to be equivalent to one another, ranged from $0.28 \%$ to $12.8 \%$ in the 4 -hr $180^{\circ} \mathrm{C}$ radioiodine desorption test) ${ }^{30}$ and, on occasion, between successive production lots of the same material. For this reason, the user should verify test results of production lots to satisfy himself that the product meets specification requirements. For conservatism, the efficiency values of Table 3.11 are recommended for design purposes. (These values are single-pass efficiencies, and the efficiency of a recirculating system would be multiplicative.)

Capacity. The capacity of a nuclear grade adsorbent is its breakthrough capacity, that is, the quantity of adsorbate (radioactive iodine or iodine compound) which, when charged to the adsorbent bed, results in the first appearance of the adsorbate in the effluent from the bed. Capacity is a function of the surface area of "active sites" in the adsorbent, and therefore of the depth and area of the beds. For impregnated activated carbons it is also a function of the nature, quantity, and condition of the impregnant.

Aging. Aging or weathering of activated carbon is the gradual deterioration of "active sites" due to oxidation of its surfaces or to desorption or chemical reaction (with environmental poisons) of its impregnant. ${ }^{30}$ This deterioration results in decreases, with time, of capacity and efficiency, and thus affects the useful life of the carbon. There are currently no valid guides for estimating the useful life of activated carbon. Tests by the British in 1966, using coal-base activated carbon impregnated with KI and TEDA, indicated a life of 18 months in a continuously on-line system, 3 years in a standby (i.e., normally off-line) system, and 5 years for carbon kept in sealed

Table 3.11. Recommended design values for single-pass methyl iodide efficiency of full-scale adsorbers containing impregnated activated carbon

2-in. bed depth, 0.2-sec minimum gas residence time

\begin{tabular}{lcc}
\hline \multirow{2}{*}{$\begin{array}{c}\text { Relative } \\
\text { humidity }\end{array}$} & \multicolumn{2}{c}{$\begin{array}{c}\text { Percent efficiency for radioiodine } \\
\text { as methyl iodide }\end{array}$} \\
\cline { 2 - 3 } & $70^{\circ} \mathrm{F}$ & $270^{\circ} \mathrm{F}$ \\
\hline 85 or less & 95 & 98 \\
90 & 90 & 90 \\
95 & 80 & 70 \\
98 & 70 & 30 \\
\hline
\end{tabular}

containers. ${ }^{31}$ Experience in the United States with coconut-base and certain experimental coal-base carbons indicates much more gradual, but still significant, aging effects. ${ }^{30,32}$ The numbers of the British tests can probably be ignored for practical purposes, but the ratio of the numbers is probably valid. That is, the carbon of a standby system can be expected to last about twice as long as the same grade of carbon when installed in a continuously on-line system. Therefore, test and experience data obtained from ERDA facilities with continuously vented containments can be extrapolated to the off-line system. Because of the uncertain life of activated carbon under normal operating conditions, NRC recommends that samples from ESF systems be taken for laboratory testing every $720 \mathrm{hr}$ of fan operation. Installation of an elapsed time meter on the fan is recommended as a means of timing the withdrawal of test samples. Regulatory Guide 1.52 gives the requirements for laboratory testing of used carbons, using the test procedures but not the acceptance criteria of RDTM 16-1 for new carbons. ${ }^{33}$

The loss in capacity for elemental iodine is much slower than that for methyl iodide. Beds exposed continuously to flowing air at one installation showed adequate remaining capacity for elemental iodine after four years of service. ${ }^{34}$ At other installations, however, exposure of the beds to paint and solvent fumes reduced capacity to the point that efficiency fell to unacceptable levels in only a few months. ${ }^{35,36}$ The loss in breakthrough capacity indicates (1) a need for routine analytical tests on samples taken from the beds to determine remaining capacity; (2) a need for conservative adsorber design; and (3) the need to protect adsorbers from unnecessary exposure to moisture, hydrocarbons, and other poisons. There is no way to rejuvenate used carbon other than by complete reactivation, a process which is impossible for carbon that has been even slightly radioactively contaminated. ${ }^{30}$

The use of a sacrificial or guard bed in advance of the active bed to protect the active bed from "tramp" contaminants was suggested several years ago and is still recommended where cost permits. ${ }^{37}$ The guard bed can be filled with a less expensive carbon and does not require costly leak tests. In any event, adsorbers should be protected against fumes, dust, and dirt during construction and maintenance operations or they may have to be replaced before startup. It is strongly recommended that, in construction situations, adsorbers not be installed until the system is ready for startup. During maintenance 
operations, if the system is not required for decontaminating air of the contained space, the filter housing should be isolated by dampers.

Performance for organic compounds is severely limited if the carbon becomes wet, as would be the case in some types of reactors if adequate demisters and/or heaters were not provided. Flooding of carbon beds with water effectively destroys their capability for trapping iodine in any form and also results in the release of any radioiodine that has been trapped. ${ }^{30}$

Retention. Because adsorbers may have to be operated for several days or weeks following an upset or accident in the contained space served by them, consideration must be given to retention of trapped gases. The retentivity of a sorbed chemical is characteristically less than $35 \%$ of the breakthrough capacity. ${ }^{38}$ So long as the quantity of iodine held in the bed is less than the retentivity limit and desorption temperature does not greatly exceed the adsorption temperature, desorption is not a significant problem. Some iodine loss will take place at higher desorption temperatures, particularly from impregnated carbons; these higher temperatures may also desorb some of the impregnant and further reduce the capacity for radioactive organic iodides. The extent of loss is still under investigation. The retentivity factor further emphasizes the need for conservative adsorber design.

High-temperature desorption is not a problem with silver-exchanged zeolites or silver-impregnated alumina because the iodine reacts with the silver to form a tightly bound chemical species, even at very high temperatures. These materials perform better at high operating temperatures, which is one reason why they are favored in certain applications.

Ignition Temperature. Adsorption systems containing activated carbon must be designed so that the decay heat generated by collected fission products cannot cause ignition of the carbon or overheating to the point of significant desorption of collected fission products or impregnant. The postaccident air cleanup system of a nuclear power reactor, for example, may contain several tons of carbon loaded in 2 -in. deep to 6 -in. deep beds. The only cooling method of consequence is forced air circulation. If, as is assumed for the DBA, $25 \%$ of the iodine inventory of the reactor core is released to the containment and is collected in the adsorption system during the first few hours following the accident, calculations and laboratory tests show that the ignition temperature might be reached if airflow through the bed is reduced to less than $5 \mathrm{fpm}$. On the other hand, if adequate airflow can be maintained, there is little doubt that the heat from deposited radioiodine can be removed without causing ignition or significant desorption from the beds. ${ }^{39}$ Calculational results by the computer program TOOHOT show that under certain conditions ignition of the carbon of an adsorber bed can take place at an airflow velocity of $4 \mathrm{fpm}^{40}$ assuming that the high-ignition-temperature carbons specified in RDT M 16-1 are used.

The ignition temperature of new charcoal is a function of ash content, impurities, internal surface area, granule size, flammability of chemical impregnants, bed depth, and airflow through the bed. ${ }^{41}$ Adsorbed substances (such as solvents and hydrocarbons) may further alter ignition temperature, which varies directly with the degree of activation and inversely with granule size. The lowest ignition point of new $\mathrm{KI}$ and $\mathrm{KI}_{3}\left(\mathrm{I}_{2}+\mathrm{KI}\right)$ impregnated coconutbase carbons is about $290^{\circ} \mathrm{C}^{42}$ The ignition of TEDA-impregnated carbons may take place at temperatures as low as 180 to $190^{\circ} \mathrm{C}$, depending on the level of impregnation, airflow, and bed depth. ${ }^{4,43,44}$ With conservative bed design (i.e., a bed that contains a large volume of charcoal relative to the airflow capacity of the system and to the amount of iodine to which it can be exposed), the specific loading of fission products will be low. This reduces the possibility of spontaneous combustion, assuming that an airflow in excess of 8 to $10 \mathrm{fpm}$ can be maintained through the system.

Airflow Capacity and Resistance. Airflow capacity is a function of bed size, configuration, and resistance. Resistance is a function of the size of carbon granules (mesh size), packing density, bed thickness, free area of the granule retaining screens, and airflow velocity. For a given bed design, resistance varies directly with airflow velocity. On the other hand, efficiency of a given bed design, assuming a minimum gas residence time to effect sorption, varies inversely with airflow velocity. Operation above the rated capacity of the adsorber, therefore, is not recommended because of the possible reduction in efficiency, as well as the more obvious penalties of higher pressure drop and operating costs. Assuming there is no change in airflow velocity and that particulate filters are provided upstream to intercept dust that might otherwise collect on the screens or in the charcoal itself, pressure drop will remain constant over the life of the adsorber. For constant gas residence time, efficiency also increases with in- 
creasing velocity; for a fixed bed depth in proportions that will maintain the' gas residence time, therefore, efficiency can be enhanced by increasing the size of the bank and the airflow velocity.

\subsubsection{Adsorber Unit Design and Construction}

Nearly all adsorbers in current commercial facilities are either of the modular tray configuration shown in Fig. 3.9, which are installed in banks as shown in Fig. 3.10, or of a permanent single-unit (PSU) fill-and-empty-in-place (often called deep bed or gasketless) design similar to the unit shown in Fig. 3.11. The pleated 1-in.-bed modular cell shown in Fig. 3.12, although still used in a number of facilities, is obsolete. Minimum requirements for pleated-bed (type I) and tray (type II) cells are specified in IES CS$8{ }^{45}$ Tray (type II) adsorber cells consist of two 2-in. thick adsorbent beds with an airspace between. Cross-sectional dimensions are standardized (IES CS-8), and the cell is installed in standardized openings (ref. 45, Appendix A) in the mounting frame of the housing. (Requirements for mounting frames are described in Chap. 5.) Length of the cell varies according to the manufacturer's particular design and may range from 26 to 32 in. It is recommended that the mounting frame have a cell support structure that can accept a cell length up to at least $32 \mathrm{in}$. to permit interchangeability with the cells of any manufacturer. Cells weigh between 80 and 100 $\mathrm{lb}$, depending on the individual design, and contain from 47 to $52 \mathrm{lb}$ of 8 - by 16-mesh ${ }^{46}$ activated carbon. No caulking, scrims, or other nonmetallic materials, except the neoprene gasket, are permitted in its construction. The cell is designed for a gas residence time of $0.25 \mathrm{sec}$ and resistance of $1.1 \mathrm{in} . \mathrm{wg}$ when operated at a volumetric airflow rate of $333 \mathrm{cfm}$. Air

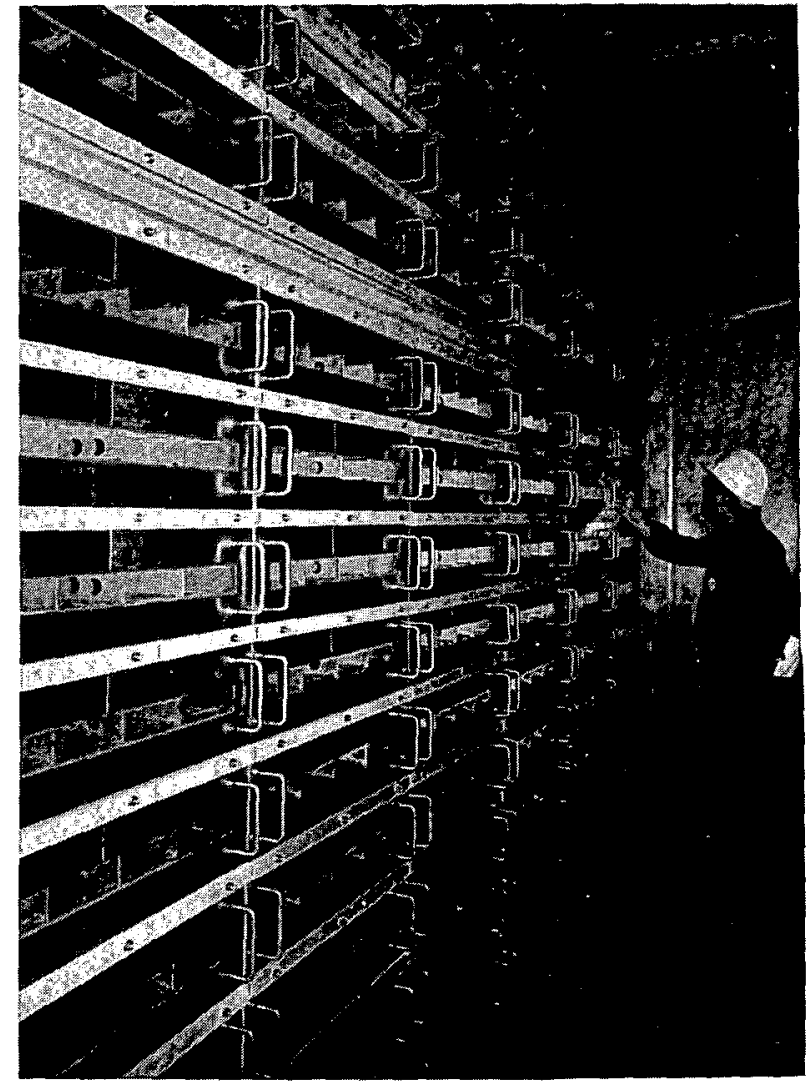

Fig. 3.10. Bank of type II adsorber cells in nuclear reactor system.

ORNL-DWG 72-7605

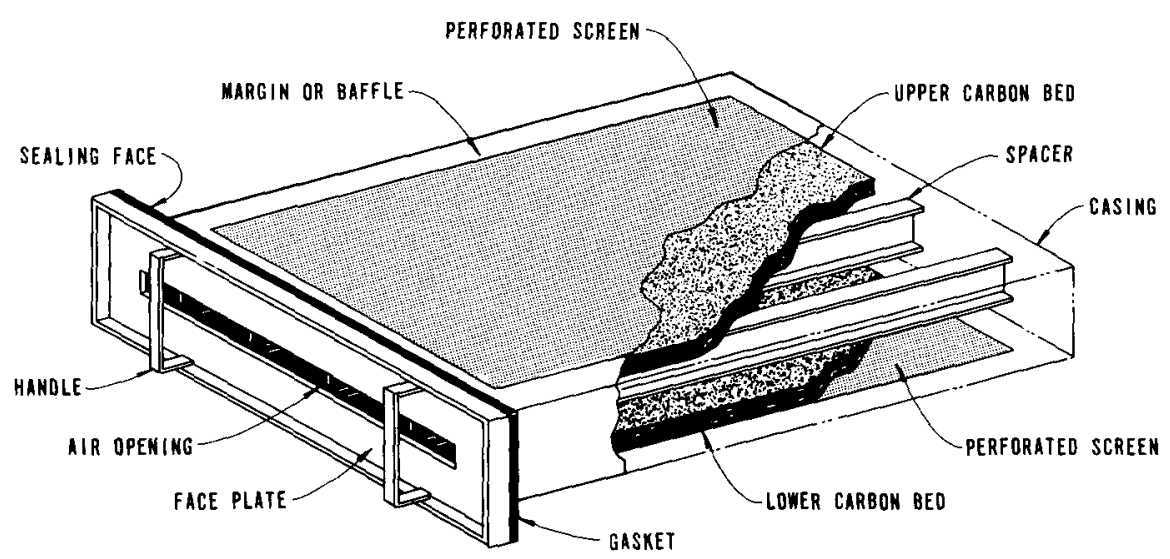

Fig. 3.9. IES CS-8 type II (unit tray or drawer type) adsorber cell. Weight, 80 to $100 \mathrm{lb}$. 


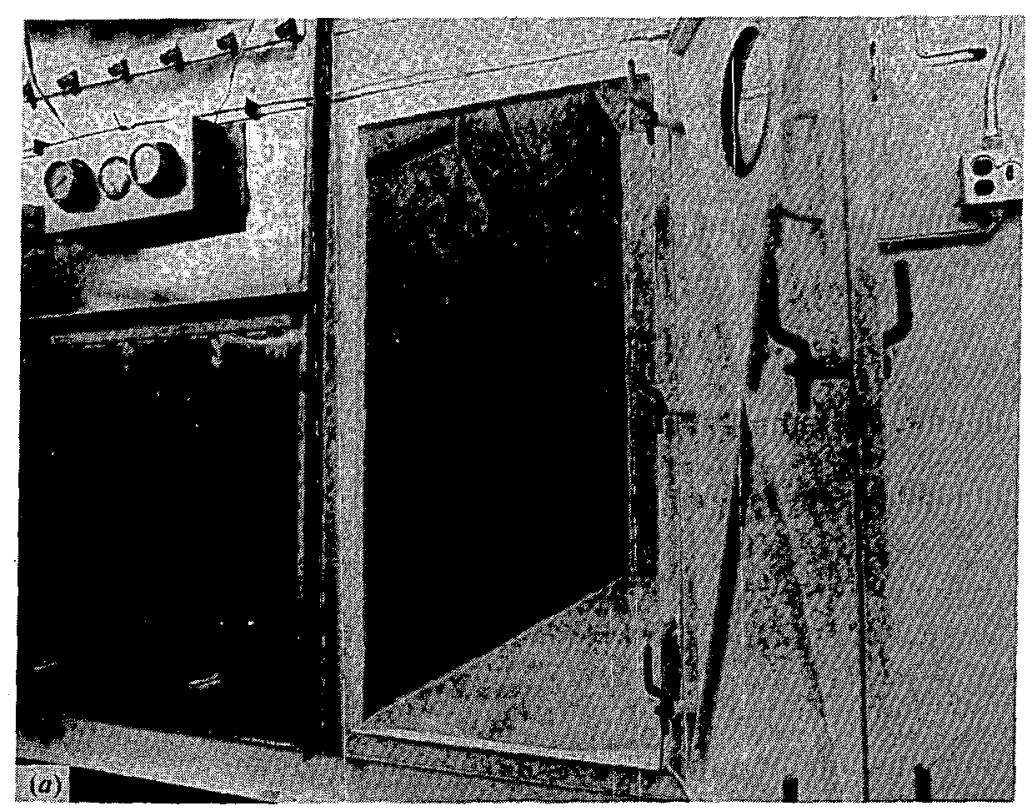

ORNL.DWG 76-A6B7

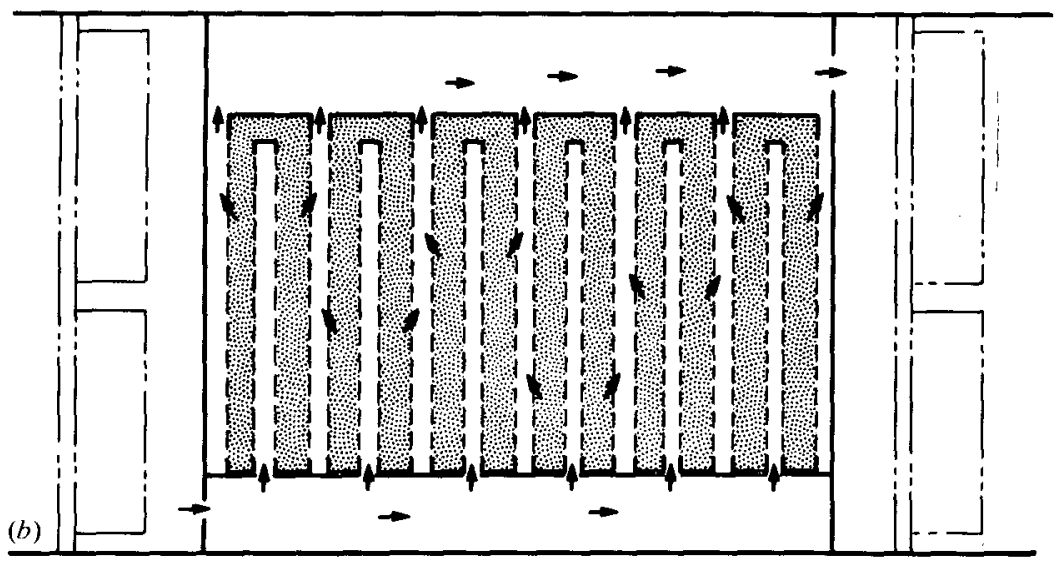

Fig. 3.11. Permanent single-unit (PSU) adsorber. PSU adsorbers are permanently installed components that are filled and emptied in place through ports (not shown) on the top and bottom. Unit is all-weld construction with no gasket seals. (a) View of downstream face of inclined bed PSU adsorber unit. Courtesy CTI-Nuclear, Inc. (b) Airflow through typical vertical bed PSU adsorber. Note HEPA filters upstream and downstream. Courtesy CVI Corp.

can flow in either direction through the slot (i.e., the cells may be installed on either the upstream or downstream side of the mounting frame). Unit cells for nuclear service must be all stainless steel; carbon steel cells used in earlier installations corroded in only a few months when exposed to moisture and air carrying significant concentrations of nitrogen oxides or other corrodents. The type II cell is suitable for containing inorganic adsorbents (e.g., silver zeolite) in addition to activated carbon.
There are currently no standards for PSU adsorbers. On the other hand, the principal provisions of IES CS-8 can be extended to these devices as long as the leaktightness, workmanship, resistance, and test requirements of IES CS-8 can be met. (IES CS-8 was written so that its governing requirements could be extended to designs other than type I and type II cells.) As with cells, all surfaces of PSU adsorbers in contact with the adsorbent are stainless steel. The outer walls of the unit are part of the pressure 
boundary of the housing in which it is installed (see Fig. 3.11). The beds, which are usually but not always deep beds (i.e., 4 in. or more), are either inclined, as shown in Fig. 3.11 a, or vertical to permit gravity flow of adsorbent to the extraction port. Packing density of the adsorbent is not as critical as in unit cells that are subject to vibration and shock during transport and handling, because any settling can be accommodated by "topping off" the unit with additional adsorbent. Emptying of the PSU adsorbers is generally by vacuum or by air fluidization plus aspiration. At least one design employs gravity emptying by opening the bottom of the beds to a hopper beneath the unit. When PSU adsorbers are

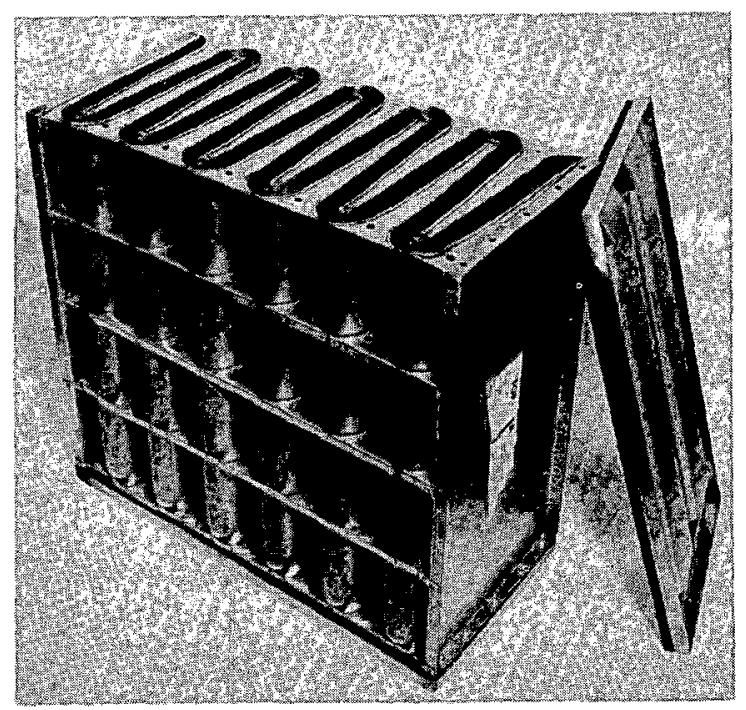

Fig. 3.12 Pleated bed (IES CS-8, type I) adsorber cell. This design is obsolete. Weighs approximately $160 \mathrm{lb}$. Courtesy Barnebey-Cheney Co.

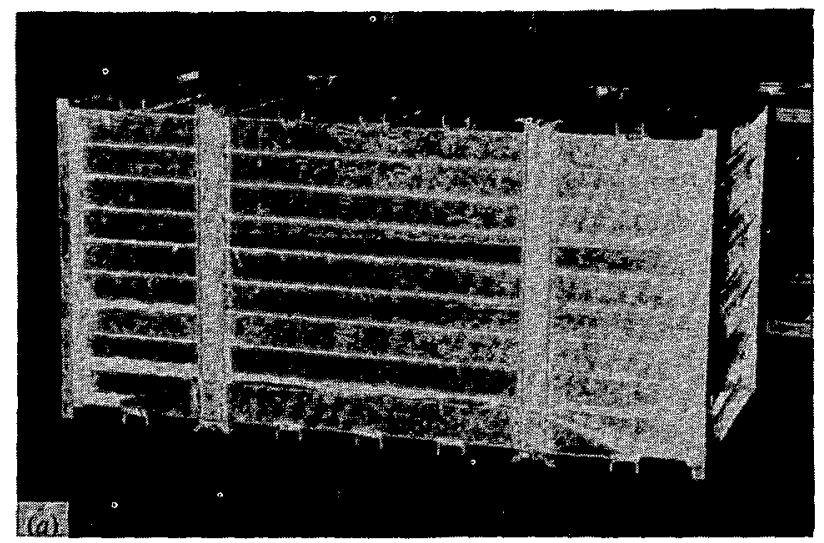

used with iodized carbon adsorbents, an important design consideration is the capability to positively extract the carbon if it gets wet. Many adsorber installations include the provision of water sprays to suppress carbon fires. If these sprays are activated, the mass of carbon can become a difficult-to-airtransport slurry, similar to wet sand. Extraction of wet iodized carbon from a PSU adsorber is essential within $24 \mathrm{hr}$ of the wetting, because corrosion is possible if it is left in contact with the stainless steel of the beds and pressure boundary.

An adsorber design used in earlier systems was the multiple-tray CBR (chemical-biological-radiological) filter unit shown in Fig. 3.13. Because of the caulked-joint construction and the inability to replace adsorbent except by replacing the entire unit, their use in nuclear applications is not recommended. Cylindrical adsorbers (Fig. 3.14) and gas mask canisters are sometimes used in small installations such as glove boxes, machine-tool hoods, and smallvolume reactor and radiochemical plant off-gas systems. However, they find no application in large adsorber systems.

\subsubsection{Adsorbents}

Detailed requirements for nuclear-grade gas phase adsorbents are specified in RDT $M 16-1 .^{28}$ The requirements are of two general types: (1) the efficiency and desorption tests discussed in Sect. 3.4 .2 and (2) physical properties that characterize the product that has been subjected to performance tests.

Coconut is the most widely used activated-carbon base material at this time. However, an increasing

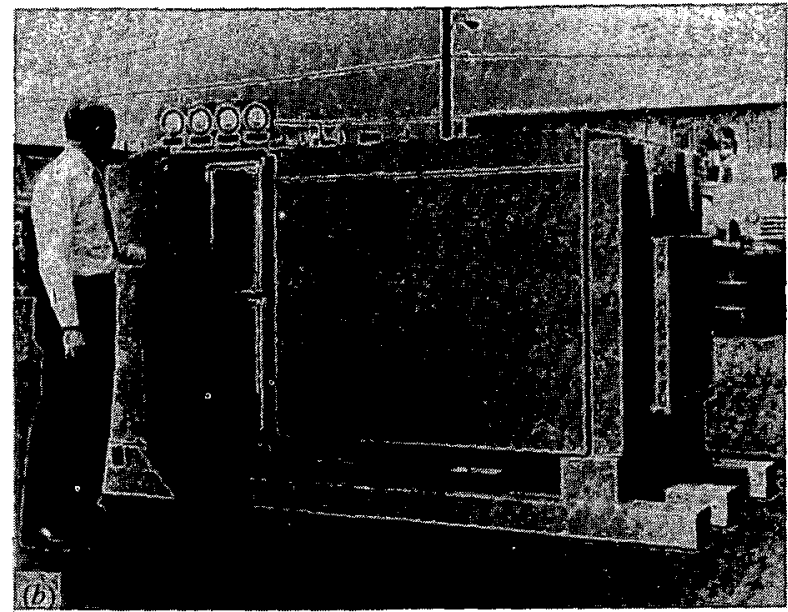

Fig. 3.13. Multiple-tray adsorber for CBR filter unit. Not recommended for nuclear applications. (a) Adsorber tray section. Note joints between trays. (b) CBR filter unit. Courtesy Farr Co. 


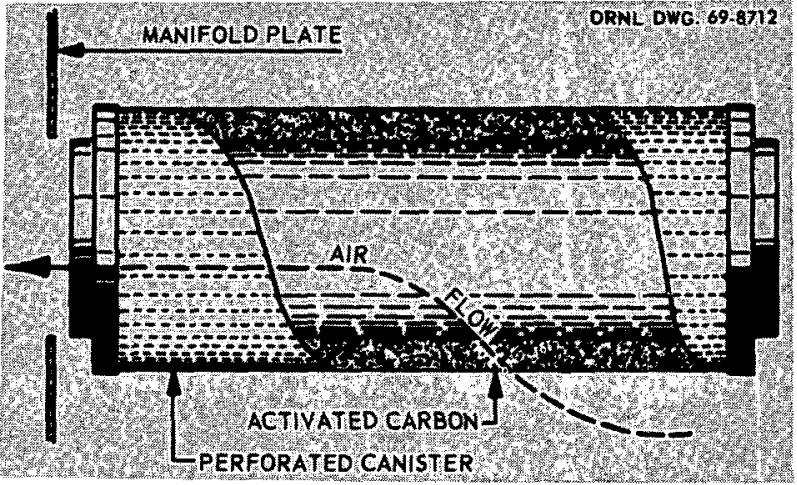

Fig. 3.14. Canister-type adsorber cell. These may be installed individually or in an array of 16 canisters gasketed into a plate with arrangement for installation of the assembly in a manner similar to that for pleated bed cells.

worldwide demand for coconut carbon has caused significant price increases, has decreased its availability, and has accentuated the need to find alternative base materials. Recent studies indicate that coal-base carbons can be impregnated to trap and retain radioactive iodine and organic iodine compounds as effectively as coconut, and these carbons may be used increasingly in the future. ${ }^{30}$ The studies included a survey of all domestically available activated-carbon base material, plus tests to evaluate properties of major interest. ${ }^{47}$

Certain physical properties are important to the user. Hardness--attrition and crushing of carbon granules under service conditions can result in dusting and subsequent escape of contaminated carbon, or in channeling through the bed with subsequent leakage of contaminated air or gas from the adsorber. Very hard carbons are desirable to resist fracture, abrasion, and crushing; a minimum hardness of $92 \%$ is recommended. Hardness is currently determined by the ball-pan (or Ro-tap) method given in RDT M 16-1. To minimize service degradation, vibration and pulsation in the air handling system must be minimized, and adsorber units should be installed with beds horizontal so that there is a minimum pressure head on the carbon granules. Carbon tetrachloride activity is difficult to relate directly to performance but to some extent is a measure of surface area and a direct measure of the degree of activation; it is, therefore, an indicator of the uniformity of product. The test is made routinely (before and after impregnation) by the manufacturer for quality assurance (QA) purposes, and test results should be specified as one of the items of data to be furnished. Minimum values that conform to the test results obtained on the qualified lot of product should be specified. Apparent density - carbon must be packed into beds so that settling does not occur during shipping, handling, installation, or operation. Packing density should approach the apparent density as determined at the time of qualification. Mesh size of the carbon is limited by the availability of perforated-steel retaining screens. Although some adsorber designs employ cotton fabric screen liners (scrims) to retain the carbon, thus permitting the use of very fine (12-by 30 -mesh) ${ }^{46}$ carbon, the practice is not recommended for nuclear exhaust applications, because the scrim could burn or deteriorate and cause the release of fines. There is also some possibility that the cotton might contribute to converting elemental iodine to an organic compound, which would increase the loading on the impregnant. For stiffness, the screens should be made from a steel sheet as heavy as possible, and the holes should be as small as possible to retain the smallest possible carbon granules. The open area of the screens should be as great as possible to minimize airflow resistance. At present, 220 holes ( 0.045 in. in diameter) per square inch (open area $=35 \%$ ) is the limit of the steel perforator's capability in No. 26 U.S. gage stainless steel and No. 24 U.S. gage carbon steel. This limits minimum carbon size to No. 16 U.S. mesh. ${ }^{46}$

\subsubsection{Inorganic Adsorbents}

Inorganic adsorbents are of interest in many nuclear applications because they are noncombustible and therefore eliminate one of the potential hazards of activated carbon. The major inorganic adsorbent of current interest is silver-exchanged zeolite. Silver oxide and silver nitrate impregnated alumina have been investigated in the laboratory and show some promise. Although retention of methyl iodide by alumina decreased strongly with increasing humidity and airflow, it increased substantially at higher temperatures. ${ }^{48}$ Impregnated aluminum silicate has been made available by one manufacturer but to date has not been used under service conditions. Silver zeolite ( $\mathrm{AgX}$ ), on the other hand, looks promising in both laboratory testing and field use. Its advantages and characteristics include (1) nonflammability and high-temperature iodine retention (samples containing adsorbed radioiodine have been heated to temperatures as high as $900^{\circ} \mathrm{C}$ before measurable amounts of radioiodine were released); (2) high-temperature operation (efficiency increases with temperature, and efficient methyl iodide adsorption is still achievable at temperatures 
as high as $400^{\circ} \mathrm{C}$ ); (3) low explosion hazard when used in air streams containing high concentrations of nitrogen oxides; (4) high efficiency under service conditions that are unfavorable to activated carbon, including high $\left(>250^{\circ} \mathrm{F}\right.$, or $\left.120^{\circ} \mathrm{C}\right)$ air temperatures and relative humidities of 90 to $95 \%$; (5) adsorbed iodine compounds form solid, insoluble products with silver zeolite, which is desirable from the standpoint of waste management; ${ }^{49}$ and (6) high $\mathrm{MPL}_{d}\left(85 \mathrm{mg} \mathrm{I} / \mathrm{g} \mathrm{AgX}\right.$ is claimed, ${ }^{50}$ although an $\mathrm{MPL}_{d}$ no greater than 45 is recommended for design purposes). Offsetting these advantages of silver zeolite are its very high cost (it may cost 40 to 50 times more per cubic foot of bed volume than comparable activated carbon) and its lower efficiency at lower (e.g., room) temperatures. For certain operations, particularly for fuel processing plant secondary treatment systems where high temperature and high concentrations of nitrogen oxides may be the rule, silver zeolite may provide an acceptable choice even at the higher cost. There is some indication, however, that service life may not be as good as claimed. In one experimental facility off-gas system, the efficiency of a silver zeolite adsorption system dropped from over $99.9 \%$ to about $91 \%$ in approximately 18 months. Although silver zeolites are not supposed to be as subject to poisoning by "tramp" contaminants in the environment as activated carbon, poisoning apparently occurred in this case. No formal explanation for the decrease has been proposed. In the case of an experimental fuel reprocessing development facility, hydrogen chloride vapors were inadvertently introduced into the air stream leading to silver zeolite adsorbers, thereby causing nearly instant incapacitation.

In Germany an inorganic silver nitrate-impregnated catalyst carrier, built of amorphous silicic acid with small additions of aluminum oxide, has been used with good success. ${ }^{51}$ The material is inexpensive and uses relatively low silver loadings, as compared with silver zeolite, and gives high removal efficiencies for radioactive iodine and methyl iodide at relative humidities on the order of $70 \%$. Efficiencies at 95 to $100 \%$ RH were poor, however. The price is reported to be about three times that of equivalent activated carbon but only $1 / 10$ that of silver zeolite.

\subsubsection{Adsorption System Design}

System properties of primary concern are (1) efficiency, (2) MPL of the adsorbent, (3) airflow capacity, and (4) airflow resistance. By assuming that an adequate adsorbent is selected, that the required gas residence time is maintained, that no poisoning or excessive aging has occurred, and that no leaks in the system have developed, system efficiency should be equivalent to the efficiency of the adsorbent, as determined by laboratory testing of samples taken from the system.

Maximum permissible loading determines the minimum quantity of adsorbent required in the system and is a function of (1) the adsorbent's capability to trap, and retain, radioiodine (or other adsorbates of interest) and (2) the quantity of adsorbent required to disperse trapped fission products so that carbon ignition becomes unlikely (i.e., $\mathrm{MPL}_{d}$ for desorption vs $\mathrm{MPL}_{i}$ for ignition). The $\mathrm{MPL}_{d}$ required to prevent break through under normal operating conditions varies with the radioiodine form (elemental or organic) and differs for different adsorbents. There is substantial disagreement as to the design value for $\mathrm{MPL}_{d}$ for each of the radioiodine forms, and currently there are insufficient data on which to base any hard numbers. MPL $d$ s as low as 2 $\mathrm{mg}$ total $\mathrm{I} / \mathrm{mg} \mathrm{C}$, to as high as $50 \mathrm{mg} \mathrm{I}_{2}$ and $5 \mathrm{mg}$ $\mathrm{CH}_{3} \mathrm{I} / \mathrm{mg} \mathrm{C}$ have been proposed for continuously online and normally off-line systems respectively. All of these values are high when desorption of iodine or ignition of carbon due to fission product heating under accident conditions is considered.

The concentration of iodine in the air that flows to the adsorption system of a reactor in the unlikely event of a major accident may range from a few parts per million to as high as $500 \mathrm{ppm}$, depending on reactor size, degree of fuel burnup at the time of the accident, and the nature of the accident. Some general assumptions in a DBA are that $50 \%$ of the fuel inventory will be released to the containment; $50 \%$ of the amount released (i.e., $25 \%$ of the fuel inventory) will plate out on the walls and floor of the containment; and the iodine that reaches the adsorbers will be distributed as particulates, organic iodides, and elemental iodine in the proportions 5, 4, and $91 .^{52}$ However, any particulate iodine will be collected on the demisters or HEPA filters upstream of the adsorbers and may desorb and subsequently collect in the adsorbers. Also, as much as $10 \%$ of the elemental iodine collected in the adsorber beds of an activated carbon system may convert to penetrating forms of iodine, primarily organic iodides, in the presence of moisture and a high radiation field.$^{53}$ For system design purposes, therefore, a distribution of 85 parts elemental iodine and 15 parts organic iodides, with zero particulates, is recommended. A substantial portion of this iodine (the exact propor- 
tion will depend on the degree of fuel burnup) will exist in the form of heat-generating radioiodine and will contribute to fission product decay heating of the carbon, which, if adequate cooling airflow is not maintained, may cause desorption of trapped iodine or ignition of carbon in the beds. Of these two possibilities, desorption of iodine is the more serious for two reasons; first because it would constitute a loss of containment for radioiodine, and secondly because bed temperatures will never reach the ignition point if sufficient airflow to prevent desorption of trapped iodine is maintained. Sufficient airflow is necessary to keep bed temperatures below $230^{\circ} \mathrm{F}$, probably on the order of 6 to $10 \mathrm{fpm}$. An MPL of 2.5 $\mathrm{mg}$ total iodine (including inert and radioactive isotopes as both elemental iodine and organic compounds) per gram of carbon is considered adequate to prevent significant fission product decay heating provided a minimum airflow of 6 to $10 \mathrm{fpm}$ under accident conditions is maintained. ${ }^{53}$

To determine the minimum quantity of carbon required in a specific system, the quantity of inert and radioactive iodine that may be trapped in the adsorbers must be estimated. Using the MPL of 2.5 $\mathrm{mg}$ total $\mathrm{I} / \mathrm{mg} \mathrm{C}$ and the assumptions on iodine distribution noted previously, the minimum quantity of carbon in the system can be estimated from the equation

$$
C=0.22 Q \text {, }
$$

where

$$
\begin{aligned}
C= & \text { minimum quantity of carbon required, } \mathrm{lb} ; \\
Q= & \text { potential iodine inventory that could be re- } \\
& \text { leased, } \mathrm{g} .
\end{aligned}
$$

Using this equation, a $1000-\mathrm{MW}(\mathrm{e})$ reactor with a potential iodine inventory of $15,000 \mathrm{~g}$. would require a minimum of $3300 \mathrm{lb}$ of carbon to provide adequate protection against desorption and ignition under accident conditions. This amount is more than adequate to meet the requirements for efficiency and retention under normal conditions.

System airflow is a function of the quantity of carbon in the system. and of gas residence time. Although the minimum gas residence time of $0.20 \mathrm{sec}$ is required for effective sorption of organic radioiodine compounds, a minimum of $0.25 \mathrm{sec}$ is recommended for design purposes. This provides the conservatism necessary to account for adsorbent bed thinning due to the nearly unavoidable warpage and camber of screens used to hold the adsorbent in modular cell and PSU adsorbers. Gas residence time can be increased by increasing bed depth or, for a fixed bed depth, by decreasing airflow velocity (i.e., by underrating). Increasing bed depth over the 2 -in. minimum employed in both PSU and modular cell adsorber designs and recommended in Regulatory Guide $1.52^{33}$ has the advantage of increasing system reliability by increasing holding capacity. It has the disadvantages of higher cost and, in activated carbon systems, of slightly decreasing ignition temperature. ${ }^{4 j}$ Ignition temperature also decreases with aging of the carbon, particularly in continuously on-line systems. Minimum operational airflow capacity can be determined from the equation

$$
C=\frac{N \operatorname{tn}(A-b)}{28.8 S T}
$$

where

$$
\begin{aligned}
C= & \text { minimum system design airflow, cfm; } \\
N= & \text { number of cells or adsorber assemblies in the } \\
& \text { bank }(N=1 \text { for a PSU); } \\
n= & \text { number of beds per adsorber assembly }(n=2 \\
& \text { for IES CS }-8 \text { type II cells }) ; \\
t= & \text { bed thickness, in. }(t=2 \text { for IES CS- } 8 \text { type II } \\
& \text { cells }) ; \\
A= & \text { total area of all bed screens of one cell or of a } \\
& \text { PSU, in. }{ }^{2} ; \\
b= & \text { total unperforated area of screens having total } \\
& \text { area } A, \text { in }{ }^{2} ; \\
S= & \text { number of screens per cell or PSU adsorber; } \\
T= & \text { minimum gas residence time required for } \\
& \text { effective sorption, sec. }
\end{aligned}
$$

For IES CS-8 type II cells and gas residence time of $0.25 \mathrm{sec}$, Eq. (3.2) reduces to

$$
C_{11}=333 \mathrm{~N} \text {. }
$$

For an installation of modular cell adsorbers, gas residence time can also be increased by providing two banks in series. This not only increases holding capacity and system reliability but avoids the decrease in carbon ignition temperature (since each bed is only 2 in. thick) and provides series redundancy. Because the first bank serves as a guard bed, most of the aging, weathering, and poisoning of the adsorbent would take place in the first bank, and service life of the second bank should be greatly extended. All these advantages are gained at increased investment costs, building space charge (because of the greater space required), and operation costs (because of the greater power requirements 
necessary to accommodate the increased system resistance).

With respect to fission product decay heating, different isotopes of iodine have different heatproducing potentials and different decay rates. For continuously on-line systems in vented containment (see Sect. 9.8) and in-containment postaccident air cleanup systems, transport of radioiodine is rapid (from a few minutes to as long as $\mathbf{3 0} \mathrm{min}$, depending on the length of the transport path) and all heatproducing isotopes must be considered in estimating the heat production potential in the adsorber system. In double containment systems the fission product transport time is much greater, thus permitting the short life heat-generating isotopes to decay to the point that their contribution to the heat-producing potential (in the adsorbers) is negligible. ${ }^{54}$ In these systems it is likely that only the contribution of I-131 (half-life of about 8 days) need be considered. In either case, the equilibrium inventories (in the fuel) of the isotopes of concern should be used for estimating the heat-generating potential in the carbon of the adsorbers.

Airflow resistance is a function of bed depth, adsorbent mesh size, and airflow velocity. The resistance of a bank of IES CS-8 type II cells, having 2-in.-thick beds filled with 8- by 16-mesh carbon and operated at a volumetric airflow rate of $333 \mathrm{cfm}(40$ lin fpm superficial velocity) is 1.1 in.wg. With HEPA filters upstream, resistance does not increase with time under service conditions.

\subsection{DEMISTERS}

\subsubsection{Introduction}

Demisters (also termed entrainment separators, mist eliminators, or moisture separators) are required in some nuclear air cleaning systems to protect the primary components (HEPA filters and adsorbers) from damage or loss of function due to entrained moisture. HEPA filters are highly efficient collectors of solid and liquid particulate matter. Because they can plug rapidly when exposed to high concentrations of liquid, the result can be decreased airflow, increased pressure drop, and perhaps rupture of the filter pack. In adsorbers, water deposition may increase pressure drop (by filling interstitial spaces between granules of adsorbent) and decrease efficiency and capacity.

Sensible moisture may stem from various sources depending on the system. In radiochemical, fuel processing, and some laboratory operations, droplets generated in scrubbers or chemical operations may be released to the airstream; or saturated gas streams, upon cooling, may produce droplets by condensation. In a water-cooled and/or water-moderated reactor, in the very unlikely event of a loss of coolant accident (LOCA), large volumes of steam will form which, upon condensation in the containment air, will pass to the postaccident air cleanup system filters and adsorbers. If sprays are provided to reduce post-LOCA pressure in the containment space of the reactor, entrained water droplets from these could also be present in the air flowing through the filters. In plants that have fire protection sprinklers in the space served by the air cleaning system, or in the duct leading to the air cleaning system, activation of the sprinklers would produce sufficient sensible moisture to adversely affect the filters in some instances. In any situation where free moisture may be present either under normal or upset conditions, a stage of demisters should be provided upstream of the filters.

With condensing steam, most of the entrained water droplets will be in the size range of 1 to $20 \mu \mathrm{m}$. With sprays and sprinklers, the steam will exist as droplets ranging from submicron diameters to 2000 $\mu \mathrm{m}$ in diameter or larger. ${ }^{55}$ Most of the droplets generated by sprays or sprinklers will be large (mass median diameter, MMD, will approach the diameters of the larger drops) and can be effectively removed by wave plates or other coarse moisture separators. However, after removal of these droplets, there may be a sufficient mass of water among the smaller drops remaining to cause plugging of unprotected HEPA filters. Droplets in the range of 1 to $20 \mu \mathrm{m}$ in diameter pose the greatest threat to HEPA filters, because droplets larger than this range are readily removed, and the mass of free water represented in droplets smaller than $1 \mu \mathrm{m}$ is too little to pose a likelihood of plugging. Within the 1-to-20$\mu \mathrm{m}$ range, however, free water cannot be removed by inertial separation alone, and use of more effective and higher pressure-drop devices is required. Filter plugging is a time-concentration phenomenon; that is, although sustained exposure to low concentrations of entrained moisture (as might exist on a seacoast or due to condensation of water vapor above a pool or wetted surface) may eventually cause plugging if the air remains saturated, such an occurrence is unlikely. However, short-term, even minutes, exposure to high concentrations of entrained water, as might exist from a spray or condensation from a broken steam line, may cause rapid plugging. In tests of plugging of HEPA filters 
by water spray ${ }^{56}$ the airflow through a 1000 -cfm filter, after being subjected to a water spray flow of $0.38 \mathrm{gpm}$ per $1000 \mathrm{cfm}$ of airflow, dropped to and leveled off at $630 \mathrm{cfm}$ in about $20 \mathrm{~min}$. With a water flow of $0.95 \mathrm{gpm}$ per $1000 \mathrm{cfm}$, airflow dropped to about $300 \mathrm{cfm}$ in $16 \mathrm{~min}$, and if the test had been continued, total plugging would probably have occurred within an hour because of the inability of the filter to drain collected water. In a test with very coarse spray (MMD $<2000 \mu \mathrm{m}$ ) at a delivery rate of $6.5 \mathrm{gpm}$ per $1000 \mathrm{cfm}$ airflow, nearly complete plugging (90\% decrease in airflow) occurred in approximately $6 \mathrm{~min}$, and spray droplets punched holes in the filter medium. In another test, ${ }^{57}$ exposure of unprotected HEPA filters to wet steam and air resulted in nearly complete plugging in less than 6 min. However, those HEPA filters protected by an adequate demister (capable of removing 1- to 5- $\mu \mathrm{m}$ diam water droplets at an efficiency of $>99 \%$, and coarse droplets at an efficiency of $100 \%$ at a water loading rate of $8 \mathrm{gpm}$ ) were still serviceable, with no observable decrease in efficiency. The serviceability determination was made after 10 days exposure to air containing a visible water fog, preceded by exposure to wet steam and airflow for $30 \mathrm{sec}$ at a flow rate of $10,000 \mathrm{cfm}$ through the 1000 -cfm demister-HEPA filter combination. Both series of tests indicated that dust collected on the HEPA filter decreases its water repellency and increases its water retention.

It can be concluded that, although the HEPA filter can drain some quantity of collected water and therefore accept some degree of water loading without plugging, there is a threshold timeconcentration value above which demisters must be provided. For clean filters, this time-concentration value appears to be such that the water retained in the filter will be less than $2 \mathrm{lb}$ per 1000-cfm airflow capacity under equilibrium conditions (i.e., water flow rate of about $0.35 \mathrm{gpm}$ per $1000 \mathrm{cfm}$ of airflow). However, such a value must be used with caution because of the increase in water retention with increasing dust loading or aging of the filter, and a time-concentration value that will produce a maximum retained water loading of $1 \mathrm{lb}$ of water under equilibrium conditions should be observed as a practical consideration (maximum water delivery rate of about $0.18 \mathrm{gpm}$ per $1000 \mathrm{cfm}$ of airflow). Sustained wetting gradually decreases the tensile strength of HEPA filter media, and intentional operation under such conditions is not recommended. Radiation also decreases the water repellency of HEPA filter media. ${ }^{58}$

\subsubsection{Demisters for Reactor Applications}

The Nuclear Regulatory Commission recommends that demisters be provided in reactor postaccident cleanup systems and in other ESF systems in which potential water or steam release may occur. ${ }^{59}$ Furthermore, it recommends that the demister be of nonflammable construction and that its design be qualified by a prototype test. Acceptable qualification procedures are described in USAEC Reports DP-812 and MSAR 71-45. ${ }^{57,60}$ Such tests should demonstrate that the demister is

1. At least $99.9 \%$ efficient, on an arrestance (i.e., weight percent) basis, for entrained water and condensed steam in the droplet range of $1 \mu \mathrm{m}$ to $2000 \mu \mathrm{m}$ in diameter, at duct velocity from 250 to $2500 \mathrm{lin} \mathrm{fpm}$, and water delivery rate of $8 \mathrm{gpm}$ per $1000 \mathrm{cfm}$ of installed HEPA filter capacity.

2. At least $99 \%$ efficient, on a count basis, for droplets in the range of $1 \mu \mathrm{m}$ to $10 \mu \mathrm{m}$ in diameter, at duct velocity from 250 to $2500 \mathrm{lin}$ fpm.

3. Nonflooding and nonreentraining atr a water-steam delivery rate of $8 \mathrm{gpm}$ at :duct velocity of 2500 lin fpm.

4. Capable of withstanding temperatures of up to $160^{\circ} \mathrm{C}$ and gamma radiation exposure up to $10^{\circ}$ rads integrated dose without visible deterioration. or embrittlement of the materials of construction.

Wire mesh demisters used in chemical processing and panel-type entrainment used in air conditioners and air washers are inefficient in the 1-to-10- $\mu \mathrm{m}$ droplet range; and the highly compressed packedfiber mist separators used for fume control in chemical plants, which are efficient in this droplet size range, have too high a resistance and too low a flooding capacity at the high air-water flow rates required for reactor postaccident air cleanup service. Wave- or bent-plate moisture separators (Fig. 3.15) are strictly impingement-type devices and are effective only against large $(>100 \mu \mathrm{m})$ drops. However, they can be useful as pre-separators to protect a more efficient demister downstream from high concentrations of large drops, similar to the application of a prefilter before a HEPA filter. Tests showed that commonly available panel filters and wire mesh demisters are useless in removing entrained water droplets in the 1-to-20- $\mu$-diam range, and one 


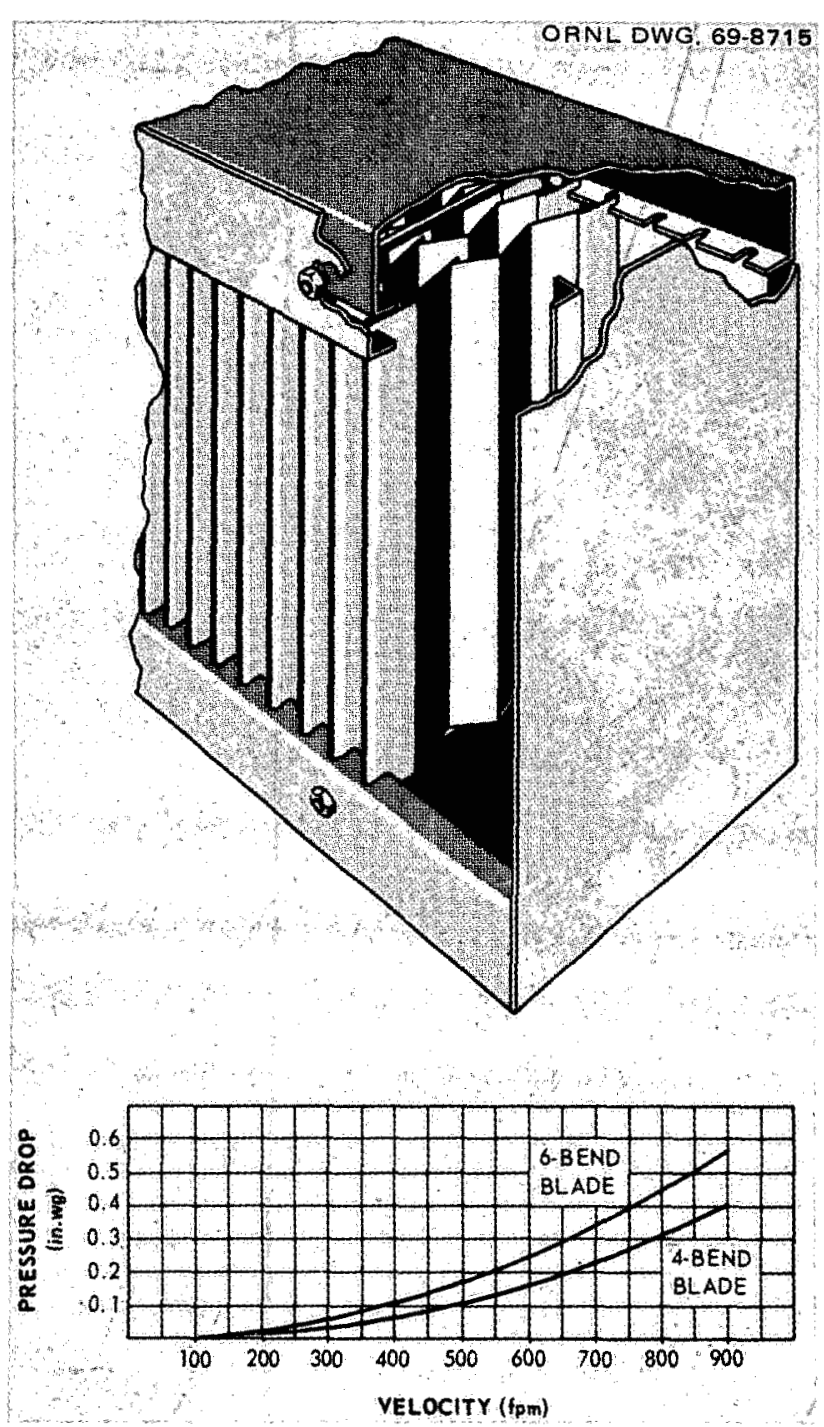

Fig. 3.15. Wave-piate separator-typical configuration and pressure-drop characteristics.

fiberglass panel filter was blown out of its retainer after only $10 \mathrm{sec}$ of exposure to wet steam. ${ }^{57}$

As of February 1976, four commercially available demister designs have been qualified for nuclear reactor service. Two of these demisters are similar and consist of an assembly of alternating coalescing and draining mats in a stainless steel case, $24 \times 24 \times 5$ to $5 \frac{1}{2}$ in. thick. The coalescing layers are knitted from fine multifilament fiberglass yarn (haxing individual filaments of less than $20 \mu \mathrm{m}$ in diameter) on a stainless steel wire matrix. The drain layers are crimped and knitted stainless steel mesh (0.011-in.diam wire). Screens on each face prevent blowout at high pressure drop, as might occur during the first few seconds of a reactor DBA. The demister weighs about $30 \mathrm{lb}$; has a rated airflow capacity of $1600 \mathrm{scfm}$ at 0.90 in.wg pressure drop when dry; and water removal rates of $650 \mathrm{lb} / \mathrm{hr}$ for drops $100 \mu \mathrm{m}$ and larger, $480 \mathrm{lb} / \mathrm{hr}$ for drops in the 10 to $70 \mu \mathrm{m}$ range, and $2.5 \mathrm{lb} / \mathrm{hr}$ for drops $10 \mu \mathrm{m}$ and smaller. Qualification of the unit shown on the left in Fig. 3.16 was reported in MSAR-71-45. ${ }^{60}$ The unit shown on the right in Fig. 3.16, available from ACS Industries, was qualified by the Harvard Air Cleaning Laboratory by using a similar procedure. ${ }^{61}$

Another demister design, made by American Air Filter and shown in Fig. 3.17, consists of a wave-plate separator followed by three 2 -in.-thick nonwoven fiberglass mats. The mats are installed in cells as shown in Fig. 3.18 and the grid assemblies, made from $1 / 8$-in.-diam stainless steel wire, prevent blowout of the mats at high overpressure ( 2 to $3 \mathrm{psi}$ ). The production model of this unit is $24 \times 24 \times 24$ in.; weighs $111 \mathrm{lb}$; has a pressure drop of $0.78 \mathrm{in.wg}$ at rated airflow when dry; and has water removal rates of $440 \mathrm{lb} / \mathrm{hr}$ for $100-\mu \mathrm{m}$-diam drops, $310 \mathrm{lb} / \mathrm{hr}$ for $\mathrm{I0-}$ to $70-\mu \mathrm{m}$-diam drops, and $6.4 \mathrm{lb} / \mathrm{hr}$ for drops smaller than $10 \mu \mathrm{m}$ in diameter ${ }^{60,62}$

The fourth demister, available from the Otto $\mathrm{H}$. York Company and used in the reactor confinement systems at Savannah River Laboratory, is a multilayer mat knitted from fine (approximately $20-\mu \mathrm{m}$ diam) Teflon $^{63}$ filament on a stainless steel wire matrix. The unit shown in Fig. 3.19 is $24 \times 24 \times 2$ in. thick and has a stainless steel case and $1 / 8$-in.-diam stainless steel reinforcing wires on each face. Qualification of this unit was described in USAEC Report DP-812.57 Objection to fluorocarbon (TFE) plastic fiber, such as Teflon, has been raised in some quarters because of its claimed unsatisfactory radiation resistance. Demisters of this design were tested at a dose rate of $6 \times 10^{6} \mathrm{rads} / \mathrm{hr}$ for $100 \mathrm{hr}$ and, although they became embrittled and would have broken if subjected to mechanical shock, they were still able to maintain their function. This dose is considered much higher than any that would be encountered in the demisters under any conceivable accident conditions, because most of the activity released under accident conditions would be in gaseous form that would pass right through the demisters, to be captured in the adsorbers downstream. ${ }^{64}$

\subsubsection{Performance}

The performance of demisters, like that of particulate filters, is defined in terms of removal efficiency for a specific particle (droplet) range, airflow 

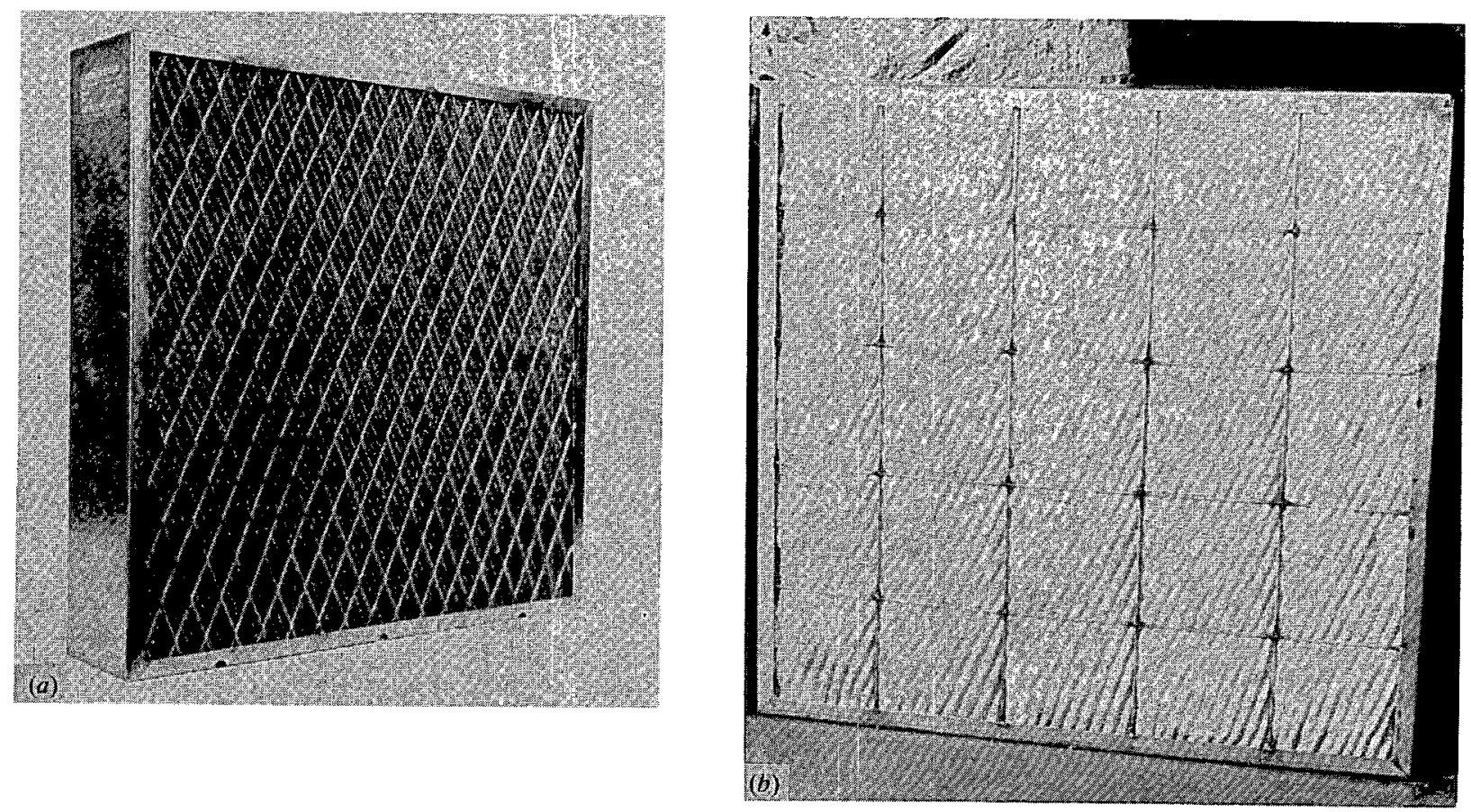

Fig. 3.16. High-efficiency demisters for nuclear service. (a) Courtesy Mine Safety Appliances Co.; (b) courtesy ACS Industries.

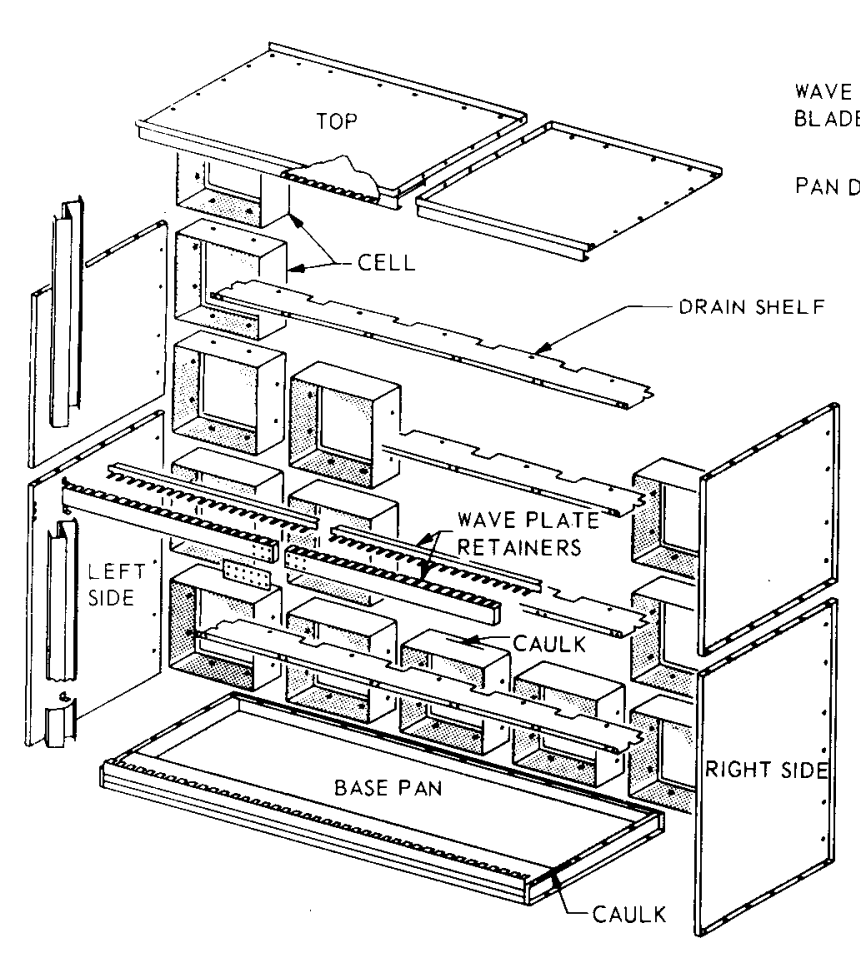

25,000-cfm SEPARATOR UNIT
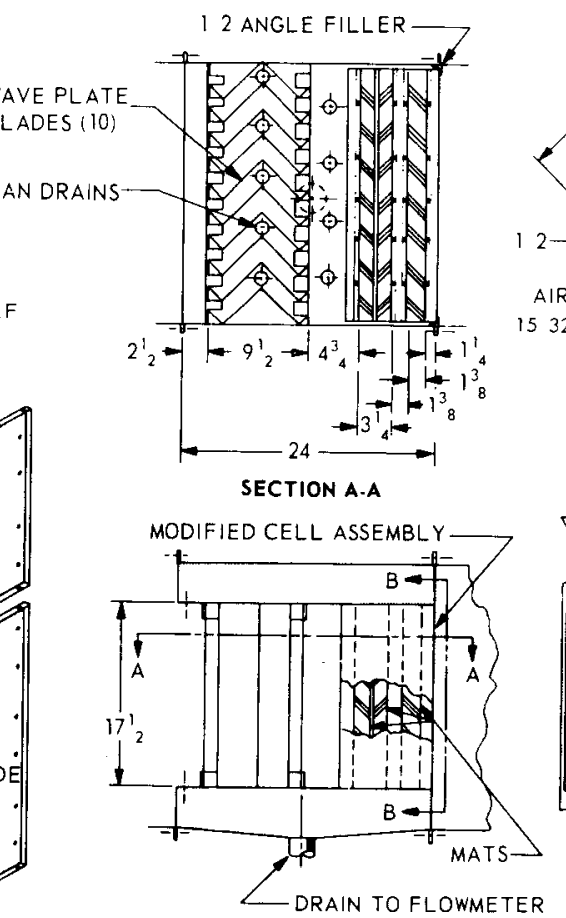

DETAILS OF 1000.cfm TEST SECTION (ALL DIMENSIONS IN INCHES)

Fig. 3.17. Exploded view and details of demister qualified for reactor postaccident air cleanup service. Courtesy American Air Filter Co. 


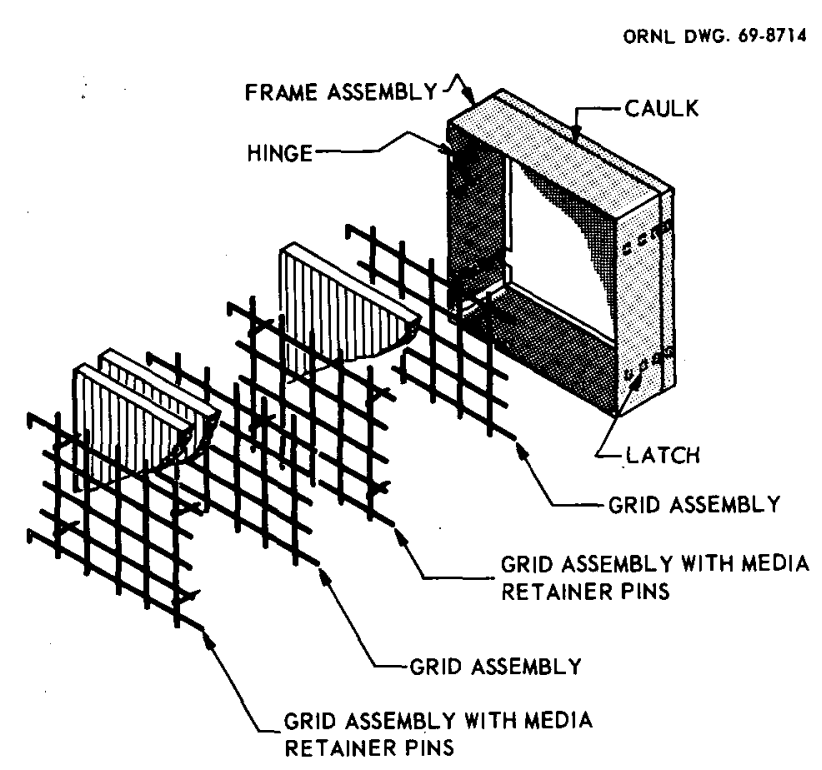

Fig. 3.18. Exploded view of typical cell assembly for demister shown in Fig. 3.17.

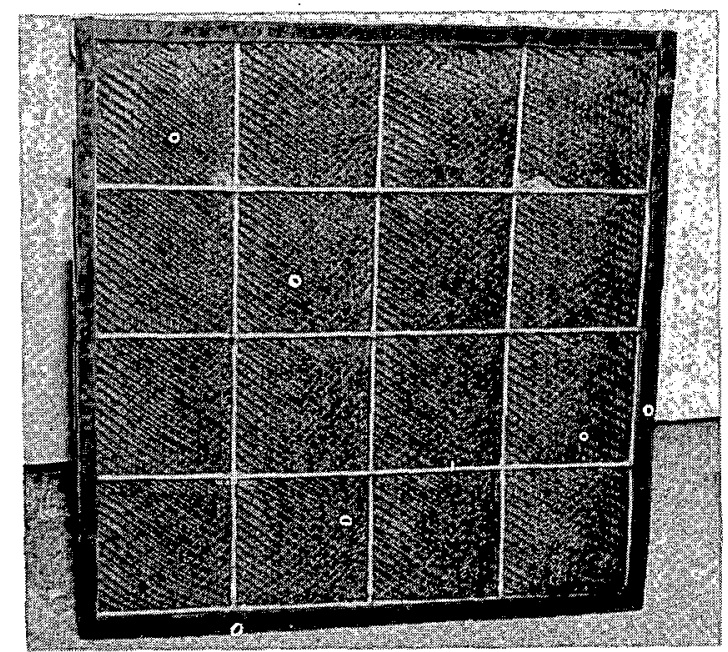

Fig. 3.19. Knitted Teflon and stainless steel wire demister used in Savannah River reactors, made by Otto H. York Co. Courtesy E. 1. du Pont, Savannah River Laboratory.

capacity, and resistance to airflow. The forces acting on liquid droplets in an air stream are identical to those acting on solid particles and the same filtration mechanisms (diffusion, impaction, and inertia) apply for both situations. In nuclear reactors, demisters must remove $99 \%$ of all droplets down to about $1 \mu \mathrm{m}$ in diameter at airflow rates of several thousand cubic feet per minute per demister, and at water delivery rates of up to 1 gal $(8.4 \mathrm{lb})$ per $1000 \mathrm{cfm}$ of steam-air mixture.
Wave-Plate (Bent-Plate) Demisters. Just as it is desirable to protect HEPA filters from high concentrations of coarse particles, it is also desirable to protect knitted or nonwoven-mat demisters from high concentrations of large water drops ( 50 to 1000 $\mu \mathrm{m}$ in diameter or larger). Because it takes more power to remove small drops (because of the higher resistance of the units capable of removing small drops) and because large quantities of water may unduly increase the water load and airflow resistance, it is uneconomical to use knitted fabric or nonwoven fiber filters to remove the large drops produced by reactor postaccident cooling sprays. Large drops are effectively removed by wave-plate demisters of the type shown in Fig. 3.15. Their efficiency is practically $100 \%$ for drops over $400 \mu \mathrm{m}$ but decreases to $60 \%$, or less, depending on blade geometry, for droplets in the range of 10 to $50 \mu \mathrm{m} .{ }^{65}$ Wave-plate demisters can handle very large volumes of air at high velocity (as high as $1500 \mathrm{fpm}$ for some types) with low pressure drop (Fig. 3.15). Because efficiency is dependent on impaction and inertial effects, operation at less than about $400 \mathrm{fpm}$ is not recommended. With most types, water carry-over may occur at velocities higher than $700 \mathrm{fpm}^{55}$

Knitted Fabric and Nonwoven Fiber Mat Demisters. Knitted fabric and nonwoven fiber demisters of the types shown in Figs. 3.16, 3.17, and 3.19 are the most satisfactory devices for removing small and intermediate-sized droplets ( 1 to $100 \mu \mathrm{m}$ ). Their design is a compromise between airflow capacity, resistance, size (i.e., materials cost), and service life. Recommendations of the manufacturer should be followed closely when supported by test data. Because drop removal is effected mainly by impingement, removal efficiency improves with increasing flow velocity and decreasing fiber diameter. The maximum fiber diameter for effective removal of droplets in the 1-to- $10-\mu \mathrm{m}$ range is about $20 \mu \mathrm{m} .{ }^{66}$ If a demister is comprised of a series of incremental layers, the droplet removal efficiency of the complete demister is the sum of the efficiencies of the individual layers. Although efficiency can be increased by increasing the thickness of the separator, this would increase the pressure drop and, therefore, operating costs; and there is a practical limit to increases in efficiency that can be attained at a given velocity. Overall efficiency can be increased to some extent by increasing the velocity, but this is at the expense of higher pressure drop and operating costs, and it also decreases efficiency for smaller droplets. Within limits, the higher the velocity, the thinner the demister (i.e., the fewer the layers) required for a 
given efficiency and the less the material required, which, in turn, results in lower procurement costs. ${ }^{66}$

For a given construction (fiber diameter and packing density), the airflow resistance of a demister at constant velocity increases directly with the number of identical layers. Therefore, there is a point of diminishing returns at which the cost of increasing the efficiency is offset by increased operating and materials costs. Efficiency can also be increased by increasing the packing density. However, airflow resistance increases even more rapidly than the improvement in efficiency. For this reason, the very efficient packed-fiber mist eliminators (Fig. 3.20) that have gained wide acceptance in the chemical industry are not recommended for nuclear reactor postaccident cleanup systems. Table 3.12 gives approximate operating velocities of the demisters discussed in this section. Knitted and crimped-wire separators are more efficient when installed vertically (i.e., horizontal airflow) than horizontally (as in most vessel offgas and scrubber installations). ${ }^{67}$

Because mat-type demisters are relatively efficient particle filters, they must be cleaned or replaced periodically if installed in continuously on-line systems. The knitted-fabric demister shown in Fig. 3.19 can be steam cleaned; the nonwoven fiber mats shown in Fig. 3.17 must be replaced. The pressure drop at which cleaning or replacement must take place is based on the capacity of the system fan. Manufacturer's recommendations on overpressure capability should be followed closely, and units should be cleaned or replaced soon enough so that an adequate margin of safety is allowed for the potential increase in pressure that would result from water loading.

The demister and its operating conditions must be tailored for each situation, and performance tests must be made under simulated conditions to obtain the optimum design of separator and system.

\subsubsection{Normal Off-Gas Demisters for Radiochemical Service}

Mist eliminators are often required in scrubber and radiochemical-operation off-gas systems to protect downstream filters from moisture and from acid or caustic fumes. Two types have given satisfactory service in radiochemical plant service.

Packed-Fiber Mist Eliminators. Packed-fiber mist eliminators of the type shown in Fig. 3.20 have given excellent performance for acid fumes in industrial service and can be tailored, by selection of fibers and materials of construction, to a wide variety of applications : :

The cylindrical element shown in Fig. 3.20a consists of a densely packed fiber bed, rigidly held between heavy corrosion-resistant screens. The unit shown is $24 \mathrm{in}$. in diameter and $120 \mathrm{in}$. long with a mounting flange for suspension from a support plate. Gas flows from the outside to the inside hollow core from which the clean gas exits at the top and the collected liquid exits at the sealed bottom through a drain pipe. Alternate designs with gas flow from the inside to the outside are also available. Fibers and other materials of construction are selected for their resistance to the reagents present in the off-gas. Operating velocities for this type of unit range from 5 to 50 lin fpm through the media, depending on design and performance requirements. Figure 3.20 shows the operating characteristics of two designs. Designs with collection efficiencies for submicron particles up to $99.98 \mathrm{wt} \%$ have been demonstrated on large-scale industrial processes. ${ }^{68,69}$ The mechanisms of mist separation for this type of element are diffusion, impaction, and inertial effects, with diffusion controlling for submicron particles.

In one radiochemical operation, cylindrical elements with 3 -in.-thick beds of $20-\mu \mathrm{m}$ fibers and fiber-packing density of $11.5 \mathrm{lb} / \mathrm{ft}^{3}$, operating at a gas velocity of $15 \mathrm{fpm}$ through the bed, gave 99.99 wt \% efficiency for droplets $3 \mu \mathrm{m}$ and larger and $99.3 \mathrm{wt} \%$ for droplets in the $0.3-$ to $-0.5-\mu \mathrm{m}$ range. ${ }^{70}$ The pressure drop of this demister was 4 in.wg when clean and was approximately $10 \mathrm{in.wg}$ after a year of operation when the elements were wet and a considerable amount of solids had been collected. The maximum temperature was $200^{\circ} \mathrm{F}$ and the measured efficiency for Cs-137 was over 96 wt \%.

High-velocity packed-fiber mist eliminators ( 250 to 500 lin fpm through the media) have found

Table 3.12. Economic operating velocities for demisters

\begin{tabular}{llc}
\hline \multicolumn{1}{c}{ Type, thickness } & Airflow & Velocity (fpm) \\
\hline Knitted fabric, 2 in. & Horizontal & $420-480$ \\
& Vertical & $280-320$ \\
Knitted fabric, 4 in. & Horizontal & $270-300$ \\
& Vertical & $220-260$ \\
Nonwoven fiber, 6 in. & Horizontal & $240-280$ \\
6-bend wave plate & Horizontal & $550-650$ \\
Wire mesh, 4 in. & Horizontal & $720-840$ \\
\hline
\end{tabular}



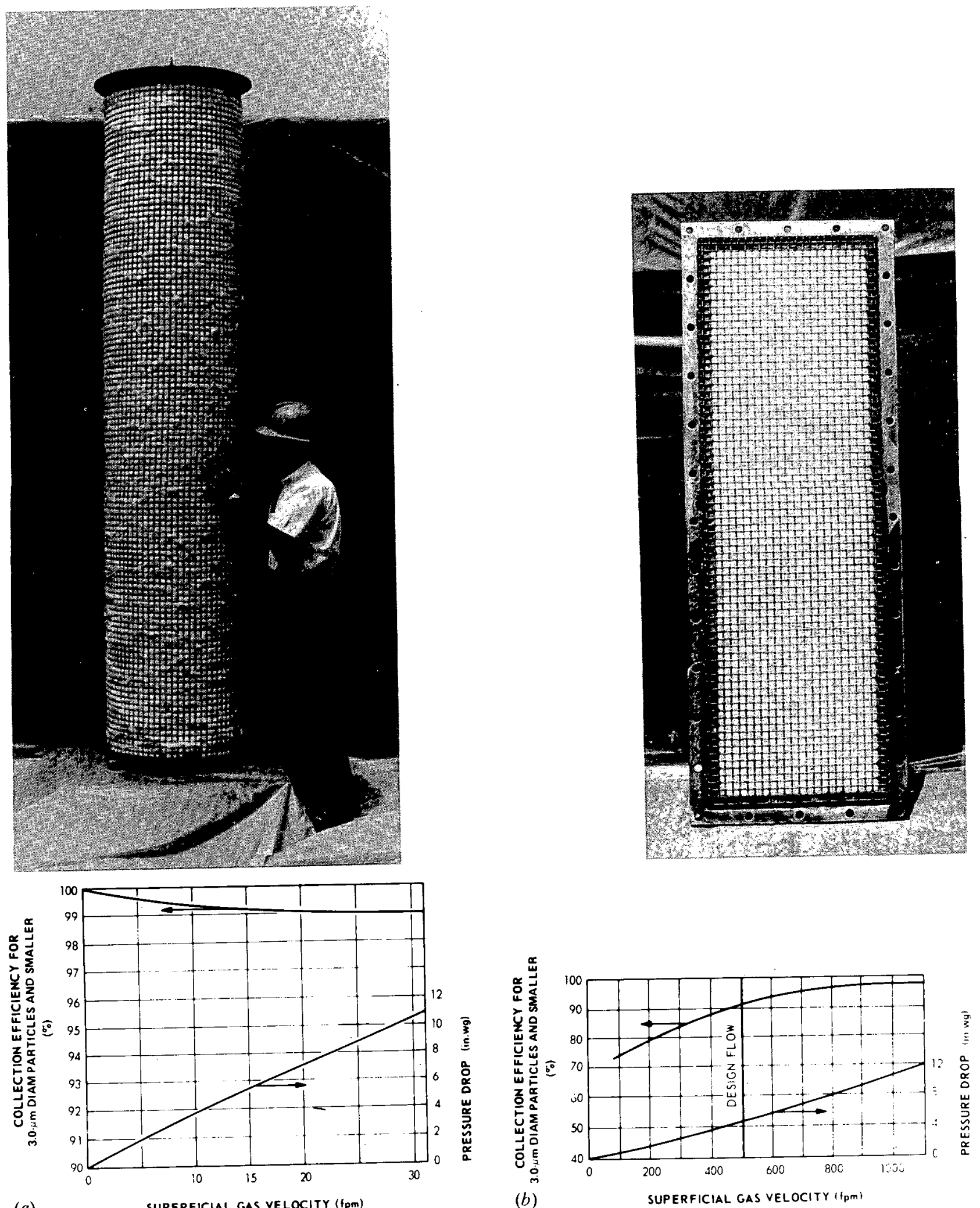

(a)

Fig. 3.20. Brink packed-fiber mist eliminators and operating characteristics. (a) High-efficiency element, $(b)$ high-velocity element. Courtesy The Monsanto Co. 
extensive application in the chemical industry. ${ }^{71}$ The rectangular element shown in Fig. $3.20 b$ has overall dimensions of $18 \frac{1}{2}$ in. by 53 in. and utilizes impaction as the controlling collection mechanism. Collection efficiencies of essentially $100 \mathrm{wt} \%$ are achieved for particles over $3 \mu \mathrm{m}$ in diameter with lower efficiencies for smaller particles. Elements similar in appearance to the high-velocity model have also been developed which have a pressure drop of 1 in.wg or less. This type, known as a "spray catcher," has essentially $100 \mathrm{wt} \%$ efficiency on particles greater than $5 \mu \mathrm{m}$ in diameter but low efficiency on smaller particles.

Packed-fiber mist eliminators are efficient solidparticle collectors but can be clogged by high dust loadings. They are sometimes made self-cleaning by adding atomized water to a gas stream containing acid or caustic fumes; under other circumstances they may have to be cleaned with steam or by backwashing. The units are particularly subject to clogging when operated completely dry, especially if viscous dusts or lint is present. In low dust concentrations this type of unit has operated for years without cleaning, which indicates the desirability of efficient building supply-air cleaning. In radioactive applications the arrangement of two units in parallel is desirable so that flow can be switched back and forth for maintenance or in the event of emergency without shutting down the system. Because water that collects on the fibers can seep through the bed, particulate carry-over is possible, as discussed in Sect. 3.5.1.

Perforated-Plate Mist Eliminators. The perforated-plate mist eliminator consists of two perforated metal sheets spot-welded together and uniformly spaced a few thousandths of an inch apart, with perforations in adjacent sheets offset so the air entering the holes in the first sheet impinges on the second sheet and must make two $90^{\circ}$ turns before it can escape. Moisture is removed by impingement of droplets on the water film flowing down between the sheets and on the face of the first sheet. The efficiency for large drops (50 $\mu \mathrm{m}$ and larger) is virtually $100 \mathrm{wt}$ $\%$, and the efficiency for $1-$ to $10-\mu \mathrm{m}$ droplets is greater than 99 wt \% at air velocities of 500 to 600 $\mathrm{fpm}$. The pressure drop is high, as shown in Fig. 3.21.

The base material is made in flat sheets, which can be welded edge to edge to form separators of any size and capacity. The material lends itself to pleating, as Fig. 3.21 shows, and can be formed easily into cones, cylinders, and other configurations (except com- pound curves) to increase the surface area per square foot of frontal area. Experience shows that the units do not clog or flood easily, but they must be cleaned regularly to give satisfactory service. The plates can be cleaned in place by irrigation with acid or caustic solutions, flushing, and scraping (on the front plate). Separation of the plates can occur if the material is bent too sharply; a minimum radius of five times the metal thickness and a minimum saw-tooth angle (Fig. 3.21 ) of $45^{\circ}$ is recommended for fabrication. The plates must be installed to allow water to flow off them easily. Saw-tooth configurations should be installed with the pleats vertical, and cones should be installed with the point up to avoid flooding. Cylinders should be vertical or installed on a steep slope.
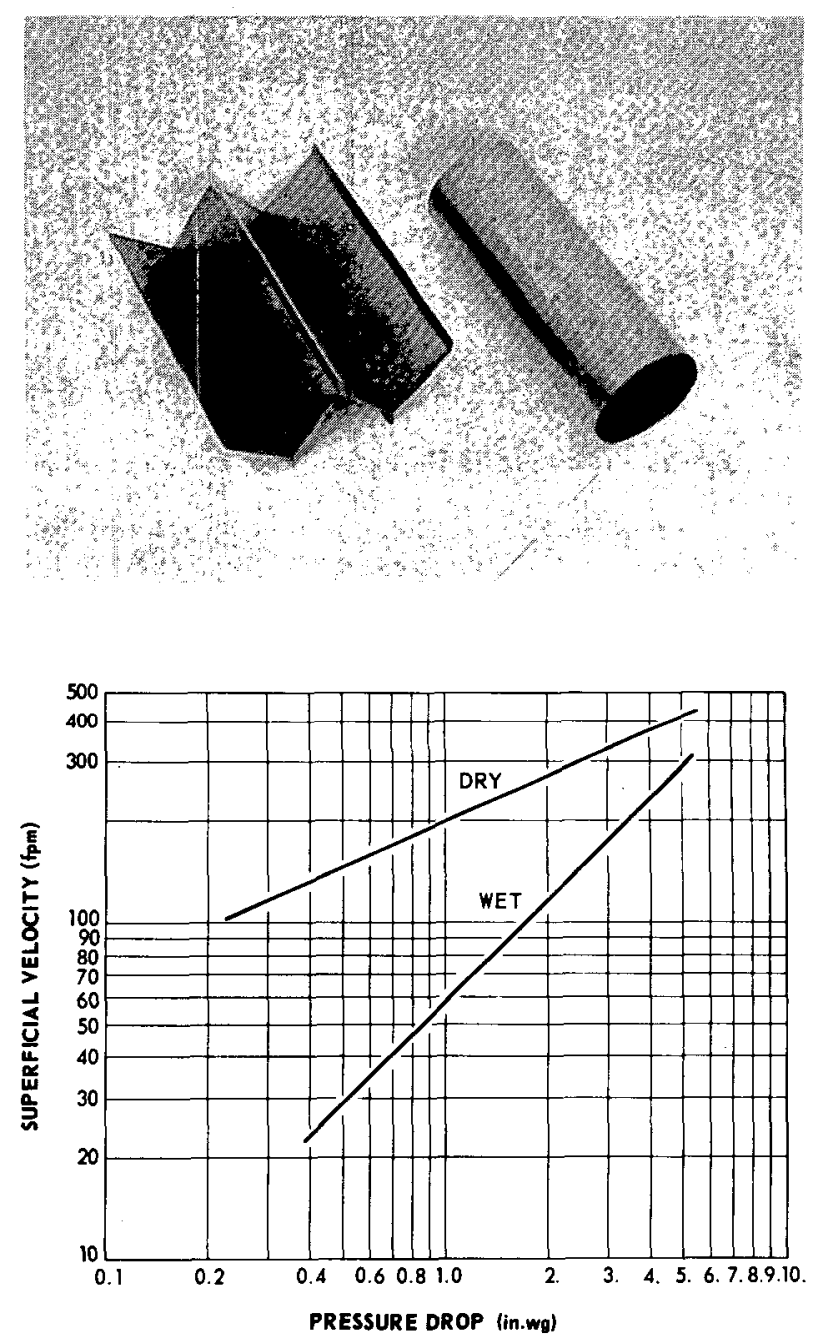

Fig. 3.21. Perforated-plate mist eliminator. Courtesy MultiMetals, Inc. 
Application. Both the packed-fiber and the perforated-plate mist eliminators have given satisfactory service in radiochemical operations, and both can be tailored to a wide range of corrosive conditions. The packed-fiber type is probably the better type where very high efficiency for small droplets at low flows is required. The perforated-plate type gives good service where flow rates are high and where extremely high efficiency for droplets smaller than about $5 \mu \mathrm{m}$ is not required. Neither type is suited to reactor postaccident cleanup applications.

\section{REFERENCES FOR CHAP. 3}

1. IES-CS-1, Standard for HEPA Filters, Institute of Environmental Sciences, Mt. Prospect, 1ll., 1968. All standards of the American Association for Contamination Control were adopted by IES when the two organizations merged in 1973 .

2. Particle-collection efficiency is the measure of the number of particles captured by the filter, expressed as a percentage of the particle concentration of the unfiltered air.

3. "Filter Unit Testing and Inspection Service," Environmental Safery and Health Information bulletin, Energy Research and Development Administration, current issue.

4. The term dust is used to denote particulate material of all types trapped by the filter.

5. C. A. Burchsted, "Environmental Properties and Installation Requirements of HEPA Filters," Proc. Symp. Treat. Airborne Radioact. Wastes, International Atomic Energy Agency, Vienna, 1968.

6. Military Specification MIL-F-51079, Filter Medium, FireResistant, High Efficiency.

7. W. L. Belvin et al., Development of New Fluoride Resistant HEPA Filter Medium, Final Report, ERDA Report TID 26649, Herty Foundation, Savannah, Ga., August 1975

8. C. C. Wright et al., The Evaluation of Substitutive Filter Framing Materials In Corrosive Environments, USAEC Report K-TL-8I, Union Carbide Corporation, Nuclear Division, May 19. 1970

9. F. E. Adley, "Progress Report, Factors Influencing High Efficiency Gasket Leakage." Proc. 9th AEC Air Clean. Conf., USAEC Report CONF-660904, 1966.

10. UL-586, High Efficiency Air Filter Units, Underwriters' Laboratories, Chicago, $2 \mathrm{~d}$ ed. 1964.

11. NFPA 90A, Standard for the Installation of Air Conditioning and Ventilation Systems Other than Residence Types, National Fire Protection Association, Boston, 1968.

12: W. L. Anderson and T. Anderson, "Effect of Shock Overpressure on High Efficiency Filter Units," Proc. 9 th AEC Air Clean. Conf., USAEC Report CONF-660904, 1966.

13. Regulatory Guide 1.76, Design Basis Tornado for Nuclear Power Plants, U.S. Atomic Energy Commission, Washington, D.C., April 1974.

14. W. S. Gregory, HEPA Filter Effectiveness During Tornado Conditions, USAEC Report LA-5352-MS, Los Alamos Scientific Laboratory, 1973.

15. IES CS-1, Standard for HEPA Filters, Institute of Environmental Sciences, Mt. Prospect, Ill., current issue.

16. C. A. Burchsted, Qualification Test for Moisture and Corrosion Resistant Separators for Air Filters, Oak Ridge National Laboratory, 1965.
17. J. A. Geer, Results of Testing of HF-Resistant Filters at Rocky Flats, Report to the Joint Government-Industry Conference on Filters and Filter Media at the 13th AEC Air Cleaning Conference, San Francisco, Calif., August 1974.

18. L. R. Jones, "High-Efficiency Particulate Air (HEPA) Filter Performance Following Service and Radiation Exposure," Proc. 13th AEC Air Clean. Conf., USAEC Report CONF-740807. 1975.

19. L. R. Jones, "Effects of Radiation on Reactor Confinement System Materials," Proc. 12th A EC Clean. Conf., USAEC Report CONF-720823, 1972.

20. J. R. Gaskill and M. W. Magee, "The HEPA-Filter Smoke Plugging Problem," Proc. 14th AEC Air Clean. Conf., ERDA Report CONF-740807, March 1975.

21. ASHRAE 52-68, Method of Testing Air Cleaning Devices Used In General Ventilation for Removing Particulate Matter, American Society of Heating, Ventilating, and Air-Conditioning Engineers, New York, 1968.

22. "Unit or Panei Type Air Filtering Devices," Code for Testing Air Cleaning Devices Used in General Ventilation, Air Filter Institute, Louisville, Ky., 1956.

23. AFI Dust Spot Test Code, Air Filter Institute, Louisville, Ky., 1960,

24. R. S. Dill, A Test Method for Air Filters, National Bureau of Standards, 1938.

25. UL-900, Air Filter Units, Underwriters' Laboratories, Chicago, current issue.

26. Building Materials List, Underwriters' Laboratories, Chicago, current issue.

27. ASTM D2652, Standard Definitions of Terms Relating to Activated Carbon, American Society for Testing and Materials, Philadelphia, current issue.

28. RDT M 16-1, Gas-Phase Adsorbents for Trapping Radioactive lodine and lodine Compounds, Energy Research and Development Administration, current issue.

29. ANSI N509, Nuclear Power Plant Air Cleaning Units and Components, American National Standards Institute, New York, 1976.

30. A. H. Dexter, A. G. Evans, and L. R. Jones, Confinement of Airborne Radioactivity, ERDA Report DP-1390, Savannah River Laboratory, October 1975.

3I. D. A. Collins et al., The Development of Impregnated Charcoals for Trapping Methyl Iodide at High Humidity, TRG Keport $1300(W)$, United Kingdom Atomic Energy Authority, London, 1967.

32. R. E. Ackley, Z. Combs, and R. E. Adams, Aging. Weathering, and Poisoning of Impregnated Charcoals Used for Trapping Radioiodine, USAEC Report ORNL-TM-2860, Oak Ridge National Laboratory, March 1970.

33. Regulatory Guide 1.52, Design, Testing, and Maintenance Criteria for Atmospheric Cleanup System Air Filtration and Adsorption Units of Light-Water-Cooled Nuclear Power Plants. U.S. Atomic Energy Commission, Washington, D.C., June 1973.

34. A. G. Evans and L. R. Jones, Iodine Retention Studies Progress Report: July 1970 -December 1970, USAEC Report DP1271, Savannah River Laboratory, 1971.

35. Letter, R. E. Adams to R. Herzel, Phillips Petroleum Co., reporting results of tests on charcoal samples removed from adsorbers of Carolina-Virginia Tube Reactor, Aug. 7, 1967.

36. Robert Blumberg, coordinating engineer for dismantling of Elk River Reactor, personal communication to C. A. Burchsted.

37. C. A. Burchsted and A. B. Fuller, Design, Construction, and Testing of High-Efficiency Air Filtration Systems for Nuclear Application, USAEC Report ORNL/NSIC-65, Oak Ridge National Laboratory, January 1970. 
38. F. R. Schwartz, Jr., President of North American Carbon Inc, personal communication to $\mathrm{C}$. A. Burchsted.

39. K. A. Lorenz, W. J. Martin, and H. Nagao, "The Behavior of Highly Radioactive Iodine on Charcoal," Proc. 13th AEC Air Clean. Conf., ERDA Report CONF-740807, May 1975.

40. E. A. Bernard and R. W. Zavadoski, "The Calculation of Charcoal Heating in Air Filtration Systems," Proc. 13th AEC Air Clean. Conf., ERDA Report CONF-740807, May 1975.

41. R. E. Adams et al., "Application of Impregnated Charcoals for Kemoving Radioiodine from Flowing Air at High Relative Humidity," Treatment of Radioactive Wastes, Proceedings of a Symposium, New York, NY, August 26-30, 1968, International Atomic Energy Agency, Vienna, 1968.

42. R. E. Adams and R. P. Shields, "Ignition of Charcoal Adsorbers by Fission Product Decay Heat," ORNL Nuclear Satety Research and Development Program Bimonthly Progress Report for November-December 1967, USAEC Report ORNLTM-2095, Oak Ridge National Laboratory, February 1968.

43. J. L. Kovach and J. E. Green, "Evaluation of the Ignition Temperature of Activated Charcoals in Dry Air," Nucl. Saf. 8 (1966).

44. A. G. Evans, Confinement of Airborne RadioactivityProgress Report July 1972 to December 1976. USAEC Report DP-1329, Savannah River Laboratory, 1973.

45. IES CS-8, High Efficiency Gas-Phase Adsorber Cells, Institute of Environmental Sciences, Mt. Prospect, Ill., current issue.

46. AS TM E11, Standard Specification for Wire-Cloth Sieves for Testing Purposes, American Society for Testing and Materials, Philadelphia, current issue.

47. V. K. Deitz and C. A. Burchsted, Survey of Domestic Charcoals for Iodine Retention, U.S. Navy NRL Memorandum Report 2960, Naval Research Laboratory, January 1975.

48. I. J. Gal et al., "Adsorption of Methyl lodide on Impregnated Alumina," Proc. 13th AEC Air Clean. Conf., ERDA Report CONF-740807, May 1975.

49. D. T. Pence et al., "Developments in the Removal of Airborne Iodine Species with Metal-Substituted Zeolites," Proc. 12th AEC Air Clean. Conf., USAEC Report CONF-720823, January 1973.

50. CRI-Nuclear Technical Data, CTI-Nuclear, Inc. Bulletin N73-001, 1975

51. J. G. Wilhelm and H. Schuettelkopf, "Inorganic Adsorber Materials for Trapping of Fission Product Iodine," Proc. 11th A EC Air Clean. Conf., USAEC Report CONF-700816, December 1970.

52. Regulatory Guide 1.3, Assumptions Used for Evaluating the Potential Radiological Consequences of a Loss of Coolant Accident for Pressurized Water Reactors, U.S. Atomic Energy Commission, Washington, D.C., June 1974.

53. A. G. Evans, "Effect of Intense Gamma Radiation on Radioiodine Retention by Activated Carbon," Proc. 12th AEC Air Clean. Conf., USAEC Report CONF-720823, 1973.
54. T. D. Anderson, "The Holdup Effect of Double ReactorContainment and its Influence on Dose from Airborne Radioactive Materials," Proc. 8th AEC Air Clean. Conf., USAEC Report TID-7677, October 1963.

55. "Phase Separation," Chap. 19 in Chemical Engineers Handbook, 4th ed., McGraw-Hill, New York, 1963.

56. J. K. Murrow, Plugging of High Efficiency Filters by Water Spray, USAEC Report TID-4500, University of California, Lawrence Livermore Laboratory, 1967.

57. A. H. Peters, Application of Demisters and Particulate Filters in Reactor Containment, USAEC Report DP-812, Savannah River Laboratory, 1962.

58. L. R. Jones, "High-Efficiency Particulate Air (HEPA) Filter Performance Following Service and Radiation Exposure," Proc. 13th AEC Air Clean. Conf., ERDA Report CONF-740807, March 1975.

59. Regulatory Guide 1.52, Design, Testing, and Maintenance Criteria for Atmosphere Cleanup System Air Filtration and Adsorption Units of Light-Water-Cooled Nuclear Power Plants, Nuclear Regulatory Commission, Washington, D.C., 1975.

60. G. H. Griwatz et al., Entrained Moisture Separators for Fine Particle Water-Air-Steam Service; Their Performance, Development, and Status, USAEC Report MSAR-71-45, MSA Kesearch Corp., March 1971.

61. M. W. First and D. H. Leith, ACS Entrainment Separator Performance for Small Droplet-Air-Steam Service, Harvard Air Cleaning Laboratory Report 75-1106, Harvard University School of Public Health, Nov. 6, 1975.

62. R. D. Rivers and J. L. Trinkle, Moisture Separator Study, USAEC Report NYO-3250-6, American Air Filter Co., June 1966.

63. Kegistered trademark, E. I. du Pont de Nemours \& Co.

64. A. G. Evans, Savannah River Laboratory, personal communication to $C$. A. Burchsted.

65. H. S. Dutcher, Air and Refrigeration Corporation, personal communication to C. A. Burchsted.

66. Г. E. Wright et al., High Velocity Filters, USAF Report WADC 55-457, AS TIA Document No. AD-142075, Donaldson Company, Inc., 1957.

67. C. G. Bell and W. Strauss, "Effectiveness of Vertical Mist Eliminators in a Cross Flow Scrubber," J. Air Pollut. Control Assoc. 23, 967-69 (November 1973).

68. J. A. Brink, "Removal of Phosphoric Acid Mists," Chap. 5 in Gas Purification Process, George Newnes, Ltd., London, 1964, Part B.

69. J. A. Brink et al., "Mist Eliminators for Sulfuric Acid Plants," Chem. Eng. Prog. 64(11), 82-86 (November 1968).

70. G. A. Johnson, Atlantic-Richfield Hanford Co., personal communication to C. A. Burchsted.

71. J. A. Rauscher et al., "Fiber Mist Eliminators for Higher Velocities," Chem. Eng. Prog. 60(11), 68-73 (November 1964). 


\section{Housing Design and Layout}

\subsection{INTRODUCTION}

This chapter discusses housing design and requirements for air cleaning units in which filters are installed in man-entry housings. Large-volume air supply and exhaust requirements may be met by a number of individual filter-blower installations operating in parallel, by a single central system, or by a combination of both. Individual filter-blower systems, shown in Fig. 4.1, have the advantages of (1) greater flexibility from the standpoint of system modification; (2) little interference with operations during filter replacement, because individual units can be shut down without affecting the remaining systems; (3) good overall control of ventilation in the event of malfunction, fire, or accident to one or a few of the individual units; and (4) easy system balancing. On the other hand, batteries of individual filterblower systems are more costly to build, operate, and maintain than a single central system of the same capacity. If individual systems discharge to individual stacks, there is also the risk of flow reversal (with the danger of contamination spread to occupied areas), when one or more fans are out of service and

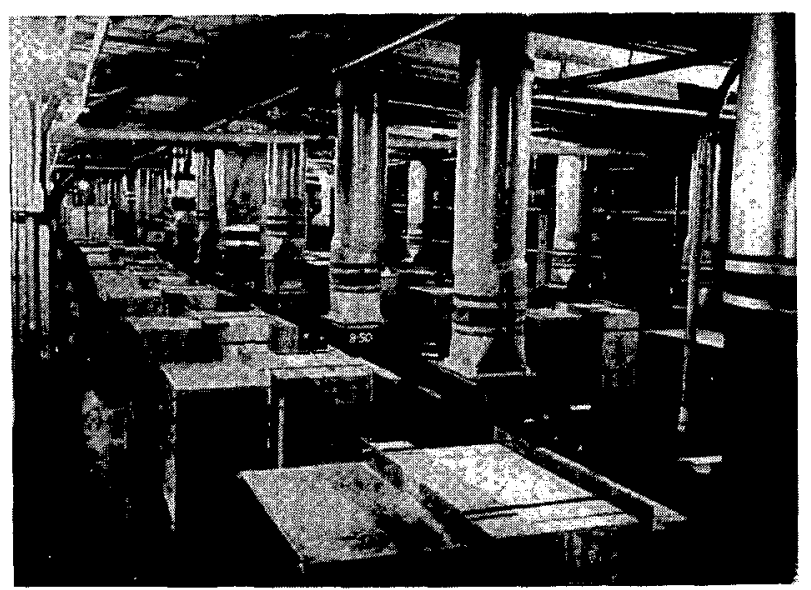

4.1. Battery of individual filter-blower systems exhausting fume hoods of a radiochemical laboratory. others continue to operate. Flow reversal during a fire in some part of the building could also result in an inadvertent and undesirable air feed to the fire, which has occurred on some occasions.

Filters of a central system may be arranged in banks in a large filter house or in a multiple singlefilter array in which a number of filters are installed between common supply and exhaust headers, as Fig. 4.2 shows. Such a multiple single-filter array must be located in a room that can be sealed off from adjacent operating, storage, and equipment areas and that lends itself to easy decontamination. In no case should a multiple single-filter array be located in an open attic or building space where problems of contamination spread could result if a filter were dropped during a change operation. The multiple single-filter arrangement has the advantages that all filters can be installed at a convenient height for replacement, and personnel do not have to enter what may be a highly contaminated filter house to change filters. The design of the individual filter installation in a multiple single-filter array is similar to that of other single-cell installations discussed in Chap. 6. Careful alignment of filter inlet and outlet connections is essential. If these connections are even slightly out of alignment, a poor seal will result, and the condition will worsen if there is system pulsation and vibration. Inlet and outlet duct axes must coincide within $\pm 1 / 16$ in.; a minimum of 2 in. (preferably 4 in.) should be allowed between filter units for access and ease of maintenance. When tape-sealed open-face filters are used, the spacing between units should be at least 6 in. to allow the workman to manipulate the tape and achieve proper adhesion. Aisle space in front and back is desirable to permit the inspection of seals. Tape-sealed connections are prone to fail, even under normal operating conditions; however, because the tape peels, these connections are not recommended.

In bank systems, a number of open-face filters are installed in parallel on a single mounting frame in a 


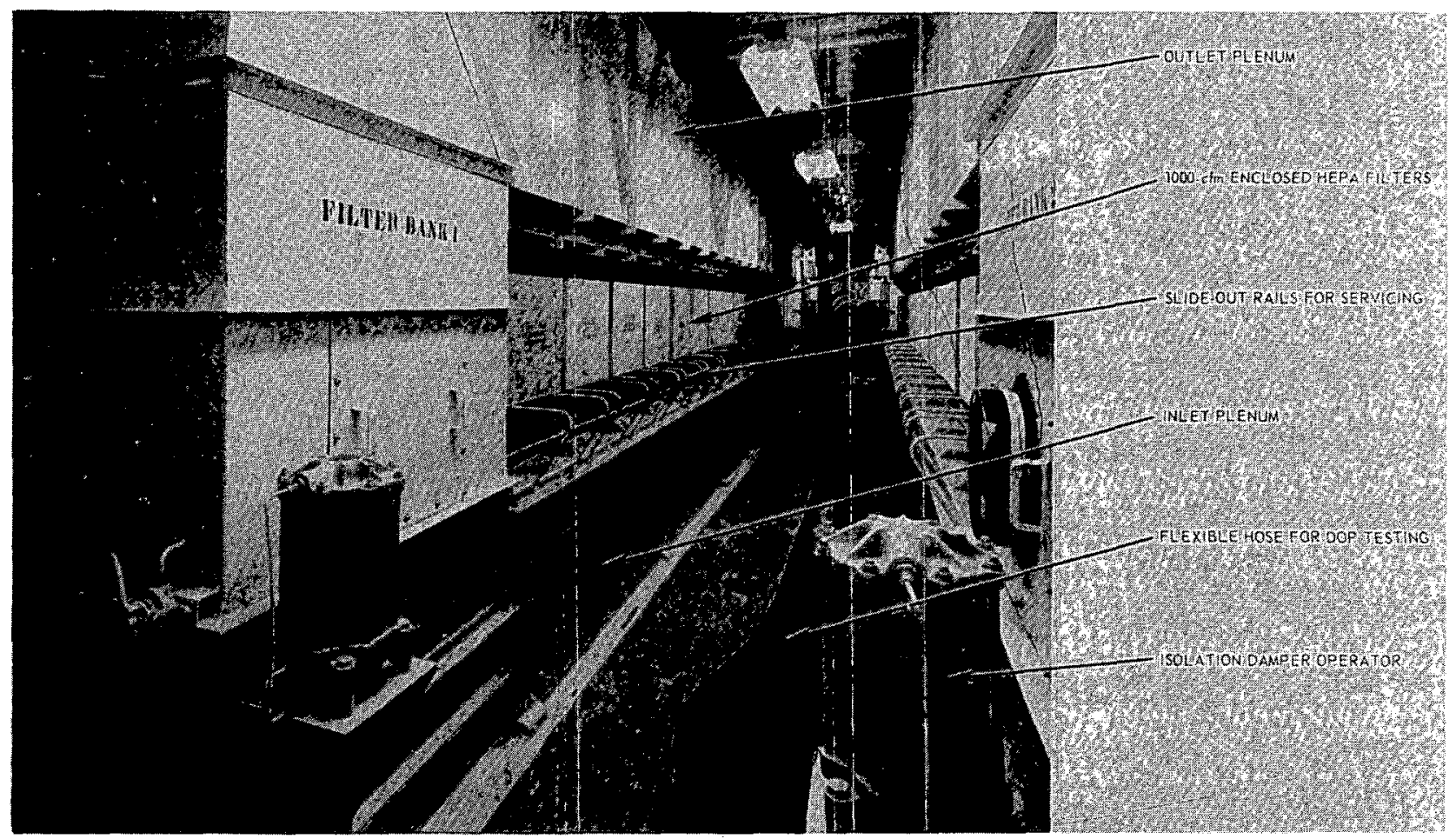

Fig. 4.2. Multiple single-filter central exhaust system installed in a Zone II contamination area. Courtesy Atomic Energy of Canada, Limited, Chalk River Laboratory.

single housing. Because banks are the more common type of large multiple-filter installation, the remainder of this chapter is devoted primarily to their design and construction, including spatial arrangement, mounting frames, housings, instruments, and testing. Although the discussion relates primarily to exhaust filter systems, most of it is equally applicable to clean-room installations and other supply-air systems that employ high-efficiency filtration. Figure 4.3 shows how large some of these installations can be, particularly in laminar-flow clean rooms and in some of the earlier central-exhaust systems of nuclear facilities. Installations containing nearly a thousand 1000-cfm HEPA filters in a single bank have been built in the past, but banks larger than 30,000-cfm nominal capacity (i.e., thirty 1000 -cfm filters) are no longer recommended for nuclear exhaust or cleanup service because of the difficulties of control (in the event of emergencies), maintenance, and testing. For exhaust and cleanup systems larger than 30,000-cfm capacity, segmentation of the system into two or more parts of equal airflow capacity, with each part in an individual housing installed in parallel, is recommended. Isolation valves on each housing are desirable for ease of system control, for isolation of individual units during an emergency, and for maintenance or testing.
Bank systems have the advantages of lower unit construction cost, lower unit operating cost, and lower space requirements when compared with multiple single-filter systems. For example, the 36,000-cfm multiple single-filter array in Fig. 4.2 occupies about $600 \mathrm{ft}^{2}$ of floor space and a volume of approximately $9000 \mathrm{ft}^{3}$, whereas a bank system of equal capacity would occupy less than $200 \mathrm{ft}^{2}$ of floor space and a volume of less than $1400 \mathrm{ft}^{3}$. The operating cost of such a multiple single-filter system may be 20 to $30 \%$ higher than that of an equivalent bank system because of friction and dynamic losses in the plenums and in the individual filter inlets and outlets.

\subsection{COMPONENT INSTALLATION}

Proper installation of HEPA filters, ad sorber cells, and demisters is critical to the reliable operation of a high-efficiency air cleaning system. Factors that must be considered in the design of such installations include:

1. structural rigidity of mounting frames:

2. rigid and positive clamping of components to the mounting frame;

3. careful specification of and strict adherence to close tolerances on alignment, flatness, and surface condition of component seating surfaces; 


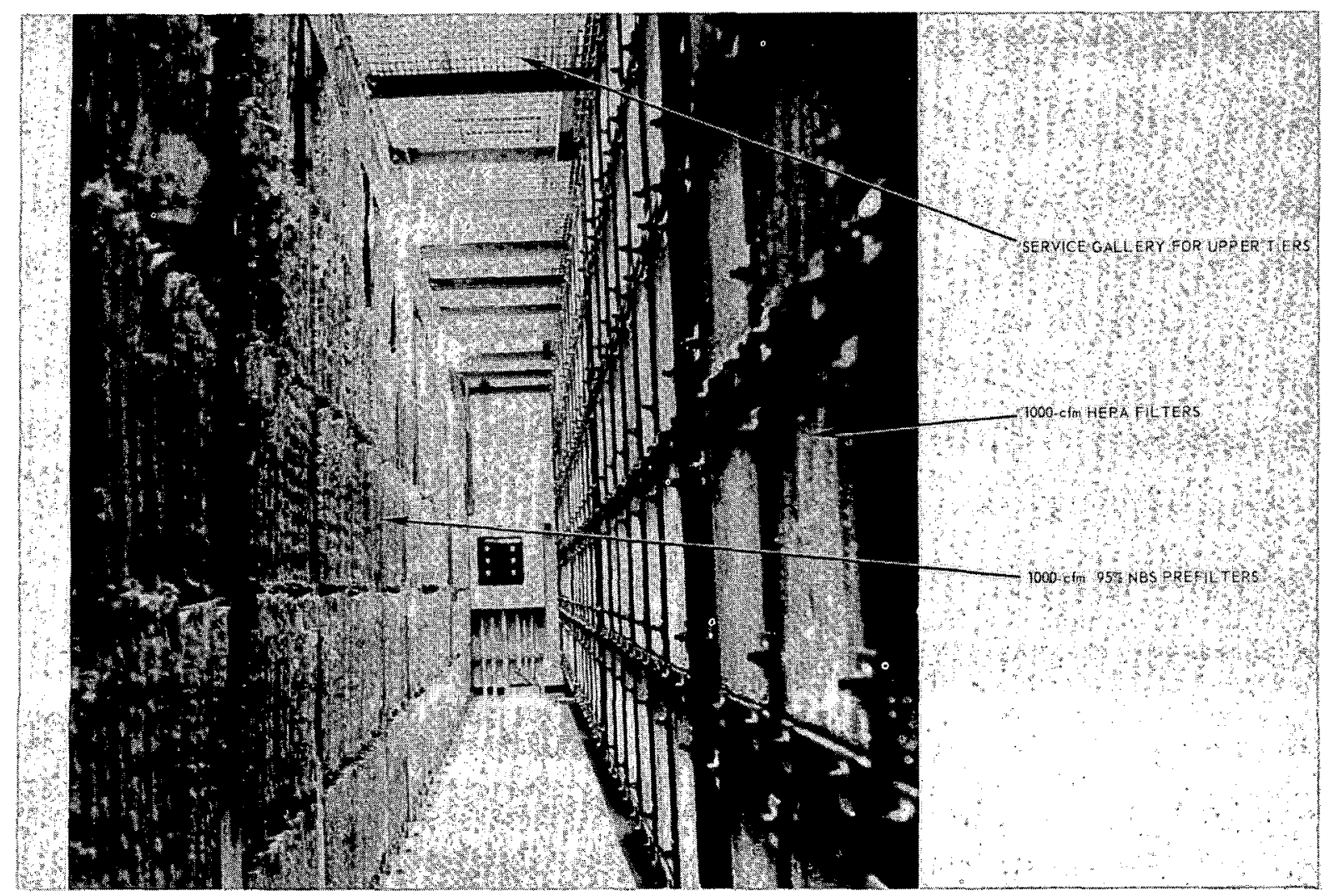

Fig. 4.3. Prefilter and HEPA filter banks of a large horizontal-flow laminar-flow clean room. Courtesy National Aeronautics and Space Administration, Greenbelt Unmanned Spaceflight Center.

4. welded-frame construction and welded seal between the mounting frame and the housing;

5. ability to inspect the interface between components and mounting frame during installation;

6. adequate spacing between components in the bank;

7. adequate spacing in the housing for men to work.

The components and mounting frame should form a continuous barrier between the contaminated zone and the clean zone of the system; any hole, crack, or defect in the mounting frame or in the seal between component and frame which permits bypassing will result in leakage of contaminated air into the clean zone and a decrease of system effectiveness. A mounting frame that is not sufficiently rigid can flex so much during operation, particularly under abnormal conditions, that leaks may develop in HEPA filters clamped to the frame (due to differential flexing of the filter case relative to the mounting frame). Cracks may also be opened between the filters and the frame, between frame members (due to weld cracking or fatigue), or between the frame and the housing. Insufficient attention to maintenance provisions in the original design can increase operating costs and reduce the reliability of the system. Once the system is installed, defects are difficult to locate, costly to repair, and may even require rebuilding the system.

The out-of-date practices of simply stacking filters (adsorbers) in a rectangular opening (using duct tape to seal gaps between filters and between filters and the opening) and of installing them in lightly constructed honeycomb mounting frames of the type shown in Fig. 4.4 are not acceptable for nuclear service. Joints between mounting frame members and between the mounting frame and the housing wall should be sealed by welding. Duct tape, mastics, resilient sealants (such as RTV silicone), and other "applied" sealing materials invariably fail from exposure to alternating heat, cold, moisture, dry air, vibration, or combinations of these conditions, which are usually encountered in high-efficiency exhaust and air cleanup systems; such materials must not be used in critical systems. Such sealing methods are prohibited in ESF systems. 'The honeycomb frame is permissible for prefilters and duct-entry installations of 


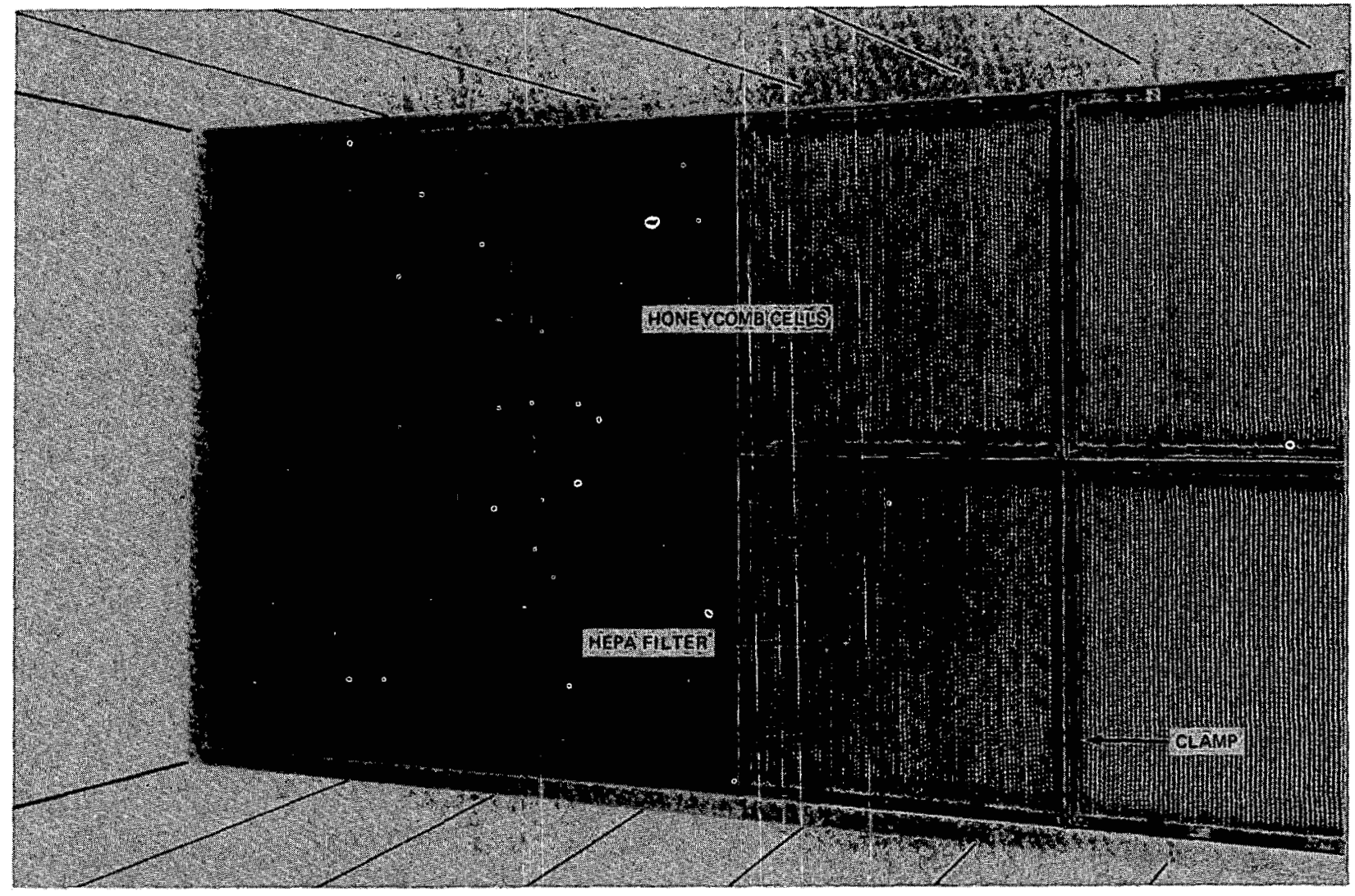

Fig. 4.4. Honeycomb mounting frame made up of individual filter cells bolted or riveted together. Note the caulked joints and the lightduty filter mounting frame and clamping devices. Mounting frames of this type are not suitable for installing HEP A filters in nuclear exhaust applications.

common air filters, but the stacked-and-taped method should not be employed for either HEPA filters or prefilters under any circumstances.

\subsection{HEPA FILTER, ADSORBER CELL, AND DEMISTER MOUNTING FRAMES}

Mounting frames for HEPA filters and other critical components should be all-welded structures of carbon or stainless steel structural shapes, plate, or heavy cold-formed sheet. Carbon steel frames should be painted or coated for corrosion resistance. Galvanized steel is not recommended because of welding difficulties and because the zinc coating does not give adequate protection in environments that may be encountered in a contaminated exhaust system. Aluminum is not recommended. Because of the high cost of surface preparation, inspection, and rework usually incurred in obtaining high-quality vinyl and epoxy coatings, stainless steel is often the best and most economic choice in radiochemical plant applications. Suitable mounting frame materials include:

carbon steel shapes and plate, ASTM A36, ${ }^{2} 499 ;{ }^{3}$ carbon steel structural tubing, ASTM A500; ${ }^{4}$ carbon steel sheet, ASTM A245, grade $D ;^{5}$ stainless steel shapes, ASTM A479, type 304L, class $C$, annealed and pickled; ${ }^{6}$

stainless steel plate, ASTM A240, type 304L, hotrolled, annealed, and pickled; ${ }^{7}$

stainless steel sheet, ASTM A240, type 304L, annealed and pickled, $2 \mathrm{D}$ or $2 \mathrm{~B}$ finish. ${ }^{7}$

Information relating to fabrication includes:

"Specification for the Design, Fabrication, and Erection of Structural Steel for Building," Manual of Steel Construction, American Institute of Steel Construction, New York, 1970.

Light-Gage Cold-Formed Steel Design Manual, American Iron and Steel Institute, New York, 4th ed., 1962. 
AWS D1.1-72, AWS Structural Welding Code, American Welding Society, Miami, 1972.

O. W. Blodgett, Design of Welded Structures, James F. Lincoln Arc Welding Foundation, Cleveland, 1966.

\subsubsection{Structural Requirements}

The mounting frame is a statically indeterminate lattice that generally consists of a set of full-length members spanning the height or width of the bank (whichever is shorter), connected by cross members that are slightly shorter than the width of individual filter (adsorber) units. The frame may be considered as an array of simply supported, uniformly loaded beams for design purposes. Experience has shown that to obtain adequate frame rigidity, these beams (frame members) should deflect no more than $0.1 \%$ of their length under a loading equivalent to 1.5 times the maximum dirty-filter pressure drop across the bank. This loading is determined from the equation

$$
W=0.036(1.5) \triangle p S,
$$

where

$$
\begin{aligned}
0.036 & =\text { conversion factor, in.wg to } \mathrm{psi} ; \\
W & =\text { uniform beam loading, } \mathrm{lb} / \mathrm{in} . ; \\
\triangle p & =\text { pressure drop across bank, in.wg; } \\
S & =\text { center-to-center spacing of filters on bank, } \\
& \text { in. }
\end{aligned}
$$

Assuming a center-to-center spacing of $26 \mathrm{in}$. for 24- by 24 -in. filters, Eq. (4.1) reduces to

$$
W=1.404 \triangle p .
$$

The value determined from Eq. (4.2) can be used in standard beam equations ${ }^{8}$ to determine the minimum moment of inertia required. Knowing the minimum moment of inertia required for the member, the size and shape can be selected directly from the tables of structural shape properties given in the AISC Manual of Steel Construction, "or can be determined by calculating the moment of inertia of a built-up or cold-formed section. For ASTM A36 steel, the standard beam equations reduce to

$$
\begin{aligned}
& \text { major frame members, } I=\frac{\Delta p L^{3}}{1.59 \times 10^{6}}, \\
& \text { cross members, } I=\frac{\Delta p}{149}
\end{aligned}
$$

where

$$
\begin{aligned}
I= & \text { minimum moment of inertia required, in. }{ }^{4} ; \\
\Delta p= & \text { maximum dirty-filter pressure drop across } \\
& \text { bank, in.wg; } \\
L= & \text { length of member, in. (cross members } \\
& \text { assumed to be } 22 \text { in. long). }
\end{aligned}
$$

In addition to flexural strength, the frame for an exhaust or air cleanup filter system should also be capable of withstanding a shock loading of at least 3 psi across the bank without exceeding the elastic limit of the frame material. In most cases, members calculated using Eqs. (4.3) and (4.4) will meet this requirement; nevertheless, they should be checked. The section moduli ( $S$ values) given in Part 1 of the AISC Manual of Steel Construction ${ }^{9}$ are then compared with the minimum values obtained from the following equations:

$$
\begin{aligned}
& \text { major frame members, } S=\frac{13 L^{2}}{f_{a}}, \\
& \text { cross members, } S=\frac{6290}{f_{a}},
\end{aligned}
$$

where

$S=$ section modulus, in. ${ }^{3}$;

$f_{a}=$ maximum allowable fiber stress, psi;

$L=$ length of member, in. (cross members assumed to be $22 \mathrm{in.} \mathrm{long).}$

For ASTM A36 steel, these equations reduce to

$$
\begin{aligned}
& \text { major frame members, } S=0.000361 L^{2} \\
& \text { cross members, } S=0.175 \text {. }
\end{aligned}
$$

For built-up and cold-formed members, the minimum $S$ value calculated from these expressions is compared with the value for the member calculated from the formula

$$
S=\frac{I}{c}
$$

where

$$
\begin{aligned}
S= & \text { section modulus, in. }{ }^{3} ; \\
I= & \text { moment of inertia of the section, in. }{ }^{4} ; \\
c= & \text { distance from neutral axis of member to } \\
& \text { extreme fiber, in. }
\end{aligned}
$$


If the $S$ values obtained from the AISC manual or calculated by using Eq. (4.9) are greater than the values calculated from Eqs. (4.5) through (4.8) (as applicable), the members selected are satisfactory.

\subsubsection{Mounting Frame Configuration}

There are three basic types of mounting frame construction: (1) face-sealed, in which the filter seals to the outermost surfaces of the frame members by means of gaskets glued to the front surface or to the flange around the face of the filter unit, as Fig. 4.5 shows; (2) pocket, in which the filter fits into an opening of the frame and seals, by means of a gasket glued to the face flange of the filter unit, on an inner flange, as Fig. 4.6 shows; and (3) drawer, in which the filter (or adsorber cell) fits through an opening and seals, by means of a gasket glued to the back of the face plate of the filter or adsorber cell, to the outermost surfaces of the frame members, as Fig. 4.7 shows. The latter configuration is an adaptation of the case used for type II adsorber cells ${ }^{10}$ and, for filters, requires a specially designed metal-case HEPA filter as shown in Fig. 4.7. The face-sealed

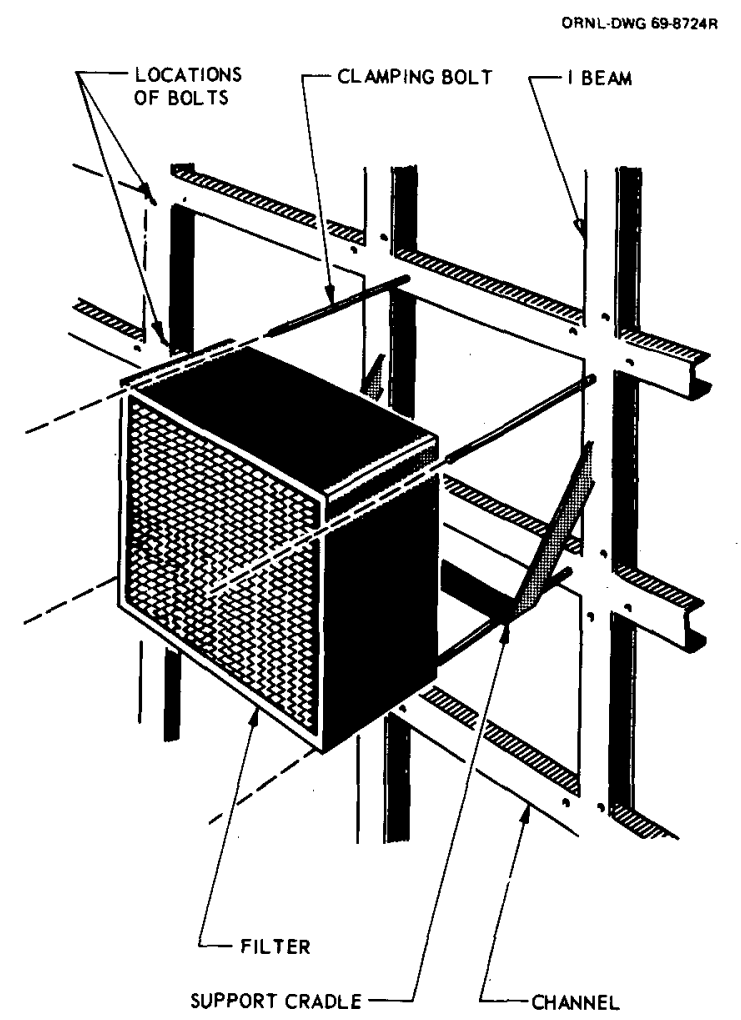

Fig. 4.5. Face-sealed mounting of HEPA filter. Note eight-bolt clamping and support cradle. Frame is all-weld construction, using structural I-beams and channels. configuration is generally recommended for conventional-design HEPA filters and type I adsorber cells. ${ }^{10}$ Pocket-type mounting frames, although satisfactory in some instances, are not recommended for the following reasons. (1) Because access to the recessed filter-sealing surfaces is often limited, removal of weld slag and spatter is difficult and often incomplete, thus preventing proper seating and sealing of filter gaskets. (2) If wood-cased filters are used and dimensions of the pocket openings are tight, swelling of the wood cases as a result of moisture may prevent easy removal of used filters. (3) Filter-sealing surfaces and gaskets are obscured during filter installation. Face-sealed mounting frames may occupy slightly more space in the filter housing than pocket frames, but they require less material and less welding, thereby presenting fewer opportunities for leakage.

A minimum face width of $4 \mathrm{in}$. is recommended for major and cross members of face-sealed HEPA filter frames. This allows 1-in.-wide filter-seating surfaces to compensate for any misalignment of the filter during installation, and allows a 2 -in. space between filters, horizontally and vertically, to give adequate room for handling (personnel replacing contaminated filters will probably have to wear double gloves), for using power tools or torque wrenches during filter change, and for manipulating a test probe between units. Although mounting frames made from members as narrow as $2 \frac{1}{2}$ in. are often used, the slight increase in material cost and building space required by the use of wider frame members will result in easier access and thereby lower cost of maintenance.

Face-widths of frame members for installing type I (pleated-bed) adsorber cells are the same as those for HEPA filters. Face-widths of frame members for installing type II (tray-type) adsorber cells may be narrower, since handles are provided on the front of the trays to facilitate installation. To provide for interchangeability of cells of different manufacture, IES CS-8 recommends the following mounting frame dimensions for the installation of type II cells (see IES CS-8 for standard cell dimensions): ${ }^{10}$

Openings: $6.37 \times 24.188$ in. (+0.063 in., -0 in.)

Space between openings: vertical, 2.5 in. minimum; horizontal, 2 in. minimum

Figure 4.8 shows a built-up all-welded type II adsorber cell mounting frame made from rectangular structural tubing; note that a structure is required 


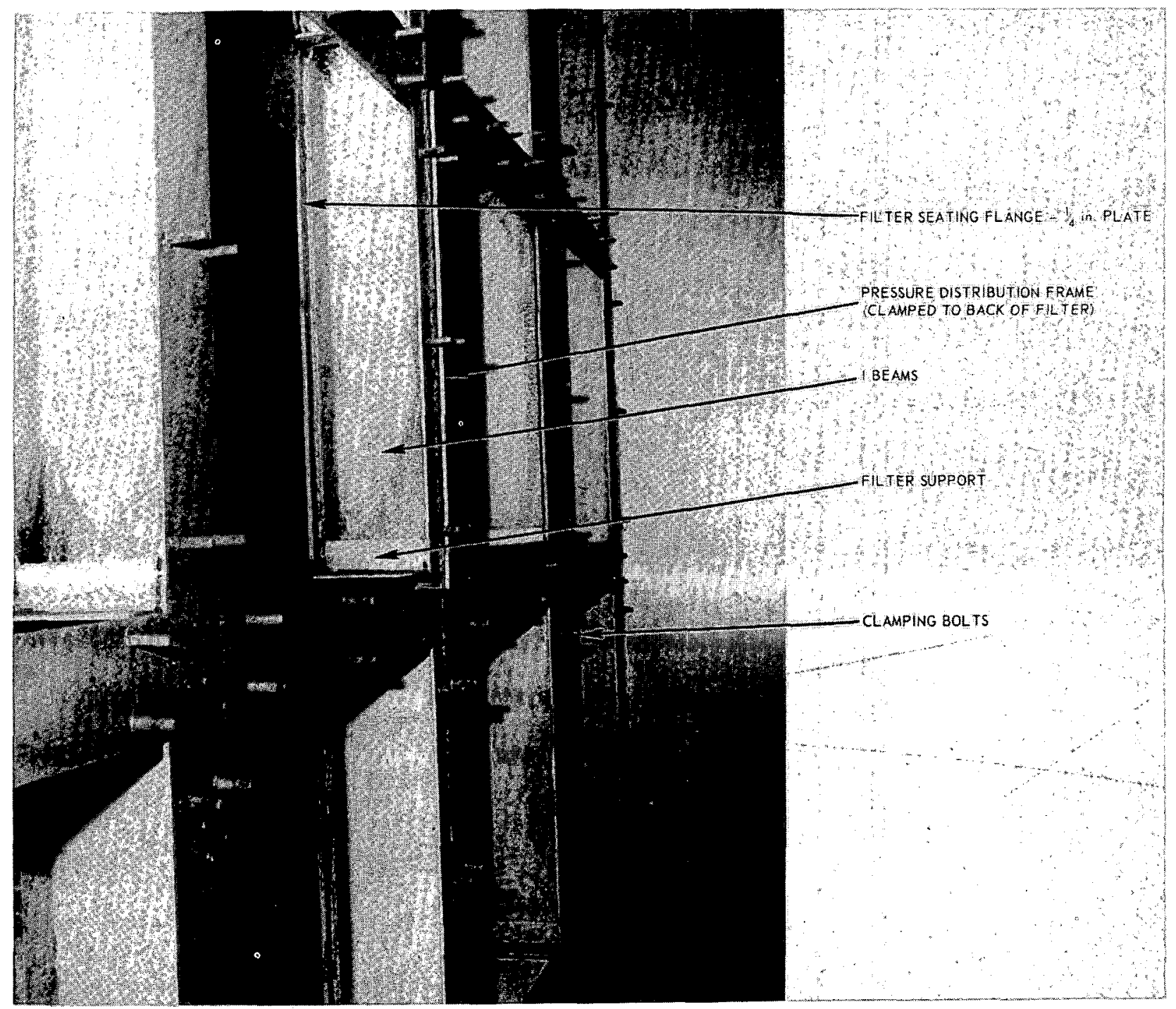

Fig. 4.6. Built-up pocket-type filter mounting frame for 500-cfm HEPA filters.

behind the frame openings to support the weight of the cells (approximately $100 \mathrm{lb}$ each). Because the length of type II cells may be different for each manufacturer, the support structure should be deep enough to take a cell up to $32 \mathrm{in}$. long to permit interchangeability of cells of different manufacture.

Satisfactory mounting frames may be made from rolled structural shapes, rectangular structural tubing, reinforced plate, or brake- or die-formed heavy gage (No. 14 U.S. gage minimum, No. 11 U.S. gage recommended) sheet metal. Figure 4.9 shows a HEPA filter frame made from 4- by 4-in. I-beams that meets all structural requirements. Minimumcost rolled structural shapes for building mounting frames are given in Table 4.1. Square structural tubing frames for HEPA filters should be made from rectangular tubing having a face width of at least 4 in.; structural tubing frames for type II adsorber cells may have narrower face widths (Fig. 4.8). Figure 4.10 shows the front and rear of a face-sealed HEPA filter frame made from a single piece of $3 / 8$-in. plate welded into a 4- by 6-in. I-beam "picture frame" and reinforced on the back with bar to provide the required structural rigidity, Note that the reinforcing bars are skip-welded to the plate to minimize distortion during welding but that a continuous seal weld is provided between the face of the frame and the "picture frame." Openings in this frame were plasmatorch cut and then finished by machining. This design 


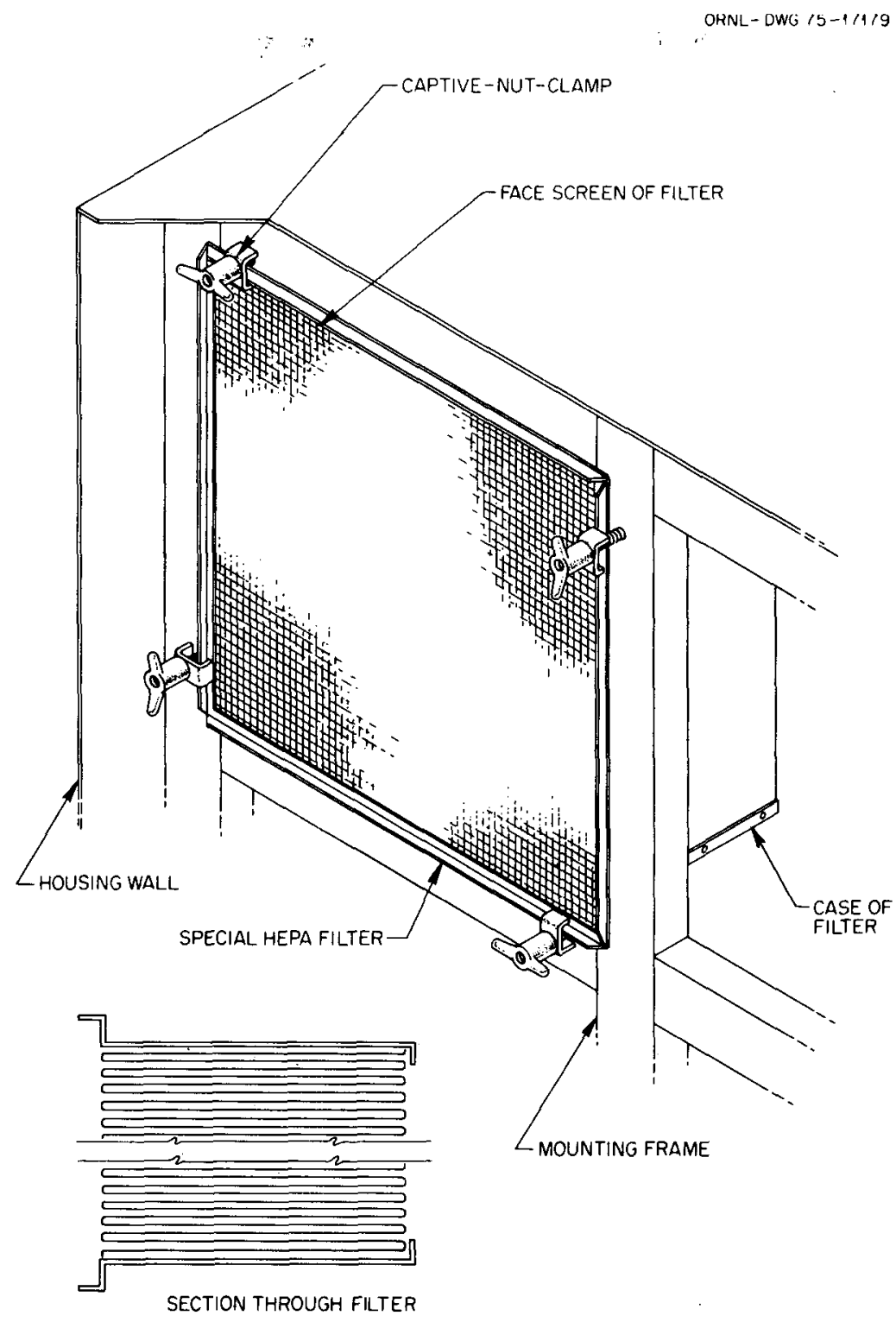

Fig. 4.7. Section of drawer-type mounting frame for HEPA filter showing details of clamping and specially designed HEPA filter. Courtesy Flanders Filter Corp.

has the advantage of requiring less material than a structural-shape frame or square tubing frame. The fact that it is made from a single piece of plate, with no welding on the face, eliminates frame-member misalignment and heat-of-welding distortion problems. Figure 4.11 shows an all-welded HEPA filter frame built up from die-formed sheet-metal members.

\subsubsection{Frame Fabrication}

Filter mounting frames should be shop-fabricated insofar as practicable, because it is nearly impossible to avoid misalignment, warping, and distortion in field fabrication. Shop fabrication is less costly than field fabrication and permits better control over assembly, welding, and dimensional tolerances. Care must be taken to avoid twisting or bending of the completed frame during handling, shipping, and field installation. For proper performance and ease of maintenance of installed filters, dimensional and surface-finish tolerances must be tight and rigidly enforced. Table 4.2 gives minimum tolerances for the installed frame. Welds on the filter-seating side of the frame must be ground flat, smooth, and flush. 
The inert-gas-shielded metal-arc (GMA) welding process and inert-gas-shielded tungsten-arc (GTA) welding process are recommended for shop and field fabrication. The GMA process is particularly well adapted to field work because it is fast, gives a reasonably good quality of weld when made by a

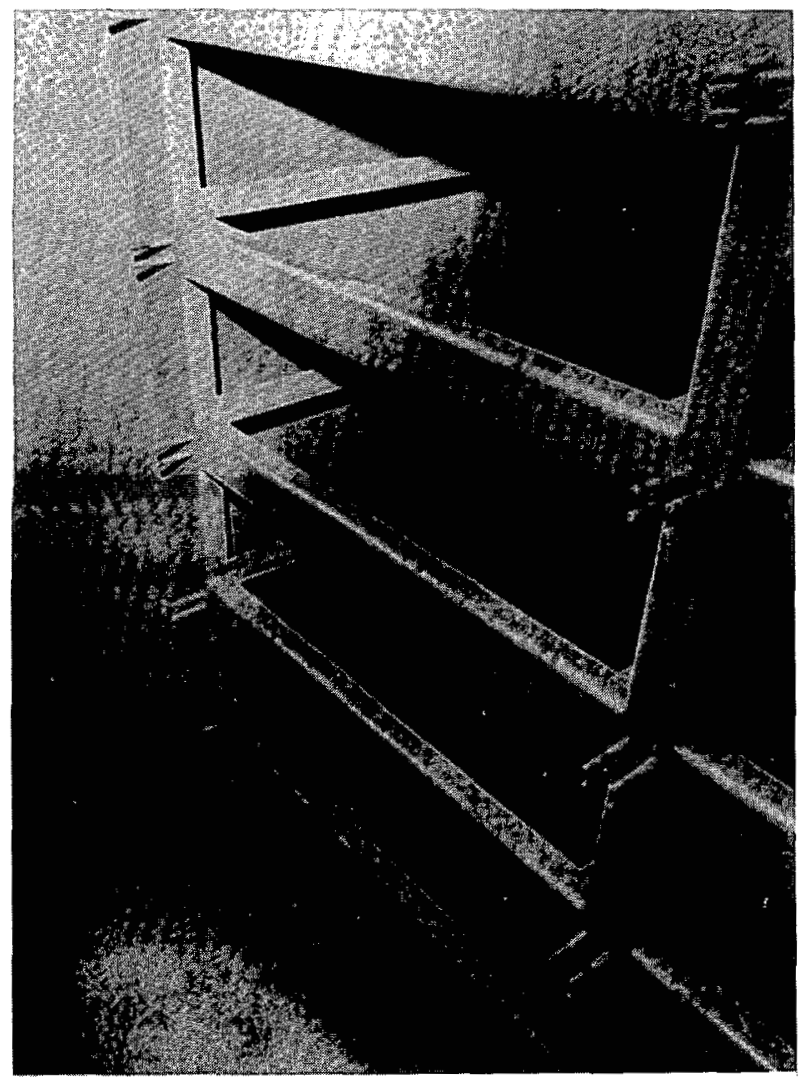

Fig. 4.8. Type II adsorber cell mounting frame made from rectangular structural tubing. Note cell support structure. qualified welder under good conditions, and has low heat input. ${ }^{11}$ Seal welds between adjoining members and between the frame and housing should be fullpenetration welds and must be made from the airentering side. Only welders qualified in accordance with the AWS Structural Welding Code or Sect. IX

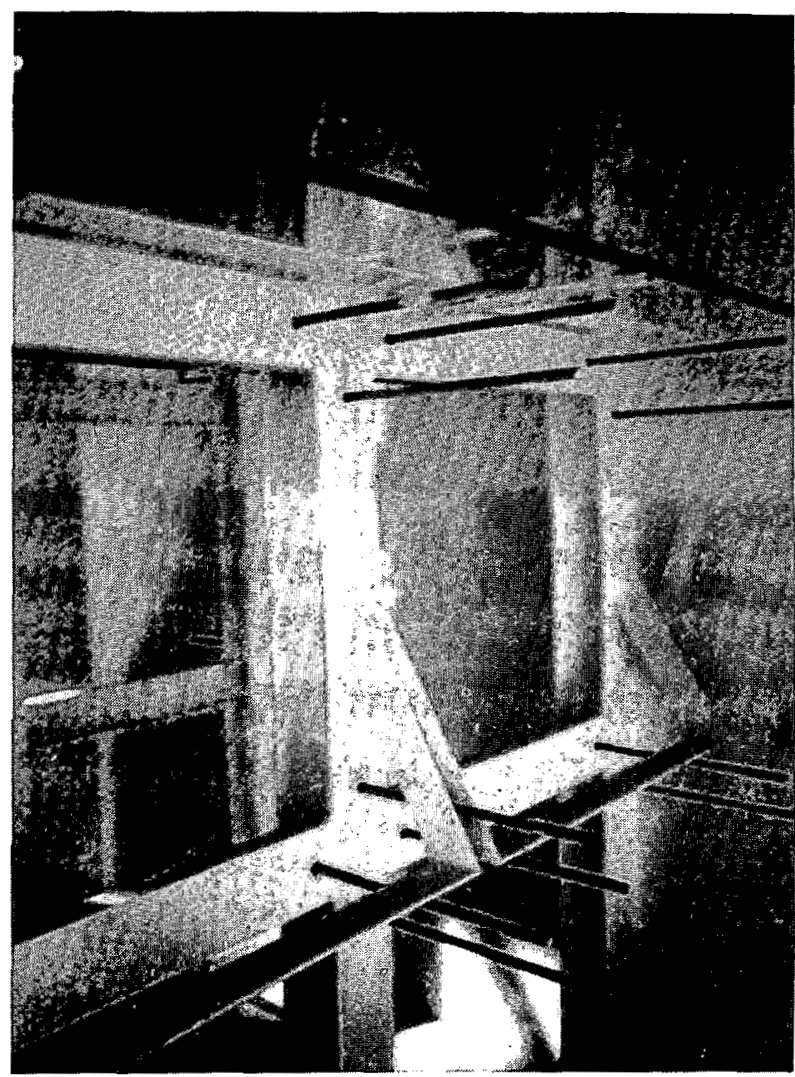

Fig. 4.9. HEPA filter mounting frame made from rolled structural shapes ( 4 - by 4 -in. I-beams).

Table 4.1. Minimum-cost structural members for HEPA filter and adsorber mounting frames

Maximum pressure drop to 12 in.wg

\begin{tabular}{|c|c|c|c|c|c|}
\hline \multirow{3}{*}{$\begin{array}{l}\text { No. of } 1000-\mathrm{cfm} \\
\text { units high }\end{array}$} & \multicolumn{3}{|c|}{ Principal member, ${ }^{a}$ I-beam } & \multirow{2}{*}{\multicolumn{2}{|c|}{$\begin{array}{l}\text { Cross member channel } \\
\quad(\operatorname{span}=22 \text { in.) }\end{array}$}} \\
\hline & \multirow{2}{*}{ Span } & \multirow{2}{*}{$\begin{array}{l}\text { Size } \\
\text { (in.) }\end{array}$} & \multirow{2}{*}{ Pounds per foot } & & \\
\hline & & & & Size (in.) & Pounds per foot \\
\hline 2 & $4 \mathrm{ft} 8$ in. & $4 \times 4 \mathrm{M}$ & 13 & $4 \times 1^{3 / 4}$ & 5.4 \\
\hline 3 & $6 \mathrm{ft} 10 \mathrm{in}$. & $4 \times 4 \mathrm{M}$ & 13 & $4 \times 1^{3 / 4}$ & 5.4 \\
\hline 4 & $9 \mathrm{ft} 0$ in. & $4 \times 4 M$ & 13 & $4 \times 1^{3 / 4}$ & 5.4 \\
\hline 6 & $13 \mathrm{ft} 4$ in. & $6 \times 4 B$ & 16 & $4 \times 1^{3 / 4}$ & 5.4 \\
\hline 8 & $17 \mathrm{ft} 8$ in. & $8 \times 4 B$ & 10 & $4 \times 1^{3 / 4}$ & 5.4 \\
\hline 10 & $22 \mathrm{ft} 0$ in. & $10 \times 4^{5 / 8}$ & 25.4 & $4 \times 1^{3 / 4}$ & 5.4 \\
\hline
\end{tabular}

${ }^{a}$ Principal members should span the shortest dimension of the bank.

${ }^{b} \mathrm{Span}=[($ number of filters $)(26)+4]$ in. 

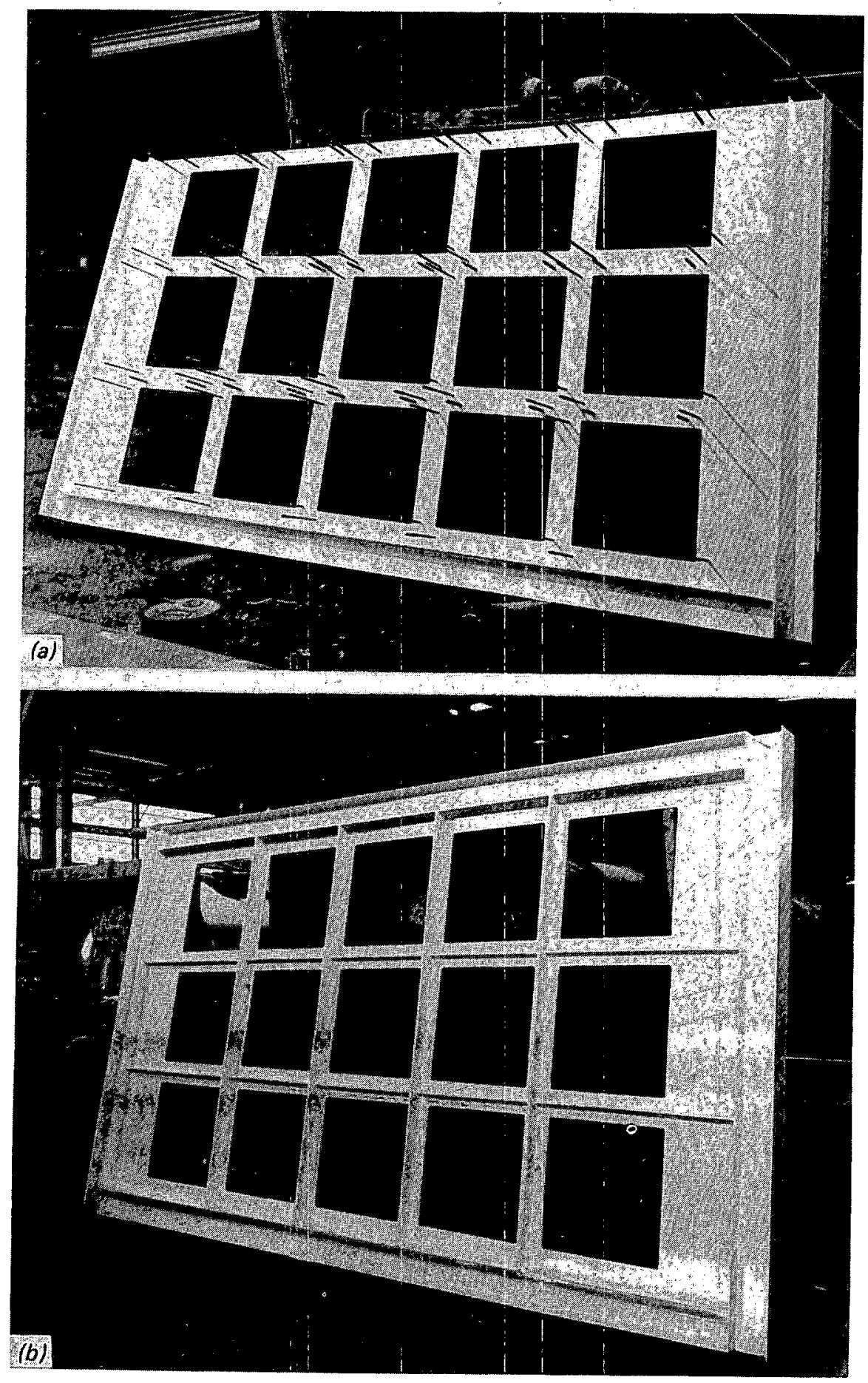

Fig. 4.10. HEPA filter mounting frame made from a single $3 / 8$-in. plate during fabrication in manufacturer's shop. Openings are torchcut, then finished by machining. "Picture frame" made from I-beams reinforces housing in addition to providing rigidity to frame itself. (a) Front; $(b)$ rear; note reinforcing bars skip-welded to single plate. Courtesy CTI-Nuclear, Inc. 


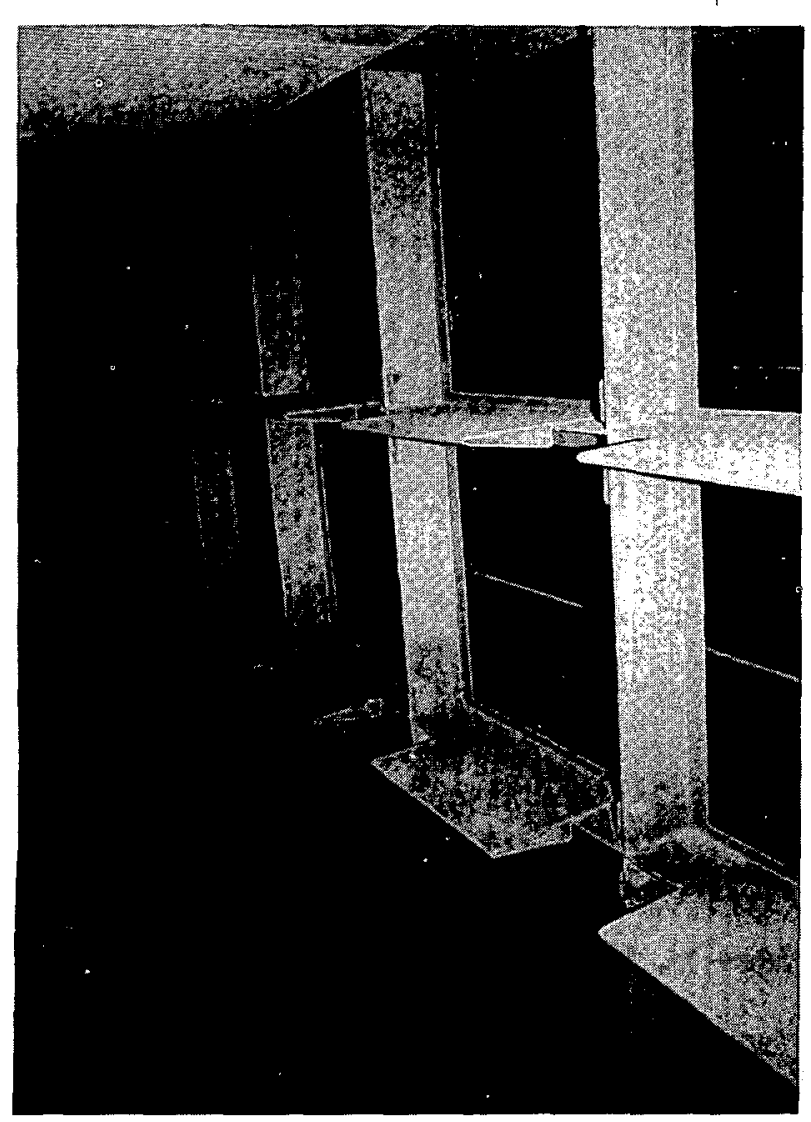

Fig. 4.11. All-welded HEPA filter frame made from die-formed sheet-metal members. Note pocket-type configuration and filter rests beneath openings. Filters are arranged in two 6-wide by $3-$ high arrays, one above the other with a permanent work gallery between.

Table 4.2. Recommended tolerances for HEPA filter and adsorber mounting frames

\begin{tabular}{|c|c|}
\hline \multirow[t]{2}{*}{ Alignment } & $\begin{array}{l}\text { Perpendicularity: maximum offset of adjoining } \\
\text { members, } 1 / 64 \mathrm{in} / \mathrm{ft} \text { or } 1 / 16 \text { in., whichever } \\
\text { is greater }\end{array}$ \\
\hline & $\begin{array}{l}\text { Planarity of adjoining members: } 1 / 64 \text { in. maximum } \\
\text { offset at any point on the joint }\end{array}$ \\
\hline \multirow[t]{2}{*}{ Flatness } & $\begin{array}{l}\text { Each filter surface shall be plane within } 1 / 16 \text { in. } \\
\text { total allowance }\end{array}$ \\
\hline & $\begin{array}{l}\text { Entire mounting fixture shall be plane within } \\
1 / 2 \text { in. total allowance in any } 8-\text { by } 8 \text {-ft area }\end{array}$ \\
\hline Dimensions & $\begin{array}{l}\text { Length and spacing of members shall be true } \\
\text { within }+0,-1 / 16 \text { in. }\end{array}$ \\
\hline Surface finish & $\begin{array}{l}\text { Filter seating surfaces: } 125 \mu \text { in. AA, maximum, } \\
\text { in accordance with USA Standard B46.1; pits, } \\
\text { roll scratches, weld spatter, and other surface } \\
\text { defects shall be ground smooth after welding, } \\
\text { and ground areas shall merge smoothly with the } \\
\text { surrounding base metal; waviness not exceeding } \\
1 / 16 \text { in. in } 6 \text { in. is permissible as long as the } \\
\text { overall flatness tolerance is not exceeded }\end{array}$ \\
\hline
\end{tabular}

of the ASME Boiler and Pressure Vessel Code ${ }^{12}$ should be permitted to make welds on HEPA filter and adsorber mounting frames. Both seal and strength welds should be visually inspected by a qualified inspector under a light level of at least 100 $\mathrm{ft}-\mathrm{c}$ on the surface being inspected. In addition, liquid penetrant or magnetic particle inspection (whichever is applicable for the base material being inspected) of the seal welds between frame members is recommended. Guides for visual, liquid penetrant, and magnetic particle inspection are given in Articles 6, 7, and 9, respectively, of Sect. V of the $A S M E$ Boiler and Pressure Code; acceptance standards for liquid penetrant and magnetic particle inspection are given in Articles 24 and 25, respectively, of Sect. V. ${ }^{13}$

Figure 4.12 illustrates pitting, scratches, and weld spatter, conditions too often observed in welded mounting frames, particularly those of the pocket type.

\subsubsection{Filter Clamping and Sealing}

HEPA filters and adsorber cells must be carefully sealed to the mounting frame to achieve the required low penetration rates and to provide for easy replacement. Except for the fluid-seal design described at the end of this section, sealants are not a satisfactory substitute for gaskets. Experience in clean rooms and contaminated exhaust and air cleanup applications has shown that flat, closed-cell neoprene gaskets, ASTM D1056 grade SCE-43, ${ }^{14}$ give the most satisfactory seal for high-efficiency filters, adsorbers, and demisters. There is no advantage in using shaped (molded) gaskets; not only are they more expensive, but research has shown that they are prone to leak. ${ }^{15,16}$ Gaskets that are too soft (i.e., less than grade SCE-43) take an excessive compression set that may permit leakage when there is relaxation of the clamping bolts. Gaskets that are too hard (i.e., harder than grade SCE-44) require such high clamping loads to effect proper sealing that the filter itself can be distorted or damaged.

As little as $20 \%$ gasket compression is needed to effect a reliable seal when the thickness of the gasket is uniform to within \pm 0.01 in. and when the seating surface of the mounting frame is plane to within \pm 0.01 in. ${ }^{16}$ However, these tolerances are much too restrictive for economical construction, and experience has shown that compressing an SCE-43 gasket at least $80 \%$ is usually necessary to effect a reliable seal over long periods. Eighty percent compression requires a loading of approximately 20 


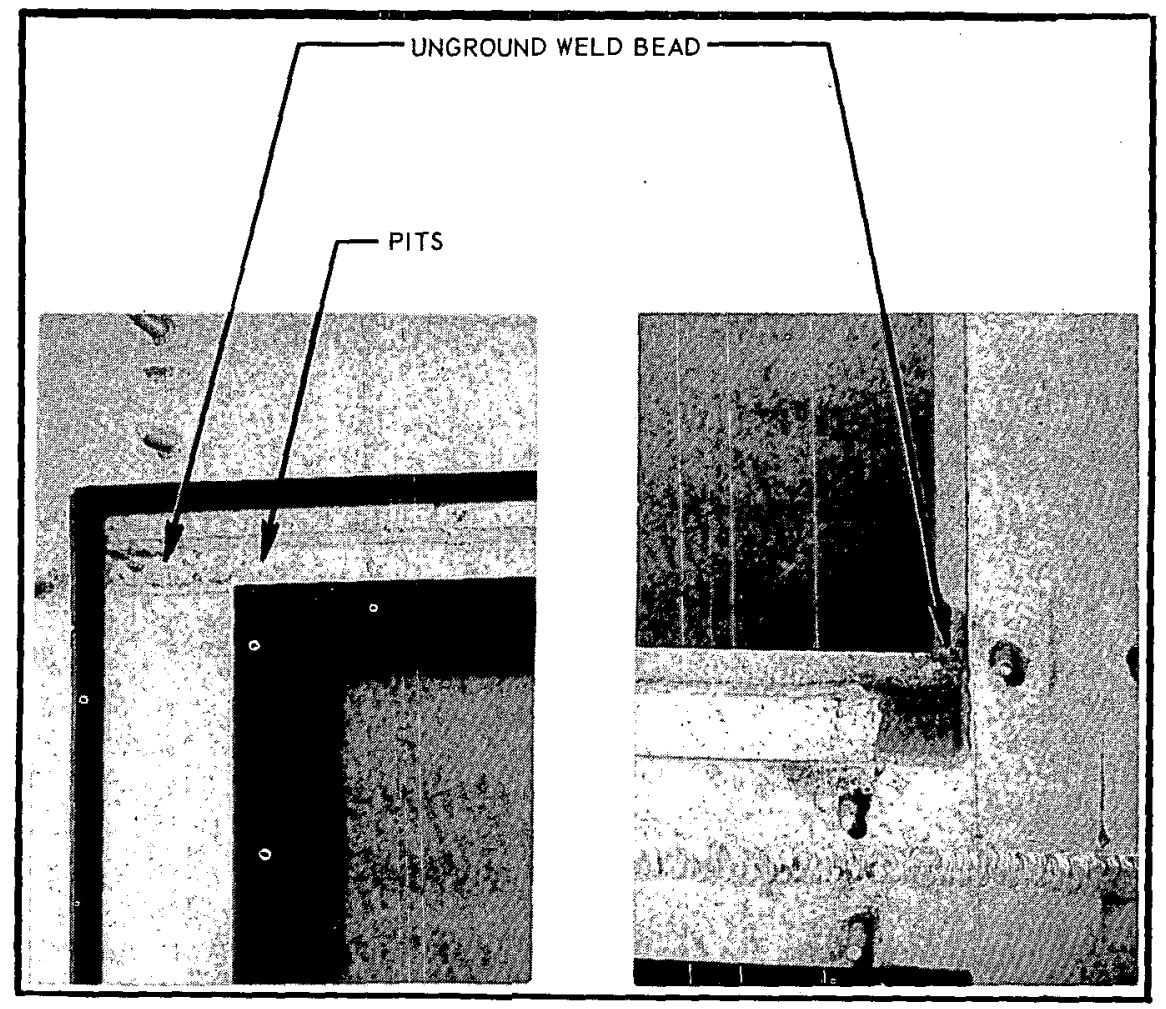

Fig. 4.12. Unacceptable filter-seating surface on HEPA filter mounting frame. Note pits, unground weld bead, and weld spatter, all of which contribute to leakage of contaminated air due to inability to properly seat the filter.

lb per square inch of gasket area, or a total clamping load of about $1400 \mathrm{lb}$ for a $24-$ by 24 -in. filter unit. The recommended procedure for installing filters is to initially torque the clamping bolts to produce $50 \%$ gasket compression and then to retorque them one or two weeks later to a total compression of about $80 \%$.

Gaskets that are too thin may not give a reliable seal with the recommended frame tolerances given in Table 4.2, whereas those that are too thick may be unstable and tend to roll or pull off the flange of the filter case as they are compressed, perhaps to the extent that sections may be extruded between the case and mounting frame and produce a serious air leak. Recommended gasket sizes are $1 / 4$ in. thick by ${ }^{3 / 4}$ in. wide and $1 / 4$ in. thick by $5 / 8$ in. wide. Gaskets must be glued to the filter element rather than to the mounting frame, because they must be replaced with each filter change. Gaskets should have cut surfaces on both faces because the "natural skin" produced by molding sometimes tends to bridge discontinuities or defects in the seating surface, and because the silicone moldrelease compounds used in the manufacture of sheet neoprene prevent proper adhesion of the gasket to the filter case.
Filter units and adsorber cells must be clamped to the mounting frame with enough pressure to enable the gasket to maintain a reliable seal when subjected to vibration, thermal expansion, frame flexure, shock, overpressure, and widely varying conditions of temperature and humidity that can be expected in service. Clamping devices must function easily and reliably after long exposure to hostile environments and, in addition, must be capable of easy operation by personnel dressed in bulky protective clothing, gloves, and respirators (or full-face gas masks) while working in close quarters. Experience has shown that a simple nut-and-bolt system gives satisfactory service under these conditions. Eccentric, cam-operated, over-center, or spring-loaded latches and other quick-opening latches, such as the window latch design shown in Fig. 4.13, are not recommended for clamping of high-integrity components such as HEPA filters and adsorber cells. If the designer insists on using such devices, means for adjusting the throw of the device must be provided to compensate for deviations in the depths of individual filter (adsorber) units. These devices too often fail, because they get out of adjustment, relax, or are damaged (during maintenance) during a period of service, 


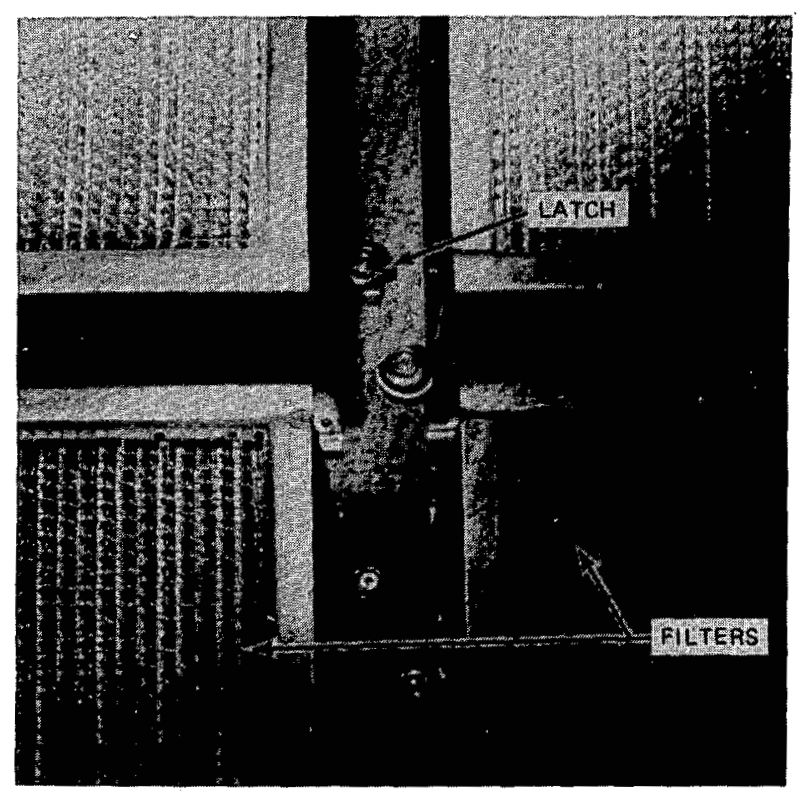

Fig. 4.13. Window latch latching devices for clamping filters. Latches bear on backs of the front flanges of the filters. This method is not reliable for installing HEPA filters.

which results in inadequate clamping pressure on the component or the need to replace the latch. In a radioactively contaminated filter system, replacement can be a hazard to personnel and to the filters and/or adsorbers installed in the system. Nut-andbolt clamping, on the other hand, entails the removal and handling of a large number of nuts; this procedure can be a problem during a filter change in a highly radioactive system.

Major requirements for filter and adsorber clamping systems are magnitude and uniformity of clamping pressure. At least four, and preferably eight, pressure points are required for HEPA filters and demisters, as shown in Figs. 4.5 and 4.9. Individual clamping of each filter unit is preferred. Common bolting in which holding clips (or bolts) bear on two or more adjacent filters or adsorber cells, as shown in Fig. 4.14, has been widely used because it is less expensive than individual clamping and requires fewer loose items to be manipulated within the confines of the housing during a filter change. However, common bolting limits the ability to adjust or replace individual filters in the bank without upsetting the seals of adjacent units. In Fig. 4.14a, for example, replacement of one of the center filters might upset the seals of eight surrounding filters. In the improved system shown in Fig. 4.14b, no clip bears on more than two filter units, and the seals of only four surrounding filters are upset when replac- ing a filter unit. The clamping system shown in Fig. 4.14b has the advantage that clips and nuts do not have to be removed to replace filters, since the clips can be rotated out of the way after the nuts have been loosened. Although this type of clamping system has been used with good success in nuclear and nonnuclear applications, many in-place test personnel object to it because of the extensive leak chasing often required before a satisfactory in-place test can be achieved. Leak chasing occurs when, on adjusting or replacing one filter, the seals of surrounding filters are upset, which results in new leaks that have to be corrected; this process is time-consuming and costly and, when conducted in a contaminated housing, can result in lengthy exposure of personnel.

Because of their weight, eight pressure points are essential for clamping type I (pleated-bed) adsorber cells. For clamping type Il (tray-type) cells, two pressure points on the top and two on the bottom edges of the front plate, with individual clamping as shown in Fig. 4.15, are needed for proper sealing. Clamping on the short sides only is not adequate. As Figs. 4.7 and 4.15 show, captive nuts reduce the number of loose items that must be manipulated within the confines of the filter housing during filter or adsorber replacement, but they must be provided with means for preventing rotation when positioned for withdrawal of the filter.

The minimum bolt size recommended for individually clamped filters is $3 / 8-16-\mathrm{UNC}$, but $1 / 2-11$ UNC or $5 / 8-11-U N C$ bolts are less prone to damage. For type I adsorbers, $5 / 8-11-U N C$ bolts are necessary. Figure 4.16 shows several methods of installing bolts to the mounting frame in which the bolts are threaded rods. Although methods $(a)$ and $(b)$ in Fig. 4.16 avoid penetration of the mounting frame (and thereby avoid potential future leaks), they present problems in alignment and location. Method $(c)$ overcomes these problems and also the problem of producing a weld bead at the base of the bolt (if too large, the weld bead would interfere with proper seating of the filter). Of the methods shown, $(b)$ is probably the least expensive and $(a)$ is the most expensive. Care must be taken with method $(c)$ to avoid pushing the base of the bolt too far through the frame, since this increases the cost of welding appreciably and makes complete seal welding difficult.

The nuts and bolts of the clamping system must be made of dissimilar materials to prevent galling and seizing. Bolting materials and clips must be resistant to corrosion. Stainless steel ( 300 series) bolts with 


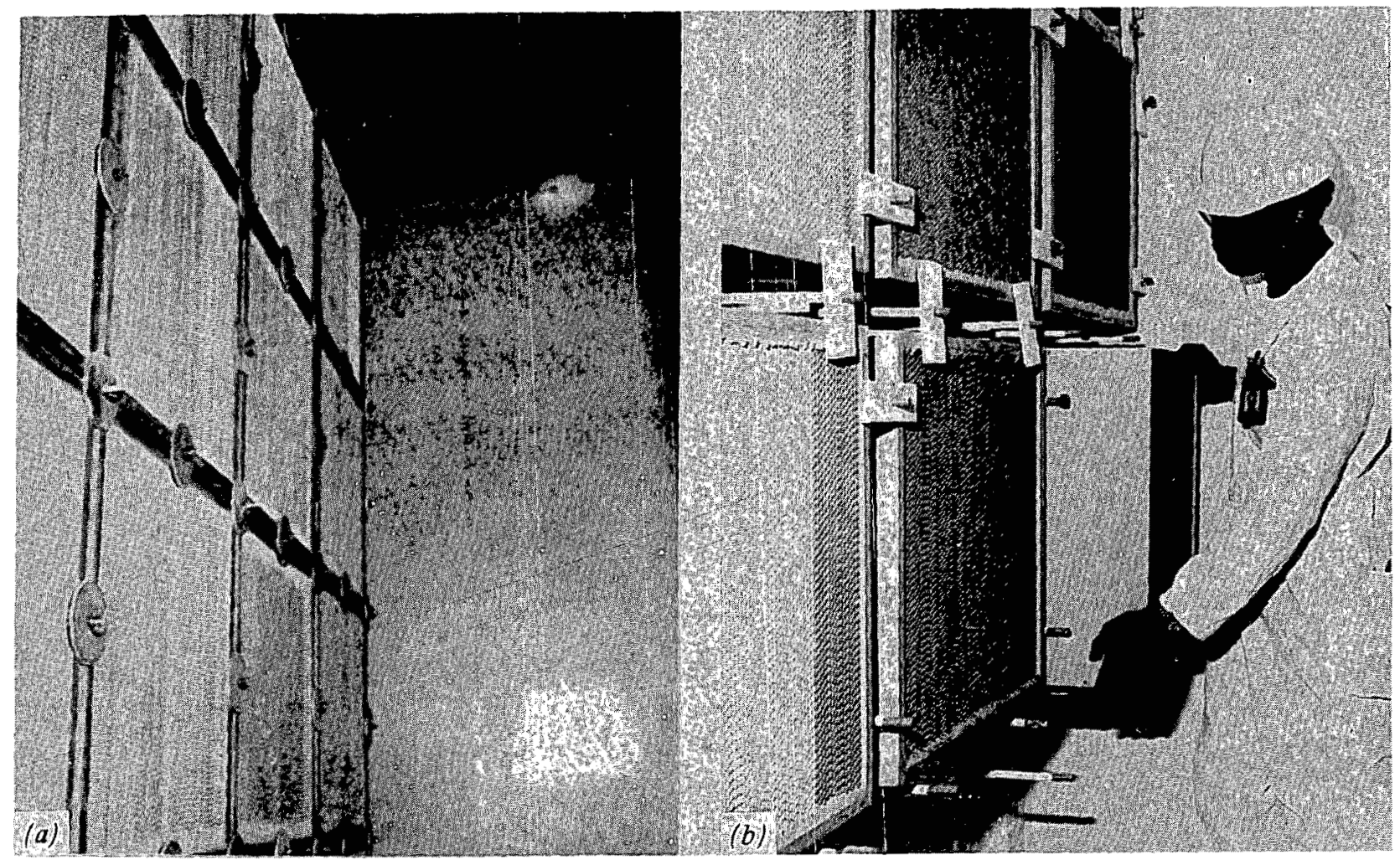

Fig. 4.14. Common filter clamping in which clamping clips bear on two or more filter units. (a) Eight-point clamping with clips installed at filter corners. Removal of a filter from this bank would disturb as many as eight surrounding filters. Clamping method is unacceptable. (b) Eight-point clamping with clips displaced from filter corners. Removal of a filter from this bank would disturb only four surrounding filters. Design is acceptable but not as good as individual filter clamping.

brass nuts are frequently used, but nuts made from a precipitation hardening $(\mathrm{PPH})$ grade of stainless steel, treated to a value substantially harder than the bolt, may be used in lieu of brass. Springs, if used, should also be made from a PPH grade of stainless steel if they are to resist corrosion and relaxation over a period of service.

Two newly developed clamping and sealing methods that deserve mention, even though they cannot be given an unqualified recommendation because of limited field experience, are both proprietary. The first, developed by American Air Filter Company, employs long levers which rotate a pair of clips that bear on the back surface of the front flange of the filter, as shown in Fig. 4.17; two of these torsion-bar assemblies are required for each filter. A possible shortcoming of the design is that the clamps bear only on the top and bottom filter flanges, and there is no pressure on the sides of the case. The manufacturer reports that the system has been employed in a number of nuclear power plant air cleaning systems with excellent results.
The second design (Fig. 4.18) encompasses a completely new type of framing and sealing. Known as Channel-Wall, ${ }^{17}$ this design employs a specialcross-section extruded-aluminum framing member (Fig. $4.18 b$ ) which presents a knife-edge sealing surface to the filter element. The filters have a channel filled with a nonflowing, nonvulcanizing silicone polymer around the sealing edge which fits into the knife edge of the mounting frame to form a positive seal between filter and frame. Rigidity of the mounting frame is not a consideration, since frame flexure cannot affect the seal or the filter. Clamping pressure need be sufficient only to hold the filter unit in place (if the filters are installed on the downstream side of the frame, clamping must be sufficient to resist displacement of the filter under normal operating filter resistance and the pressures produced by shock loadings in the system). Experience with this system has been primarily in clean room applications. Test results verify that the silicone fluid does not flow when tested under the conditions of the UL-586 hotair test (which would, of course, destroy the filter-toframe seal) and that the system can maintain sealing 


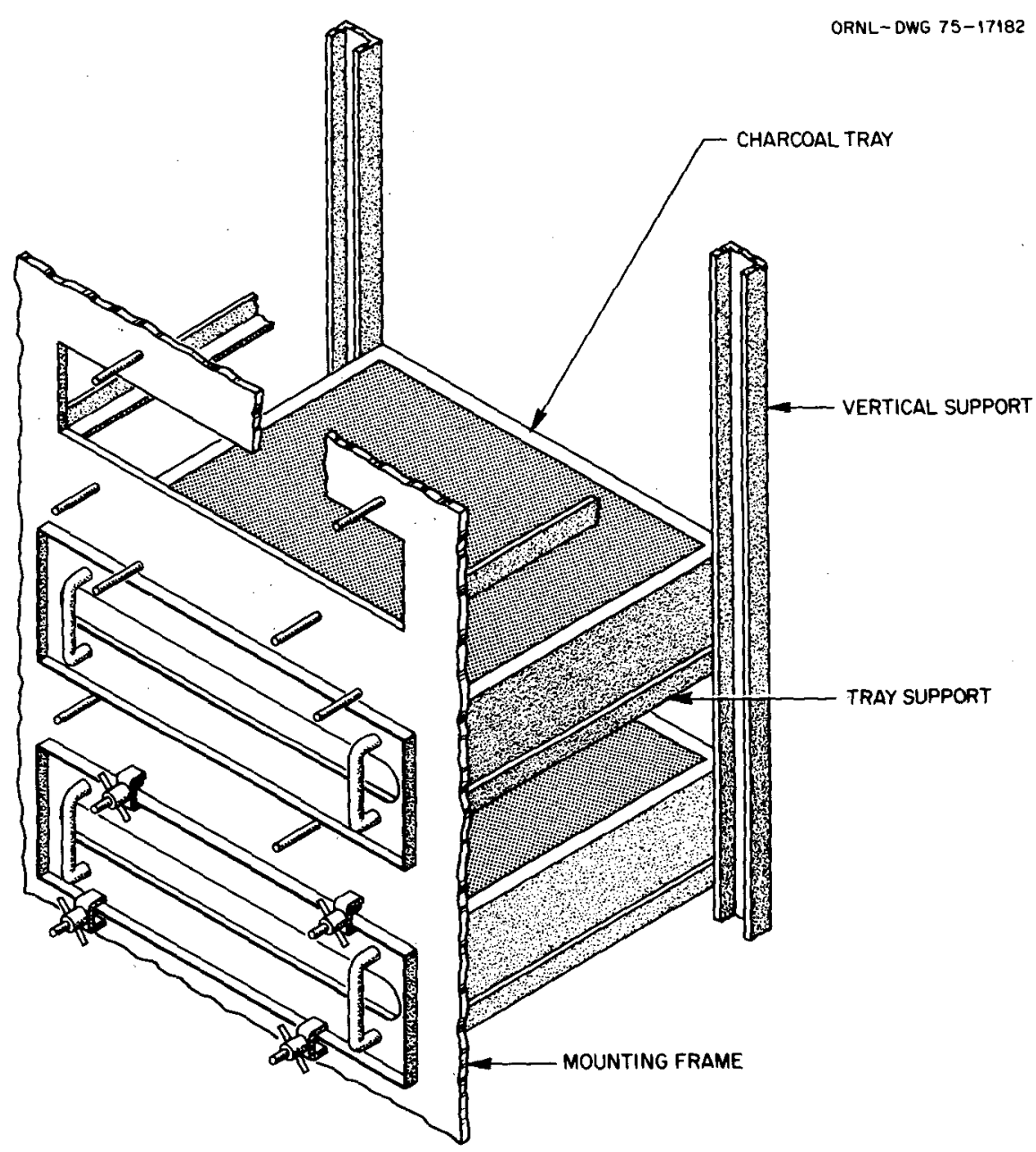

Fig. 4.15. Four-point individual clamping of type II adsorber cell. Note captive nuts to reduce number of loose parts to be handled during cell change.

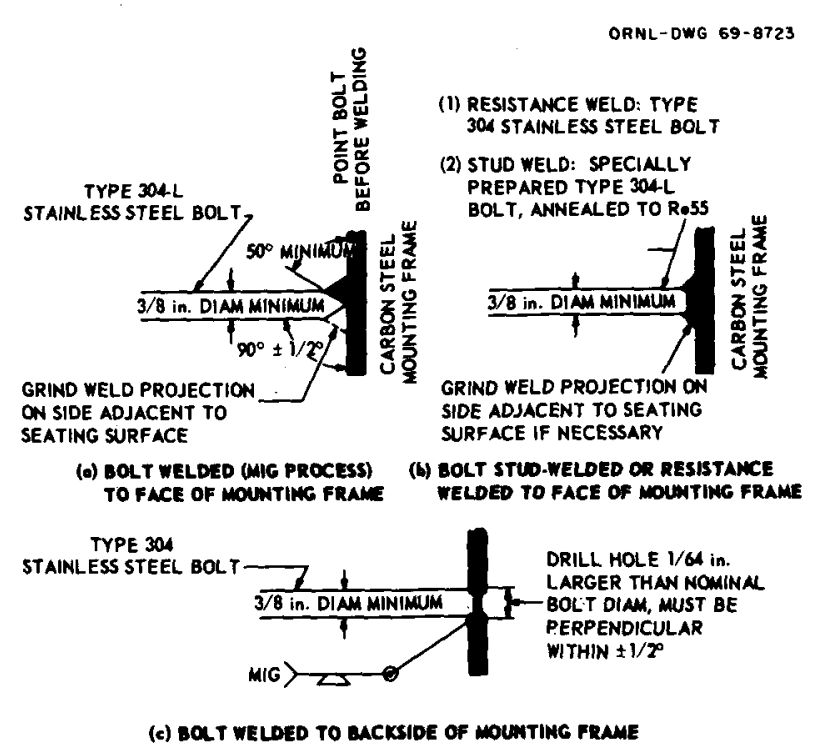

Fig. 4.16. Methods of welding clamping bolts to filter mounting frames. integrity at filter-resistance values in excess of 10 in.wg. Although high levels of radiation $\left(5\right.$ to $10 \times 10^{6}$ rads) tend to solidify the fluid, the seal is apparently not affected. It has been demonstrated that filter replacement requires substantially less time than that required for gasket-sealed systems.

\subsubsection{Filter Support}

A desirable feature from the standpoint of maintenance is a cradle or other support for the filter element as it is moved into position on the frame. The cradle should not obscure any more of the filter-toframe interface than is necessary, to avoid interference with inspection as the filter is installed. Figure 4.5 shows an acceptable cradle design. The support shown in Fig. 4.19 is better because it obscures less of the gasket-frame interface. The cradle in Fig. 4.20 is unacceptable, because it obscures too much of the interface. In some installations, filters have been 


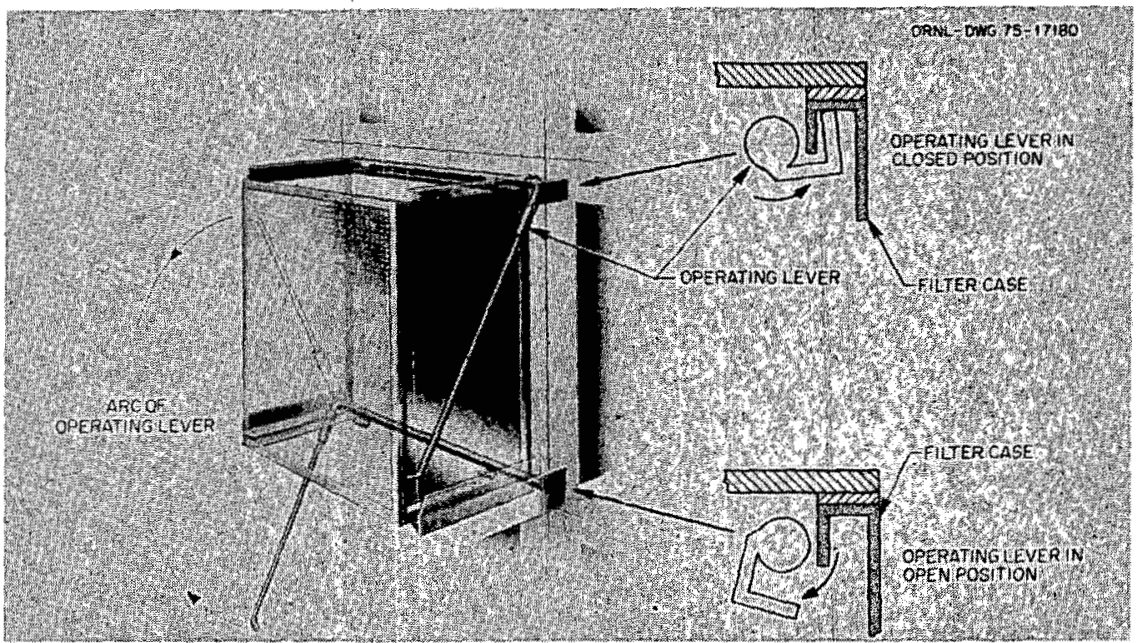

Fig. 4.17. Torsion bar clamping system. Details show end views of operating lever in closed and open positions. Note that clamping is on gasket flange of filter, and that clamping pressure is applied to only two sides of the filter. The operating levers are slightly bent when locked, which provides the spring pressure needed to compress the gasket. This method can be used only with steel-cased filters. It eliminates tools and loose items in the filter housing during a filter change and is easy and fast to operate. Courtesy American Air Filter Co.

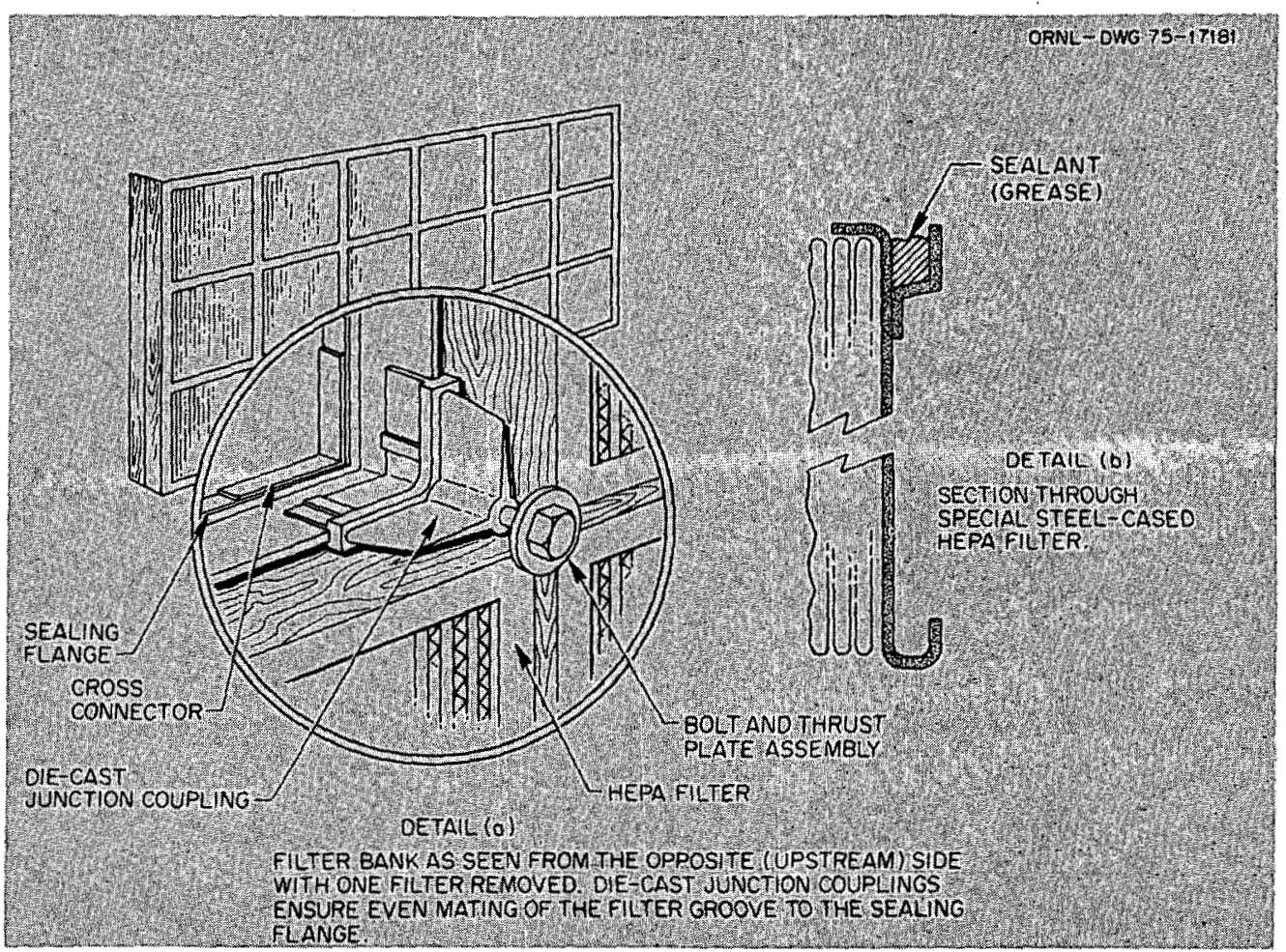

Fig. 4.18. Channel-Wall filter-sealing and mounting frame system. Design is proprietary and requires special filter units as shown in (b). Wood-cased filters have a $3 / 8$ - by ${ }^{3} / 4$-in.-deep groove routed in the sealing edge, in which the sealant is placed. (b) Section through special steelcased HEPA filter. Courtesy Flanders Filter Corp. 


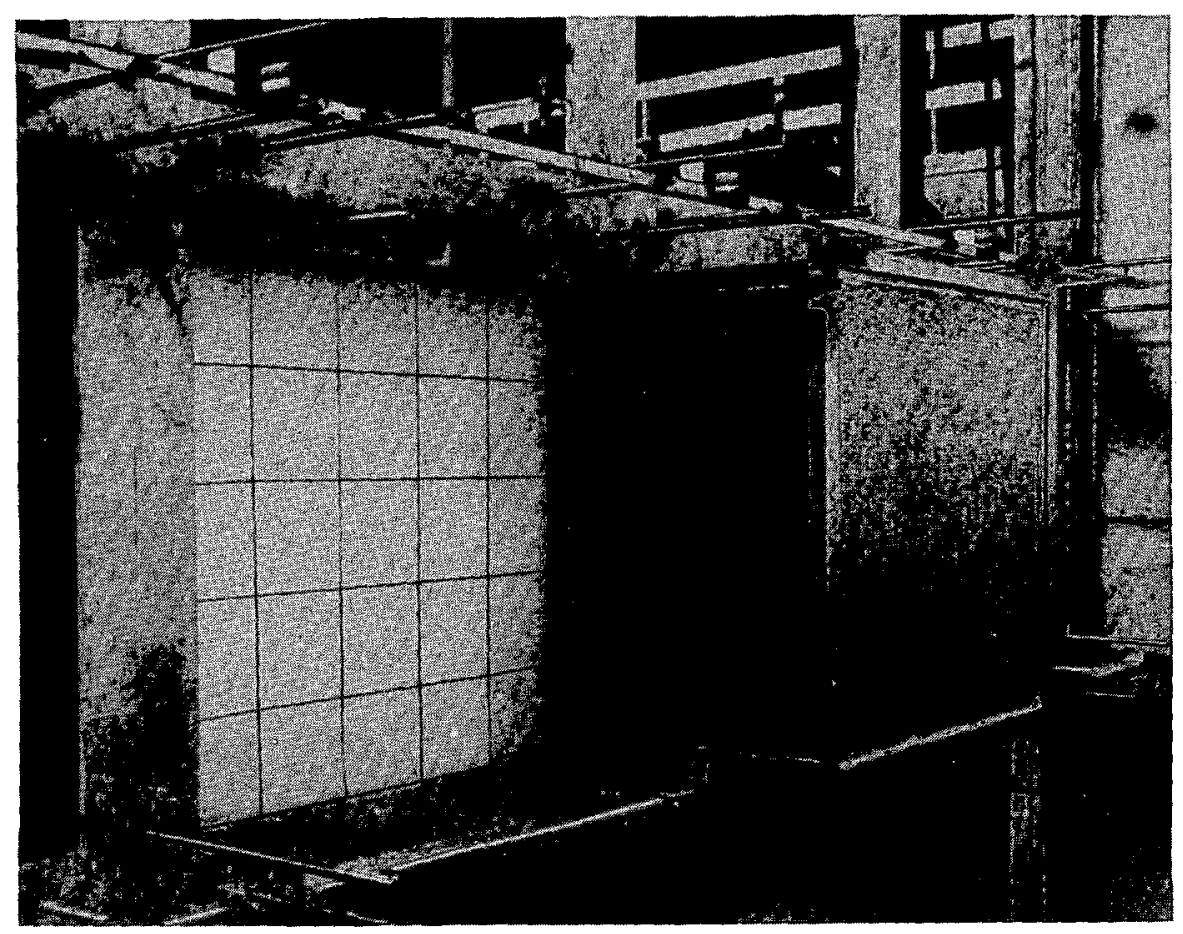

Fig. 4.19. Good filter-support-cradle design. Note all-around access to gasket-frame interface. Courtesy CTl-Nuclear, inc.

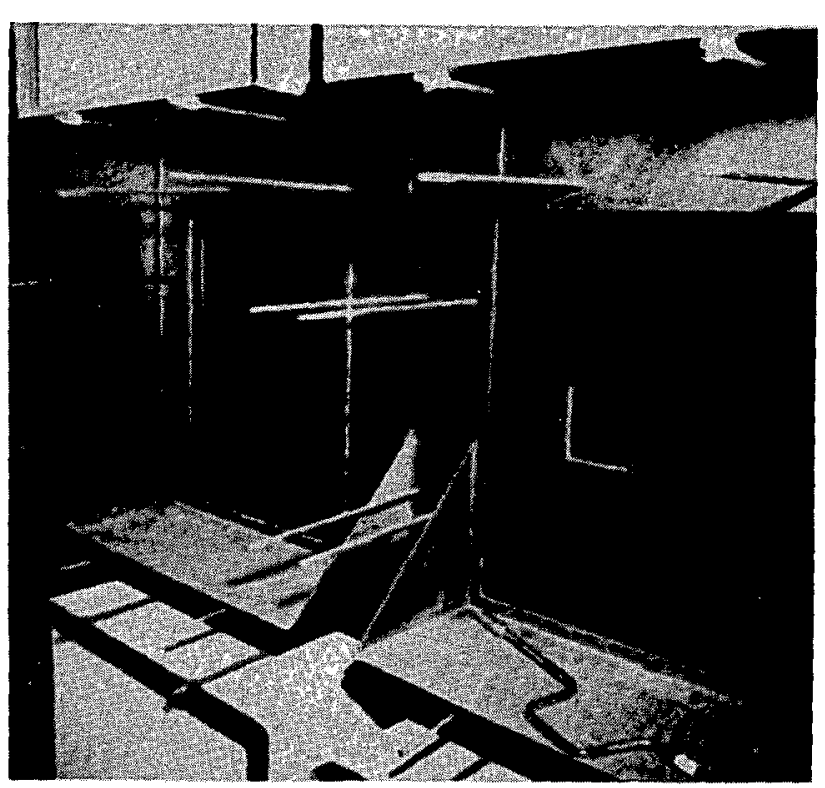

Fig. 4.20. Unacceptable filter-support cradle. Note that the cradle obscures much of the filter mounting frame interface.

supported on the bottom clamping bolts; this risks damage to the threads of the clamping bolts and is not recommended. Figure 4.21 shows a filter mounting frame in which a second frame structure, spaced a few inches ahead of the mounting frame, provides the function of a support cradle. Filter clamping bolts are attached to the front structures, which permits the use of short bolts and avoids penetration of or welding on the mounting frame itself.

\subsection{SIZE AND ARRANGEMENT OF FILTER AND ADSORBER BANKS}

The size (nominal airflow capacity) and orientation of filter banks (vertical or horizontal), the location of filters on the bank (upstream or downstream side), and the floor plan and height of the bank all affect the reliability, performance, maintainability, and testability of the air cleaning system. Savings gained by designing for minimum space and materials can be wiped out many times over by the higher operational, maintenance, and testing costs that will result from higher pressure drop and cramped working space in the filter housing.

\subsubsection{Vertical Filter Banks}

Vertical banks with horizontal airflow are preferred in contaminated exhaust systems, because the filters are more favorably oriented with respect to ease of handling, mechanical strength of the filters, and collection of condensate. In horizontal banks, filter pleats can collect moisture, which in time may 


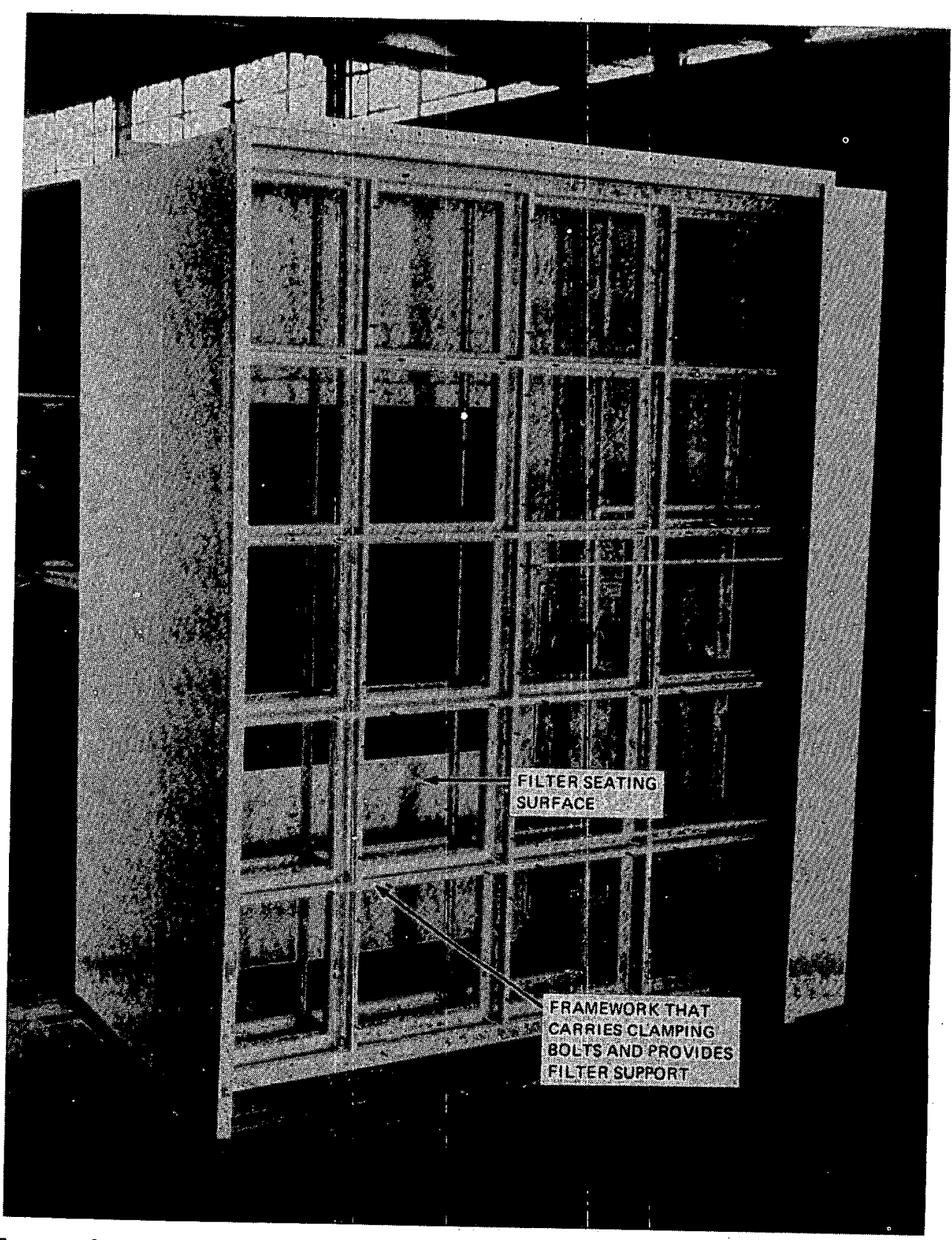

Fig. 4.21. Two-part, face-sealed HEPA-filter mounting frame. Filters seal to rear frame. Front frame provides filter support during maintenance and carries clamping bolts. This arrangement permits shorter clamping bolts (which decreases the possibility of misalignment of filters seal. The 4-wide by 5-high array is pont also prevents the bolts from being welded on or penetrating the primary frame to which the desirable. Courtesy MSA Research Corp. 
cause deterioration of the medium, separators, adhesives, and filter casings. In vertical banks, filters must be installed with vertical pleats and separators because horizontal pleats tend to sag over a period of time, as can be seen in Fig. 4.22. The pleats of type I adsorber cells and the beds of type II cells, on the other hand, must be installed horizontally to avoid settling of adsorbent in the cells.

\subsubsection{Horizontal Filter Banks}

If filter banks must be horizontal, upflow is preferred to downflow, because filter-core sagging is offset to some extent by air pressure and because there is less chance of cross-contamination from the dirty side to the clean side of the system. With downflow, contaminated dust dislodged during a filter change will fall into the clean side of the system. Also, liquid collected in filter pleats of a downflow system will eventually seep through the medium and carry dissolved contaminants into the clean side of the system. On the other hand, upflow systems may

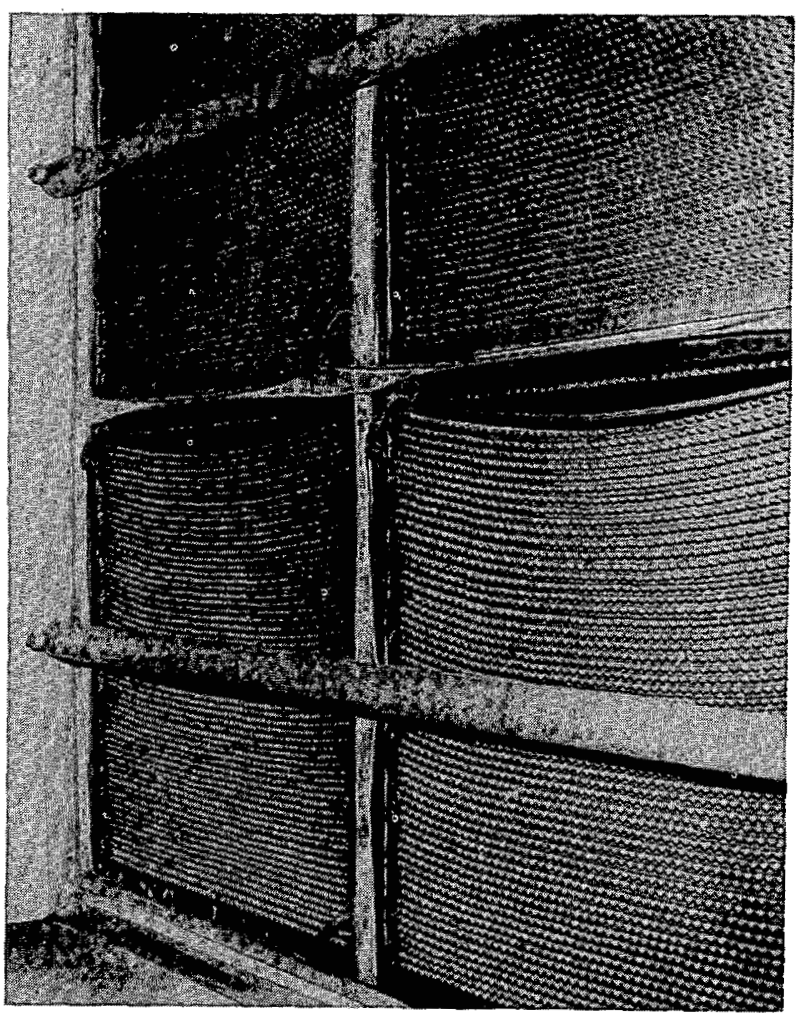

Fig. 4.22. Improperly installed HEPA filters. Horizontal pleats have sagged nearly 2 in. after six to eight months of service. Pleats should be vertical. Also note peeling duct tape used to seal bet ween filters and between filters and housing, which have been simply stacked into the duct opening. These practices are unacceptable for any type of filter installation. require withdrawal of contaminated filters into the clean zone. When horizontal installation must be used, filters should be mounted on the upper side of the mounting frame so that their weight will load rather than unload the gaskets. Replacement of filters from above is easier and less costly than replacement from below. The discussion of structural strength of filter mounting frames was based on vertical banks. The design of a horizontal mounting frame must also take into consideration the weight of the filters (Table 4.2) and the weight of the frame; in addition to air pressure loading. Otherwise, tie rods between the frame and the ceiling, or other means of supporting the frame, must be provided.

\subsubsection{Location of Filters on Mounting Frame}

No clear-cut preference can be justified for mounting filters on the upstream side or the downstream side of the mounting frame; both methods have been used successfully. ERDA usually recommends downstream mounting, whereas many ERDA contractors prefer upstream mounting. The following advantages are cited for upstream mounting of filters:

1. Filters are withdrawn into and handled within the contaminated side of the system during a filter change. No contaminated materials need be brought into the clean side of the system, so there is more complete separation of the clean and dirty sides of the system.

2. Airflow tends to load the filter gaskets during operation, so there is less likelihood of leaks.

Disadvantages of upstream mounting are (1) personnel have to work within a highly contaminated zone during a filter change; (2) there is the possibility that contamination can be tracked or carried out of the contaminated zone by workmen unless there is careful planning and execution of a filter change; and (3) filter clamping devices are located in the dirty side of the system where they are most exposed to corrosion and dirt.

The following advantages are cited for downstream mounting of filters:

1. Filters are withdrawn into and handled within the clean side of the system; therefore, there is less likelihood of tracking or carrying contamination into the building during a filter change.

2. Personnel are not required to work in a highly contaminated portion of the housing during a filter change. 
3. Filter clamping devices are located on the clean side of the system and are therefore less subject to corrosion.

4. Leak probing of installed filters is more sensitive. If there are gasket or casing leaks, the driving force of air entering the filter forces the test aerosol through the leak, and it is readily detected. With upstream mounting, on the other hand, any test aerosol that goes through a leak in a gasket or filter case mixes with the air and test aerosol passing through the opening in the mounting frame, thus obscuring the leaks. Although the existence of a leak may be disclosed by a test, the location of the leak cannot be easily determined by probing.

5. Only the upstream face of the filter is contaminated during operation; the outer surfaces of the filter case and the downstream face of the filter pack are not usually contaminated.

The disadvantages of downstream mounting are (1) filter gaskets tend to be unloaded by air pressure during operation, thus increasing the likelihood of gasket blowby; and (2) the contaminated filters must be withdrawn into the clean side of the system in a filter change. The second disadvantage can be offset by "fixing" the contaminated dust by spraying the upstream side of the filter pack with paint or acrylic spray or by taping cardboard over the upstream face of the filter; however, this procedure would require personnel to enter the contaminated chamber of the housing, and the possibility still exists of dislodging contaminated dust into the clean side of the system, either from the filter itself or from the edges of the frame opening (which is exposed to contaminated air during operation).

Filters have been mounted on both sides of a mounting frame in some installations when two-stage filtration was specified. Although this dual mounting saves space and prevents carry-over of contamination to the clean side of the system when the upstream filter is replaced, it makes reliable in-place testing of filters impossible. When new filters are installed, the first set that is installed can be tested, and then the two sets together can be tested as a single bank. However, the first set will obscure the deficiencies of the second set during the second test. In addition, the set of filters installed first, and found satisfactory by test, could be damaged during installation of the second set, and the damage could escape discovery. Double mounting of filters has the additional disadvantage that fire in the upstream filter will readily jump to the second set, so that any advantage of double filtration during the fire would be lost. Therefore, double mounting is not recommended and is prohibited by ERDA where it has the authority to control design.

Similar problems exist when prefilters or adsorber cells are installed back-to-back with HEPA filters (Fig. 4.23), since probing for leaks between components is impossible and there is risk of damage to the fragile and critical HEPA filter. With back-toback installation of HEPA filters, the reliability of the individual filters is unknown. A cardinal rule in contaminated exhaust systems is that no credit is granted for untested and untestable filters. Therefore, although two sets of filters may be provided by double mounting, the operator cannot take credit for two-stage filtration or series redundancy. A third

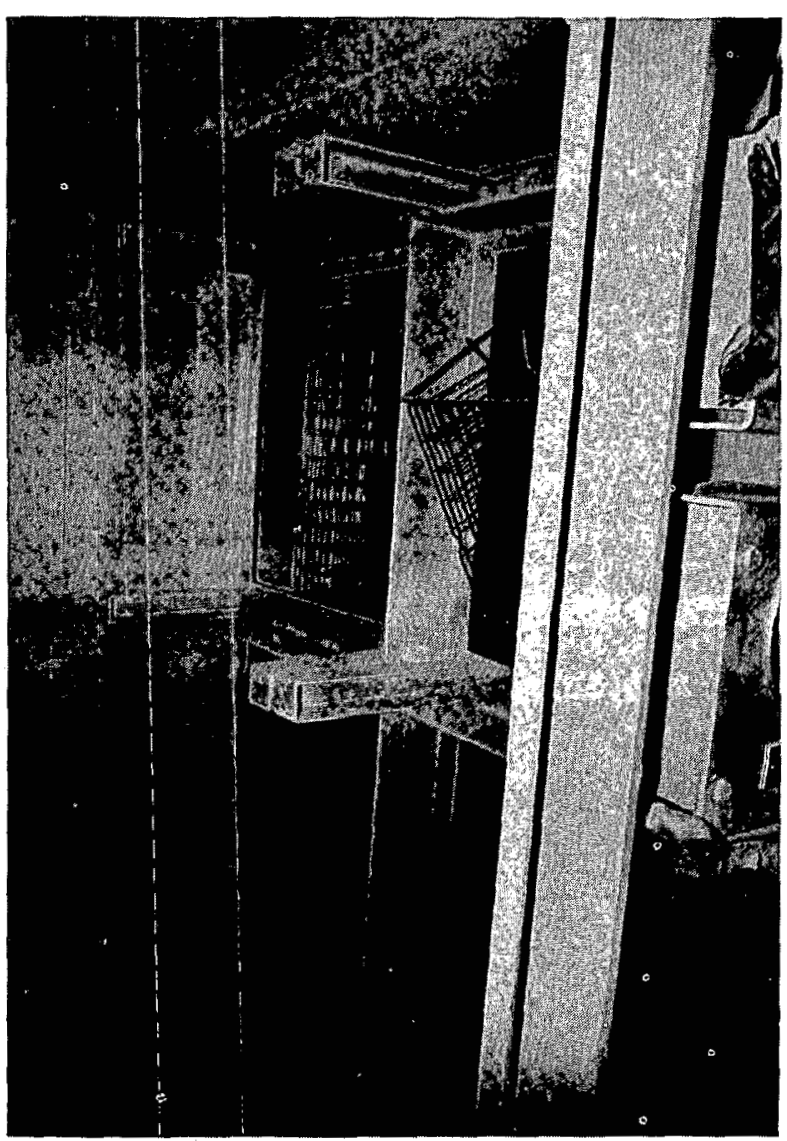

Fig. 4.23. Back-to-back installation of prefilters and HEPA filters prevents access to upstream face of HEPAs. Note potential for damage to HEPA filters when installing prefilters. Inspection of upstream face of HEPA filters is impossible unless prefilters are removed. Back-to-back installation of prefilters to HEPA filters, HEPA filters to HEPA filters, or HEPA filters to adsorbers is not recommended. 
bank of filters in series with the double bank would be necessary by this rule, and, in fact, this type of wasteful multiplication of filter banks has occurred in some systems.

\subsubsection{Size of Banks}

A nominal limit of $30,000 \mathrm{cfm}$ is recommended by ERDA and NRC for any single filter or adsorber bank. For larger systems, this limit requires that the system be segmented into two or more.smaller subsystems, each contained in an individual housing and having an installed capacity of $30,000 \mathrm{cfm}$ or less. The purpose of this requirement is to facilitate maintenance and in-place testing, to improve control in the event of a system upset, and to enhance reliability of the total system. A $30,000-\mathrm{cfm}$ bank is about the largest that can be in-place tested conveniently (Chap. 8). In addition, by having the system broken into two or more air cleaning units, testing and filter replacement can be conducted in one unit while the other unit remains on-line. NRC Regulatory Guide 1.52 recommends such redundancy for ESF air cleaning systems in reactors. ${ }^{18}$ The designer may also choose to segment a system into units of substantially less than $30,000 \mathrm{cfm}$ when redundancy is desired to achieve advantages of control, maintainability, and testability.

\subsubsection{Arrangement of Banks}

Spatial arrangement of filters on a mounting frame influences operating performance and maintenance. If one were to specify twelve 1000 -cfm filters $(24 \times 24$ $\times 11^{1 / 2}$ in.) arranged in a 6-wide by 2-high array, it would create a difficult installation and maintenance situation, because personnel would be forced to crawl or work stooped over in the filter house. On the other hand, arranging the same bank in a 2-wide by 6-high array would make it impossible for one to reach the upper filters without bringing ladders or temporary scaffolding into the housing (a major source of filter damage) or providing a permanently installed work gallery. If the filters were arranged 3 wide by 4 high, there would still be the problem of access to the top tier of filters. The best solution is to arrange the filters in a 4-wide by 3-high array. For similar reasons, the best arrangement for a 6000 -cfm system would be a 2wide by 3-high array.

Insofar as possible, banks should be laid out in an array of three filters high or nine type II adsorber cells high. Where floor space is at a premium, the bank may be arranged with one 3-high array above another, with a service gallery between, as shown in Fig. 4.11. Thus an 18,000-cfm bank might be arranged in an array 6 wide by 3 high or 3 wide by 6 high, with a service gallery between the third and fourth tiers. The arrangement of a 24,000 -cfm bank in a 6-wide by 4-high array would be undesirable. A better arrangement is an array 8 wide by 3 high, or, if the floor space is at a premium, two 4-wide by 3-high arrays, one above the other, separated by a service gallery. In no case should filter changing require the use of ladders or temporary scaffolding. To require a workman dressed in bulky protective clothing (with sight obscured by a respirator or gas mask and sense of feel dulled by double gloves) to manipulate a ladder or scaffold within the confines of a filter house is an open invitation to filter damage and personnel injury. Based on the 95th-percentile man, ${ }^{19}$ the maximum height at which a man can operate hand tools effectively is $78 \mathrm{in}$., and the maximum load he can handle at a height of $5 \mathrm{ft}$ or more is $40 \mathrm{lb}^{20}$ Therefore, provision for access to the higher tiers of filters is necessary. At costs of $\$ 80$ to $\$ 120$ per filter unit, any savings realized in first costs by not providing a permanent service gallery could be offset in only one or two filter changes by the cost of damaged filters alone. Service galleries in high banks reduce the costs of preparing for and cleaning up after a filter change. The pay-out period for a gallery in a 7wide by 6-high filter bank was estimated to be about two years, based on labor savings only and taking no credit for prevention of potential filter damage. ${ }^{21}$

Filter banks should be rectangular. The use of oddshaped banks, such as the one shown on the left in Fig. 4.24, in order to limit installed filter capacity to calculated system airflow requirements, increases construction costs significantly. By filling out the rectangle, as shown at the right of Fig. 4.24, construction costs will be less. In addition, if all nine spaces are filled with filters, operating costs may also be reduced, because the additional filter would permit operation at a lower flow rate per unit, with attendant longer filter life and reduced filter-change frequency, as discussed in Chap. 2. For the purposes of laying out adsorber banks, three type II (tray) adsorbers will fit vertically into the space occupied by one 24- by 24-in. HEPA filter.

\subsubsection{Floor Plan of Filter Banks}

Vertical banks may be arranged in a plane or in a $U$-shaped or stepped pattern to permit more filter units to be installed in a given housing width. Although there is no appreciable saving in floor space

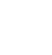

.

(1)

(1)

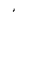


with U-shaped or stepped banks (Fig. 4.25), such arrangements may lend themselves to more favorable layout of nearby equipment. The use of a U-shaped or stepped bank in large systems may also have the advantage of improving inlet conditions to the fan or reducing the size and cost of duct transitions to the housing. Judicious layout of a bank can often reduce pressure losses in the system and bring about more uniform dust loading of filters, the reby equalizing the utilization of filters installed in the bank. If the open side of a U-arrangement is centered on the fan inlet, for example, the distances from the filters to the fan are more or less equalized, and the bank may, in effect, form an inlet box which enhances fan-inlet conditions and produces more uniform pressure drop across, and loading of, the filters. On the other hand, straight (plane) banks are safer from the standpoint

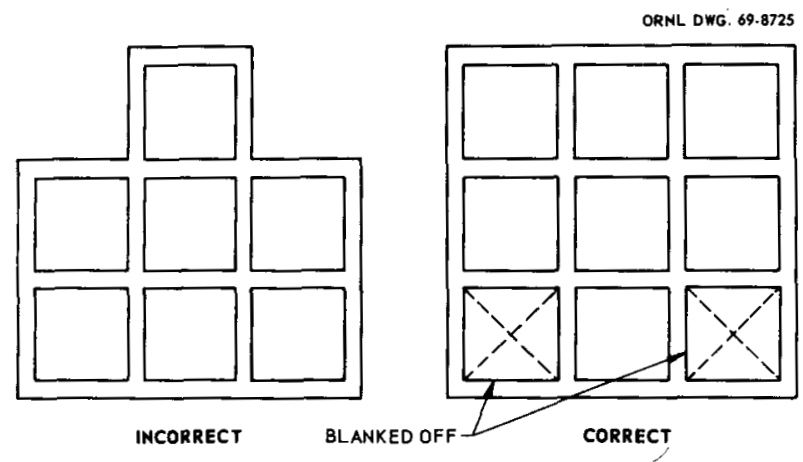

Fig. 4.24. Filter bank layout. Layout at left is much more expensive. If necessary to match installed filter capacity to calculated airflow requirements, fill out the rectangle and blank off some of the openings, as shown at right.

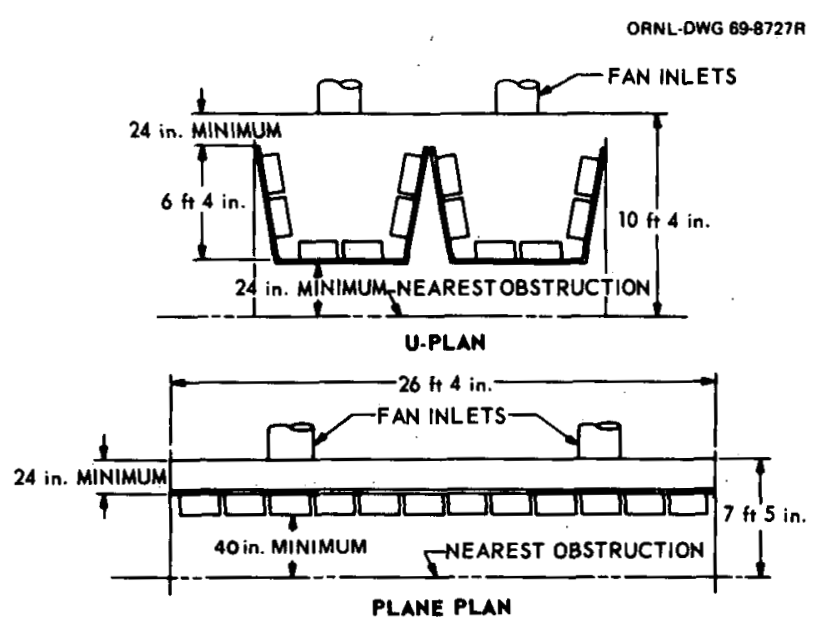

Fig. 4.25. Filter bank floor plans showing minimum clearances and floor space required. of fire-spread than U-shaped or stepped
arrangements. $^{22}$

The procedures that will be required for construction and operational maintenance must be considered in planning. Adequate clearances for access must be maintained at turning points and between the bank and the nearest obstruction. Passageways between banks and between banks and the housing wall must be wide enough for welders to operate effectively and for workmen, dressed in bulky clothing, to get in to change filters; both welders and workmen will have to kneel or stoop to get to the bottom tier. A 95th-percentile man, in a kneeling position, requires a minimum clearance of $36 \mathrm{in}$. from the face of the filters to the nearest obstruction, not including withdrawal space for the filter unit itself. A minimum clearance of $40 \mathrm{in}$. is therefore recommended between the face of one bank and the nearest obstruction. The principal dimensions for U-shaped arrangements are shown in Fig. 4.26. A maximum angle of 2 in 12 is recommended. Stepped banks are usually laid out at right angles.

\subsection{HOUSINGS}

\subsubsection{General}

Large man-entry housings may be steel, concrete, or masonry; and they may be shop-built or fieldfabricated. The trend, particularly in ESF systems, is increasingly toward shop-built steel housings. Carbon steel is the most common material of construction; however, stainless steel may be used when there is a potential for corrosion. Aluminum is not suitable in most cases because of the difficulty in obtaining reliable welds and the severe surface pitting that often occurs under service conditions. Aluminum is never used in reactor containments that employ caustic sprays. Galvanized steel may be used, but particular care must be paid to thorough removal of the coating prior to welding and to thorough cleaning and recoating of the weld area after welds are completed. Galvanized steel is not likely to give adequate corrosion protection in many continuously on-line filter systems. When ionizing radiation is present, concrete or steel-lined concrete housings or filter pits are necessary.

\subsubsection{Arrangement and Location}

Maintainability is a major consideration when laying out large filter housings. Although some systems may have only a single bank of HEPA filters, 

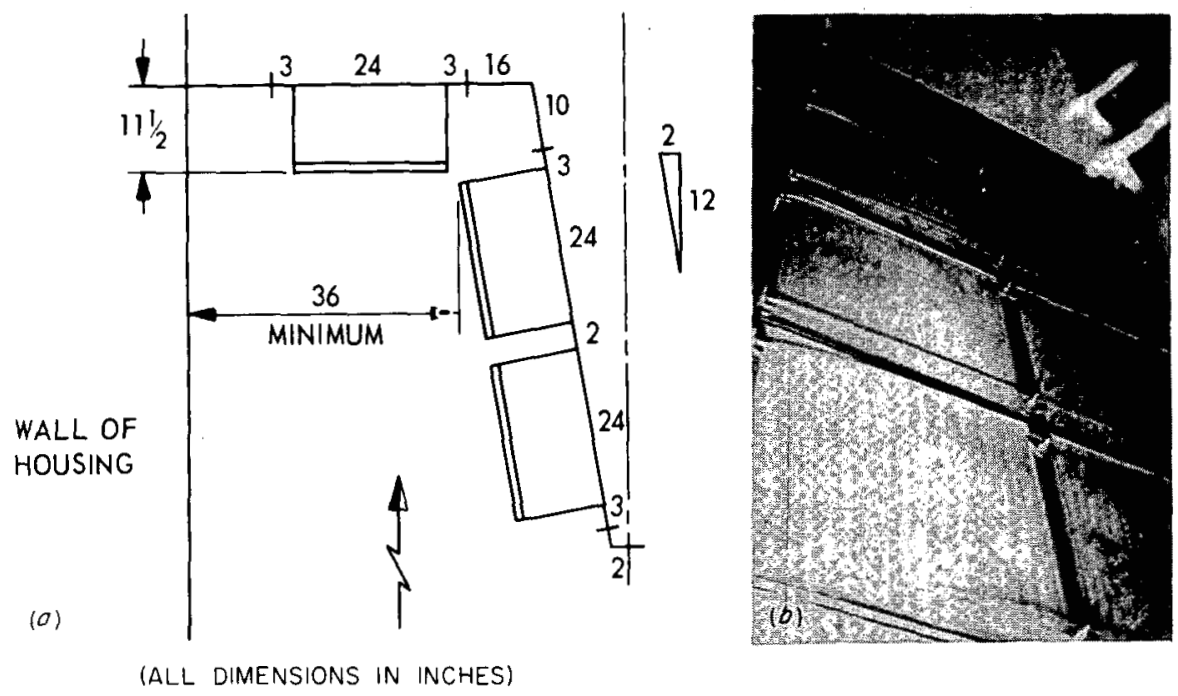

Fig. 4.26. Layout of U-and V-banks. (a) Recommended dimensions for laying out a U-shaped or V-shaped filter bank. (b) Poorly laid out $\mathrm{V}$-bank. Note inadequate working space at apex of bank, 5-high filter layout with no service gallery, and minimal spacing of filters on the mounting frame.

most will have at least an additional bank of prefilters, and many will have multiple banks of HEPA filters. Those systems in which radioiodine releases must be controlled will also require one or more banks of adsorbers. Often a bank of demisters is required, with the result that there could be as many as six or more banks of components in a single housing. There must be sufficient clear corridor space adjacent to the housing for handling filters during a filter change and also adequate corridors to and from the housing. Dollies are often used to transport filters to and from the housing area. This practice makes for safer operations from the stand point of both injury to personnel and contamination spread from dropped filters. When dollies are used, space must be allowed to get the dollies in and out and for loading and unloading. Additional space is desirable for stacking new filters (in their cartons) adjacent to the work area. Recommended clearances for housings and adjacent aisles or air locks are given in Fig. 4.27.

A factor sometimes overlooked is proper access to the filter housing. Too frequently, housings have been situated among machinery and equipment where workmen are required to climb between, over, or under obstructions to get to the door of the housing and then have inadequate work space. In some installations it has been necessary to carry filters, one at a time, over a roof and then rely on rope slings to transfer them from or to a waiting truck. It is essential to preplan the route for getting filters and adsorbers to and from the housing and to provide elevators or cranes when they have to be hoisted to an upper level.

High-risk operations often require segmented systems having two or more housings ducted in parallel that exhaust from the same area and vent to the same stack. Each housing must have inlet and outlet isolation dampers to permit one to be held in standby or, when both are normally operated simultaneously, to allow one housing to be shut down for maintenance or during an emergency. When highactivity alpha emitters such as plutonium or transuranic elements are handled, it may also be desirable to compartment the system in series, with separate housings for prefilters and HEPA filters, as well as in parallel for extra safety (Fig. 2.12).

Another important consideration in housing layout is the uniformity of airflow through the installed components. This is especially important for adsorbers, since flow through those components must be no greater than $120 \%$ of the specified rate ( $333 \mathrm{cfm}$ per cell for type II adsorbers) to achieve the gas residence time required for efficient adsorption of radioactive organic iodine compounds. Figure 4.28 shows a good housing transition in a standby gas treatment system for a nuclear reactor; such long transitions are difficult, particularly in large housings. Nevertheless, every effort should be made to locate and design inlets and outlets to avoid 

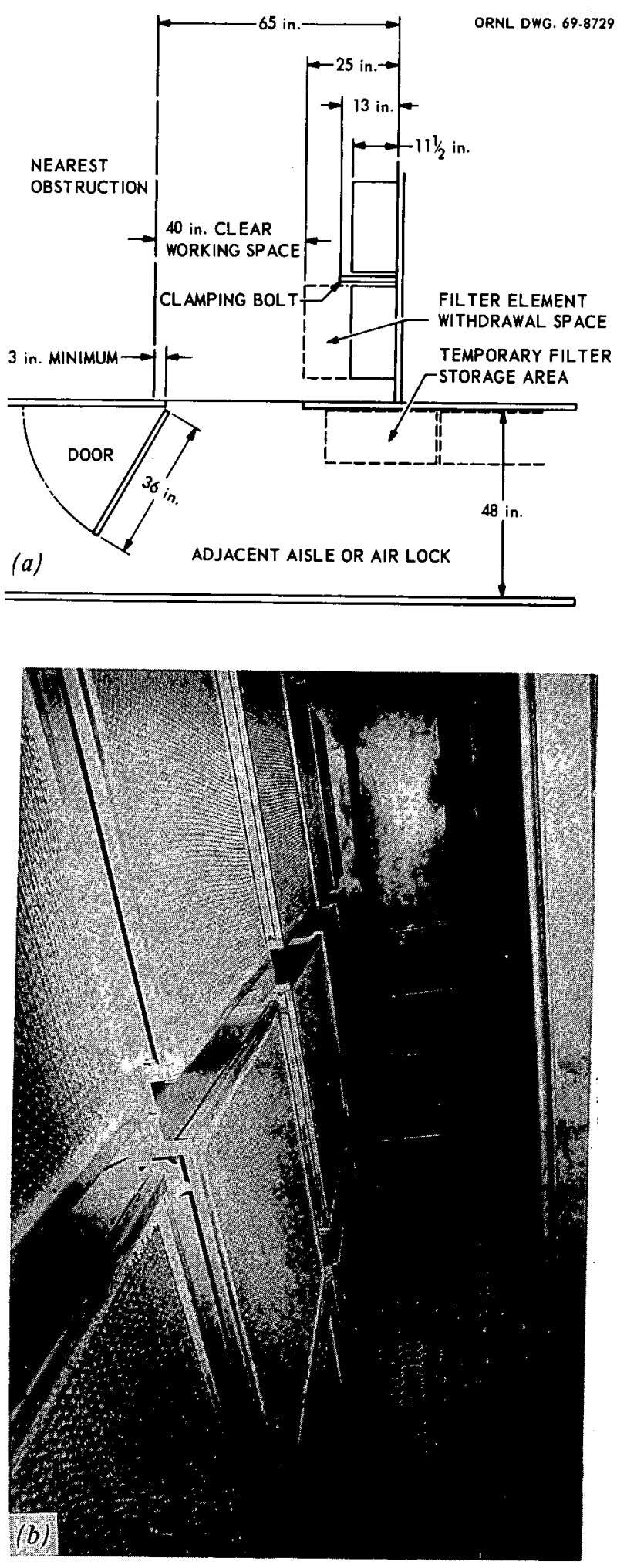

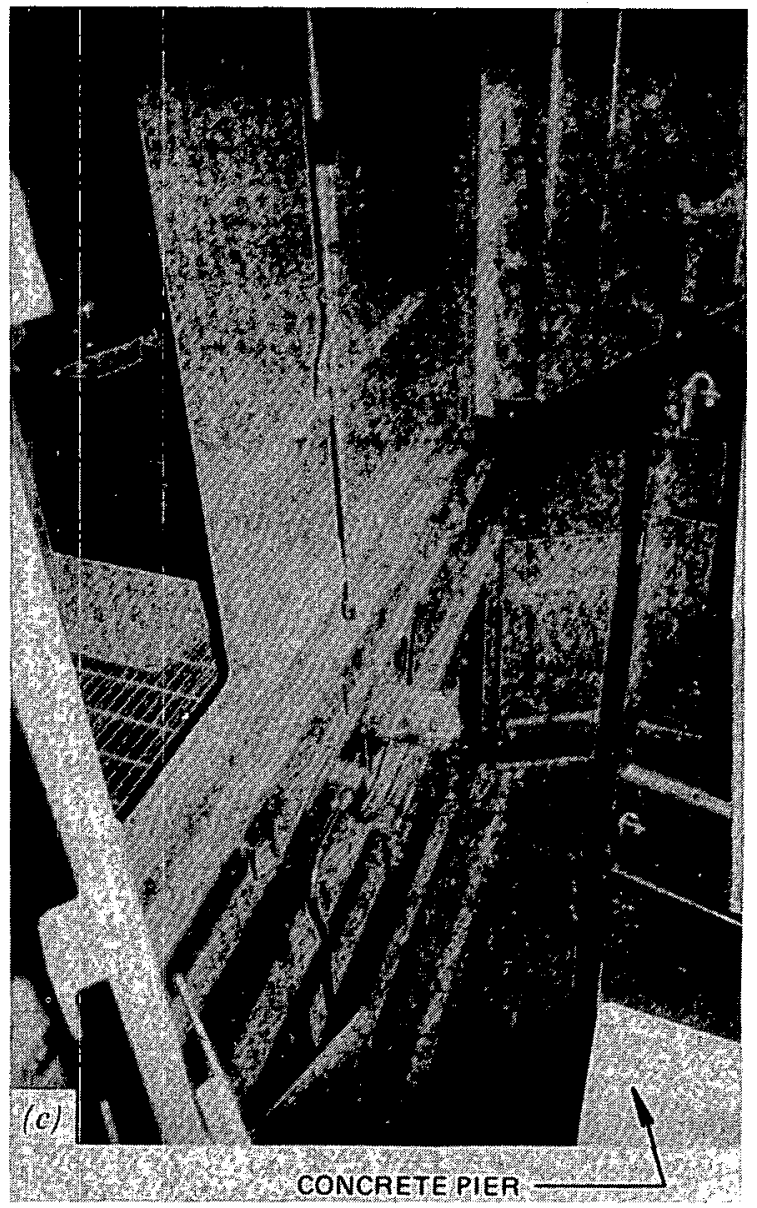

Fig. 4.27. Layout of housings and aisles. (a) Recommended space and clearances in and adjacent to the filter housing. Note that clear working space does not include component withdrawal space. (b) Inadequate space within a filter housing. Note safety hazard on floor of service gallery. (c) Inadequate space adjacent to filter housing. Note concrete pier at lower right corner, which restricts door swing, and lack of gallery at second floor level. 


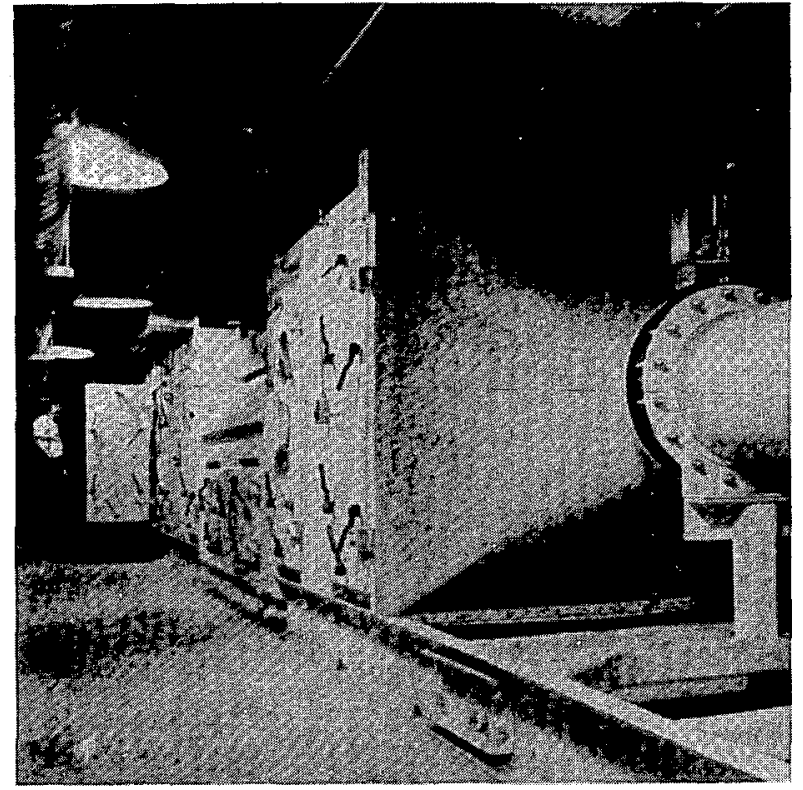

Fig. 4.28. Shop-built filter housing for nuclear reactor standby gas treatment system. Note long transition from duct to housing, which promotes uniformity of airflow through installed components. Courtesy CVI Corp.

stratification and to enhance the uniformity of airflow through components.

\subsubsection{Steel Housings}

Design practices used for conventional air conditioning and ventilation system ductwork and equipment casings are not adequate for highreliability, high-efficiency contaminated-exhaust and air cleanup systems. Experience has shown that, under system upset and shutdown conditions, housing leaks can result in the escape of contamination to clean areas. Even with fans operating, reverse leakage of particles from the low-pressure side of a system (i.e., the interior of the housing or duct) to the highpressure side (i.e., occupied area of the building) can sometimes occur because of dynamic and aspiration effects. Outleakage may also occur when the system is shut down. Filter housings for contaminated exhaust service must be able to withstand negative pressures, without damage or permanent deformation, at least up to fan cutoff, which may be $\geqslant 20$ in.wg in many systems. A pressure differential of 2 in.wg between the inside and outside of a housing prođuces a load of more than $1000 \mathrm{lb}$ over every $10 \mathrm{ft}^{2}$ of the housing wall. If the filters are operated to economical pressure drops, the housing may have to withstand ten or more times this load without appreciable deflection. Pulsation and vibration may aggravate the condition. In addition, the housing should be able to withstand design shock loads without damage.

The references cited in Sect. 4.3 for the design, fabrication, and welding of mounting frames are also applicable to steel housings. Housings should be of all-welded construction, with bolted flange or welded inlet and outlet connections to the ducts and fans. Table 4.3 gives minimum sheet-metal thicknesses for sheet steel housings, and Table 4.4 gives minimum moments of inertia for steel reinforcing members. Sheet-metal thicknesses in Table 4.3 are based on a maximum deflection of $1 / 4 \mathrm{in}$./ lin $\mathrm{ft}$ at a pressure differential between the interior of the housing and atmosphere equivalent to 1.5 times the maximum pressure at fan cutoff. The moments of inertia for reinforcing members listed in Table 4.4 were selected so as not to exceed the allowable stress of the steel. Members up to 20 in. long were considered to be uniformly loaded beams with fixed ends, whereas members longer than $20 \mathrm{in}$. were considered to be uniformly loaded beams with simply supported ends. Sheet-metal thicknesses in Table 4.3 are given in U.S. gage numbers for sheet and fractional inches for plate.

Table 4.3. Minimum sheet-metal thicknesses ${ }^{a}$ for welded steel ${ }^{b}$ filter housings under negative pressure

\begin{tabular}{|c|c|c|c|c|c|c|c|}
\hline \multicolumn{2}{|c|}{$\begin{array}{c}\text { Dimensions of } \\
\text { largest } \\
\text { unsupported } \\
\text { panel (in.) }\end{array}$} & \multicolumn{6}{|c|}{$\begin{array}{l}\text { Thickness (U.S. gage for sheet, fractional inches for plate } \\
\text { for negative pressure (relative to outside) of - }\end{array}$} \\
\hline & & \multirow{2}{*}{4 in. $w g$} & \multirow{2}{*}{8 in.wg } & \multirow{2}{*}{12 in.wg } & \multirow{2}{*}{20 in.wg } & \multirow{2}{*}{1 psi } & \multirow[t]{2}{*}{2 psi } \\
\hline $\begin{array}{l}\text { Long } \\
\text { side }^{d}\end{array}$ & $\begin{array}{c}\text { Short } \\
\text { side }\end{array}$ & & & & & & \\
\hline \multirow[t]{4}{*}{$54(2)$} & 12 & 18 & 18 & 14 & 16 & 14 & 11 \\
\hline & 24 & 18 & 14 & 11 & 12 & 8 & $1 / 4$ \\
\hline & 36 & 16 & 12 & $x$ & 11 & $1 / 4$ & $3 / 8$ \\
\hline & 48 & 14 & 12 & 6 & $x$ & $1 / 4$ & $3 / 8$ \\
\hline \multirow[t]{4}{*}{$80(3)$} & 12 & 18 & 16 & 14 & 16 & 14 & 11 \\
\hline & 24 & 18 & 14 & 11 & 12 & 8 & $1 / 4$ \\
\hline & 36 & 16 & 12 & 6 & 11 & $1 / 4$ & $3 / 8$ \\
\hline & 48 & 14 & 12 & 6 & 8 & $1 / 4$ & $3 / 4$ \\
\hline \multirow[t]{4}{*}{$106(4)$} & 12 & 18 & 16 & 16 & 14 & 14 & 11 \\
\hline & 24 & 18 & 14 & 12 & 11 & 8 & $1 / 4$ \\
\hline & 36 & 16 & 12 & 8 & 6 & $1 / 4$ & $3 / x$ \\
\hline & 48 & 16 & 10 & 6 & $1 / 4$ & $3 / k$ & \\
\hline
\end{tabular}

"Based on flat plate, edges held but not fixed (F. J. Roark, Formulas for Stress and Strain, 4th ed., McGraw-Hill. New York, 1965), and maximum deflection of $0.25 \mathrm{in}$. $\mathrm{ft}$ between reinforcements.

' 30,000 to 38,000 psi yield strength.

Metal thicknesses less than No. 18 U.S. gage are not recommended because of welding problems.

${ }^{d}$ Length based on 2 -in. spacing between 24 - by 24 -in. filter units; the numbers within parentheses denote number of filter units. The metal thicknesses are adequate for panel lengths within \pm 10 in. of the length shown. 
Table 4.4. Recommended minimum moments of inertia for selecting reinforcing members for steel filter housings under negative pressure ${ }^{a, b}$

\begin{tabular}{|c|c|c|c|c|c|c|c|}
\hline \multicolumn{2}{|c|}{ Reinforcement } & \multicolumn{6}{|c|}{$\begin{array}{l}\left.\text { Moment of inertia (in. }{ }^{4}\right)^{d} \text { for } \\
\text { negative pressure (relative to outside) of - }\end{array}$} \\
\hline $\begin{array}{l}\text { Length }^{c} \\
\text { (in.) }\end{array}$ & $\begin{array}{l}\text { Spacing } \\
\text { (in.) }\end{array}$ & 4 in.wg & 8 in.wg & 12 in.wg & 20 in.wg & $1 \mathrm{psi}$ & $2 \mathrm{psi}$ \\
\hline \multirow[t]{4}{*}{$54(2)$} & 12 & 0.04 & 0.04 & 0.04 & 0.04 & 0.04 & 0.08 \\
\hline & 24 & 0.04 & 0.04 & 0.04 & 0.06 & 0.08 & 0.16 \\
\hline & 36 & 0.04 & 0.04 & 0.05 & 0.09 & 0.12 & 0.24 \\
\hline & 48 & 0.04 & 0.05 & 0.07 & 0.12 & 0.16 & 0.32 \\
\hline \multirow[t]{4}{*}{$80(3)$} & 12 & 0.04 & 0.04 & 0.05 & 0.08 & 0.11 & 0.21 \\
\hline & 24 & 0.04 & 0.06 & 0.09 & 0.16 & 0.21 & 0.43 \\
\hline & 36 & 0.05 & 0.10 & 0.14 & 0.24 & 0.32 & 0.63 \\
\hline & 48 & 0.06 & 0.13 & 0.19 & 0.32 & 0.42 & 0.86 \\
\hline \multirow[t]{4}{*}{$106(4)$} & 12 & 0.04 & 0.09 & 0.13 & 0.22 & 0.30 & 0.60 \\
\hline & 24 & 0.09 & 0.18 & 0.26 & 0.44 & 0.60 & 1.19 \\
\hline & 36 & 0.13 & 0.27 & 0.39 & 0.66 & 0.90 & 1.79 \\
\hline & 48 & 0.18 & 0.36 & 0.52 & 0.88 & 1.19 & 2.38 \\
\hline \multirow[t]{4}{*}{$132(5)$} & 12 & 0.09 & 0.17 & 0.26 & 0.51 & 0.69 & 1.39 \\
\hline & 24 & 0.18 & 0.34 & 0.52 & 1.02 & 1.39 & 2.78 \\
\hline & 36 & 0.27 & 0.51 & 0.78 & 1.53 & 2.08 & 4.17 \\
\hline & 48 & 0.36 & 0.68 & 1.04 & 2.04 & 2.76 & 5.55 \\
\hline \multirow[t]{4}{*}{$158(6)$} & 12 & 0.15 & 0.29 & 0.44 & 0.73 & 1.0 & 2.0 \\
\hline & 24 & 0.29 & 0.59 & 0.88 & 1.46 & 2.0 & 4.0 \\
\hline & 36 & 0.44 & 0.87 & 1.32 & 2.19 & 3.0 & 6.0 \\
\hline & 48 & 0.58 & 1.16 & 1.76 & 2.19 & 4.0 & 8.0 \\
\hline
\end{tabular}

${ }^{a}$ Based on permissible deflection of $1 / 8$ in. $/ \mathrm{ft}$.

${ }^{b}$ Uniformly loaded beam, $50 \%$ simply supported and $50 \%$ fixed ends assumed.

'Length based on 2-in. spacing between 24- by 24-in. filter units; the numbers within parentheses denote number of filter units. The data given are adequate for any length within \pm 10 in. of length given.

${ }^{d}$ Structural angles can be chosen from the tables given in the AISC Manual of Steel Construction.

Housings installed inside a reactor containment may experience a pressure lag during rapid pressurization of the containment following a major accident. Unless the housings are equipped with pressure-relief dampers, this lag could result in a substantial enough pressure differential between the housing and containment to collapse the housing.

Reinforcing members should be spaced to minimize vibration and audible drumming of the housing walls which can be transmitted through the system. Reinforcements should be installed on the outside of the housing, when possible, to eliminate interior ledges and projections that collect dust and constitute hazards to personnel working in the housing. All sharp corners, welds, weld spatter, and projections inside the housing should be ground smooth. The housing design must minimize cracks and crevices that are difficult to clean and that may collect moisture that can cause corrosion.
Mastics and caulking compounds, including silicone-base, RTV sealants, deteriorate in service and should not be used for sealing between panels and sections of a contaminated exhaust housing. Lock seams, rivets, and bolts used in conventional construction for joining panels do not produce leaktight joints. Leaks upstream of the filters are not permissible because of possible outleakage of contamination, and inleakage of air downstream of the filters results in reduced system performance. When bolted flange joints are used between the housing and ducts, $1^{1 / 1} / 2 \times 1^{1 / 1} \times 1 / 4$-in.-angle flanges with ASTM D1056 grade SCE-45 or 30-40 Shore-A durometer neoprene gaskets are minimums. ${ }^{14}$ Maximum bolt spacing of $4 \mathrm{in}$. is recommended for flanges.

Shop fabrication of housings is recommended over field fabrication because of the superior workmanship and control possible under shop conditions. Figure 4.29 , which shows two redundant 


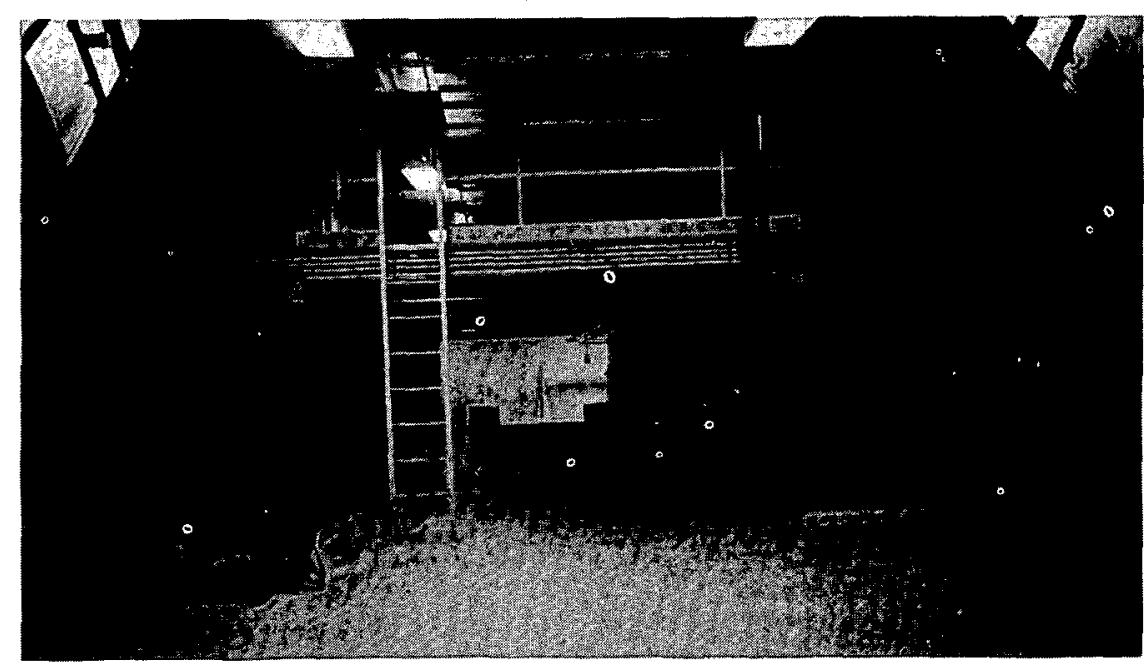

Fig. 4.29. Redundant units of nuclear reactor auxiliary-building ventilation system. Sections of the housings were shop-fabricated and assembled in the field. Each housing has $32,000 \mathrm{cfm}$ capacity in a 4 -wide by 8 -high filter array. Note service gallery between housings and installation in a room that can be isolated as a contamination zone.

32,000-cfm units of an auxiliary building ventilation system for a nuclear reactor, illustrates that shop fabrication is practical, even for very large housings. Note the service gallery between units, which continues on into the housings. These housings were built in sections and assembled in the field. Field joints for such housings should be seal-welded, since mastic and gasket-sealed joints cannot be considered reliable for permanent installations.

\subsubsection{Masonry and Concrete Housings}

Filter housings for low-gamma-activity systems and vaults for high- (or potentially high-) gammaactivity systems are sometimes built as an integral part of the building structure. Concrete block construction that meets requirements for at least a 2$\mathrm{hr}$ fire rating ${ }^{23}$ is suitable for low-activity systems, but cinder block and other low-density material should not be used. Suitable block and ceiling constructions for low-activity system housings are shown in the UL Building Materials List . $^{24}$ Columns and bearing walls beneath masonry housings must have at least a $3-\mathrm{hr}$ fire rating, and a 4-hr rating is preferred. Interior walls and ceilings should be plastered with $1 / 2$ in. of gypsum plaster and coated with epoxy or other impermeable paint to seal the surfaces and facilitate cleaning and decontamination. Blocks must be sealed to the floors along the entire perimeter. After sealing is completed, housing and mounting-frame leak tests should be made as described in Chap. 8. Areas particularly susceptible to leakage are door frames and flush-mounted items, such as light switches, on interior or exterior surfaces. This type of construction is suitable for secondary containment rooms in which large housings or multiple single-filter systems are installed (see Figs. 4.2 and 4.29).

In high-radiation-level or potentially highradiation-level systems (e.g., a reactor or fuel reprocessing plant), filters may have to be installed in poured concrete housings or underground concrete pits. Figure 2.13 shows a typical installation of this type. When unusually leaktight construction is required, as for a filter system connected directly to the containment vessel of a nuclear reactor, a complete steel lining may be required inside the pit. Concrete housings and pits must be designed in accordance with recognized radiation shielding principles, ${ }^{25}$ in addition to standards of the American Concrete Institute (ACI) and the Concrete Reinforcing Steel Institute (CRSI). Barite, magnetite, or other highdensity concrete is recommended for shielding blocks and portions of the housing or pit that extend aboveground. ANSI N101.6 provides guidance to the construction of concrete radiation shields. ${ }^{26}$

Particular care must be taken with concrete construction to avoid spalling and cracking that could result in the leakage of unfiltered air and rough surfaces that are difficult to decontaminate. Surfaces that are exposed to radioactive substances must have a smooth finish that is resistant to wetting and free of defects that can trap contaminants. An example of undesirable cracking, spalling, and surface roughness, such as that frequently found in concrete 
construction, is shown in Fig. 4.30. Cracks such as those shown can be repaired by heavy undercutting and grouting with epoxy; however, this process is time-consuming, costly, and subject to the deficiencies of poor workmanship. Cracks can be minimized by the use of high-strength concrete $(3000 \mathrm{lb}$ at 28 days) and the liberal use of reinforcing steel. Highstrength concrete also minimizes spalling problems. Curbs and steel embedments should be provided for the installation of filters, as discussed in the following section, and interior corners should be rounded or coved with a 2 -in. minimum radius to facilitate painting and decontamination.

\subsubsection{Seal Between Mounting Frame and Housing}

A critical point in housing construction is the seal between the mounting frame and housing. A seal weld should be employed, except for remotely maintained systems in which a gasketed seal between a removable mounting frame and the housing is used to enable removal of the entire assembly of mounting frame and filters following an accident (see Fig. 9.12). Figure 4.31 shows the usual method of welding the mounting frame into a steel housing. The perimeter angle is welded to the housing on both sides all around. The frame-to-housing seal weld sometimes fatigues and cracks under service conditions, particularly when the housing is subjected to excessive vibration, shock loading, and frequently when materials of construction of frame or housing are too light. Two alternate frame-installation methods that minimize the possibility of bypassing the filters in the

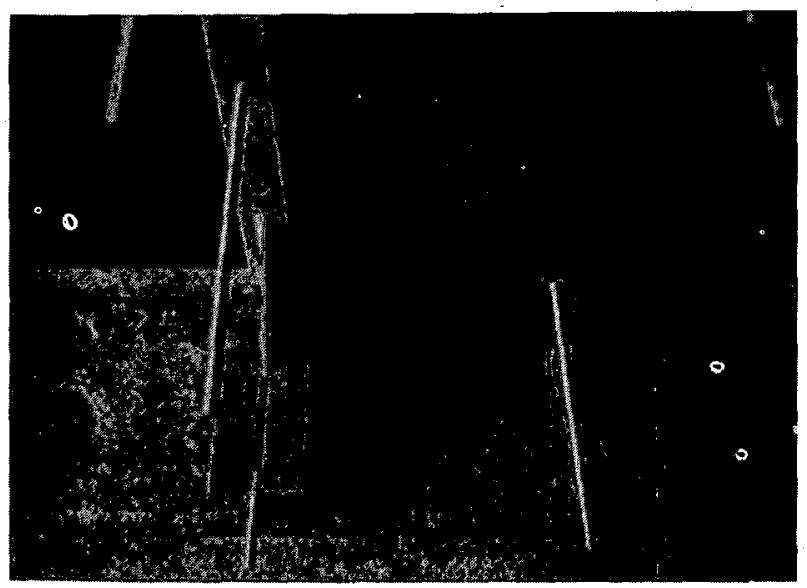

Fig. 4.30. An unacceptable concrete surface. Note cracks, spalls, and surface roughness that could result in leakage under the filter frame, trap contaminated material, and interfere with decontamination.

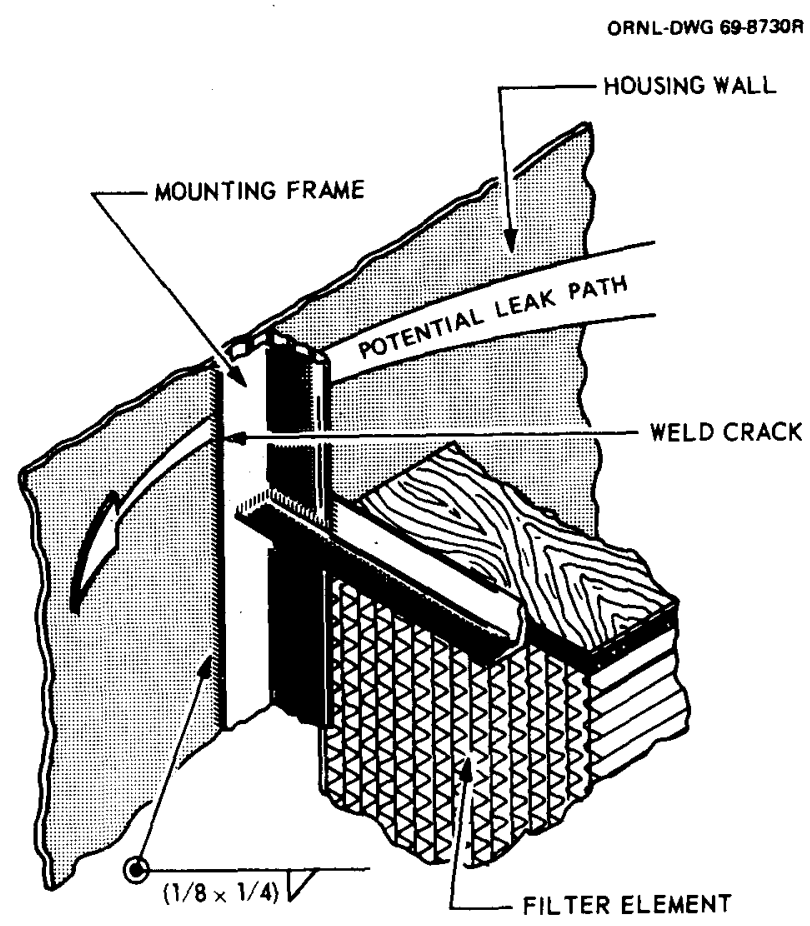

Fig. 4.31. Acceptable method for seal-welding mounting frame to housing. Weld crack and leak path show how a fatigued weld could result in bypassing the filters.

event of seal-weld cracks are shown in Fig. 4.32. Any of the methods shown are acceptable.

When a housing is installed on a concrete floor, the seal between mounting frame and floor is made by welding to a structural member embedded in the floor, as Fig. 4.33 shows. An angle (as shown) or a channel or I-beam with web vertical may be used to provide a labyrinth seal between the embedded member and concrete. Channels and I-beams should not be embedded with flanges pointing down, since this would trap air and cause voids in the concrete, thus providing a possible leakage path. Anchors (shown in Fig. 4.33) are desirable with angles or channels to oppose overturning forces on the mounting frame due to air pressure. For masonry and concrete housings and pits, embedments in walls and ceilings (shown in Fig. 4.34) are also required. Wall, ceiling, and floor embedments are welded together at the corners to form a continuously sealed surround for the mounting frame.

\subsubsection{Housing Floor}

Steel housings should, if possible, have steel floors welded continuously to the walls of the housing. In no case should the housing be installed on a wood floor 

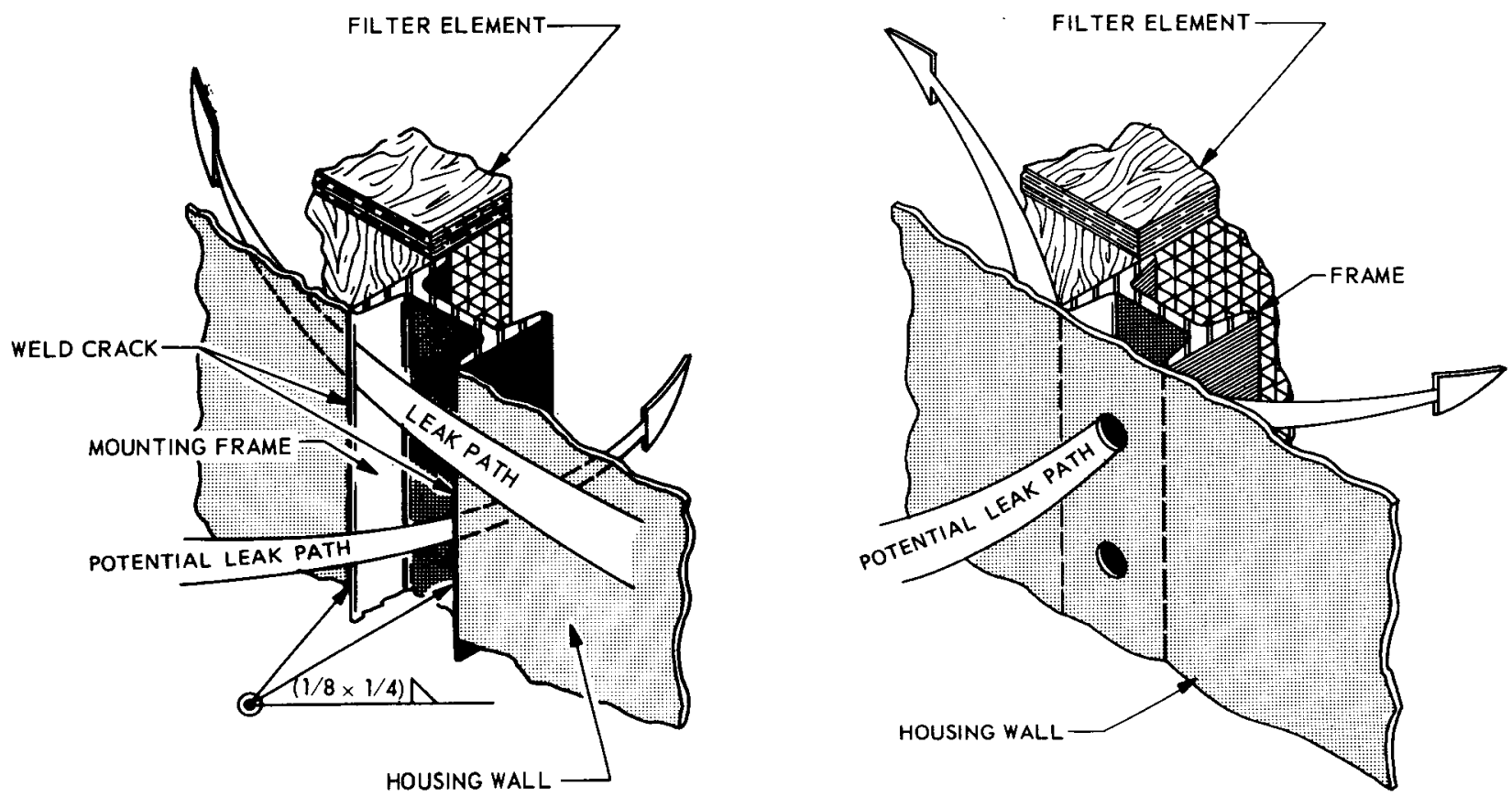

Fig. 4.32. Methods of welding frame to housing to ensure that weld cracks result in inleakage instead of outleakage. In both installation methods, both legs of the I-beam are seal-welded to the housing. Because pressure inside the housing, both upstream and downstream of the filters, is below atmospheric pressure, a crack in either seal weld will result in inleakage to, rather than outleakage from, the housing.

ORNL. DWG. 69.8732

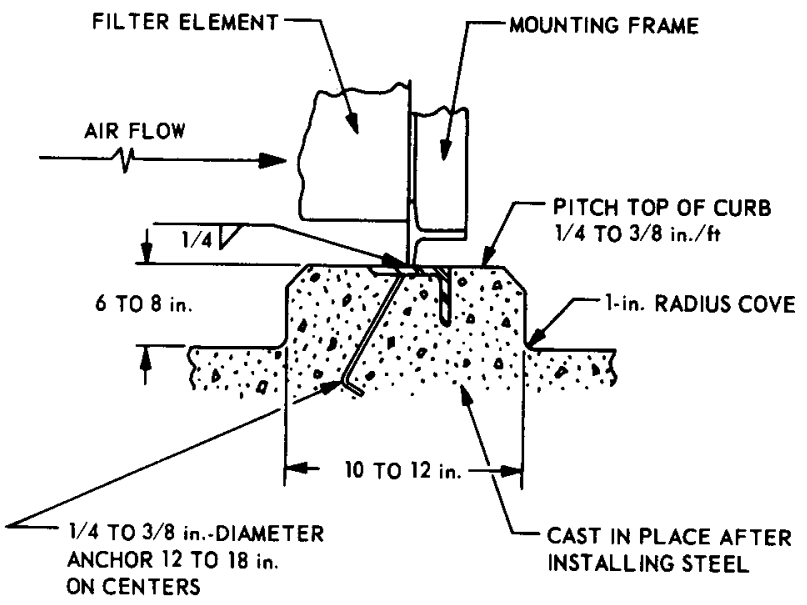

Fig. 4.33. Embedded structural member for seal-welding filter mounting frame to a concrete floor. Note anchor bolts, curbs, and seal weld from air-entering side.

or on a floor having less than a 3-hr fire rating. A channel or I-beam curb, welded to the floor, is recommended to raise the filter mounting frame off the floor. When steel housings are installed on concrete floors, curbs should be provided under the mounting frame (Fig. 4.33) and walls. An embedded member must be provided in the curb under the mounting frame.

Floors of filter housings should preferably be pitched slightly toward a drain. The section of flooring between two banks of components must be considered a separate floor to be drained independently. Floors should be free of obstructions and raised items that would be hazardous to workmen. Concrete floors must be smooth and free of cracks and spalls, and tops of curbs must be pitched away from the steel to prevent corrosion from standing water.

\subsubsection{Housing Doors}

Easily opened doors are essential on large housings, and more than one door is generally needed. A door should be provided to each compartment (space between banks) where maintenance, testing, or inspection may take place. To save the cost of access doors by requiring a workman to gain access to the downstream side of a bank by crawling through a filter opening, as the inspector is required to do in Fig. 4.35, is poor economy and can result in cross contamination of the system, damage to installed filters, and injury to personnel. The use of bolted-on 


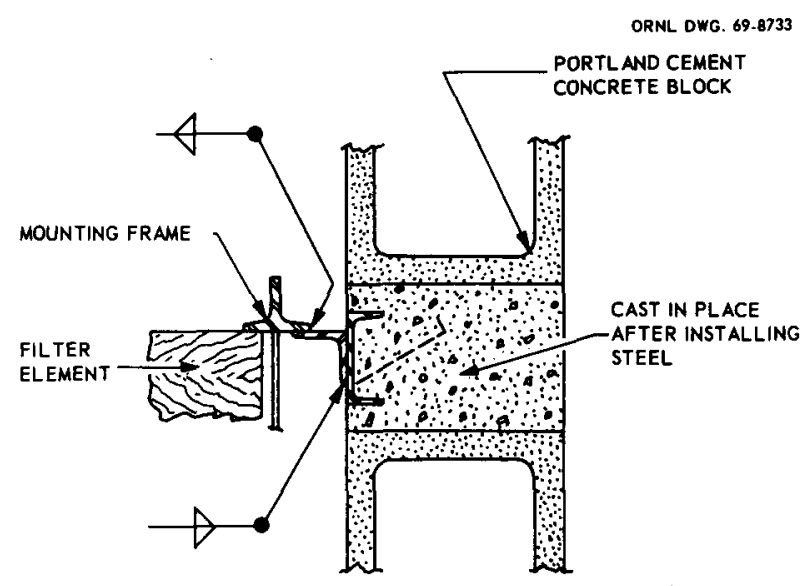

Fig. 4.34. Embedded structural member for walls and ceilings of masonry (shown) or concrete housing. Channel with flanges down is not suitable for floors, because trapped air would cause voids.

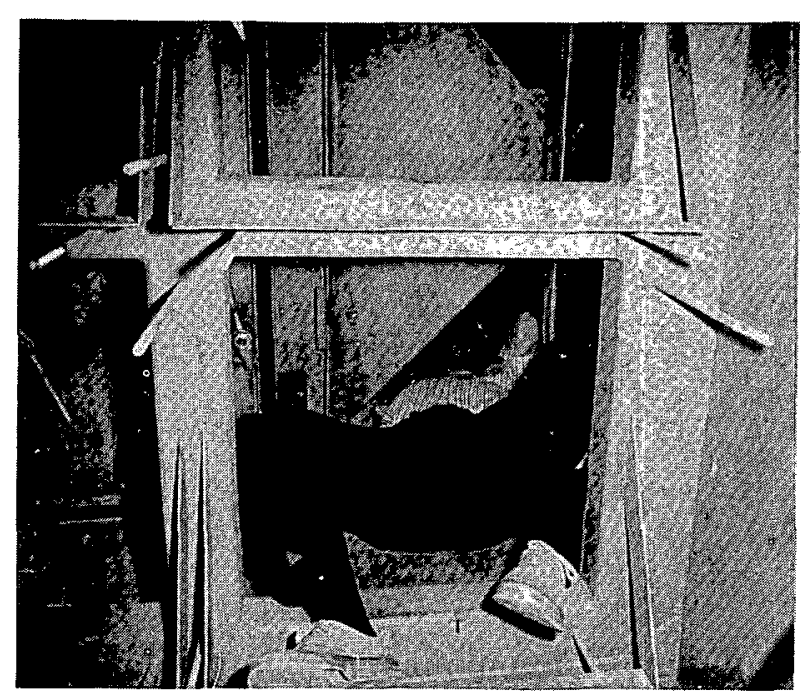

Fig. 4.35. Housing with inadequate provision for access. Inspector is required to crawl through a HEPA filter opening to gain access to downstream side of filters and to adsorbers.

removable panels for access to filter compartments should be avoided for even the smallest filter housings because of the time loss when necessary to get into the housing (the time loss could be disastrous in an emergency) and because nuts tend to rust or freeze after a few months of service. There is also the problem that lost nuts are often not replaced, which eventually results in reduced leaktightness of the panel. Sliding doors should never be used for filter housings, because they cannot be sealed and because they jam after any distortion of the housing.

Sturdy double-pin-hinged doors with rigid, closefitting casings and positive latches, such as the marine bulkhead-type shown in Fig. 4.36, should be provided on man-entry housings, particularly those for ESF and other high-hazard service. Doors and gaskets must be designed to maintain a hermetic seal under positive and negative pressures equal to at least the fan cut-off pressure. Doors of negative pressure systems must open outward and, since they may have to be opened against the negative pressure, means for breaking the vacuum or for mechanically assisted opening are desirable. Doors should have heavy-duty hinges and positive latching devices operable from inside and outside. Means for locking, preferably a padlock, should be provided to prevent unauthorized entry. The stiffness of doors is important, since flexible doors can be sprung when opened against negative pressure or allowed to slam shut under load. An air lock at the entry to the housing will eliminate problems of opening doors against negative pressure and slamming, and also, if large enough, will provide an intermediate work area for personnel during a filter change.

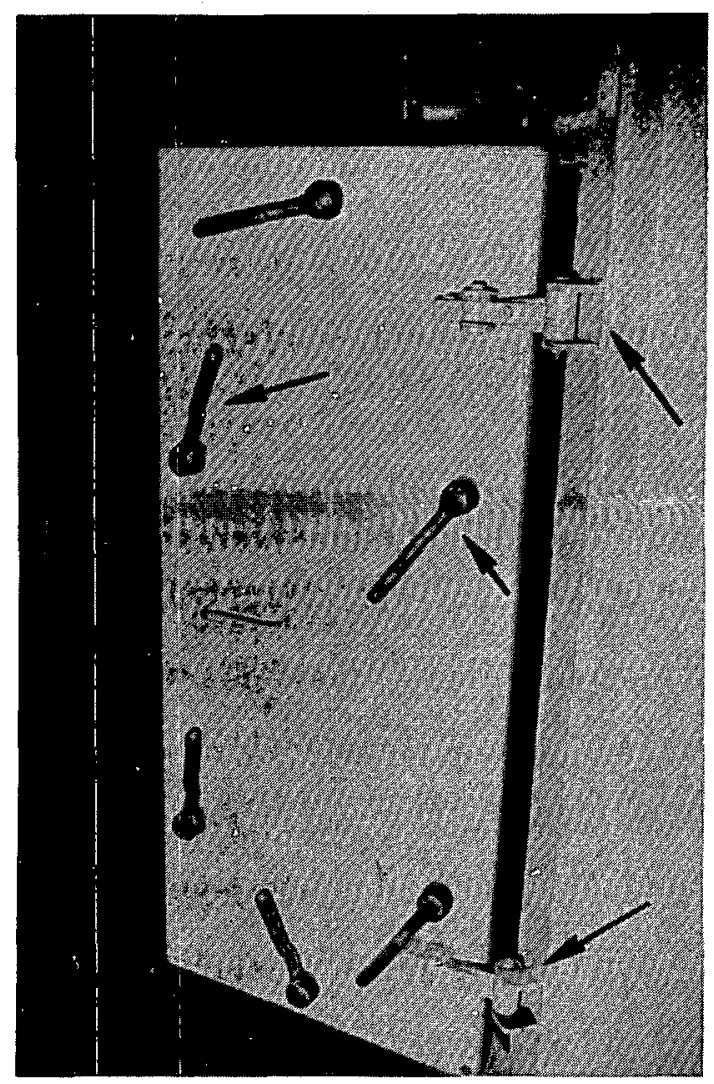

Fig. 4.36. Marine bulkhead-type housing door. Note rigid casing, slip-pin hinges, and latching dogs operable from both sides. The dogs at the top and bottom are probably unnecessary, since four would have been sufficient to seal a door this size. 
Housing doors of the type shown in Fig. 4.36 require a minimum of two latching dogs on each side. Lighter-construction doors require additional latches to achieve a satisfactory seal. Latching dogs should be operable from inside and outside the housing, and shafts must be fitted with O-rings, glands, or stuffing boxes to prevent leakage. Door hinges should be of the double-pin, loose-pin, or other type that will permit the full plane of the door to move perpendicularly to the plane of the door frame during the last fraction of an inch of closure. Single-pin hinges,

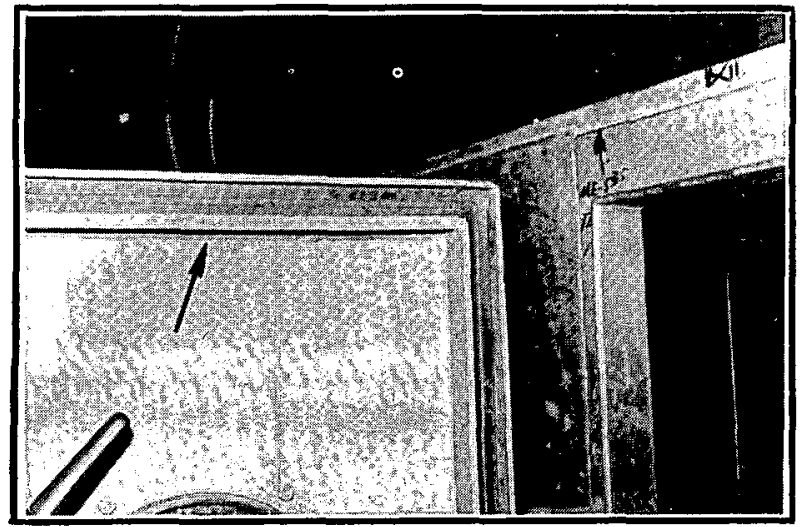

Fig. 4.37. Single-pin hinge causes gasket roll on hinge side of door. Door cannot be sealed. which result in angular motion throughout the door closing arc, do not permit the door to seal properly and may cause the gasket to be rolled out of its groove after a period of use, as shown in Fig. 4.37, thus resulting in the loss of housing leaktightness. If door gaskets are too hard they will be incompressible, and the door cannot be sealed properly even with leverand-wedge latching dogs of the type shown in Fig. 4.36. If too soft, the gasket will rapidly take a compression set and lose its ability to seal. Solid neoprene or silicone rubber of about 30-40 Shore-A durometer is recommended.

A compromise may have to be made in sizing doors for man-entry housings. On the one hand, the door must be large enough for easy access to personnel dressed in bulky protective clothing, wearing gas masks or respirators, and perhaps carrying $24 \times 24 \times$ 12-in. filters weighing up to $40 \mathrm{lb}$, or $26 \times 6 \times 30$-in. adsorber cells weighing up to $130 \mathrm{lb}$ (dimensions of the door through which a 95th-percentile man can pass erect, carrying such loads, are shown in Fig. 4.38). On the other hand, the larger the door the more difficult it is to seal, and the more likely it or its frame can be damaged if allowed to slam under load. The door should be as large as possible for easy access, but in no event should it be any less than $26 \mathrm{in}$. wide by 48 in. high. A coaming ( 2 in. high minimum to 6 in. high
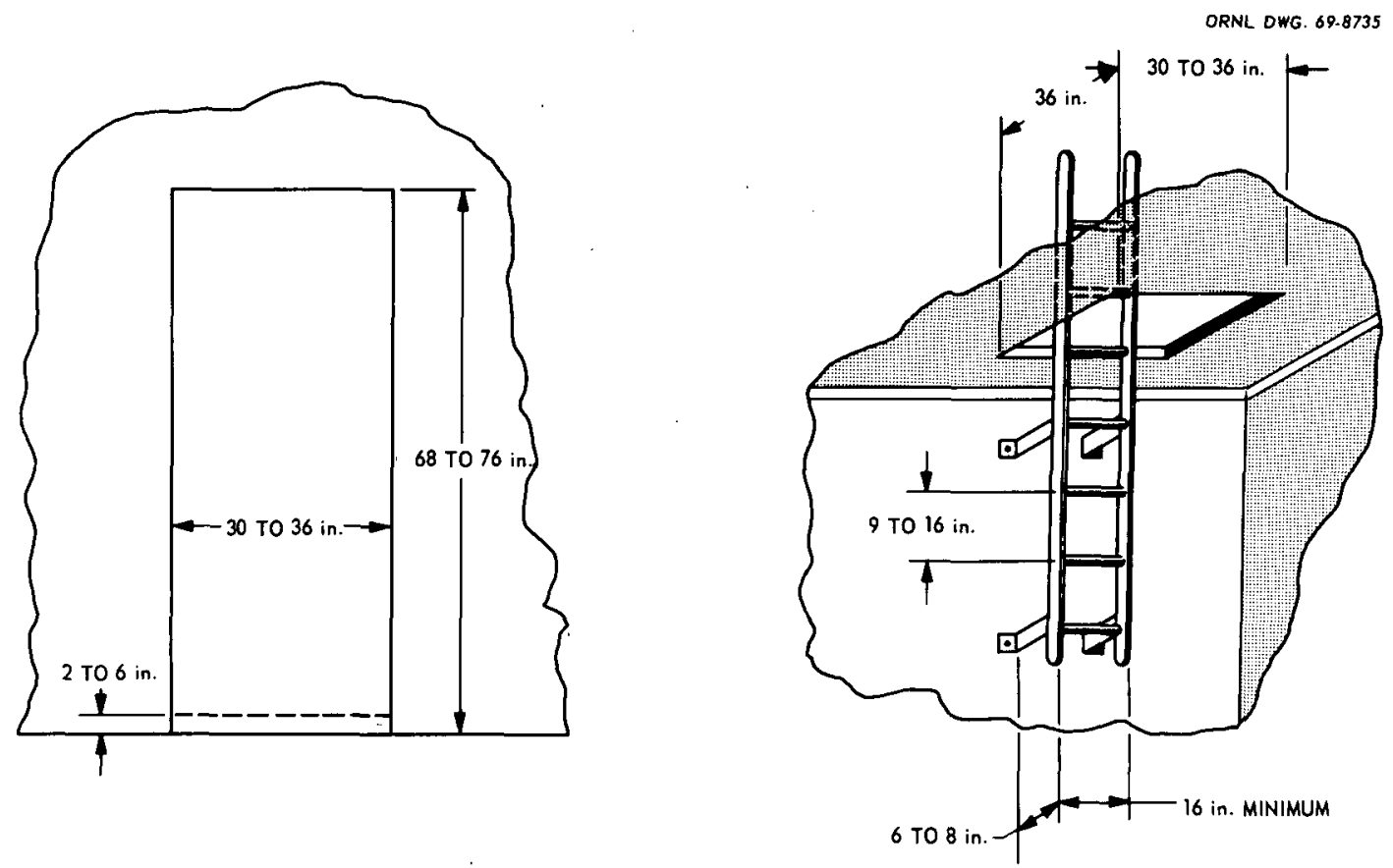

Fig. 4.38. Maximum filter-house door and hatch dimensions. Door dimensions based on erect 95th percentile man. 
maximum) should be provided at all doors to prevent the outflow of contaminated water should the housing ever be flooded. The ladder and hatch dimensions shown in Fig. 4.38 are suggested for access hatches in work galleries, for high banks, and for hatches of underground filter pits.

\subsubsection{Housing Drains}

Floor drains are essential in contaminated-exhaust filter housings, particularly when sprinkler protection is provided. Even if moisture or condensation is not expected under normal conditions, occasional washdown may be required for decontamination, and water will be needed in the event of a fire. When the housing is above grade, the minimum provision for drainage is a Chicago half coupling, sealed with a bronze pipe plug, using tetrafluorethylene (TFE) plastic "ribbon dope" so the plug can be easily removed when needed. When the filter is at or below grade, drains should be piped to an underground contaminated-waste system during initial construction, since later installation is likely to be very costly. Drains from ESF systems must also be piped to the radioactive waste system. In cold climates, water seals, traps, and drain lines must be protected against freezing if they are above the frost line. When fire sprinklers are installed in the filter house, the drains must be sized to carry away the maximum sprinkler flow without water backup in the housing.

A separate drain is needed for each chamber of the filter house, and each drain must have its own water seal or trap. The spaces between two banks of components in series and between a bank and the housing are considered separate chambers. When piped to a common drain system, drain lines from the individual chambers of the housing must be valved, sealed, or otherwise protected to prevent bypassing of contaminated air around filters or adsorbers through the drain system.

\subsubsection{Housing Leaktightness}

Contaminated filter housings must be leaktight to prevent contamination of adjacent service and operating areas. Although it is commonly assumed that all leakage will be inleakage in a negative pressure system, outleakage can occur under some conditions, even when the system is operating at its design negative pressure, and particularly when the system is down. The recommended maximum permissible leak rates given in Table 4.5 are based on the criteria of the Code of Federal Regulations 10 CFR
Table 4.5. Recommended maximum permissible leak rates for contaminated exhaust and air cleanup filter housings $(\Delta p=10$ in.wg)

\begin{tabular}{lc}
\hline Construction & $\begin{array}{c}\text { Maximum permissible } \\
\text { leak rate (percent } \\
\text { housing volume } \\
\text { per hour) }\end{array}$ \\
\hline Unlined concrete & 60 \\
$\begin{array}{l}\text { Painted concrete, masonry } \\
\text { Conventional sheet metal } \\
\text { housing, no attention to } \\
\text { doors, etc. }\end{array}$ & $30-36$ \\
$\begin{array}{l}\text { All-welded man-entry steel } \\
\text { housing }\end{array}$ & $30-36$ \\
$\begin{array}{l}\text { Small single-filter housing } \\
\text { Housing of recirculating } \\
\text { system located within } \\
\text { containment building }\end{array}$ & 0.2 \\
$\begin{array}{l}\text { Boiling water reactor/gas } \\
\text { cooled reactor } \\
\text { containment }\end{array}$ & 0.05 \\
$\begin{array}{l}\text { Pressurized water reactor } \\
\text { containment }\end{array}$ & Not critical \\
\hline
\end{tabular}

20 and 10 CFR 100 and the current ALARA requirements. Leak testing of filter housings is covered in Chap. 8 and ANSI N510. ${ }^{27}$

\subsubsection{Other Housing Requirements}

Figure 4.39 illustrates a number of features that are desirable in a contaminated exhaust or air cleaning housing. The housing is all-welded construction with No. 11 U.S. gage $\left(\sim^{1 / 8}\right.$ in.) walls and ceiling, $1 / 4-$ in. steel floor, and $3 \times 3 \times 1 / 4-$ in. reinforcing angles on $24-$ in. centers. The housing is one of six that were shopfabricated for a series-parallel prefilter/HEPA filter installation for a high-hazard exhaust system at a research laboratory. Each housing contains either a bank of nine 1000 -cfm prefilters or nine 1000 -cfm HEPA filters. The housings are dampered as shown in Fig. 2.12. Features of the housing include

1. shop fabrication;

2. permanently installed DOP injection nozzles (inside housing) and probe and sample ports;

3. wired-glass viewports on each side of filter bank for visual inspection without entering the housing;

4. permanently installed lights on each side of filter bank, two in parallel on each side, with switch at 


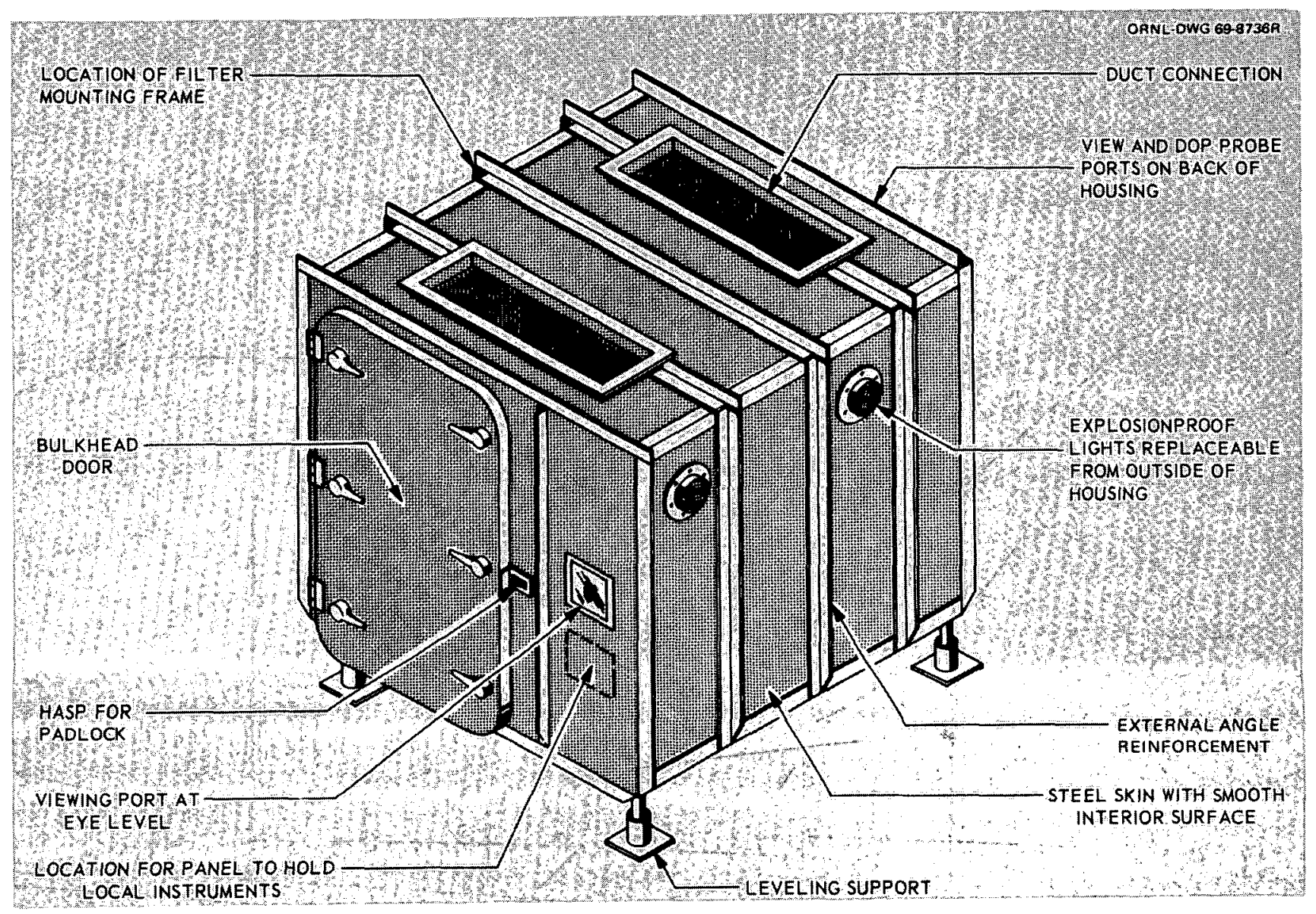

Fig. 4.39. Steel filter housing showing many desirable features.

door and second switch under viewport on back of housing; lights on independent switches;

5. lights installed in vapor-tight globes, replaceable from outside of the housing;

6. wiring installed on outside of the housing (penetrations for wiring are a common source of leakage);

7. shock-mounted instrument panel close to housing door, with pressure-drop manometer across each bank of filters;

8. large marine bulkhead door with dogs operable from inside and outside of the housing;

9. ample space (approximately 4 by $7 \mathrm{ft}$ ) inside of housing for personnel to work during a filter change;

10. all reinforcements on outside of housing;

11. housing opens on aisle that can be controlled and that serves as workspace during a filter change;

12. compartmented system-any housing of the six can be isolated for service or emergency without necessitating shutdown of system;
13. housing is isolated from the building, leveling feet for adjustment;

14. all-weld construction eliminates leaks to occupied areas.

\subsubsection{Paints and Coatings}

The mounting frame and housing interior of carbon steel and masonry must be painted to protect against corrosion and to facilitate cleaning and decontamination. Surfaces must be prepared properly, and prime and top coats must be applied in strict accordance with the paint manufacturer's directions in order to obtain the necessary wet-film and dry-film thicknesses. Film thicknesses should be tested during and after application. Steel surfaces should be abrasive-blasted to white metal to a profile of 1 to 2 mils in accordance with Surface Preparation Specification 5 of the Steel Structures Painting Council. ${ }^{28}$ The prime coat must be applied within 2 to $3 \mathrm{hr}$ after grit-blasting and in no case should be delayed to the next day. Hand- or power-tool 
cleaning (Surface Preparation Specifications 2 and 3 of the Steel Structures Painting Council) is usually sufficient for exterior steel surfaces. Ambient temperature and metal temperature should be at least 10 to $20^{\circ} \mathrm{F}$ above the dew point before starting to paint, and there must be adequate drying time between coats. Thick runs and streaks must be avoided, particularly on gasket seating surfaces, where they may chip off and leave uneven surfaces that will interfere with proper sealing of the filter. After painting, gasket seating surfaces should be coated with a silicone oil or grease to prevent the filter gasket from adhering to the paint after a period of service. Clamping bolts should not be painted because the paint will scrape off and jam the nuts. Threads should be masked during painting and then coated with a silicone grease.

High-build epoxy-polyamide or modifiedphenolic coating systems have proven satisfactory for interior steel and masonry surfaces. Although inorganic zinc primers are often recommended for steel, their use is not recommended for housing interiors because the zinc particles are difficult to hold in suspension properly and they tend to surface, causing blistering and peeling of the top coats. ${ }^{29}$ An airless spray is recommended for applying prime and top coats. Guidance in the selection of coatings and paints for nuclear service is given in ANSI N512. ${ }^{30}$ For purposes of selection, the classification "moderate exposure" is recommended for highactivity (or potentially high-activity) systems, and the classification "light exposure" is recommended for low-activity systems. The recommendations on quality assurance during application of paints and coatings, given in ANSI N101.4, ${ }^{31}$ are suggested for ESF and other high-activity (or potentially highactivity) systems.

Inorganic zinc primers are acceptable for exterior steel surfaces, but complete curing may take from two days to six weeks, depending on temperature and humidity conditions. One or two coats of high-build epoxy, vinyl, acrylic, or silicone paint are recommended for exterior steel surfaces exposed to the weather. Epoxy-polyamide coatings are superior to epoxy amines for water and salt resistance and have better tolerance for moisture during application. Vinyls are excellent for general marine and chemical plant exposures and do not chalk as much as the epoxies when exposed to sunlight. On the other hand, they are inferior to the epoxies in abrasion resistance, solvent resistance, and resistance to severe water or brine splashing. For out-of-doors service, acrylic coatings give the best protection against chalking and discoloration from sunlight and ultraviolet but are suitable only as top coats over an intermediate epoxy or vinyl coating. Silicone-base paints are useful for high-temperature applications, and aluminum-filled silicones give good protection up to $1000^{\circ} \mathrm{F}$. For a housing or duct that is located indoors and is exposed only to normal building atmospheres, an acrylic paint is suitable and gives good protection against color fading.

Because of difficulties in applying high-quality coatings and their often unsatisfactory performance in service, the designer should seriously consider stainless steel for mounting frames and housing in applications where corrosion or frequent decontamination will be encountered. Although quoted prices for carbon steel construction with high-quality coating systems generally run about 20 to $25 \%$ of the cost of stainless steel construction, experience shows that delays and difficulties in proper application often raise the final cost of coated carbon steel to as much as or more than stainless steel.

\section{REFERENCES FOR CHAP. 4}

1. ANSI N509, Nuclear Power Plant Air Cleaning Units and Components, American National Standards Institute, New York, current issue.

2. ASTM A36, Specification for Structural Steel, American Society for Testing and Materials, Philadelphia, current issue.

3. ASTM A499, Specification for Hot-Rolled Rail Carbon Steel Bars and Shapes, American Society for Testing and Materials, Philadelphia, current issue.

4. ASIM A 500 , Specification for Cold-Formed Welded and Seamless Carbon Steel Structural Tubing in Rounds and Shapes, American Society for Testing and Materials, Philadelphia, current issue.

5. ASTM A245, Specification for Flat-Rolled Carbon Steel Sheets, American Society for Testing and Materials, Philadelphia, current issue.

6. ASTM A479, Specification for Stainless and Heat-Resisting Steel Bars and Shapes, American Society for Testing and Materials, Philadelphia, current issue.

7. ASTM A240, Specification for Corrosion-Resisting Chromium and Chromium-Nickel Steel Plate, Sheet, and Strip, American Society for Testing and Materials, Philadelphia, current issue.

8. "Beam and Girder Design," Sect. 2 in Manual of Steel Construction, American Institute of Steel Construction, New York, current issue.

9. Ref. 8, Sect. 1, "Dimensions and Properties."

10. IES CS-8, Standard for High-Efficiency Gas-Phase Adsorber Cells, Institute of Environmental Sciences, Mt. Prospect, III., current issue.

11. Welding Handbook, Sect. 2, "Welding Processes," American Welding Society, Miami, current issue. 
12. ASME Boiler and Pressure Vessel Code, Sect. IX, "Welder Qualification," American Society of Mechanical Engineers, New York, current issue.

13. ASME Boiler and Pressure Vessel Code, Sect. V, "Nondestructive Examination," American Society of Mechanical Engineers, New York, current issue.

14. ASTM D1056, Specification and Tests for Sponge and Expanded Cellular Products, American Society for Testing and Materials, Philadelphia, current issue.

15. F. E. Adley, "Factors Influencing High Efficiency Gasket Leakage," Proc. Ninth Air Clean. Conf., USAEC Report CONF660904, September 1966.

16. L. L. Sims, Molded HEPA Filter Gasket Leakage, Hanford Environmental Health Foundation Report HOHF-11, October 1969.

17. Trademark of Flanders Filters, Inc.

18. Regulatory Guide 1.52, Design, Testing, and Maintenance Criteria for Atmospheric Cleanup System Air Filtration and Adsorption Units of Light-Water-Cooled Nuclear Power Plants, U.S. Atomic Energy Commission, Washington, D.C., 1973.

19. The percentile value indicates the portion of the population which falls at or below a particular value of measurement; in this case, only $5 \%$ of the population is tall enough to handle tools at heights over $78 \mathrm{in}$.

20. C. D. Morgan et al., Human Engineering Guide to Equipment Design, McGraw-Hill, New York, 1963.

21. W. V. Thompson, High Efficiency Particulate Filter History and Activities, USAEC Report RL-REA-1000, General Electric Co., Kichland, Wash., March 1965.
22. P. D. Erickson et al., Evaluation of Filter Flammability and Filter Bank Fire Detection Systems, USAEC Report RFP-222, Dow Chemical Co., Rocky Flats, Colo., 1961.

23. UL-263, Fire Tests of Building Construction and Materials, Underwriters' Laboratories, Chicago, 1959.

24. Building Materials List, Underwriters' Laboratories, Chicago, current issue.

25. T. Rockwell III (ed.), Reactor Shielding Design Manual, Van Nostrand, Princeton, N.J., 1956.

26. ANSI N101.6, Concrete Radiation Shields, American National Standards Institute, New York, current issue.

27. ANSI N510, Testing of Nuclear Air Cleaning Systems, American National Standards Institute, New York, 1975.

28. Surface Preparation Specification 5, "White Metal Blast Cleaning," Steel Structures Painting Manual, vol. 2, Steel Structures Painting Council, Pittsburgh, current issue.

29. J. P. Jarvis, project engineer, Oak Ridge National Laboratory, personal communication to C. A. Burchsted.

30. ANSI N512, Protective Coatings (Paints) for the Nuclear Industry, American National Standards Institute, New York, 1975.

31. ANSI N101.4, Quality Assurance for Protective Coatings Applied to Nuclear Facilities, American National Standards Institute, New York, 1972. 


\section{External Components}

\subsection{INTRODUCTION}

External components of the air cleaning system include fans, ductwork, dampers, housings, stacks, instruments, and other items of the system concerned with the movement, control, conveying, and monitoring of airflow and/or gas flow, as opposed to the internal components discussed in Chap. 3. In most cases, external components constitute a portion of the pressure boundary or are external to the pressure boundary of the system.

\subsection{DUCTWORK}

\subsubsection{Functional Design}

The sizing and layout of ductwork to provide desired air distribution, ventilation rates, transport velocities, and other functional requirements of the ventilation system are covered by ASHRAE handbooks, ${ }^{1}$ ACGIH's Industrial Ventilation, ${ }^{2}$ and ANSI Z9.2. ${ }^{3}$ The purpose of this section is to review the physical aspects of the duct system in relation to nuclear air cleaning. The least expensive first-cost duct layout may not be the most economical when total annual cost of operating the system is considered. Short-radius elbows and other shortcuts in ductwork may seriously increase system resistance, requiring, for instance, the use of a larger fan and/or fan motor and resulting in higher operating costs, or conversely, making it impossible for the system, as installed, to operate at the desired level of performance. The physical layout of ductwork in a building is often compromised to conform to the confines of a building structure or design. This may be unavoidable when installing new ducts in an existing building. In new construction, consideration should be given to providing adequate space and optimizing configuration for duct layout in the earliest phases of building layout long before the building design has been finalized. Easy access to filter housings, fans, dampers, and other components is vital to maintainability and testability, and therefore to reliability of the system. The allowance of adequate space for well-designed elbows, size transitions, and fan inlets and outlets is vital to least-cost operation.

\subsubsection{Mechanical Design}

Duct cost is influenced by the size and quantities of ductwork, materials of construction, coatings used for protection against corrosion, construction methods (seams, joints, etc.), airtightness requirements, the sequence of erection (including a consideration of space limitations, posterection cleaning requirements, etc.), and the number and type of field connections and supports (hangers, anchors, etc.) required. Consideration should be given to future modification, dismantling, and disposal of contaminated ductwork, particularly in the design of systems for laboratories, experimental facilities, and other operations where change of the ductwork can be expected. The provision for adding on or changing ductwork is a consideration often overlooked in initial design.

Where space permits, round duct is generally preferred to rectangular duct because it is stronger (particularly under negative or collapsing pressure), is more economical for high pressure construction often required for nuclear applications, provides more uniform airflow, and is easier to join and seal than rectangular duct. The principal disadvantages of round duct' are that it makes less efficient use of building space and that it is sometimes difficult to make satisfactory branch connections. Any duct system that carries radioactive material, or that could carry radioactive material under upset conditions, should be considered as a safety-related system. The level of radioactivity will largely determine the quality of duct construction required. Although it is sometimes assumed that all leakage in negative pressure ductwork will be inleakage, this is not 
necessarily true. In the event of fire or explosion in a contained space (room, enclosure, hot cell, glove box, or containment structure) served by the system, ductwork can become positively pressured (with resultant outleakage). Outleakage can also be caused by a rapidly closing damper or by dynamic effects (in a poorly laid out system) under normal operating conditions. Under system shutdown conditions or during maintenance, the possibility of outleakage from normally negative-pressure ductwork also exists. The engineer must consider these possibilities in the design and specification of permissible leak rates for negative-pressure portions of safety-related systems. In addition, ducts that normally carry clean air can sometimes become contaminated. Ducts must be sized for the transport velocities needed to convey, without settling, all particulate contaminants. Recommended transport velocities are given in Sect. 5 of Industrial Ventilation. ${ }^{2}$ Ducts for most nuclear exhaust and postaccident air cleanup systems should be sized for a minimum duct velocity of $2500 \mathrm{fpm}$.

Tables 5.1 through 5.4 list suggested sheet metal gages and reinforcement for negative-pressure ducts operating at pressures below 2 in.wg negative. Suggested gages and reinforcement for positivepressure ducts are given in the SMACNA standards. ${ }^{4}$

Table 5.1. Recommended sheet-metal thicknesses for round duct under negative pressure Factor of safety $=3$ over code based on ultimate strength for ducts with diameters up to 24 in. and 5 over code for ducts with diameters over 24 in., based on paragraph UG-28 in Sect. VII of the ASME Boiler and Pressure Vessel Code

\begin{tabular}{|c|c|c|c|c|c|c|c|c|c|c|}
\hline \multirow{2}{*}{$\begin{array}{l}\text { Negative } \\
\text { pressure } \\
\text { in duct }\end{array}$} & \multirow{2}{*}{$\begin{array}{l}\text { Reinforcement } \\
\text { spacing } \\
\text { (in.) }\end{array}$} & \multicolumn{9}{|c|}{ Sheet-metal thickness (U.S. gage no.) ${ }^{a}$ for duct diameter of -} \\
\hline & & 4 in. & 8 in. & $12 \mathrm{in.}$ & $16 \mathrm{in.}$ & 20 in. & $24 \mathrm{in.}$ & 36 in. & $48 \mathrm{in.}$ & $60 \mathrm{in.}$ \\
\hline \multirow[t]{4}{*}{4 in.wg } & $\infty^{b}$ & 24 & 24 & 20 & 18 & 16 & 14 & 10 & 8 & 4 \\
\hline & 96 & 24 & 24 & 24 & 22 & 20 & 18 & 16 & 14 & 14 \\
\hline & 48 & 24 & 24 & 24 & 24 & 24 & 22 & 20 & 18 & 16 \\
\hline & 24 & 24 & 24 & 24 & 24 & 24 & 24 & 22 & 20 & 18 \\
\hline \multirow[t]{4}{*}{8 in.wg } & $\infty$ & 24 & 22 & 18 & 16 & 14 & 12 & 8 & 4 & \\
\hline & 96 & 24 & 22 & 22 & 18 & 18 & 18 & 14 & 12 & 12 \\
\hline & 48 & 24 & 24 & 24 & 22 & 20 & 20 & 16 & 14 & 14 \\
\hline & 24 & 24 & 24 & 24 & 24 & 22 & 22 & 18 & 16 & 16 \\
\hline \multirow[t]{4}{*}{12 in.wg } & $\infty$ & 24 & 20 & 16 & 14 & 12 & 12 & 6 & 2 & \\
\hline & 96 & 24 & 22 & 18 & 18 & 16 & 16 & 12 & 11 & 11 \\
\hline & 48 & 24 & 22 & 22 & 20 & 18 & 18 & 14 & 14 & 12 \\
\hline & 24 & 24 & 24 & 24 & 22 & 22 & 22 & 16 & 16 & 16 \\
\hline \multirow[t]{5}{*}{20 in.wg } & $\infty$ & 24 & 18 & 14 & 12 & 11 & 8 & 4 & & \\
\hline & 96 & 24 & 20 & 16 & 16 & 14 & 14 & 11 & 11 & 8 \\
\hline & 48 & 24 & 22 & 20 & 18 & 16 & 16 & 14 & 12 & 11 \\
\hline & 24 & 24 & 24 & 22 & 20 & 18 & 18 & 16 & 14 & 12 \\
\hline & 12 & & & & & & & & 20 & 16 \\
\hline \multirow[t]{5}{*}{ I psi } & $\infty$ & 20 & 14 & 12 & 10 & 8 & 6 & & & \\
\hline & 96 & 24 & 18 & 16 & 14 & 12 & 12 & 10 & 8 & 6 \\
\hline & 48 & 24 & 20 & 18 & 18 & 16 & 16 & 12 & 11 & 11 \\
\hline & 24 & 24 & 24 & 22 & 20 & 18 & 18 & 14 & 12 & 12 \\
\hline & 12 & & & & & & & & 16 & 14 \\
\hline \multirow[t]{5}{*}{$2 \mathrm{psi}$} & $\infty$ & 18 & 12 & 11 & 8 & 4 & 2 & & & \\
\hline & 96 & 22 & 16 & 14 & 12 & 12 & 11 & 6 & 6 & 4 \\
\hline & 48 & 24 & 18 & 16 & 14 & 14 & 12 & 10 & 8 & 6 \\
\hline & 24 & 24 & 20 & 18 & 18 & 16 & 16 & 11 & 11 & 11 \\
\hline & 12 & & & & & & & 14 & 12 & 12 \\
\hline
\end{tabular}

${ }^{a}$ Minimum sheet-metal thickness for shop-welded duct is No. 18 U.S. gage. Minimum sheet-metal thickness for field-welded duct is No. 16 U.S. gage.

${ }^{b}$ Where $\infty$ is shown, no reinforcement is required. 
Table 5.2. Recommended ASTM A36 angle reinforcement for round duct under negative pressure

\begin{tabular}{lllllllllll}
\hline $\begin{array}{l}\text { Negative } \\
\text { pressure } \\
\text { in duct }\end{array}$ & \multicolumn{8}{c}{ Angle size ${ }^{a}$ for duct diameter of -} \\
\cline { 2 - 11 } & 4 in. & 8 in. & 12 in. & 16 in. & 20 in. & 24 in. & 36 in. & 48 in. & 60 in. \\
\hline 4 in.wg & A & A & A & B & B & B & B & C & C \\
8 in.wg & A & A & A & B & B & B & B & C & C \\
12 in.wg & A & A & A & B & B & B & B & C & C \\
20 in.wg & A & A & A & B & B & B & B & C & C \\
1 psi & A & A & A & B & B & C & C & C & C \\
2 psi & A & A & A & B & B & C & C & D & D \\
4 psi & A & A & A & B & B & C & C & D & D \\
\hline
\end{tabular}

${ }^{a}$ Symbol for angle size (in.): $A=1 \times 1 \times 3 / 16 ; B=1 \frac{1}{1 / 2} \times 1^{1 / 1} \times{ }^{1 / 1} ; C$ C $=2 \times 2 \times 1 / 4 ; D=2 \frac{1}{2} \times$ $2^{1 / 2} \times 1 / 4$.

Source: Based on R. J. Roark, Formulas for Stress and Strain, 4th ed., McGraw-Hill, 1965, Formula 12, Table XV.

Table 5.3. Recommended sheet-metal thicknesses for rectangular welded duct under negative pressure

\begin{tabular}{|c|c|c|c|c|c|c|}
\hline \multirow{2}{*}{$\begin{array}{l}\text { Negative } \\
\text { pressure } \\
\text { in duct } \\
\text { (in.wg) }\end{array}$} & \multirow{2}{*}{$\begin{array}{l}\text { Reinforcement } \\
\text { spacing } \\
\text { (in.) }\end{array}$} & \multicolumn{5}{|c|}{$\begin{array}{c}\left.\text { Sheet-metal thickness }{ }^{a} \text { (U.S. gage No. }\right)^{b} \text { for } \\
\text { longest side of length - }\end{array}$} \\
\hline & & $12 \mathrm{in.}$ & 24 in. & $36 \mathrm{in.}$ & $48 \mathrm{in.}$ & $60 \mathrm{in.}$ \\
\hline 4 & 48 & 18 & 18 & 16 & 14 & \\
\hline 4 & 24 & 18 & 18 & 18 & 16 & 16 \\
\hline 4 & 12 & 18 & 18 & 18 & 18 & 16 \\
\hline 8 & 48 & 18 & 14 & 12 & 12 & \\
\hline 8 & 24 & 18 & 16 & 16 & 14 & 14 \\
\hline 8 & 12 & 18 & 18 & 18 & 18 & 18 \\
\hline 12 & 48 & 18 & 12 & 8 & 11 & \\
\hline 12 & 24 & 18 & 16 & 12 & 12 & 12 \\
\hline 12 & 12 & 18 & 18 & 18 & 18 & 18 \\
\hline 20 & 48 & 14 & 11 & 6 & 6 & \\
\hline 20 & 24 & 14 & 14 & 11 & 11 & 11 \\
\hline 20 & 12 & 18 & 14 & 14 & 14 & 14 \\
\hline \multirow[t]{3}{*}{$1 \mathrm{psi}$} & 48 & 12 & 10 & & & \\
\hline & 24 & 16 & 12 & 11 & 10 & \\
\hline & 12 & 18 & 14 & 12 & 11 & \\
\hline \multirow[t]{3}{*}{$2 \mathrm{psi}$} & 48 & 12 & 10 & & & \\
\hline & 24 & 14 & 11 & 10 & 8 & \\
\hline & 12 & 16 & 12 & 11 & 10 & \\
\hline
\end{tabular}

${ }^{a}$ For maximum deflection of $1 / 16 \mathrm{in} . / \mathrm{ft}$ in the long dimension.

${ }^{b}$ Minimum sheet-metal thickness for field-welded duct is No. 16 U.S. gage.

Source: Based on R. J. Roark, flat plate formula for edges held but not fixed, Formulas for Stress and Strain, 4th ed., McGraw-Hill, 1965, p. 246. 
Table 5.4. Recommended ASTM A36 angle reinforcement for rectangular ducts under negative pressure Based on uniformly loaded beam with $50 \%$ simple support, $50 \%$ fixed ends, and deflection of $1 / 8$ in./ $\mathrm{ft}$

\begin{tabular}{|c|c|c|c|c|c|c|c|c|c|c|c|c|}
\hline \multirow{3}{*}{$\begin{array}{l}\text { Negative } \\
\text { pressure } \\
\text { in duct } \\
\text { (in.wg) }\end{array}$} & \multicolumn{12}{|c|}{ Angle size ${ }^{a}$ for ducts with maximum panel size of - } \\
\hline & \multicolumn{5}{|c|}{12 in. by - } & \multicolumn{4}{|c|}{24 in. by - } & \multicolumn{3}{|c|}{48 in. by -} \\
\hline & 12 in. & $24 \mathrm{in.}$ & 36 in. & $48 \mathrm{in.}$ & $60 \mathrm{in.}$ & $24 \mathrm{in.}$ & 36 in. & 48 in. & $60 \mathrm{in.}$ & 36 in. & 48 in. & $60 \mathrm{in.}$ \\
\hline 4 & E & $\mathbf{E}$ & $\mathrm{E}$ & $\mathbf{F}$ & F & $\mathrm{E}$ & G & G & $\mathrm{G}$ & $\mathbf{H}$ & $\mathbf{H}$ & $\mathbf{H}$ \\
\hline 8 & $\mathrm{E}$ & E & E & F & $\mathbf{F}$ & E & G & G & G & $\mathbf{H}$ & $\mathrm{H}$ & $\mathrm{H}$ \\
\hline 12 & E & E & E & F & F & E & $\mathrm{G}$ & $\mathrm{G}$ & $\mathrm{G}$ & $\mathrm{H}$ & $\mathbf{H}$ & H \\
\hline 20 & E & $\mathrm{F}$ & $\mathbf{H}$ & H & & $\mathrm{G}$ & $\mathrm{H}$ & $\mathbf{J}$ & & & & \\
\hline $1 \mathrm{psi}$ & $F$ & $\mathrm{G}$ & $\mathbf{H}$ & $\mathrm{J}$ & & $\mathbf{H}$ & $\mathbf{J}$ & K & & & & \\
\hline $2 \mathrm{psi}$ & G & $\mathbf{H}$ & $\mathbf{J}$ & L & & $\mathrm{J}$ & $\mathbf{K}$ & $\mathrm{L}$ & & & & \\
\hline
\end{tabular}

${ }^{a}$ Symbol for angle size (in.): $\mathrm{E}=1 \times 1 \times 3 / 16 ; \mathrm{F}=1 \frac{1}{4} \times 1 \times 1 \frac{1}{4} \times{ }^{3} / 16 ; \mathrm{G}=1 \frac{1}{2} \times 1 \frac{1}{1 / 2} \times{ }^{3} / 16 ; \mathrm{H}=2 \times 2 \times 3 / 16$; $\mathrm{J}=2 \frac{1}{2} \times 2 \frac{1}{2} \times{ }^{1 / 4} ; \mathrm{K}=3 \times 2 \frac{1}{2} \times 1 / 4 ; \mathrm{L}=4 \times 3 \times 3 / 8$.

For ducts to be field-fabricated by welding, a minimum of No. 18 U.S. gage, and preferably No. 16 U.S. gage, sheet metal is recommended because of the difficulty of making reliable field welds in thinner material. Section 5.10 of ANSI N509 recognizes several levels or grades of duct construction but does not define them (in terms of specific requirements) or distinguish clearly between them. ${ }^{5}$ Because a nuclear facility may contain spaces of widely differing potential hazard level (see discussion of zoning, Sect. 2.2.1), the type of duct construction required may vary from one part of the plant to another. Questions that must be answered to establish the type of duct construction needed for a particular application include

1. Is the system safety-related?

2. If safety-related, is the level of radiation that exists in the duct, or that could exist in the duct in the event of a system upset, low? Intermediate? High?

3. Must the air cleaning system remain operable in the event of a system upset (power outage, accident, malfunction) or can it be shut down?

4. Where will the ductwork be located relative to (1) the contained space served by the system and (2) occupied spaces of the building? (Building spaces that are not normally occupied, but which are occasionally entered for repair or service of equipment, are considered to be occupied.)

5. Is the system once-through or recirculating?
6. Is it an ESF system (as defined by Regulatory Guide 1.52$)^{6}$ intended to mitigate the consequences of an accident?

7. What are the environmental considerations, that is, pressure, temperature, corrosion, etc.?

According to the answers to these questions, the duct should be constructed to conform to one of the several grades outlined in Table 5.5 and the leaktightness recommendations of Table 5.6. Recommended construction requirements are as follows:

Level 1: In accordance with SMACNA Low Velocity Duct Construction Standards, ${ }^{4}$ except that button-punch and snap-lock seam and joint construction are not permitted; these constructions are considered unsuitable, even for low-pressure construction. ${ }^{7}$ Companion-angle or bolted (or screwed) standing-seam transverse joints are recommended. Standing edges of seams or joints and reinforcement should be on the outside of the duct. ${ }^{8}$ The use of level 1 ductwork is limited to systems serving administrative areas and other nonsafety-related applications in which maximum static pressure does not exceed 2 in.wg.

Level 2: In accordance with SMACNA High Velocity Duct Construction Standards, ${ }^{4}$ except that (1) button-punch and snap-lock construction are not permitted; (2) only bolted flanged joints, companion-angle flanged joints, welded-flanged joints (in accordance with Fig. 5.1), or welded joints are permitted for transverse connections; (3) tie rods and cross braking are not permitted on 
Table 5.5. Guide for selecting recommended duct construction levels for various applications in nuclear facilities ${ }^{\circ}$

\begin{tabular}{|c|c|c|c|c|c|c|}
\hline \multirow{3}{*}{$\begin{array}{l}\text { Contamination } \\
\text { level and/or } \\
\text { function }\end{array}$} & \multirow{3}{*}{$\begin{array}{l}\text { Operating } \\
\text { mode }^{c}\end{array}$} & \multicolumn{4}{|c|}{ System type, duct location } & \multirow{3}{*}{$\begin{array}{l}\text { HVAC, supply, } \\
\text { recirculating } \\
\text { portion within } \\
\text { contained space }\end{array}$} \\
\hline & & \multicolumn{4}{|c|}{$\begin{array}{l}\text { Outside contained space, all } \\
\text { systems, duct located in- }\end{array}$} & \\
\hline & & Zone IV & Zone III & Zone II & Zone 1 & \\
\hline \multirow[t]{2}{*}{ None, supply, HVAC } & A & 1 & 1 & 2 & 2 & 2 \\
\hline & B & 1 & 1 & 1 & 1 & 1 \\
\hline \multirow[t]{2}{*}{ Low (class 4) } & A & 3 & 2 & 2 & 2 & 2 \\
\hline & B & 1 & 1 & 2 & 2 & 1 \\
\hline \multirow[t]{2}{*}{ Moderate (class 3) } & $\mathbf{A}$ & 4 & 3 & 2 & 2 & 2 \\
\hline & B & 4 & 2 & 2 & 2 & 1 \\
\hline \multirow[t]{2}{*}{ High (class 2) } & A & 4 & 4 & 4 & 4 & 2 \\
\hline & $\mathbf{B}$ & 4 & 4 & 4 & 4 & 2 \\
\hline \multirow[t]{2}{*}{ Very high (class 4) } & $\mathbf{A}$ & 4 & 4 & 4 & 4 & 2 \\
\hline & B & 4 & 4 & 4 & 4 & 2 \\
\hline \multirow[t]{2}{*}{ Process off-gas } & A & 5 & 5 & 5 & 4 & 2 \\
\hline & B & 5 & 5 & 4 & 4 & 2 \\
\hline \multirow[t]{2}{*}{ Controlled atmosphere } & $\mathbf{A}$ & 5 & 5 & 5 & 5 & 5 \\
\hline & B & 5 & 5 & 5 & 5 & 5 \\
\hline ESF control ${ }^{8}$ & $\mathbf{A}$ & 4 & 4 & 4 & 4 & $2^{n}$ \\
\hline ESF control room & $\mathbf{A}$ & 4 & 4 & 4 & 4 & $2^{k}$ \\
\hline Other ESF & $A$ & 4 & 3 & 3 & 3 & $2^{h}$ \\
\hline
\end{tabular}

${ }^{a}$ Duct construction level: 1, SMACNA low velocity; 2, SMACNA high velocity; 3, improved SMACNA high velocity; 4, welded; 5, pipe or welded duct, zero leak.

${ }^{b}$ Contamination levels, from Table 2.1.

'Operating mode: A, system to operate following upset or accident; B, system shutdown in event of upset or accident.

${ }^{d}$ HVAC: heating, ventilating, and air conditioning; building enclosure zones, from Table 2.3 and Sect. 2.2.1.

${ }^{e}$ Contained space: the building area or enclosure served by the system.

${ }^{\prime}$ Inert gas, desiccated air, or other controlled medium.

${ }^{8}$ HGTS, shield building exhaust, or other primary or secondary containment postaccident air cleanup systems.

${ }^{h}$ Duct must be structurally designed to function following a DBA or safe shutdown earthquake.

negative-pressure ducts; (4) standing edges and reinforcement of seams and joints are on the outside of ducts only; (5) sheet-metal thicknesses and reinforcement of negative-pressure ducts should be in accordance with Tables 5.1 through 5.5; and (6) radiation-resistant sealants (e.g., silicone room temperature vulcanizing) are required in the makeup of unwelded seams and in penetrations of safety-related ductwork. The use of level 2 ductwork is limited to systems serving administrative areas and Zone I and II areas, in which the radiotoxicity of materials handled or that could be released to the ductwork does not exceed hazard class 2 (Tables 2.1 through 2.3 ), and in which negative pressure does not exceed 10 in.wg static.
Level 3: Same as level 2 except that (1) transverse joints shall have full-flanged face width, $1 / 4$-in.thick gaskets (ASTM D1056" grade SCE-45 cellular neoprene; $30-40$ durometer, Shore-A, solid neoprene; or equivalent silicone elastomer) with interlocking notched corners (Fig. 5.1); and (2) nonwelded longitudinal seams, transverse joints, or the entire exterior may have hard-cast treatment (polyvinyl acetate and gypsum tape system) or comparable fire-resistant, corrosion-resistant, radiation-resistant, nonpeeling, leaktight treatment.

Level 4: All-welded construction except with sufficient mechanical transverse joints to facilitate coating (painting), erection, and future modifica- 
Table 5.6. Recommended maximum permissible duct leak rates at 2 in. $\mathrm{wg}^{a}$ negative (by methods of ANSI N510)

\begin{tabular}{|c|c|}
\hline Duct class & Maximum permissible leak rate \\
\hline Level 1 & $5 \%$ of system airflow per minute \\
\hline Level 2 & $1 \%$ of system airflow per minute \\
\hline Level 3 & $0.2 \%$ of volume per minute ${ }^{b}$ \\
\hline Level 4 & $0.1 \%$ of volume per minute ${ }^{b}$ \\
\hline Level 5 & $\begin{array}{l}\text { Zero detectable leak at any } \\
\text { test pressure up to } 20 \text { in.wg }\end{array}$ \\
\hline Recirculating & $\begin{array}{l}\text { Leak test not required if totally } \\
\text { within contained space served } \\
\text { by air cleaning system }\end{array}$ \\
\hline
\end{tabular}

${ }^{a}$ Maximum permissible leak rate at pressures greater than 2 in.wg is found from the equation

$$
L_{p} \times L_{2} \sqrt{P^{\prime} / 2}
$$

where

$L_{p}=$ permissible leak at higher pressure,

$L_{2}=$ permissible leak at 2 in.wg, from table,

$P^{\prime}=$ higher pressure

${ }^{b}$ Based on volume of portion of system under test.

ORNL-DWG 69-8704A

(a)

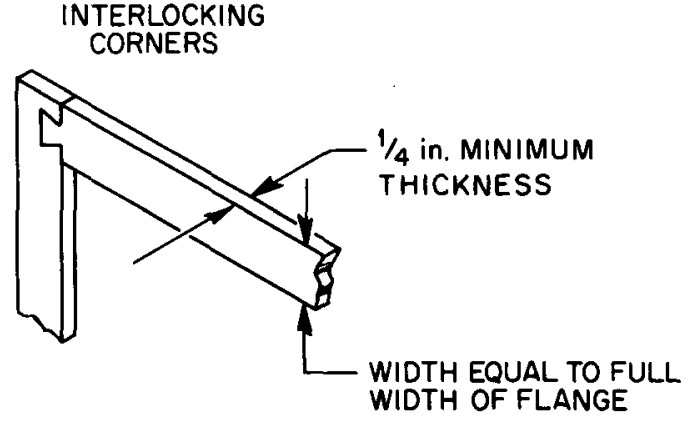

DUCT FLANGE GASKET DETAIL

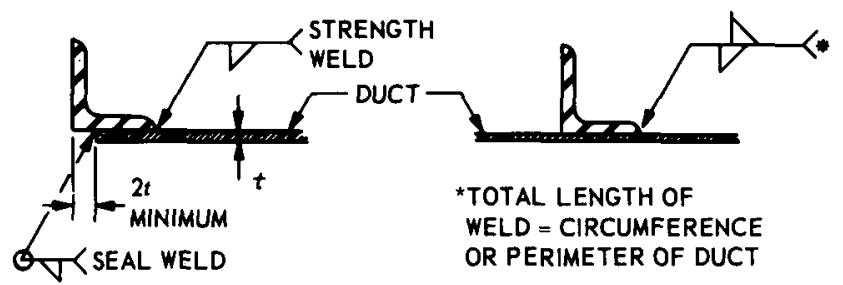

END REINFORCEMENT

INTERMEDIATE REINFORCEMENT (b)

Fig. 5.1. Method of welding reinforcing and flange angles to negative pressure ductwork. Note skip welds on back of angles to prevent duct casing from pulling away from angle. tion and/or dismantling. Mechanical transverse joints to conform to Fig. 5.1. Sheet-metal thicknesses and reinforcement taken from Tables 5.1 through 5.4 or determined by engineering analysis. ESF ductwork to meet requirements of Sect. 5.10 of ANSI N509. ${ }^{5}$

Level 5: Level 4 ductwork meeting requirements for leaktightness of level 5 duct (Table 5.6), or pipe meeting requirements of the American National Standard for Pressure Piping ${ }^{10}$ or the ASME Boiler and Pressure Vessel Code. ${ }^{11}$

\subsubsection{Engineering Analysis}

When sheet-metal thickness and reinforcement are established from engineering analysis rather than from Tables 5.1 through 5.4, a design pressure of at least 1.25 times the normal operating pressure is necessary for level 1, 2, and 3 constructions. A design pressure of 1.5 times the maximum negative pressure that can exist in the particular run of duct, under the most adverse conditions to which it can be subjected under any conceivable conditions, including the DBA and safe shutdown earthquake (SSE), is recommended. The maximum negative pressure is generally the fan shutoff pressure. In the engineering analysis, the following loadings should be considered as applicable to the particular system under consideration:

1. Differential pressure across the duct wall, as affected by maximum internal and external pressures that could prevail during testing and under normal and abnormal operating conditions, and any increase or decrease in the pressure due to inadvertent closure of a damper or plugging of an internal component. For duct work located within the containment vessel of a reactor, the external pressure under DBA conditions, due to the lag of pressure rise within the ductwork during the pressure transient in the containment vessel, must also be considered (such overpressures may be alleviated through the use of pressure-relief dampers that discharge to the containment space).

2. Effects of natural phenomena, including tornado and earthquake (Chap. 9), for ESF ductwork.

3. Thermal expansion.

4. Weight of the ductwork, including all attachments. 
5. Weight of personnel walking on large ductwork only. Where this situation is likely to occur, duct sections with exposed top surfaces should be capable of supporting a $250-1 \mathrm{~b}$ weight concentrated midway between the hangers or reinforcement, without permanent deformation. The out-of-roundness produced by such loading could lead to a sudden collapse of round duct when operating under negative pressure.

A maximum allowable stress of 0.7 times the elastic limit is recommended for the design of ductwork; maximum deflections under normal operating conditions should be

Rectangular duct: 0.125 in./ft of maximum unsupported panel span in the direction of airflow but not greater than 0.75 in. Deflection of reinforcement $-0.125 \mathrm{in}$./ $\mathrm{ft}$ of span but not more than $0.75 \mathrm{in}$. across total span.

Round duct: $0.0625 \mathrm{in}$./ft of diameter, but not more than 0.5 in. at any point.

\subsubsection{Materials of Construction}

Ductwork may be made from painted or coated carbon steel, galvanized steel, aluminum, stainless steel, or any combination of these materials required to resist corrosion in the service environment. Glassfiber-reinforced plastic (GFRP) and epoxy ducts have been used in corrosive environments where fire and safety requirements permit and may be less expensive than stainless steel, lined carbon steel, or epoxy- or vinyl-coated carbon steel. Although GFRP duct has been approved by the National Fire Protection Association and Underwriters' Laboratories for commercial and industrial use, ${ }^{12}$ even high-temperature resins will soften under brief exposure to temperatures of $350^{\circ}$ to $450^{\circ} \mathrm{F}^{13}$ Softening of GFRP duct can lead to rapid collapse or distortion, followed by loss of air cleaning function. GFRP and other plastic ductwork should not be used for level 3,4 , or 5 construction and should be used with caution for levels 1 and 2 .

\subsubsection{Paints and Protective Coatings}

Coating and paint requirements must be consistent with corrosion that can be expected in the particular application and with the size of the duct. Corrosionand radiation-resistant paints and coatings should, as a minimum, meet the requirements of ANSI N512 for "light exposure." " Unless special spray heads are used, spray coating of the interior of ducts smaller than $12 \mathrm{in}$. in diameter is often unreliable, because it is difficult to obtain satisfactory coating and to inspect for defects and "holidays." The interior of duct 8 in. and smaller cannot be satisfactorily brush-painted; therefore, dip coating is recommended. Ducts to be brush-painted should be no longer than 5 or $6 \mathrm{ft}$ to ensure proper coverage. When special coatings, such as the high-build vinyls and epoxys, are specified, the designer must keep in mind that the difficulties in surface preparation, application, and inspection may increase the cost of coated carbon steel to the point that stainless steel may be economically competitive, as well as perhaps providing better protection. An important point is that high-build coatings and paints can be damaged during handling and shipping and that corrosion can begin under such damaged areas without the user's knowledge. Painted and coated ductwork must be inspected carefully during the painting (coating) operation and also on receipt. Galvanized coatings and plates should also be carefully inspected, particularly on sheared edges and welds. Defects and "holidays" in such areas must be touched up with zinc-rich primer and top coat before the system is placed in service (see also Sect. 4.5.11).

\subsubsection{Hangers, Supports, and Anchors}

Non-ESF ductwork can be hung, supported, and anchored in accordance with the recommendations of Chap. 5 of the SMACNA High Velocity Duct Construction Standards, ${ }^{4}$ with the following exception: anchors and attachments which rely on an interference-fit between, or deformation of, the base material (concrete roof deck, beam, etc.) and the attachment device (as is the case for powder-actuated drive bolts and studs and for concrete anchors) should not be used for safety-related ductwork. Support requirements for ESF ducts and other ductwork that must remain in place in the event of an earthquake or major accident must be established by modeling or engineering analysis. Such analysis must be based on the inputs (forces, accelerations) to the building element to which the duct is fastened or from which it is hung (i.e., floor, wall, roof deck, etc.) that will be produced by a DBA or SSE, or both. NonESF ductwork located above or adjacent to other ESF equipment of the facility, which could damage such equipment if it fell, is also subject to this restriction.

\subsubsection{Acoustic Treatment of Duct}

Acoustic linings and duct silencers are not permitted in safety-related ducts or ducts which carry, or 
may carry, moisture or corrosive fumes. Acoustic treatment, if -required, must be attached to the exterior of the duct.

\subsubsection{Duct Leakage}

Leaktightness of ductwork, particularly in systems that carry or have the potential of carrying radioactive material, is extremely important. Duct leakage wastes power and thermal energy (i.e., energy required to heat, cool, or dehumidify air), causes noise, prevents correct airflow to outlets from inlets, makes difficult the balancing of systems and controlling of temperature and humidity, and produces unsightly dirt collections and radioactive contamination at leakage sites. The Carrier Corporation's System Design Manual, in discussing ductwork equivalent to levels 1 and 2, states: ${ }^{15}$

Experience indicates that the average air leakage from the entire length of low velocity [positive pressure] ducts, whether large or small systems, averages around $10 \%$ of the supply air quantity. Smaller leakage per foot of length for larger perimeter ducts appears to be counterbalanced by the longer length of run. Individual workmanship is the greatest variable, and duct leakages from $5 \%$ to $30 \%$ have been found.... High velocity duct systems [Level 2] usually limit leakage to $1 \%$.

Even $1 \%$ is excessive for systems that carry or have the potential of carrying intermediate- to high-level radioactivity. Leak rates based on the percentage of airflow are meaningless and subject to misinterpretation. Duct tightness is generally tested by sealing off sections of the system which are then individually tested by either the direct-measurement or pressuredecay method of ANSI N510. ${ }^{16}$ With such procedures, a leakage criterion based simply on percentage of airflow can produce anomalous results. By such a criterion, two duct systems built to the same construction standards and having the same volume and surface area but different airflow rates could have widely differing permissible leakages (PL). Conversely, if the airflow rates are the same but the volumes differ, they could have widely differing PLs. For this reason, a PL based on duct volume, as has been used in AEC (subsequently ERDA) installations for many years, or a PL based on the surface area of the pressure boundary of the section under test is recommended. Table 5.6 gives permissible leak rates for the various levels of construction, including the values that have been recommended over the years for nuclear grade ductwork. ${ }^{17}$ The values for levels 3, 4, and 5 ductwork are more stringent than those recommended for ductwork in nuclear power plants by ANSI N509.

The leak rates cited by Carrier $^{17}$ were for positivepressure ductwork; the same ducts, tested at the same degree of negative pressure, would have leaked many times more. In tests conducted at an ERDA facility, ${ }^{18}$ sections of level 2 ductwork tested alternately at 2.5 in.wg positive and $2.5 \mathrm{in}$.wg negative by the pressuredecay method showed no pressure loss in $15 \mathrm{~min}$ under positive pressure but a loss of $2 \mathrm{in}$.wg in $15 \mathrm{~min}$ under negative pressure. This tendency for the same ductwork to leak substantially more under negative pressure than under positive pressure is confirmed by SMACNA. ${ }^{19}$ It is recommended that leak tests be made under negative pressure if possible and at the normal discharge pressure or suction pressure of the fan insofar as is practicable. These leak rates are predicated on the potential for outleakage of contamination to occupied areas of the facility should the ductwork or filter housing become pressurized under system upset conditions.

\subsection{DAMPERS}

\subsubsection{Damper Specification}

Dampers are the valves of the air cleaning and ventilation system. By definition, a damper is any device that controls pressure, direction, or volume of airflow in a ventilation system, including those items normally classed as valves when used in piping systems. ${ }^{5}$ Clear, concise specifications must be established for mechanical strength, for leakage rate at maximum (i.e., DBA) operating conditions, and for the ability to perform under required operational and emergency conditions. Operability of linkages must be assured through specification of and requirement for cycling at minimum torque requirements under full load; static testing of the closed damper should be required, where applicable, for those to be used in critical applications to verify strength and leaktightness. All features important to proper operation should be stipulated in detail, including materials of construction, permissible lubricants, bearings, blade design and edgings (if permitted), indicating and locking quadrant, supports, operator type and capability, and the accessibility of operator, linkages, blades, and bearings for maintenance.

Factors that must be considered in the selection or design of dampers for nuclear applications include function of damper; type of construction; dimensions 
and space limitations; pressure drop across closed damper; normal blade operating position; method of mounting damper; blade orientation relative to damper case; operator type and power source; seismic requirements; requirements for position indicator, limit switches, and other appurtenances; configuration of damper; permissible leakage through closed damper; space required for service; airstream temperature range; orientation of damper in duct; direction of airflow; failure mode and blade position; maximum closing and opening times; and method of shaft sealing.

In conventional air conditioning and ventilating applications, procurement of dampers has generally been accomplished by specifying little more than the manufacturer's make and model number "or approved equal." This is poor specification practice under any circumstances and is inadequate for nuclear and other potentially high-risk applications. Therefore, a method of damper specification based on classification of important features was developed for this handbook. The classification method was further refined by ANSI Subcommittee N45.8 on components and testing of nuclear air cleaning systems and is included in ANSI N509. ${ }^{5}$ The classification enables the designer to make a rational selection of dampers, independent of manufacturer's make and model number, for a specific application. By appropriate selection of the classifications from Tables 5.7 through 5.11, a specification can be written to serve as the basis for both design and procurement.

\subsubsection{Description and Application of Dampers}

Requirements for a damper for a specific application can be stated by combining the classification symbols; for example, fc/so-1-C-III-E specifies a flow-control, shutoff, parallel-blade, industrialquality, leak group III damper with electric motor operator. Having established the requirements of the damper, plus the duct dimensions, airflow requirements, and system static pressure, the vendor can then select an item from his line which is suitable for the application. Table 5.12 gives recommended damper requirements for various types of nuclear air cleaning systems. Typical dampers used in critical service applications are shown in Figs. 5.2 and 5.3. A large butterfly damper is shown in Fig. 5.4.

\subsubsection{Damper Design and Fabrication}

Class A and B dampers differ only in that class A must meet the requirements for materials, fabrication, inspection, and testing of the ASME Code. ${ }^{20}$
Table 5.7. Classification of dampers by function

\begin{tabular}{|c|c|}
\hline Designation & Function \\
\hline fc & $\begin{array}{l}\text { Flow control damper. One which can be con- } \\
\text { tinuously modulated to vary or maintain a given } \\
\text { level of airflow in the system in response to a } \\
\text { feedback signal from the system or from a signal } \\
\text { fed to the damper operator by means of a } \\
\text { manually actuated control or switch. }\end{array}$ \\
\hline $\mathrm{pc}$ & $\begin{array}{l}\text { Pressure control damper. One which can be } \\
\text { continuously modulated to vary or maintain a } \\
\text { given pressure or pressure differential in the air } \\
\text { cleaning system or in a building space served by } \\
\text { the system in response to a signal. }\end{array}$ \\
\hline b & $\begin{array}{l}\text { Balancing damper. One which is set (usually } \\
\text { manually) in a fixed position to establish a } \\
\text { baseline flow or pressure relationship in the air } \\
\text { cleaning system or in building spaces served by } \\
\text { the system. }\end{array}$ \\
\hline so & $\begin{array}{l}\text { Shutoff damper. One which can be completely } \\
\text { closed to stop airflow through some portion of } \\
\text { the system, or opened partially or fully to permit } \\
\text { airflow (the fc damper may also serve this } \\
\text { function). }\end{array}$ \\
\hline $\mathrm{i}$ & $\begin{array}{l}\text { Isolation damper. A high-integrity shutoff } \\
\text { damper used to completely isolate a portion of a } \\
\text { system from a contained space or from the } \\
\text { remainder of the system with a leaktight seal. }\end{array}$ \\
\hline bd & $\begin{array}{l}\text { Back draft damper. One which closes } \\
\text { automatically or in response to a signal to prevent } \\
\text { flow reversal. }\end{array}$ \\
\hline $\mathrm{pr}$ & $\begin{array}{l}\text { Pressure-relief damper. One which is normally } \\
\text { closed but will open in response to overpressure } \\
\text { in the system or in the contained space served by } \\
\text { the system in order to prevent damage to the } \\
\text { system. }\end{array}$ \\
\hline
\end{tabular}

Class A and B dampers may be either forged or castbody, liquid-service, pipeline valves, or heavy-duty, industrial-quality, conventional units that can meet the leaktightness and pressure requirements as specified. Body and flanges of fabricated class B dampers are generally of welded construction and made from structural shapes; otherwise, requirements are similar to those for class $\mathrm{C}$ dampers.

Class $\mathrm{C}$ dampers are of heavy industrial-quality construction, with bodies made from structural channels or channels die- or roll-formed from heavy steel sheet or plate. The body should be no less than 4 in. wide with 1.25-in.-wide (or wider) flanges on both faces. Deflection of the body sides under the maximum differential pressure to which the damper will be subjected under normal or accident conditions should not exceed $0.3 \%$ of the length of the side. 
Table 5.8. Classification of dampers by configuration

\begin{tabular}{|c|c|}
\hline Designation & Configuration \\
\hline 1 & $\begin{array}{l}\text { Parallel blade damper. A multiblade damper } \\
\text { having blades that rotate in the same direction } \\
\text { (AMCA 500). }{ }^{a}\end{array}$ \\
\hline 2 & $\begin{array}{l}\text { Opposed blade damper. A multiblade damper } \\
\text { having adjacent blades that rotate in opposite } \\
\text { directions (AMCA } 500 \text { ). }{ }^{a}\end{array}$ \\
\hline 3 & $\begin{array}{l}\text { Butterfly damper. A heavily constructed } \\
\text { damper, often a valve used in piping systems and } \\
\text { usually round in cross section, designed for high- } \\
\text { pressure service ( } 25 \text { psi minimum pressure } \\
\text { rating), with one centrally pivoted blade that can } \\
\text { be sealed to meet the requirements of Leak Group } \\
\text { I (Table } 5.10 \text { ). }\end{array}$ \\
\hline 4 & $\begin{array}{l}\text { Single-blade balanced damper. A damper, } \\
\text { usually round in cross section, with one centrally } \\
\text { pivoted blade. }\end{array}$ \\
\hline 5 & $\begin{array}{l}\text { Single-blade unbalanced damper. An accurately } \\
\text { fabricated, often counterbalanced damper, usu- } \\
\text { ally rectangular in cross section, with one eccen- } \\
\text { trically or edge-pivoted blade. }\end{array}$ \\
\hline 6 & $\begin{array}{l}\text { Folding blade or wing blade damper. A damper } \\
\text { with two blades, pivoted from opposite sides of a } \\
\text { central post, which open in the direction of } \\
\text { airflow. }\end{array}$ \\
\hline 7 & $\begin{array}{l}\text { Poppet damper. A weight or spring-loaded pop- } \\
\text { pet device that opens when the pressure differen- } \\
\text { tial across it exceeds a predetermined value. }\end{array}$ \\
\hline 8 & $\begin{array}{l}\text { Slide or gate damper. A damper similar to a gate } \\
\text { valve, with a single blade that can be retracted } \\
\text { into a housing at the side of the damper to } \\
\text { partially or fully open the damper. }\end{array}$ \\
\hline
\end{tabular}

${ }^{a}$ AMCA 500, Test Methods for Louvers, Dampers, and Shutters, Air Moving and Conditioning Association, Arlington Heights, Ill., 1975.

Blade shafts should be made from solid steel bar and should extend the full width of the blade and journals. Blades should be made from heavy steel sheet (generally at least No. 11 U.S. gage) or plate and should be welded or through-bolted to the shafts in such a manner that the integrity of the attachment can be verified by visual inspection after assembly of the damper. Deflection of blades and shafts should be no more than $0.3 \%$ of the free span with the blades in the closed position and under a differential pressure of at least 1.5 times the design pressure to which they will be subjected under maximum service conditions (DBA conditions for those dampers that must operate during and following an emergency or accident). Sealing materials applied to blade edges
Table 5.9. Classification of dampers by construction

\begin{tabular}{cl}
\hline Designation & \multicolumn{1}{c}{ Construction } \\
\hline A & Code damper. A valve meeting the requirements \\
& for class 2 or 3 components (whichever is \\
& specified) of Sect. Ill of the $A S M E$ Boiler and \\
& Pressure Vessel Code ${ }^{a}$ or a heavy-duty \\
& fabricated-construction damper designed for a \\
& service rating of at least 25 psi, having a die- \\
& formed or structural-shape body and flanges and \\
& meeting the requirements for materials, fabrica- \\
& tion, inspection, and testing in accordance with \\
& the requirements for class 2 or 3 valves of Sect. III \\
& of the $A S M E$ Boiler and Pressure Vessel Code. \\
& High-pressure damper. A valve meeting the re- \\
& quirements of ANSI B31.1; or a heavy-duty \\
fabricated-construction damper designed for a & service rating of at least 25 psi, having a die- \\
& formed or structural-shape body and flanges and \\
& meeting the requirements for materials, fabrica- \\
tion, and inspection of ANSI B31.1.
\end{tabular}

${ }^{a}$ ASME Boiler and Pressure Vessel Code, Sect. III, Div. 1, "Nuclear Power Plant Components," Subsection NC, "Class 2 Components," and Subsection ND, "Class 3 Components," American Society of Mechanical Engineers, New York, current issue.

${ }^{b}$ ANS1 B31.1, Power Piping, American National Standards Institute, New York, current issue.

and seats must be radiation-resistant and readily replaceable. Linkages of class $C$ dampers should be located outside the airstream, should be made from steel bar, and should be structurally designed to transmit at least twice the maximum force that can be produced by the operator without exceeding an allowable stress of 0.7 times the yield strength of any part. The minimum length of any linkage arm of an industrial-quality damper should be 3 in. Bearings should be of the flange-mounted, lubricantimpregnated, sintered-bronze journal type or rollingelement type and should be designed to operate at the specified service temperature or $200^{\circ} \mathrm{F}$, whichever is higher. Rolling-element bearings should be used for service temperatures higher than $200^{\circ} \mathrm{F}$ and should have grease fittings that are accessible from the outside of the damper after it has been installed in the duct system. Shafts must be sealed to maintain a 
Table 5.10. Classification of dampers by leaktightness

\begin{tabular}{|c|c|c|c|c|c|}
\hline \multirow{3}{*}{$\begin{array}{l}\text { Damper blade } \\
\text { length or diameter } \\
\text { (in.) }\end{array}$} & \multicolumn{5}{|c|}{ Designation } \\
\hline & \multicolumn{5}{|c|}{$\begin{array}{c}\text { Maximum permissible leak rate }\left(\mathrm{scfm} / \mathrm{ft}^{2}\right) \text { of internal cross section } \\
\text { at } 1 \text { in.wg } \Delta p \text { across closed damper }{ }^{a}\end{array}$} \\
\hline & Group I & Group I-A & Group II & Group III & Group IV \\
\hline 12 & Bubble-tight & 2 & 15 & 60 & $b$ \\
\hline 24 & at pressure & 3 & 10 & 40 & $b$ \\
\hline 36 & specified by & 4 & 8 & 32 & $b$ \\
\hline 48 & purchaser & 4 & 6 & 32 & $b$ \\
\hline 60 & & 4 & 6 & 27 & $b$ \\
\hline 72 & & 4 & 5 & 25 & $b$ \\
\hline
\end{tabular}

\footnotetext{
${ }^{a}$ Leak tests are not applicable to balancing dampers unless specified by purchaser. Interpolation may be used to find permissible leak rates for intermediate-size dampers. Use multiplying factors below to find permissible leak rates at higher differential pressures; class $A$ and $B$ dampers should be tested at design pressure or $12 \mathrm{in.wg}$, whichever is lower. Class $C$ dampers should be tested at design pressure.

${ }^{b}$ Damper leakage is not a factor; a leak test is not required.
}

$\begin{array}{cccc}\text { Differential pressure } & \text { Multiplying factor } & \text { Differential pressure } & \text { Multiplying factor }\end{array}$

\begin{tabular}{cc|cc}
\hline 2 & 1.4 & 7 & 2.6 \\
3 & 1.7 & 8 & 2.8 \\
4 & 2.0 & 9 & 3.0 \\
5 & 2.2 & 10 & 3.2 \\
6 & 2.4 & 12 & 3.5 \\
\hline
\end{tabular}

Table 5.11. Classification of dampers by operator type

\begin{tabular}{cl}
\hline Designation & \multicolumn{1}{c}{ Operator type } \\
\hline M & $\begin{array}{l}\text { Manual operator-lever on damper with in- } \\
\text { dicating quadrant }\end{array}$ \\
C & $\begin{array}{l}\text { Manually controlled chain operator to permit } \\
\text { remote adjustment }\end{array}$ \\
E & Electric motor operator \\
H & Hydraulic operator \\
P & Air (pneumatic) operator \\
\hline
\end{tabular}

degree of leaktightness commensurate with leakage for level 3, 4, and 5 duct systems.

Class D dampers are of light sheet-metal, commercial-quality construction. Dampers for level 2 and higher ductwork must have flanges to permit mounting between sections of duct. Flange installation is also preferred in level 1 ductwork. Damper body should be made from No. 16 U.S. gage or heavier sheet metal, die- or roll-formed into channel cross section. Flange width should be $1 \frac{1}{4}$ to $1 \frac{1}{2}$ in. as required to meet duct construction standards (Fig. 3-15 in ref. 4, SMACNA High Velocity Duct Construction Standards). Singlethickness blades should be made from at least No. 16

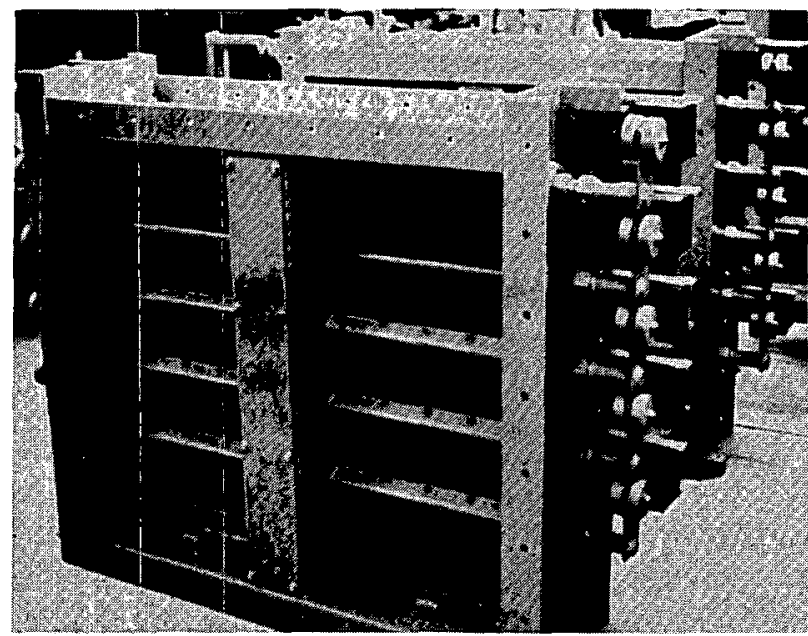

Fig. 5.2 Heavy-duty, industrial-grade, parallel blade damper.

U.S. gage sheet metal, and double-thickness blades should be at least 18 gage. The maximum unsupported blade length should be 48 in., and the blade width of multiblade dampers should not exceed $9 \mathrm{in}$. Shafts should be at least $7 / 16$ in. in diameter and fitted with lubricant-impregnated, sintered-bronze journal bearings or rolling-element bearings. Linkages may be mounted either in or outside of the 
Table 5.12. Damper requirements ${ }^{a}$

\begin{tabular}{|c|c|c|c|c|c|}
\hline \multirow{3}{*}{ Damper function ${ }^{b}$} & \multicolumn{5}{|c|}{ Damper properties ${ }^{c}$} \\
\hline & \multicolumn{5}{|c|}{ Level of duct system (Table 5.6) } \\
\hline & 1 & 2 & 3 & 4 & 5 \\
\hline \multicolumn{6}{|l|}{ Flow or pressure control } \\
\hline Construction class & D & D & D & $\mathrm{C}$ & $\mathrm{A}, \mathrm{B}$ \\
\hline Leakage group & III & III & Il & II & I \\
\hline Suggested configuration & $2,4,8$ & 2,4 & 2,4 & 2,4 & 2,4 \\
\hline \multicolumn{6}{|l|}{ Balancing } \\
\hline Construction class & $\mathrm{D}$ & $\mathrm{D}$ & $\mathrm{D}$ & $\mathrm{C}$ & $\mathrm{A}, \mathrm{B}$ \\
\hline Leakage group & IV & IV & IV & IV & VI \\
\hline Suggested configuration & $1,4,8$ & 1,4 & 1,4 & $1,2,4$ & $1,2,4$ \\
\hline \multicolumn{6}{|l|}{ Shutoff } \\
\hline Construction class & $\mathrm{D}$ & $\mathrm{D}$ & $\mathrm{D}$ & $\mathrm{B}, \mathrm{C}$ & $A, B$ \\
\hline Leakage group & III & III & II & I-A & I \\
\hline Suggested configuration & $1,2,4,8$ & $1,2,4$ & $1,2,4$ & 3 & 3 \\
\hline \multicolumn{6}{|l|}{ Containment isolation } \\
\hline Construction class & & & & A & A \\
\hline Leakage group & & & & I & 1 \\
\hline Suggested configuration & & & & 3 & 3 \\
\hline \multicolumn{6}{|l|}{ Unit or system isolation } \\
\hline Construction class & $\mathrm{D}$ & $\mathrm{C}$ & C & B & $\mathrm{A}, \mathrm{B}$ \\
\hline Leakage group & II & I-A & I-A & 1 & 1 \\
\hline Suggested configuration & $1,2,4,8$ & $1,2,4,8$ & $1,2,4$ & 3 & 3 \\
\hline \multicolumn{6}{|l|}{ Back draft prevention } \\
\hline Construction group & D & D & D & C & $\mathrm{A}, \mathrm{B}$ \\
\hline Leakage class & III & III & II & I-A & 1 \\
\hline Suggested configuration & $1,5,6$ & $1,5,6$ & $1,5,6$ & $1,5,6$ & 5,6 \\
\hline \multicolumn{6}{|l|}{ Pressure relief } \\
\hline Construction group & D & D & D & & \\
\hline Leakage class & III & II & I-A & & \\
\hline Suggested configuration & $1,5-7$ & $1,5-7$ & $1,5-7$ & & \\
\hline
\end{tabular}

${ }^{a}$ Requirements for damper classifications as defined in Sect. 5.3.

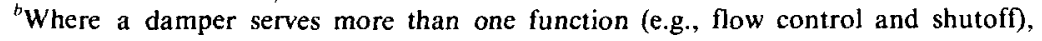
requirements for the more stringent service apply.

'Configuration other than those shown may be used if leakage characteristics and construction are equivalent or better.

airstream and should have a minimum lever-arm length of $1 \mathrm{in.}$ in any member.

\subsubsection{Damper Operator}

In most cases operators should be installed outside of the airstream, and they should always be factorymounted by the damper manufacturer. Torque requirements based on operating conditions specified by the user should be established by the damper manufacturer. The operator should be capable (1) of producing a minimum of 1.5 times the torque required to move the blades from full-open to fullclosed and (2) of meeting the specified leaktightness in the closed position under the maximum service conditions (normal operating or system upset, whichever is specified) the damper will have to operate. Dampers in which the blade shafts penetrate the body should have a mechanical positionindicating device (e.g., pointer and escutcheon plate). Dampers that will be controlled remotely should also be equipped with switches, relays, or other devices to produce a position-indication signal that can be transmitted to the central control station.

\subsubsection{Qualification and Acceptance Testing}

Qualification consists of prototype or preproduction model tests to verify design and/or to establish 


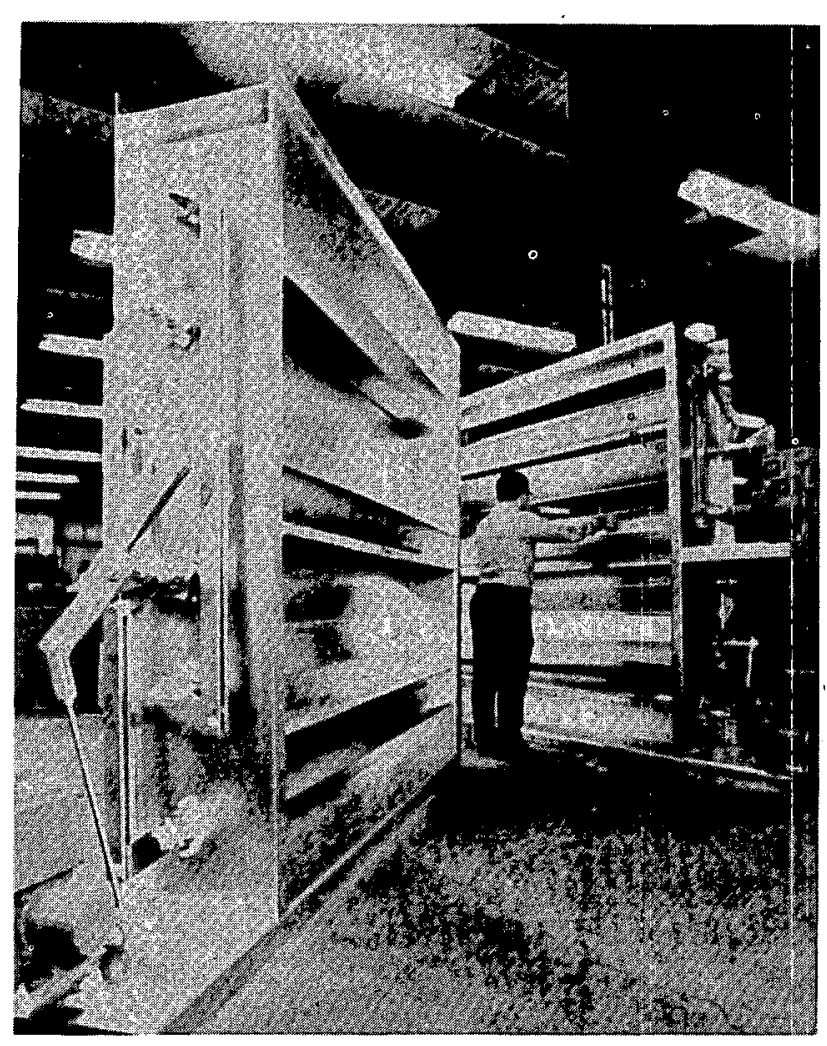

Fig. 5.3 Heavy-duty, industrial-grade, opposed blade dampers for nuclear service. Courtesy American Warming and Ventilating Co.

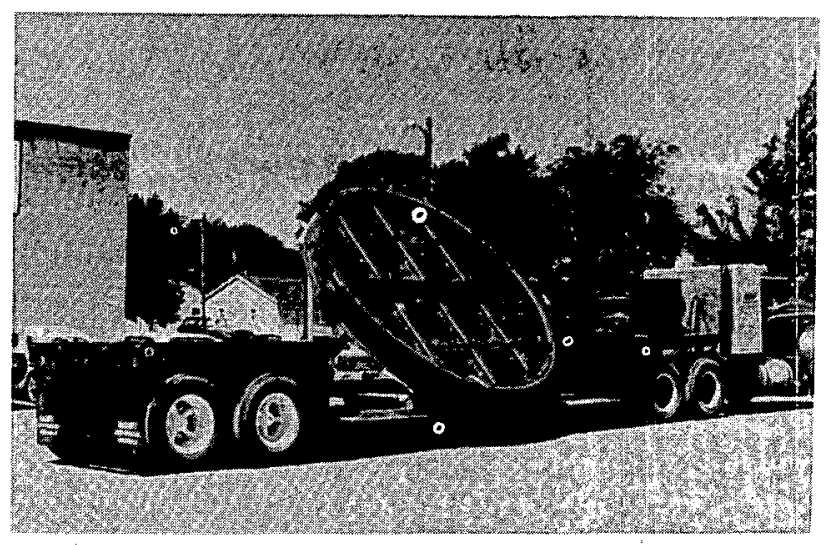

Fig. 5.4. Large butterfly damper for nuclear isolation service. Courtesy American Warming and Ventilating Co.

performance and operational characteristics of the design. In the case of AMCA-rated dampers, ${ }^{21}$ these tests consist essentially of pressure-drop and airflow determinations at various degrees of blade opening. ${ }^{22}$ The AMCA rating is generally sufficient evidence that suitable qualification tests have been made. For dampers not listed by AMCA, the manufacturer should be required to provide performance data obtained under conditions equivalent to those used in the AMCA test code. ${ }^{22}$ For valves (construction classes $A$ and $B$ ), equivalent test data are often available for both water and gas flow. A particularly important piece of information to be obtained by qualification testing is the resistance of the fully open damper and the resistance vs blade-position curve from full-open to full-closed. These resistances must be included in the air cleaning system design calculations in the same manner as other system resistances.

Production units are subject to acceptance tests to verify that the units are in good operating condition. Repetition of qualification tests to demonstrate operational characteristics is generally unnecessary and unwarranted. A damper to be used in a level 4 or 5 duct system that will be or may be exposed to high levels of contamination should be cycled through the full range at least 25 times, with all accessories attached, to verify the free and correct operation of all parts and the correct adjustment, positioning, and seating of the blades. Adjustments should be made as necessary during the test to correct deficiencies. Shop leakage tests may be desirable, particularly for zeroleak (group I) and some group II dampers. Such tests should be made after successful completion of the cycling test. Dampers that perform a containmentvessel-isolation function should always be leak-tested in the shop prior to acceptance. Because damper operators are generally furnished to the damper manufacturer as a purchased item, a test to verify the torque characteristics of the operator, after installation on the damper in its service position, is desirable, particularly for control, shutoff, and isolation dampers at critical locations in the system.

\subsection{FANS}

\subsubsection{Fan and System Curves}

A major requirement for a fan operating in a highefficiency air cleaning system is its ability to perform safely and efficiently over a much larger variation of resistance than more conventional ventilation systems. This variation of resistance is caused by dust loading of the HEPA filters and may double from the time of filter installation to the time of filter change, or may increase as much as five times in some systems (see discussion of operation to high pressure drop, Sect. 2.3.5). The increase in resistance across the HEPA filters is usually the major factor influencing 
the pressure-flow relationships (represented by the numbered curves in Fig. 5.5) of high-efficiency air cleaning systems. Fan performance (airflow vs pressure capability) and system resistance vs airflow are represented by characteristic curves such as curves A, 1, and 2 of Fig. 5.5. The volume of air that can be delivered by the fan is determined by the intersection of the fan and system characteristic curves; the flow represented by this point of intersection is the only flow that can be delivered by the fan under the given operating conditions. In most cases, a fan with a steeply rising characteristic (curve A, Fig. 5.5 ) is desirable to maintain reasonably constant airflow in the system over the entire life of the HEPA filters. If a fan with a broad, flat characteristic is chosen, it will be less able to deliver the required airflow as the filters become dust-loaded (curve 1 to curve 2), and either system performance (i.e., airflow) or filter life will have to be sacrificed. Any decrease in filter life will, of course, be accompanied by higher change frequency and therefore increased operating (maintenance) costs. If a pressure-equalizing device (damper) is installed to balance system pressure against filter pressure drop in order to maintain a

ORNL-DWG 69-8709A

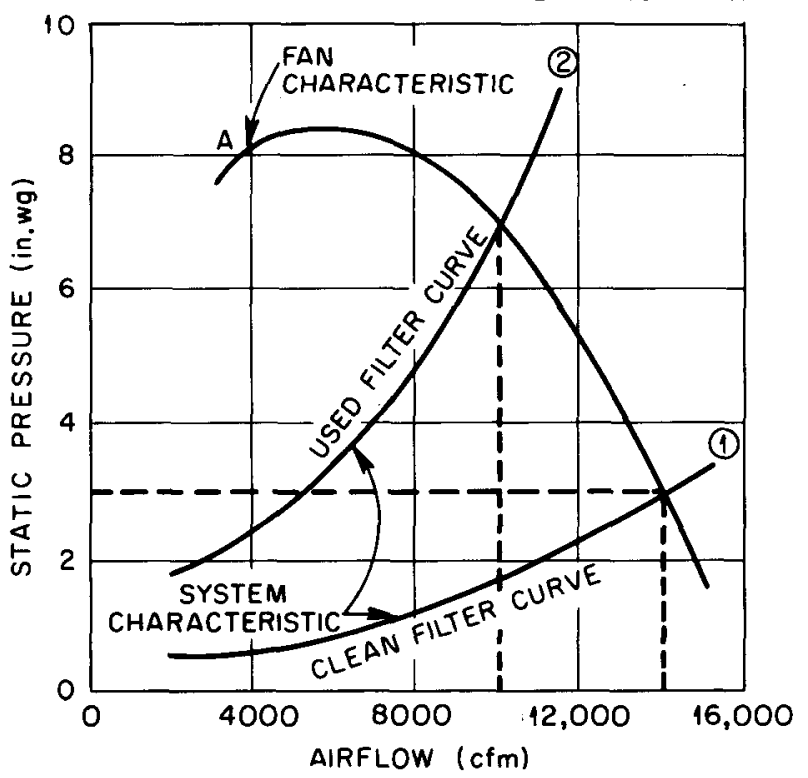

Fig. 5.5. Fan and system characteristic curves showing relationships between airflow delivery and resistance. With no dampers to adjust system resistance, airflow drops from 14,000 $\mathrm{cfm}$, when new filters are installed (intersection of curves $\mathrm{A}$ and 1 , $\Delta p=3$ in.wg) to $10,000 \mathrm{cfm}$ when they are replaced at an economic filter resistance increase of 4 in. $\mathrm{wg}$ (intersection of curves A and 2 , system $\Delta p=7$ in.wg). constant pressure-airflow relationship in the system, a penalty in operating (power) costs will result.

\subsubsection{Fan Performance-System Effect}

The inability of fans to perform in the field in accordance with published ratings has long troubled the industry. The problem arises partly because the ratings are based on idealized laboratory conditions that are rarely encountered in the field, and partly because of design and/or field compromises made to accommodate the field situation. Many of the problems of fan operation stem from poorly designed connections to the duct. Close-coupling, too short transitions between unmatched (in size) duct and fan inlets, square-to-round connections, and poorly designed inlet boxes create a vortical or eccentric flow into the fan impeller and result in noise, vibration, and reduced efficiency. A $45^{\circ}$ spin in the direction opposite fan rotation may reduce fan delivery as much as $25 \%$ and require a compensating increase in fan pressure of from 50 to $55 \%$. Figure 5.6 shows the effects of various inlet conditions on fan performance and the resultant increase in fan capability (fan static pressure) to compensate for these effects. Too often these effects are not taken into account when calculating fan requirements, with the result that neither the fan nor the filters can perform to the desired design levels. Outlet connections also affect fan performance, as Fig. 5.7 shows.

To alleviate the situation, AMCA has published a Fan Application Manual, Part 1 of which includes a set of "system effect curves" by which the designer can predict the effects of design features (such as the inlet and outlet conditions illustrated in Figs. 5.6 and 5.7) on fan performance and, when needed, make allowance for them in initial fan selection. ${ }^{23}$ System effects are the losses in fan performance that result from the fan being installed in a less than ideal configuration. These effects must be taken into account by the designer if a realistic estimate of fan performance under conditions of the "real life" system is to be made. Figure 5.8 illustrates a deficient fan-system interaction resulting from one or more undesirable design conditions. The assumption is made that pressure losses in the duct system have been accurately estimated (point 1 , curve $\mathrm{A}$ ), and a suitable fan, based on published ratings, has been selected for operation at that point. However, no allowance has been made for the effect of the fan connections on fan performance, that is, of the interaction between the fan and the system as designed. To compensate for the system effect loss 


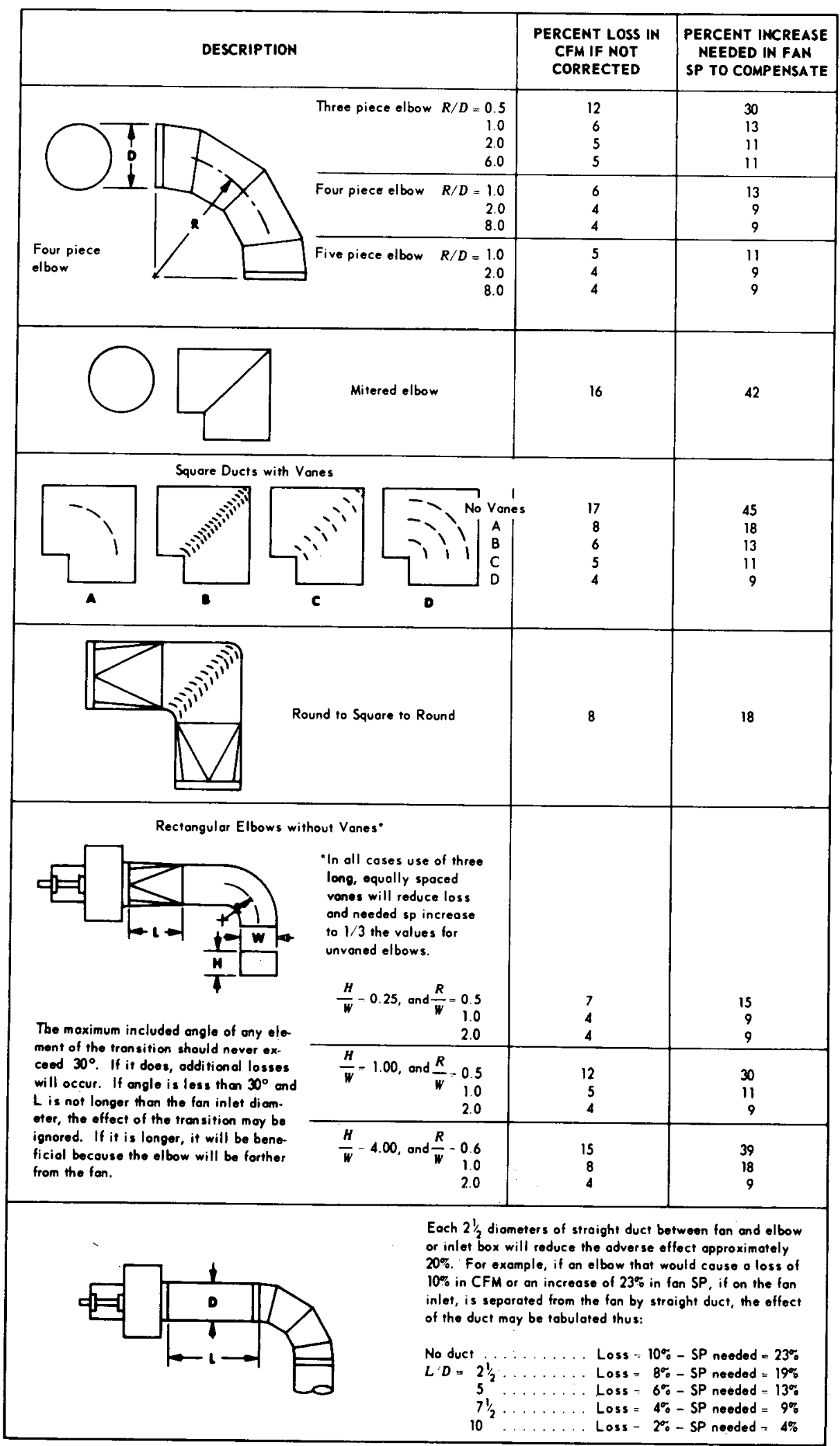

Fig. 5.6. Effect of fan inlet on fan performance. From C. J. Trickler, "Is the System Correctly Designed," Air Cond. Heat. Vent. 57,87 (May 1960). 


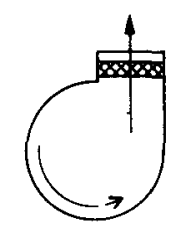

LOSS $=50 \%$ OF FAN OUTLET VELOCITY PRESSURE
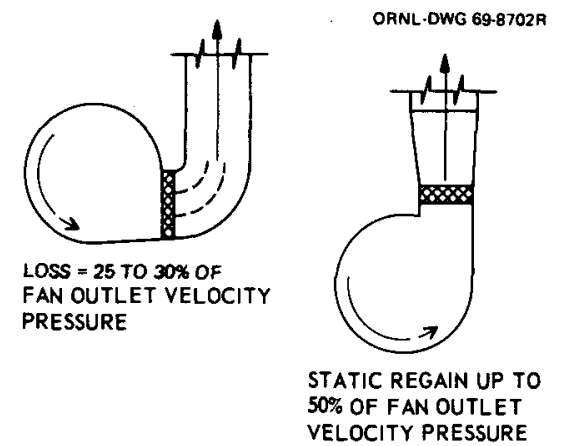

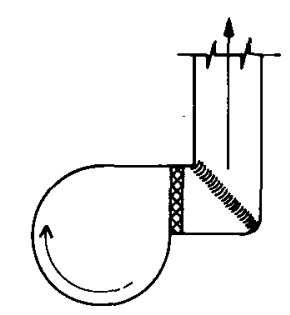

LOSS $=100 \%$ OR MORE OF FAN OUTLET VELOCITY PRESSURE

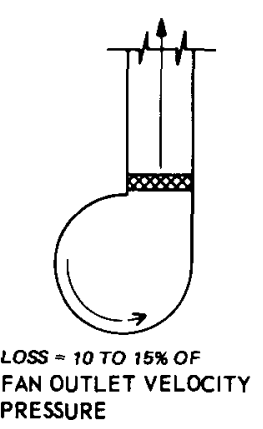

PRESSURE
Fig. 5.7. Effect of fan outlet connection on fan performance.

because of the unfavorable interaction between the fan and its connections, it will be necessary to add a system effect factor to the calculated system pressure losses to determine the actual system characteristic curve and to determine the actual fan required to produce the required operating characteristics.

Testing to establish the capability of the fan in a nuclear air cleaning system as originally installed is recommended by ANSI N510. ${ }^{16}$ Part 3 of the AMCA Fan Application Manual ${ }^{24}$ provides guidelines for such testing, including examples of the application of system effect factors for various system configurations. Planes of measurement, measurements to be made, average test readings, calculation of test results, and corrections to overcome deficiencies disclosed by the tests are all covered in detail. It is preferable to apply system effect factors before selection, purchase, and installation of a fan to prevent the incorporation of unfavorable features into the system design. In applying system effect factors, it must be recognized that those factors given in the AMCA manual are only guidelines and general approximations, although many have been obtained from research studies. Fans of different types and fans of the same type, but made by different manufacturers, will not necessarily interact with the system in exactly the same way. It is necessary, therefore, to apply judgment based on experience in applying system effect factors. The appendixes to Part 3 of the AMCA Fan Application Manual $^{24}$ provide the background for such judgment factors.

\subsubsection{Multiple Fan Installation}

The installation of two fans in series is sometimes desirable where a steeply rising pressure-airflow characteristic is needed; ${ }^{25}$ however, caution must be exercised in such design. In theory, the combined pressure-volume characteristic of two fans operating in series is obtained by adding the fan pressures at the same volumetric airflow as shown in Fig. 5.9. Care must be taken in designing the connection between the fans, because a significant loss of efficiency can occur in the second-stage fan due to nonuniform flow into its inlet, particularly if the two fans are closely coupled.

Two or more fans are often operated in parallel to move large volumes of air, to enhance the control of segmented air cleaning facilities, or to limit the installed capacity (i.e., filters, adsorbers) of any one unit of the air cleaning system. The combined volume-pressure curve in this case is obtained by adding the volumetric capacity of each fan at the same pressure (Fig. 5.10). If the ducts that feed the fans are independent (as, for example, arrangements 1c, $2 c$, or 3c of Fig. 2.9), the outputs of the fans will be additive. If the fans are fed from a common inlet box, as shown in Fig. 5.11, adverse interaction can take place, which may substantially reduce the output of the combination.

One concern in parallel fan installations is that some fans have a positive slope in their characteristic curves to the left of the peak pressure point (Fig. 5.10). If the fans are operated in the pressure-volume regime of this positive slope, unstable operation may result, as shown by the closed loop to the left of the peak pressure point in Fig. 5.10 (this loop is obtained by plotting all of the possible combinations of flow at each pressure). If the system characteristic curve intersects the fan characteristic in the area of this loop, more than one point of operation is possible; this may cause one of the fans to handle more of the system load (airflow) than the other and result in a motor overload. The unbalanced flow condition tends to shift rapidly so that the fans intermittently load and unload. The pulsing that results from such loading and unloading generates noise and vibration and may cause damage to the fans, motors, 


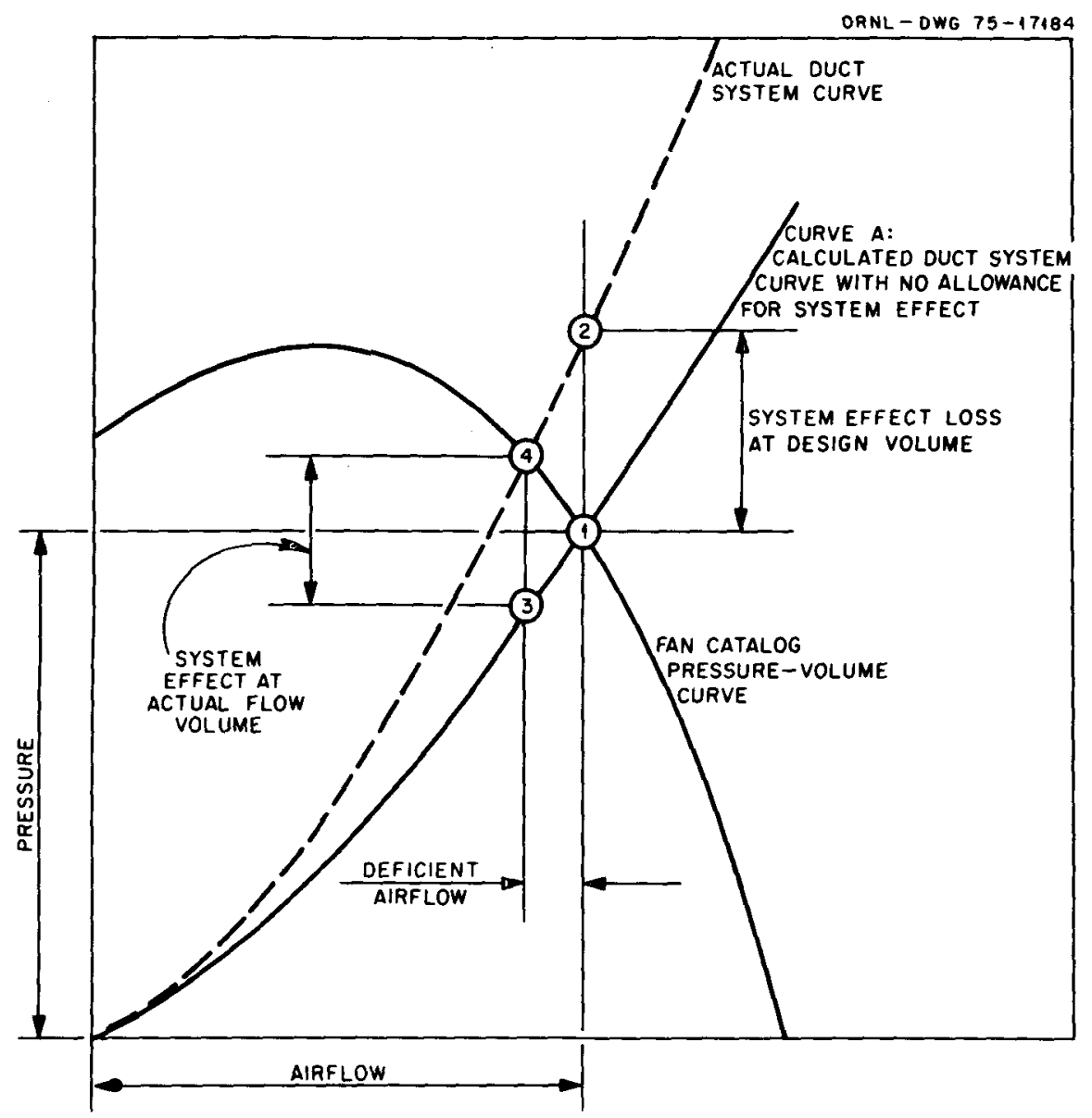

Fig. 5.8. Result of selecting a fan on the basis of manufacturer's ratings. Poor interaction between selected fan and system, due to poor inlet and/or outlet design, results in system effect loss at design volume. This loss must be added to the calculated system resistances to determine the total system losses on which to base fan selection. Courtesy Air Moving and Conditioning Association.

ductwork, or filters. Aileron controls in the fan outlets, or the provision of dampers near the inlets or outlets, may sometimes be sufficient to correct unbalanced flow or to eliminate pulsing and/or reversing operation.

\subsubsection{Fan Capacity}

Experience has shown that fans are often unable to meet the actual demands of the system as they are installed; therefore, sacrifices in performance and efficiency result. Poor fan selection may be a result of either underestimating actual system losses or failure to recognize fan/system interaction (i.e.; system effects). In push-pull systems (i.e., systems containing both supply and exhaust fans that operate at the same time), exhaust fan capacity should be at least $10 \%$ greater than supply fan capacity to compensate for infiltration, pressure surges, wind effects (i.e., pressure variations in the building and ductwork due to variable wind conditions exterior to the building), and temperature variations, and to eliminate any possibility of overpressurizing the building by the supply fans.

Improper fan operation can be avoided by carefully evaluating system pressure drops and interactions under all predictable operating conditions and by specifying a type and size of fan that matches the demands of the duct system as installed. Control must be exercised over the installation of ducts and fans to preverit field compromises that can only result in the reduced ability of the system to perform as intended.

\subsubsection{Fan Reliability and Maintenance}

Air movers in nuclear air cleaning facilities must provide trouble-free reliable service, often for long periods of time and with a minimum of maintenance. Years of successful operation have proven that 


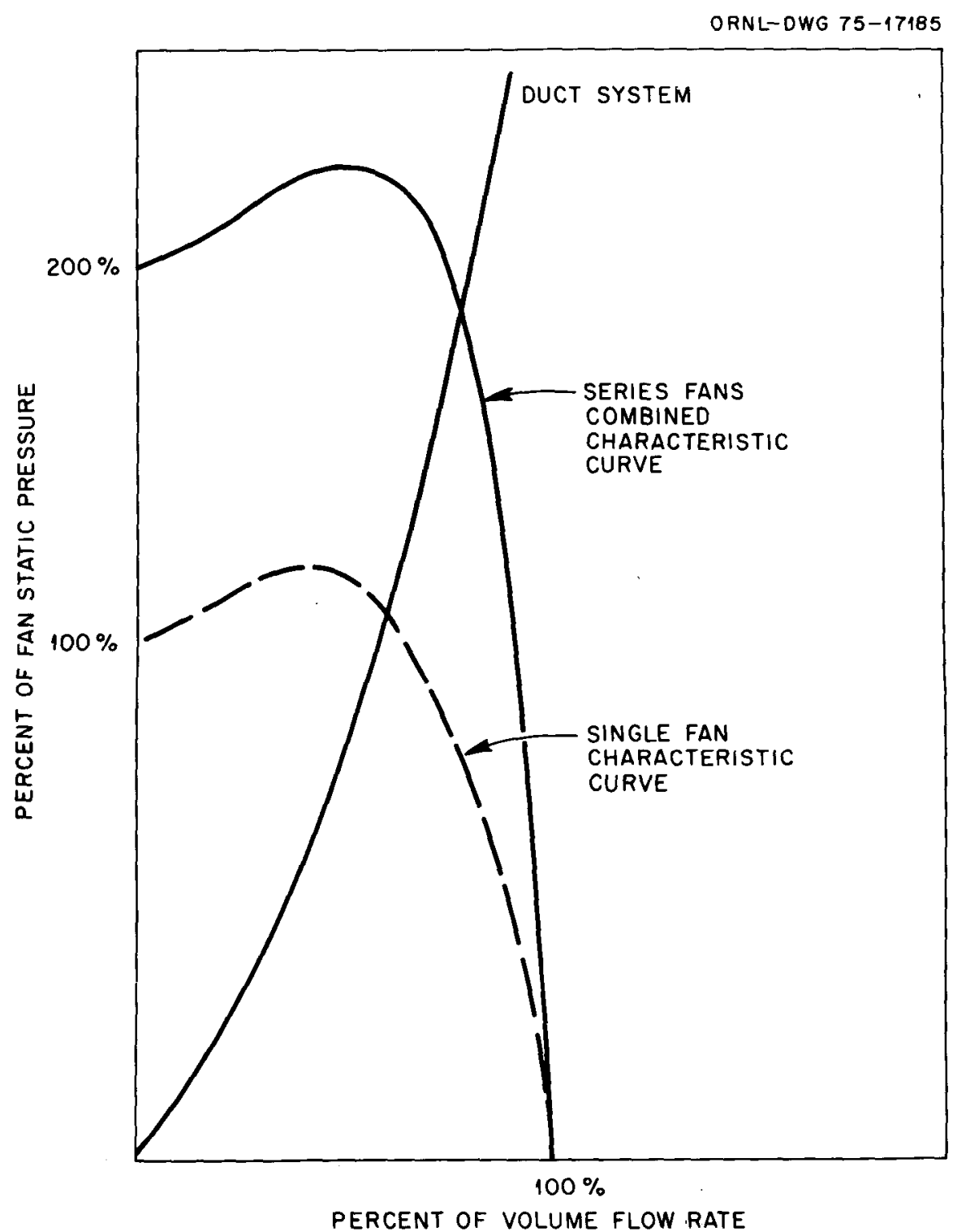

Fig. 5.9. Typical characteristic curve of two fans operating in series. From the AMCA Fan Application Manual.

carefully chosen centrifugal fans are capable of providing such service. In recent years, in-linecentrifugal and vane-axial fans have also been used successfully in an increasing number of applications. These fans require less volume and space for a given volume-pressure duty, and their installed weight is generally less than other conventional-air-moving devices. In addition, because of the straight-through design, they can withstand shock waves in the duct system better than the conventional centrifugal fan, and they can tolerate high humidities and temperatures without failure or loss of efficiency.
This ability to endure severe duty has been proven in tests designed specifically to simulate all conditions, including seismic shaking, which are likely to be encountered in a nuclear reactor containment during and after a DBA. With an integral semiopen motor of proper design that incorporates radiation-resistant windings and insulation, an independent cooling water system is not required, nor is a sealed-off environment, for the motor. The design requires no heavy complicated support structure to satisfy seismic or other loadings postulated for the most stringent service in a nuclear facility and is simple to 


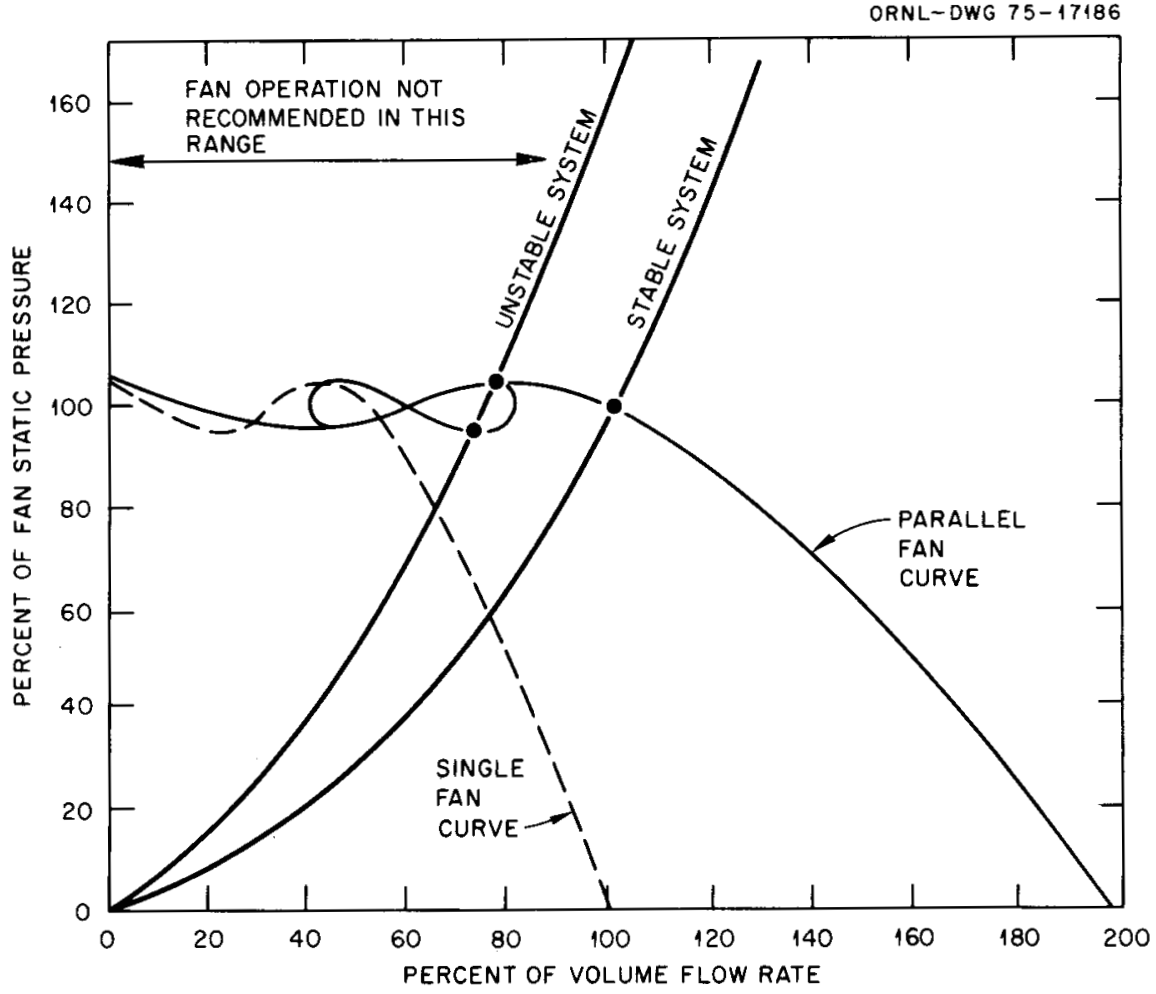

Fig. 5.10. Typical characteristic curve of two fans operating in parallel. From the AMCA Fan Application Manual.

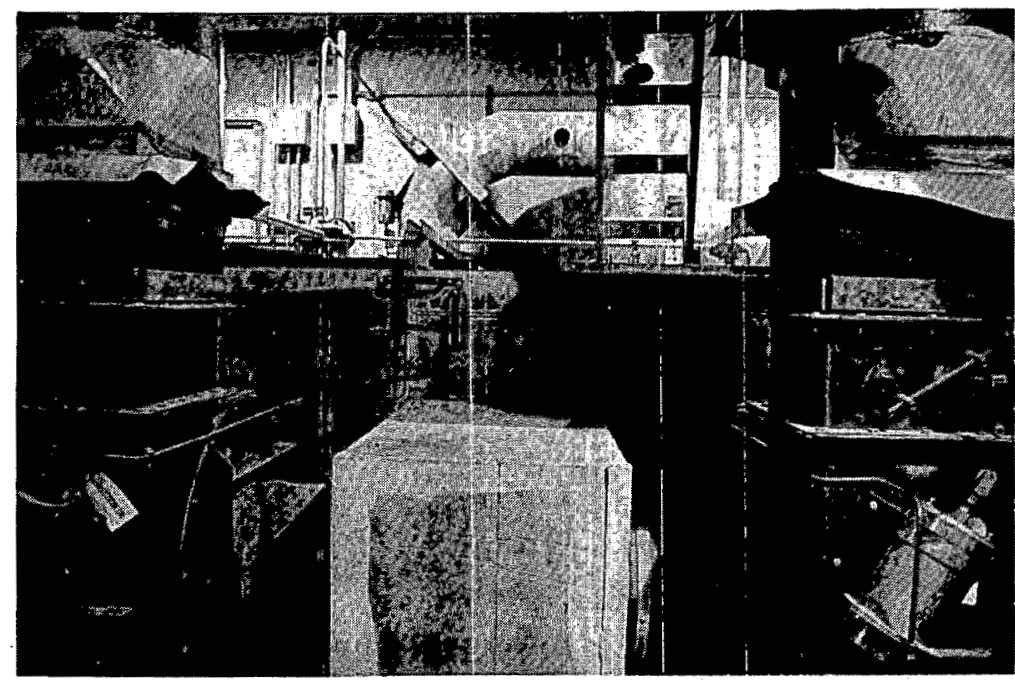

Fig. 5.11. Parallel fans with common inlet box.

operate and maintain. Additional information on fan types and their application is given in refs. 26 through 29.

Fans for nuclear power plant postaccident cleanup systems present special problems. If the fan and motor are located inside the primary containment, they must be able to operate continuously for long periods of time at both normal containment conditions (usually negative pressure with respect to atmosphere and temperatures of 100 to $120^{\circ} \mathrm{F}$ ) and following a DBA or SSE. The DBA service environment includes pressure as high as $50 \mathrm{psig}$, temperature 
as high as $280^{\circ} \mathrm{F}$, air density as much as three times normal, and exposure to heavy-rain conditions if containment sprays are provided. Water cooling may be required for the motor(s), with an alternate water supply and an alternate power supply in the event of failure of the normal supply. Alternate-redundant air cleaning units, fans, motors, or combinations of these may be required to ensure high probability of continuous function in the event of a maximum incident (i.e., DBA or SSE). Because motors and fans to meet these conditions require special design, the plant owner must obtain documented proof, based on reliable model or prototype testing, of their ability to perform under such conditions, including calculations and test data pertaining to all components. Acceptance tests are essential to verify that the equipment, as furnished, is capable of performing as promised by the qualification test data. In addition, regular and routine operational checks should be made in accordance with a preplanned schedule to verify the continued reliability of the system.

Dependability of operation is an important consideration in the selection of fans for nuclear applications. Even when the system is planned for part-time or intermittent operation, continuous operation may be required after the system goes into service and should be considered as the norm for design purposes. Savings in capital costs achieved through the specification of light-duty equipment are offset quickly by high maintenance costs after the system goes into service, even for a brief period. Roller bearings are preferable to journal bearings in fans and motors because of their superior operating characteristics, lower maintenance, and greater availability of replacements. Direct drive is generally more reliable than V-belt drive, although it is not as flexible for the adjustable flow rates often demanded by changing system requirements in laboratory and experimental facility applications. When V-belt drive is specified, at least $25 \%$ extra belt capacity should be required over that required to carry the starting load of the motor; this extra capacity gives better wear characteristics and ensures continued operation in the event of partial belt failure. AMCA drive arrangement 4 or 8 is recommended. ${ }^{29}$

\subsubsection{Fan Installation}

Proper mounting of the fan will minimize noise and vibration and reduce maintenance costs. Noise is objectionable in supply and exhaust systems and is often difficult and costly to eliminate after the system goes into service. Excessive noise in exhaust and air cleanup systems is often accompanied by vibration and pulsation that may be harmful to filters, adsorbers, and other components. Flutter or "reeding" of HEPA filter separators, for example, is a common cause of filter failure, and vibration of activatedcarbon-filled adsorbers can cause settling and crushing of the granules and, eventually, carbon loss that can cause bypassing of contaminated air.

When practicable, mounting of the fan and motor on a common base designed for isolation of vibration is recommended. Figure 5.12 shows a typical base for large fans. The fan and motor are mounted on a concrete pad that acts as an inertial mat to limit the amplitude of vibration and to dissipate vibrational energy. The pad is mounted on spring isolators, which will provide a high degree ( $99 \%$ or more) of vibrational damping. For some systems, positive amplitude limiters may be required to restrain the base from excessive movement under extreme conditions (such as the accelerations imposed by an SSE or DBA). In some cases, fans may be hard-mounted, as shown in Fig. 5.13. Careful balancing of the fan shaft and impeller to minimize vibrations that cannot be isolated via installation design is particularly important in this latter design.

Floors and walls adjacent to the fan and filter housing should be designed for minimum resonance. This can be done for simple structures by first determining floor deflection through the use of standard beam formulas ${ }^{30}$ and then by determining the vibrational frequency of the structure from the equation $^{31}$

$$
f=\frac{187.7}{y^{1 / 2}}
$$

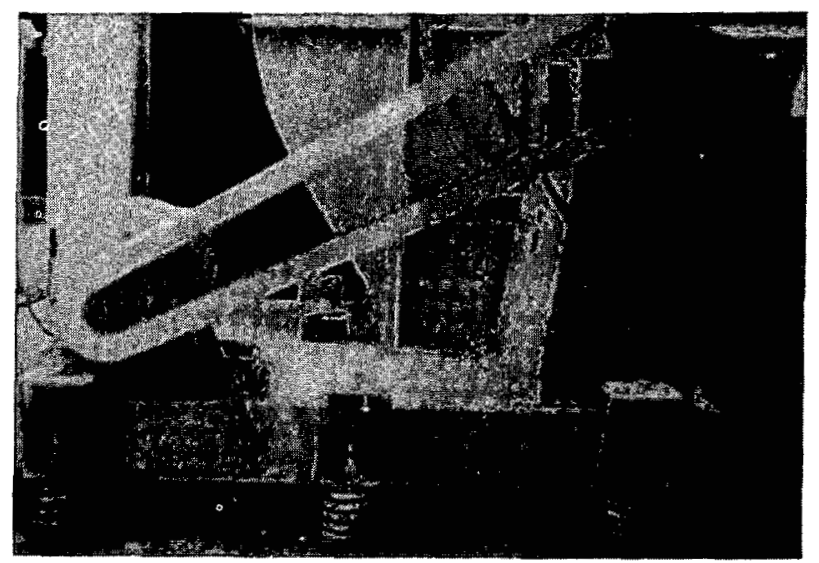

Fig. 5.12. Inertial mounting for tan and motor in buldaing exhaust system of a transuranium laboratory facility. 


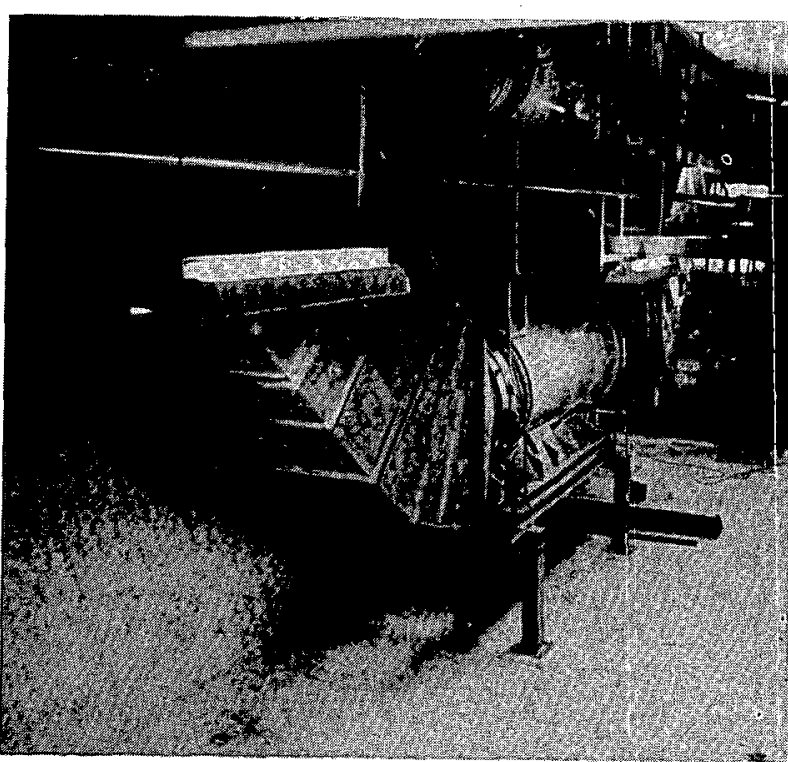

Fig. 5.13. Hard-mounted axial-centrifugal fan in reactor ESF exhaust system.

where

$f=$ frequency of vibration of the structure, $\mathrm{Hz}$;

$y=$ deflection of supporting beams or floor under normal load, as determined from beam formulas, ${ }^{30}$ in.

For minimum vibration, the speed of the motor and fan should be at least $25 \%$ less than the vibration frequency of the structure. Walls and plenums can be checked by a similar method, by using the deflection due to static pressure in the plenum for finding the vibration frequency. Where practicable, the fan should be mounted directly over a column to obtain maximum rigidity. A fan that is to develop a static pressure of 4 in.wg or more should be tested at the factory and checked for vibrations at the bearings and fan housing extremities. ${ }^{31}$

Vibration created by fans, motors, and drives can be isolated through the use of flexible connections between the fan and ductwork. Where such connections are used, a frequent problem has been tearing and pulling-out of the fabric (from which the flexible connection is made) at the connector clamp. The flexible connection design shown in Fig. 5.14 can overcome these problems. The fabric shown consists of two layers of 30-oz neoprene-impregnated fiberglass cloth, lapped so that the ends are displaced from one another, and glued.
ORNL DWG. 69.8703

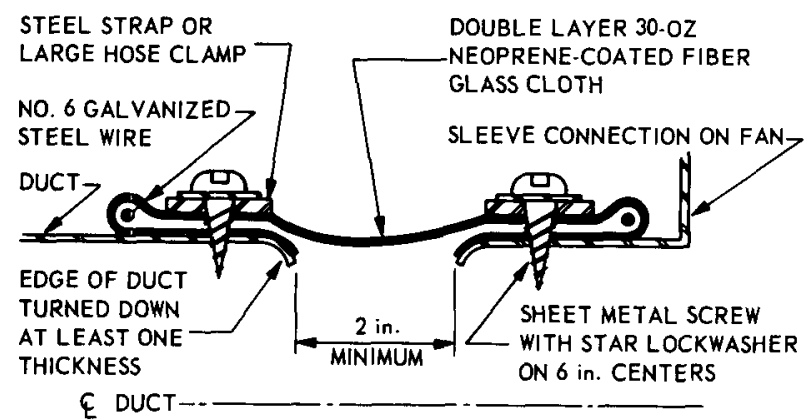

SLEEVE END ON FAN OR DUCT

DOUBLE LAYER 30-OZ NEOPRENE-COATED FIBER GLASS CLOTH7

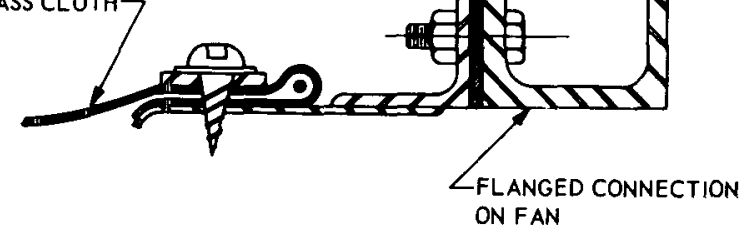

E DUCT-

FLANGE ON FAN OR DUCT

Fig. 5.14. Recommended design for flexible connections between fan and ductwork.

\subsubsection{Location of Fan}

Fan location has a direct bearing on ventilation and air cleaning system performance. Fans in contaminated exhaust systems are normally installed downstream of the filters and as close to the stack as possible. This places the fan in the most favorable location with respect to duct cleanness and the protection of personnel from contamination during maintenance. This position also provides negative pressure in as much of the duct system as possible in order to minimize outleakage of contamination to occupied areas of the building. Locating a fan within the filter housing reduces duct transmission of noise and vibration, eliminates shaft leakage problems and some ducting, and makes a flexible connection between fan and ductwork unnecessary; however, this may be a poor location from the standpoints of access and fan-entrant pressure losses. The location of a fan in a potentially contaminated area can also cause problems. The flexible connections between 
fan and ductwork and fan and shaft penetrations are obvious leakage paths which could permit contaminated air from the room to bypass the air cleaning facilities if the fan is located on the suction side of those facilities.

The location of a fan outside a building is not recommended. Even with a shed to protect it from the elements, there is a tendency for the fans to receive less attention (i.e., inspection, maintenance, and timely service). When fans are located outside the building, service may be inhibited during inclement weather and condensation problems often develop. Except for the lack of service during inclement weather, the same problems exist for fans installed inside but in unheated off-corners of the building. Reasonable access for maintenance and service is imperative, and fans installed above floor level must have sufficient clear space around and below for personnel to get to them with the aid of ladders and/or scaffolding.

\subsection{AIR INTAKES AND STACKS}

\subsubsection{General}

The design and location of exhaust stacks and air intakes have an important bearing on system performance. If air intakes are too close to the ground, blowing sand, dust, grass clippings, and other particulate matter may be drawn into the building, plug the supply-air filters, and/or reduce their life. Exhaust fumes from vehicles passing nearby or standing close to the building may also be drawn into the building supply-air system if the intake is too close to the ground. Intakes must be sited to protect them from snow, ice, and freezing rain during the winter months, and baffles or louvers must be provided to give protection from driving rain and to minimize the effect of wind. Although horizontal louvers are preferred by architects for aesthetic reasons, vertical louvers are more effective for removing and draining water drawn into the intake. Wind pressure can have an appreciable effect on flow rates in a low-head ventilation system and can cause pulsations that may disrupt or reverse differential pressure conditions between zones of the building.

The average wind direction and weather conditions that are likely to cause stack discharges to come close to the ground (the phenomena known as looping and fumigation) must be analyzed when establishing the location of stacks and intakes to ensure that stack effluents cannot be drawn back into the building or into an adjacent building. Intakes should be located upwind of stacks (i.e., based on prevailing wind for the site). Intakes downwind of shipping docks may be prone to drawing vehicle exhaust fumes into the building. Intakes located close to a roof or in a roof penthouse may have the same problems as those located too close to the ground.

\subsubsection{Stacks}

Low exhaust stacks should be avoided because nearby buildings and/or the unevenness of the ground may cause eddies, whorls, or stagnant air pockets (e.g., the presence of a hill having a slope steeper than $15^{\circ}$ is sufficient to create an adjacent stagnant zone). A stack height of two and one-half times the highest point of the building or any adjacent building within $500 \mathrm{ft}$ is recommended; ${ }^{32}$ however, in no case should the stack end be less than $12 \mathrm{ft}$ above the building or any adjacent building. Stacks must be structurally designed to withstand maximum predicted wind loads; they may be single-wall or double-wall. The single-wall stack is initially the least expensive design and can have riveted or, preferably, welded seams. Wall thickness may vary from a minimum of No. 16 U.S. gage for sizes up to $14 \mathrm{in}$. in diameter (or $14 \mathrm{in}$. for the longest side of rectangular stacks) to No. 10 U.S. gage or heavier for stacks over 18 in. in diameter. Number 12 U.S. gage is recommended for single-wall stacks between 14 and $18 \mathrm{in}$. in diameter (or the widest side). Stainless steel is recommended where corrosion may be a problem (type 409 or 430 where corrosive fumes are discharged only occasionally, type 304 or 316 where corrosion is a serious problem). Porcelain-lined stacks are also available; they should have two coats of enamel to avoid early failure.

Double-wall stacks have a substantially higher first cost but may be more economical in the long run when condensation is a problem. Double-wall stacks are generally prefabricated and have two distinct advantages over single-wall construction: (1) the stack does not lose as much heat as a single-wall stack; instead, heat remains in the gas and prevents excessive cooling and the resultant loss of plume rise; and (2) the relatively thin inner liner is rapidly heated to a temperature above the dew point during startup, thus minimizing condensation and hastening evaporation. ${ }^{33}$

The "Chinese hat," "goose neck," and similar stack-end treatments that divert discharged air downward should be avoided because of the potential 
for creating a personnel hazard in the event of a breach in the air cleaning system. Such treatments impose an unnecessary back pressure on the system, prevent straight-up discharge, and minimize effective stack height. In addition, they are often ineffective in preventing rain from dripping down the stack. The concentric-sleeve vertical-discharge stack end shown in Fig. 5.15 is recommended; the offset stack-end designs shown in Industrial Ventilation are also suitable but will create some additional pressure loss. ${ }^{34}$
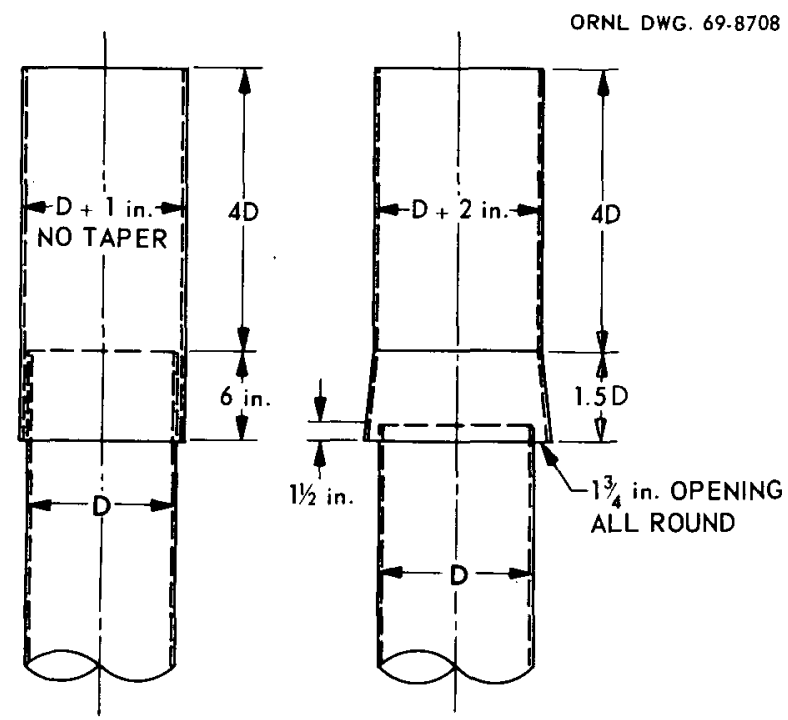

BRACKET UPPER STACK TO DISCHARGE DUCT

Fig. 5.15. Concentric-sleeve stack-end dimensions. Design on the right is preferred but may be more expensive.

\subsection{VENTILATION SYSTEM CONTROL AND INSTRUMENTATION}

\subsubsection{Introduction}

The parameters of major interest in nuclear ventilation systems are airflow and pressure. The usual control procedure for an exhaust system is to maintain constant airflow and monitor pressure to ensure safe operation. Control limits for safe operation are determined in the design stage, and the system is operated within those limits. Any modification of the ventilation system requires a reevaluation of the control limits and, for autnmatic control systems, an evaluation of the change on the control system. Control system design, instrument selection, location and installation, and instrument sensing and actuating line location and installation must consider the consequences of single-component failure; singlesystem failure; catastrophes such as fire, explosion, earthquake, tornado, and flooding of the contained space; ventilation system deterioration or failure; failure of power or actuating media (e.g., instrument air); and the pressure and temperature ratings of the equipment and contained spaces served by the ventilation system. Airflow control can be achieved by (1) control dampers, (2) variable inlet vanes on the fan, (3) variable speed fan, or a combination of these.

\subsubsection{Damper Control}

The throttling damper (Sect. 5.3) is the simplest, lowest first cost, highest operating cost, and most widely used method of ventilation system control. If the pressure available from the fan to compensate for dust loading of the filters is small (less than $25 \%$ of the fan static pressure at the operating point, as shown in Fig. 5.16a), throttling dampers may be used without an unreasonable operating cost penalty. Single-blade (e.g., butterfly) dampers should be used insofar as practicable in automatic control systems because hysteresis (the difference in linkage or blade stroke when the blade moves from the full-open to the fullclosed position and when it moves from the fullclosed back to the full-open position) in multiblade dampers makes it difficult, if not impossible, for the instruments to correlate blade position (i.e., damper opening) with stroke. The result, in an automatically controlled system with multiblade dampers, is "hunting" of the control system and dampers, and oscillation of airflow.

\subsubsection{Variable Inlet Vane Control}

If the differential pressure available from the fan to compensate for dust loading is $40 \%$ or more of the fan static pressure at the operating point, inlet vane control is desirable. An inlet vane control damper costs about three times more than equivalent parallelblade or opposed-blade dampers, but, at a capacity reduction of $50 \%$ or less, it produces power savings that may average $25 \%$ as compared to the parallelblade or opposed-blade control damper. ${ }^{35}$ Another factor that recommends the inlet vane damper over a control damper in the duct is that it permits operation of the fan for long periods at much below the fan's maximum efficiency. Full-open inlet vane dampers cause the fan to operate at some penalty to airflow, static pressure, and horsepower (Fig. 5.16b). One tube-axial fan manufacturer indicates a $10 \%$ increase in brake horsepower, a $3 \%$ decrease in airflow, and a 

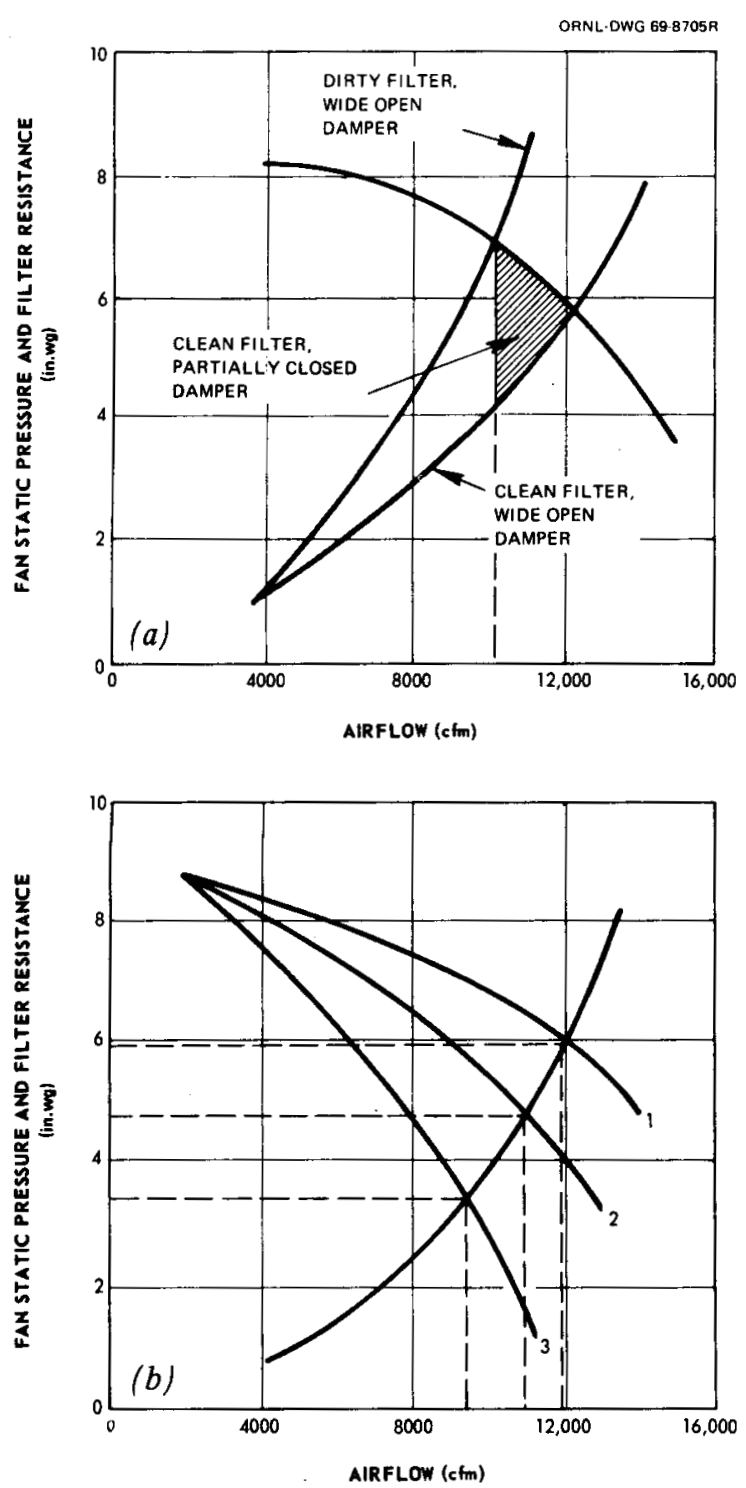

$6 \%$ decrease in static pressure when the fan is operated with its inlet vane damper in the full-open position, as compared with operation without an inlet vane damper. ${ }^{36}$ Other fan types, including centrifugals, show a similar decrease in performance when operated with an inlet vane damper in the fullopen position (see Fig. 5.17).

The AMCA Fan Application Manual recommends the use of variable vane inlet dampers when the fan is to be operated for long periods at reduced flow. ${ }^{23}$ The effectiveness of this damper stems from the fact that the inlet vanes generate a forced inlet vortex that rotates in the same direction as the fan impeller; similarly, any restriction of the

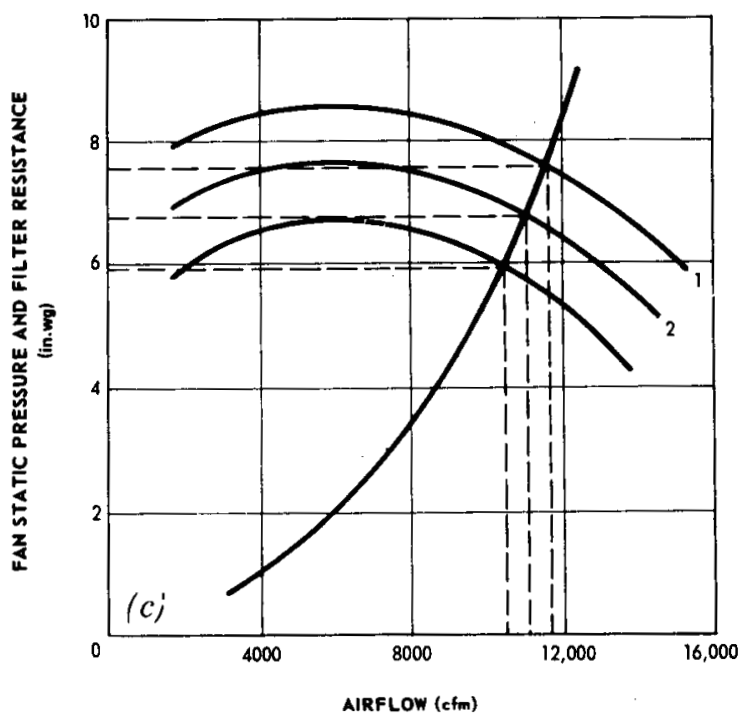

Fig. 5.16. Comparison of fan and system operating characteristics with different methods of airflow control. (a) Damper control; (b) inlet vane control; (c) variable speed control.

fan inlet reduces the fan performance. Inlet vane dampers are of two types: integral or built-in, and add-on. The resistance and system effect of inlet vane dampers in the wide-open position must be considered in the original fan selection and system functional design. System effects of inlet vane dampers should be available from the fan manufacturer; if not, the system effect curves of AMCA $201^{23}$ should be applied to account for pressure losses due to the use of these dampers.

Although variable vane inlet dampers generally provide smooth airflow control down to less than $30 \%$ of operating-point flow, there have been instances of severe vibration on large fans when the 


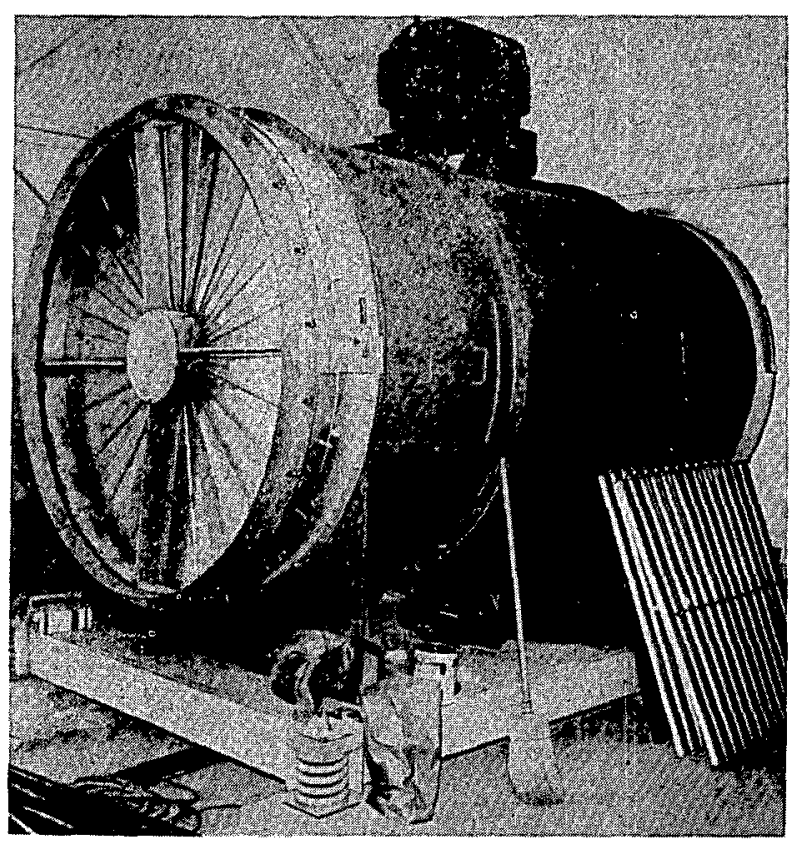

Fig. 5.17. Vane axial fan with variable inlet vane control damper. Damper is add-on type that could be made by other than the fan manufacturer. Damper is in full-closed position to protect fan during construction. Courtesy Mound Laboratory.

vanes were positioned between 30 and $60 \%$ opening. Because vibration is aggravated by system turbulence, consideration must be given to ways of ensuring smooth airflow patterns in the duct entering the damper and leaving the fan when inlet vane dampers are employed in high-velocity systems.

\subsubsection{Variable Speed Control}

Variable speed fans represent the highest-first-cost but lowest-operating-cost means of controlling airflow. Speed control may be achieved by variable pitch sheaves on the fan and motor, hydraulic drive, or variable speed motor. Variable-pitch-sheave-drive is the least expensive but most trouble-prone method. Hydraulic drive costs less than variable speed motors, permits starting of the motor under high torque, and is generally trouble free. However, operation of the fan at less than full speed is inefficient; some hydraulic drive clutches are difficult to operate near the full-speed position; and all require an auxiliary lube oil system. Throttling dampers are required in most systems for branch line control, apart from the requirement for total system airflow control. The effect of these branch line dampers on the system must be considered in motor and fan selection. Figure $5.16 c$ shows the system effect of variable speed control.

\subsubsection{Automatic Control}

High reliability of the ventilation control system is essential, because malfunction of that system during an emergency or power outage could cause malfunction or failure of the ventilation system, thus jeopardizing the safety of operating personnel and perhaps permitting a release of contamination to the environs. Automatic control of the ventilation system is desirable, if not mandatory, when rapid response is required to variations in system control parameters, malfunctions, or operational upsets in the contained spaces served by the system. To maintain the pressure differentials between various building spaces necessary for directional control of airflow, many ventilation systems require continuous fan operation, and immediate switching to an alternate fan in the event of an emergency or failure of the on-line fan or its power supply. Automatic control is also desirable because of the shortcomings of human nature. Despite the best of procedures and administrative controls, operational personnel in the spaces served by the ventilation system will, in most cases, be primarily concerned with performance of the day-today duties relating to the function of the facility and can be expected to pay no more than minimal attention to proper operation of the ventilation system. In an emergency, the first reaction of many will be to run rather than to stay in the area of possibly high danger to make adjustments necessary for control of a manually controlled system.

On the other hand, automatic control is expensive and has been reported to give less than satisfactory results in some nuclear facilities. Investigations have shown serious shortcomings in some areas of presentday automatic control system design and operation. Four features are essential to the reliability of an automatic control system for nuclear applications. First, a reliable automatic control system must be designed by thoroughly competent instrument engineers who have demonstrated their ability to recognize and accommodate the requirements and peculiarities of this type of system. Second, only components of known reliability can be used; the ordinary instruments and dampers used in residential and commercial heating and air-conditioning service and in many ind ustrial applications have no place in a high-reliability nuclear automatic control system. Third, skilled and competent instrument technicians must be available to correct system anomalies. Where technicians are not available for full-time employment, instrument and control system service can sometimes be contracted but is less desirable. If 
the services of well-trained, competent instrument technicians are not available to the facility, the use of automatic control is questionable. Fourth, modifications to the ventilation system must be evaluated with respect to their effect on the automatic control system. Many cases of automatic control system malfunction and unreliability have been traced to changes made in the ventilation system that were never brought to the attention of the instrument department. Such changes may include adding or dropping glove boxes to or from a glove box line; replacing a glove box with a chemical fume hood; or simply dampering a glove box, cell, or building space out of a system. Any of these procedures can cause unbalances that will affect the operation of the control system. Automatic control systems are costly and reportedly are costly to maintain (although investigations of current systems suggest that high maintenance costs, in well-designed and -installed control systems, may be a myth). However, in the wake of a serious incident or catastrophe, no investigating committee will seriously consider the validity of cost as a determining factor in the design, procurement, or operation of a control system. Problems sometimes arise in automatic control systems because designers fail to recognize that dampers, measuring devices, and other restrictions in the duct are essentially orifices, and that duct entering and leaving such devices must be designed for smooth airflow upstream and downstream for proper operation of the device. Other problem areas often overlooked are hysteresis in control dampers (Sect. 5.5.3) and sizing and installation of instrument sensing and actuator-fluid lines (Sect. 5.6.7).

\subsubsection{Central Control}

Monitoring and control of all but the most simple ventilation facilities is desirable in both automatically controlled and manually controlled systems. Most changes that take place in a high-efficiency air cleaning system occur very slowly and can be monitored by a routine check of pressure, differential pressure, and airflow by operating personnel who are primarily responsible for other functions in the plant. Abnormal conditions are best signaled by an alarm which triggers when a monitored parameter rises above or falls below some predetermined set point. Central control has the advantage thatsuch readings can be made, the effect of changes to the monitored parameters can be determined, and corrective action can be taken without a trip to the field. However, sufficient local instruments at the point of interest should also be provided to facilitate maintenance and inspection and to provide a check on the central instrumentation. Centralized control, particularly if instruments and control switches (e.g., for fans and dampers) are laid out on a graphic display panel, enables the operator to rapidly assess a situation, to determine the cause of an upset condition, to determine its interaction with other systems and its safety ramifications, and to take rapid action when necessary. The system response to corrective action can be monitored, reassessed, and modified in minimum time without entering potentially contaminated spaces of the building. Centralization of control also provides a focal point where operational information can be funnelled under normal operating conditions, for feedback purposes, and where knowledgeable personnel can be contacted in an emergency.

\subsubsection{Instrumentation}

Safe and reliable operation of a ventilation system, whether automatically or manually controlled, requires instruments to monitor critical operating parameters. As a minimum, such instrumentation includes pressure drop across each individual bank of HEPA filters (not just a single instrument to read pressure drop across the total filtration system), and airflow rates at critical points in the duct. Pressures in critical operating areas of the facility and pressure differentials between areas may also have to be monitored. Quality instruments, with accurately engraved and legible scales, rugged enough to withstand continuous operation under less than ideal conditions without loss of accuracy, and durable enough to last for the life of the installation, are essential. As noted earlier, unreliability in automatic control systems has more than once been traceable to poor choices in the selection of instruments. The principal requisite for locating instruments is accessibility. An instrument that is out of easy reach or is not easily readable will not be maintained or used. Instruments should be located at eye level or only slightly above or below. Panel mounts should be provided for fragile items and for those that require service entry from the back. Instruments that are adversely affected by vibration, particularly those with delicate electrical contacts or springs, should be installed on vibration isolators or on panels that are mounted on vibration isolators. Where stable support is not available, the panel should be mounted on its own standard. Instruments with related function should be grouped on a single panel or adjacent to one another, as indicated in Fig. 5.18, so that operators can correlate related readings, such as

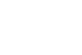




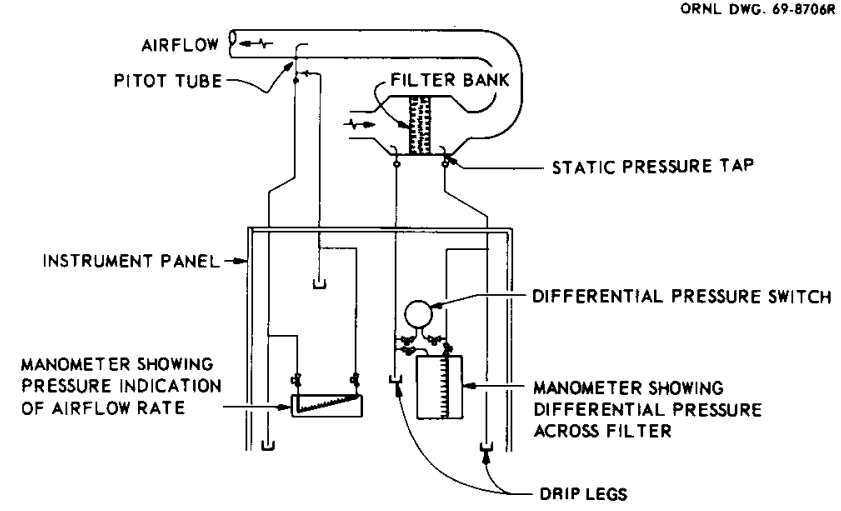

Fig. 5.18. Grouping of instruments with related function.

pressure drop across filters and airflow in the ducts, without going to several locations. Critical ventilation systems that cannot be permitted to fail or to be misunderstood (because of erroneous instrument readings) may be fitted with redundant instrumentation for certain critical parameters. Redundant instruments must be totally independent so that failure of one, from a cause either internal or external to the instrument/ventilation system, cannot affect the other.

Installation of instruments out-of-doors should be avoided when possible but not at the expense of greatly extending sensing lines that would decrease sensibility or reliability, or at the expense of inaccessibility. When located out-of-doors, instruments must be protected from the weather. Indicating fluids in manometers must be of a type that cannot freeze or boil under the temperature extremes that can occur at the site, and that will not change color if exposed to sunlight. Plastic instruments, instrument cases, and instrument cover glasses should not be used in outdoor installations. Raintight electrical cabinets, NEMA class 3 , are recommended. ${ }^{37}$

In low-hazard areas of the building where easy access is possible, the requirement for pressure drop and airflow readings for simple, noncritical systems can often be met by providing for temporary attachment or installation of portable instruments. For pressure drop readings, a sealable length of tubing that penetrates and is welded to the duct wall is satisfactory. A simple hole in the duct, sealable by tape, may be sufficient for inserting a hand-held pitot tube. For critical systems, however, permanently installed instruments must be provided in accessible locations as close to the monitoring point as practicable, with usually a remote slave instrument in the central control room. For critical systems, the location, number, and size of instrument taps and instruments must be specified in the original design, and any changes after the system goes into operation should be recorded in the "as built" drawings.

Actuating fluid (e.g., instrument air) and sensing lines should be large enough that they cannot be plugged due to freezing of condensed water collected in them, or from contaminants that inadvertently get into the lines. A minimum tubing size of $3 / 8$ in. OD is essential for outdoor lines and is recommended for all lines, indoor or outdoor. Sensing lines must be kept as short as possible to minimize the time response to parameter changes, and they should have a minimum number of bends or flow restrictions. Sensing lines should be rigid to prevent expansion under pressure or temperature extremes that could result in false readings or multiply short-term parameter variations. Preferably, lines should be run and instruments located above the ducts to minimize condensation problems. Because such locations are often impossible, however, as a minimum, lines should be sloped to low points fitted with drip legs, and instruments should be fitted with drip legs. Figure 5.19 illustrates good installation practices for sensing lines. Where sensing lines are located in or are serving contaminated spaces or spaces in which caustic or acid fumes are present, they should be made of stainless steel, and instruments should be located far enough away from the contaminated space that migration of such contaminants to the instrument is minimized. In particularly critical applications (e.g., hot cells), a very-low-velocity purge line may be attached to the actuating fluid or sensing line; the purge flow must be very low, of course, to avoid influencing operation of the device or affecting readings.

ORNL DWG. 69-8707

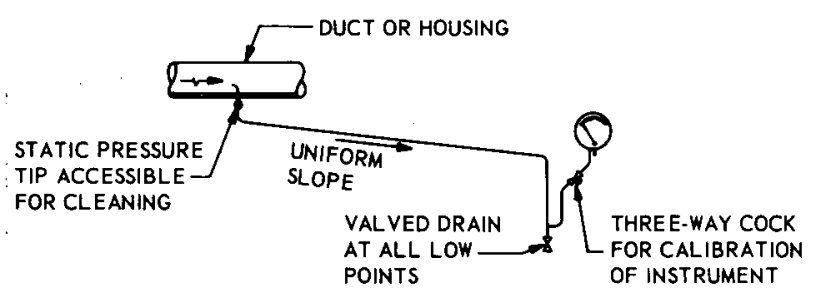

Fig. 5.19. Proper orientation of instrument sensing lines. 


\section{REFERENCES FOR CHAP. 5}

I. ASHRAE Handbook and Directory-1973 Systems, Chap. 21, "Ventilation of the Industrial Environment," and Chap. 22, "Industrial Exhaust Systems," American Society of Heating, Refrigerating, and Air-Conditioning Engineers, Inc., New York, 1973.

2. Industrial Ventilation, American Conference of Governmental Industrial Hygienists, 13th ed., Ann Arbor, Mich.,1974.

3. ANSI Z9.2, Fundamentals Governing the Design and Operation of Local Exhaust Systems, American National Standards Institute, New York, 1971.

4. High Velocity Duct Construction Standards and Low Velocity Duct Construction Standards, Sheet Metal and Air Conditioning Contractors' National Association, Arlington, Va., current issues.

5. ANSI N509, Standard for Nuclear Power Plant Air Cleaning Units and Components, American National Standards Institute, New York, 1975.

6. Kegulatory Guide 1.52, Design, Testing and Maintenance Criteria for Atmosphere Cleanup System Air Filtration and Adsorption Units of Light-Water-Cooled Nuclear Power Plants, U.S. Atomic Energy Commission, Washington, D.C., 1973.

7. Letter to editor from M. R. Morton, J. Inst. Heating and Ventilating Eng. 37, A-5 (March 1970).

8. John Stratton, Director of Research, Sheet Metal and Air Conditioning Contractors' National Association, personal communication to C. A. Burchsted.

9. ASTM DI056, Standard Specification for Sponge and Expanded Cellular Rubber Products, American Society for Testing and Materials, Philadelphia, current issue.

10. ANSI B3I, American National Standard for Pressure Piping, American National Standards Institute, New York, current issue of applicable section.

11. ASME Boiler and Pressure Vessel Code, American Society of Mechanical Engineers, New York, current issue of applicable section.

12. R. E. Stevens, National Fire Protection Association, personal communication to C. A. Burchsted.

13. M. W. First, Harvard Air Cleaning Laboratory, Harvard University, personal communication to $\mathrm{C}$. A. Burchsted.

14. ANSI N512, Protective Coatings (Paints) for the Nuclear Industry, American National Standards Institute, New York, 1974.

15. System Design Manual, Part 1, "Loading Estimating," Carrier Corp., York, Pa., 1960, pp. 1-110 and 1-111.

16. ANSI N510, Testing of Nuclear Air Cleaning Svstems, American National Standards Institute, New York, 1975.

17. W. D. Burch and T. A. Arehart, "Safety Review Procedures for Hot-Cell and Radiochemical Processing Facilities at ORNL," Proc. 15th Conf. Remote Systems Technol., American Nuclear Society, 1967.

18. Unreported tests at Mound Laboratory, Monsanto Research Corp., in which C. A. Burchsted served as consultant.
19. John Stratton, Director of Research and Development, Sheet Metal and Air Conditioning Contractors' National Association, personal communication to C. A. Burchsted.

20. ASME Boiler and Pressure Vessel Code, Sect. III, Div. 1, "Nuclear Power Plant Components," Subsection NC, "Class 2 Components," and Subsection ND, "Class 3 Components," American Society of Mechanical Engineers, New York, current issue.

21. Directory of Products Licensed to Bear the AMCA Certified Rating Seal, Air Moving and Conditioning Association, Publication 261, Arlington Heights, Ill., current issue.

22. AMCA 500, Test Methods for Louvers, Dampers, and Shutters, Air Moving and Conditioning Association, Arlington Heights, III., 1975.

23. "Fans and Systems," Part 1 of the Fan Application Manual, Air Moving and Conditioning Association, Publication 201, Arlington Heights, 11l., 1973.

24. "Field Performance Measurements Guide for Fans," Part 3 of the Fan Application Manual, Air Moving and Conditioning Association, Publication 203, Arlington Heights, Ill., 1975.

25. Ref. 23, pp. 41-42.

26. R. Pollak, "Selecting Fans and Blowers," pp. 86-94 in Chemical Engineering, McGraw-Hill, New York, Jan. 22, 1973.

27. H. F. Farquhar, Fans and Blowers, Lau Industries, Dayton, Ohio, 1974.

28. ASHRAE Handbook-Equipment, Chap. 3, "Fans," American Society of Heating, Refrigerating, and AirConditioning Engineers, New York, 1975.

29. AMCA AS-2404, Drive Arrangements for Centrifugal Fans, Air Moving and Conditioning Association, Arlington Heights, Ill., 1966.

30. Manual of Steel Construction, American Institute of Steel Construction, New York, 7th ed., 1970, pp. 2-196 to 2-212.

31. C. J. Trickler, "Cause and Prevention of Fan Vibration," NYB Engineering Letter No. E-9-r, New York Blower Co., Chicago.

32. G. A. Briggs, Plume Rise, AEC Critical Review Series, USAEC Report TID-25075, 1969.

33. F. Fields, "The Plant Chimney-Failure Causes and Keplacement Choices," Plant Eng. 29, 265-66 (April 17, 1975).

34. Industrial Ventilation-A Manual of Recommended Practice, American Conference of Governmental Industrial Hygienists, 13th ed., 1974, p. 8-5.

35. K. H. Seibert, More Tips on Fan Application, Air Conditioning, Heating and Manufacturing Co., Inc., 4th ed., 1975, p. 12.

36. Engineering Guide, for Ventilating Systems, Western Engineering and Manufacturing Co., 4th ed., 1975.

37. NEMA IS 1.1, Enclosures for Controls and Sistems, National Electrical Manufacturers' Association, New York, current edition. 


\section{Small Air Cleaning Units}

\subsection{INTRODUCTION}

This chapter discusses the installation of internal components, primarily HEPA filters, in systems that require only a single filter per stage of each air cleaning unit (Sect. 2.4.9). Although installation requirements are generally the same as those for multifilter housings (Chap. 4), the use of questionable practices in some older systems and the proliferation of commercially built off-the-shelf housings (caissons) make a separate discussion of this subject desirable. Single-filter (i.e., nonparallel) installations are employed in the supply, exhaust, and recirculating air cleanup systems of rooms, glove boxes, hot cells, chemical fume hoods, and other contained spaces; in the off-gas lines of process vessels and radiochemical operations; and in other applications in which the airflow is $1500 \mathrm{cfm}$ or less. Single-filter installation for glove boxes is a separate topic and is covered in Chap. 7. Although the discussion in this chapter centers about the installation of HEPA filters, it also applies to adsorber cells and other components for which a better than average installation is necessary.

The design of the filter (adsorber) installation is a function of the configuration of the filters (adsorbers) used. Section 3.2 describes three general HEPA filter configurations: open-face rectangular (with wood case or with steel case having double-turned flanges on each face, as shown in Fig. 3.2), open-face cylindrical (with molded-phenolic or metal case, with or without flanges on one or both faces), and enclosed (Fig. 3.3). The enclosed configuration may be either rectangular or cylindrical; it consists of a core, identical to that of the equivalent open-face filter, which is sealed into an elongated case with a closed end and nipple on one or, more often, both ends. The nipples of the enclosed filter may be plain, as shown in Fig. 3.3, or flanged. The rectangular open-face filter is most commonly used in both large-volume (multifilter) and low-volume (single-filter) applications, and this chapter deals mostly with this type of filter. The installation of cylindrical open-face filters and enclosed filters is discussed in Sects. 6.4 and 6.3 respectively. Any HEPA filter size other than those listed in Table 3.1 should be considered as a "special" with respect to procurement.

Single-filter installations can be grouped into three broad categories: in-wall (filter mounted in or to a wall penetration of a room, glove box, hot cell, or other contained space); in-duct (filter installed "in the line" between two sections of duct, with or without transitions); and duct-entrance (filter installed at opening of duct leading from a room, glove box, hot cell, or other contained space). In-wall installations are generally employed to clean the air entering a contained space, to prevent the backflow of contamination in the event the contained space becomes pressurized, or both. The filter may be installed bare (sides of case exposed) or in a partial enclosure. As in other installations, a prefilter is recommended upstream of the HEPA filter. Duct-entrance filters are strongly recommended to maintain the cleanliness of contaminated exhaust and air cleanup ducts. They should be mounted in or close to the entrance of the duct and, like the in-wall type installation, may be installed either bare, as Fig. 6.1 shows, or in a partial enclosure.

In-duct open-face filters should be installed in totally enclosing housings or caissons as shown in Fig. 6.2. Common practice in the past, however, has been merely to tape or clamp the filter between two sections of duct or a pair of duct transitions, with the case exposed, as shown in Fig. 6.3. Such installations provide no secondary containment in the event of a breach of the filter case or gaskets, or the tape seals, and, particularly for wood-cased filters, these installations fail to meet the requirements of UL-181 and NFPA $90 \mathrm{~A}^{1,2}$ 
ORNL DWG. 69.8767

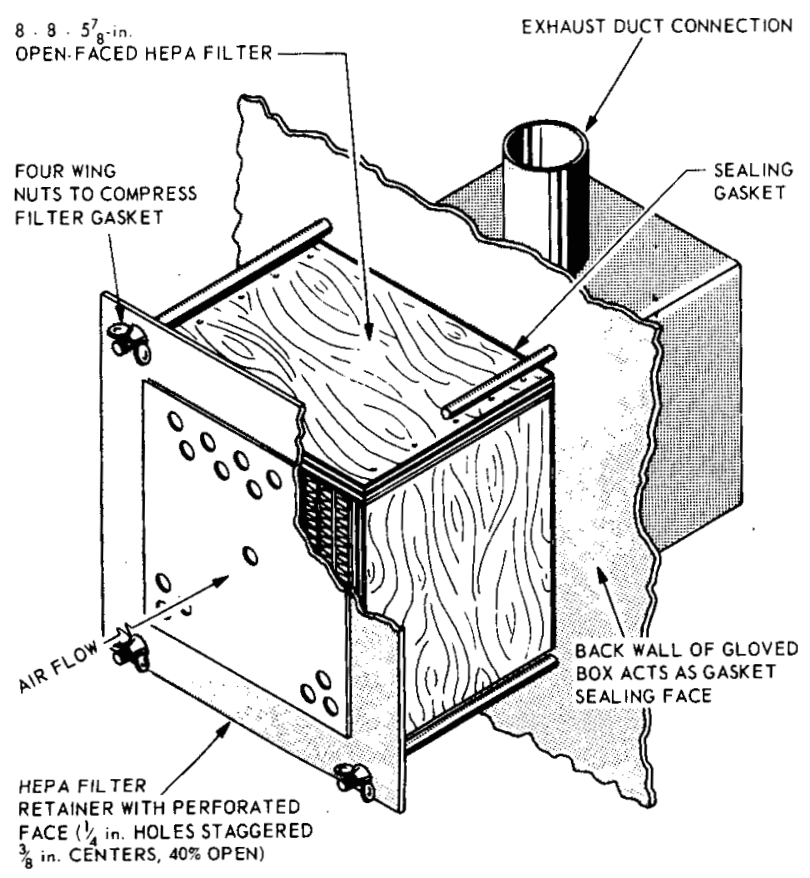

Fig. 6.1. Duct-entrance or in-wall HEPA filter installation without enclosure. Note protective perforated plate on exposed face to prevent physical damage to fragile core of filter.

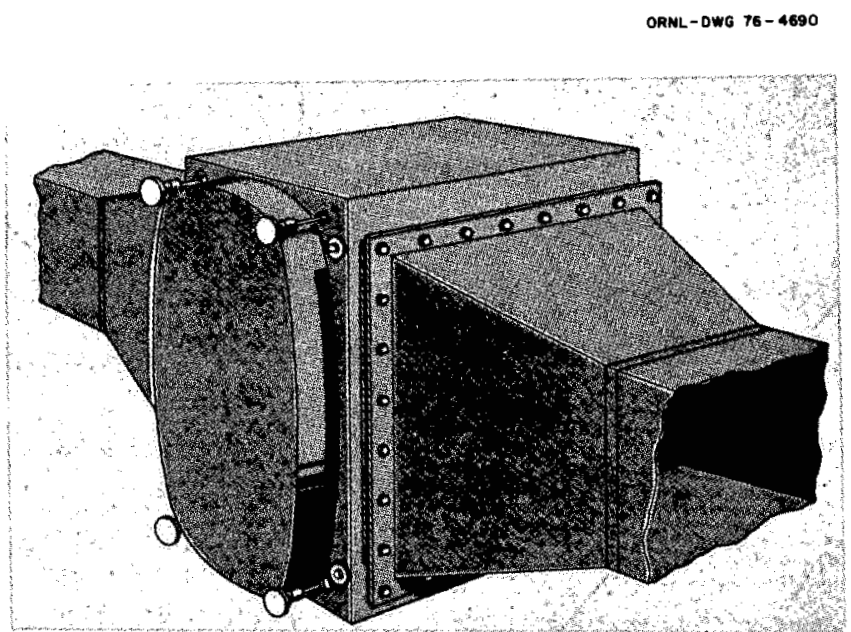

Fig. 6.2. In-duct HEPA filter installation. Filter installed in total enclosure or caisson.
NOTE EDGE GASKETS ON THE FILTER

CASING ARE NOT REQUIRED. THE

TAPE ADHERES TO THE METAL DUCT

AND THE ADJOINING WOOD FACE OF

THE FILTER CASING.

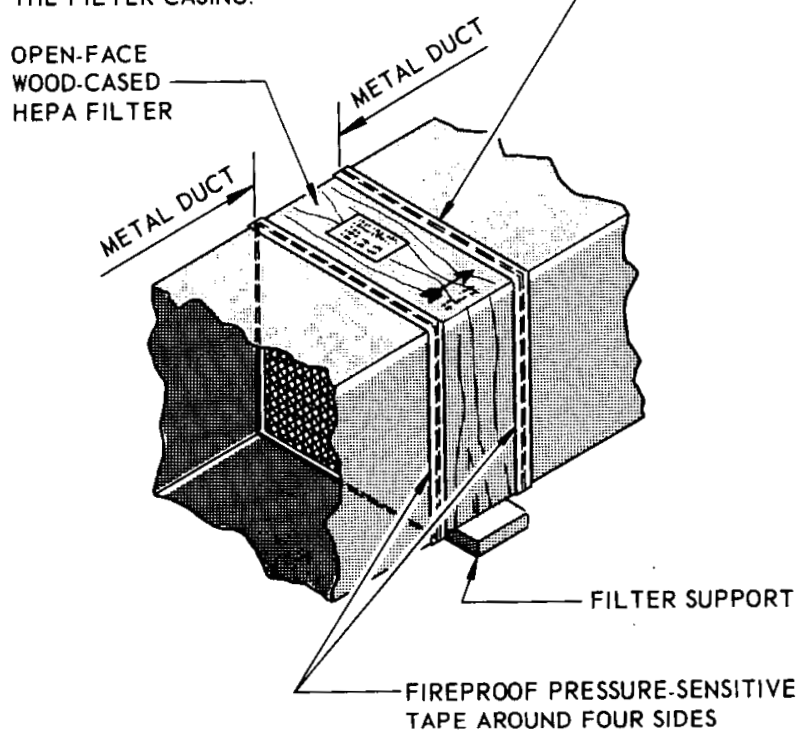

(a) FIREPROOF TAPE

CLAMPING BOLTS MUST COMPRESS BOTH GASKETS (EQUALLY) AS WELL AS DISPLACE DUCT MAKING TORQUING OF NUTS ALMOST MEANINGLESS.

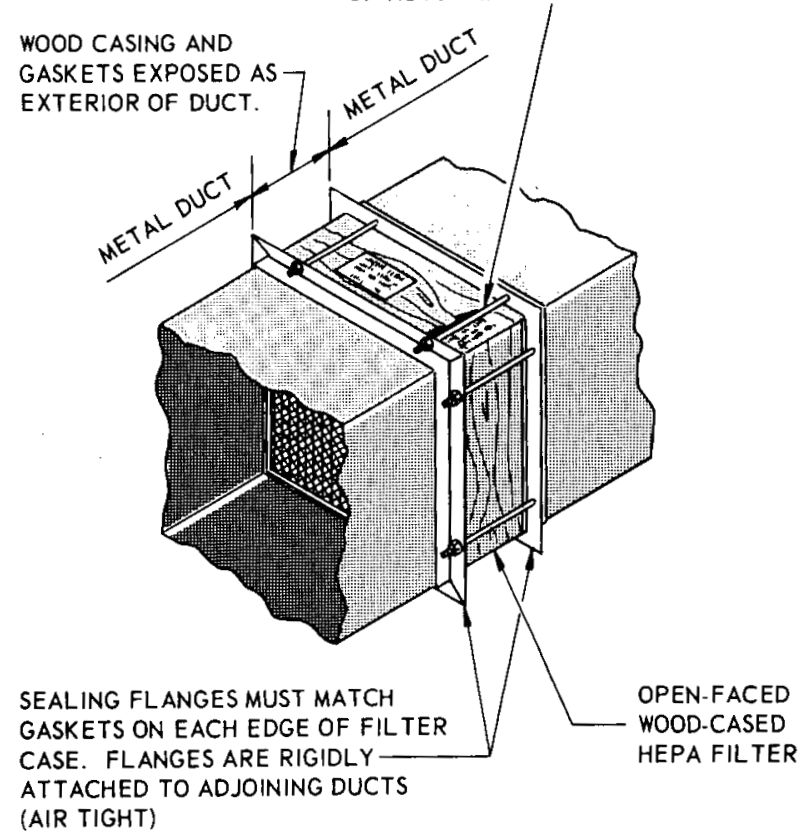

(b) SANDWICHING FLANGES

Fig. 6.3. Common in-duct filter installation methods. These are not recommemded for contaminated exhaust or recirculating air cleanup applications where high integrity is required. 


\subsection{HOUSINGS}

Housings for in-duct installations may be as small as the mini-caisson for a 25-cfm HEPA filter or as large as the complete multistage air cleaning unit, containing demister, prefilter, two stages of HEPA filters, and adsorber, shown in Fig. 6.4. Figure 2.13 shows a concrete-enclosed single-component air cleaning unit. Probably the most common singlecomponent housing today is the bag in, bag out caisson which is commercially manufactured by a number of companies to a more or less standard configuration. Figure 6.5 shows several commercial single-filter housings.

Commercially made caissons, like other air cleaning system components, are not items to be selected "on faith." Designers have been prone to look upon these as "black boxes," believing that, because they are off-the-shelf items, they can be assumed to be of adequate design and to be suitable for any nuclear application. Such is not the case, and some users have been faced with replacing or upgrading many such commercial enclosures over the past several years. Features that must be checked carefully when purchasing standard commercial housings include the filter (component) mounting frame and clamping device, the rigidity of the box and its cover, the method of cover sealing and clamping, access to the installed component, the rigidity and construction of duct connections, and the materials of construction of all parts, including the component clamping mechanism. These same features are of importance in the design of one-of-a-kind shop-built housings.

\subsubsection{Component Installation}

Requirements for installation of components are basically the same as those for bank installations (Sect. 4.2) and include structural rigidity, flatness, and accuracy of construction of the mounting frame; positive and reliable seal of the component to the frame; specification of and strict adherence to close tolerances in fabrication; and leaktight welded construction. A minimum sheet-metal thickness of 0.078 in. (No. 14 U.S. gage) and preferably 0.125 in. (No. 11 U.S. gage) is recommended for mounting frames of commercially made and shop-fabricated housings. The mounting frame must be seal-welded into the housing in such a manner that no warping of the filter (component) seating surface will result. There should be a right-angle bend all around the seating surface to provide reinforcement and to ensure flatness. Figure 6.6 shows a portion of the turned-angle filter seating surface of a commercial housing and the four-bar-

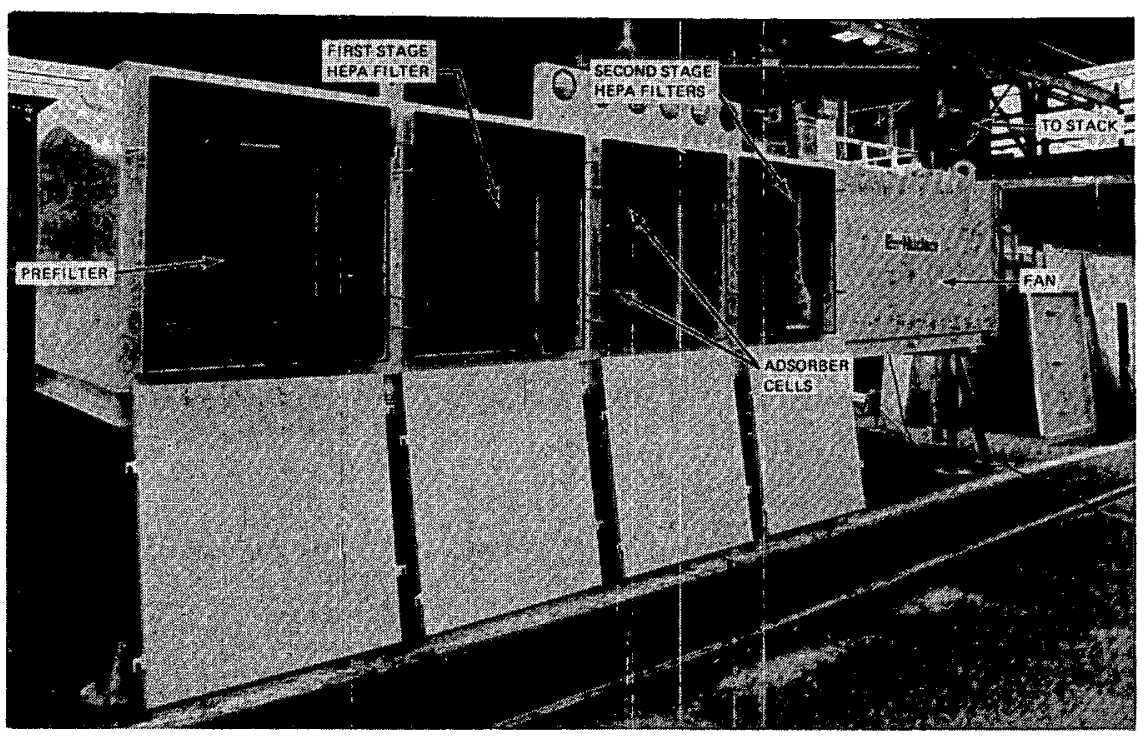

Fig. 6.4. Self-contained, unitized air cleaning unit with prefilter, HEPA filter, two type II adsorber cells, and second HEPA filter. Fan located within housing at right, connections to inlet and stack flanges on top of unit. As shown, unit is designed for airflow of $333 \mathrm{cfm}$ with adsorbers arranged in series. With a single stage of adsorption, unit could be rearranged for 1000 -cfm airflow by removing the baffle between the adsorber cells. Courtesy U.S. Army, Aberdeen Proving Ground-EA. 

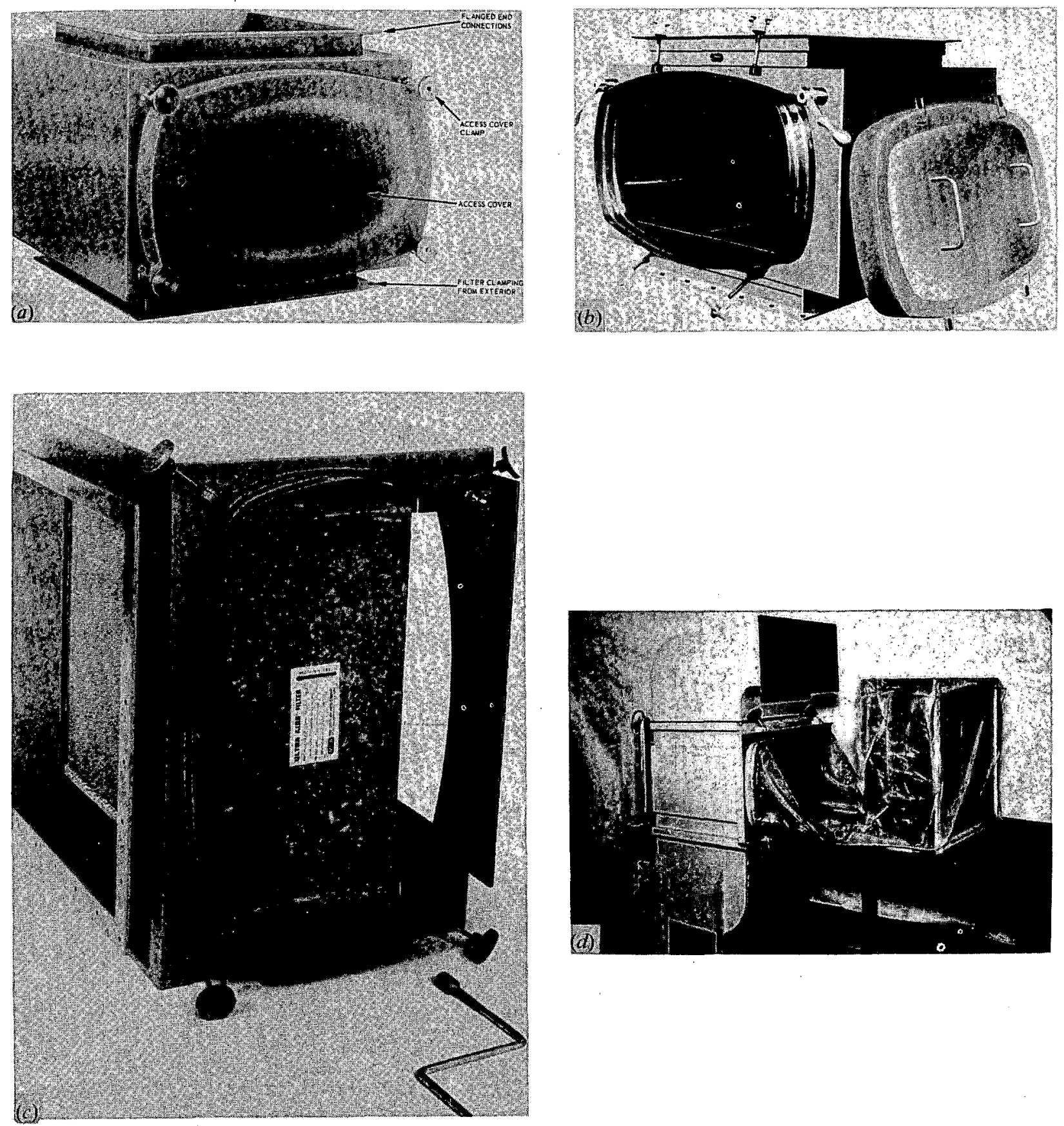

Fig. 6.5. Commercially manufactured single-filter housings. (a) SGN Caisson, made by Barnebey-Cheney, Columbus, Ohio. (b) NCI modular filter enclosure, made by Nuclear Containers, Inc., Elizabethton, Tennessee. (c) MSA Ultra-lok, made by MSA Co. Note design to accommodate filter in either vertical (preferred) or horizontal orientation. Note crank for operating four-bar-linkage clamping mechanism from outside of the housing. (d) Vokes UNIPAK, made by Vokes, Ltd., England. Courtesy United Kingdom Atomic Energy Authority. 

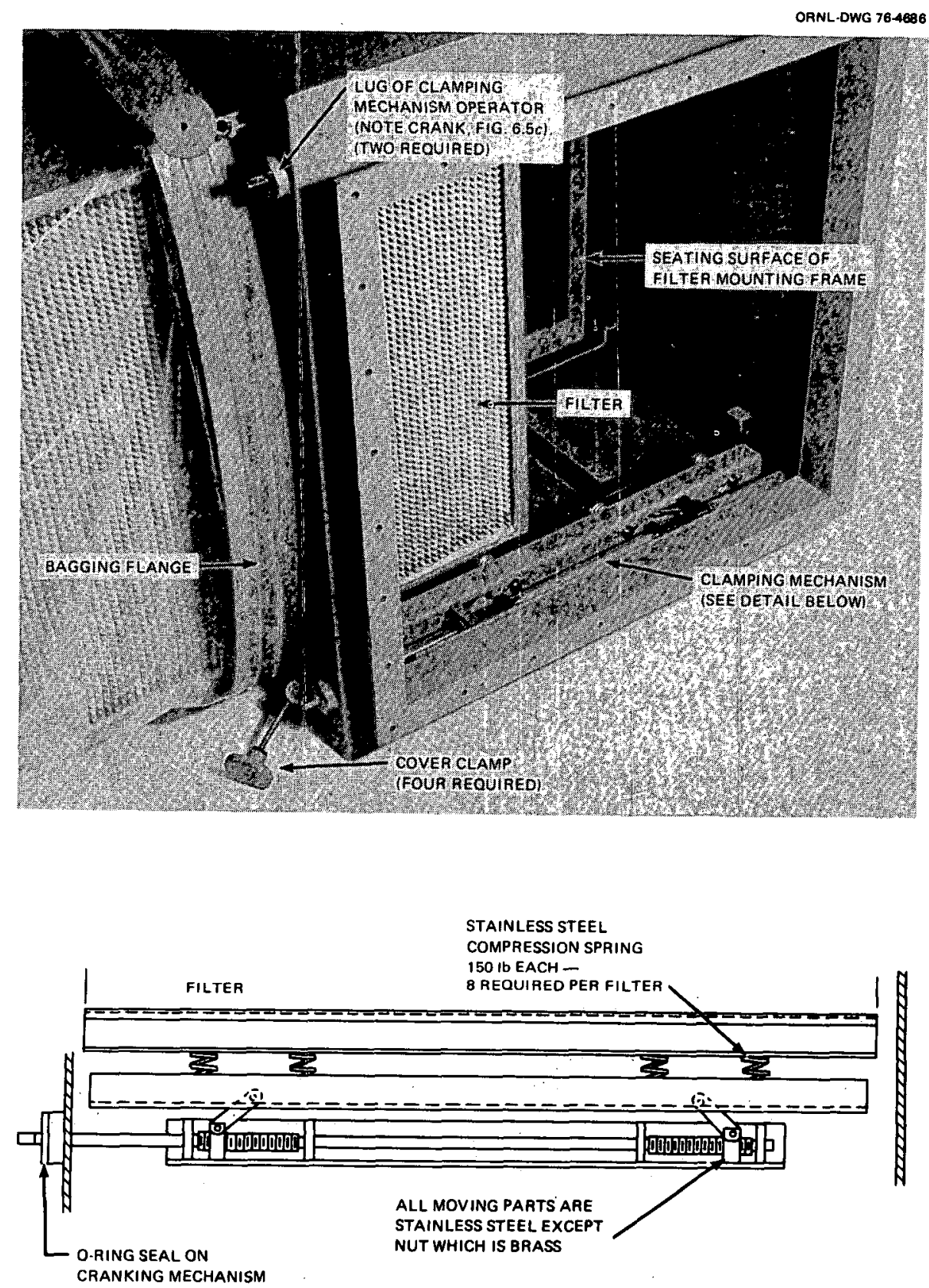

Fig. 6.6. Typical commercial single-filter housing showing important features and details of a typical clamping device. Courtesy MSA Research Corp. 
linkage clamping mechanism that is operated by means of a wrench (Fig. 6.5c) from outside the cabinet. Structural requirements of the mounting frame will be met if the 14-gage or 11-gage steel is used, particularly if the stiffening flange (right-angle bend) is used. Face mounting of the filter to the mounting frame is recommended, with a "finger space" of at least 2 in. on two sides, as shown in Fig. $6.5 c$, for ease of access and filter replacement. Access space within the housing is particularly important in bag in, bag out housings. The frame fabrication and tolerance recommendations of Sect. 4.3.3 are applicable to both commercial and shop-built mounting frames. Either the flat-gasket-to-flat-flange or the fluid seal described in Sect. 4.3.4 is recommended. Flat-gasket-to-knife-edge seals tend to leak excessively if the knife edge is nicked, or if there is any lack of parallelism between the knife edge and the filter face; the compression set produced by a knife edge in only a portion of the gasket also results in leakage if there is any degree of relaxation of the clamping device.

Figure 6.7 shows a nonwelded mounting frame consisting of a single $1 / 4-i n$. plate that is sealed by gaskets between the flanges of the body and transition of a field-assembled housing. The filter is clamped by bolts and installed through a hatch in the side of the housing. As noted in Chap. 4, bolted clamping provides the maximum in reliability (provided the bolts are large enough) and ability to adjust to dimensional variations of the filters. As noted in Sect. 4.3.4, a gasket compression of at least $80 \%$ is needed to effect a reliable seal between a highefficiency device such as a HEPA filter or radioiodine adsorption cell; this requires a gasket loading of something over $20 \mathrm{lb}$ per square inch of gasket area, or a total loading of over $1400 \mathrm{lb}$ for a 24 - by 24 -in. filter. Such loadings are easily accomplished with the bolted clamping method shown in Fig. 6.7. Most commercial single-filter housings, however, employ a four-bar linkage or cam arrangement, of which those noted in Figs. 6.5 and 6.6 are typical. Some are adjustable to accommodate dimensional variations that occur from one filter element to the next, and some are not adjustable. It is important for the designer to verify that the clamping mechanism of the commercial housing he is considering can develop the loading required and is adjustable. All parts of the mechanism should be stainless steel to prevent rusting and seizing under operational conditions. This includes springs, which tend to break when rusted. The only exception to this rule is that nuts, if used, should be brass, bronze, or other material that will not gall in contact with the stainless malethreaded part (Fig. 6.6). Suggested values for critical dimensions of the mounting frame are given in the table of Fig. 6.7. Clamping mechanisms should be on the clean side of the filter, and operator shafts, when required, must be sealed by O-rings, as Fig. 6.6 shows, or glands. A rest, guides, stops, or other means for aligning the filter prior to clamping should be provided within the housing.

The clamping pressure required to properly seal a gasket-sealed HEPA filter or adsorber cell must be both high and uniform, as noted in Sect. 4.3.4. However, this requirement is substantially relaxed when the fluid seal system ${ }^{3}$ is used. As Fig. 4.18 shows, the filter element has a groove filled with a non-Newtonian (i.e., nonflowing) fluid. The filter is pushed against the knife edge flange of the mounting frame so that the fluid comes in contact with some fraction of an inch of the knife edge, thus forming an airtight seal. Clamping pressure need only be sufficient to prevent the filter from backing away from the knife edge (which would break the seal) under any foreseeable differential pressure across the filter under either normal operating or system upset conditions. The fluid, a silicone compound, has been tested and found to be capable of maintaining an adequate seal under the fire and hot air conditions of $U L-586^{4}$ and the radiation exposure requirement of MIL-F-51068-D. ${ }^{5}$

\subsubsection{Housing Construction}

The walls of the housing must be sufficiently strong to prevent "oil canning" and overstressing under an alternating positive and negative pressure equal to at least 1.5 times the maximum absolute gage pressure to which the housing will be subjected, under the most severe conditions for which it is intended (i.e., usually a DBA); a minimum design pressure of 10 in.wg is recommended. In general, the design and fabrication recommendations of Sect. 4.5.2, and the leaktightness recommendations of Sect. 4.5 .8 are applicable to housings of these smaller dimensions. In purchasing commercial housings, the designer should check the details of construction to verify that the design proposed is, in fact, adequate for his application; that is, that the walls of the housing (or the cover) will not "oil can" and that stresses in the walls or clamping mechanism will not exceed a value of 0.7 times the yield strength of the material from 


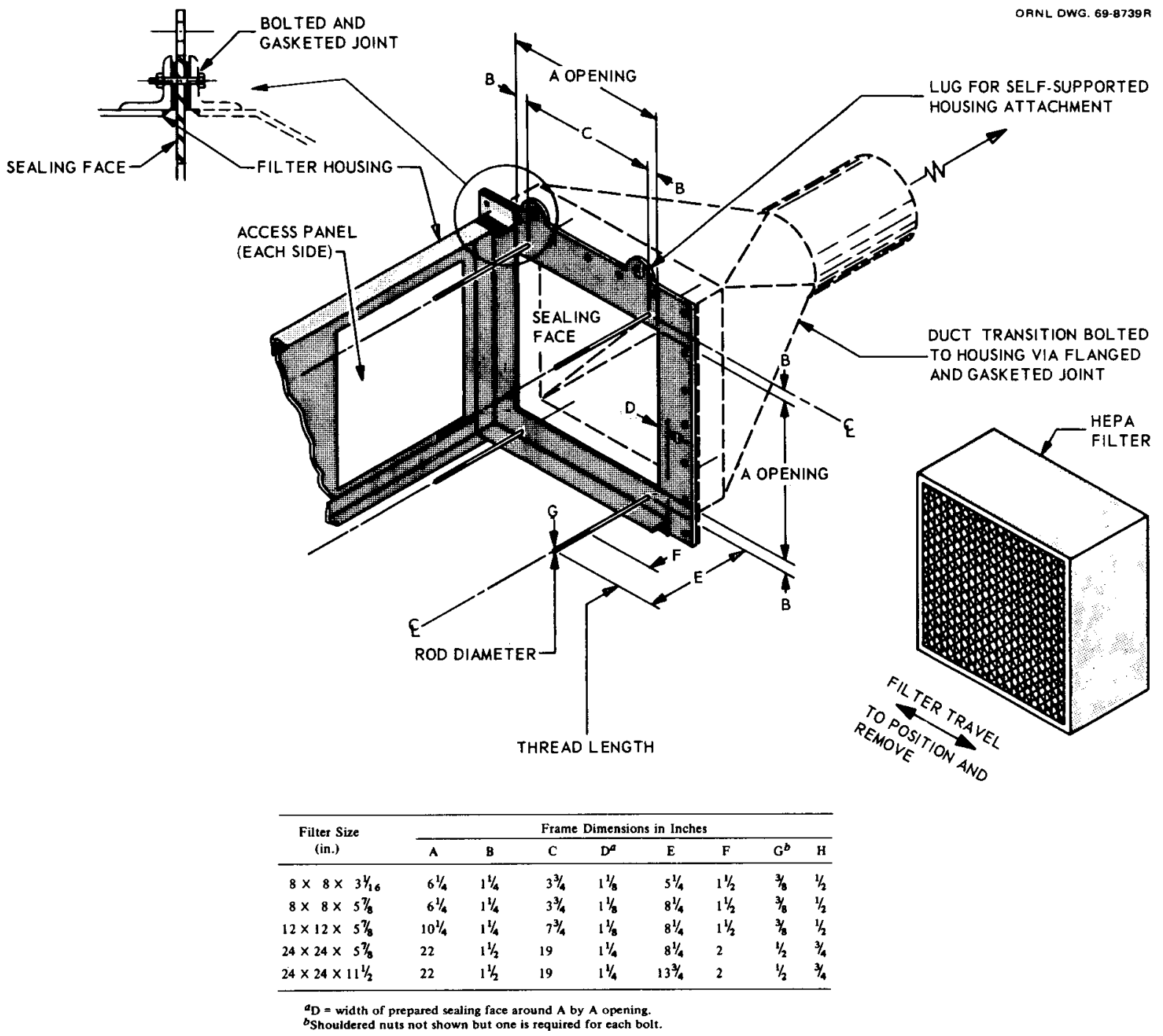

Fig. 6.7. Details of typical owner-designed single-filter housing showing important dimensions applicable to commercial and one-of-akind housings.

which they are made under a housing pressure of 1.5 times the design pressure.

Many failures of commercial housings can be traced to corrosion. The filter housing is a common point where corrodents tend to condense, collect, and concentrate. When the filter housing is to be installed in a line which may, under either normal or abnormal conditions, contain corrosive fumes or vapors, stainless steel construction should be employed. In any event, all parts of the clamping device (including springs, but not nuts) should be of stainless steel. The designer should determine what coating has to be used and establish, to his personal satisfaction, that it is adequate for his application ( 10 to 20 years service life is often considered normal for such housings; 40 years is a figure often expressed for nuclear power reactors). Results of chemical-resistance tests, as specified in ANSI N512, ${ }^{6}$ should be reviewed in such evaluations.

The housing cover should be attached by hand nuts of the type shown in Figs. 6.5 and 6.6. Attachment of covers with machine bolts or nuts may be cheaper but will be a constant problem to the user. Nuts get lost and threaded bolts get damaged under service conditions; the result is often the inability to seal the housing properly, and the need to remove and replace 
a large number of nuts or bolts inhibits access and proper service. For hand-nut cover clamping, the cover must have a 2-in.-deep lip or flange all around for stiffening (Fig. 6.5). The cover must also be stiff enough, or reinforced, so that it will not "oil can" under pressure variations to which it may be subjected. The cover and the cover-clamping mechanism must be capable of sealing the cover opening whether or not a bag is in place.

\subsubsection{Bagging}

Most commercially manufactured and some oneof-a-kind shop-built housings are designed for bag in, bag out filter replacement. Figure 6.8 illustrates a step-by-step description of this procedure. As noted in the drawings, shutoff dampers are needed upstream and downstream of the filter (or other component being replaced) to permit isolation of the housing during the change, and to prevent ballooning or sucking in of the bag, when the cover is opened, due to a pressure differential between the inside and outside of the bag. A small, valved, breather vent is required on the clean side of the filter to control pressure in the housing; a slight negative pressure $(1 / 4$ to $1 / 2$ in.wg) helps ensure inward leakage should the housing become pressurized due to pumping of the bag. When heat-sealing plastic bags, two seals about 1 to 2 in. apart are usually made so that when the bag is cut between them both the housing opening and the plastic-enclosed filter are sealed from the room environment. One operator makes three heat seals and cuts the bag on the middle one, which makes for a better seal and gives added protection in the event one of the outer seals is defective. Heat sealers capable of spanning at least a third of the bag width are available commercially.

Bags must be of clear plastic to permit the worker to see what he is doing (in the Vokes and Flanders housing designs, for example, the worker must manipulate the filter clamping mechanism through the bag.) Bagging materials are polyethylene or polyvinyl chloride, 0.006 to 0.008 in. thick. Because the bags tear and abrade easily, particularly when used with metal-cased filters or adsorbers, the thicker material is preferable, and care must be taken when carrying out the procedure with larger $(24 \times 24 \times$ $11^{1 / 2-i n}$.) items. Although bag-out housing manufacturers often advertise that a major advantage of their design is that housings can be installed inside the laboratory with no additional protection, the fact that bags tear readily makes this a dubious statement. Housings should be installed in a room that can be isolated as a contamination or radiation zone in the event of a bag tear and resultant spill. The excess bag material that remains after a new filter is placed into the housing is folded carefully against the side of the filter element, as shown in Step 8 (Fig. 6.8), to prevent any portion of it from getting into the airstream or being pinched between the housing cover and bagging ring. The bag, after it is folded within the filter housing, must be isolated from system airflow on the clean side of the filter, because the plastic can be damaged from continued exposure to the airstream. Covers of bag-out housings must be capable of sealing the housing with and without the bag installed and must be kept closed when the system is in operation to protect the bag that remains in the housing. Bagging should not be considered an automatic solution to the contamination hazard, and the user is cautioned to take proper precautions during filter changes. Figure 6.9 shows the proper dress for personnel engaged in a bag-out filter change when there is the possibility of high contamination levels. Note the full body cover and gas mask.

\subsubsection{Housing Installation}

For multifilter installations (Sect. 4.4.1), horizontal airflow, with filter faces vertical, is recommended for large (24- by 24-in. face dimensions) HEPA filters. This recommendation is not so important for smaller filters that have sufficient media support inherent in their design to resist gravitational pull on filter core and collected dust. When vertical airflow (face of filter horizontal) is unavoidable, upflow is recommended for all sizes for the reasons given in Sect. 4.4.2. Unlike multifilter installations, installation of the filter on the clean side (i.e., downstream) of the mounting frame is always recommended for single-filter installations. Except for the common practice of installing furnace filters directly to or adjacent to the HEPA filter, back-to-back installation of prefilters or adsorbers to HEPA filters should be avoided. Because of test and fire safety considerations, a distance of 4 to $5 \mathrm{ft}$ between the HEPA filter and a prefilter or adsorber cell is recommended. If a flame arrester is provided in the system, it must be far enough upstream (usually 1 to $2 \mathrm{ft}$ ) of the prefilter or HEPA filter to diffuse any flame that penetrates the arrester.

For multistage installation, components may be installed in a single housing, as Fig. 6.4 shows, or in 


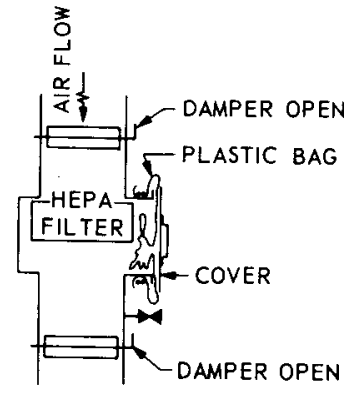

ORNL DWG. $69-8738$

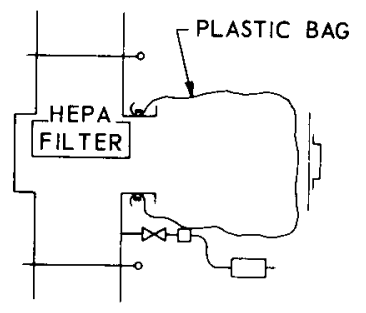

1. NORMAL POSITION. FILTER IN PLACE. AIR FLOWING

3. COVER UNCLAMPED. BAG EXTENDED. HEPA FILTER UNCL AMPED ON OUTSIDE OF HOUSING. BREATHER REMAINS OPEN TO.PERMIT INFLOW THAT COMPENSATES FOR DAMPER LEAKAGE AND LIMITS NEGATIVE PRESSURE IN HOUSING SO PLASTIC BAG IS WORKABLE.

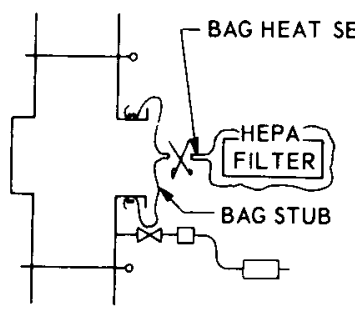

5. DIRTY FILTER SEPARATED FROM SYSTEM*
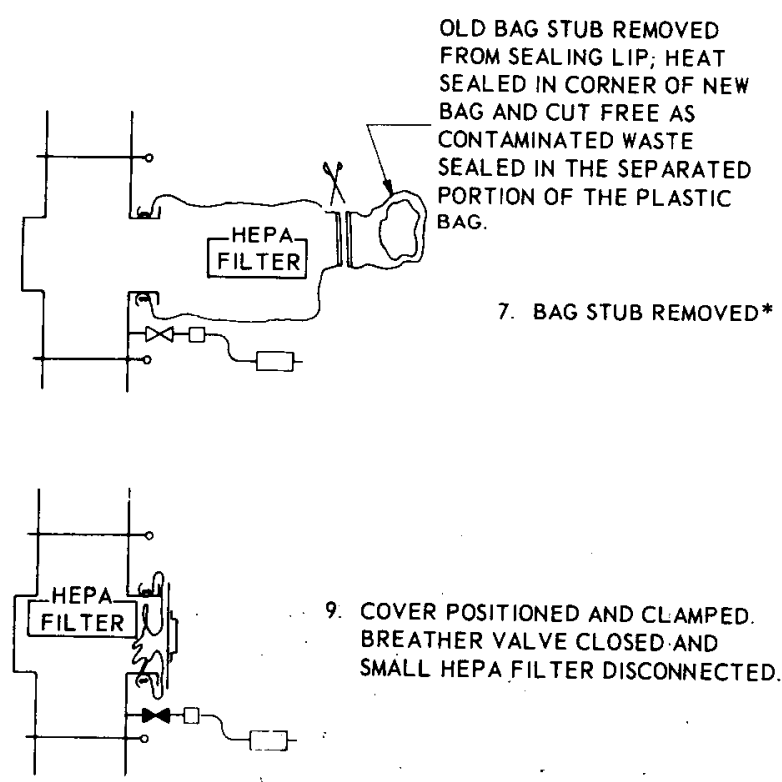

9. COVER POSITIONED AND CLAMPED. BREATHER VALVE CLOSED.AND SMALL HEPA FILTER DISCONNECTED.

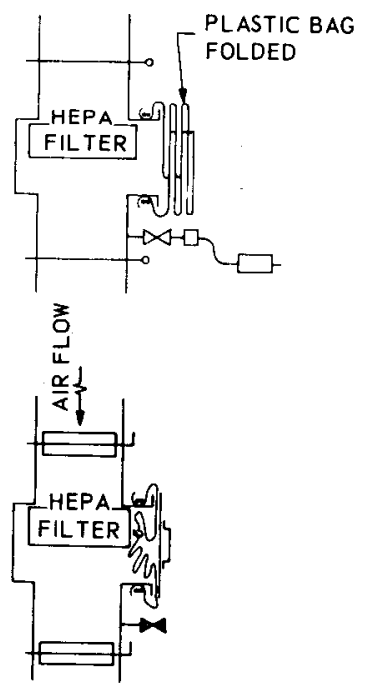

6. NEW BAG CONTAINING NEW HEPA FILTER ATTACHED TO SEALING LIP OVER OLD BAG STUB.

8. CLEAN HEPA FILTER POSITIONED IN HOUSING THEN CLAMPED BY OPERATOR FROM OUTSIDE OF HOUSING. BAG IS FOLDED TO FIT WITHIN OPENING WITHOUT RESTRICTING AIR FLOW PATH.

10. UPSTREAM DAMPER OPENED TO DESIRED POSITION FOR SYSTEM FLOW, THEN DOWNSTREAM DAMPER OPENED FULL TO RESTORE SYSTEM FLOW.

Fig. 6.8. Procedure for bag in, bag out filter (adsorber cell) change. ${ }^{*}$ One operator makes three heat seals and cuts the bag on the middle one; if carefully done, this procedure leaves no contaminated surface. 


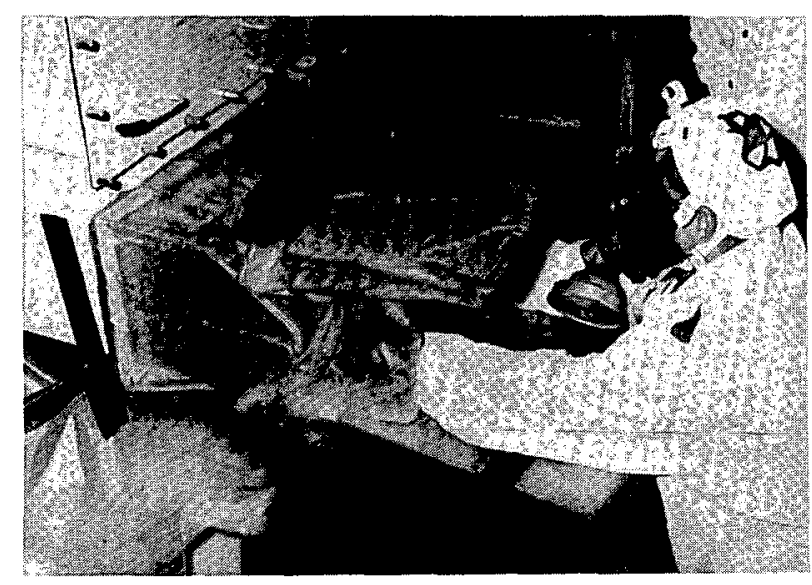

Fig. 6.9. Recommended attire for personnel engaged in contaminated filter handling, including bag-out filter change. Note full body covering and respiratory protection. Courtesy Director, Atomic Weapons Research Establishment, Aldermaston, United Kingdom.

interconnected individual housings as Fig. 6.10 shows. Although bolted, gasketed joints (Fig. 6.11) are recommended, flexible connections (Fig. 5.14) are suitable for housings connected directly to a fan. Duct-tape seals between housings and ductwork are unacceptable. Multistage installations create problems from the standpoint of periodic surveillance testing of HEPA filters and adsorber cells (Chap. 8). As Fig. 6.11 shows, even though a flangeto-flange installation is undoubtedly the least expensive from the standpoint of materials and space occupancy, there may be insufficient room between components to introduce a well-mixed test agent, to obtain a satisfactory upstream sample, or to probe for leaks on the downstream faces of the components. Careful planning of filter and adsorber test procedures, before design of the installation is completed, is essential, particularly for multistage installations. Although some housing specifications require, and some vendors routinely furnish, sample ports in the housing itself, such ports should not be automatically considered as meeting the requirement for preplanned and preinstalled test ports. As noted in Chap. 8, the test agent injection port must be located well upstream of the filter or adsorber to achieve good mixing of air and agent. Upstream samples must be taken from a point in the duct that is immediately upstream of the filter or adsorber. Downstream samples must be taken at a point far enough downstream to obtain good mixing of the air and test agent that penetrates the filter or adsorber. This point is at least 10 duct diameters downstream, or preferably downstream of the fan. Figure 6.12 shows features that should be considered in the design of a single-filter installation. Fire protection is discussed in Sects. 2.5.2 and 9.5.

\subsection{ENCLOSED FILTER INSTALLATION}

Enclosed HEPA filters often appear to offer an ideal solution to some in-duct requirements. They are, by design, unitary; they do not require enclosures; and, after removal from the duct, they can be sealed and handled without personnel coming in contact with the contaminated filter core. However, they must be used with caution. First, the wood-cased type, because the case is part of the system pressure boundary, does not meet the requirements of the National Fire Codes (specifically NFPA 90A) ${ }^{2}$ and should not be used in any application where the potential of a filter fire exists. Second, steel-cased enclosed filters are notorious for leaky cases and

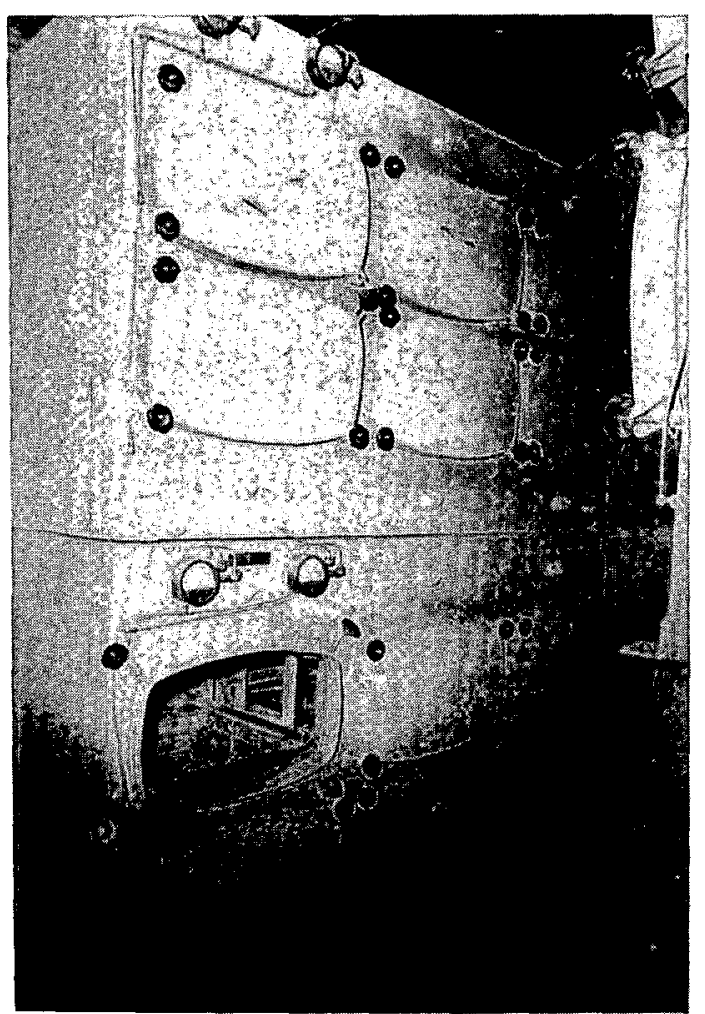

Fig. 6.10. Large capacity air cleaning system employing bag in, bag out multifilter caissons. Courtesy Allied Chemical Co., Idaho Falls, Idaho. 


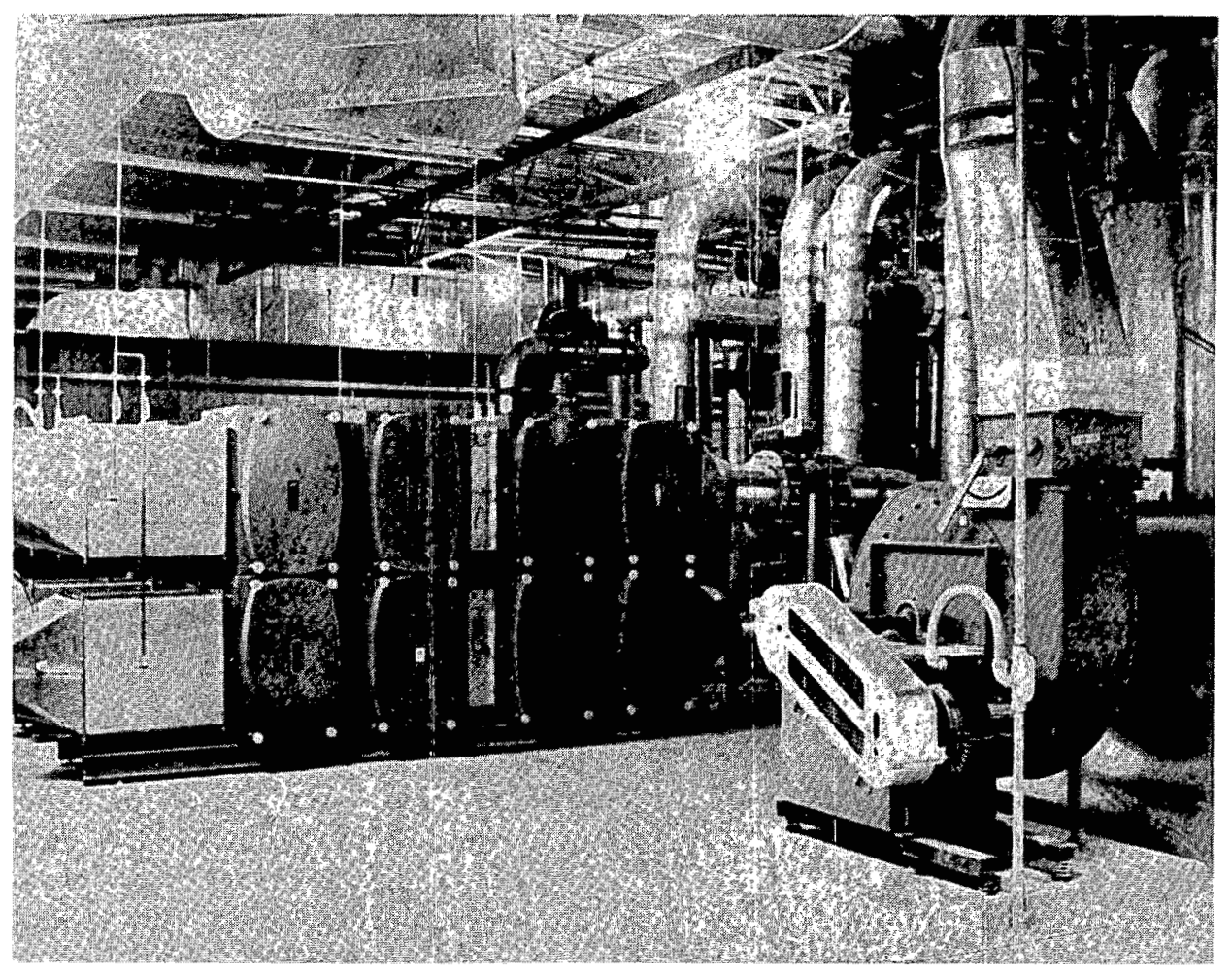

Fig. 6.11. Two-branched multistage air cleaning system employing individual single-filter caissons. Courtesy MSA Co.

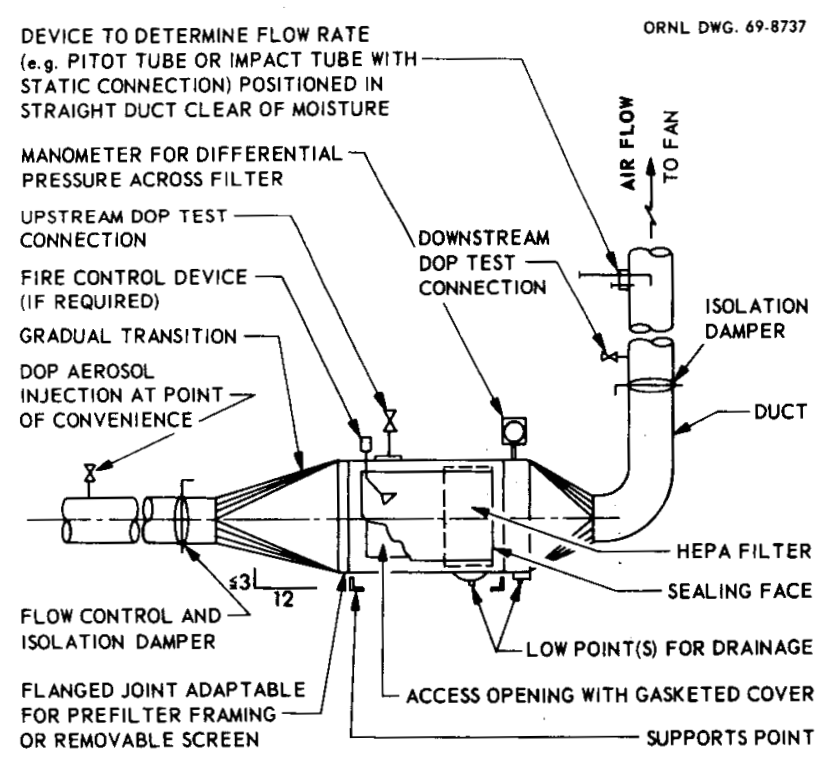

Fig. 6.12. Features of single-filter housing installation. should not be used in positive-pressure applications or where the filter case could be pressurized under system-upset conditions. The case is part of the system pressure boundary. Although the filter core is adequately sealed into the case to prevent leakage between case and core under normal airflow, air can leak through the joints of the case, which are simply riveted through a layer of compressed glass-fiber matting. This seal is not airtight and, under positive pressure, is not an effective particle filter. For this reason, it is recommended that when steel-cased enclosed filters are to be used in systems in which there are, or could be, significant levels of contamination, the filters be bagged at all times, as shown in Fig. 6.13 .

Enclosed filters are most commonly furnished with plain nipple ends, as Fig. 3.3 shows, and are generally sealed into the duct by means of (1) flexible tubing and clamp rings (Fig. 6.14); (2) specially designed elastomer sealing glands or cuffs (Fig. 6.15); or (3) a 


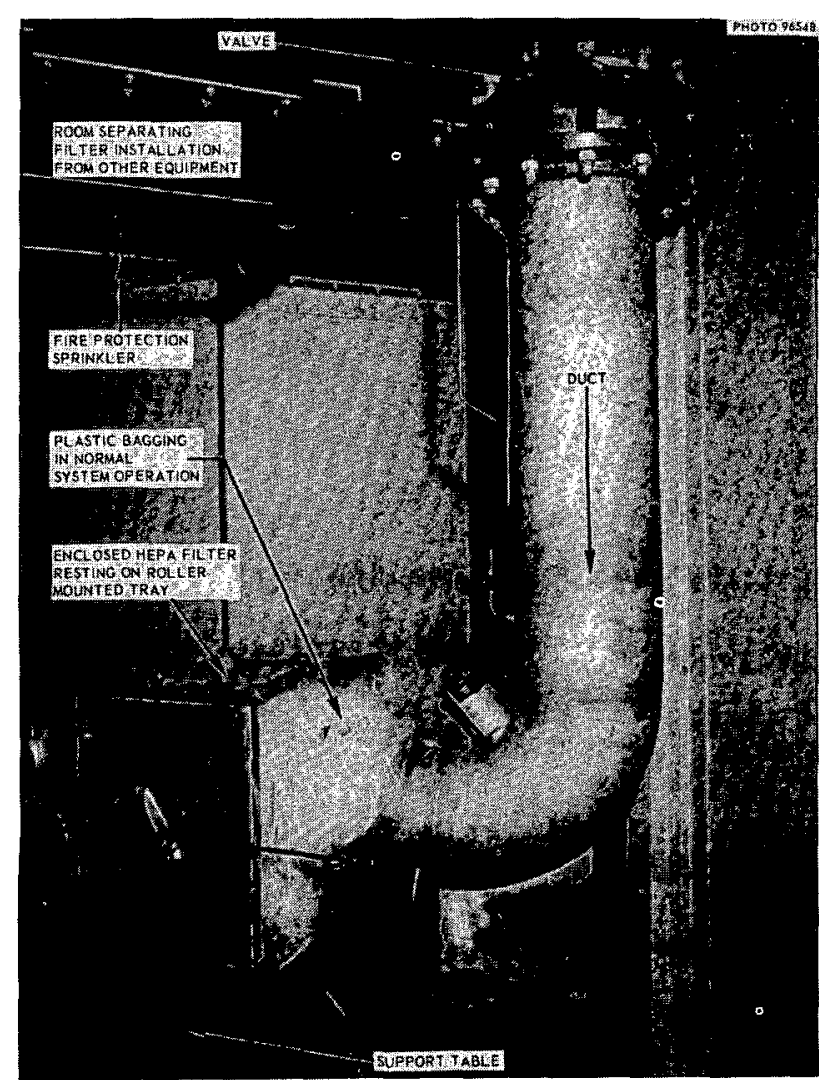

Fig. 6.13. Steel-cased enclosed-filter installation showing plastic bagging to prevent outleakage of particulates and inleakage of air.

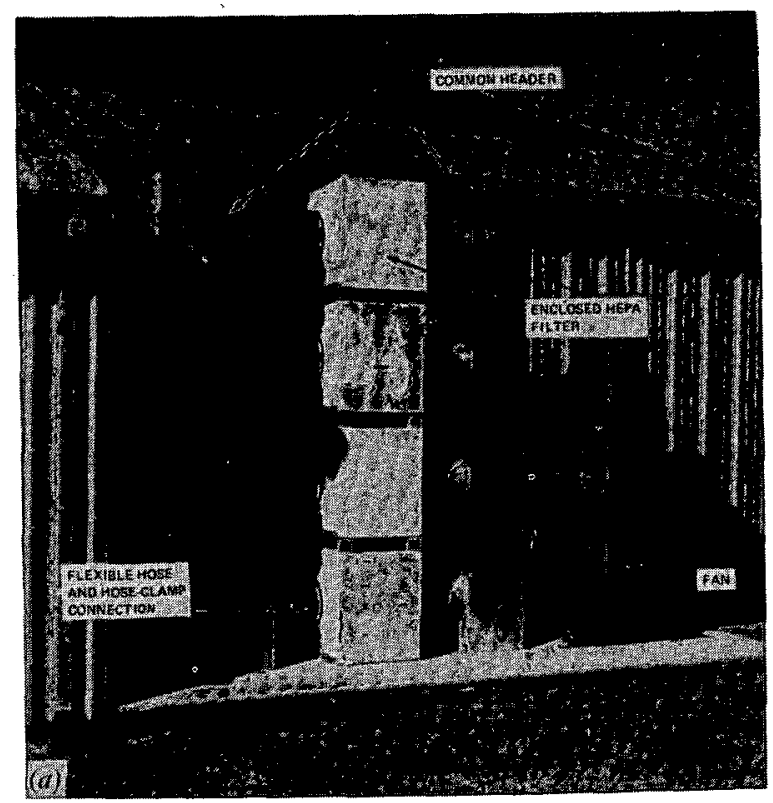

wrap of duct tape. The third method is not reliable and, if used, the tape should be protected with a metal collar as Fig. 6.16 shows. All of these seals are subject to failure when exposed to fire or hot air and cannot be recommended for applications where high levels of radioactivity may be present. Flanged joints are the only type that can withstand extended exposure (more than $10 \mathrm{~min}$ ) to high temperature air or to fire imposed from either inside or outside the duct.

Individual filter supports, such as those in Fig. 4.2, are recommended. In the installation shown in Fig. $6.14 a$, temporary supports for the upper units would have to be built to permit replacement of one of the lower filters. As with open-face filters, horizontal airflow through enclosed filters is recommended.

\subsection{CYLINDRICAL FILTER ELEMENTS}

The cylindrical HEPA filter is another configuration that often appears to offer ideal solutions to certain installation requirements. However, this type of filter must be used with caution because of

Fig. 6.14. Typical flexible-tubing and clamping-ring installation of enclosed HEPA filters. (a) Multiple installation of wood-cased enclosed filters between common supply and exhaust plenums. Note lack of individual filter supports. $(b)$ Typical installation of steel-cased enclosed filter in glove box application. Note support bracket and straps to secure filter element.

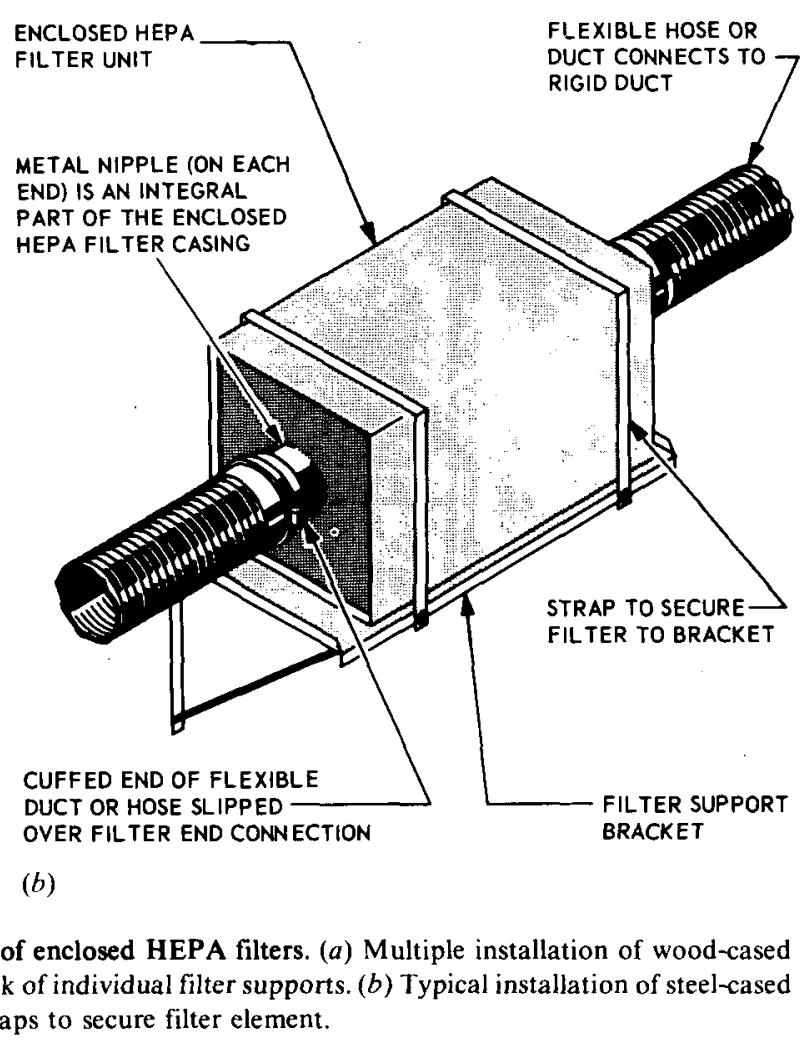


shortcomings in its construction. One manufacturer makes a spiral of the filter material and a separator; the others make a conventional pleated-mediumand-separator core that is trimmed to cylindrical shape. In both designs, the core is slipped into a molded or welded-seam cylinder and sealed by catalyst-activated plastic foam or an adhesive. There is no interference or pressure fit between core and

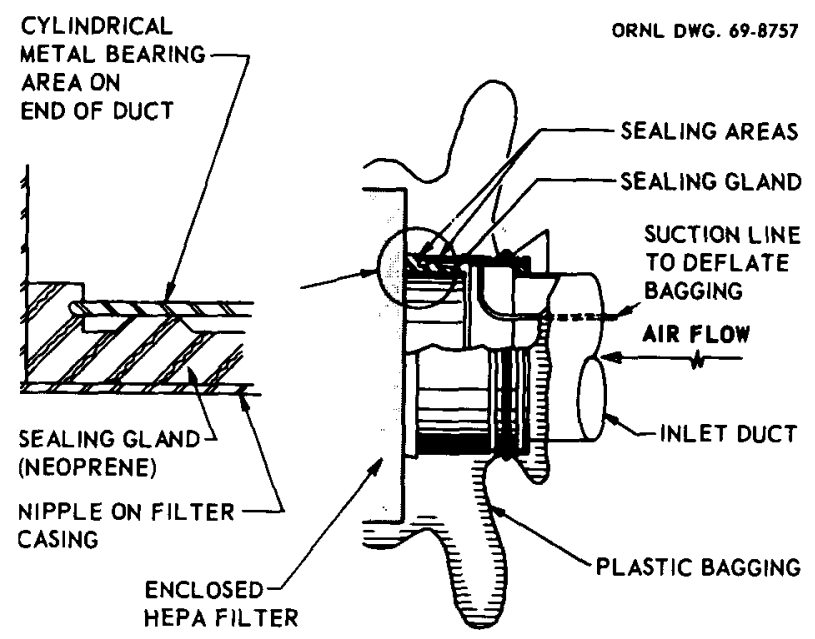

Fig. 6.15. Specially designed elastomeric gland for sealing enclosed filter into duct. casing, as in the open-face and enclosed rectangular configurations, because the core would be damaged when fitted into the case. In addition, because the cases are often made from light gage, easily deformed sheet metal, they are often considerably out-of-round. The result is often a filter element that leaves much to be desired from the standpoints of leak integrity after a period of service and resistance to the exigencies of air cleaning system service.

Cylindrical HEPA filters can be obtained with or without flanges on one or both ends. The filters without flanges are used in push-through (so-called incessant) installations; the filters are sealed into a cylindrical opening with one or more half-round circumferential gaskets (fixed to the filter) which make a slight interference fit with the receiver. Inasmuch as the filters are often out-of-round and a reliable interference fit between filter and receiver is impracticable, push-through installations are often unreliable under system-upset conditions. Pushthrough filters are subject to being blown out of the receiver if pressure differentials become high. Flanged cylindrical HEPA filters can be installed in openings of pipes by bolting them to a flange on the pipe or by clamping the filter flange between mating pipe flanges. Conventional neoprene sponge gaskets
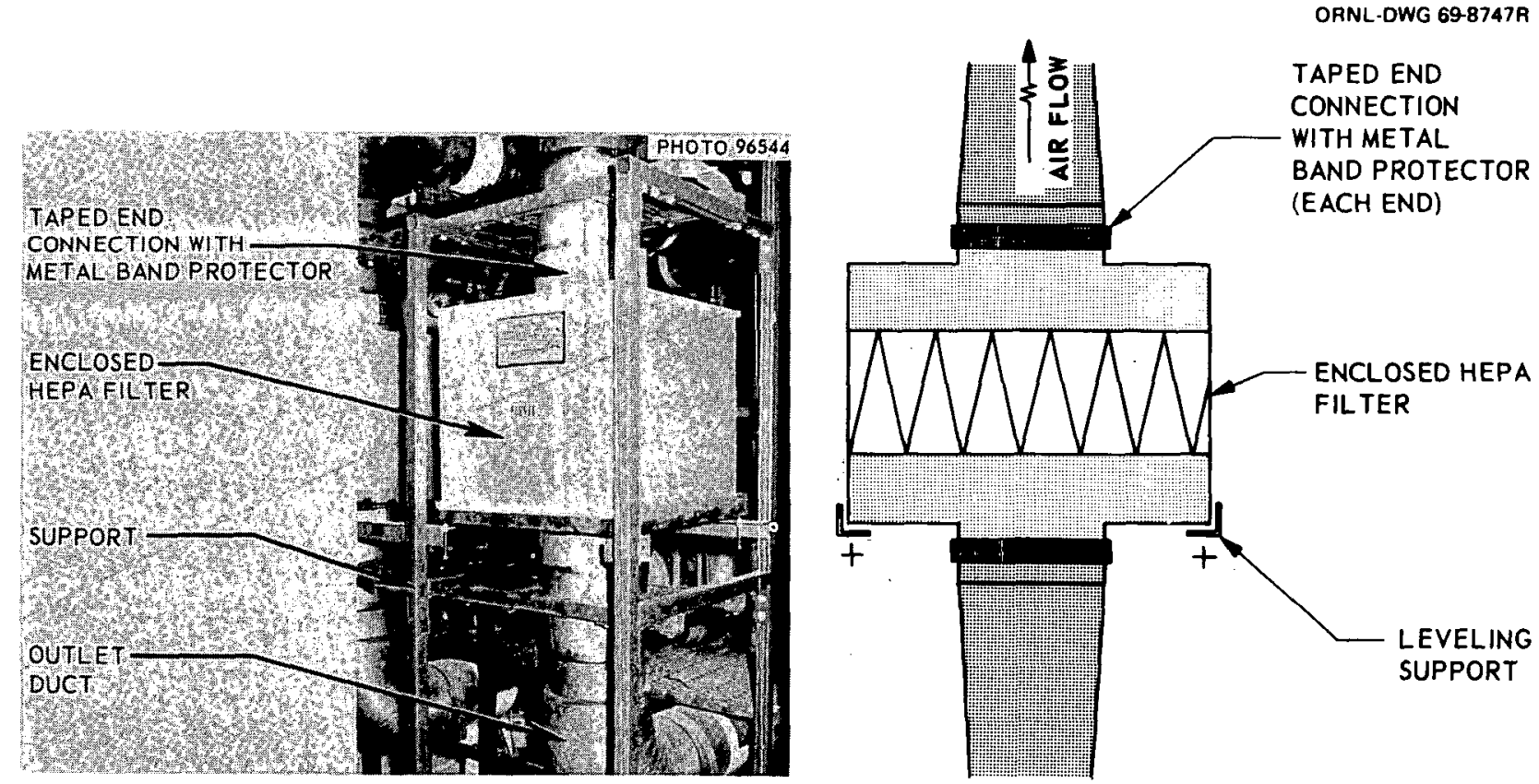

Fig. 6.16. Enclosed filter installation. Note taped connections with metal-band protectors, support for filter. Downward airflow is poor, because (1) airflow and gravity combine to impose an unnecessary pressure condition on the filter core, and (2) if condensation occurs in the pleats of the filter, contamination can seep through with the condensed liquid and contaminate the clean side of the system. 
are used for sealing (Sect. 4.3.4). Because filter flanges and cases are characteristically made from light-gage sheet metal with the flange seal-welded to the cylinder, these filters often leak at the flange-tocase weld. The flange often becomes deformed. Either condition results in an installation that is difficult to seal.

Cylindrical HEPA filters cost substantially more than rectangular HEPA filters of equivalent airflow capacity. In most cases they should be considered as "specials," because manufacturers generally do not stock them. There are no standard dimensions or airflow capacities, and those listed in the manufacturer's catalogs are generally simply those that have been special-ordered by another purchaser at an earlier date. No cylindrical filters are rated under UL$586^{4}$ or listed in any of the standard specifications for HEPA filters (e.g., MIL-F-51068, IES CS-1). Cylindrical HEPA filters are characteristically of relatively small diameter (less than $12 \mathrm{in}$.) and airflow capacity. The authors know of only one 1000-cfm cylindrical HEPA filter installation. In this case the filter was installed horizontally with downward airflow; it had to be replaced frequently because of mechanical damage resulting from high moisture loadings, the unfavorable filter configuration, and unfavorable installation practices. The operator eventually replaced the cylindrical filter with open-face rectangular units which could be bought from stock at a fraction of the price of the special cylindrical elements.
Figure 6.17 shows two methods of installing cylindrical filters, one a duct-entrance design and the other a hot-cell exhaust design. In the hot-cell exhaust design, the mounting is sloped to permit runoff of any liquid accidentally spilled on the shield that protects the filter, and to facilitate handling by the cell electromechanical manipulators. Where cylindrical HEPA filters are used, liberal clearance (at least $1 / 8$ in. all around) between the case and receiver is necessary to accommodate the characteristic out-of-roundness. The advantage of cylindrical filters is close conformance to round ducts and pipes, which can permit the use of smaller and cheaper duct transitions and less space requirement. For in-line installations, however, except where the filter has flanges on both faces and is installed as a spool piece, provision must be made to extract the filter from the duct or pipe after the connection is broken, with the result that the space advantage over an equivalent open-face rectangular filter may be lost. Spool-piece filters must have flanges that can withstand the forces imposed by the duct or piping system, and the flange bolting.

Cylindrical filters are often used in radioactive vacuum cleaners and portable air purifiers (Fig. 6.18). The air purifier shown is a single-use device that is discarded when the contamination level or pressure drop of the collectors becomes greater than some preestablished design level, or the arresting efficiency of the collectors drops below a preestablished design level (usually $99.97 \%$ for the

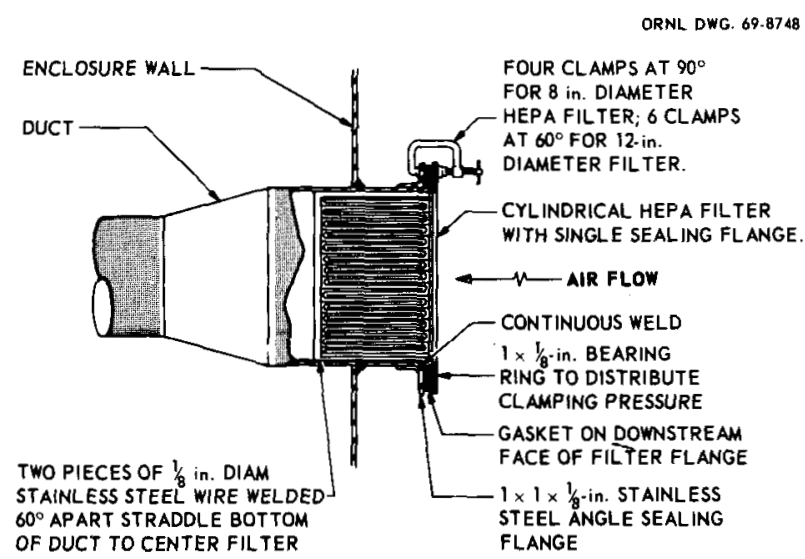

(a)

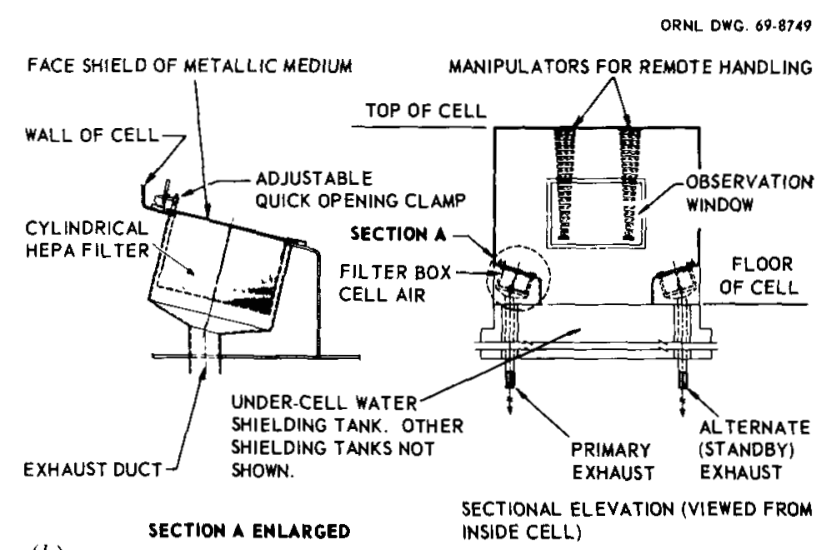

(b)

Fig. 6.17. Typical cylindrical HEPA filter installation. (a) Installation designed for contact maintenance. (b) Installation designed for remote maintenance. 


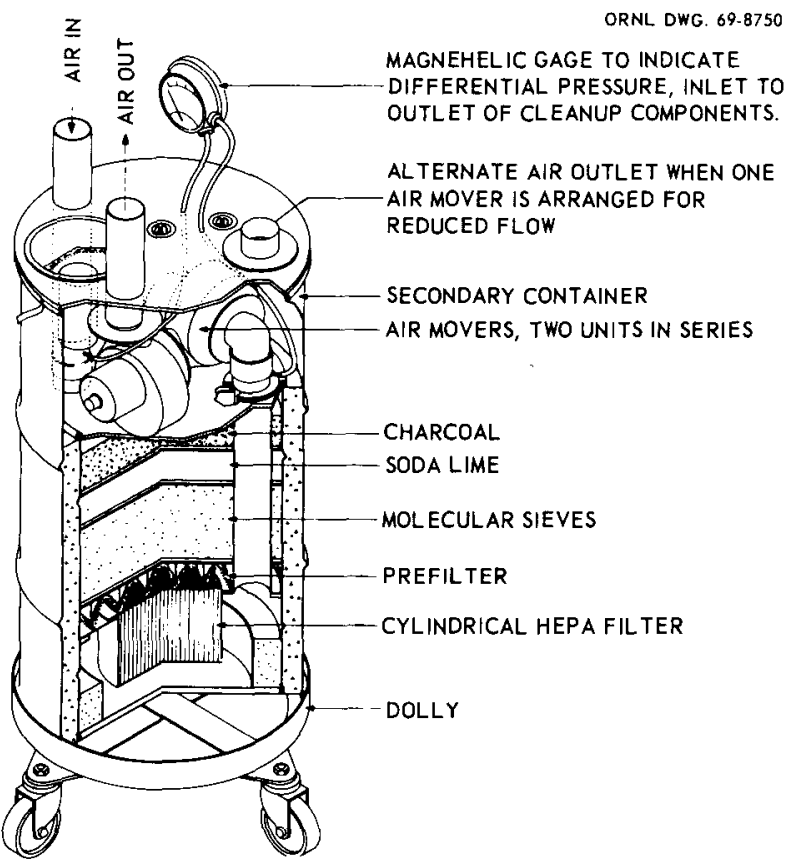

Fig. 6.18. Dry air purifier employing cylindrical HEPA filter.

filter). The air purifier was provided to clean a lowvolume flow of off-gas evolved during the processing of high-level transcurium elements. Although an open-face rectangular filter element could have been used in a somewhat different arrangement, the use of the cylindrical element was a designer's option, not a requirement of the application. As a general rule, the use of the rectangular filter is recommended wherever practicable.

\subsection{INSTALLATION}

\subsubsection{Human Factors}

The recommendation to install filters vertically, with horizontal airflow, is discussed in Chap. 4. When practicable, single-filter installations should be located where they can be reached for service and testing without requiring workers to climb ladders or scaffolding. This requires a consideration of human engineering factors, that is, the reaching and lifting capability of the average man. Figure 6.19 shows dimensions for the 5th and 95th percentile man. Dimensions of female workers are substantially smaller. ${ }^{8}$ Extrapolation of the recommended weight limits given in Fig. 6.20 indicates that handling a 1000-cfm HEPA filter, in the body positions often encountered in filter-change operations, is at the upper range of personnel capability (higher loadings result in lower man-efficiency) and that handling of adsorber cells is well beyond the limits for one person. Consideration must be given to the positions that a worker must assume to perform the required task. If he must hold his hands overhead for any length of time, he becomes fatigued. If he has to crouch, bend, or squat, he will soon become stiff, which will contribute to his loss of efficiency. If a worker has to hold a heavy weight while performing a precision operation (e.g., supporting the weight of a filter or adsorber cell while trying to fit it between duct transitions or into a restricted opening), the stress of the combined task becomes fatiguing and he is apt to make a mistake. ${ }^{8}$ All of these factors are compounded when the worker must wear protective clothing and respiratory protection. In addition, protective clothing adds to his spatial requirements and limits his mobility. For HEPA filter and adsorber cell installations, location of the filter or housing at an elevation between knee and shoulder height (Fig. 6.19 ) is recommended.

\subsubsection{Fume Hood Filter Installations}

The wide and often unpredictable variety of chemical operations conducted in laboratory fume hoods makes the selection and installation of HEPA filters difficult and uncertain. Corrosive fumes may damage the filter and its mounting, and moisture and heat from hooc operations may accelerate the damage. Operations that produce steam or moisture should be restricted to minimize condensation in, or the carry-over of water and/or chemical droplets to, the filter core.

Figure 6.21 shows a wood-cased enclosed HEPA filter installed immediately downstream of a fume hood and within the laboratory. This installation presents a threat to the laboratory in the event of a spill during a filter change and necessitates interruption of laboratory operations when the filter is changed or tested. Some facilities install the fume hood filters in the attic, usually directly above the hood served, as shown in Fig. 4.1. Where such design is employed, the attic space should be designed as a contamination zone, providing for ease of cleanup in the event of a spill, and should not be used for extraneous purposes such as storage and experimental work when radioactive materials are handled in the hood. 


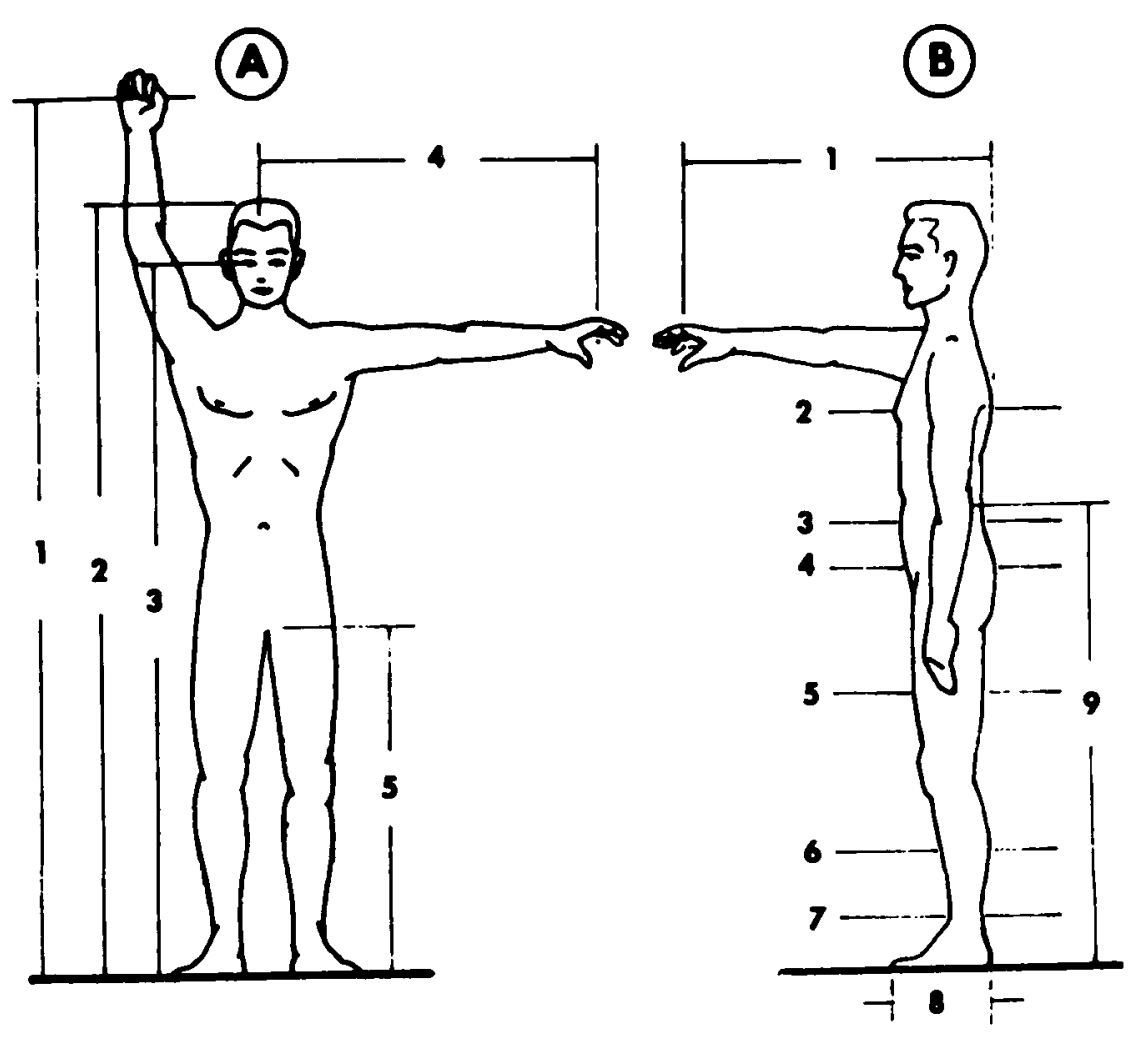

MALE HUMAN BODY DIMENSIONS

Selected dimensions of the human body (oges 18 to 45) suitable for initial design of crew spoce and equipment. Locations of dimensions correspond to illustrotions on the page al the left.

\begin{tabular}{|c|c|c|c|}
\hline & \multirow{2}{*}{ DIMENSIONAL ELEMENT } & \multicolumn{2}{|c|}{$\begin{array}{l}\text { DIMENSION } \\
\text { (in inches excepl where noted) }\end{array}$} \\
\hline & & Sih PERCENTILE & 95th PERCENTILE \\
\hline & Weight & $132 \mathrm{LB}$ & 20118 \\
\hline $\begin{array}{l}1 \\
2 \\
3 \\
4 \\
5\end{array}$ & $\begin{array}{l}\text { Vertical reach } \\
\text { Stature } \\
\text { Eye to foor } \\
\text { Side arm reach from Cl of body } \\
\text { Croteh to hoor }\end{array}$ & $\begin{array}{l}77.0 \\
65.0 \\
61.0 \\
29.0 \\
30.0\end{array}$ & $\begin{array}{l}89.0 \\
73.0 \\
69.0 \\
34.0 \\
36.0\end{array}$ \\
\hline $\begin{array}{l}1 \\
2 \\
3 \\
4 \\
5 \\
6 \\
7 \\
9 \\
9\end{array}$ & $\begin{array}{l}\text { Fonward arm reach } \\
\text { Chest circumforence } \\
\text { Woist circumference } \\
\text { Hip circumference } \\
\text { Thigh circumference } \\
\text { Colf circumference } \\
\text { Ankle circumference } \\
\text { Foot length } \\
\text { Elbow to foor }\end{array}$ & $\begin{array}{r}28.0 \\
35.0 \\
28.0 \\
34.0 \\
20.0 \\
13.0 \\
8.0 \\
9.8 \\
41.0\end{array}$ & $\begin{array}{l}33.0 \\
43.0 \\
38.0 \\
42.0 \\
25.0 \\
16.0 \\
10.0 \\
11.3 \\
46.0\end{array}$ \\
\hline
\end{tabular}

Fig. 6.19. Male human body dimensions. From W. E. Woodson, Human Engineering Guide for Equipment Designers, University of California Press, 1966. 


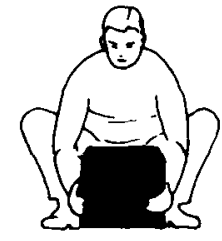

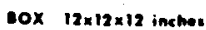
LOAD DIMENSIONS
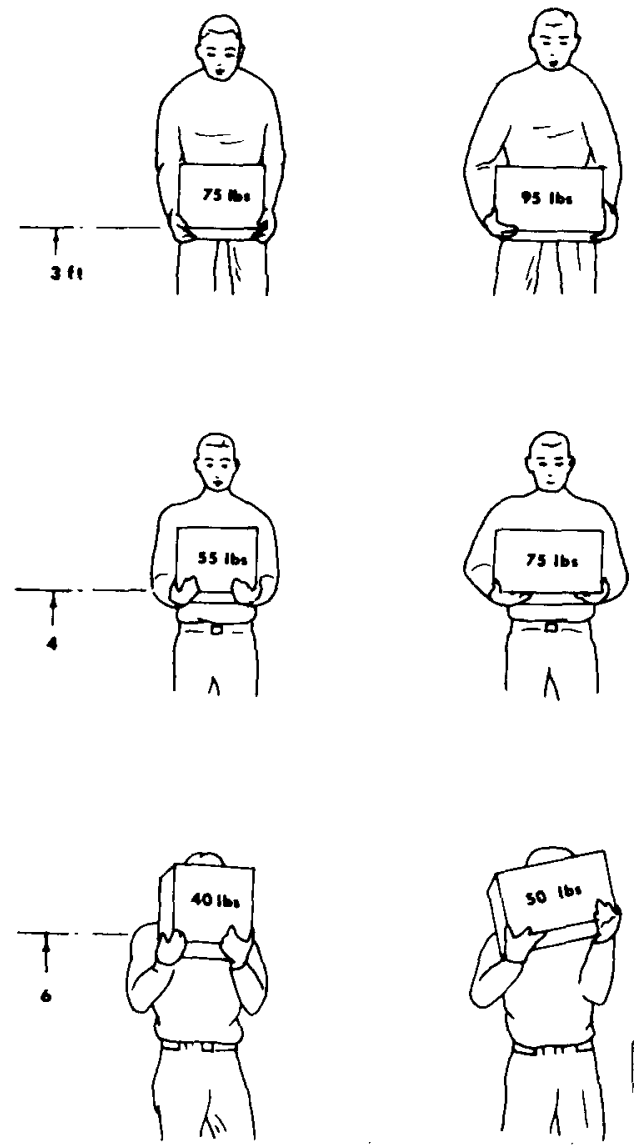

Fig. 6.20. Recommended weight limits for 95 th percentile male and maximum loads that should be handled in various body-load orientations and load-heights. From W. E. Woodson, Human Engineering Guide for Equipment Designers, University of California Press, 1966.

Hoods in which perchloric acid and certain other chemicals are handled should be provided with washdown facilities to permit periodic decontamination of the hood and ductwork (perchloric acid hoods should not be used for handling other materials because of the explosion hazard). Off-gas scrubbers are often provided in hoods. Both washdown facilities and scrubbers generate substantial quan- tities of sensible water droplets. Provision of demisters that meet the requirements given in Sect. 3.5 should be considered for protection of the filters and their mountings. Moisture collected in the demister should be conducted to a hood drain rather than permitted to fall back into the work space of the hood. Demisters should have adequate handling space and be easily accessible for cleaning, inspection, and replacement. Where incandescent particles or flaming trash can be released to the hood exhaust stream, a spark arrestor may be needed to protect the HEPA filter. This arrestor can be either a commercial flame arrestor, a metal-mesh graded-density demister, or as a minimum, a piece of 40 -mesh metal cloth. In any event, the arrestor must be located at least 1 to $2 \mathrm{ft}$ ahead of the HEPA filter and must have easy access for cleaning, inspection, and replacement.

Heat sources such as heating mantles, furnaces, and Bunsen burners are common equipment in laboratory fume hoods and should be planned for in the initial hood and exhaust system design. Extended lengths of duct between the hood and filter can

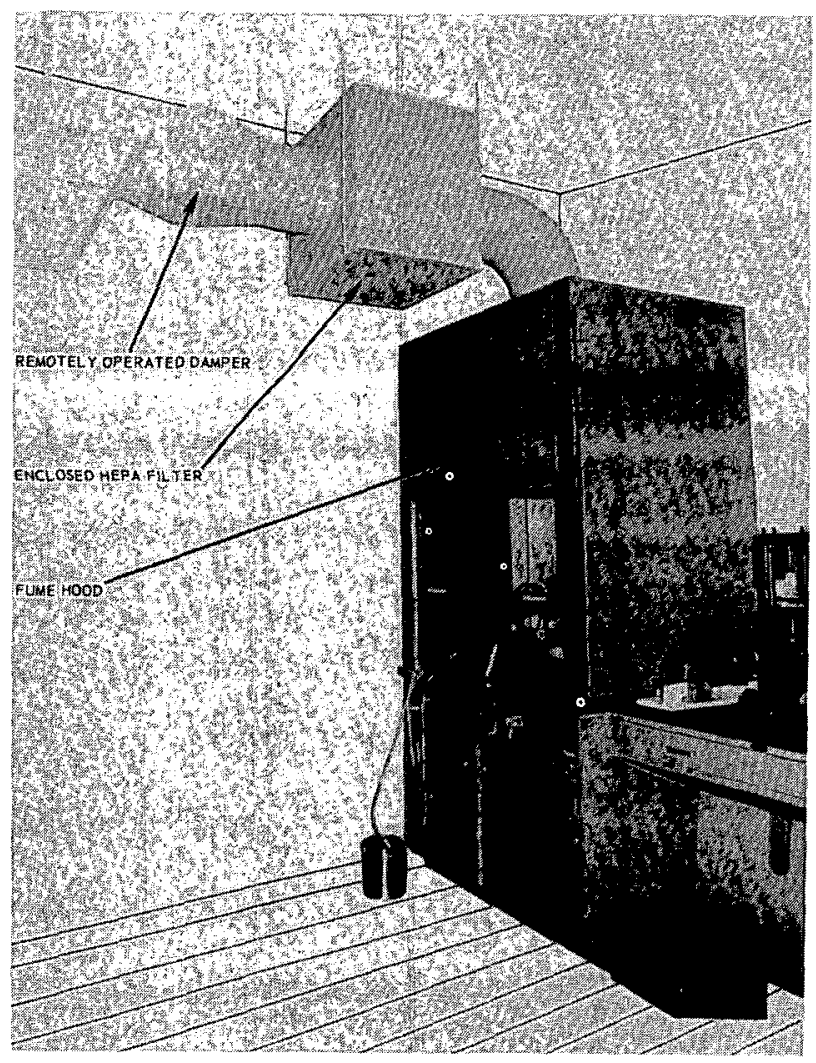

Fig. 6.21. Wood-cased enclosed HEPA filter installation for laboratory fume hood. 


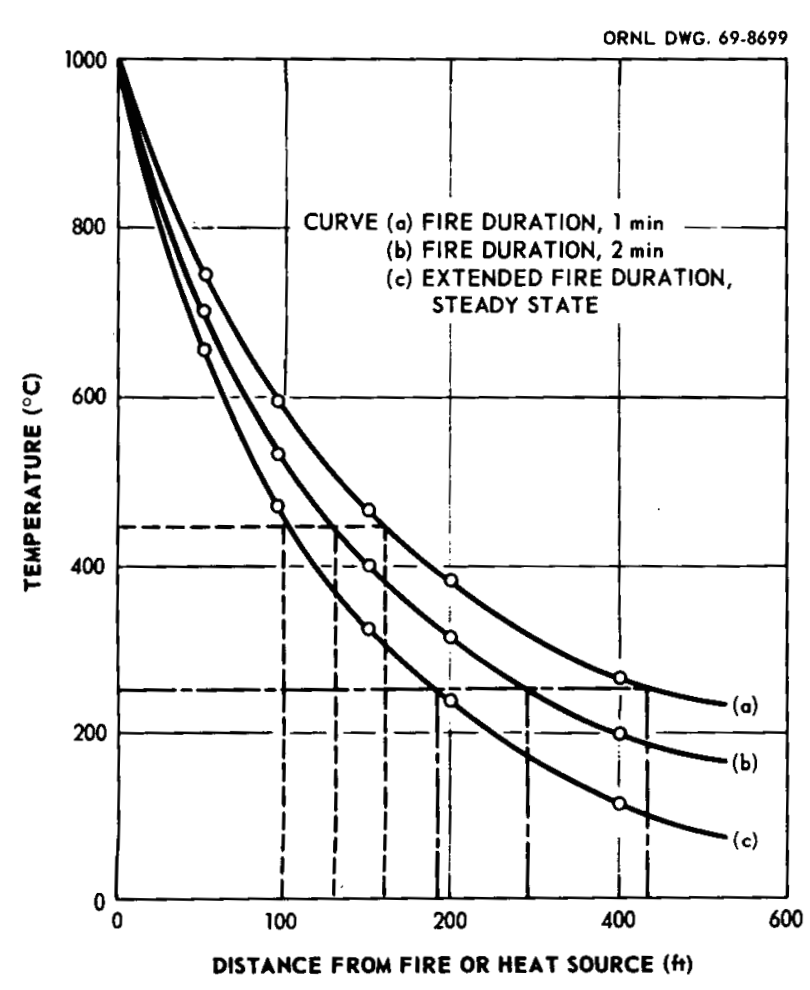

Fig. 6.22. Cooling rate of air in a 12-in.-diam duct carrying 1000 cfm of air. Dashed lines show the length required to cool the air from an initial temperature of $1000^{\circ} \mathrm{C}\left(1832^{\circ} \mathrm{F}\right)$ to $450^{\circ} \mathrm{C}\left(842^{\circ} \mathrm{F}\right)$ and $250^{\circ} \mathrm{C}\left(482^{\circ} \mathrm{F}\right)$ for various fire conditions. From S. E. Smith et al., Protection Against Fire Hazards in the Design of Filtered Ventilation Systems of Radioactive and Toxic Gas Process Buildings, UKAEA Report AWRE O-24/65, Atomic Weapons Research Establishment, United Kingdom, July 1965. provide substantial cooling, but to be really effective, a length of at least $100 \mathrm{ft}$ is needed (Fig. 6.22). Such a length is often impractical, and control of heatproducing operations by limiting the size of heat sources, insulation of furnaces, etc., or use of air cooling methods such as sprays, must be relied upon. Chapter 9 discusses operational control for fire prevention and heat control in HEPA filter systems.

\subsubsection{Emergency and Portable Air Cleaning Units}

Figures 6.23 and 6.24 show portable air cleaning units used for emergency and temporary air cleanup applications. The emergency system in Fig. 6.23 is an army CBR unit and fan installed on a common skid that can be transported by truck or airplane. The CBR unit, which contains a HEPA filter $(1000 \mathrm{cfm})$ and a multitray adsorption unit, was designed for attachment to a small reactor containment structure in the event of an accident. The portable unit in Fig. 6.24 is conceptually the same but employs a HEPA filter and either a prefilter or pleated-bed (type I) adsorber cell installed in individual commercially built caissons. The selection and installation of enclosures (caissons) and components in units such as these should adhere to the same principles discussed earlier. Because the unit in Fig. 6.24 can accommodate only a $1-i n$. pleated-bed adsorber cell, it is not satisfactory for service in which high arresting efficiency for radioactive organic iodine compounds is required. Figure 6.25 shows a superior design,

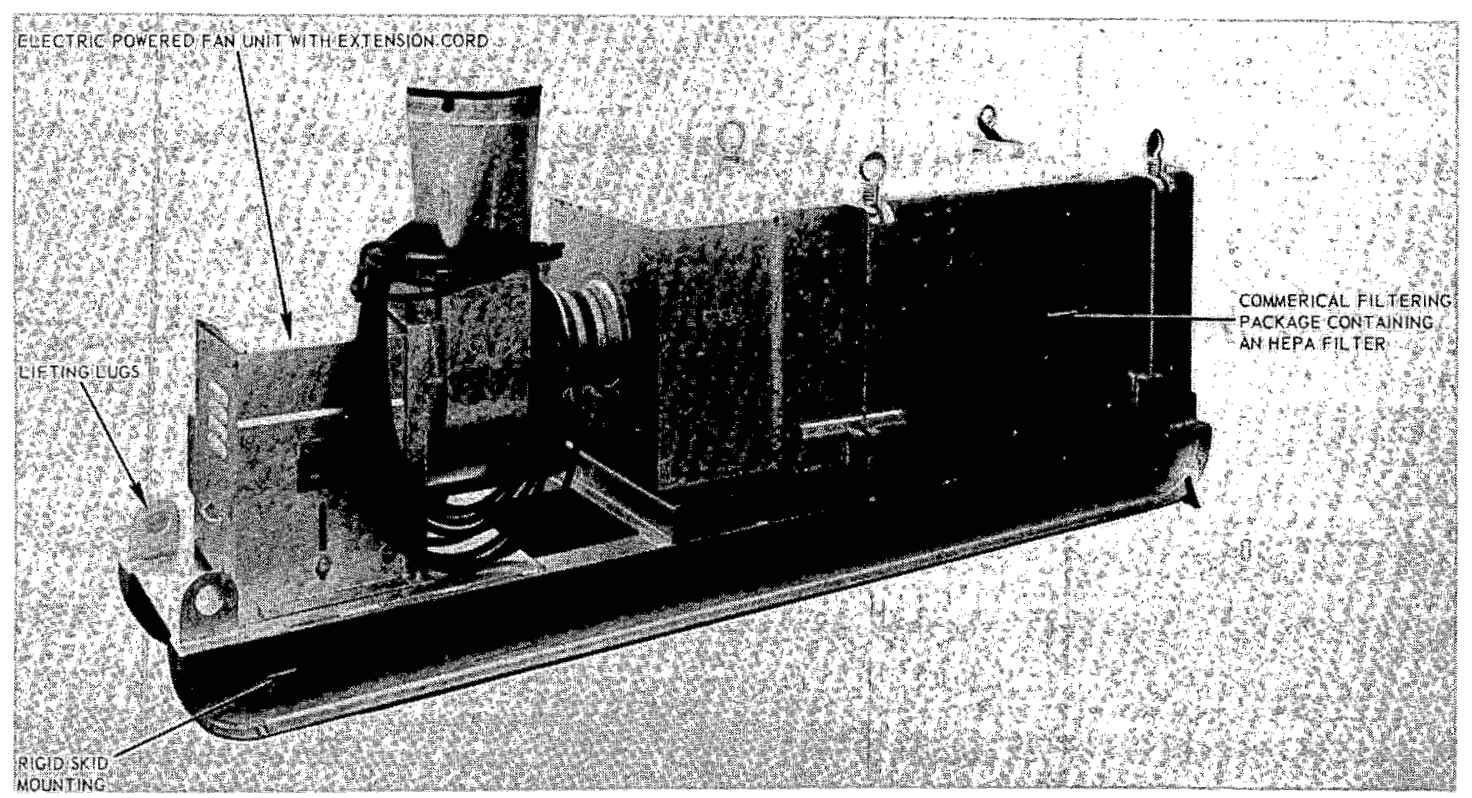

Fig. 6.23. Emergency air cleaning unit consisting of a CBR unit and fan mounted on a common skid, transportable by truck or plane. Courtesy Lawrence Livermore Laboratory. 


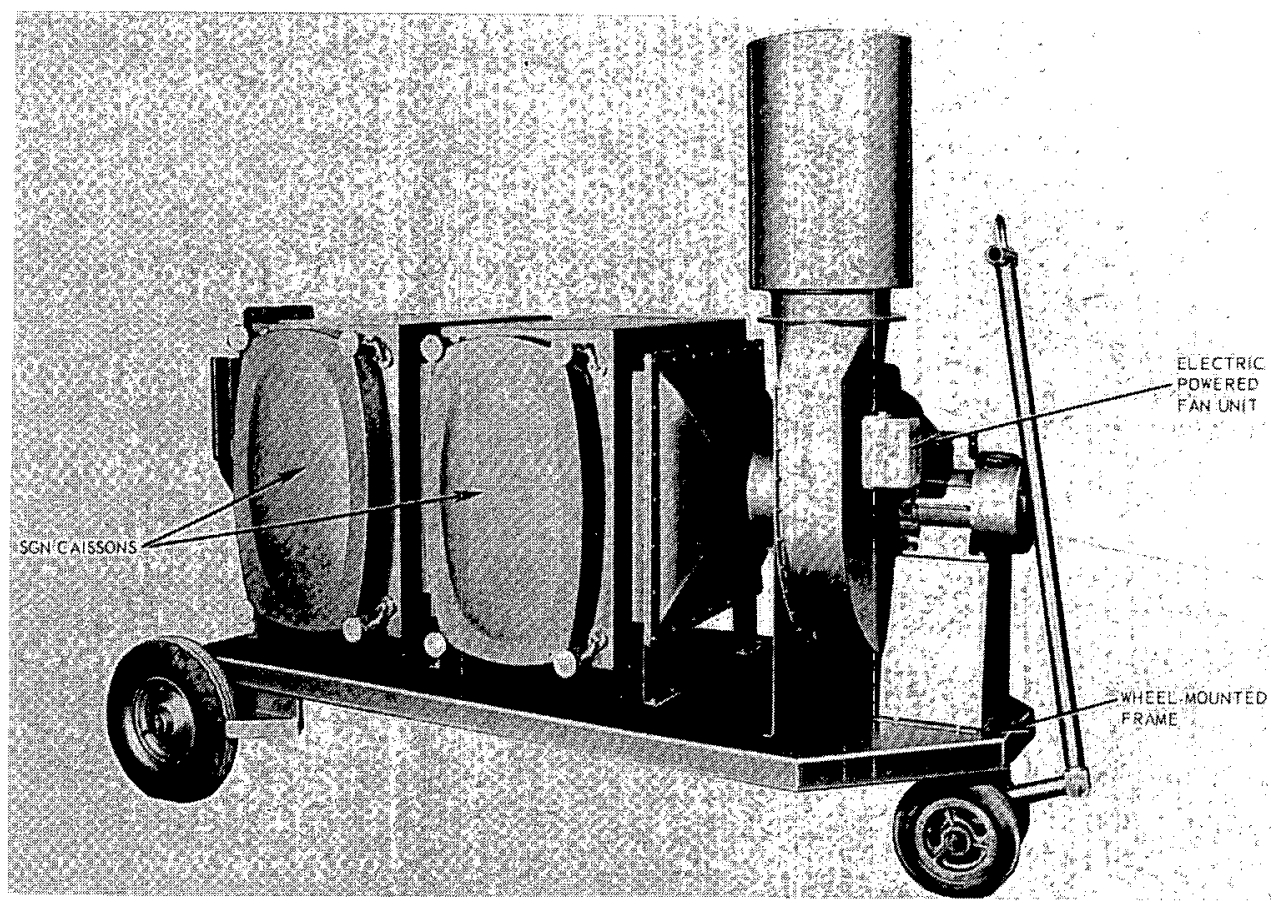

Fig. 6.24. Portable air cleaning unit consisting of two 1000 -cfm caissons and fan mounted on a cart. Courtesy Sainte Gobain Nucléaire.

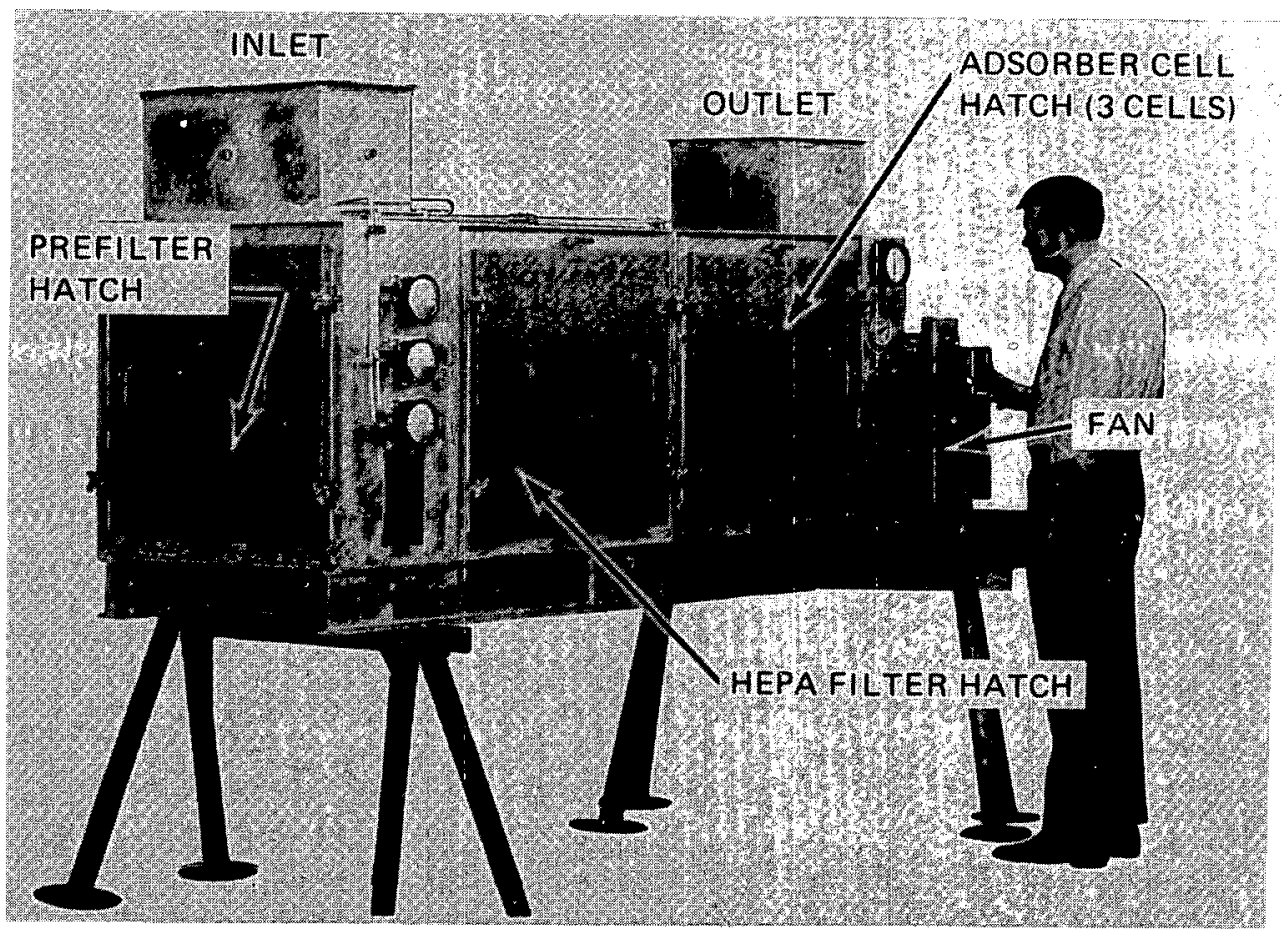

Fig. 6.25. Transportable air cleaning unit consisting of a heavy-duty enclosure containing prefilter, HEPA filter, three type II adsorber cells, and fan mounted on a common skid. Transportable by truck or plane. Courtesy CTI-Nuclear, Inc. 
employing a prefilter, 1000-cfm HEPA filter, and three 2-in.-bed tray-type (CS-8 type II) adsoiber cells.

Emergency and portable air cleaning units require the same periodic inspection and in-place leak testing as permanently installed systems. The rough handling and shock they can be expected to experience during transport makes careful inspections and functional tests desirable prior to each use. The skid and framing assembly must be rigid and capable of absorbing the shocks that can be expected from the transport environment and handling.

Figure 6.26 shows a portable HEPA filter installation that can be used for temporary exhaust service. Portable hoods and enclosures are often used in nuclear plants for spot protection of temporary operations or inadvertently contaminated areas. These situations do not warrant the expense of a complete air cleaning unit and the need can often be met by a simple arrangement such as the one showr in Fig. 6.26. This device can be connected to any duct of a central exhaust system by a flexible hose and used to ventilate contained spaces such as a manhole, tank, vault, hood, or enclosure. For such temporary installations, sealing the filter into its housing using a wrap of duct tape is satisfactory. If the portable

\section{REFERENCES FOR CHAP. 6}

1. UL-181, Safety Standard for Air Duct Materials, Factory Made, and Air Duct Connections, Underwriters' Laboratories, Chicago, 1974.

2. NFPA $90 \mathrm{~A}$, "Air Conditioning and Ventilating Systems," National Fire Codes, National Fire Protection Association, vol. 9 , Boston, 1975.

3. Copyright, Flanders Filters, Inc.

4. UL-586, Safety Standard for High Efficiency Air Filter Units, Underwriters' Laboratories, Chicago, current issue.

5. MIL-51086-D, Filter, Particulate, High-Efficiency, Fire Resistant, U.S. Department of Defense, Washington, D.C., current issue. installation becomes contaminated, the whole assembly can be discarded as a throwaway item and replaced at moderate cost.

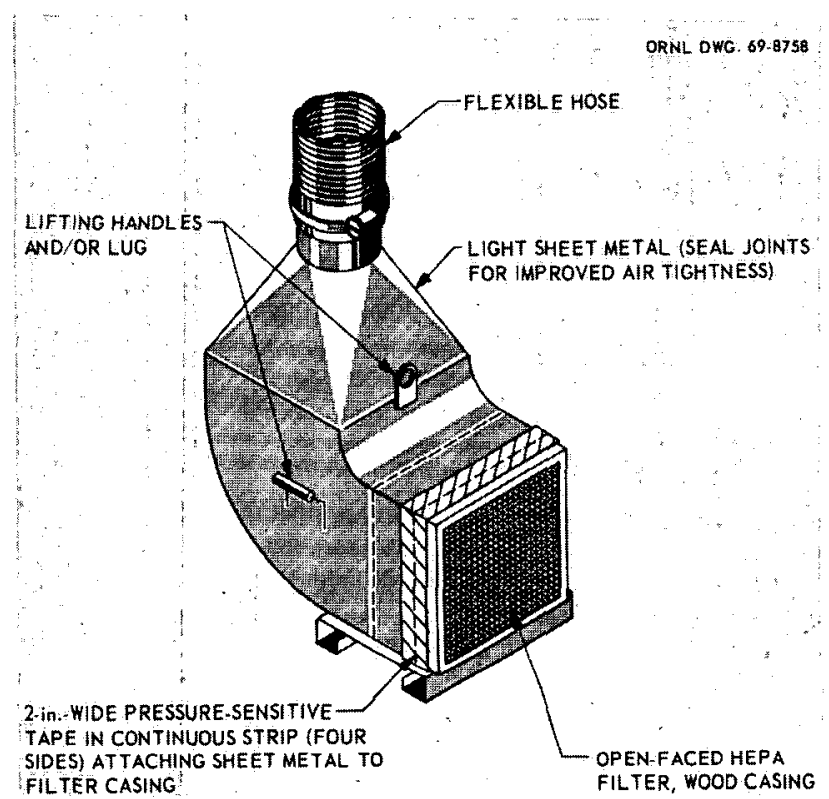

Fig. 6.26. Low-cost filter enclosure for temporary service.

6. ANSI N512, Protective Coatings (Paints) for the Nuclear Industry, American National Standards Institute, New York, 1974.

7. J. Young, W. T. Pearce, and T. C. Parsons, Dry Scrubber Unit for Low-Peak Ventilation Systems, USAEC Report UCRL10953, Lawrence Radiation Laboratory, University of California, Berkeley, August 1963.

8. W. E. Woodson and D. W. Conover, Human Engineering Guide for Equipment Designers, University of California Press, Berkeley, 1966. 


\section{Glove Box Filtration}

\subsection{INTRODUCTION}

Glove boxes occupy a middle ground between chemical fume hoods and hot cells as primary containment areas for working with radioactive materials. Glove boxes are Zone I or Zone II spaces for work with materials involving greater risk than would be appropriate for a fume hood but not of sufficient risk to require a hot cell. Zoning is discussed in Sect. 2.2.1. Material appropriate for glove box handling generally presents little or no penetrating radiation hazard. Glove boxes mainly protect against the inhalation of radioactive materials, and glove box filtration plays a significant role in such protection. The escape of radioactive material without sufficient treatment nullifies the protection provided by the glove box. This chapter discusses the filtration of air or other gases associated with the ventilation of these enclosures. To understand the importance of glove box filtration, a clear understanding of the functioning of the glove box itself is necessary.

\subsubsection{Description of Glove Boxes}

A glove box (Fig. 7.1) is a windowed, airtight enclosure equipped with one or more flexible gloves for manual handling of material inside the enclosure from the outside. Figure 7.2 defines glove boxes and focuses attention on their use in the nuclear industry. Glove boxes are also used in other industries and laboratories to enable the safe handling of pathogenic and toxic materials or to prevent contamination of a material being handled. The main purpose for using a glove box is to permit the use of the hands in manipulating hazardous materials through a membrane (the glove) while preventing release of the material to the environment.

There is no commercial standard to cover the construction of glove boxes. As a result, many

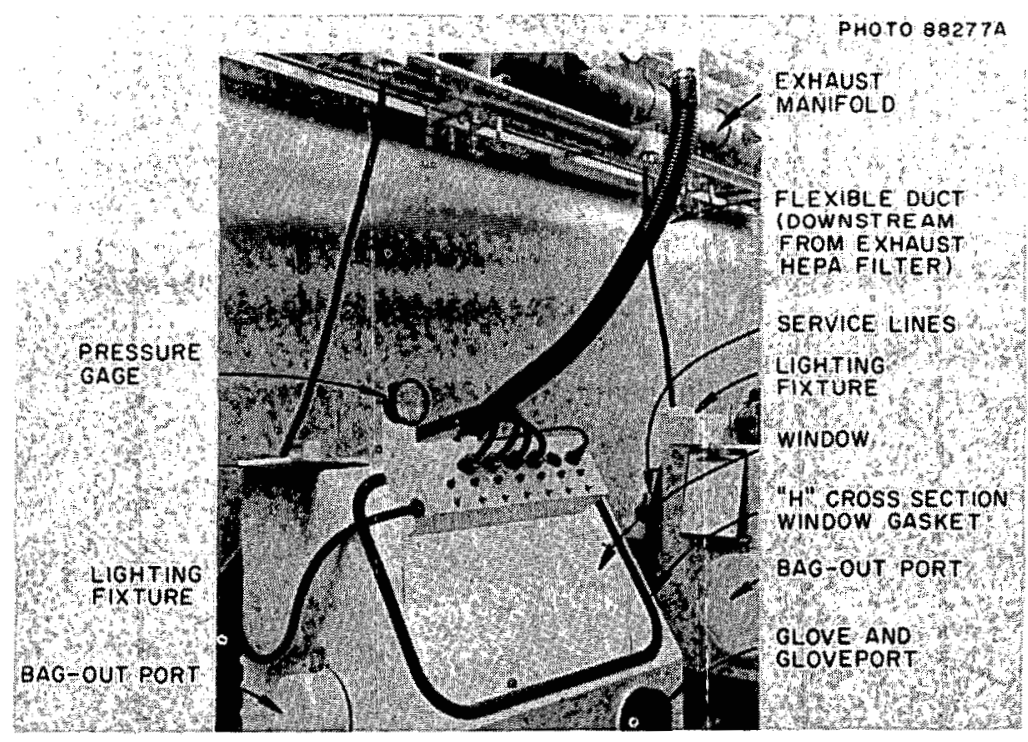

Fig. 7.1. Typical glove box showing major features. Box is mounted on wheels. All services are brought into back of box. Note differences in lighting fixtures and bag-out ports of two boxes that can be seen. Flexible duct is permissible only downstream of the glove box exhaust HEPA filter, mounted on the back of the box. 
1. DEFINITION

2. PURPOSE

3. REASON

NECESSARY

4. HOW

ACCOMPLISHED

5. RELATED

WORKSPACES

6. WORKSPACE

HANDLING

7. EXCLUSION

METHOD

\section{GLOVE.BOX ATMOSPHERE \\ 9. Destination \\ OF BOX}

AN ENCLOSURE WITH CONNECTED GLOVES TO ALLOW PEOPLE ON THE OUTSIDE TO HANDLE MATERIAL WITHIN WORKSPACE TO PROCESS MATERIAL AND CONTROL INTERACTION OF SURROUNDINGS AND MATERIAL

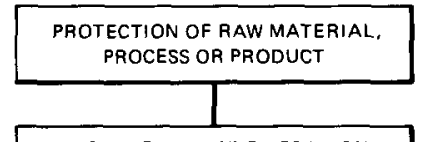

EXCLUDE UNWANTED PORTION
OF SURROUNDINGS FROM MATERIAL

BIOSPHERE FROM MATERIAL

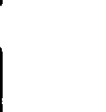

EXCLUDE DANGEROUS POBTION

EXCLUDE DANGEROUS PORTION
OF MATERIAL FROM SURROUNDINGS
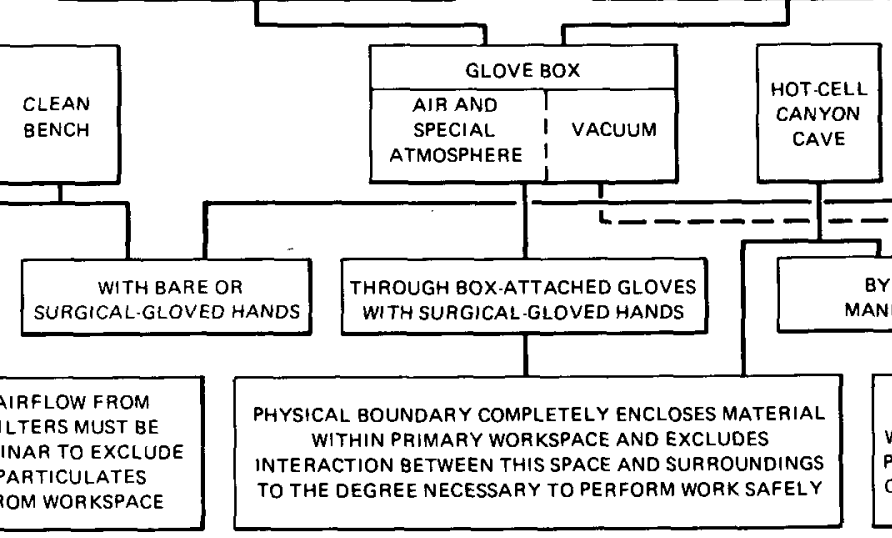

HOT.CELL
GANYON

CAVE

FUME

HOOD

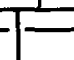

L-ー--- $=--7$

\section{THROUGH BOX-ATTACHED GLOVES}

WITH SURGICAL-GLOVED HANDS

$$
\text { (1 }
$$

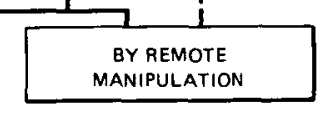

PHYSICAL BOUNDARY COMPLETELY ENCLOSES MATERIAL WITHIN PRIMARY WORKSPACE AND EXCLUDES NTERACTION BETWEEN THIS SPACE AND SURROUNDINGS

TO THE DEGREE NECESSAR

AIRFLOW TOWARD WORKSPACE AWAY FRO

PERSONNEL (EXHAUSTED
OUT AFTER TREATMENT)

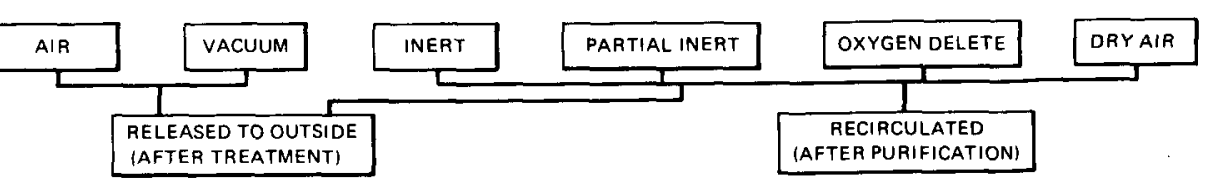

Fig. 7.2. Characteristics of glove boxes.

\section{COMMENT}

GLOVE BOX IS SPACE FOR WORK TO BE PERFORMED WHILE EXCLUOING UNWANTED EFFECTS OF SURROUNDINGS IN MATEAAL OR MATERALS ON SURAOUNDINGS. PROTECT THE SURROUNDINGS

VACUUM GLOVE BOX EVACUAATED FOR DURATION OF VACUUM GLOVE BOX EVATCUATED OTHERWISE

IN VACUUM GLOVE BOXES, MATERIAL IS HANDLED REMOTELY UNDER VACUUM. THROUGH BOX GLOVES WHEN VACUUM RELIEVED

PHYSICAL BOUNDARY MAY BE SHEET METAL WALLS. GLASS WINDOWS, RUBBER GLOVES, GASKETS, PIPE SERVICE CONNECTIONS. OR INLET OR EXHAUST. FILTER MEDIA

GLOVE-BOX ATMOSPHERES OTHER THAN AIR ARE USED TO PROTECT MATERIAL OR PREVENT IT FROM BECOMING LESS STABLE 
different designs exist. Fabricators in the United States build and sell glove boxes and related equipment either of their own design or to customer specifications. Few parts of such boxes are interchangeable. Even though some standardization has resulted from recurring requirements of single users, few features of glove box design are universally accepted in the nuclear industry. Clear evidence of the differences in need and opinion on this subject is given in Glove Boxes and Containment Enclosures, ${ }^{1}$ in which considerable design and operational data were documented in 1962.

Most glove boxes contain no special radiation shielding. Their volume varies from 20 to $100 \mathrm{ft}^{3}$, with geometry that makes them little wider than an outstretched arm. They contain one or more safetyglass, laminated-glass, or plastic viewing windows on at least one side, with glove ports, usually in multiples of two, at various locations in the walls. Interior workspace is reserved for primary operating purposes on the box floor between the glove ports and within reach of a gloved hand and arm. Remote handling capability, other than tool extensions for the gloved hand, is usually not provided. Glove boxes are normally kept at a negative pressure of 0.3 to 1.0 in.wg relative to their surroundings. The maximum safe differential pressure between the interior and exterior of the box is usually less than 10 in.wg; greater differential pressure may damage or rupture a glove or window, with subsequent loss of box integrity. Material transfers between the box interior and exterior, commonly made through a bag-out port, are time-consuming and tedious. The associated HEPA filter installation must adapt to these limitations and still provide reliable service. Hybrid glove box-shielded cells, vacuum glove boxes, roomhigh glove boxes, glove box "trains," etc., are often encountered, and all require reliable filter installations.

Special atmospheres, such as inert gas and dry air, are often used in glove boxes to enhance safety or to provide a necessary processing environment within the box. Inert Atmospheres, ${ }^{2}$ by White and Smith, provides design information on special atmosphere boxes. This chapter is mainly concerned with airventilated boxes, since their application is more general and the principles can be applied, with only slight modification, to other atmospheres.

\subsubsection{Importance of Glove Box Ventilation and Filtration}

Operations conducted in glove boxes often provide the elements for unstable conditions (e.g., fire and pressurization). A properly designed and operated glove box ventilation system minimizes these instabilities and the possibility of an accidental release of airborne radioactive material. Air is a safe glove box atmosphere for most operations. On the other hand, operations on pyrophoric materials such as plutonium, or the presence of reactive gases such as hydrogen, may require a special atmosphere (e.g., low oxygen, inert gas, vacuum).

For air-atmosphere boxes, ventilation at relatively low flow rates provides sufficient dilution of the limited combustible volatiles found in well-operated glove boxes. The correct airflow quantity and pattern, along with the proper location of supply and exhaust connections, minimizes the likelihood of fire while providing sufficient dilution to prevent the buildup of explosive gases (Sect. 7.2.1). Air circulation also removes some of the equipment heat generated inside the box and helps maintain reasonable working temperatures for the operator (Sect. 7.2.2). However, this convective cooling may not be sufficient to remove all the process heat generated in the box, and auxiliary cooling may be required. A well-designed glove box ventilation system includes vacuum-and pressure-surge relief (Sect. 7.2.6) and adequate pressure control to maintain proper pressure differentials between the glove box and its surroundings (Sect. 7.2.5). If a glove should tear or accidentally come off, there should be an assured flow of air through the opening into the box, sufficient to prevent outleakage of contamination until the port is closed (Sect. 7.2.4). Instruments to give foreknowledge of imminent filter plugging and the ability to change filters without undue delay or effort are necessities of a well-designed and -operated glove box installation. In short, the glove box ventilation and filtration system must be capable of reliable performance to assure the glove box technician that he may safely operate the box without fear of airborne contamination to himself, his neighbors, and the environment.

\subsection{DESIGN OF GLOVE BOX VENTILATION SYSTEMS}

The air exchange rate is an important consideration in all glove boxes. In large boxes $\left(>100 \mathrm{ft}^{3}\right)$, distribution and correct internal flow patterns are also important. Spaces likely to be poorly ventilated (e.g., centrifuge wells) must be given special consideration to avoid stagnant pockets.

For normal operations, flow rate is based on dilution of evolved combustible or corrosive gases and heat dissipation, and is often based on prior 
experience. Exhaust capability must be sufficient to provide safety under postulated abnormal conditions, including the loss of a glove.

Operating personnel, industrial hygienists, and radiation specialists can assist the designer in establishing realistic requirements, particularly when an existing system is being replaced or revised. The types and quantities of materials to be used inside the box and their toxicity and state (wet slurry, dry powder, etc.) must be considered when establishing the air exchange rate and velocity. When exposed radioactive material is handled inside a glove box, the box becomes the primary containment. Therefore, the glove box should under no circumstances be at a positive pressure relative to its surroundings, A pressurized glove box provides a motive force for airborne contamination to leak from the box into the workroom. Negative pressure inside the box is essential when working with radioactive material.

\subsubsection{Dilution of Evolved Gases}

A high air exchange rate is often required to dilute fumes generated in an air-ventilated glove box. When evolved gases, vapors, and particles are not flammable, toxic, or corrosive, flow rates just sufficient to maintain a negative pressure, with differentials from 0.3 to 1.0 in.wg in the box, may be employed. However, when fumes or vapors are hazardous, a higher ventilation rate is necessary. The maximum generation rate of hazardous substances must be determined to establish the minimum airflow rates needed for dilution. The following equations can be used to determine minimum safe airflow rates. ${ }^{3}$

$$
Q=\frac{R\left(10^{6}\right)(S)}{L}
$$

where

$$
\begin{aligned}
Q= & \text { required dilution flow rate, cfm; } \\
R= & \text { contaminant generation rate, cfm; } \\
S= & \text { safety factor ( } 4 \text { to } 10 \text { suggested, depending on } \\
& \text { volatility, flash-point temperature, degree of } \\
& \text { mixing, and risk); } \\
L= & \text { limit value of contaminant, volume parts per } \\
& \text { million (vpm) [use threshold limit value (TLV) } \\
& \text { for toxic vapors and lower explosive limit } \\
& \text { (LEL) }{ }^{4} \text { converted to vpm, for combustible } \\
& \text { vapors]. }
\end{aligned}
$$

If the contaminant vapor is evaporated from a liquid, the contaminant generation rate, $R$, can be determined using the rate of liquid evaporated.

$$
R=\frac{W}{M}(359) \frac{t+460}{492}
$$

where

$$
\begin{aligned}
W= & \text { liquid evaporation rate, } \mathrm{lb} \text { of solvent per } \\
& \text { minute; } \\
M= & \text { molecular weight of contaminant; } \\
t= & \text { air temperature, }{ }^{\circ} \mathrm{F} .
\end{aligned}
$$

Equation (7.2) assumes that a pound mole of gas will occupy $359 \mathrm{ft}^{3}$ at $32^{\circ} \mathrm{F}$ and standard pressure. The dilution flow rate, $Q$, in Eq. (7.1) assumes that the dilution air is free of the contaminant under consideration; otherwise the background concentration of the contaminant in the dilution air (in vpm) must be subtracted from the limit value, $L$, in the denominator.

Concentration gradients can easily be formed during rapid vaporization if the hazardous gas is much lighter or heavier than air and there is poor mixing; ${ }^{5}$ safety factors above 7 should be used in such cases. For example, $1 \mathrm{lb}$ of acetone evaporated in a box in $1 \mathrm{hr}$ requires a dilution rate of $5.1 \mathrm{cfm}$ times the safety factor, $S$, for assurance of dilution below the lower explosive limit. ${ }^{6}$ Since acetone evaporates rapidly and has a flash point of $0^{\circ} \mathrm{F}$ and an LEL of $2.2 \%$, a safety factor of 10 should be used. In operation, as little as possible of a solvent like acetone should be permitted in the glove box at any one time.

\subsubsection{Heat Dissipation}

Many glove box operations generate heat. High temperatures within the box cause a worker's hands and arms to perspire heavily within the gloves, thus lowering his efficiency. For worker comfort, sufficient air should be circulated through the box to limit the inside temperature to no more than $15^{\circ} \mathrm{F}$ above room temperature. When the calculated airflow rate for cooling exceeds one air change per minute, consideration should be given to supplementary cooling, better insulation of heat sources, recirculating coolers, chill blocks for hot materials, or decreasing the generation of heat by the intermittent operation of equipment. There are practical limits to the amount of cooling that can be accomplished by airflow, since high airflow rates create strong air currents that interfere with delicate work such as weighing, dispensing liquids by dropper, and handling light powders. Where possible, operators should be protected from objectionable sources of radiant heat by surrounding the heat source with reflective

\section{(1)}


shields or conductive jackets. Exhaust air streams may be routed through such shields to permit the maximum pickup of convected heat before leaving the box.

When heat transfer rates to the box atmosphere have been determined, the required cooling airflow rate to dilute the hot gases is calculated from the equation

$$
Q=\frac{H}{C\left(t_{2}-t_{1}\right)}
$$

where

$$
\begin{aligned}
Q= & \text { airflow, cfm; } \\
H= & \text { sensible heat change (by convection), Btu } / \mathrm{hr} \\
& (1 \mathrm{~W}=3.41 \mathrm{Btu} / \mathrm{hr}) ; \\
t_{1}= & \text { temperature of entering air, }{ }^{\circ} \mathrm{F} ; \\
t_{2}= & \text { desired average air temperature inside box, } \\
& { }^{\circ} \mathrm{F} ; \\
C= & \text { conversion factor for sensible heat change for } \\
& \text { air, Btu } /(\mathrm{cfm})(\mathrm{hr})\left({ }^{\circ} \mathrm{F}\right)=\text { (density) (specific } \\
& \text { heat) }(60 \mathrm{~min} / \mathrm{hr}) .
\end{aligned}
$$

Both the density and specific heat of air at room conditions depend on the humidity ratio of the air. The density also depends on temperature. In a room at $75^{\circ} \mathrm{F}$ and $50 \%$ relative humidity, the air density is $0.073 \mathrm{lb} / \mathrm{ft}^{3}$ and specific heat is $0.24 \mathrm{Btu} / \mathrm{lb}$. Therefore $C$ is $1.1 \mathrm{Btu} /(\mathrm{cfm})(\mathrm{hr})\left({ }^{\circ} \mathrm{F}\right)$ and $\mathrm{Eq}$. (7.3) becomes

$$
Q=\frac{H}{1.1\left(t_{2}-t_{1}\right)}
$$

Long-term operation of high-heat-producing equipment can damage filters when exhaust air temperatures approach the temperature limit of the filters for continuous exposure to heat (see Tables 3.4 and 3.5).

\subsubsection{Empirical Flow Rates}

It is often necessary to establish glove box flow rates from working experience, without knowledge of the specific operation or the equipment that will occupy the box. Forty air changes is a reasonable rate for establishing ventilation-system airflow capacity.

In actual box operations, airflow rate is more likely to be reduced than increased from design rates. Normal airflow from each box on a manifold system can easily be reduced by a factor of as much as 10 , without difficulty, if a properly controlled and filtered bleed system is provided. Doubling the design airflow from each box on a manifold system may be impossible without major revision to the equipment and ducting.

\subsubsection{Exhaust Requirements}

The maximum airflow rate from the glove box determines the required capacity of the filters and determines the size of the equipment for the entire downstream portion of the ventilation system. The airflow resistance of the exhaust-air path must be sufficiently low so that the pumping of gloves by personnel conducting operations in the box will not result in pressurization. In small, low-flow boxes without air inlets, such as inert atmosphere types, pressure surges due to glove pumping may be a serious problem. Fast inward movement of the gloves (i.e., pumping) can cause pressure pulsations in a small box which overcome pressure differentials as slight as -0.3 in.wg, whereas differentials greater than 1.0 in.wg will cause gloves to become stiff and tiring to use. Delicate operations are more difficult to perform at high pressure differentials because operators lose much of their sense of touch when gloves separate from the fingers and then expand into the box when hands are removed.

The maximum rate of exhaust flow from an airventilated glove box is usually based on the required inlet flow when a glove is ruptured or inadvertently removed. The air velocity into the open port should be $100 \mathrm{fpm}$ or greater. For an 8-in.-diam glove port opening, $100 \mathrm{fpm}$ corresponds to a flow rate of 35 $\mathrm{cfm}$. Good contamination control is more easily achieved in a glove box having low air leakage. Glove boxes should have leakage of less than 0.01 box volume per hour, when tested at 1.0 in.wg pressure differential to the outside, to be suitable as primary containers for hazard class 1 or class 2 (Sect. 2.2.1) materials. This basic leaktightness requirement aids the achievement of a suitable working negative pressure in a glove box, since little air must be exhausted to maintain the proper differential pressure. A requirement for greater leaktightness may be needed when it is necessary to exclude air or moisture in special atmosphere boxes, or to otherwise enhance containment. Glove box construction features are described in USAEC Report TID-16020 and other publications. ${ }^{2,-9}$ For inert-atmosphere glove boxes, construction requirements to control 
atmospheric purity, leakage, and pressure regulation are more critical than for air-ventilated boxes.

\subsubsection{Vacuum- and Pressure-Surge Relief}

Glove boxes must be protected against physical damage resulting from excessive pressure or vacuum. An exhaust manifold and inlet supply system must be able to handle slowly manifested pressure or vacuum disturbances. As pressure and vacuum transients become more rapid, distant control devices become less able to maintain the box differential pressure at or close to equilibrium, and the box may be subject to structural damage. Maintaining a safe differential pressure becomes more difficult as box leakage, flow rate, and free volume (i.e., volume not enclosed by equipment within the box) decrease. Pressure surges are of particular concern in high-purity (extremely low-leakage) glove boxes.

Each glove box that contains service connections or internal equipment whose malfunction might cause a pressure surge should be equipped for prompt surge relief. The response time and pressure-flow characteristics of the surge-relief device will depend on the flow and pressure characteristics of the pressure source, the free volume, and the relative strength of the glove box. The relative strength is defined as the lowest pressure differential that will cause rupture of the glove box pressure boundary at its weakest point (e.g., the failure of a glove or window). Depending on the design of the box, the weakest point may be a window, an inlet filter, or a glove. Since inlet and exhaust connections may be shut off when a surge occurs, a conservative approach to surge-relief design is to neglect the normal inlet- and exhaust-flow capacity. The surge-relief device can be a liquid-filled U-tube, as shown in Fig. 7.3. The surge-relief flow capability should exceed the flow from the largest possible source of pressurization at the design relief pressure. The HEPA-filtered surge-relief line should not be connected to a glove box exhaust manifold, because this line will be subjected to the same pressure as the normal glove box exhaust connection. For the relief device in Fig. 7.3, a liquid storage reservoir is provided to handle the blown seal fluid. The filter should be protected from impingement of the seal fluid. If room aị cannot be tolerated in the glove box, as is the case in some inert-atmosphere applications, a different vacuum surge-relief system must be used. A U-tube can be devised to restore its seal after relieving the surge, but such a system must include a feature to alert the operator that a pressure surge has occurred so that he may make necessary safety checks. The NASA-devised vent $\operatorname{cover}^{10}$ shown in Fig. 7.4 may be used for pressure-surge relief (or separately as vacuum-surge relief at the in-box end) following a HEPA filter. An inlet filter may provide surge relief if no backflow device or other restriction is provided. The filter-face area would have to be about four times the area of an unfiltered port to achieve equal venting effect. ${ }^{11}$

Explosion venting is not covered in this handbook. NFPA $68^{12}$ contains information on explosion venting, with over 70 references. Concerning explosions, emphasis should be placed on prevention and not on

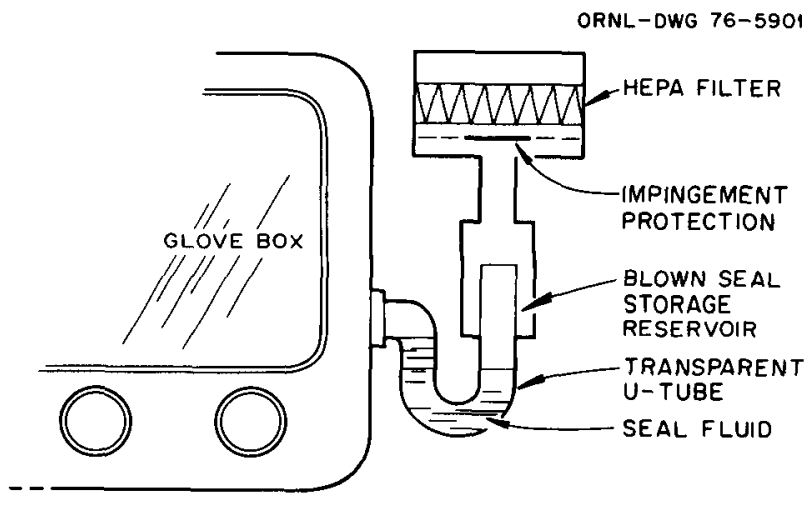

Fig. 7.3. Glove box vacuum-pressure surge-relief device.

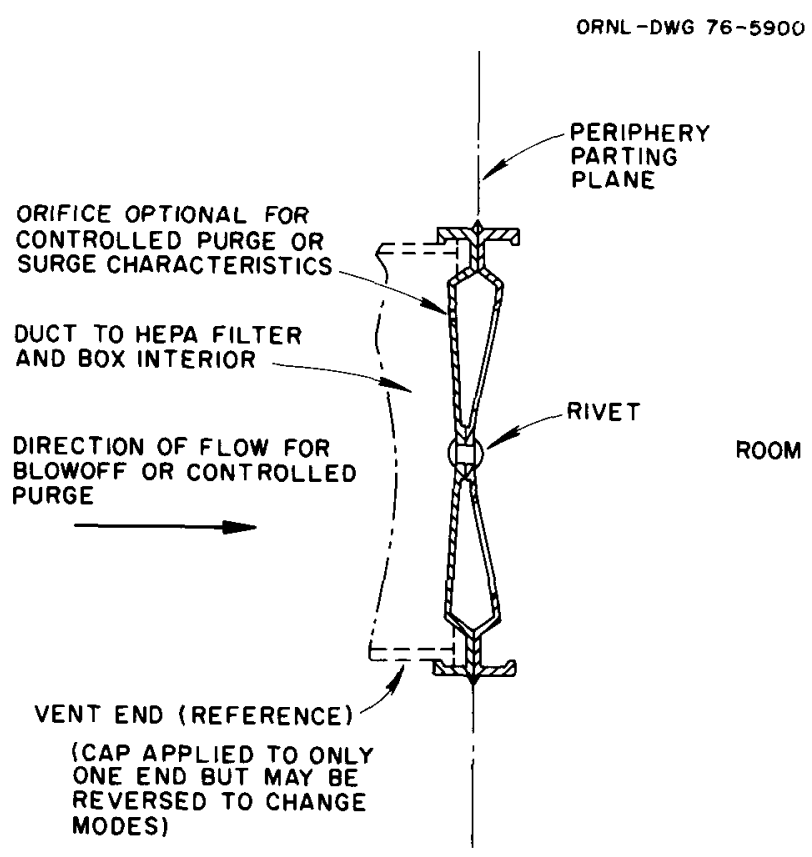

Fig. 7.4. Pressure surge-relief cap. Cap may be repositioned for vacuum relief service. 
postoccurrence treatment. Glovebox Fire Safety ${ }^{y}$ contains a short section on glove box explosion suppression, with the warning that the use of such systems remains largely on an experimental basis.

\subsubsection{Glove Box Exhaust Manifold}

The glove box exhaust manifold includes all of the glove box exhaust system downstream from the point where the exhaust from two or more boxes joins and the flow is no longer separated. Glove box exhaust manifolds are discussed here to the extent of providing proper perspective to the importance of the HEPA filter in the safe operation of the glove box. Sections 7.2, 7.3, and 7.4 and refs. 1, 2, 8, 9, and 12 of this chapter discuss details of the exhaust system and illustrate working examples.

The glove box exhaust manifold draws air or exhaust gas from each connected box at a controlled pressure and flow (interdependently), houses secondary treatment facilities, and transmits the air for further treatment or exhausts it to the outside atmosphere. Primary exhaust treatment should be applied inside or as close to the glove box as possible and, in all cases, prior to the connection of the exhaust line to the exhaust manifold. In some systems, a portion or most of the cleaned exhaust gas may be recirculated back to the glove boxes.

The manifold system should be sized and controlled to accept a range of flow whose high extreme is the sum of (1) the maximum normal flow from each box (Sects. 7.2.1, 7.2.2, and 7.2.3), (2) the largest maximum flow under removed glove conditions from one of each five connected boxes (Sect. 7.2.4), and (3) an allowance for system growth. The low extreme is the summation of the minimum flows from each box. An allowance for system growth should be provided at not less than $20 \%$ of (1) plus (2) above for a new system. If this allowance exceeds $50 \%$ of (1) plus (2), other provisions, such as installing an equivalent dummy flow, should be considered.

Filter installation in a glove box exhaust manifold should resemble those described in Chap. 6. Prefilters may be unnecessary for the manifold system since this is the second HEPA filter the exhaust gas enters. Prefilters, if furnished, should precede the first HEPA filter if they are to serve their primary function. The provision of prefilters for fire protection of the manifold HEPA filters should be considered, as discussed in Sect. 9.5. Exhaust fans should be downstream of the HEPA filters.
Shutoff suction pressure of the fan should preferably be less than the failure pressure of the weakest component of all items upstream, including the connected glove boxes. However, system dynamics and pressure-flow characteristics for normal and emergency conditions may preclude the use of such a fan. If the fan shutoff pressure exceeds the failure pressure of the weakest component, the excess should be as small as possible. This recommendation assumes a wide-open exhaust duct, without controls or safety bleeds, and is aimed at providing an inherently safe, ideally fail-safe system. Exhaust gascleaning requirements may render this recommendation unattainable without adding inordinate size, complexity, and cost to the system. Nevertheless, when possible, this fan-pressure limitation should be considered. The use of positive-displacement blowers with air-ventilated glove boxes should be avoided, because these blowers are inappropriate for oncethrough glove box exhaust service. Positive displacement blower systems depend on external devices to limit the negative pressure attainable, especially under low or no-flow conditions, and can produce greater pressure differentials than those produced by other exhaust gas movers. Specification of too high a maximum pressure differential is potentially more dangerous to a system than overdesign of an attainable flow.

\subsubsection{Exhaust Cleanup Requirements}

Each particulate radioactive nuclide that might be present in the gas exhausted from a nuclear facility has a limit of acceptable concentration, as discussed in Sect. 2.2.1. The number of HEPA filter stages required in a glove box exhaust stream is a function of the permissible concentrations, in air, of the nuclides that will be in the subject exhaust stream, the decontamination factor achievable by the filter series (Sect. 2.6.2), and a safety factor for ALARA considerations discussed in Sect. 2.2.1. The fire protection scheme selected for the glove box filters may also affect the number of stages of filtration, in that it might be assumed that a stage preceding the final stage of filters is breached as a result of a fire. Occasionally, more HEPA-filter stages than necessary are installed in an attempt to increase reliability (see series redundancy, Sect. 2.6.1). The stages that follow the first-stage HEPA filter do not have to be in the immediate vicinity of the glove box being ventilated. The glove box exhaust is preferably ducted to a filter room remote from the room in which the glove box is located. This arrangement 
gives more space and facilitates the maintenance and testing of HEPA filters downstream from the glove box HEPA filter. One ERDA contractor has developed criteria for new glove box facilities, in which service space is equal to the operating space and about $40 \%$ of the service space is occupied by ducts, plenums, and ventilation equipment.

When corrosive gases or vapors are in the exhaust airstream, all filters in a series will be exposed. The widely held impression that the life expectancy of a group of HEPA filters arranged in series is directly proportional to the number of filters in the series may be false when chemical or heat degradation occurs. Under these conditions, when the first stage fails, there is a potential for others to fail from the same cause. Corrosive gases and mists from vats, scrubbers, and similar equipment must be neutralized and removed before they reach the HEPA filters.

The benefit of two or more filter stages cannot be realized unless each stage is kept in serviceable condition. Therefore, each stage of HEPA filters must have built-in provisions for routine in-place testing. (In-place testing of glove box filters is covered in Sect. 7.5.4.) The detection of damaged filters by monitoring airflow resistance is ineffective and hazardous.

\subsection{DESIGN OF FILTER SYSTEMS}

The glove box filter systems discussed in this section, for the most part, are first-stage HEPA filters, although second-stage filters located upstream from the exhaust manifold connection are also discussed.

Filters must pass the correct flow (with as few flow controllers as possible) when they are either clean or dirty. A maximum dirty-filter resistance of three times clean-filter resistance for HEPA filters and two times clean-filter resistance for prefilters is generally used for design purposes. Figure 7.5 gives the approximate airflow and pressure-drop relationships for clean open-face HEPA filters. Figure 7.6 shows common locations for HEPA filters near or inside glove boxes. Type $2 \mathrm{C}$ shows the installation of inlet and exhaust filters inside the glove box.

\subsubsection{Exhaust HEPA Filters}

A detailed discussion of filter performance and materials of construction is given in Sect. 3.2. Operational experience with a particular system is the most reliable basis for filter selection for a particular

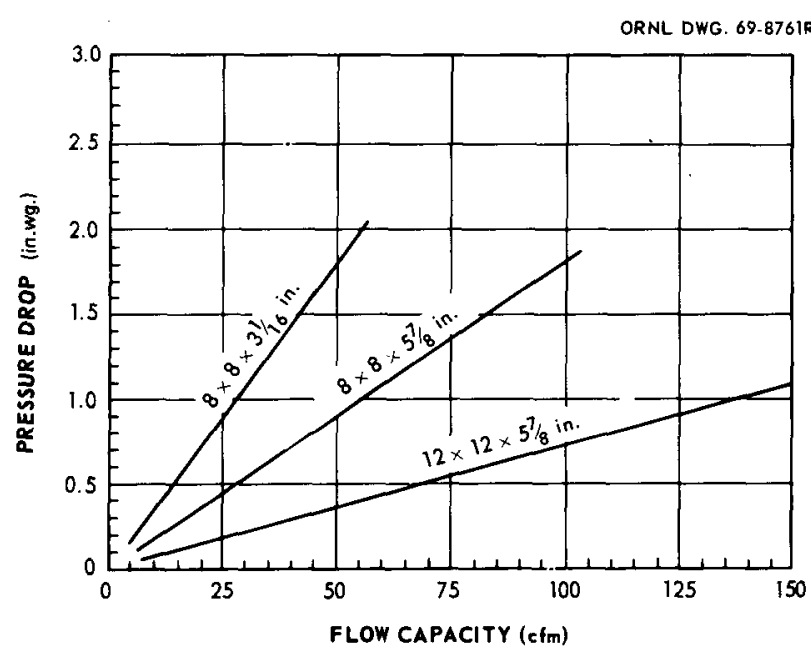

Fig 7.5. Flow vs pressure drop relationship for small, clean open-face HEPA filters. Based on air velocity of $5 \mathrm{fpm}$ through the medium at nominal airflow for filter.

ORNL DWG. 69.8765

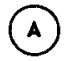

(B)
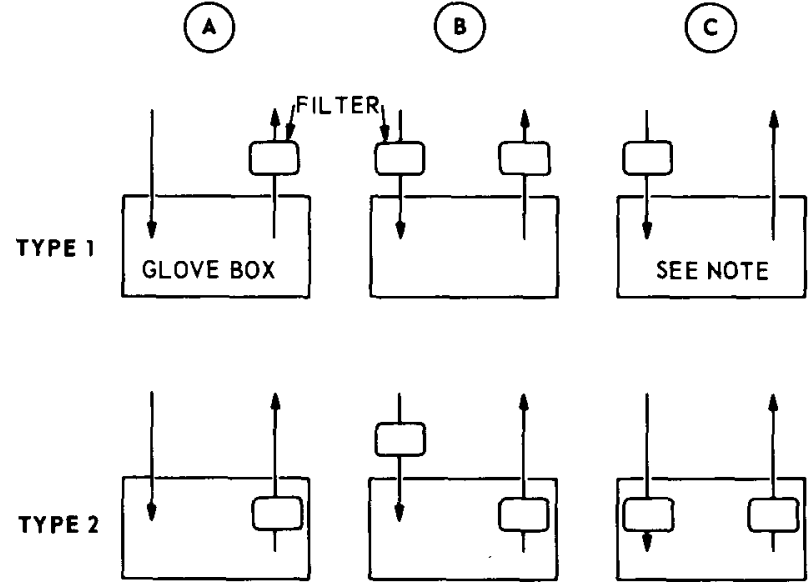

NOTE: TYPE IC IS NOT SUITED FOR CONTAINMENT OF RADIOACTIVE MATERIALS WITHIN THE GLOVE BOX BUT IS APPLICABLE WHERE THE INTENT IS TO EXCLUDE AIRBORNE PARTICLES FROM THE GLOVE BOX SPACE.

Fig. 7.6. Possible arrangements of fiiters near or inside glove boxes.

service. For new and untried systems, the initial choice should be limited to the traditional rectangular, open-face, pleat, and separator construction (Sect. 3.2). Adoption of new types of HEPA filters with special features, size, or materials can result in uneconomical and unreliable system operation. The susceptibility of some nonstandard filters to deterioration in certain environments and the inability to readily obtain replacements have 
presented problems to operators. If exhaust streams are kept chemically neutral, as they should be for reliable exhaust-system operation, HEPA filters of standard construction usually afford the most economical service.

A single-HEPA-filtered exhaust path is usually acceptable when work within the glove box does not involve highly toxic aerosols and does not require continuous cooling or dilution of vapors. When continuous airflow is essential, two exhaust connections should be provided to avoid interruption of exhaust flow during a filter change and to provide standby protection in the event of system upset. The purpose of multiple exhaust connections is lost unless all filtered paths are kept in serviceable condition so that a standby connection is always ready during an emergency. Figure 7.7 illustrates single-and multiplefiltered exhaust connections for a glove box.
The safety value of multiple-filtered exhaust connections can be realized easily in a line of interconnected glove boxes or in a large enclosure with several compartmented work areas. Compartmenting doors between work areas or between single boxes in an interconnected line must not isolate a work area with only one filtered exhaust connection. The multiple exhaust points required to handle total airflow in a line of interconnected boxes must be sized for maximum flow and valved individually for flow control. Current ERDA philosophy is to discourage long lines of interconnected glove boxes. Where they are necessary, fire doors between boxes should be provided.

The designer of equipment to be used in and around glove boxes must understand and respect the limitations of the human body. Past experience often reflects a lack of appreciation for these limitations.

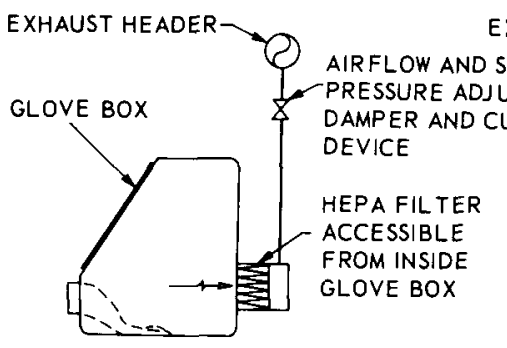

(a)

SINGLE EXHAUST CONNECTION
EXHAUST HEADER

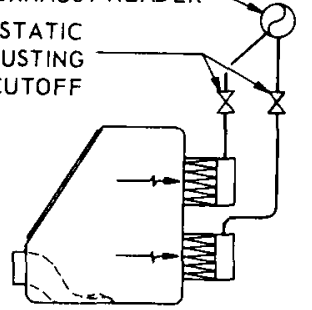

(b)

MULTIPLE EXHAUST CONNECTIONS IN PARALLEL

SINGLE STAGE OF FILTRATION

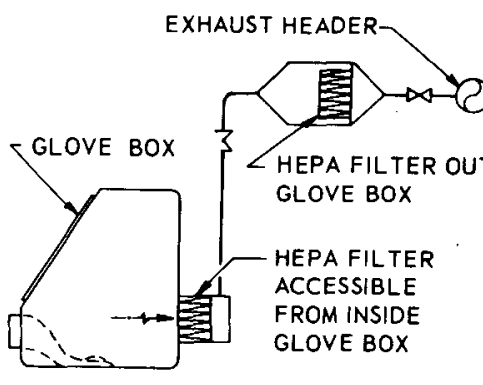

(c)

SINGLE EXHAUST CONNECTIONS FILTER STAGES IN SERIES.

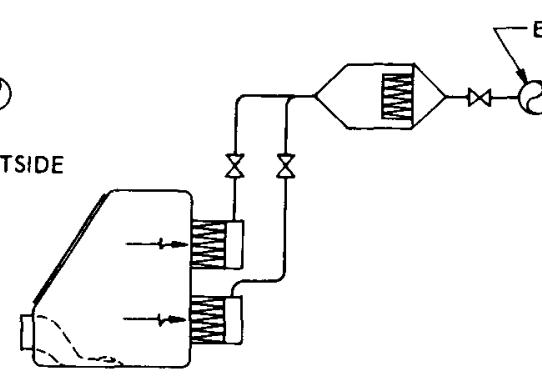

(d)

MULTIPLE EXHAUST CONNECTIONS TO BOX IN PARALLEL SECOND-STAGE FILTER COMMON TO BOTH CONNECTIONS
ORNL DWG. 69.8759R

Fig. 7.7. Suggested arrangements for single- and parallel-filtered exhaust connections for glove boxes. 
Tasks beyond the worker's reach or out of his sight and objects too heavy to lift readily at arms length impose unsafe conditions and lead to the neglect of items that are difficult to maintain. Working within an enclosure with hands covered by full-length flexible gloves limits the use of the senses. The required depth of reach into an enclosure should be limited to between 6 and 22 in., as shown in Fig. 7.8. A depth reach less than 6 in. allows little working room, while a depth from 22 to $29 \mathrm{in}$. is difficult and tiring; a depth reach over $29 \mathrm{in}$. makes manipulation impossible. The nominal limit for the coordinated use of both hands is a depth reach of 25 in. Figure 7.8 shows other critical dimensions for operators in and around glove box equipment. The dimensions shown are based on values that are convenient for $95 \%$ of adult males. ${ }^{13}$

\subsubsection{Exhaust Filter Located Inside the Glove Box}

The arrangement of filters inside glove boxes must be convenient and safe for the operator. The designer must thoroughly study the planned location and operation of process equipment to be certain that inbox filters are properly located with respect to airflow and do not impede the functioning of the box. The

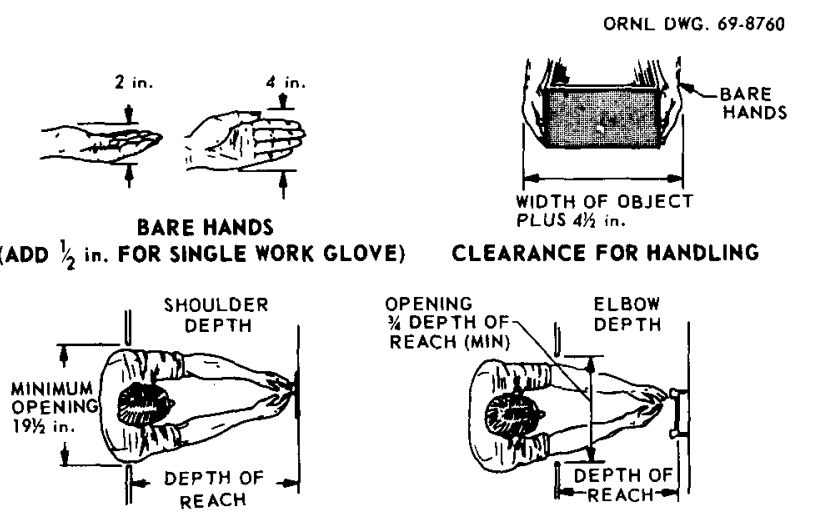

REACH THROUGH OPENINGS
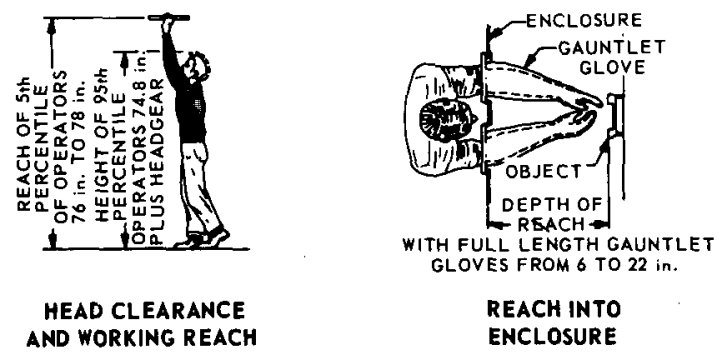

Fig. 7.8. Dimensional guide of critical work limits for male operators in and around glove boxes. filters must be located for convenient maintenance, testing, and inspection and still be protected from splashing liquids, flying missiles, and areas of great fire potential.

The largest HEPA filter installed inside a box is usually $12 \times 12 \times 5 \%$ in. Larger filters are impractical because safe handling may require special internal equipment, the filter would occupy too much space, and removal and replacement would require access ports that are too large.

Filter installation inside a glove box permits the handling of highly contaminated filters within an acceptable containment, minimizes direct contact of personnel with collected material, and permits the capture of airborne particles as close to their source as possible. On the other hand, the glove box is the focal point of the work to be accomplished, and its interior space is expensive, including any volume occupied by an in-box filter. Glove boxes are estimated to cost $\$ 500$ per $\mathrm{ft}^{2}$ of floor area and $\$ 146$ per $\mathrm{ft}^{3}$ of volume. ${ }^{14}$ Box interior space costs exceed the cost of the operating area in which the glove box is installed by 5 to 10 times. Also, because work goes on in close quarters, the filter is more liable to be damaged inside the box; removal and replacement of filters interfere with glove box operations and are more time-consuming and tedious than for filters mounted outside the box.

Figure 7.9 shows a typical mounting for an openface HEPA exhaust filter in the back-or sidewall of a glove box. A perforated retainer plate guards the open face of the filter. A prefilter is needed only when airborne particles are large enough to make it worthwhile (Sect. 7.3.6). The wall of the box serves as the sealing face for the filter gasket. Box wall flatness and dimensional stability are needed for good gasket seating. To change the filter the operator removes the four wing nuts and perforated faceplate to free the filter. The wing nuts must be large enough for glovedhand operation. When a filter is mounted against the backwall of a glove box, it reduces available workspace, especially when the filter is located directly opposite a glove station. Less workspace is lost if the filter is mounted to the immediate right or left of the glove station. Addition of an extra glove can often improve access and further reduce the consumption of prime space by the filter.

Figure 7.10 shows another method of mounting open-face HEPA filters inside a glove box. By having a separable exit box, this type of inside mounting permits the filter assembly to be relocated to accom- 
modate changes in box use. The assembly is supported by the 2 -in. pipe that protrudes through the wall of the glove box. However, the filter sealing

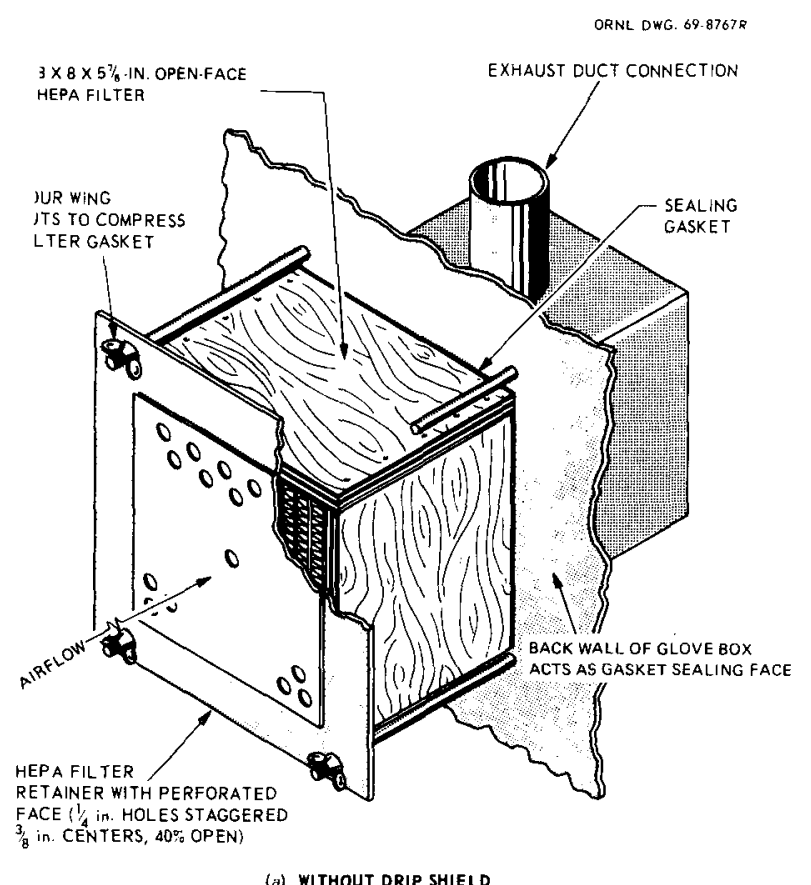

(a) WITHOUT DRIP SHIELD
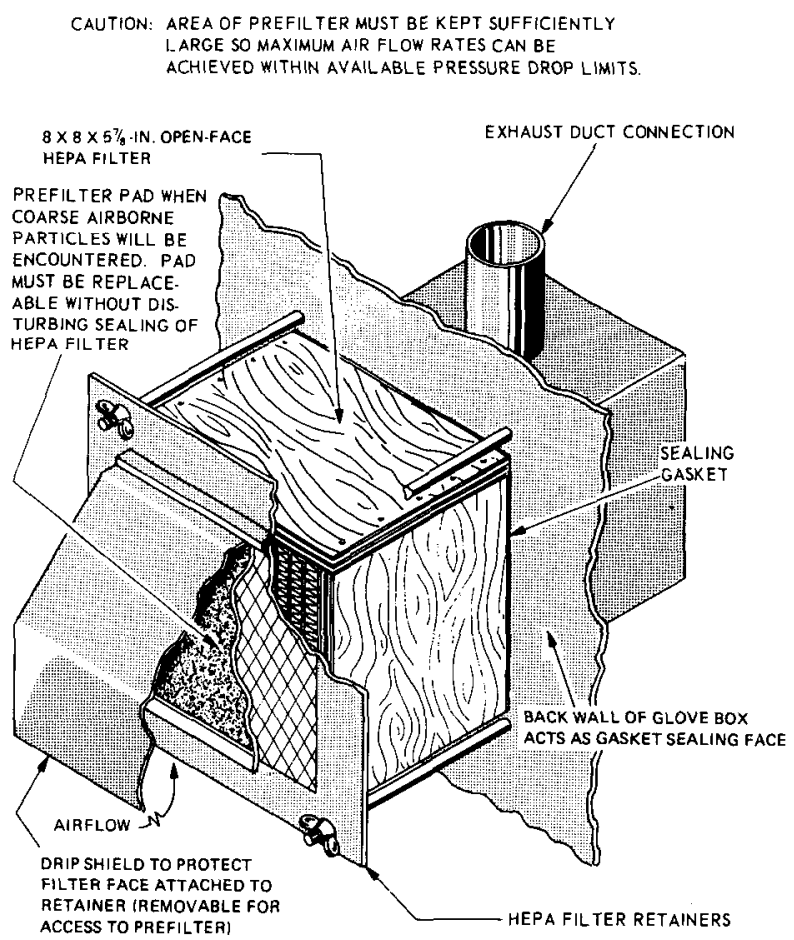

(b) WITH DRIP SHIELD AND PROVISION FOR PREFILTER

Fig. 7.9. Recommended methods for mounting open-face HEPA exhaust filter inside glove box (in-box). gasket is not visible for inspection in this arrangement. This mounting method is a companion to the inlet-air-filter mounting shown in Fig. 7.11, which uses accessories of identical design. The similarity of inlet- and outlet-filter mounting designs reduces the number of different parts that must be kept on hand.

Fire-resistant adhesive tape has often been used for sealing in-box filters. Gaskets are not used, and the weight of the filter is supported by a ledge or niche on the box wall. Filters installed in this way can leak seriously when subjected to heat or other disturbance that might displace the tape. Even under favorable environmental conditions, the tape peels and tends to fail after a period of time. The use of tape sealing should be limited to low-level contamination systems where leakage can be tolerated and fire potential is nil.

Figure 7.12 shows several desirable features of a good in-box filter installation. The design

- uses a standard-size HEPA filter located in the back- or end-wall of the glove box;

- maximizes inside box space by partially recessing the filter in the wall;

- has a simple clamping method with no removable pieces and is operable with a gloved

ORNL DWG. $69.8768 R$

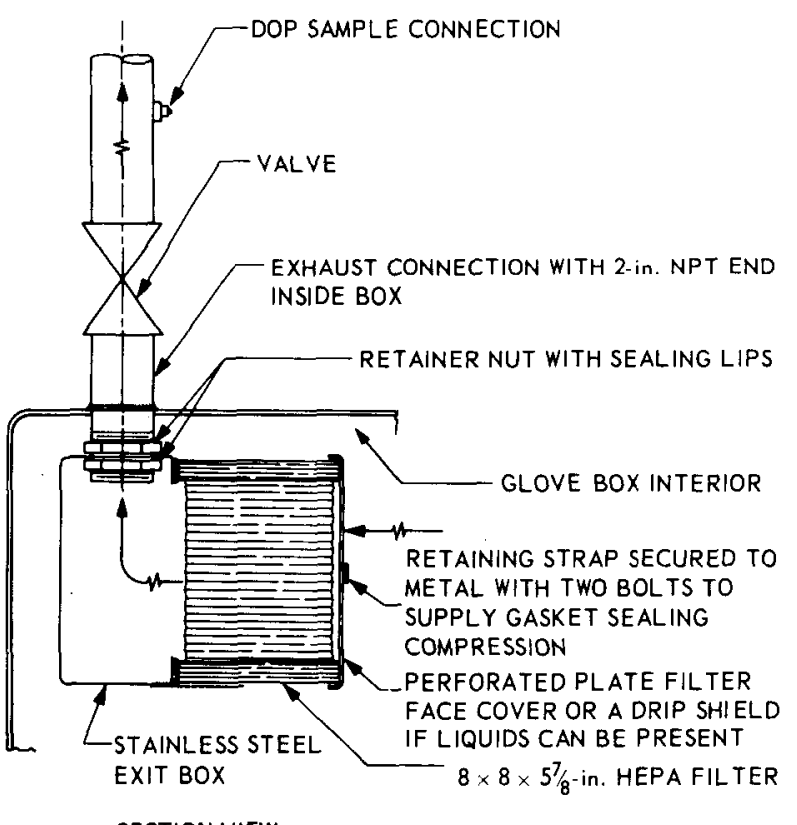

SECTION VIEW

Fig. 7.10. Open-face HEPA filter with exit box mounted inside glove box (in-box) for exhaust service. 
ORNL DWG. 69-8763R

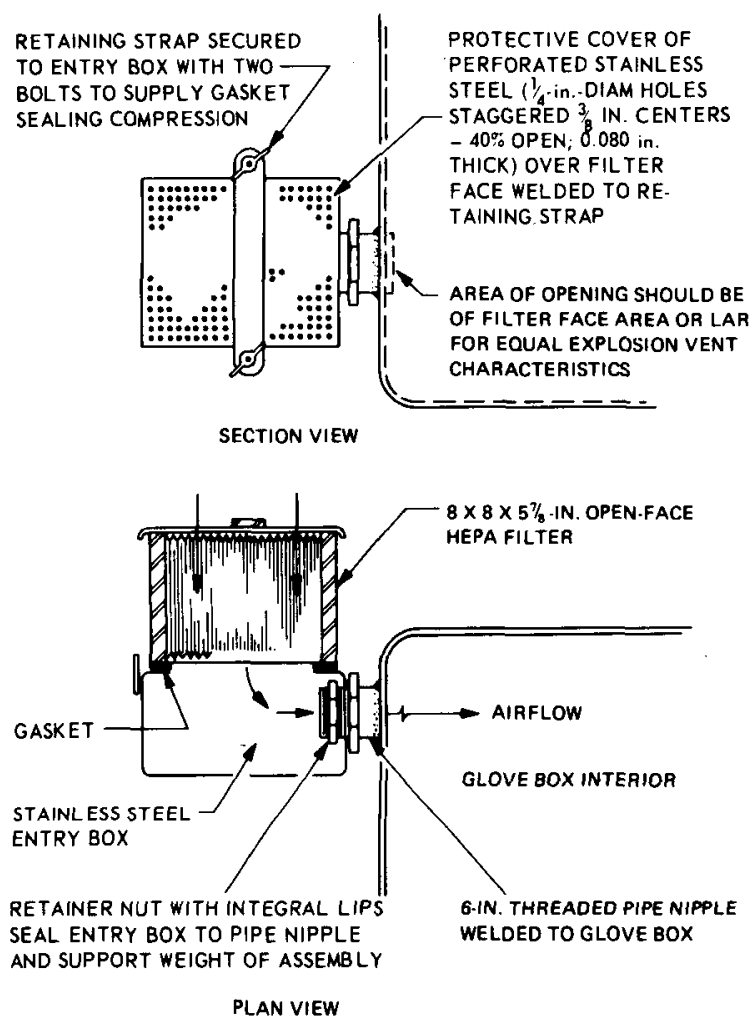

Fig. 7.11. Glove box air inlet with HEPA filter.

hand by actuating a spring-loaded (snap-overcenter), easily replaceable cam latch on each side of the filter;

- has a retainer that serves as a face shield for the filter and permits attachment of a steel-cased prefilter by a flexible magnetic strip (accessible from the front); the filter remains in position after being unclamped because of the folded lip at the top.

A general drawback of this arrangement is the inability to inspect the gasket and sealing-face area while the filter is in place; there could be other drawbacks for specific applications. Unfortunately, there is no single mounting arrangement without some disadvantages, and an intelligent compromise must be made for each application.

The construction materials used for filter mounting devices and associated hardware inside the glove box must provide an operating life equal to that of the box, unless the parts can be easily removed and replaced. Stainless steel should be used for bolts, latches, and other moving parts and for items that must resist corrosion. Dimensions and tolerances of filter sealing faces and mounting devices for glove boxes are the same as those for mounting frames in multiple-filter systems given in Table 4.2. Where the mounting is an integral part of the glove box, construction tolerances must also be consistent with those of the box but never more lax than those values given in Table 4.2 if high-efficiency performance is to be attained.

Bag-out port sizes necessary for the transfer of standard open-face HEPA filters are given in Table 7.1.

\subsubsection{Exhaust Filter Located Outside the Glove Box}

The advantages of outside filter mounting include better fire protection for the filters (space available

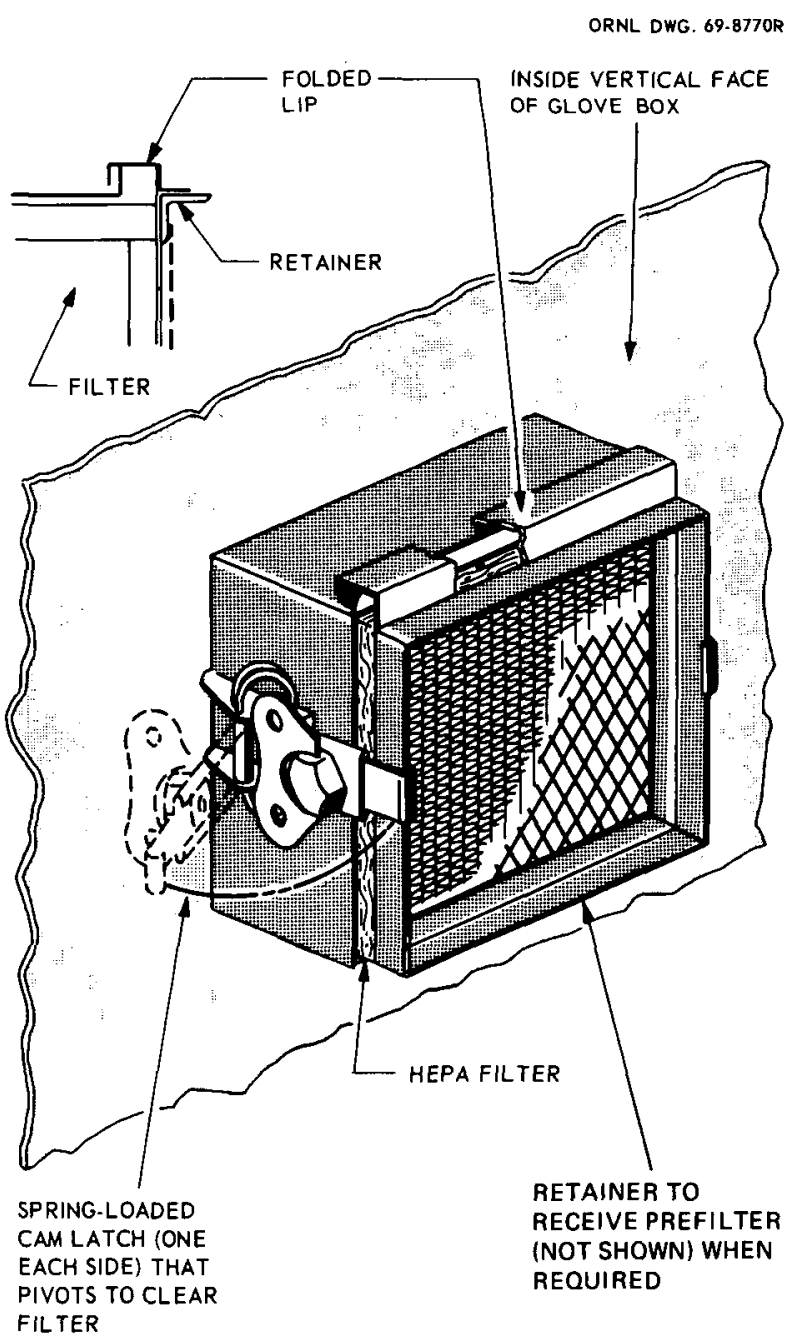

Fig. 7.12. Inside glove box open-face HEPA filter mounting arrangement with cam latches. 
Table 7.1. Glove box port sizes for transfer of standard open-face HEPA filters

\begin{tabular}{lll}
\hline \multirow{2}{*}{ Filter size } & \multicolumn{2}{c}{ Required port size (in.) } \\
\cline { 2 - 3 } & Round (diameter) & Rectangular \\
\hline $8 \times 8 \times 3^{1 / 16}$ & $9^{3 / 4}$ & $8^{1 / 2} \times 4^{1 / 2}$ \\
$8 \times 8 \times 5^{7 / 8}$ & $10^{3 / 4}$ & $8^{1 / 2} \times 6^{1 / 2}$ \\
$12 \times 12 \times 5^{7 / 8}$ & 14 & $12^{1 / 2} \times 6^{1 / 2}$ \\
$24 \times 24 \times 5^{7 / 8}$ & 26 & $25 \times 6^{1 / 2}$ \\
$24 \times 24 \times 11^{1 / 2}$ & $27^{3 / 4}$ & $25 \times 12$ \\
\hline
\end{tabular}

for fire shield, surrounding space more readily sprinkled, etc.), conservation of in-box space, easier handling, less interference with glove box operations, and lower cost. Outside filter mounting does not automatically provide these advantages; a poor mounting arrangement can create serious handling problems and can compromise the containment of the box. When installed outside the glove box, an enclosed filter may be better able than an open-face filter to hold dust and moisture particles within the casing when the spent filter is removed from the duct. Removal of a used open-face filter requires more care and preparation to ensure that contamination will not be spread during the procedure. Sections 3.2 and 6.3 provide information on enclosed filters. Both open-face and enclosed filters have been used for external installations.

For multistage filter installations, it is sometimes desirable to locate the second stage close to the glove box so that exhaust airflow can be maintained during a filter change without spreading contamination too far down the duct. However, the second filter should be located beyond the range of likely damage. Unless a specific reason exists, it is usually better to locate the second-stage filter in a service area remote from the glove box.

Two commonly used methods of outside glove box filter mounting, shown in Fig. 7.13, are not recommended except for very-low-level applications (class 4, Table 2.2). Although simple in appearance and less costly than installations in separate housings, these mounting methods make filter replacement a very tedious and delicate operation if contaminant spills are to be avoided. Because these filters are outside and near the glove box, the risk of contaminating the local area is higher than for housings such as those shown in Fig. 7.14 or the use of enclosed filters (Fig. 7.15). Filters should be located within convenient reach, especially if tools must be used and bolts removed. High locations that require ladders or scaffolds for access are a hazard to personnel and to the glove boxes nearby (Fig. 7.8). When filters are sandwiched between two flanged faces, as shown in Fig. 7.13 , the precise alignment of gasket seating surfaces is essential to ensure uniform gasket compression. Misalignment of surfaces will not only cause nonuniform gasket compression but will increase the chance of leakage at the gasket. Rigid ducting can cause bolt pull-up forces to strain the wall

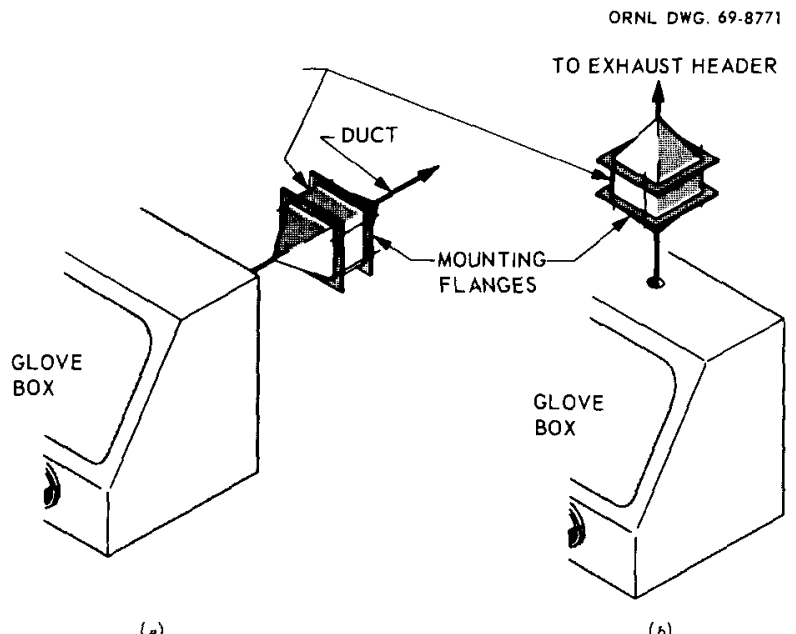

Fig. 7.13. Two examples of methods for mounting HEPA filters outside glove boxes. These methods are not recommended.

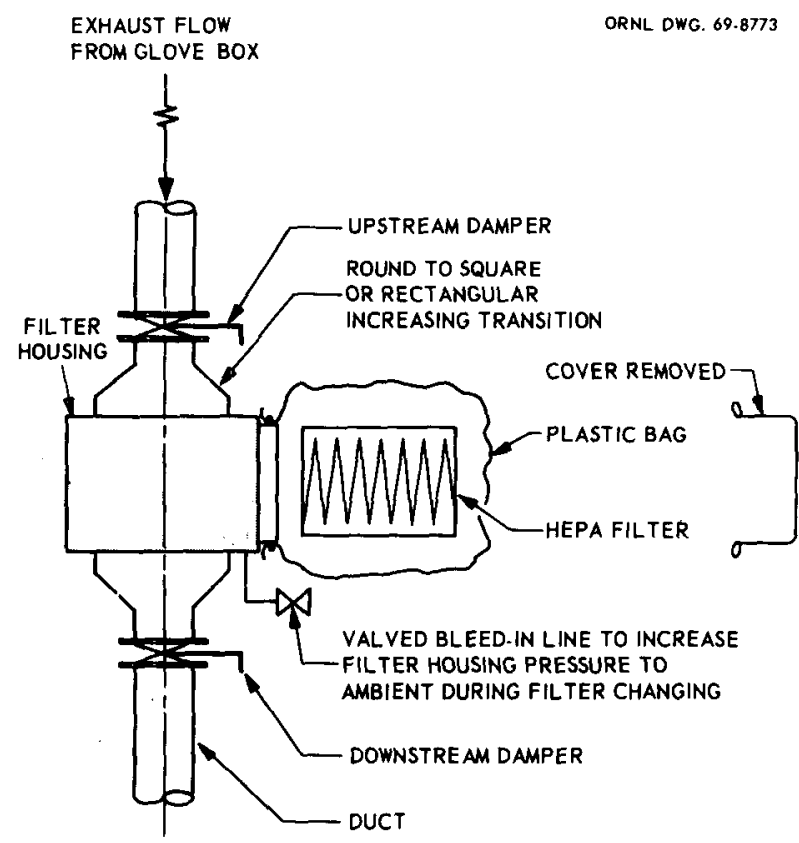

Fig. 7.14. Typical connection for a single-filter housing in a glove box exhaust stream. 


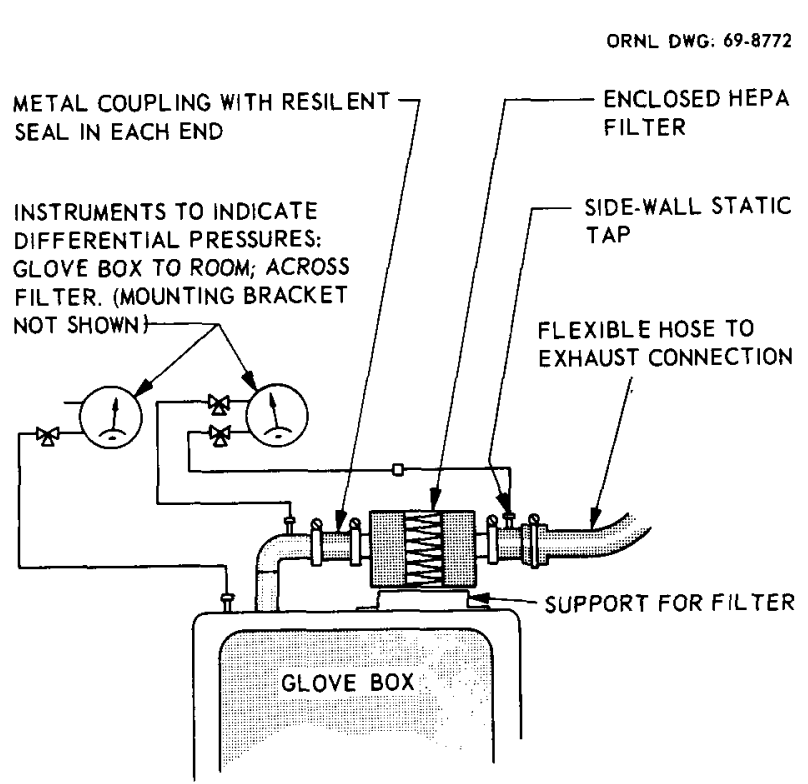

Fig. 7.15. Installation of an enclosed HEPA filter mounted outside the glove box.

of the glove box or the joints in the ducting. Pull-up forces for double-gasket mountings are significantly greater than for single-gasket mountings. A short section of cuffed flexible hose on the downstream side of the filter is one method of relieving strains, but it does nothing to decrease the required gasket sealing forces. Nonmetallic flexible hoses lessen the integrity of the exhaust system because of the vulnerability of the hose to fire, accidental tearing, or penetration. Use of hoses is not advised in hazard class 1 and 2 (Table 2.2) systems. The use of nonmetallic hoses is less objectionable when there is a rigidly mounted HEPA filter between the box and the hose. Experience has clearly shown that wood and steel filter casings can be damaged by excessive pull-up. Wood casings will take greater longitudinal (i.e., face-toface) loadings than steel casings.

Much operating experience has been gained relevant to the use of enclosed HEPA filters outside glove boxes. Figure 7.15 shows a typical installation. Although flanged and nipple-end enclosed filters have both been used, nipple-end enclosed filters are easier to install. The integral casing caps help enclose the dirty filter medium and make filter changing a less difficult and risky task than with open-face filters. In the system shown in Fig. 7.15, the exhaust ducts are flexible hoses with cuffed ends sized to fit the nipples of the enclosed filter. Initial installation costs of enclosed HEPA filters are less than those for separate filter housings.
The important features of a bag in, bag out filter installation are shown in Fig. 7.14. If filter change by bagging is not necessary for contamination control, the operation can be simplified by direct handling methods and the valved bleed-in line is not needed. The arrangement for the mini-caisson housing shown in Fig. 7.16 is comparable to the arrangement shown in Fig. 7.14 except for the absence of a valved bleed-in line and a damper to permit isolation of the housing from the duct. For applications that require filters to be bagged in and out, the procedure is like that described in Sect. 6.2.3 and shown in Fig. 6.8.

There is a limited choice of commercially available prefabricated filter enclosures suitable for glove box operations. One such enclosure is the mini-caisson shown in Fig. 7.17. This housing is made for openface HEPA filters with dimensions of $8 \times 8 \times 5^{7} / 8$ in. and $12 \times 12 \times 5^{7} / 8$ in. The unit is flanged into the duct and is designed for bag in, bag out filter change. To prevent leakage of contaminated air during a filter change, this housing must be provided with an isolation damper in the downstream side of the duct. Although not essential for most glove box service, a second damper may be desirable on the upstream side to close off negative pressure that hampers filter bagging caused by other exhaust connections serving the same glove box (multiple connections) or glove box line.

The first cost of commercial housings may be more than five times the cost for a comparable enclosed HEPA filter installation, but filter replacement costs are lower. A comparison can be made, using purchase

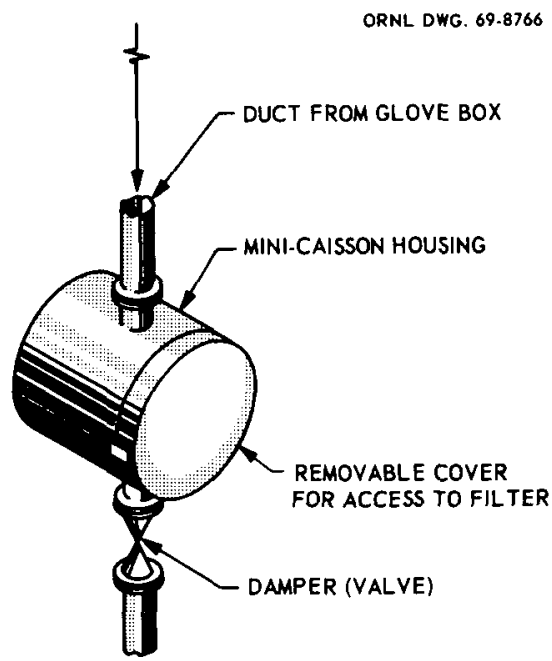

Fig. 7.16. Typical connections for SGN mini-caisson manufactured by Barneby-Cheney Co. 
and service costs, to show how many filter changes would have to be made before the price of changing the open-face filters of a permanent commercial housing would equal the price of the same number of enclosed HEPA filters. In 1969, nine filter changes were required before capital costs were equalized. Since then, filter unit costs have almost doubled. Since relative savings will be dependent upon the frequency of filter change and the life of the installation, the designer must consider such time factors in making any comparison. Experience at many installations indicates that the majority of HEPA filters used in glove box systems last longer than six months of continuous operation. When conditions are unusually clean or use is intermittent, as is true for most glove boxes used in research applications, they may last longer than 18 months. Using a filter change frequency of six months as a
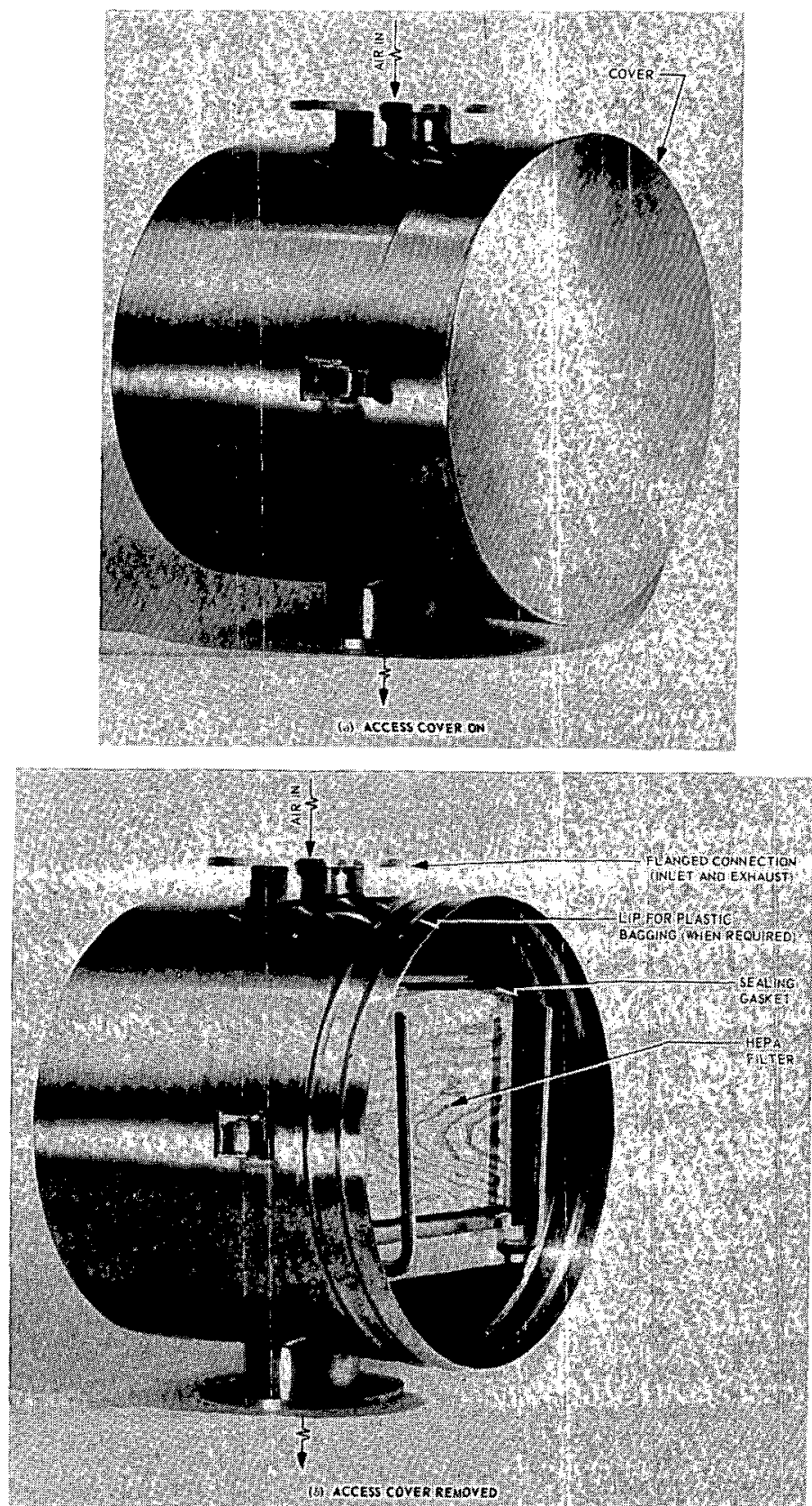

Fig. 7.17. Mini-caisson housing for open-face HEPA filters. Courtesy Barneby-Cheney Co. 
basis, there is no equipment cost advantage for using a commercial housing unless the life expectancy of the installation is more than five years.

Although difficult to estimate, the total costs (installation and operating) for HEPA filters installed inside glove boxes may be less than those of out-of-box installations using commercial enclosures, if the cost of in-box space is neglected. However, total costs for in-box filter installations (using standard-sized filters) are about equal to those for installations using enclosed HEPA filters in ducting outside the glove box, again neglecting boxspace cost. The cost advantages for any type of installation can easily be overruled by special operational requirements, or lack of space can make the desired installation method impractical.

\subsubsection{Inlet HEPA Filters}

Work performed in glove boxes frequently requires that supply air be kept free of airborne contaminants. Inlet HEPA filters help to maintain clean conditions inside and, when chosen properly, serve three other useful functions: (1) they extend the service life of the exhaust filter by protecting them from atmospheric dirt loading; (2) they prevent the spread of contamination from the glove box to the room in the event of a box pressurization; and (3) they provide overpressure relief.

The design of the inlet filter installation is relatively simple for air-ventilated nonrecirculating glove boxes. Since no duct connections are required, openface filters may be used with an installation and clamping method that leaves one face completely exposed. Typical methods of installation are shown in Figs. 7.11 and 7.18. Because they are less likely to be contaminated, inlet air filters are easier to replace than exhaust filters; therefore, they provide fewer problems and less risk during changes. Whether mounted inside or outside the glove box (outside mounting is preferred), the same high quality of mounting, clamping, and sealing is required.

The open face of the filter must be protected from physical damage and fire. Plugging of the inlet filter by smoke is of secondary concern, however, since one recommendation for glove box fire suppression is to reduce normal airflow. Locating the inlet connection (or an attached inlet duct) high in the box tends to reduce the amount of air drawn into the box during a fire because of the chimney effect.
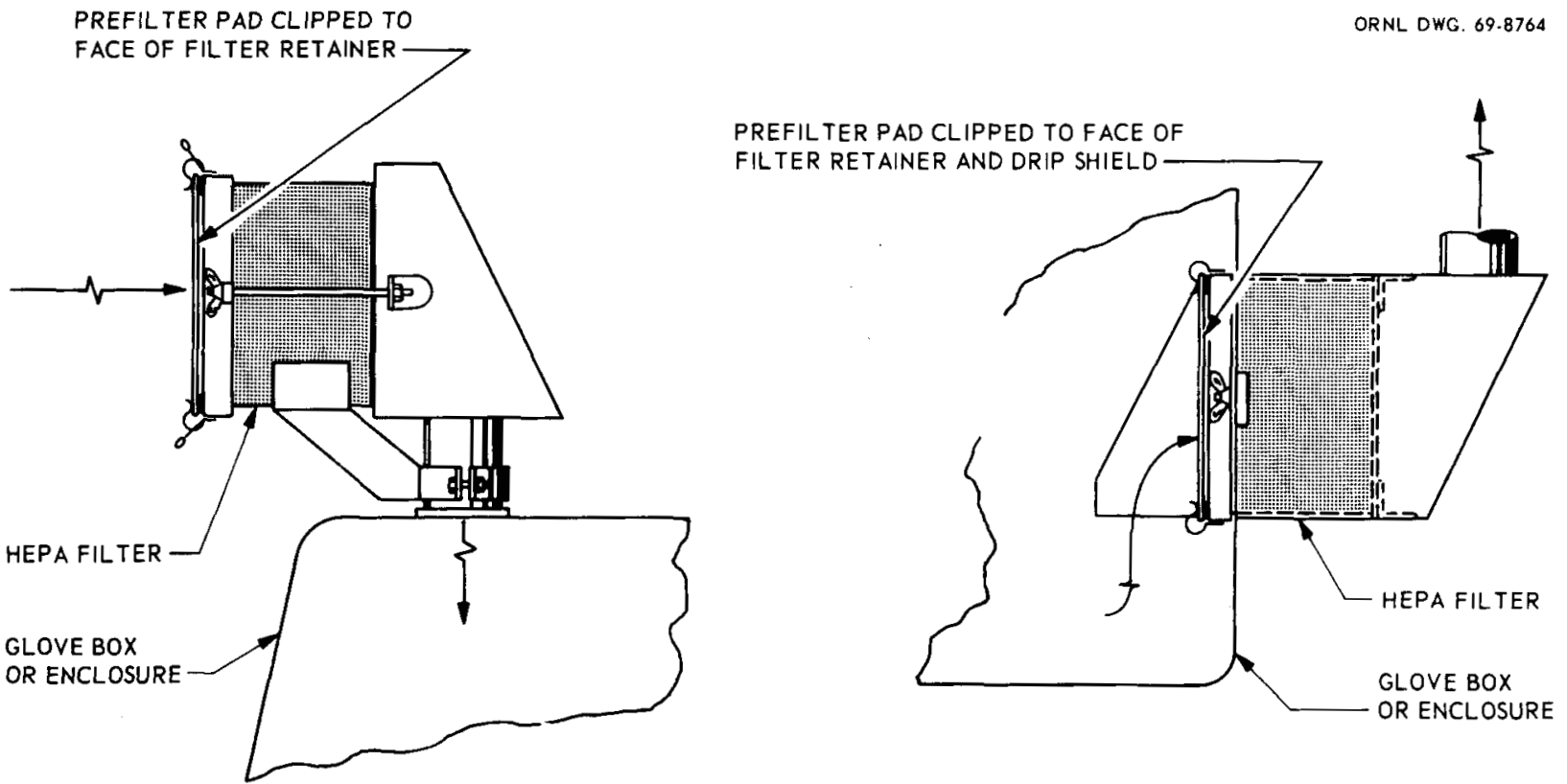

INLET AIR FILTER INSTALLATION

IN-BOX EXHAUST FILTER INSTALLATION

Fig. 7.18. Typical installations of prefilter pads on face of HEPA filters. 


\subsubsection{HEPA Filter Selection}

The number of types and sizes of HEPA filters used at an installation should be minimized for logistical and operating economy. All HEPA filters should be of fire-resistant construction. The sizes of HEPA filters most often used in glove box systems are $8 \times 8$ $\times 3^{1 / 16}$ in., $8 \times 8 \times 5^{7} / 8$ in., and $12 \times 12 \times 5^{7} / 8$ in. with nominal airflow capacity of 25,50 , and $125 \mathrm{cfm}$ respectively. The size and number of filters required for a glove box are determined by the maximum flow requirements and the pressure differential available to overcome air resistance. Glove box filters are customarily operated at flow rates below their nominal rating. Wood-cased fire-resistant HEPA filters are less expensive and should be considered wherever the operating environment (temperature, humidity, etc.) permits.

Undesirable features of both the enclosed and openface HEPA filters include the following:

- insufficient capacity for large amounts of dust;

- chemical fumes such as caustic hydrofluoric acid mist can destroy filter medium separators and adhesives;

- sharp corners and edges of metal casings can damage protective bagging;

- in dry atmospheres ( $<2 \%$ relative humidity) the plywood of wood-cased HEPA filters may shrink and delaminate, causing eventual failure of the filter. Very low moisture levels may cause a shrinkage problem for particle board casings as well. This could be an acute problem in inert atmospheres where very low moisture levels ( $<50 \mathrm{ppm})$ have to be maintained. In such systems, steel-cased filters should be used.

Open-face HEPA filters have the following additional shortcomings:

- more vulnerable to damage during handling and storage;

- lack of handle or gripping area for ease of withdrawal from an enclosure;

- difficult to replace damaged face gaskets.

Enclosed HEPA filters have the following additional shortcomings:

- lack UL certification;

- reeding (induced vibration of separators caused by air motion) at high flow rates is worse than in open-face filters, because the entering air impinges on a smaller area of the filter pack;

-greater weight than open-face filters (see Table 3.2 for comparison);

- substantially greater cost than open-face filters;

-greater space requirements;

- problem of air leakage with steel cases, especially in inert atmosphere and high-pressure applications;

-no visible means of detecting damage to the medium.

\subsubsection{Prefilters}

As in larger systems, prefilters may be used in both the inlet and exhaust air streams to extend the life of the HEPA filters used in glove box filtration systems. Prefilters are sacrificial items and the decision to use them requires that the designer evaluate the advantage of longer HEPA filter life against the problems of limited space frequently encountered in glove box systems. Prefilters attached directly to the face of the HEPA filter provide no fire protection for that HEPA filter. Glove box prefilter service often requires that filters be subjected to periods of high temperature, moisture, dust, and corrosive agents that shorten their effective life and mounting.

Experience with prefilters in glove box ventilation systems has shown the use of metal media to be impractical. Without viscous coatings the fil:ering efficiency of metal-media prefilters is poor, and these filters are often almost impossible to clean and decontaminate. Adhesives and oil coatings that improve particle retention reduce in-box cleanness and fire resistance. Experience clearly indicates that use of conventional types of prefilters that require cleaning or decontamination or both before reuse is impractical. Throwaway filters with simple installation methods are preferred. After use, the units are discarded as contaminated waste unless collected materials must be reclaimed. Glass-fiber-media prefilters are preferred because serviceability is good, costs are low, and combustible content is small.

Inlet airstreams with HEPA filters should be fitted with prefilters when using atmospheric air. However, when the room air has been cleaned of the bulk of its airborne dust by building supply-air systems, when local room activities do not generate dust and lint that can be drawn into the box, and when airflow 
through the HEPA filter is less than $75 \%$ of its rated capacity, there may be no need for a prefilter.

A common method of prefiltering is to clip a thin $(1 / 8$ to $1 / 4$ in.) fiberglass pad to both the inlet and exhaust HEPA filters, as Fig. 7.18 shows. Neither plastic foam nor organic fiber should be used because both are flammable. The pad is cut to fit the face of the HEPA filter and is clipped to the filter retainer. This method of attachment permits easy removal of the prefilter pad without disturbing the seal of the HEPA filter. Normal usage generally requires frequent replacement of the prefilter pads, which do not have much dirt-holding capacity and can quickly become plugged by house dust and lint. Convenient methods of attaching the prefilter pads are essential. Frequent replacement of prefilter pads assures that

- air resistance (pressure drop) does not change rapidly, which thus allows airflows to remain more nearly constant without frequent manipulation of dampers;

- the accumulation of combustible dust in the exhaust path is less, thereby providing better fire protection for the HEPA filter downstream if the prefilter is not applied directly to the face of the HEPA filter;

- the exhaust path can pass a greater flow of air in relieving an emergency condition.

Fiberglass pads $(1 / 4$ in. thick or less) can provide average atmospheric dust collection efficiency up to $20 \%$ (as determined by ASHRAE 52-68), ${ }^{15}$ with low airflow resistance. Thin ( $1 / 4$ in. thick or less) clean fiberglass pads used at air velocities of $35 \mathrm{fpm}$ will create an initial pressure drop in the range of 0.03 to 0.15 in.wg. Table 7.2 lists several types of media that can be used as prefilter pads.
For applications where long-term continuous processes hamper regular maintenance of in-box filters, the designer must include

-provision for greater suction pressure (well below the limit that would subject glove or box integrity to unsafe differential levels) controlled by the damper to allow for longer use of prefilters;

-provision for more prefilter area; or

- selection of a prefilter with less initial resistance to permit longer use, even though lowering collection efficiency.

\subsection{FILTER REPLACEMENT}

The safe replacement of a contaminated glove box filter must be planned in the design stage to facilitate proper execution. The designer should prepare a written preliminary filter change procedure along with the design documents. Because they are prepared for construction purposes, the intended operating and maintenance procedures envisioned by the designer may not always be implicit or obvious in the design drawings and specifications. By writing a preliminary filter change procedure, the designer assures himself that the changeout can be performed safely.

Crew members directly involved in a contaminated filter change must wear appropriate respiratory protection. Filters installed inside the glove box are accessible by the use of gloves on the box. When the total activity of contaminants is high, additional protective measures may be necessary to reduce worker exposure. One method of controlling the spread of contamination, while preserving the in-

Table 7.2. Prefilter pad materials

\begin{tabular}{lcccccc}
\hline Medium & $\begin{array}{c}\text { Thickness } \\
\text { (in.) }\end{array}$ & $\begin{array}{c}\text { Initial } \\
\text { pressure } \\
\text { drop(in.wg) }\end{array}$ & $\begin{array}{c}\text { Velocity } \\
\text { (fpm) }\end{array}$ & $\begin{array}{c}\text { Collection efficiency } \\
\text { NBS } \\
(\%)\end{array}$ & $\begin{array}{c}\text { AFI }^{b} \\
(\%)\end{array}$ & Remarks \\
\hline $\begin{array}{l}\text { Owens-Corning Fiberglas } \\
\text { Corp., RE-1 }\end{array}$ & $3 / 16$ & 0.05 & 35 & 20 & & \\
$\begin{array}{l}\text { American Air Filter Co. } \\
\text { Type G Airmat }\end{array}$ & $\begin{array}{l}3 / 32 \\
\text { No. 12 Airmat }\end{array}$ & 0.03 & 35 & & 87.5 & UL class 1 rating \\
\hline
\end{tabular}

${ }^{a}$ Clean efficiency-ASHRAE 52-68 test method using atmospheric dust.

${ }^{b}$ Average arrestance- ASHRAE 52-68 test method using synthetic dust.

'UL-900, Safety Standard for Air Filters, Underwriters' Laboratories, current issue. 
tegrity of the closed box and the system, is bagging the filters in and out of the glove box, as shown in Fig. 7.19. The plastic bagging materials used are discussed in Sect. 6.2.3. When inert-atmosphere or oxygen-free environments are used inside the glove box, additional provisions may be required to prevent air leakage into the box.

Replacement of a HEPA filter inside an airventilated box entails many steps that must be performed sequentially. Each step must be carefully planned and completed in a methodical manner to preserve containment of the system. Close coordination between maintenance and operating personnel is necessary to establish a mutually satisfactory date and time for the filter change, to identify the boxes and systems involved, to procure the necessary materials, and to schedule personnel. The health and safety requirements of the industrial hygienist, health physicist, and safety engineer must be established; one of these specialists should be designated the health and safety supervisor and should be available to monitor the operation and to assist as necessary.
When the necessary materials and tools are ready and all personnel have been instructed in their specific duties, final permission must be secured from the responsible operator to alter the airflow and replace the filters. The flow path of the exhaust system should be thoroughly understood, and persons responsible for related exhaust systems that will be affected should be forewarned. For instance, if two glove box exhaust systems manifold to the same blower, final filters, and stack, the removal of one system from service for a filter change will have an effect on system flow and pressure characteristics of the other system. Safety clothing and respiratory protection should be worn as directed by the health and safety supervisor. Typical steps required to change a filter and place a box back in service are as follows:

1. Cease all glove box operations and store unsafe materials in suitable containers.

2. Cut off gas flow to the glove box affected and adjust flow through the remaining branches to
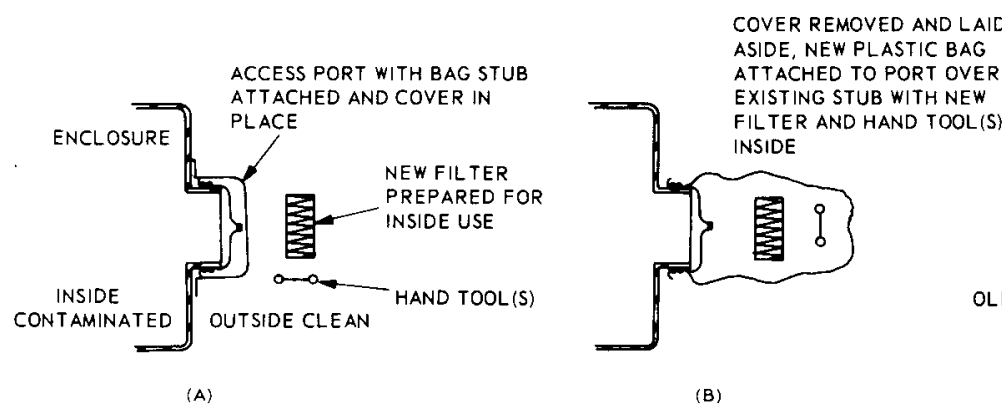

(B)

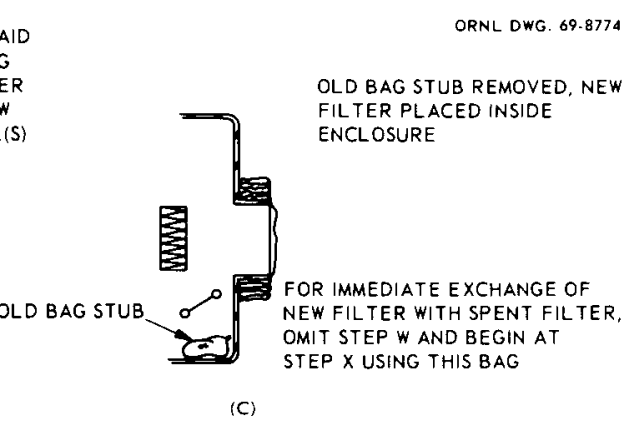

NEW FILTER BAGGED INTO CONTAMINATED ENCLOSURE

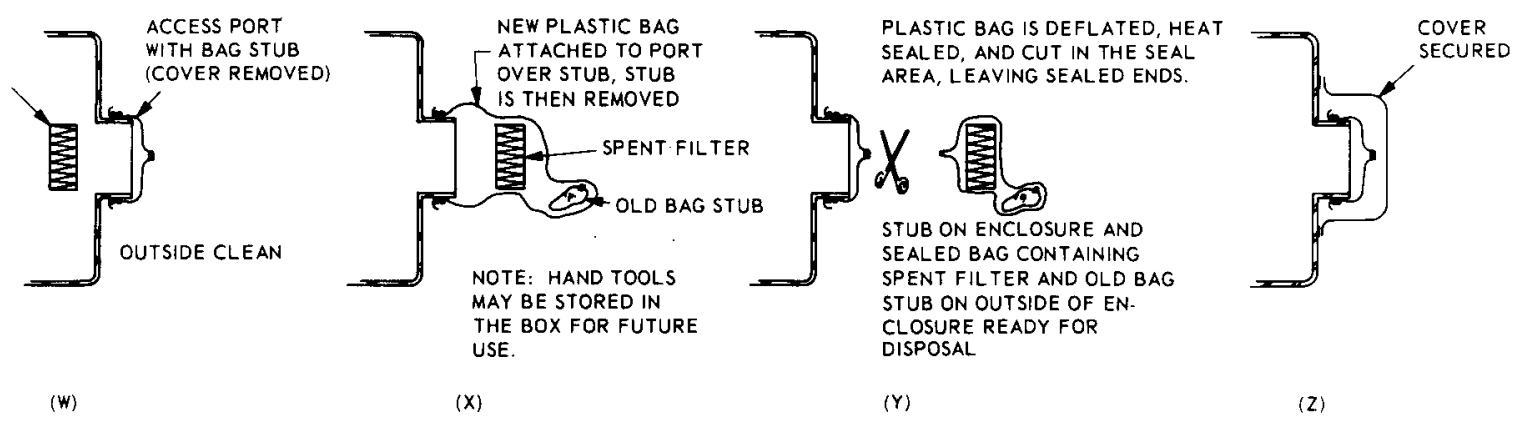

SPENT FILTER BAGGED OUT OF CONTAMINATED ENCLOSURE

Fig. 7.19. Technique for changing filters installed inside contaminated enclosures. 
restore a safe negative pressure and flow rate in each.

3. Bag in (a) a clean replacement filter (and prefilter if used); (b) a small, clear plastic bag and sufficient tape to hold the spent filter and prefilter; and (c) all hand tools required, as shown in steps A, B, and C of Fig. 7.19. It is recommended that hand tools needed for filter changing be introduced the first time filters are changed and then left in the glove box for subsequent use if space and environment permit. Decontamination is often more costly than replacement of tools.

4. Using the box gloves, remove the dirty filter and prefilter from their mounting frame.

5. Insert the dirty filter and prefilter into an empty plastic bag, slowly expel excess air, and seal with tape.

6. Inspect gasket sealing face of the mounting frame and clean if necessary. Place replacement filter in position and secure clamping devices. Place new prefilter in position and secure.

7. Remove the dirty filters and all debris from the glove box, as shown in steps $\mathrm{W}, \mathrm{X}, \mathrm{Y}$, and Z of Fig. 7.19, and place removed items in a container for contaminated waste disposal.

8. Restore airflow through the glove box and adjust flow and negative pressure throughout the system.

9. Before glove box operations are resumed, test the newly installed HEPA filter with DOP, using the permanent test connections on the housing. If the test result is not satisfactory, stop the flow and inspect the filter for damage. If no damage is apparent, reposition the filter, restore the flow, and retest the filter. If the second DOP test is unsatisfactory, the filter should be replaced and steps 3 through 9 repeated. Continued leakage suggests a failure of the mounting frame or a faulty test, and each possibility should be examined in detail until the fault is discovered and corrected.

10. Decontaminate the area.

11. After successful filter replacement, notify the responsible operator.

Filters located outside a glove box require convenient access for changing, and it is usually necessary to interrupt airflow during the change. Since they are located outside the glove box, highly contaminated filters must be bagged during the change. Different bagging techniques provide different degrees of protection. The technique shown in Fig. 7.20 employs the principle of total containment when even minute leakage cannot be permitted. This method seals both ends of the air ducts, and no flow can occur downstream while the filter is removed. When uninterrupted airflow through a box is required, this method of filter change necessitates the use of multiple exhaust connections on the box. An out-of-box filter in the process of being removed from a system by the procedure illustrated in Fig. 7.20 (step 3) is shown in Fig. 7.21.

For other methods where bagging does not block the airflow path (e.g., using the housings represented by Fig. 7.14) but merely encapsulates the filter being removed or replaced, there is a dependence on the damper in the duct to prevent blow-by (leakage) during a filter change. The total containment method (illustrated in Fig. 7.20) disconnects the exhaust duct and does not depend on damper tightness. The technique of bagging filters from housings (Fig. 7.14) offers protection only for local personnel and the service area where the filter mounting device is located. The side of the system downstream of the filter is protected not by bagging but by leakproof dampers and flawless handling of the dirty filter. Because any dislodged particles will be swept downstream when airflow is restored, downstream HEPA filters should be provided to intercept these particles.

\subsection{GLOVE BOX SAFETY}

\subsubsection{Protection Against Fire and Explosion}

Fire and explosion protection has received much attention. Some useful references include:

A. J. Hill, Jr., Automatic Fire Extinguishing Systems for Glove Boxes and Shielded Cells at the Savannah River Laboratory, DP-1261, Savannah River Laboratory, June 1971.

C. Jackson, T. W. Hodge, D. H. Swingler, and A. J. Smith, Some Aspects of Fires in Glove Boxes, AERE-R 3067, United Kingdom Atomic Energy Authority, October 1959.

S. E. Smith, F. J. Hall, W. E. Holmes, and A. F. George, Protection Against Fire Hazards in the Design of Filtered Ventilation Systems of Radioac- 
ORNL DWG. 69.8775

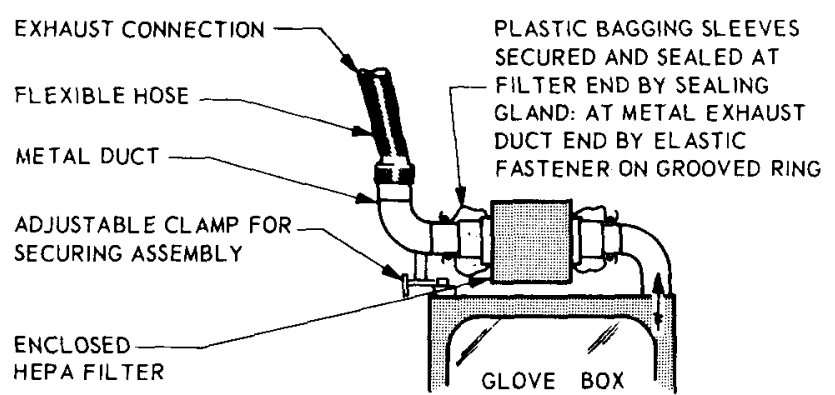

(1)

NORMAL POSITION

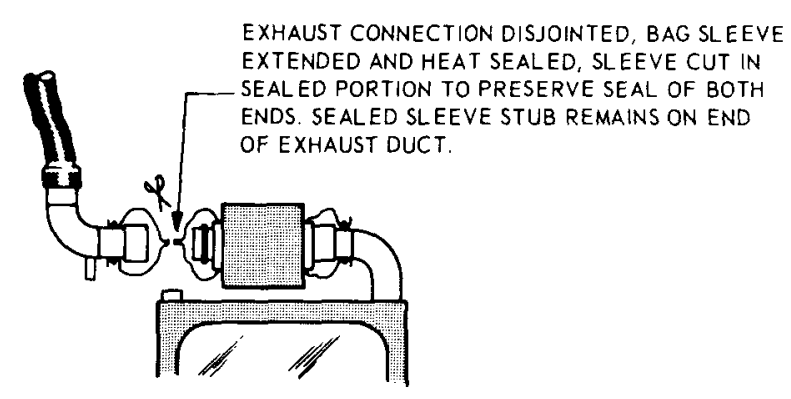

(2)

EXHAUST CONNECTION SEVERED

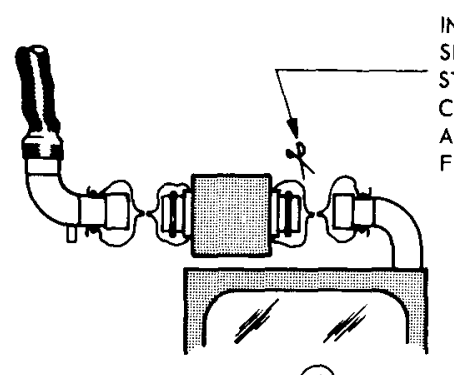

(3)

INLET END OF FILTER IS DISJOINTED, HEAT SEALED AND CUT FROM SYSTEM SIMILAR TO STEP 2, PRESERVING SEAL ON GLOVE BOX CONNECTION. INSERT DIRTY FILTER IN ADDITIONAL PLASTIC BAG AND SEAL END FOR DISPOSAL AS CONTAMINATED WASTE.

DIRTY FILTER SEPARATED FROM SYSTEM

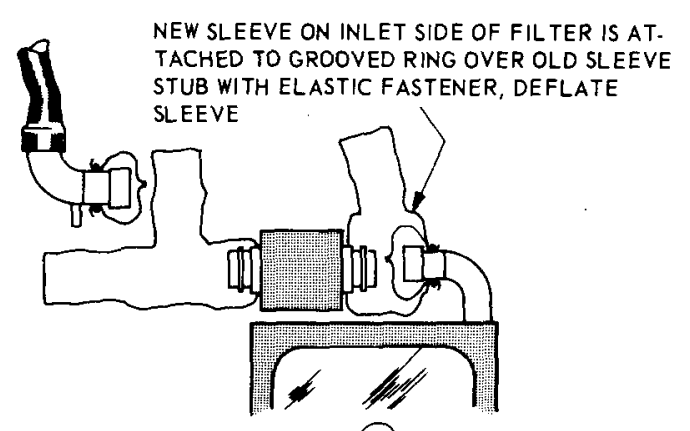

(4)

CLEAN FILTER INLET SEALED OVER SLEEVE STUB ON GLOVE BOX

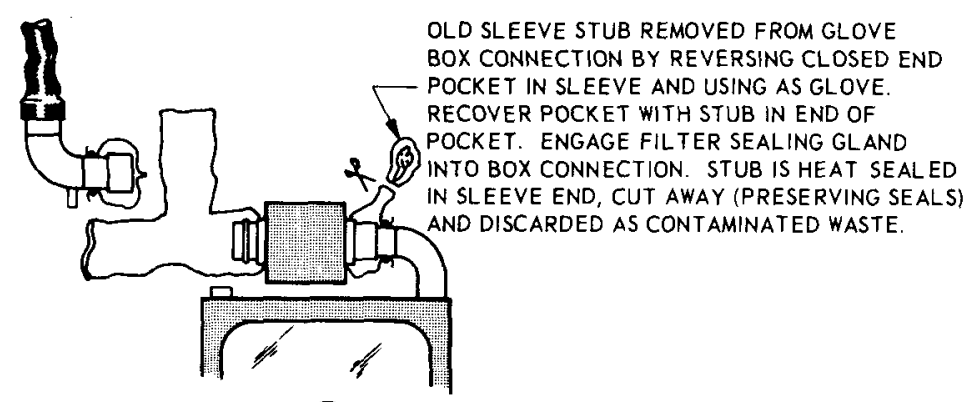

(5)

CLEAN FILTER SEALED TO GLOVE BOX EXHAUST CONNECTION

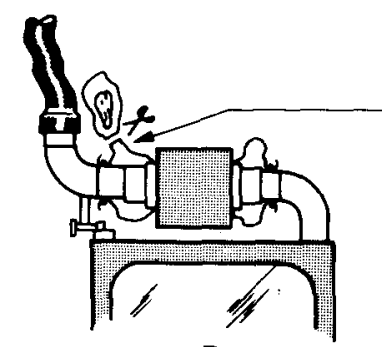

(6)

EXHAUST CONNECTION SEALED TO FILTER, AIR FLOW RESUMED
EXHAUST DUCT IS JOINED TO OUTLET END OF CLEAR FILTER SIMILAR TO STEP 5. PARTICULAR CAUTION NECESSARY TO PREVENT LOOSE STUB FROM BEING SUCKED UP DUCT. ALLOW BAGGING TO DEFLATE BEFORE CONNECTING DUCT. SECURE ASSEMBLY WITH ADJUSTABLE CLAMP

Fig. 7.20. Bagging technique for enclosed HEPA filter located outside the glove box.

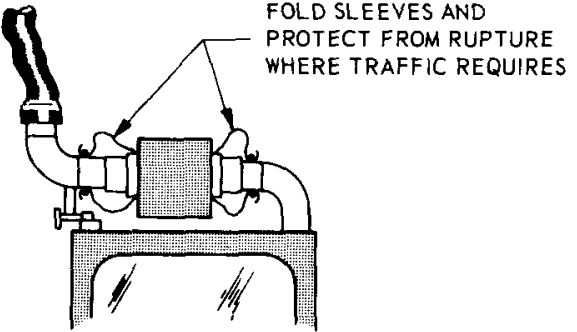

(7)

NORMAL POSITION RESTORED WITH CLEAN FILTER IN PLACE

CLEAN ENCLOSED HEPA FILTER PREPARED IN ADVANCE WITH PLASTIC BAGGING SLEEVES AND SEALING GLANDS ON FILTER CASING NIPPLES 


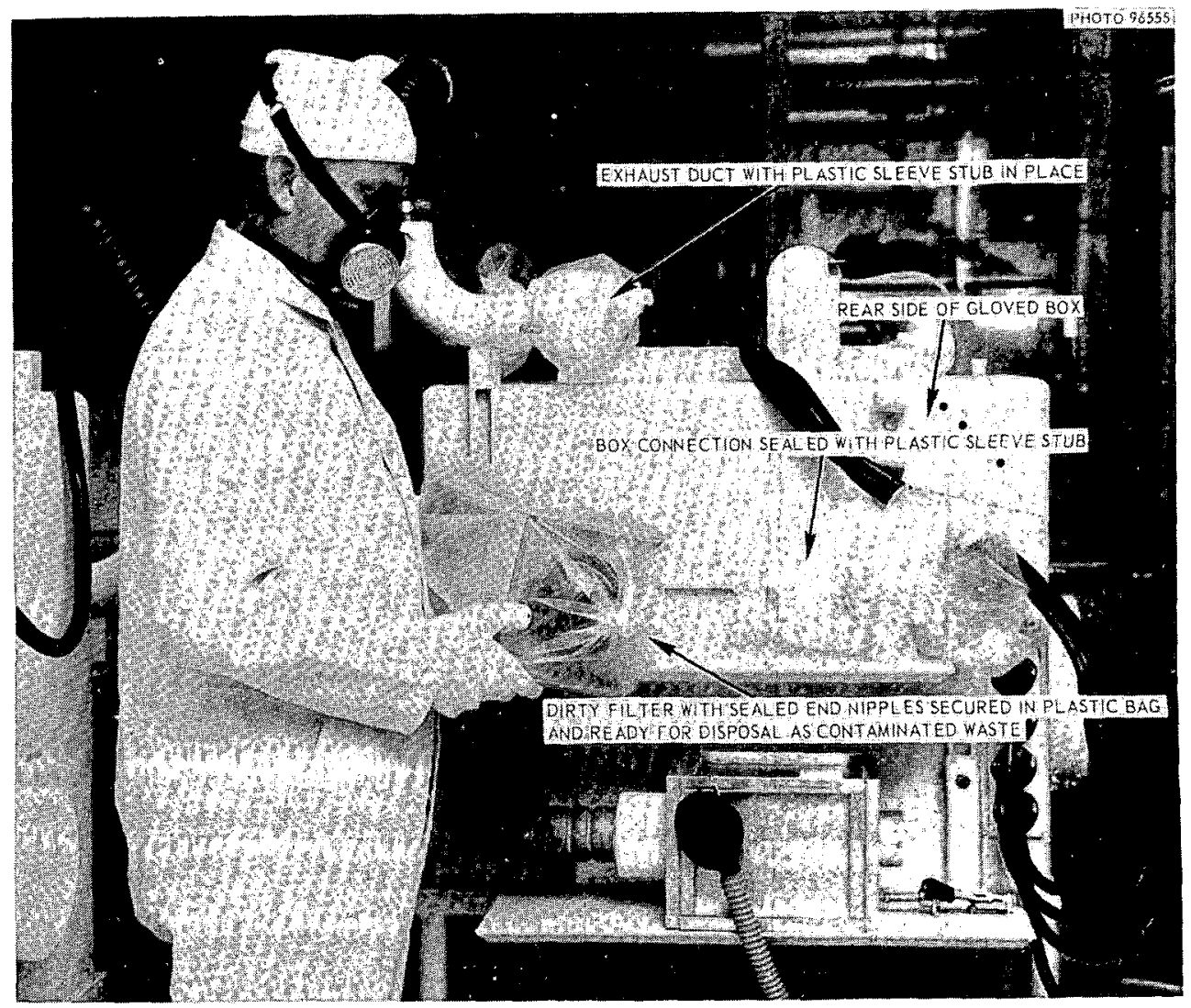

Fig. 7.21. Enclosed HEPA filter being removed from exhaust stream outside glove box by bagging. Courtesy Oak Ridge National Laboratory.

tive and Toxic Process Buildings, AWRE 0-24/65, UKAEA, July 1965.

J. Young, Engineering Study of Radiological Fire Prevention at Lawrence Laboratory, Berkeley, California, UCRL-19465, Lawrence Radiation Laboratory, January 1970.

W. E. Domning and R. W. Woodward, Glovebox Fire Tests, RFP-1557, Rocky Flats Plant, Nov. 6, 1970.

L. R. Kelman, W. D. Wilkinson, A. B. Shuck, and R. G. Goertz, Safe Handling of RadioactivePyrophoric Materials, ANL-5509, Argonne National Laboratory, December 1955.

S. H. Pitts, Jr., "Factors Influencing the Ignition of Metallic Plutonium," Nucl. Saf. 9(2), 112-19 (March-April 1968).

R. E. Felt, Burning and Extinguishing Characteristics of Plutonium Metal Fires, 1SO-756, Richland, Wash., August 1967.
H. V. Rhude, "Fire and Explosion Tests of Plutonium Glove Boxes," pp. 305-11 in Proc. Hot Lab. Equip. Conf. 10th, Washington, D.C., Nov. 26-28, 1962.

A. J. Hill, Jr., Fire Prevention and Protection in Hot Cells and Canyons, DP-1242, Savannah River Laboratory, April 1971.

H. A. Lee, Guide to Fire Protection in Caves, Canyons, and Hot Cells, ARH-3020, Richland, Wash., July 1974.

NFPA 801, Recommended Fire Protection Practice for Facilities Handling Radioactive Materials, National Fire Protection Association, Boston, 1970.

R. E. Giebel and R. L. Riegel, Drybox Gloves-Evaluation and Procurement, RFP-1286, Rocky Flats Plant, June 23, 1971.

P. E. Johnson, Evaluation of Improved Fire Resistant Glove Materials for Gloveboxes, USAEC 
Report TID-25086, Factory Mutual Research Corp., Boston, 1969.

C. W. Jacoby, Glovebox Window Materials, RFP1424, Rocky Flats Plant, Mar. 13, 1970.

Glovebox Window Materials, USAEC Report TID-24896, Factory Mutual Research Corp., Boston, 1969.

The task of fire and explosion protection can be divided into prevention, detection, and suppression. These areas are discussed below as they apply to glove box operations and in Chap. 9.

Protection. Fire prevention in glove boxes is mainly accomplished by the following methods:

1. Using nonflammable materials in construction. ${ }^{1,2,8,9}$ Gloves and windows have received the most attention, since they are the most necessary and vulnerable parts of the glove box.

2. Strict adherence to acceptable housekeeping practices.

3. Avoiding the use of flammable materials within the box wherever possible and limiting the amount of flammables to the minimum required for immediate use when no suitable nonhazardous substance can be substituted. Using containers for flammable substances that are as safe as can be found for the planned operation.

4. Maintaining a current in-box material inventory and not using the box for storage. Boxes usually are inappropriate for storage, especially for chemicals.

5. Establishing a safer, nonoperative box configuration and periodically checking to make sure that nonoperating boxes are in safe condition. Precautions include isolating boxes by closing fire stops, checking through-flow, checking port covers, disconnecting electrical equipment, and removing corrosives.

6. Designing the box with downdraft ventilation (high air inlet, low outlet), to inhibit combustion while still purging the box.

7. Providing a protective atmosphere (Sect. 7.5.2). This measure was listed last because those preceding it are applicable to all glove boxes, whereas inerting is used only when there is too much risk involved in operating without a protective atmosphere. Assessing the degree of risk involved in an operation is often a subjective evaluation.

Detection. A glove box fire detection system is recommended when there is high risk of fire. If flammable solvents, coolants, packaging materials, etc., must be present during operation and especially in unattended boxes, a heat detector should be located within the box. Regulatory Guide $3.12^{16}$ calls for heat detectors and combustible-gas or vapordetection meters on glove boxes wherever fire or explosion hazards exist.

Fire detectors are required in plutonium glove boxes. ${ }^{17}$ A description of currently available detectors is given in the Factory Mutual safe practice guide. ${ }^{9}$ Report DP- $1242^{18}$ contains a useful section on evaluating detectors.

Suppression. Since a fire within a glove box may be of paper, chemical, electrical, or pyrophoric metal origin, there is no single suppression method that is best for all glove boxes. Halon-1301 ${ }^{19}$ is an effective general extinguishing agent; it is better than carbon dioxide $^{20}$ on chemical and electrical fires but ineffective against pyrophoric metal fires; ${ }^{21}$ and it decomposes above $750^{\circ} \mathrm{F}$. The use of carbon microspheres for extinguishing metal fires is discussed in Sect. 9.5.4. The complete exclusion of oxygen with rapid heat removal to below its ignition temperature $\left(500^{\circ} \mathrm{C}\right)$ is effective for extinguishing plutonium fires. ${ }^{21}$

There is no assurance that filters will remain functional during and following exposure to fire, smoke, or burning debris. The temperature reached during a fire, the quantity and density of smoke released, and the duration of the fire determine the destructive effects on prefilters and HEPA filters.

HEPA filters can withstand $750^{\circ} \mathrm{F}$ temperatures for periods up to $10 \mathrm{~min}$ but should not be subjected to indefinite exposure to temperatures higher than $275^{\circ} \mathrm{F}$ (Tables 3.4 and 3.5). Longer filter life and more reliable service can be obtained when normal operating temperatures are below $200^{\circ} \mathrm{F}$ and high temperature extremes are avoided.

The selection and arrangement of HEPA filters on, in, or near glove boxes and similar enclosures are limited by the type of fire control equipment used, because HEPA filters and most prefilters are not compatible with all types of fire extinguishing systems. Dry chemical extinguishers employ finely divided solids that can clog filters. Ideally, the 
discharge point of dry chemical agents should be selected to blanket the fire zone effectively without becoming airborne.

When large amounts of carbon dioxide $\left(\mathrm{CO}_{2}\right)$ are released from a cylinder, moisture in the glove box atmosphere may form ice crystals that can clog filters after only a few minutes of operation. Should this happen, introduction of more $\mathrm{CO}_{2}$ is likely to overpressurize or perhaps rupture the box. Carbon dioxide is a poor fire extinguishing agent for glove boxes because of its tendency to clog exhaust filters, reduce airflow, and obscure vision when moisture is present.

When foaming agents or spray droplets from fire extinguishing systems reach a filter, it is quickly clogged if free moisture cannot be evaporated into the air passing through the filter. This limits the use of foam generators and water fogs to ventilation systems where emergency devices are actuated and controlled manually, or where continuous airflow through the filter (or filters) during an emergency is not required. The standard for high expansion foam systems is NFPA $11 \mathrm{~A},{ }^{22}$ and for synthetic foams, NFPA 11 B. $^{23}$

\subsubsection{Protective Atmospheres--Inerting}

The difference between a protective atmosphere and an extinguishing agent is that the protective atmosphere prevents the fire condition from occurring, whereas the extinguishing agent is applied as a response after a fire has started. Argon, carbon dioxide, nitrogen, and helium have all been used as inerting agents. The protective atmosphere system is designed for continuous operation, whereas the extinguishing system usually has a one- or two-shot, single-incident application before reservicing is required to return the system to the ready state.

Inerting with smothering agents may require that less than $1 \%$ oxygen be present in the glove-box atmosphere. Process and product-purity considerations may require as little as $100 \mathrm{ppm}$ of total atmospheric impurities within the glove box for successful operation. Since many of the detailed considerations are similar for high-purity and fireprotection inerting, and because of the widespread application of high-purity inerting, most of this discussion will involve high-purity systems. The best single reference for design, construction, and operational information is Inert Atmospheres ${ }^{2}$ by White and Smith.
Inert-atmosphere glove boxes that contain radioactive material are operated at pressure differentials of 0.3 to $1.0 \mathrm{in}$.wg negative relative to the surroundings. Gas flow rate is usually determined by the atmospheric purity required and the purity of the incoming gas. The box atmosphere purity can be compromised by air leakage into the box or into service connections or leakage from process equipment in the box.

Filter installation requirements in inertatmosphere glove boxes are more stringent than for air-ventilated boxes, because acceptable box airleakage rates are generally less than 0.0005 box volume $/ \mathrm{hr}^{2}$ To attain this standard, joints and fastenings between items of equipment and materials (gaskets and seals) must have extremely low gas permeability. Full-welded joints are recommended for all permanent fixtures. Gasketed joints may deteriorate in service, and this imposes continuing costs for periodic testing and repair.

Low-leak systems require quality construction for all components including boxes, filters, and associated ducts. Any inleakage associated with the filter mounting or connecting duct will adversely affect the quality of the inert atmosphere that can be maintained in the box and therefore the cost of inert gas purification. Penetrations must be minimized in both number and size. The use of smaller HEPA filters allows smaller ports for maintenance. Filter changes should be planned for times when other maintenance operations (routine or special) are taking place inside the box to reduce interruptions to operations, to reduce the loss of inert gas, and to minimize the time required to recondition box spaces.

For fire protection, the preventive step of inerting is more satisfactory, though more expensive, than extinguishing a fire if it does occur. However, oxygen must be reduced below $1 \%$ before it fails to support the burning of some pyrophoric metal. ${ }^{9}$ The use of dry air (relative humidity less than $20 \%$ ) reduces the hazard of pyrophoric metal fires but does not eliminate it. Moisture in the presence of heated pyrophoric or reactive metals, such as finely divided plutonium, increases the possibility of explosion by generating hydrogen. The suitability and cost of an inert gas for the process are significant factors when selecting this type of fire control. The gas flow rate in most inert gas boxes is kept as low as possible to be consistent with required box-atmosphere purity levels; low-capacity filters are frequently used. The

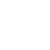


inert gas may be purged on a once-through basis or recirculated through a purification unit. A word of caution concerning commercially available (off-theshelf) recirculating glove boxes: at one ERDA installation, over half of the problems with glove boxes during the last five years involved this type of installation, even though commercial boxes represented less than one-tenth of the boxes on hand. Some problems were caused by vacuum-pump oilmist plugging of a HEPA filter, others by the failure of vacuum relief solenoids. Factory Mutual Research Corporation published a report on oil mists from vacuum pumps. ${ }^{24}$ The statistics point up again that off-the-shelf items cannot be used in a containmenttype ventilation system without evaluation, nor can they be applied as "black boxes" by those responsible for operational safety.

\subsubsection{Control and Instrumentation}

Glove box instrumentation may range from simple indicators and alarms to sophisticated control systems. The type of control or instrument used will depend on the characteristic to be monitored, the relative hazard, and the method and time available to correct an upset condition. Operational characteristics to be measured should always include the differential pressure between box and surroundings, the filter resistance, the gas flow rate through the box, and the box atmospheric temperature. In addition to instruments on the box, it may be necessary to indicate and provide for readout and/or alarm at a central panel for oxygen content, liquid level, neutron flux, gamma flux, fire, or explosive gas mixture inside the box.

When, for safety, a monitored characteristic requires annunciation when the level of a monitored parameter passes some predetermined point (e.g., an alarm when the glove box pressure differential becomes less negative than $0.3 \mathrm{in} . \mathrm{wg}$ relative to the surroundings), the alarm may be local, that is, alerting the operator to the upset condition, or it may signal an annuciator panel in an adjoining "cold" area by the entry door to the glove box room or in a control room, or both. There should be a written procedure in addition to sufficient information on the current contents of each box to allow evaluation of the hazard when an alarm sounds and to plan corrective action.

The minimum instrumentation for a glove box ventilation system should include devices to indicate the differential pressure between the box and its surroundings, and to indicate filter resistance, total exhaust flow rate, and exhaust air temperature. Figure 7.22 shows the arrangement of indicating devices in a glove box ventilation system. The items shown above the double-dashed line (Fig. 7.22) indicate the types of instruments commonly used to supplement the minimum instrumentation necessary to improve safety for a particular operation or circumstance. For example, when box operators are not in full-time attendance for a continuous process, a sensor can be provided to monitor abnormal pressure or temperature and to actuate an alarm at a remote point where an attendant is stationed.

Figure 7.23 shows a typical local mounting for an aneroid differential pressure gage on top of a glove box. The instrument should be mounted near eye level, and the indicating face should always be located so that the operator has a clear view while manipulating the gloves. Sensing lines should be short and sloped directly back to the box so that moisture will not pocket in the tube. Tubing should be at least $3 / 16$ in. ID to allow the instrument to respond quickly to rapid changes in pressure. Use of a three-way vent valve at the gage permits easy calibration (zeroing) without disconnecting the sensing tube. Calibration of glove box differential pressure gages should be a weekly routine.

Most users prefer an instrument pressure range of 0 to $\mathrm{lin} . \mathrm{wg}$; however, the instrument must have a proof pressure greater than the maximum system pressure (negative or positive) so that it will not become inoperable or leak during a system pressure transient. Liquid-filled devices are not recommended for glove box pressure indicators, because liquids can be sucked into the box or filter if the safety traps on the manometer leak, as they often do after long exposure to high pressure. The aneroid gage has a diaphragm that seals the path against contamination leakage.

Inlet air filters on air-ventilated glove boxes do not require differential pressure gages. The pressure drop across the filter is approximately the differential pressure across the box pressure boundary. Inlet flow rates can be measured periodically and with acceptable accuracy $( \pm 15 \%)$ with a portable instrument, such as a thermal anemometer (see Chap. 9, Industrial Ventilation) ${ }^{25}$ When inlet filters become clogged enough to limit the inlet flow below the volume needed to serve the box, they must be changed. 


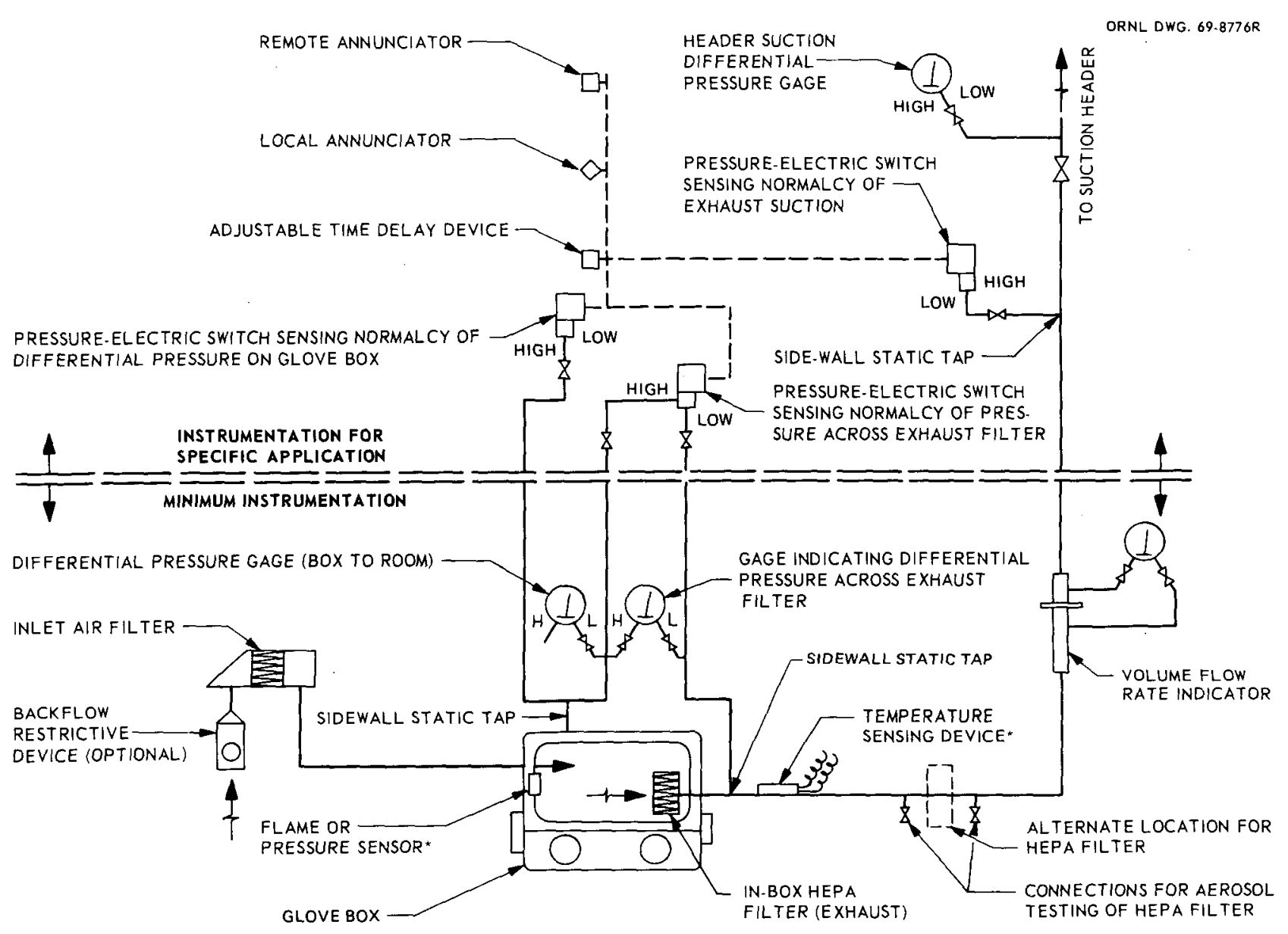

-APPLIED WHERE SPECIFIC BOX USE INCLUDES FLAMMABLE OR EXPLOSIVE MATERIALS TO ACTUATE ALARM AND PROTECTIVE SYSTEM

Fig. 7.22. Instruments in a glove box ventilation system.

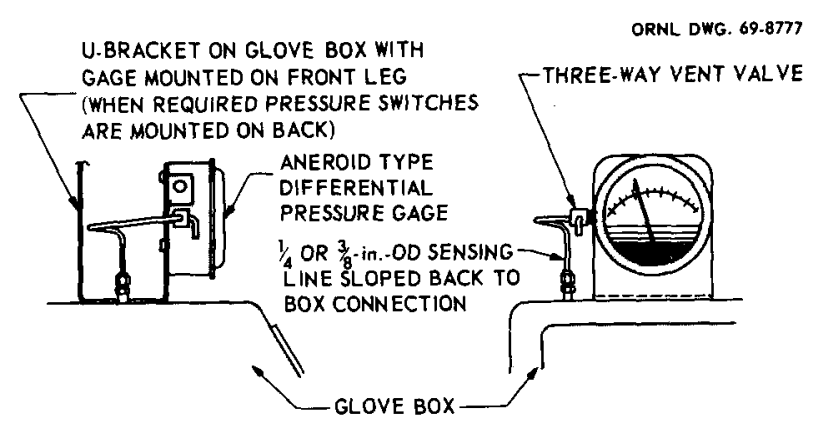

Fig. 7.23. Aneroid gage to indicate differential pressure between the glove box and the laboratory.

A differential pressure gage should be provided for each exhaust HEPA-filter stage to indicate filter resistance. Pressure-sensing connections can be provided to permit the use of portable instruments. As Fig. 7.22 shows, a dual (high-low) pressure switch can be used to actuate an alarm when a predetermined resistance limit is exceeded or a loss of suction is experienced. Suitable alarms or controls that can function on small pressure differentials $(\leqslant 0.25 \mathrm{in.wg})$ are difficult to keep in proper calibration and are often expensive. Figure 7.24 shows a method of indicating pressure drop through a filter. Section 5.6 gives further information on differential pressure instrumentation.

Instruments used to measure airflow rates from glove boxes include an orifice plate, venturi meter, flow nozzle, and calibrated pitot tube. The important point is to use a simple, trouble-free device that gives reliable readings within $\pm 15 \%$ accuracy. When free moisture is absent, a pitot tube is the least expensive and most adaptable device for the small volume flow rates associated with glove box ventilation. Velocity pressure measurements (corrected for pitot-tube 
single centerline location $^{26}$ ) for air flows and duct sizes common in glove box applications are given in Fig. 7.25. The corrections shown are for air at $60^{\circ} \mathrm{F}$ and 14.7 psia and neglect the pitot-tube coefficient. Pitot tubes are available with coefficients of 1.00 , but there is an advantage in using the more common commercial pitot tube with a coefficient of 0.825 at low flow velocities. The equation for measuring velocity with a pitot tube is

$$
V=K(2 g h)^{1 / 2},
$$

where

$V=$ fluid velocity, $\mathrm{ft} / \mathrm{sec}$;

$K=$ coefficient of the pitot tube;

$g=$ acceleration of gravity, $32.17 \mathrm{ft} / \mathrm{sec}^{2}$;

$h=$ velocity pressure $(\mathrm{ft})$ of the air-gas stream.

The following equation is used for air at standard conditions:

$$
V=4005 K\left(h_{w}\right)^{1 / 2},
$$

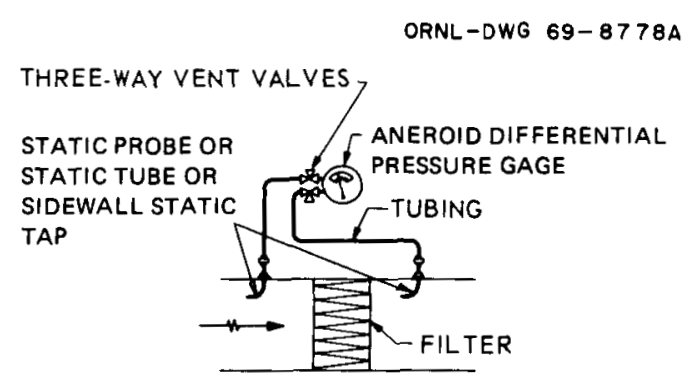

Fig. 7.24. Method of indicating pressure drop across a filter. Liquid-filled manometer is not recommended for this service.

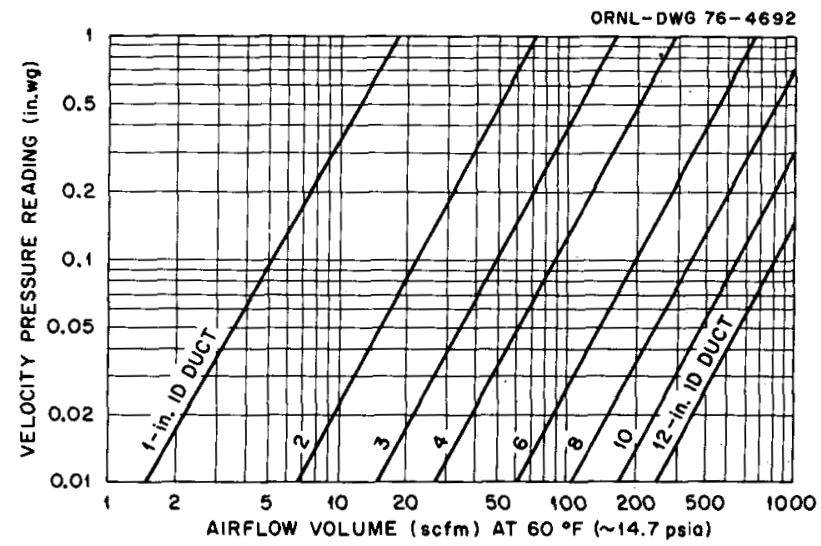

Fig. 7.25. Single centerline pitot-tube corrected airflow volume measurements. where

$V=$ fluid velocity, fpm;

$h_{w}=$ velocity pressure, in.wg.

A pitot tube with a coefficient of 0.825 has a velocity pressure reading that is 1.47 times the velocity pressure reading of the pitot tube with a coefficient of 1.00 for the same fluid velocity. This pressure differential allows the low velocities often encountered in glove box ventilation to be measured more easily.

Figure 7.26 shows the arrangement of a round orifice in a straight section of metal duct. Either method (pitot tube or orifice) can be used to read the flow volume directly on a properly calibrated gage. For a thin square-edge round concentric orifice with the properties given in Fig. 7.27, flow rate can be determined, with sufficient accuracy for glove box applications, by the following equation:

$$
Q=14 d^{2} h^{1 / 2},
$$

where

$$
\begin{aligned}
& Q=\text { airflow, cfm; } \\
& d=\text { orifice diameter, in.; } \\
& h=\text { pressure drop across orifice, in.wg. }
\end{aligned}
$$

Assumptions inherent in the constant 14 used in Eq. (7.7) are (1) air at standard temperature and

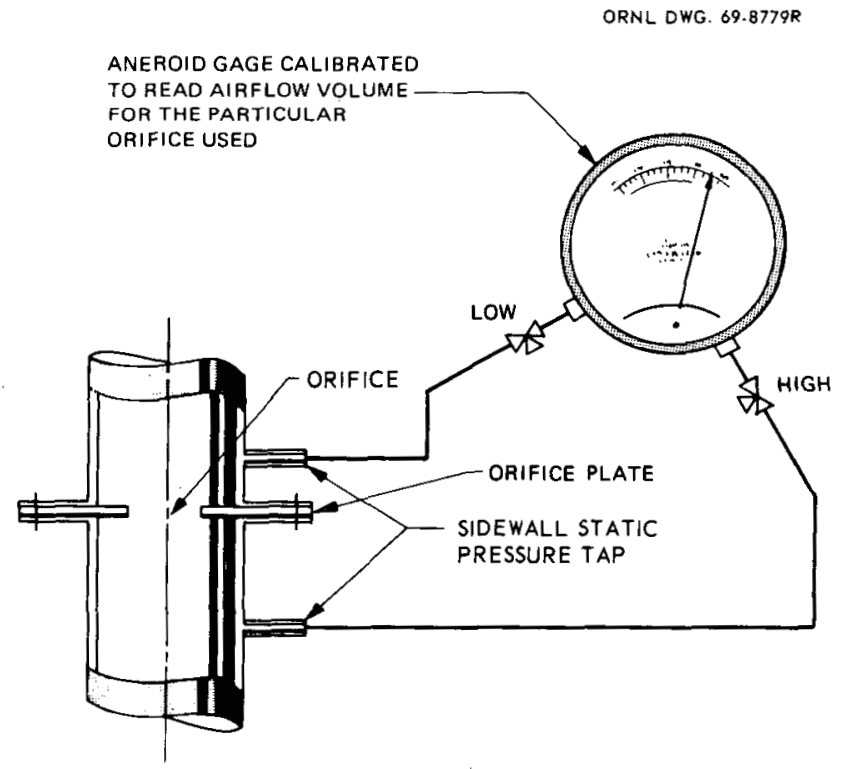

Fig. 7.26. Orifice meter method of measuring volume flow rate in small ducts. 
ORNL DWG 69-8780R

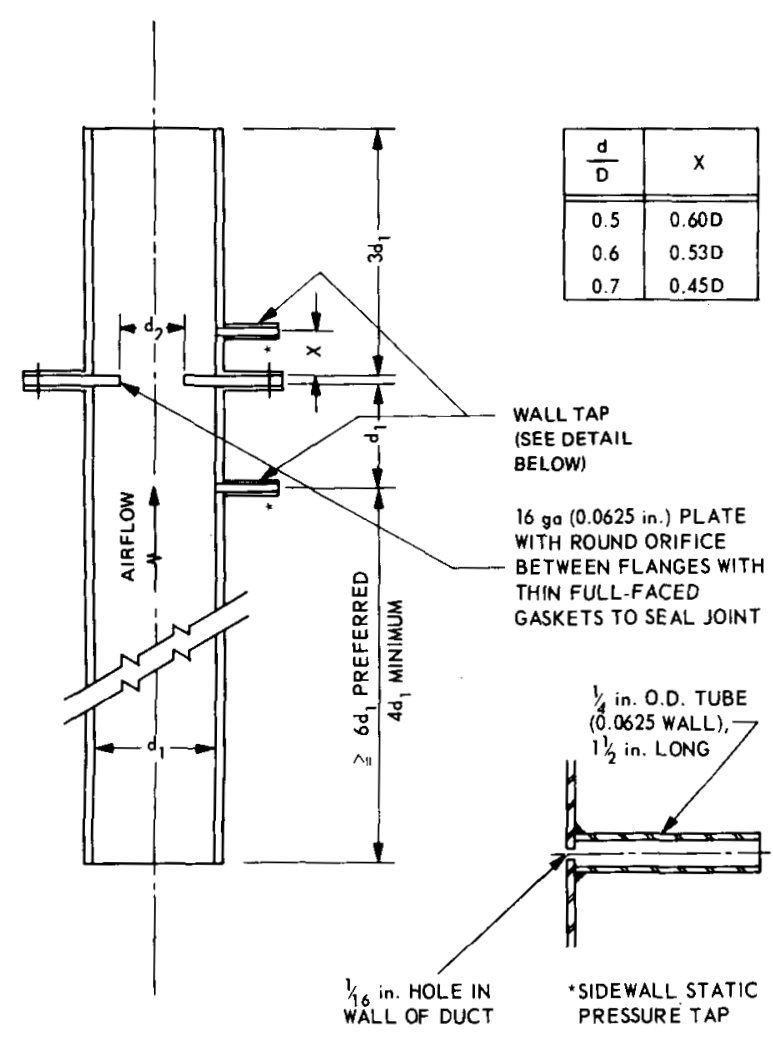

Fig. 7.27. Arrangement of thin, sharp-edge concentric orifice in small duct.

pressure, (2) flow coefficient for orifice $=0.65$, and (3) ratio of orifice diameter to smooth-duct diameter, $D$, $=0.2 \leqslant d / D \leqslant 0.7$. The practical use of this formula can be shown by the following example.

Determine the orifice size necessary for a $20-\mathrm{cfm}$ airflow rate that would give a reading near the center of scale on a 0 - to 0.50 -in.-range gage.

$$
\begin{aligned}
& Q=20 \mathrm{cfm} \\
& h=\frac{0.50}{2}=0.25 \mathrm{in} \cdot \mathrm{wg} \\
& d=\frac{Q}{14 h^{1 / 2}}=\frac{20}{14(0.25)^{1 / 2}} \\
& d=1.79 \mathrm{in} .
\end{aligned}
$$

For 3-in. sched 10 stainless steel pipe $(I D=3.260 \mathrm{in}$.), the $d / D$ ratio is $1.79 / 3.26=0.55$, which is within the acceptable range.

A shortcoming of the thin-plate orifice is the loss of head of the air flowing through the device. The following tabulation gives the loss of head of concentric orifices for various $d / D$ ratios:

$\begin{array}{cc}\begin{array}{c}d / D \\ \text { ratio }\end{array} & \begin{array}{c}\text { Fraction of velocity } \\ \text { head not regained }\end{array} \\ 0.2 & 0.95 \\ 0.3 & 0.89 \\ 0.4 & 0.83 \\ 0.5 & 0.74 \\ 0.7 & 0.53\end{array}$

In the example above, $0.70 \times 0.20=0.14$ in.wg. is the pressure loss when $20 \mathrm{cfm}$ flows through the orifice of $d / D=0.55$.

Immediately after installation, while filters are still clean, the measured pressure drop across the HEPA filter can be used to check airflow to a good degree of accuracy by proportioning the measured pressure drop to that stamped on the filter case at the time of predelivery testing. The pressure drop across the filter is no longer a dependable indication of gas flow rate after the filter has accumulated dust. After a filter has been in service for a period of time, it is necessary to measure both the pressure drop across the filter and the airflow through it to evaluate the filter's status and relationship to the whole ventilation system.

Written procedures for periodically testing each alarm, control, and emergency system serving the glove box and its ventilation system are essential.

\subsubsection{DOP Testing of Glove Box Filters}

HEPA filters must be tested immediately after installation and then periodically to assure that air cleanup capability and containment integrity remain intact. The principles of DOP testing of HEPA filters are given in Chap. 8. The HEPA filters used in glove box systems are often inconvenient to test because DOP must be injected into the inlet duct or glove box. Figure 7.28 shows the usual methods for injecting DOP and extracting samples for glove boxes. DOP cannot be fed into the inlet of the box to test the exhaust-side filters if high-efficiency filters are used in the inlet. Methods A and B (Fig. 7.28) require that DOP be drawn into the glove box by the suction of the exhaust system. However, when glove boxes house apparatus with open or exposed optical lenses and with highly polished surfaces, delicate balances, crystalline structures, sensitive conductors, or similar equipment or products, DOP should not be injected into the box. In such cases, the filter should be installed in the duct downstream of the glove box so that the injected DOP aerosol will not back up into 


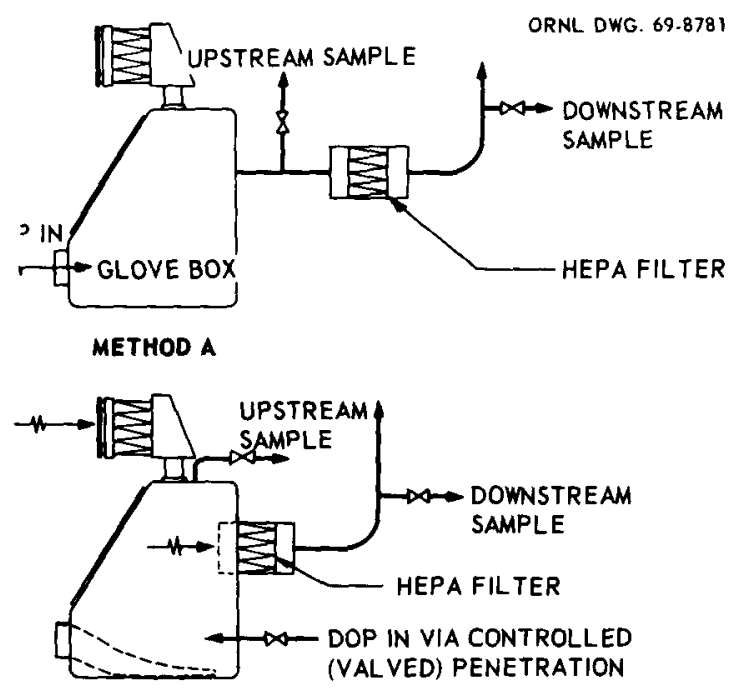

METHOD B

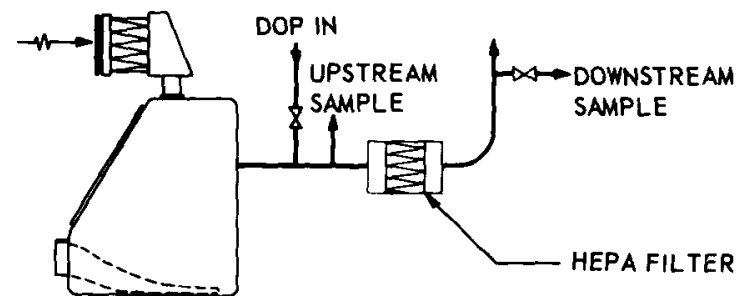

METHOD C

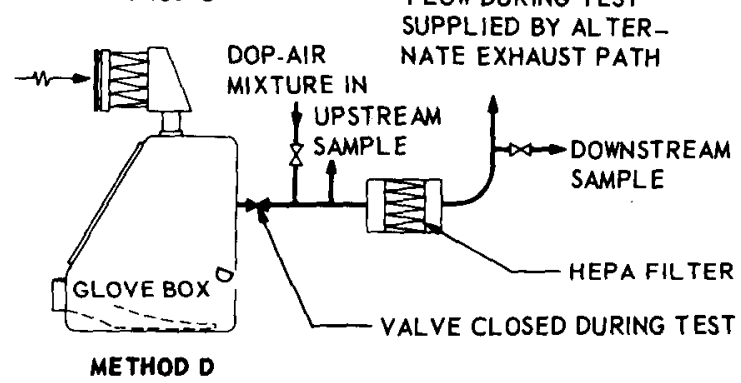

Fig. 7.28. Four methods of DOP testing HEPA filters in glovebox exhaust streams.

the glove box proper. Method C (Fig. 7.28) may then be used for DOP testing the exhaust HEPA filter.

Where new or replacement exhaust filters are required to be tested before restarting the ventilation system, method D may be used. Note in this method that the exhaust path from the glove box is closed and that the DOP-air mixture for filter testing is drawn from a separate valved path. The side path is closed and sealed after testing is completed.

Methods A and B (Fig. 7.28) require DOP-air mixtures to be injected into the glove box via some convenient opening. A glove port can be used if

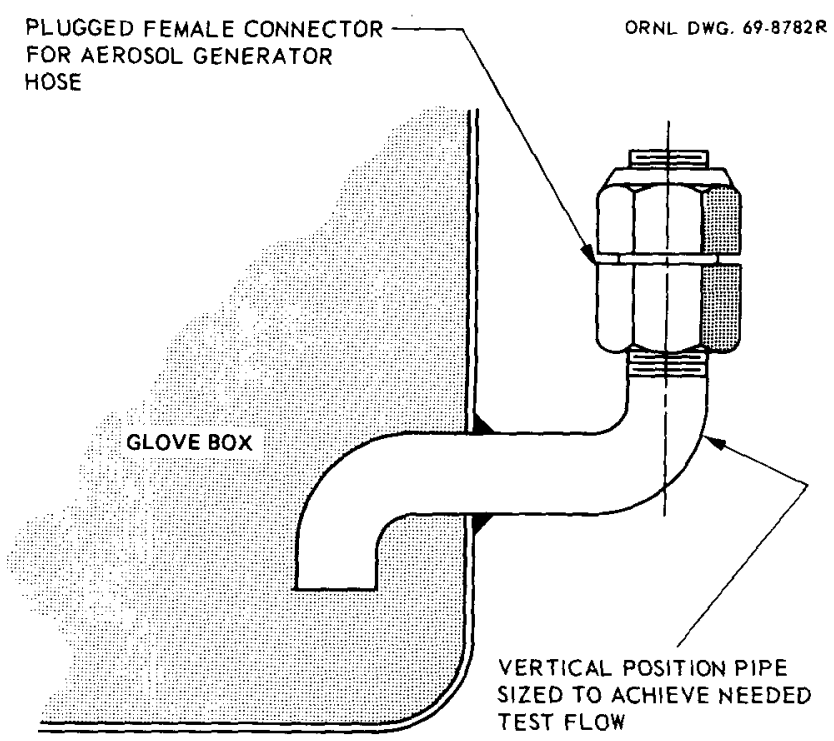

Fig. 7.29. Connection for introducing DOP into glove box.

containment is not critical during testing. Otherwise, a connection can be prepared (Fig. 7.29) or an alternate method can be devised. Methods C and D (Fig. 7.28) do not require the introduction of DOP into the glove box. The DOP inlet connection must be sized to pass the DOP or DOP-air mixture. The connection for concentrated DOP in method $\mathrm{C}$ must admit 2 to $5 \mathrm{cfm}$, while the connection in method D must accommodate the total DOP-air mixture used for the test.

\subsubsection{Glove.Box Shielding}

Some glove boxes may require gamma or neutron shielding because of the nuclide used and the amount of material involved. Boxes handling kilogram quantities of plutonium can be shielded by providing leadimpregnated gloves and by installing lead sheets over the metal portion, lead glass over the windows, and lead-hinged covers over the ports. ${ }^{27}$ The operating, shielding, removal, and replacement requirements of the glove box HEPA filter must also be considered when glove box shielding is required.

\section{REFERENCES FOR CHAP. 7}

1. N. B. Garden, ed.,"Report on Glove Boxes and Containment Enclosures," Health and Safety, USAEC Report TID-16020, June $20,1962$.

2. P. A. F. White and S. E. Smith, Inert Atmospheres, Butterworth and Co., Washington, D.C., 1962.

3. ASHRAE Handbook and Product Guide-Systems, American Society of Heating, Refrigerating and AirConditioning Engineers, New York, 1973. 
4. Fire Protection Guide on Hazardous Materials, National Fire Protection Association, Boston, current issue.

5. J. Young and W. D. Phillips, Explosion Tests in a Gloved Box with Ether Air Mixtures, USAEC Report UCRL-16259, Berkeley, Calif., 1965.

6. N. I. Sax, Dangerous Properties of Industrial Materials, 4th ed., Van Nostrand Reinhold, New York, 1975.

7. C. J. Barton, Review of Glove Box Construction and Experimentation, ORNL-3070, Oak Ridge National Laboratory, May 31, 1961.

8. G. N. Walton et al., Glove Boxes and Shielded Cells, Butterworth and Co., London, 1958.

9. Glovebox Fire Safety, A Guide for Safe Practices in Design, Protection, and Operation, USAEC Report TID-24236, Factory Mutual Research Corp., Boston, 1967.

10. An Economical Vent Cover, NASA Tech Brief B72-11348, Marshall Space Flight Center, Huntsville, Ala., July 1972.

I1. C. Yao, J. deRis, S. N. Bajpai, and J. L. Buckley, Evaluation of Protection from Explosive Overpressure in AEC Glove Boxes, FMLC Bulletin 16215.1, RC69-T-23, Factory Mutual Research Corp., Boston, 1969.

12. NFPA 68, Explosion Venting, National Fire Protection Association, Boston, 1974.

13. W. E. Woodson and D. W. Conover, Human Engineering Guide for Equipment Designers, University of California Press, Berkeley, 1966.

14. G. H. Llewellyn, Computer Programs for the Determination of Capital Costs for HTGR-RFDP Process Evaluation Project, ORNL/TM-4613, Oak Ridge National Laboratory, March 1975.

15. ASHRAE 52-68, Method of Testing Air Cleaning Devices Used in General Ventilation for Removing Particulate Matter, American Society of Heating, Refrigerating and Air-Conditioning Engineers, New York, current issue.
16. Regulatory Guide 3.12, General Design Guide for Ventilation Systems of Plutonium Processing and Fuel Fabrication Plants, U.S. Atomic Energy Commission, Washington, D.C., August 1973.

17. ERDA Manual, "General Design Criteria, Plutonium Facilities," Appendix 6301, Part II, Sect. I, Sept. 17, 1974, p. 84.

18. A. J. Hill, Jr., Fire Prevention and Protection in Hot Cells and Canyons, DP-1242, Savannah River Laboratory, April 1971.

19. Tradename of E. I. du Pont de Nemours and Co., Inc.; Halon-1301 is bromotrifluoromethane, $\mathrm{CBrF}_{3}$.

20. A. J. Hill, Jr., Automatic Fire Extinguishing Systems for Glove Boxes and Shielded Cells at the Savannah River Laboratory, DP-1261, Savannah River Laboratory, June 1971.

21. R. E. Felt, Burning and Extinguishing Characteristics of Plutonium Metal Fires, 1S0-756, Richland, Wash., August 1967.

22. NFPA IIA Standard for High Expansion Foam Systems (Expansion Ratios 100:1 to 1000:1), National Fire Protection Association, Boston, 1970.

23. NFPA IIB Standard on Synthetic Foam and Combined Systems, National Fire Protection Association, Boston, 1974.

24. R. D. Edwards, P. E. Johnson, and C. Yao, Control and Reduction of Oil Mists from Mechanical Vacuum Pumps, USAEC Report TID-25085, prepared by Factory Mutual Research Corp., Boston, 1969.

25. Industrial Ventilation, A Manual of Recommended Practice, American Conference of Governmental Industrial Hygienists, Ann Arbor, Mich., current issue.

26. L. K. Spink, Principles and Practices of Flow Meter Engineering, 9th ed., Foxboro Co., Foxboro, Mass., 1972.

27. J. B. Owen, Control of Personnel Exposures to External Radiations in a Plutonium Chemical Plant, RFP-1254, Rocky Flats Plant, 1968. 


\section{Testing}

\subsection{INTRODUCTION}

Stringent testing is necessary to achieve and maintain the high performance levels required of a high-efficiency nuclear air cleaning system. By definition, such systems must exhibit an installed decontamination factor (DF) of at least 2000, that is, an efficiency of $99.95 \%$, for aerosols having a number median diameter (NMD) less than $1 \mu \mathrm{m}$. Decontamination factors near 2000 may be required for gaseous radioiodine compounds. Processes that involve the handling of plutonium and other transuranic elements may require total system DFs of $10^{6}$ or higher. These factors are achieved by providing two or more HEPA filters in series and compare with DFs of only 4 to 6.5 for particles in this size range attainable in most industrial and conventional ventilation air cleaning systems.'

System tests fall in two broad categories: (1) prestartup acceptance tests which verify that components have been installed properly and without damage, and demonstrate that the system can operate as intended and (2) surveillance tests made periodically after the system has been placed in operation to demonstrate its continuing ability to perform its intended air cleaning function. Surveillance tests are leak tests of the HEPA filter and adsorber installations. Details of major acceptance and surveillance tests, in sufficient detail to provide guidance for the preparation of test procedures, are given in ANSI N510. ${ }^{2}$ In all cases, tests should be preceded by careful visual inspection, as discussed in Sect. 8.4

\subsection{ACCEPTANCE TESTS}

Acceptance tests fall into two broad categories: (1) those that relate to the permanent elements of the system, the ducts, housing, mounting frames, and location of test ports, and (2) those that verify the installation and condition of the primary air cleaning
components-HEPA filters and adsorbers. Acceptance tests of HEPA filter and adsorber installations are identical to the surveillance tests of those elements and are covered in Sect. 8.3. Tests in the first category include leak tests of ducts, housings, and primarycomponent mounting frames; airflow capacity and distribution tests; gas residence time tests for systems containing adsorbers; duct-heater tests for systems containing heaters; and air-test agent mixinguniformity tests. The acceptance test program for a particular system may contain any or all of these tests, depending on the nature of the system and its importance, that is, the potential consequence of a failure of, leakage from, or release from the system.

Nuclear Regulatory Commission guides recommend the full battery of acceptance tests for ESF systems. ${ }^{3}$ Lesser systems may not warrant such stringent testing. On the other hand, these tests, which are made only once at the time of acceptance of a new or rebuilt system, provide an assurance of system reliability that cannot be obtained in any other way, and the American National Standards Committee N45-8 (responsible for ANSI N510) recommends that they be considered for any highreliability system.

\subsubsection{Duct and Housing Leak Tests}

The level of duct and housing leaktightness, and therefore the acceptance criterion for the test, is based on the type of construction and the potential hazard (consequence) of a leak. Recommended maximum permissible leak rates for various duct and housing constructions are given in Tables 4.5 (for ducts) and 5.6 (for housings). The designer may specify tighter requirements, based on the containment requirements of the system, but is cautioned against specifying a degree of leaktightness that cannot be met on a practical basis.

Duct leak tests may be made by testing the entire ductwork system at one time or by testing one section 
at a time, blanking off the ends of the section under test. The second method is more practical for large systems. When segmented, the permissible leak rate for the individual sections is based on the proportionate volume of that section. The apparatus and procedure for leak testing level 1 and 2 ducts are described in the SMACNA High Velocity Duct Construction Standards. ${ }^{4}$ Test methods for level 3,4, and 5 ducts and for housings are described in Sect. 6 of ANSI N510. If the specified leaktightness cannot be met, leaks are located and repaired by one of the methods described in Sect. 6 of ANSI N510 before making the final leak rate test for record. Halogen and mass spectrometer leak-detection methods are generally too sensitive for this application; furthermore, the halogen method introduces a contaminant that may interfere with subsequent in-place testing of adsorbers.

\subsubsection{Mounting Frame Leak Tests}

These tests are made to verify that there are no leaks through HEPA filter and adsorber mounting frames or through the seal between the mounting frames and the housing. The tests also verify that there is no bypassing of the mounting frames through electrical conduits, drains, compressed air connections, common anterooms of the housing, or other inadvertent leak paths. Familiar sources of leaks are weld cracks and incomplete welds. A properly designed mounting frame should have no penetrations (by conduits, piping, or ducts), and lighting, drain, and other ancillary systems should be designed so that no bypassing of the HEPA filters and adsorbers can occur. Nevertheless, unauthorized modifications are often made in the field. The purpose of this test is to disclose such items as well as leaks caused by poor workmanship or shipping damage. The test is recommended for any installation, whether duct and housing leak tests are made or not, but it is particularly necessary when subsequent in-place tests of the HEPA filter and adsorber stages are to be made by a shrouded method (Sect. 8.3.1).

The test is first made by blanking off all openings for filters and adsorbers, closing or blanking off all openings in the housing, and conducting a soapbubble or spray-DOP leak test around all welds and other potential leak paths, as described in Sect. 7 of ANSI N510. After all leaks have been repaired, individual chambers of the housing should be checked by a pressure leak rate test to verify that there are no bypasses that have not been disclosed by the leak detection check. It is not necessary that these tests be made from the upstream side of the mounting frame, and it is quite acceptable to test two mounting frames simultaneously by blanking off the openings of both and pressurizing the space between. Because the mounting-frame-pressure leak test is a chamber-bychamber test of the housing, it can replace the need for a housing leak test (Sect. 8.2.1).

\subsubsection{Airflow Capacity Test}

The purpose of this test is to verify that the system of fans, ducts, housings, filters, dampers, and other specified components, as installed, will produce specified airflows under the clean-filter and dustloaded-filter conditions that the system will encounter in service. Filter resistance should, in nearly all cases, be the only uncontrollable variable in the system, and the system must be capable of producing the design airflow during all stages of normal filter life. Filter resistance will be encountered in pad-type demisters, in prefilters, and in the HEPA filters. There should be no pressure buildup due to dust in adsorbers properly protected by HEPA filters. The allowable resistance increase for demisters and prefilters should be obtained from the manufacturer. The maximum clean-filter pressure drop across a new HEPA filter, at rated airflow, is 1 in.wg or less. Artificial resistance can be added for the second part of the test by blanking off filters until the desired pressure drop is achieved.

Airflow capacity tests should be made in accordance with Sect. 8 of ANSI N510 and Chap. 9 of Industrial Ventilation. ${ }^{5}$ Measurements are generally made at a single point in the duct upstream of the filter housing. When flow velocities in various parts of the system are important, additional tests at critical points in the system may be desirable. Capture velocities at hood and duct openings and transport velocities in ducts will vary according to the nature of the material to be conveyed. These velocities are discussed in Sects. 4 and 5, respectively, of Industrial Ventilation. ${ }^{5}$

\subsubsection{Airflow Distribution Test- Adsorber Residence Time}

Maldistribution and stratification of airflow in the housing of a high-efficiency air cleaning system can cause various problems. In demisters, which depend on high velocities and impaction for effective operation, excessive penetration of small droplets may occur in low-velocity areas of the demister bank, whereas reentrainment may occur in areas subject to 
higher-than-design velocity. In prefilter and HEPA filter banks, variable distribution of airflow across the bank may result in underutilization of some of the filters and perhaps variable performance. A jet effect has been observed opposite the inlets to some large filter banks, which causes the filters directly in the path of the jet from the housing inlet to become more heavily dust loaded than those not in the path. In some cases, filter damage has been observed in the impacted filters. Variable airflow distribution across a bank of adsorber cells results in high penetration of cells in high-velocity areas, because effective operation for organic radioiodide adsorption is directly related to gas residence time. CS- 8 type II (tray) ${ }^{6}$ adsorber cells are designed to produce an average residence time of $0.25 \mathrm{sec}$ when filled with 8 - by 16 mesh activated carbon and operated at a volumetric airflow of $333 \mathrm{cfm}$. The minimum residence time for trapping organic radioiodides is considered to be 0.20 sec. Conformance to the requirements of Sect. 8 of ANSI N510, which permits a variation of $+20 \%$ from the design airflow at any point of internal components, will ensure that the required residence time can be achieved in any adsorber cell in the bank.

\subsubsection{Air-Test Agent Mixing Tests-Testability}

No safety credit can be claimed for HEPA filters or adsorbers that are not tested regularly to verify that they continue to meet performance requirements. Although individual filter units and adsorber cells are tested by the manufacturer, in-place testing after installation is essential because of the damage and deterioration that can take place during shipping, handling, installation, and service. Therefore, an important phase of acceptance testing is to verify that HEPA filter and adsorber installations can be tested satisfactorily. The design of many older systems permitted an acceptance test of the HEPA filters, but these designs were such that tests after the system went into operation were nearly impossible, particularly if the system had become contaminated. Such designs are not acceptable in high-reliability applications.

In-place tests are made by introducing a test agent, DOP for HEPA filters and fluorocarbon refrigerant gas for adsorbers, upstream of the bank to be tested. The concentrations of test agent upstream and downstream (upstream concentration is considered $100 \%$ ) are then determined, and penetration is calculated from the ratio of concentrations. Reliability of the test is determined by (1) the ability to properly introduce the test agent and to obtain representative samples and (2) physical access to the banks being tested. The first can be verified by an air-aerosol mixing test. This test is made once, at the time of acceptance testing, and its satisfactory completion is a prerequisite to both acceptance and future surveillance in-place testing of HEPA filters and adsorbers.

Good testability requires the provision of permanent test agent injection and sample ports, or other planned and preestablished means for injecting the test agent and for taking reliable, well-mixed samples. Details of the air-aerosol mixing test are described in Sect. 9 of ANSI N510. ${ }^{2}$ It is essential that the air and test agent mixture charged to the filters (adsorbers) is thoroughly mixed so that concentrations entering all points of the filters, including the upstream and downstream sample points, are essentially uniform. Adequate mixing upstream usually can be obtained by introducing the test agent at least 10 duct diameters upstream of the filters or adsorbers, or by introducing it upstream of baffles or turning vanes in the duct. When neither of these methods is practicable, a Stairmand disk ${ }^{7}$ located 4 to 6 duct diameters upstream will give satisfactory mixing. When duct arrangement makes it necessary to introduce the test agent directly into the filter housing, a design such as that discussed under multistage housings (Sect. 8.3.3) may be required. Extraction of the downstream sample at a point several duct diameters downstream of the fan will usually provide a well-mixed sample. Fan-shaft leakage is a consideration in sampling downstream of the fan. Since leakage at the shaft will be inleakage, sufficient air to excessively dilute the downstream sample can be drawn in if the shaft annulus is large (thus giving a low downstream concentration reading), or dust may be drawn into the fan which will provide a high downstream reading (which may be particularly prevalent during construction). A shaft seal, or at least a temporary seal to be applied during testing; is recommended.

The second aspect of testability, access, requires space for personnel and equipment; space to manipulate equipment without damaging filters or creating hazards for personnel; passages for getting personnel and equipment to the point they are needed; provision for getting services (electrical, compressed air) to the equipment; access to both faces of filters and adsorbers; adequate lighting; viewports; and other features that facilitate safe 
testing. These testability requirements are all matters of design that should be checked before the start of construction, and they should be confirmed as part of the acceptance check. The space, access provisions, service galleries, and filter-array recommendations of Chap. 4 are essential for effective in-place testing in man-entry housings. Lights should be provided between each chamber of the housing (space between banks or between bank and end wall) and should be sufficient to produce a light intensity of at least $5 \mathrm{ft}-\mathrm{c}$, and preferably twice that value, on the faces of the filters and adsorbers. Reliance on flashlights and portable lamps is a hazard that should be avoided in man-entry housings; either can be dropped, leaving the workman in the dark and under adverse conditions. A portable light usually gives too little light to be useful, and if dropped from a service gallery, is a danger to workmen below. Also, a drop cord is an additional hindrance in what is often an already toocrowded working space.
Switches for permanent lights should be installed on the outside of the housing, adjacent to the door of the illuminated chamber. Thus when viewports are provided the filters can be checked without entering the contaminated housing. Figure 8.1 illustrates the advantage of viewports when sufficient internal lighting is provided, whereas Figs. 8.2 and 8.3 illustrate the problems encountered by service personnel in poorly designed, poorly laid out, and poorly lighted housings. In Fig. 8.2, there is no possible access to the upper two tiers of filters without bringing ladders or scaffolding into the housing, a hazard to installed filters and to personnel. ${ }^{8}$ The layout in Fig. 8.3 is not only hazardous to personnel from the standpoint of very limited working space but also because of the thoughtless placement of the piping element just above the service gallery floor. Effective testability also demands a limit on bank size from the standpoints of generating sufficient test agent and the time required to test a single bank of

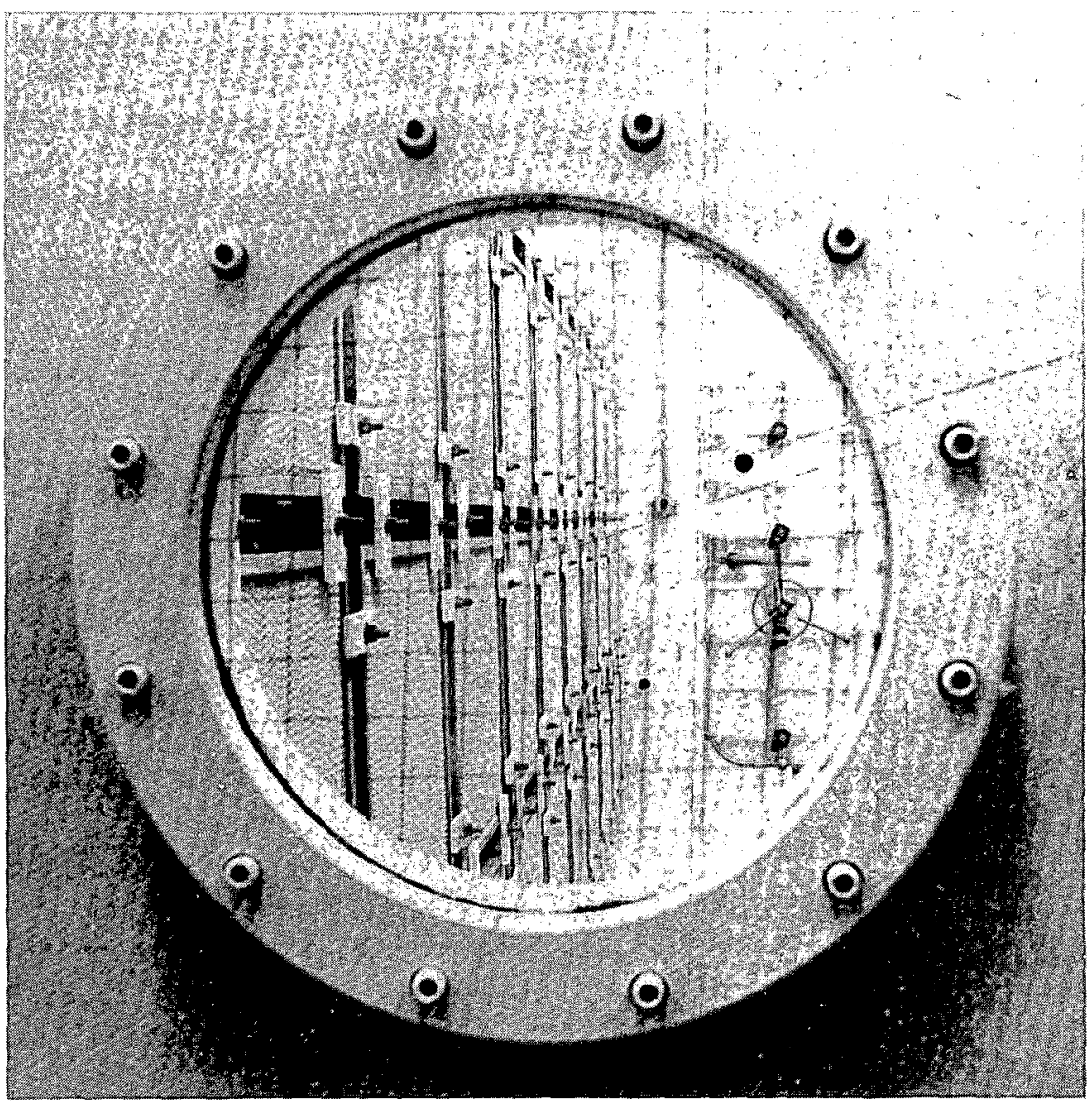

Fig. 8.1. Housing viewport. Note excellent lighting within the housing. Courtesy Rockwell International, Rocky Flats Plant. 


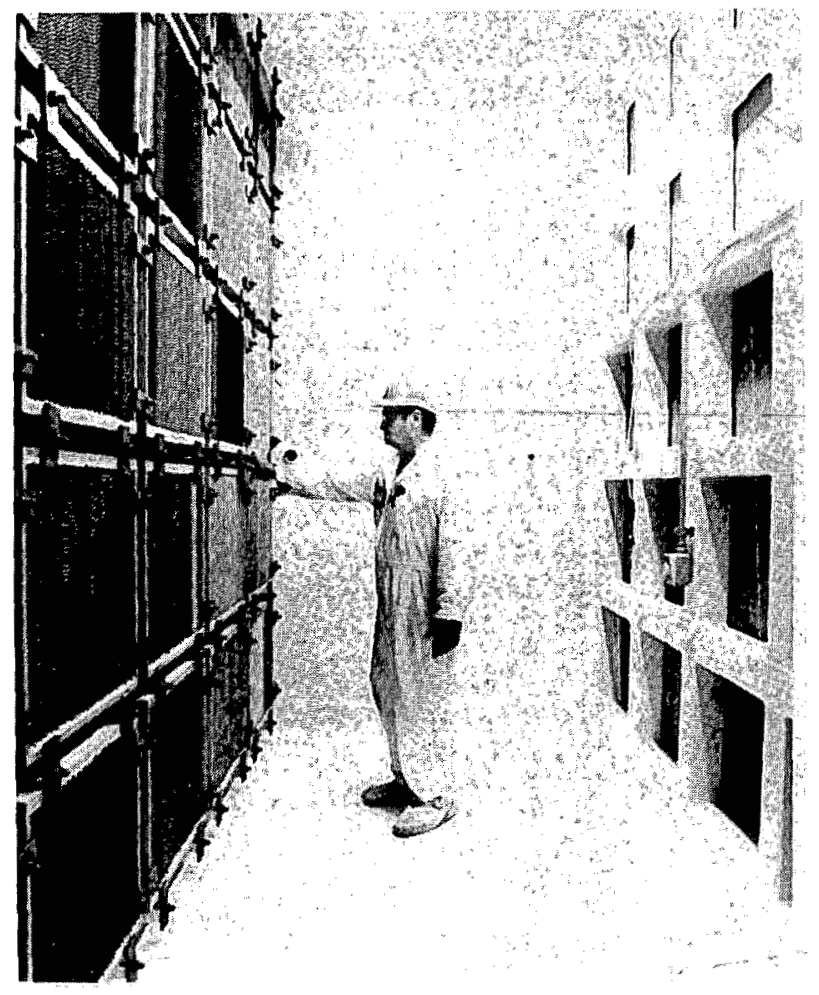

Fig. 8.2. Excessively high filter bank. Note that temporary ladders or scaffolding would be necessary to gain access to the two upper tiers of filters.

filters or adsorbers. A limit of $30,000 \mathrm{cfm}$ installed capacity is recommended. ${ }^{3,8}$ Electrical and compressed-air connections should be provided close to but not inside the housing, and provision should be made for bringing cables and air lines (when required for testing) into the housing without going through the door. A cable chase can be made by drilling a hole through the housing wall and welding a 2-to 3 -in. half nipple on the outside; the nipple is capped (using fluorocarbon tape to prevent galling) when not in use (such passthroughs should be included in the original design). Chapter 4 recommends door latches operable from both sides in man-entry housings; these latches are essential to prevent personnel from being trapped inside. If a fan is located inside the housing, guards must be provided on its inlet, belts, and shaft extensions. Finally, testability demands preplanned, written test procedures that have been carefully reviewed and checked as part of the acceptance program.

\subsection{SURVEILLANCE TESTING}

Surveillance tests are of three types: in-place leak tests of HEPA filter stages using DOP, in-place leak tests of adsorber stages using a slightly adsorbable gas such as fluorocarbon refrigerant-11 (R-11), and laboratory tests of samples of adsorbent withdrawn from the system to establish its remaining adsorption capacity. These tests are also employed as part of the acceptance procedure for new installations, except that laboratory tests are made on samples of adsorbent taken from batch material as furnished.

Surveillance tests of HEPA filter and adsorber systems should be made at regular intervals after installation to detect deterioration and leaks that may develop under service conditions. Regular in-place

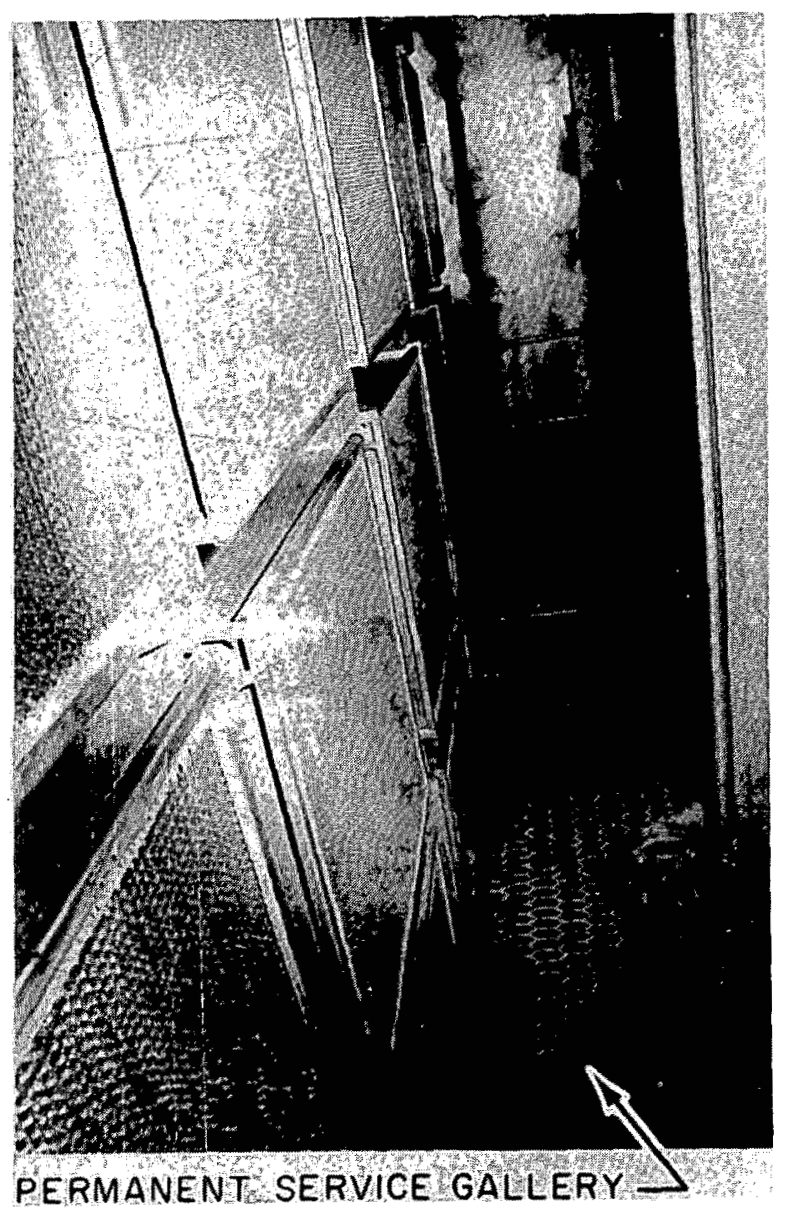

Fig. 8.3. Inadequate space between banks of components. Note stumbling hazard on floor of service gallery. 
testing of standby systems is necessary because deterioration can take place even when systems are not being operated. Aside from component damage, frequently discovered causes of failure to meet inplace test requirements include loose clamping bolts, inadequate clamping devices, foreign material trapped between gaskets and mounting frame, rough or warped mounting-frame surfaces, cracked welds, unwelded joints in mounting frames, incorrectly installed components (e.g., HEPA filters installed with horizontal pleats), inadequate seals between mounting frame and housing, poorly designed mounting frames, and bypassing through or around conduits, ducts, or pipes that penetrate or bypass the mounting frames. Tests of 50 HEPA filter banks at one ERDA installation, prior to initiating a routine testing program, revealed 31 banks $(62 \%)$ that could not meet the maximum system penetration of $0.05 \%{ }^{9}$ After repair or rebuilding of the unsatisfactory banks, they have routinely exhibited penetrations within specified values; of some 670 HEPA filter installations at Oak Ridge National Laboratory, most routinely exhibit penetrations of less than $0.008 \% .^{10}$

\subsubsection{In-Place Testing for HEPA Filters}

In-place tests of HEPA filter installations are made with an aerosol of polydispersed DOP consisting of droplets having a light-scattering NMD of $0.7 \mu \mathrm{m}$ and a size range of approximately 0.1 to $3.0 \mu \mathrm{m} .{ }^{11}$ The DOP used for efficiency testing by manufacturers and ERDA Quality Assurance Stations is a monodispersed aerosol having an NMD of $0.3 \pm 0.03$ $\mu \mathrm{m}$. The in-place test is made by charging the upstream side of the filter or filter bank with DOP smoke, then measuring and comparing (using a lightscattering photometer) the DOP concentration in samples of filtered and unfiltered air (Fig. 8.4). If the

ORNL-DWG 69-8783R

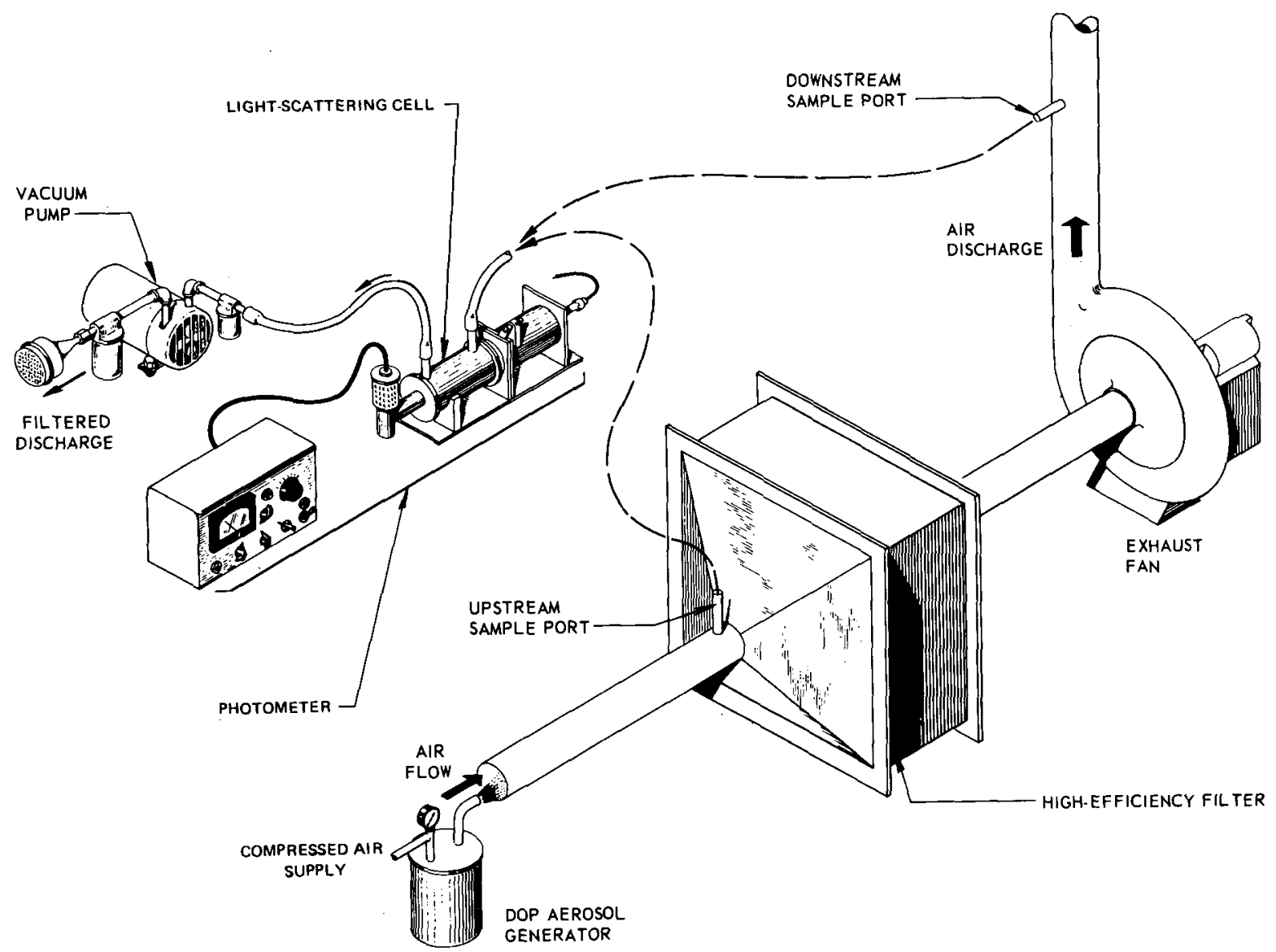

Fig. 8.4. Equipment arrangement, in-place test of HEPA filters. 
system exceeds the specified maximum permissible penetration value, the downstream faces of the filters and mounting frame can be scanned with the photometer probe to locate localized high DOP concentrations which indicate leaks.

Figure 8.4 shows basic equipment and a schematic of a typical test arrangement. The instrument shown is the Naval Research Laboratory (NRL) linear readout, forward-light-scattering photometer. An instrument having a threshold sensitivity of at least $10^{-3} \mu \mathrm{g} /$ liter for $0.2-$ to $1.0-\mu \mathrm{m}$ particles and a sampling rate of at least $1 \mathrm{cfm}$ is recommended. ${ }^{11}$ The instrument should be capable of measuring concentrations $10^{5}$ times the threshold value. Compact selfcontained instrument packages are commercially available (Fig. 8.5). Polydispersed DOP aerosol may be generated thermally ${ }^{12}$ or by compressed air. Compressed-air generators are widely used for testing small systems. They are commercially available or can be homemade in sizes from 1 to 24 nozzles. Figure 8.6 shows details of a six-element Laskin-nozzle generator installed in a paint bucket. A rule of thumb for determining generator capacity is to allow one Laskin nozzle per $500 \mathrm{cfm}$ of installed filter capacity. Compressed-air DOP generators are suitable for systems up to about $3000 \mathrm{cfm}$; above this size they become cumbersome. ${ }^{8}$ Although gasthermal generators are generally used for testing systems of $20,000 \mathrm{cfm}$ installed capacity and larger, they have too much output for small systems (Fig. 8.7). The engineer must not confuse this type of

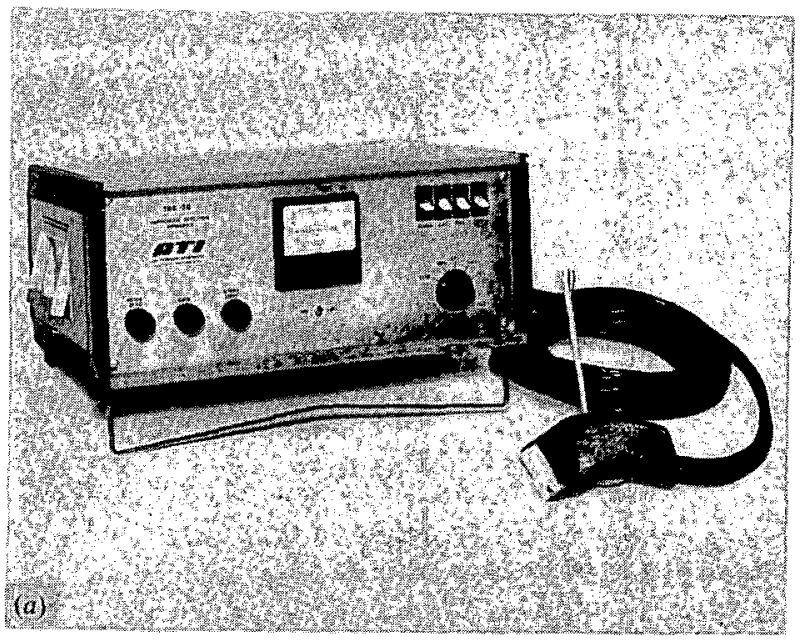

generator with the equipment used by manufacturers or ERDA Quality Assurance Stations for predelivery efficiency testing of HEPA filters (Fig. 8.8). The gasthermal generator produces a polydispersed aerosol of about the same NMD and size range as the compressed-air generator. It is small and can generally produce enough aerosol at a concentration of 80 to $100 \mu \mathrm{g} \mathrm{DOP} /$ liter to test banks up to $30,000 \mathrm{cfm}$ installed capacity. Gas-thermal generators capable of producing enough aerosol to test a system of 200,000 cfm installed capacity at a concentration of $40 \mu \mathrm{g}$ $\mathrm{DOP} /$ liter are said to be commercially available. ${ }^{13}$

A detailed description of the procedure for conducting an in-place test of HEPA filters is given in Sect. 10 of ANSI N510. ${ }^{2}$ Prerequisite to the test is the demonstrated ability to achieve good mixing of the DOP and air at the upstream and downstream sample points (Sect. 9, ANSI N510). For systems in which good mixing cannot be achieved, multipoint sampling and averaging, in accordance with Sect. 11 of ANSI N510, may be used. Figure 8.9 shows a wellplanned test-port installation. Figure 8.10 illustrates the type of improvisations the operator may have to resort to when the designer fails to take testing into consideration in the original layout of the system.

For installations designed in accordance with this handbook and employing HEPA filters that have been tested by one of the ERDA Quality Assurance Stations, an acceptance criterion of $0.03 \%$ maximum penetration is recommended for the in-place DOP test. This value is equivalent to the minimum

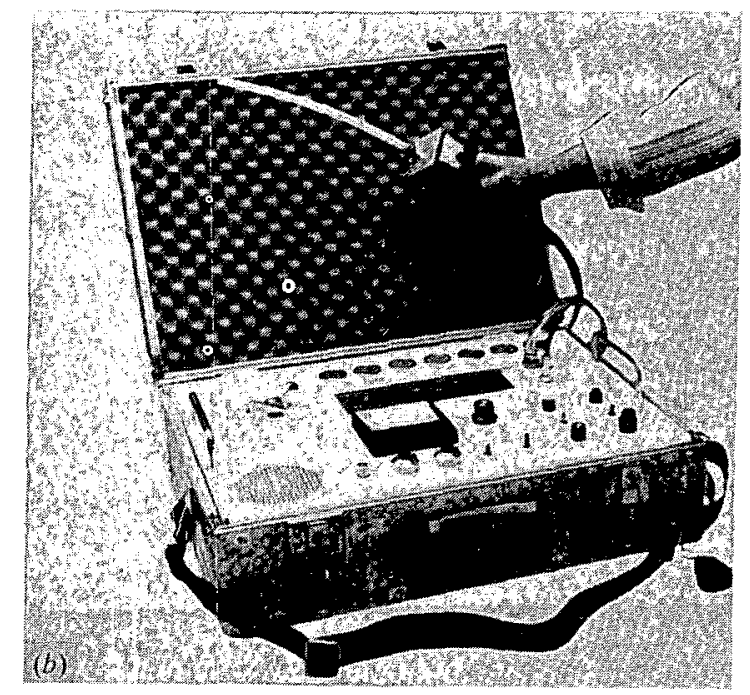

Fig. 8.5. Commercially available packaged forward-light-scattering instruments for HEPA filter testing. (a) Air Techniques, Inc., Model TDA-2D; $(b)$ Frontier Enterprises, Inc., Model FE973. 


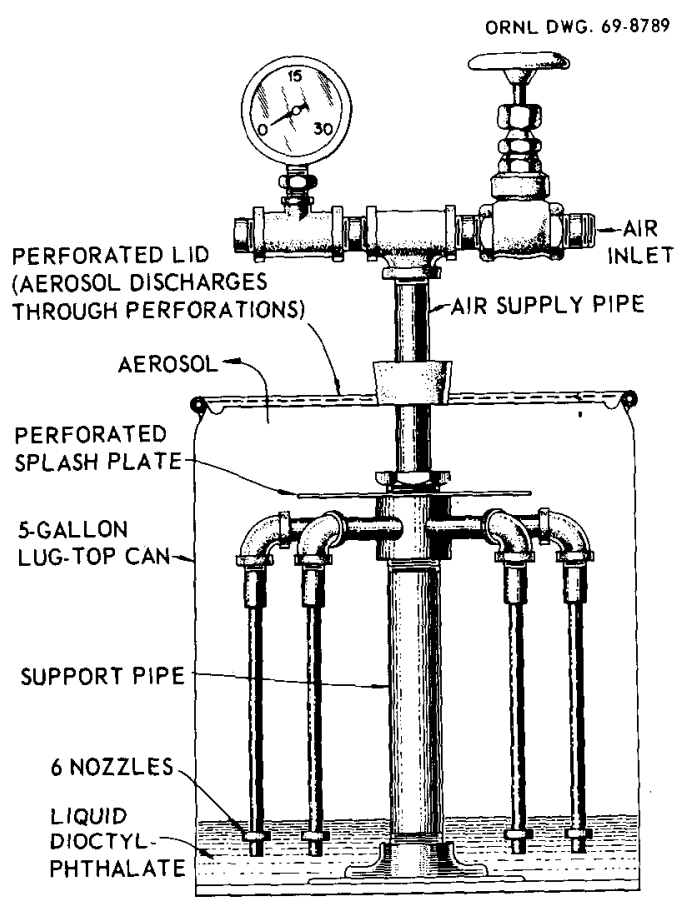

(a) 6-NOZZLE GENERATOR
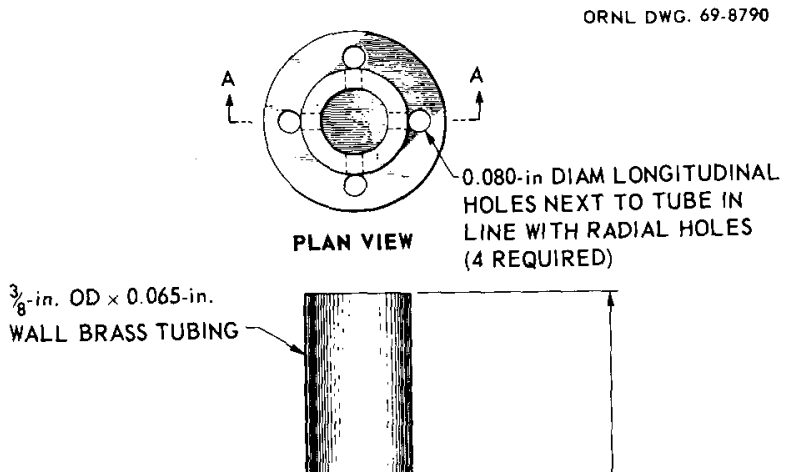

$5 / 8$-in. OD BRASS COLLAR

SILVER BRAZE TO

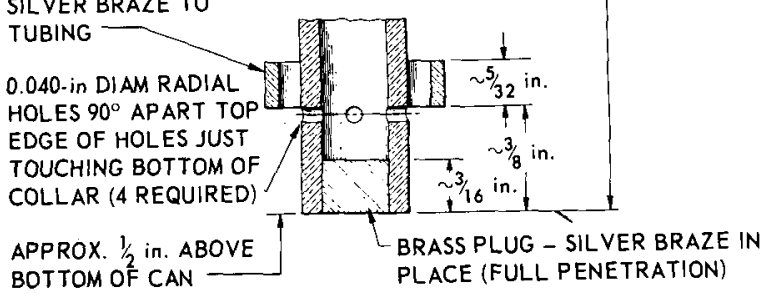

SECTION VIEW A.A

(b) DETAILS OF LASKIN NOZZLE

Fig. 8.6. Compressed-air-operated DOP aerosol generator.

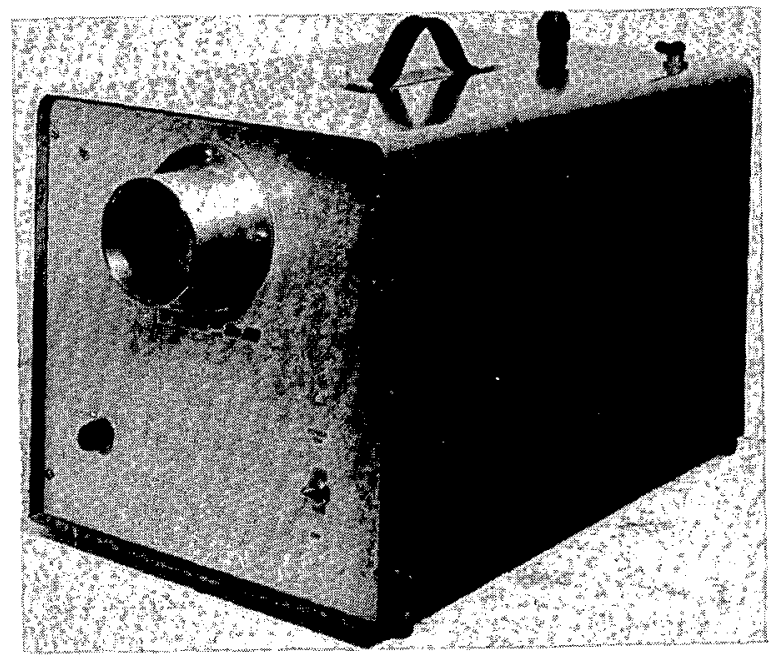

Fig. 8.7. Gas-thermal DOP generator for in-place testing of HEPA filter systems. Generator produces a polydispersed smoke of approximately 0.7 NMD.

efficiency of new filters. A lesser criterion $(0.05 \%)$ is allowed for nuclear power plants ${ }^{2}$ and most older installations. However, tests by an ERDA contractor show that the latter penetration can be met in a 20,000-cfm system despite a hole in one of the filters nearly 1 in. in diameter. ${ }^{14}$ Experience in repeated surveillance tests of nearly 1000 individual filter systems at another ERDA facility indicates that a properly designed HEPA filter system can routinely meet an acceptance criterion as low as $0.01 \%$ penetration. ${ }^{15}$ Most HEPA filters tested by the ERDA Quality Assurance Stations now exhibit penetrations in the $0.01 \%$ or lower range. ${ }^{16}$

The in-place test can be made at rated system airflow or at reduced flow. Because diffusion is the primary mechanism of small-particle collection, the test at reduced flow is often more sensitive than the full test. The actual rate of airflow for the reduced flow test is a function of the sensitivity of the photometer; some test agencies test at as low as 5 to $10 \%$ of rated system airflow. Reduced flow testing also has the advantage that less DOP is required.

\subsubsection{In-Place Testing for Adsorbers}

The effectiveness of an adsorber can be impaired by poisoning (i.e., adsorption of or chemical reaction with paint fumes, solvent vapors, hydrocarbons, and many chemical fumes); by wetting; by settling of the adsorbent in the bed due to vibration or air pulsation; and by design and installation defects. There are two basic methods of in-place testing adsorber systems. 


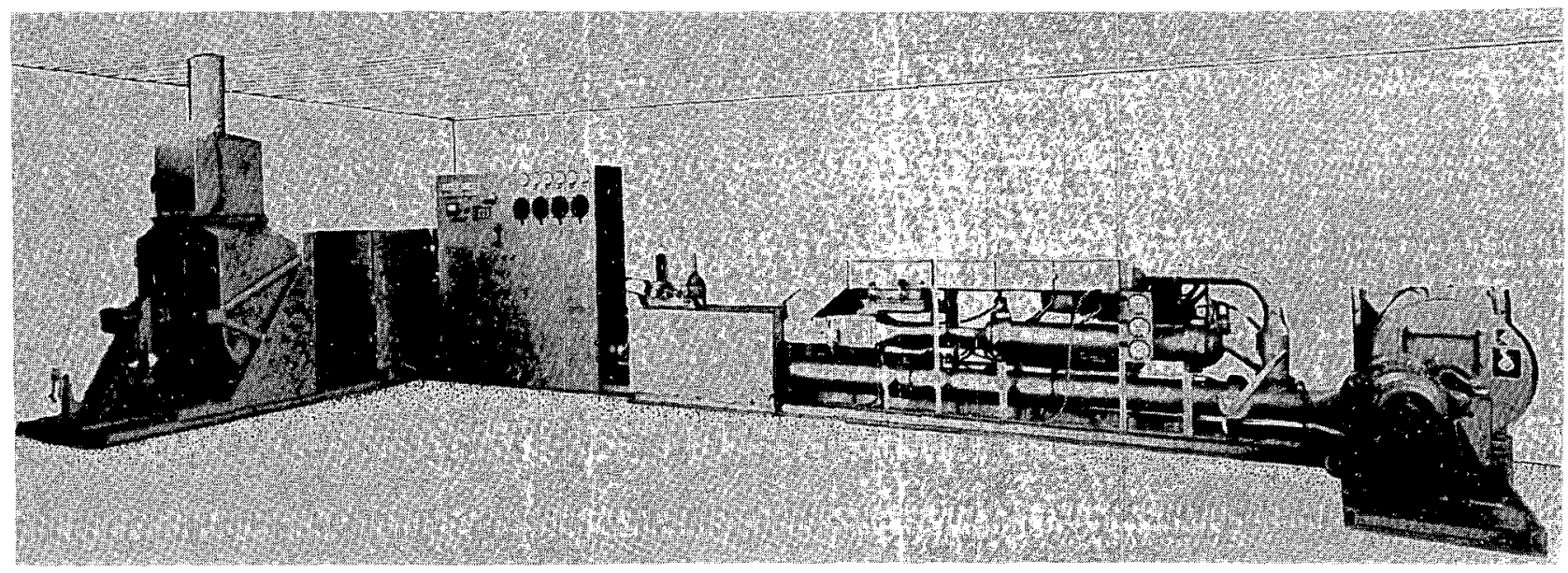

Fig. 8.8. U.S. Army Q-107 penetrometer for efficiency testing of HEPA filters. Equipment contains a thermal DOP generator capable of producing a monodispersed aerosol.

One method uses a fluorocarbon refrigerant gas and the other uses a radioactive tracer gas (iodine or methyl iodide). The first, developed by the Savannah River Laboratory, ${ }^{17}$ is the most commonly used, particularly in commercial applications. The second method involves the use of radioactive isotopes and personnel licensed to handle them. Radioiodine tracer methods were developed primarily for ERDA installations. ${ }^{18,19}$ Both in-place tests are leak tests rather than efficiency tests, and they must be supplemented with laboratory tests of samples taken from the adsorbers at the time of the in-place test to determine system efficiency and remaining capacity for iodine. Maximum penetration in the test is based on bed depth and the location of the adsorber. ${ }^{3}$ For adsorbers designed to operate inside the primary containment of a reactor, permissible penetration is $0.1 \%$, regardless of bed depth. For systems located outside such containment, the maximum permissible penetration is $0.1 \%$ for 2 -in.-thick beds and $0.05 \%$ for thicker beds.

Refrigerant Gas Test. The first test, commonly referred to as the Freon ${ }^{20}$ test, is made by charging the upstream side of the adsorber with a slightly adsorbable and readily desorbed fluorocarbon gas (usually $\mathrm{R}-11$, trichloro[mono] fluoromethane), and then determining the concentrations immediately upstream and at a point downstream where satisfactory mixing with the air occurs. Penetration is calculated from the ratio of downstream to upstream readings. Refrigerant-112 was originally used but is no longer made. The sampling system consists of a pump to draw upstream and downstream air samples from the adsorber system, two identical gas chromatographs with electron-capture detectors for measuring refrigerant gas concentrations, a timer, and several rotameters for determining sample dilution factors. The chromatographs should have a linear range of about 1 to $100 \mathrm{ppb}$ (by volume) for detection of the refrigerant gas. Since the upstream concentration exceeds the linear range of the instrument, the sample must be diluted with a known volume of air to bring it within the detection range of the chromatograph. Calibrated rotameters are used to determine the dilution factors. Some organizations combine equipment for adsorber testing with DOP equipment used for testing filters. The equipment used for adsorber and DOP tests is shown in Fig. 8.11. Figure 8.12 shows a schematic of the test setup. Prefilters and HEPA filters in the duct have no effect on the Freon test. The test is relatively easy to conduct by persons experienced in the use of the gas chromatograph, but it must be conducted in accordance with prescribed procedures (Sect. 12, ANSI N510). ${ }^{2}$ Precise adjustment of the airflow rate, the $\mathrm{R}-11$ injection rate, and the chromatographs is not required, but the cross calibration of the two chromatographs is necessary for accurate results. The use of the mixer shown in Fig. 8.12 is not necessary if samples can be taken far enough downstream (approximately 10 duct diameters) to ensure good mixing. Where good mixing cannot be achieved, a multiple sampling technique must be used (Sect. 11, ANSI N510). ${ }^{2}$

One problem in the use of chromatographs is the instrument's sensitivity to oxygen and to oxygencontaining compounds such as $\mathrm{CO}_{2}$. Also, the 


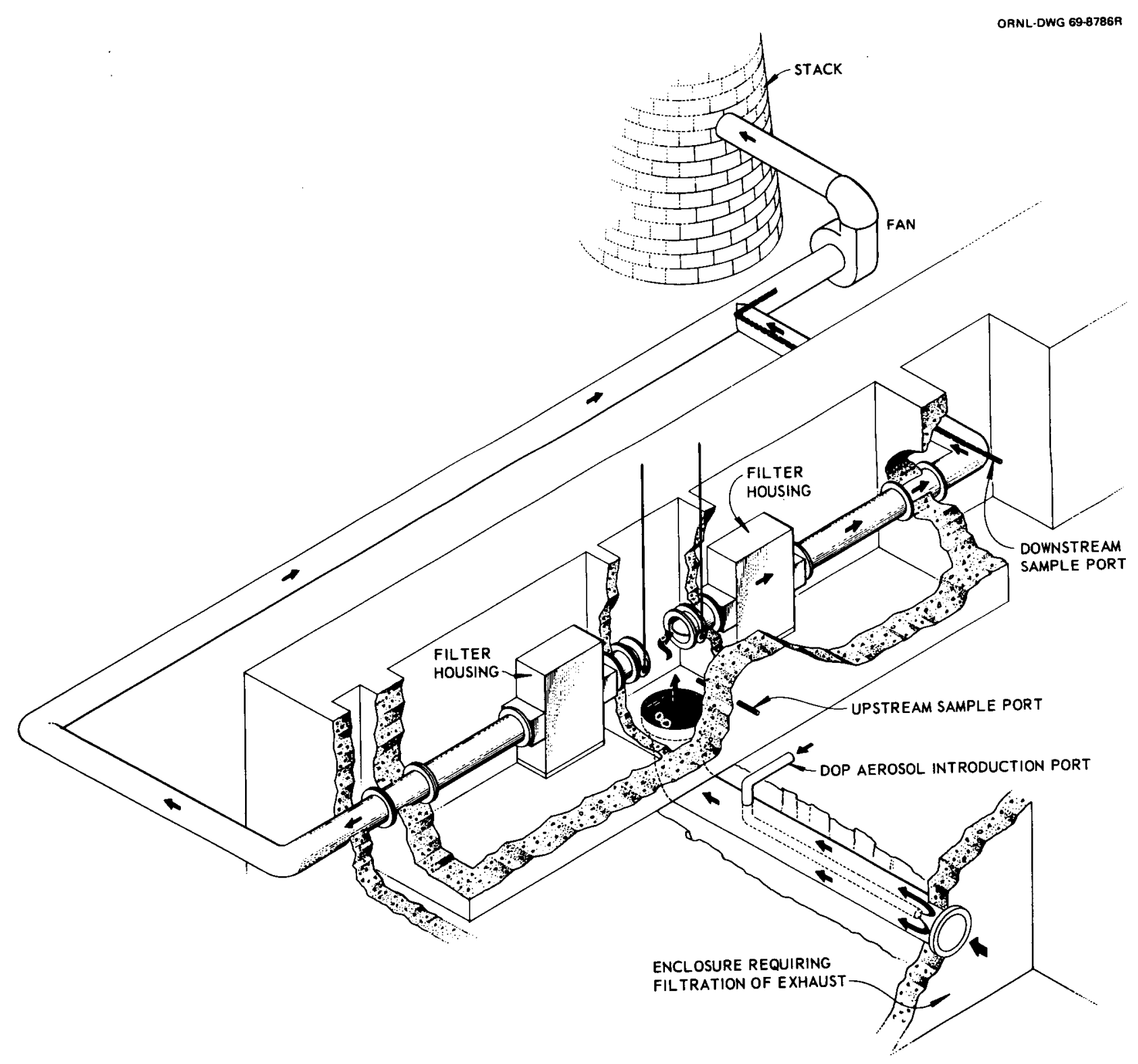

Fig. 8.9. Preplanned arrangement for testing filters in a large laboratory facility. 
ORNL DWG. 69-8787

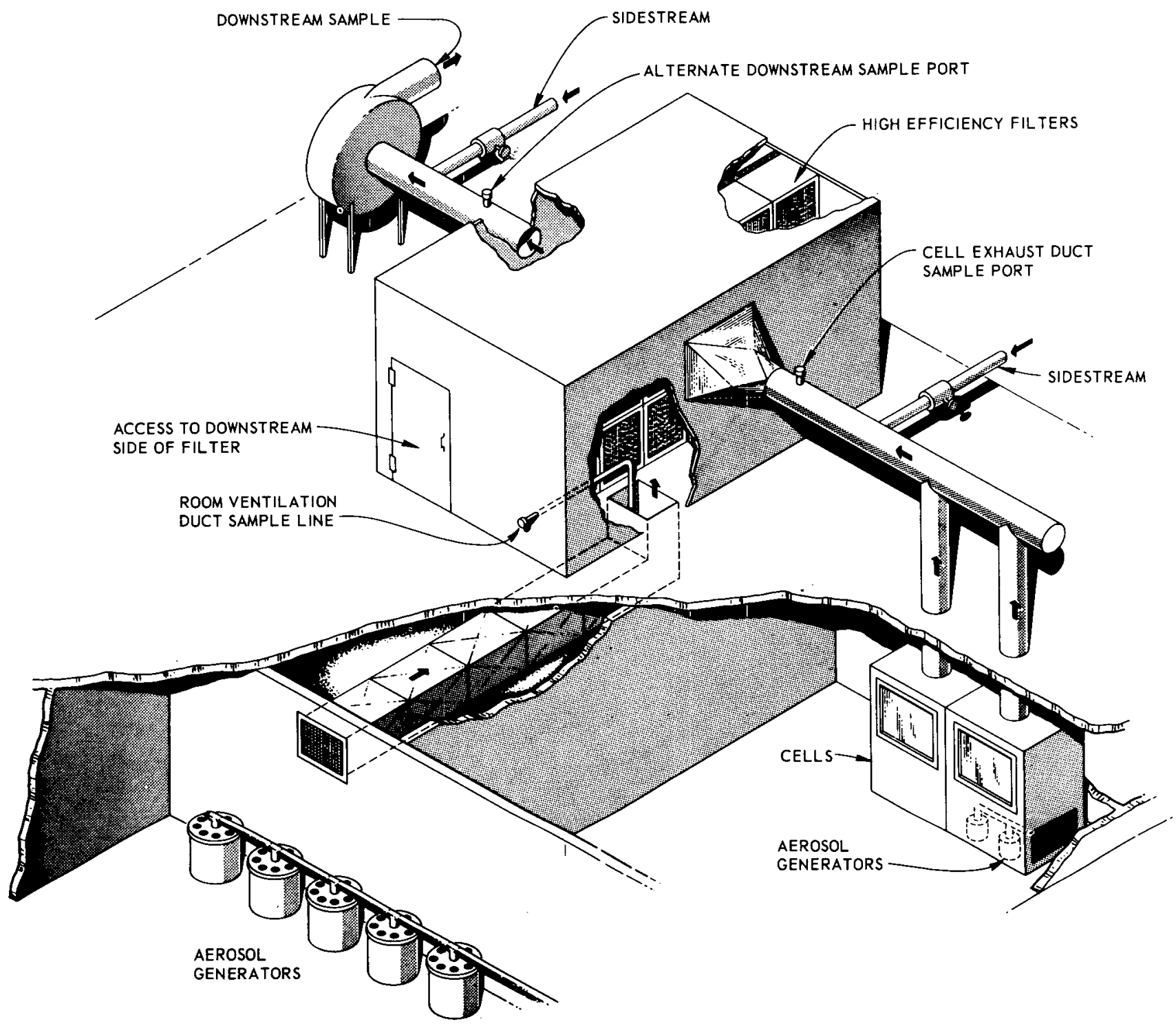

Fig. 8.10. Improvised arrangement for testing filters. No plan for surveillance testing was considered in the original system design. Aerosol generator capacity in cells and room must be balanced to obtain reasonable uniformity of air and DOP in the filter housing. 


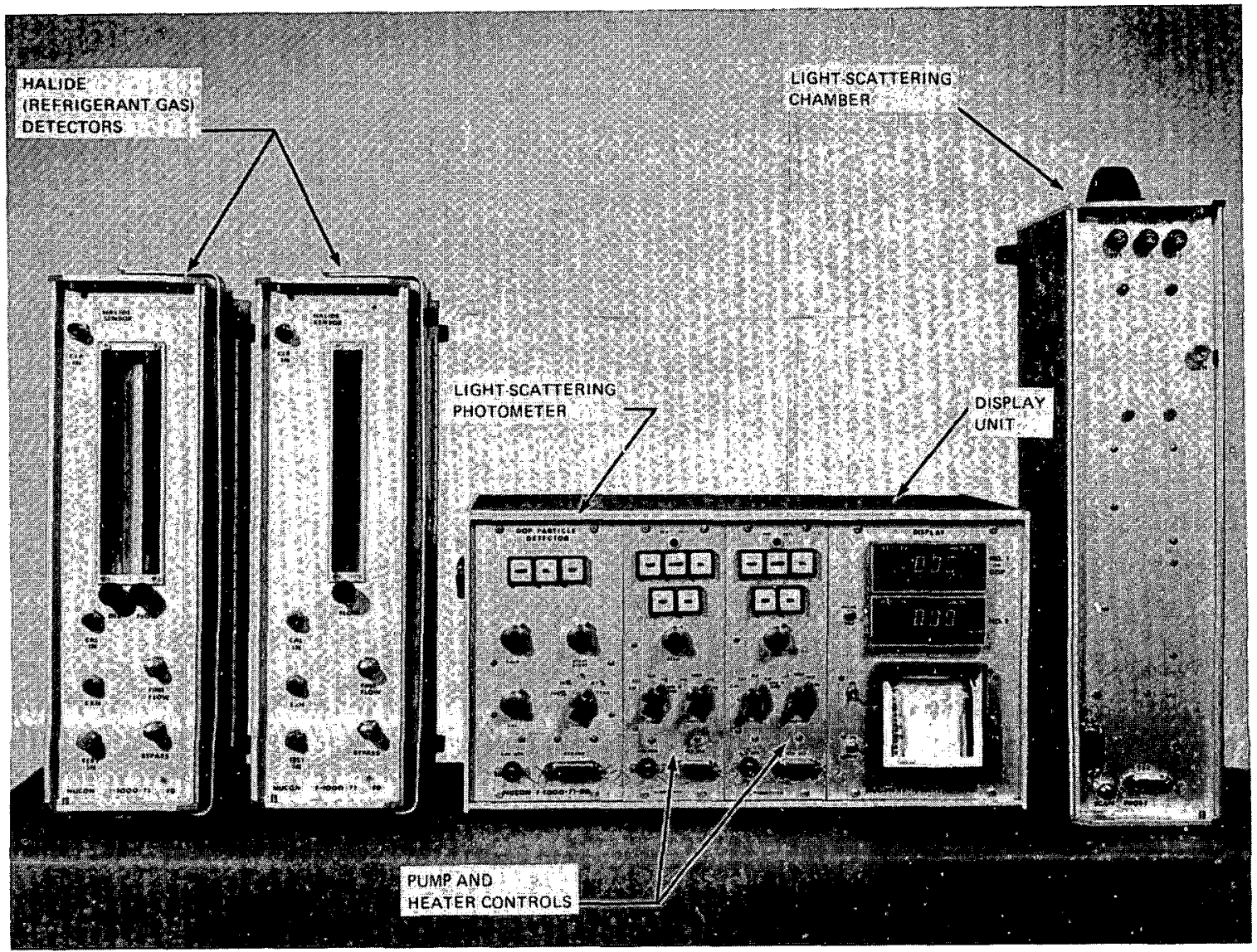

Fig. 8.11. Test equipment for Freon and DOP tests. Courtesy Nuclear Consulting Service, Inc.

ORNL-DWG 698791P

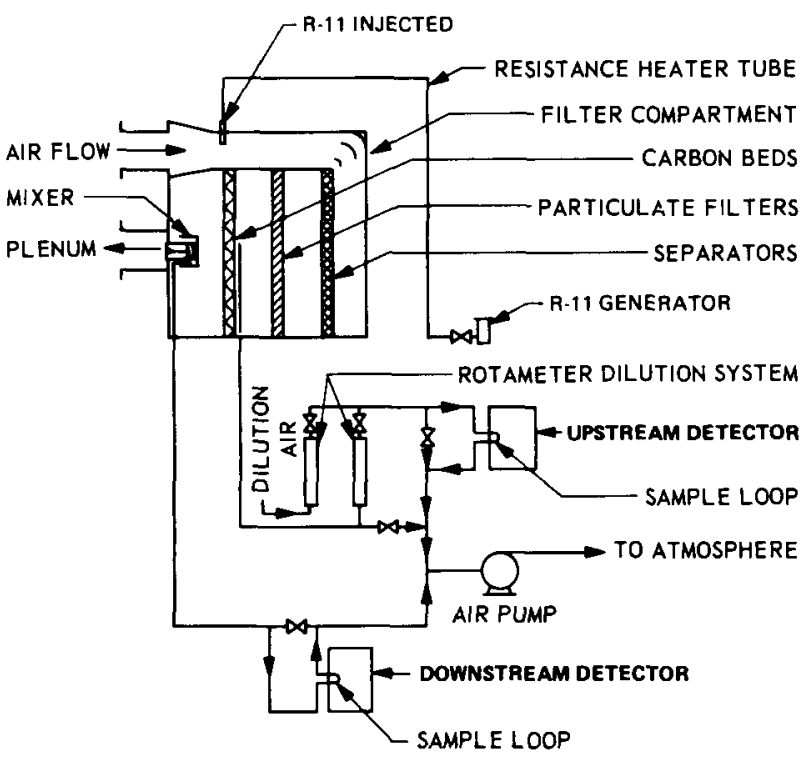

Fig. 8.12. Schematic of Freon (R-11) test arrangement. accuracy of the test depends on the operator's skill in interpreting peaks on the chromatograph output.

Radioactive Iodine Tests. These tests are currently used for routine adsorber-bank testing at Oak Ridge National Laboratory (ORNL) and the Hanford (Richland, Washington) facilities of ERDA. Two tests are used, one with radioactively traced elemental iodine and the second with radioactively traced methyl iodide. Equipment requirements for the elemental iodine test include an iodine injection tube (Fig. 8.13), two sampling units (Fig. 8.14), a sample extraction pump, and three calibrated rotameters for controlling the injection and sampling flows. The sampling units are filled with charcoal of known efficiency for elemental iodine. The test aerosol is ${ }^{127} \mathrm{I}_{2}$ containing ${ }^{131} \mathrm{I}_{2}$ tracer. A combination of injected radioactivity (in microcuries), sampling rate, and counting technique (usually dictated by the kind of counting equipment available) must be developed to give the required test precision. At ORNL, a combination of sampling and injection rates is selected 
ORNL DWG. 69.8792

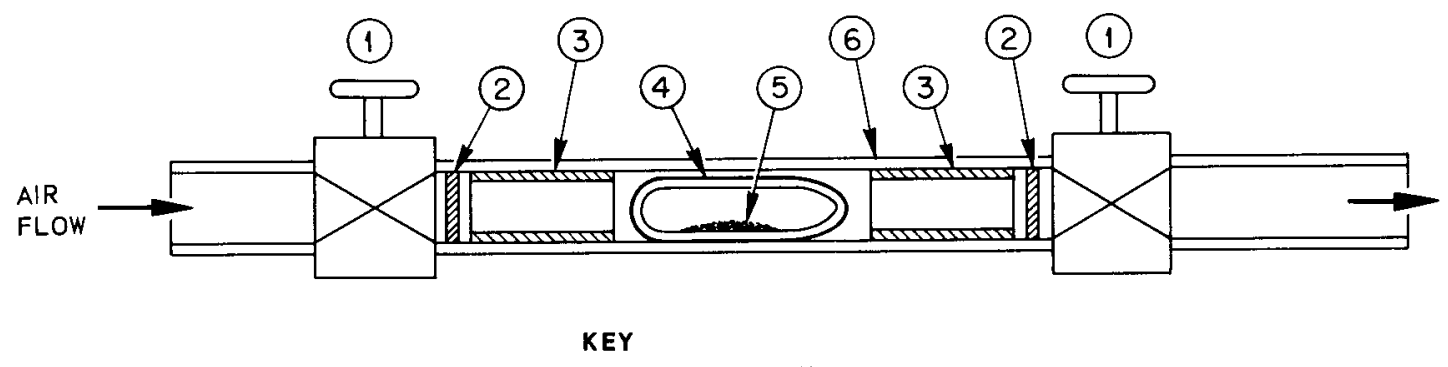

$\begin{array}{ll}1 & \text { VALVE, SEMI-NEEDLE } \\ 2 & \text { WIRE SCREEN } \\ 3 & \text { SPACER, GLASS TUBE } \\ 4 & \text { IODINE AMPUL, GLASS } \\ 5 & \text { IODINE CRYSTALS } \\ 6 & \text { TUBING, STAINLESS STEEL }\end{array}$

Fig. 8.13. Injector tube for radioiodine tracer test.

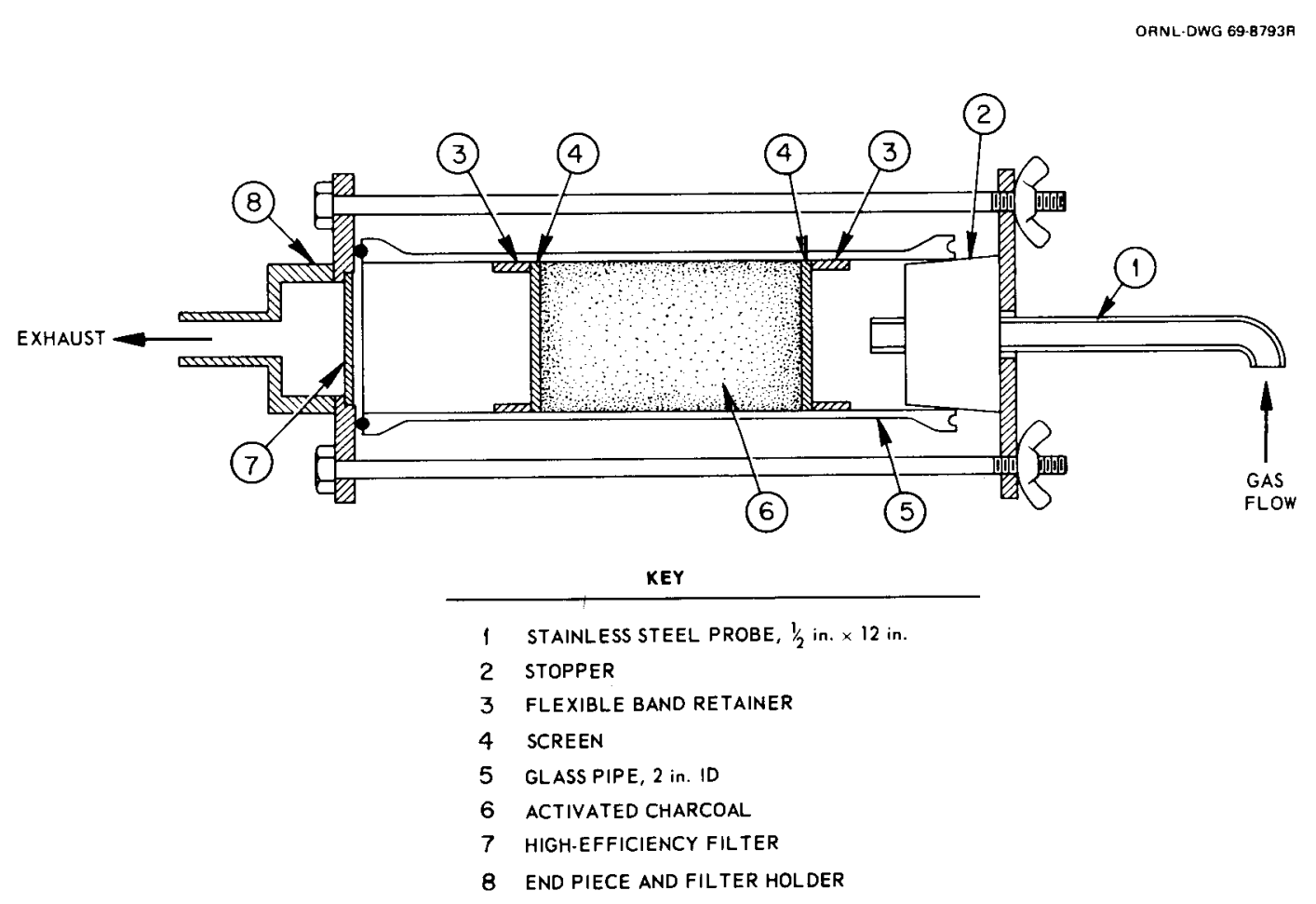

Fig. 8.14. Sampling element for radioiodine tracer test. 
which, with available counting equipment, will produce an upstream sampler radioactivity count between $8 \times 10^{5}$ and $5 \times 10^{6}$ counts $/ \mathrm{min}$. These are not rigid limits but are convenient target values which have considerable latitude. Satisfactory tests have been made at sampling rates as low as $0.03 \%$ of the system flow rate, but sampling rates of about $1 \mathrm{cfm}$ per $1000 \mathrm{cfm}(0.1 \%)$ of rated adsorber capacity are recommended.

The amount of iodine required and the size of the injector tube are not critical. The amount of ${ }^{127} \mathbf{I}_{2}$ is invariably $100 \mathrm{mg}$ in the ORNL tests, although this amount may be doubled if excessive plateout in the upstream duct or housing occurs. The amount of ${ }^{131} I_{2}$ tracer must be adjusted to give the radioactivity count noted above. The radioactive iodine source is prepared by mixing the required quantities of ${ }^{1-27} \mathrm{I}$ and ${ }^{131}$ I as NaI, precipitating the iodine fraction as $\mathrm{PdI}_{2}$ by treatment with acidified $\mathrm{PdCl}_{2}$, then decomposing the $\mathbf{P d I}_{2}$ under vacuum. The liberated ${ }^{127+131} I_{2}$ is collected in a liquid-nitrogen-cooled U-tube and transferred to a glass ampule that is installed in the injector (Fig. 8.13). Preparation of the iodine and loading of the injector must be carried out in a laboratory equipped for handling radioactive materials. To inject iodine during the test, the injector tube is crushed, breaking the ampule and releasing the iodine vapor. Compressed air is passed through the tube at a carefully controlled rate for about $2 \mathrm{hr}$. During the final half hour, heat is applied to the injection tube to drive out the remaining iodine.

Figure 8.15 shows a typical in-place radioiodinetracer test setup. After system flow and background

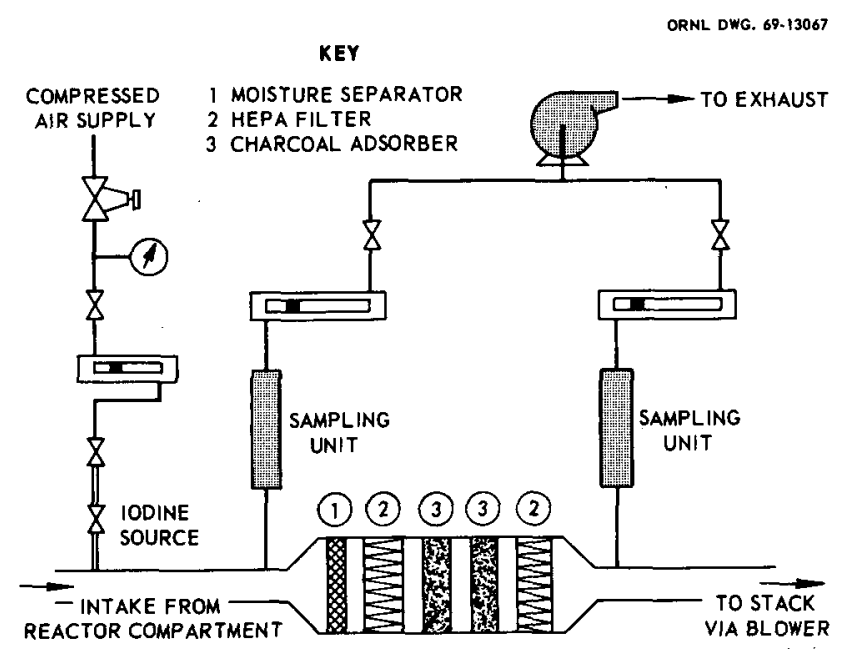

Fig. 8.15. Test setup for radioiodine tracer tests. radioactivity levels are established, iodine is injected far enough upstream to ensure adequate mixing with the main airstream, and samples are withdrawn simultaneously through the upstream and downstream sampling units. The injection of iodine is continued for approximately $2 \mathrm{hr}$, but system airflow and downstream sampling are continued for another $2 \mathrm{hr}$ to catch any iodine that may desorb from the beds, in addition to that which penetrates immediately. Exhaust air from the sampling units is usually dumped back into the upstream side of the main system.

The iodine content of the carbon in the samplers is determined by direct gamma spectroscopy, and the efficiency is determined from the equation

$$
E=\left(1-\frac{C_{d}}{C_{u}-B}\right),
$$

where

$E=$ efficiency, $\%$;

$C_{d}=$ iodine content of downstream unit, dis/min;

$C_{u}=$ Iodine content of upstream unit, dis $/ \mathrm{min}$;

$B=$ background due to impurity iodine in charcoal, $\mathrm{dis} / \mathrm{min}$.

The methyl iodide test for determining the efficiency of adsorbers for organic radioiodine compounds is similar to the test for elemental iodine and uses the same equipment except for the injector. The injector used for the methyl iodide test is a U-tube and vapor expansion chamber. Sampling and analytical procedures are the same as those for the elemental iodine test. The test vapor is $\mathrm{CH}_{3}{ }^{127} \mathrm{I}$ containing $\mathrm{CH}_{3}{ }^{131}$ I tracer. Because the methyl iodide test determines a different property of the adsorbent and is dependent on a different sorption mechanism, it cannot be used in place of the elemental iodine test, and both tests are required for a complete evaluation of impregnated charcoal adsorbers. Both of these tests suffer from the limitations of using radioactive tracers in the field and from the number of variables that must be controlled to achieve reliable results.

\subsubsection{In-Place Testing for Multistage Systems}

Systems that contain two or more HEPA filter stages and/or two or more adsorber stages in series in the same housing give special problems because of the difficulty of obtaining a representative single-point sample downstream of the first bank and the difficulty of introducing the second-stage test agent at a 
point where good mixing can be achieved. Series banks are usually so close that neither of these objectives can be achieved in the normal manner. Because of the very high collection efficiency of the first-stage elements, sufficient test agent cannot be introduced upstream of the first stage to permit effective testing of the second stage. It has been shown that DOP has no adverse effect on activated carbon or other adsorbents when used for testing nuclear air cleaning systems, ${ }^{21}$ and the refrigerant gases used to date have no adverse effect on HEPA filters.

First-Stage Downstream Sample. The first-stage downstream sample can be obtained either by a multiple sampling technique (Sect. 11, ANSI N510) or by providing a temporary jumper duct to bypass airflow around the second stage to either the system fan, as Fig. 8.16 shows, or to a temporary auxiliary fan. In the arrangement shown in Fig. 8.16, the downstream housing damper is closed so that no air is brought through the components downstream of the bypass connection. The downstream sample can be taken either upstream of the fan, in the temporary bypass duct (if that duct is long enough to ensure good mixing), or downstream of the fan. Note that the bypass duct ports must be capped and sealed when not in use.

For testing multistage HEPA filter banks, one ERDA facility scans the downstream face of the stage to be tested in accordance with the procedure outlined in Sect. 4 of IES CS-2. ${ }^{11}$ The recommended scanning pattern for each filter in the bank is shown in Fig. 8.17..$^{14}$ Prior to the start of scanning, the upstream side of the stage is flooded with DOP and the photometer is adjusted to read $100 \%$ at full-scale reading on the least-sensitive scale of the instrument. A high concentration will always exist directly downstream of a leak. During the downstream scan, the relative magnitude of each leak is determined by turning the scale shift knob of the instrument until a reading about halfway between half scale and full scale is obtained. The reading is recorded and the leak flow for that point is calculated from the equation

leak-probe meter reading, $\%$

upstream concentration, $\%$

$$
\times \text { probe flow rate }=\text { leak flow, }
$$

where probe flow is the airflow capacity of the instrument. The percent penetration of the total bank is calculated from the equation

$$
\text { penetration }=\frac{\Sigma_{n} \text { leak flows }}{\text { total flow }}
$$

Defective filters are replaced and installation deficiencies are corrected before making the final test. This method is said to be more sensitive than the usual method of HEPA filter testing, and it is recommended for multistage systems having plutonium or transuranic-element source terms. ${ }^{14}$

Test Agent Injection, Second-Stage Upstream Sample. Figure 8.16 shows how a temporary auxiliary duct may be used to introduce a test agent to a multistage system. This figure shows the DOP being injected for a first-stage filter test; however, a similar method can be used to introduce a test agent into the space between the first and second stages. For the second stage, the downstream damper of the housing is opened and the system fan draws directly through the bank under test. If for some reason the system fan cannot be used, an auxiliary fan may be used to establish airflow, as shown in Fig. 8.18.

When the test agent is introduced through an auxiliary duct (Fig. 8.16), the upstream sample can be taken any place in the auxiliary duct (upstream of the bank to be tested), assuming that the auxiliary duct is long enough to ensure good mixing. When using an auxiliary blower, a downstream sample can be taken downstream of the blower, as shown in Fig. 8.18.

Another method of obtaining proper mixing of test agent with air is to shroud adjacent filters (adsorbers) and introduce the agent to each filter element (adsorber cell) individually by using a multiple discharge distributor, as shown in Fig. 8.19. The upstream sample is taken downstream of the perforated distribution plate. The downstream sample is taken with a multipoint sampling probe (Fig. 8.20). The penetrations of the individual filters (adsorbers) are averaged to find the gross bank penetration. This method requires that a mounting-frame pressure leak test be made, usually at the time of acceptance testing, ${ }^{8}$ and that air containing test agent be passed through one unit (filter or adsorber cell) or group of units one at a time. The method has the advantage of substantially reducing the total quantity of test agent 


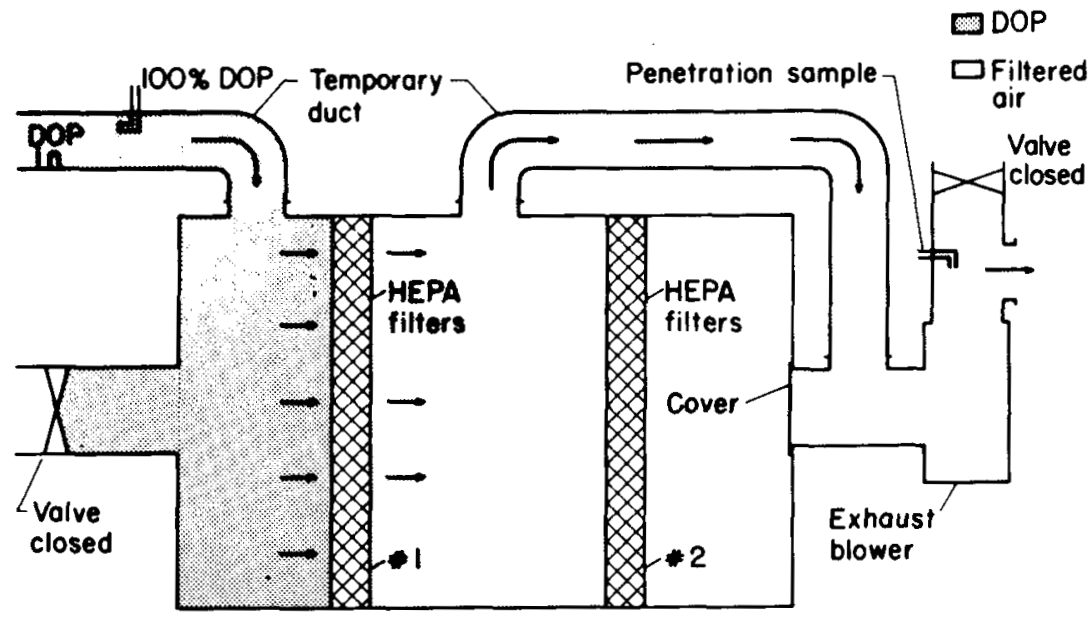

(a)

Room Air Filter System

Filter Bank 1 Test

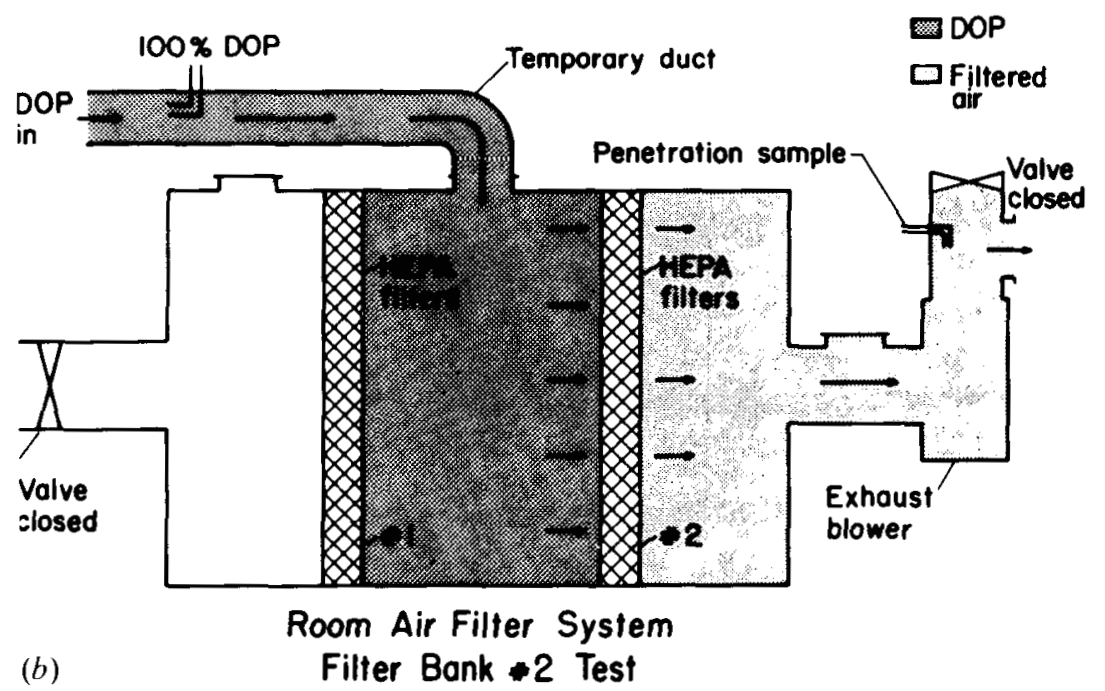

Fig. 8.16. Testing of multistage HEPA filter installation, permitting individual testing of each bank. Test can be made using an auxiliary blower, at reduced flow, instead of the system fan (see Fig. 8.18). (a) Test setup for testing upstream bank of filters. DOP generator located at entrance to temporary duct on right. Temporary duct on left provides bypass of second-stage filters to system fan. (b) Test setup for testing downstream bank of filters. DOP generator located at entrance to temporary duct; no bypass duct required. From R. Mitchell et al., "Design of Ventilation and Air Cleaning Systems for the New Los Alamos Plutonium Facility," Proc. I3th A EC Air Clean. Conf., ERDA Report CONF-740807, March 1975. 


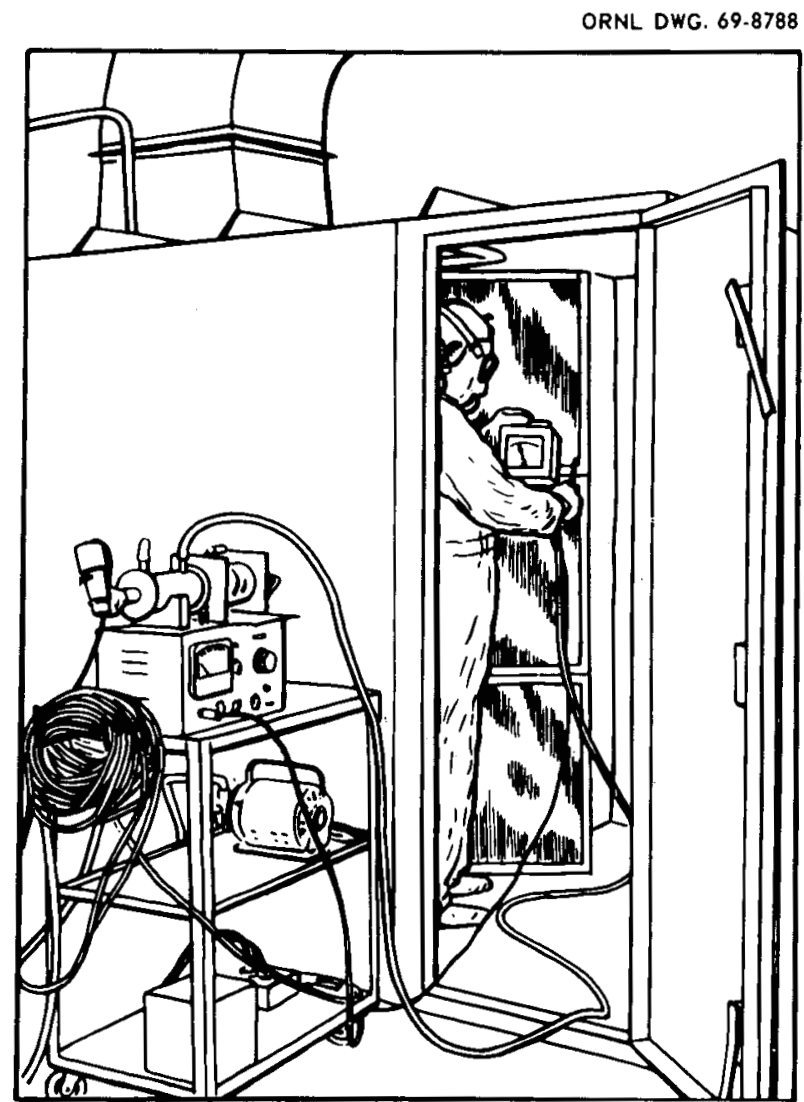

(a)

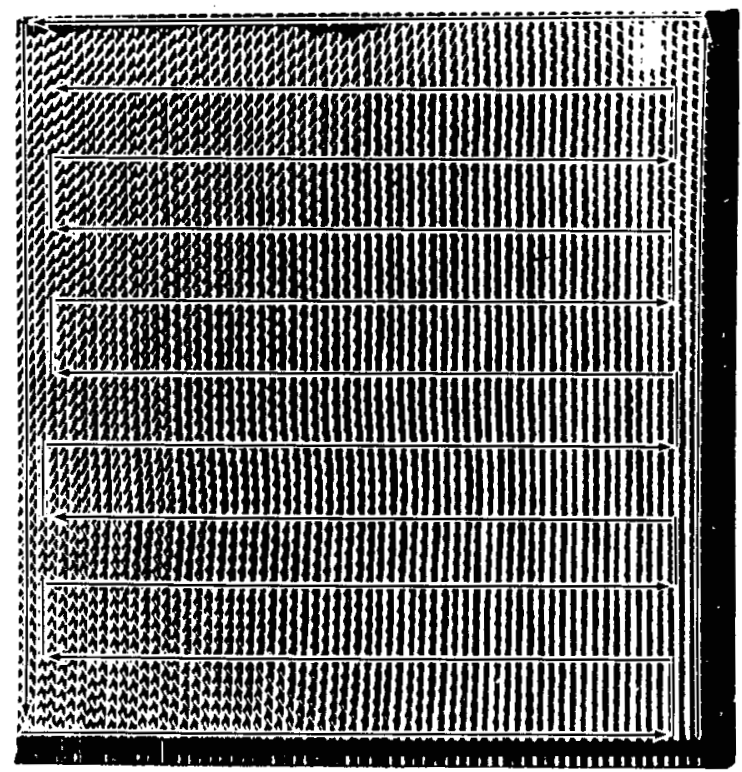

(b)

Fig. 8.17. Scanning HEPA filters to locate leaks. ( $a$ ) Leak probing HEPA filter bank; extension meter used to measure a sharp increase in DOP, which would indicate a leak; $(b)$ suggested probe-scanning pattern for 24 - by 24 -in. HEPA filter.

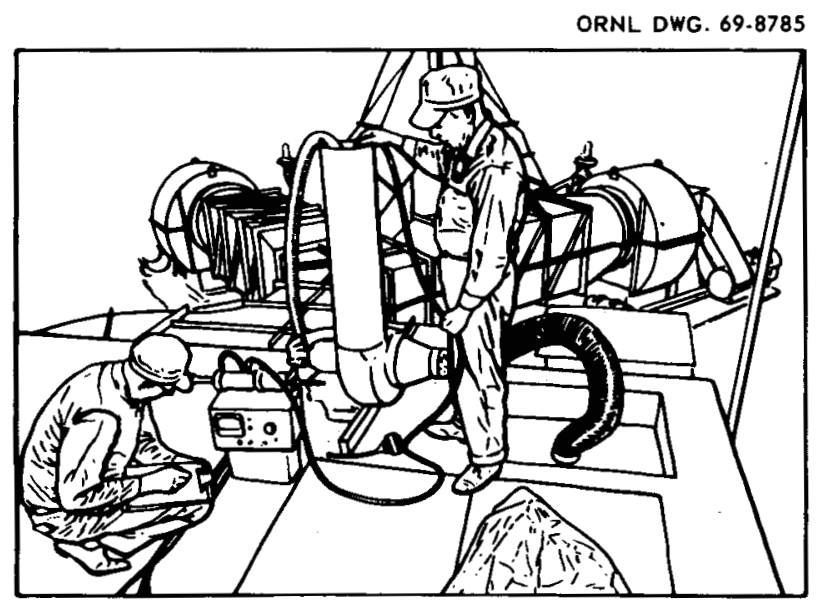

Fig. 8.18. Auxiliary blower being used for testing first-stage HEPA filter bank in a 15,000 -cfm air cleaning system. DOP is injected in duct upstream of housing, and air is extracted between bank of adsorbers and second stage of HEPA filters. Downstream sample is taken in stack of auxiliary blower. introduced to the system if scanning is required to locate leaks; however, it requires more time than the usual method of taking single-point upstream and downstream samples.

Because DOP has no adverse effect on activated carbon and refrigerant gases have no adverse effect on HEPA filters, it is possible to inject DOP upstream of the adsorbers when testing a secondstage HEPA filter bank and to inject refrigerant gas upstream of the HEPA filters when testing adsorbers.

\subsubsection{Adsorbent Sampling and Testing}

For licensed reactors, laboratory tests of adsorbent should be made in accordance with the performance (iodine) test requirements of RDT $\mathrm{M} \mathrm{I6-1}^{22}$ and should meet the acceptance criteria given in Table 8.1. Laboratory testing is costly and is usually kept to a minimum. Samples taken for testing must be as 


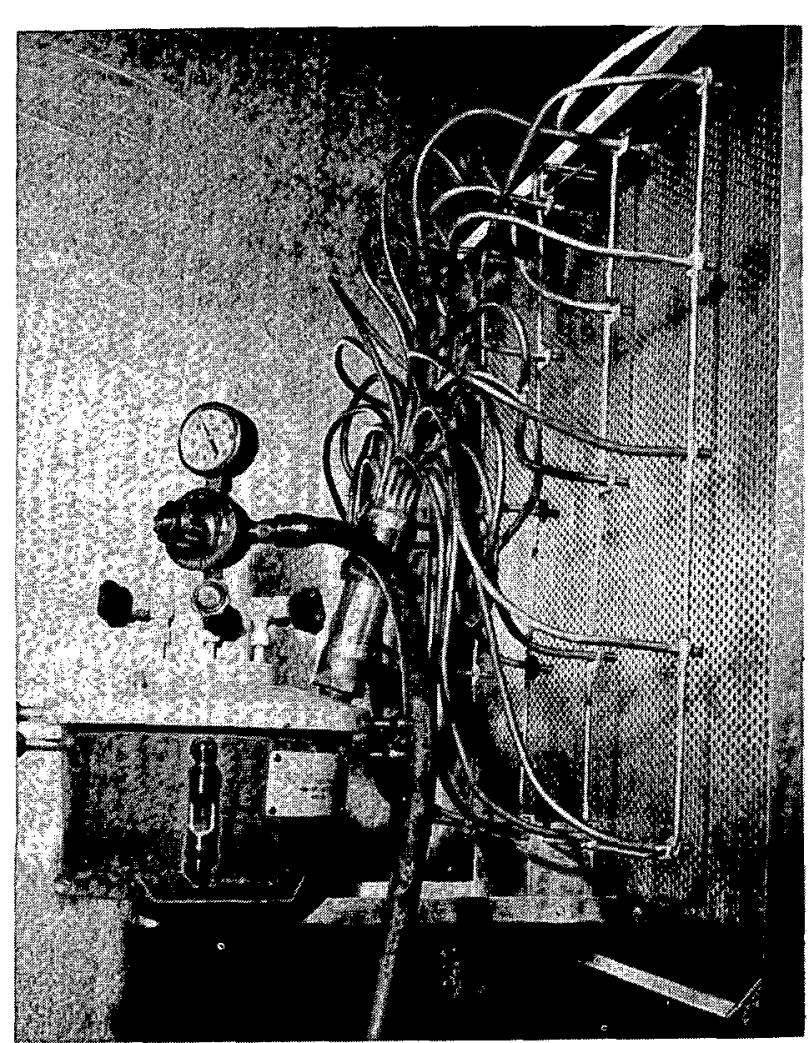

Fig. 8.19. DOP smoke distributor mounted in temporary shroud. Courtesy American Air Filter Co.

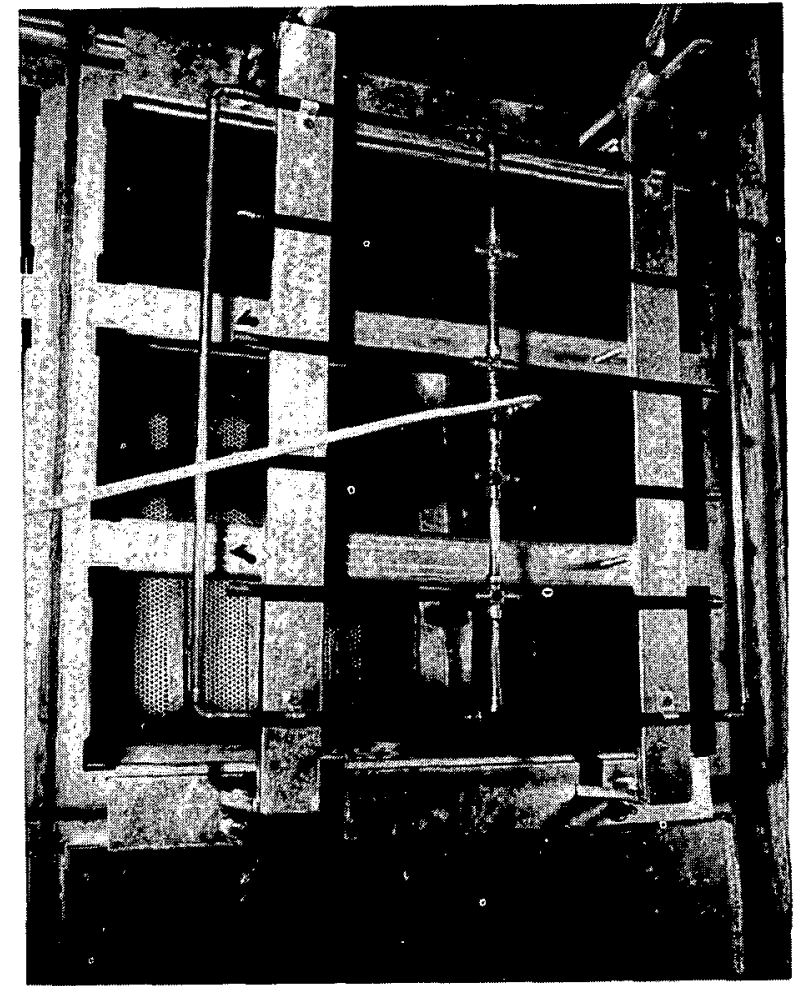

Fig. 8.20. Multipoint sampling probe. Courtesy American Air Filter Co.

Table 8.1. Laboratory surveillance test requirements for adsorbents ${ }^{a}$

\begin{tabular}{ll}
\hline \multicolumn{1}{|c}{ Activated carbon bed depth } & \multicolumn{1}{c}{$\begin{array}{c}\text { Laboratory test requirements } \\
\text { for a representative sample }\end{array}$} \\
\hline $\begin{array}{l}2 \text { in.; airfiltration system designed to op- } \\
\text { erate inside primary containment }\end{array}$ & $\begin{array}{l}\text { Test initially and yearly thereafter under } \\
95 \% \text { relative humidity, maximum design } \\
\text { temperature, and design face velocity for } \\
\text { an elemental iodine penetration of }<1.0 \%\end{array}$ \\
and <10\% for methyl iodide
\end{tabular}

\footnotetext{
${ }^{a}$ Test requirements proposed for reissue of Regulatory Guide 1.52, Design, Testing, and Maintenance Criteria for Atmospheric Cleanup System Air Filtration and Adsorption Units of Light-Water-Cooled Nuclear Power Plants, U.S. Atomic Energy Commission, Washington, D.C., 1973.

${ }^{b}$ Maximum postulated DBA temperature $\left({ }^{\circ} \mathrm{F}\right)$ rounded to the next highest decade (e.g., $181^{\circ} \mathrm{F}$ should be rounded to $190^{\circ} \mathrm{F}$ ).
} 
representative as possible, which means that they must have been subjected to the same airflow, temperature, humidity, and poisoning conditions as the remainder of the adsorbent in the same stage. Ideally, a large sample should be taken from a point close to the center of the stage, since this area probably receives the most severe environmental exposure. Such a sample is often not practical because of the difficulty of extracting the sample and of returning the system to its original condition afterwards. Therefore, a number of sample cartridge designs have been developed, a few of which are shown in Fig. 8.21. Sample cartridges must be provided in sufficient number to permit taking samples at specified intervals for the life of the adsorbent. British tests indicated a maximum life of coal-base activated carbon of three years for nonoperating or standby systems and 18 months for continuously on-line systems. ${ }^{23}$ No guides have been established for the adsorbents used in this country. Sample cartridges must be designed so that bed depth of, airflow through, and pressure drop across the cartridge are essentially the same as for the adsorber stage itself. For this reason, the zero-flow hang-on cartridges shown in Fig. 8.22 are not acceptable. Flow-through cartridges must be provided and installed in an area of the bank where air will flow through them and not in obvious low-flow areas such as the outside edge of the mounting frame. Cartridges must be accessible to service personnel without the necessity of climbing up component banks or bringing in temporary ladders or scaffolding.

If sample cartridges are not provided, other means of sampling are necessary. In a multicell system such as that shown in Fig. 8.23, samples can be obtained by removing and emptying a cell, taking a sample of the loose adsorbent, refilling the cell (using a qualified filling procedure to ensure proper loading), and reinstalling it in the bank. For some PSU adsorbers, it may be possible to take a thief sample ${ }^{22}$ from a point close to the center of the unit. In small adsorber installations, when considering the cost of the tests, some users have found it more economical to simply replace the adsorbent at the stipulated sampling frequency rather than make surveillance sample tests. (Regulatory Guide 1.52 currently calls for adsorbent testing at intervals of $720 \mathrm{hr}$ of operation until experience shows that longer intervals between testing are justified on the basis of life curves developed from the test results.) ${ }^{3}$

\subsubsection{Frequency of Testing}

The following test schedule is suggested for both continuously and intermittently on-line systems designed in accordance with this handbook.

Application

All systems

Radiochemical plants, fuel reprocessing plants, and laboratory fume hoods

Reactor postaccident cleanup systems and ESF postaccident cleanup systems of fuel reprocessing plants

Zone III contamination areas of facilities handling moderate to large quantities of radioactive materials

Zone I and II contamination areas of plants and laboratories handling moderate to large quantities of radioactive materials

Zone IV contamination areas (glove-box lines, hot-cell exhaust, etc.) of laboratories and plants handling moderate to large quantities of radioactive materials

Systems continually on standby but operated only occasionally during plant maintenance to ventilate the system
Semiannually unless experience indicates that annual testing is sufficient. If filters (adsorbers) are replaced at short (less than six month) intervals to limit exposure of personnel to radiation during a filter (adsorber) change or to permit contact maintenance of system by limiting the amount of radiation that can be collected in the filters (adsorbers), systems should be in-place (i.e., leak-) tested following each filter (adsorber) change. Laboratory testing of adsorbents may not be necessary if the adsorbent is replaced frequently

At least biannually 


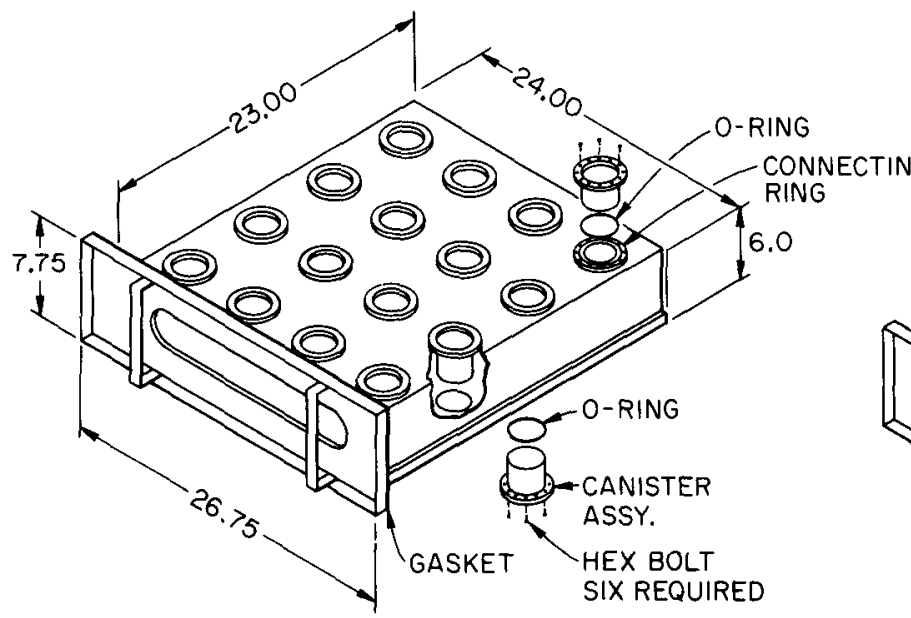

(a)

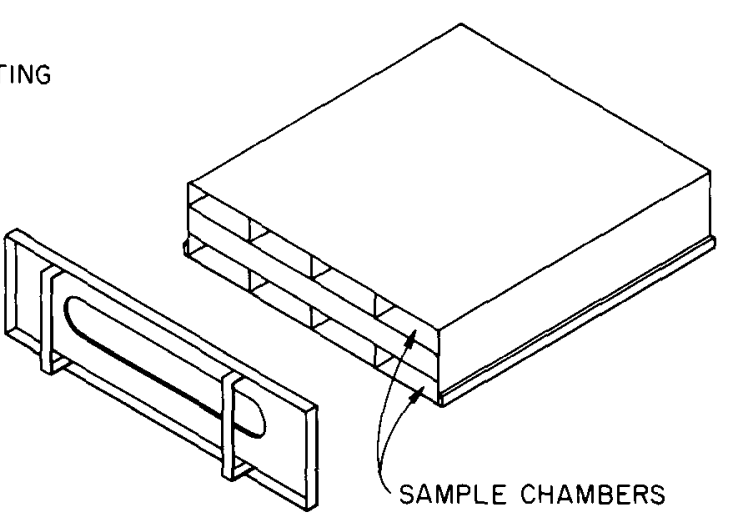

(b)

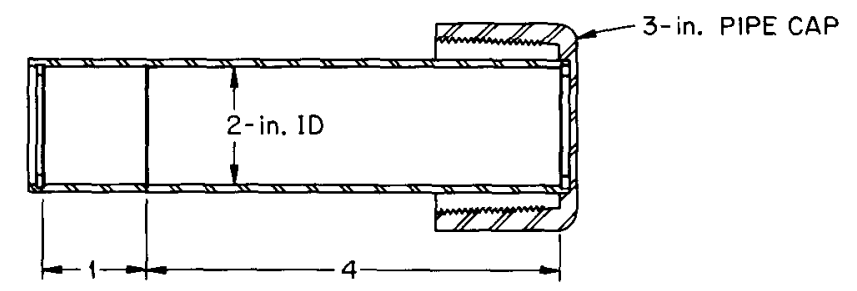

(c)
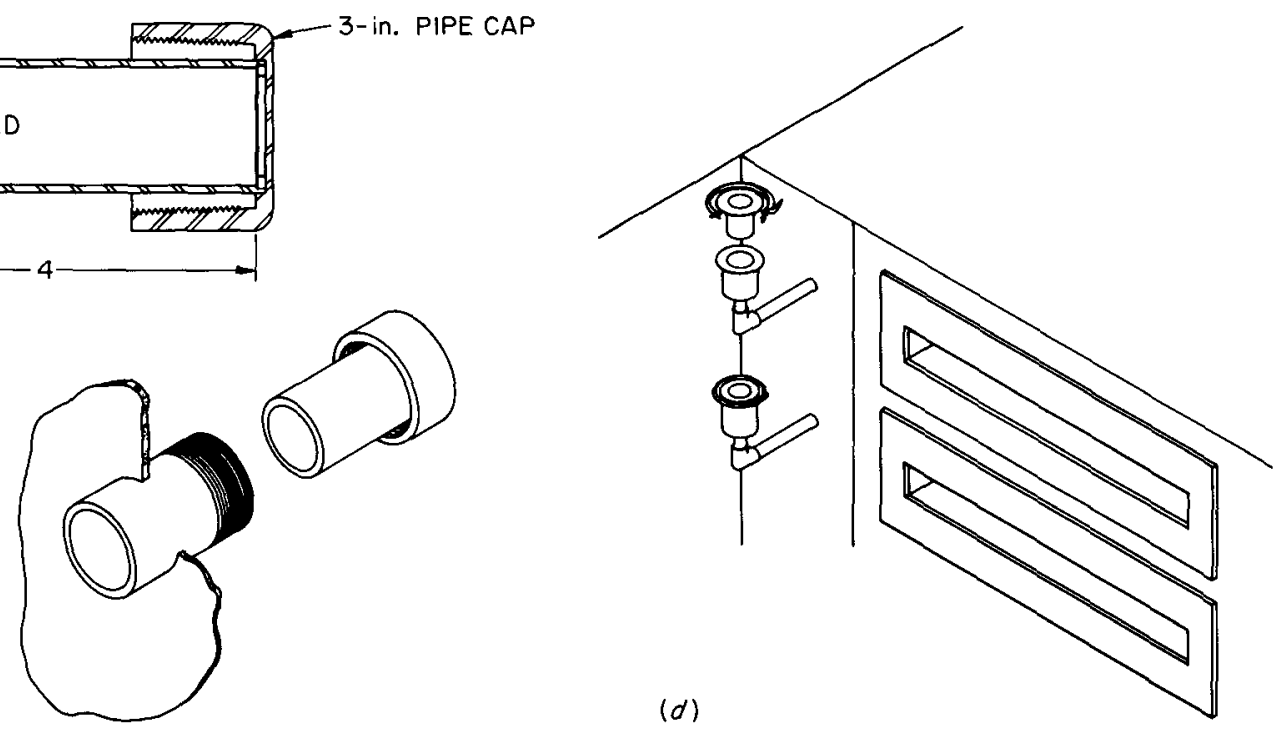

DIMENSIONS ARE INCHES

Fig. 8.21. Typical adsorbent sample canister designs. (a) Special type Il cell with 32 sample canisters. One canister is removed and replaced each time a sample is taken (blanking off openings, instead of canister replacement, would result in increasing airflow through remaining canisters). (b) Special type II cell with eight sample chambers. One of eight chambers is emptied and refilled each time a sample is to be taken. $(c)$ Through-wall sample canister for 4 -in. deep-bed gasketless adsorber with 1 -in. guard bed. Canister fits into pipe nipple, sealwelded into wall of adsorber unit. Nipple is blanked of or canister is replaced with a new canister each time a sample is taken. $(d)$ Throughmounting-frame test canister installation using sample units of same design shown in (a). Holder is blanked off or refilled with a new sample unit when a sample is taken. Sampler tops in mounting frame should be closer to center of bank to achieve more representative airflow through samples. 

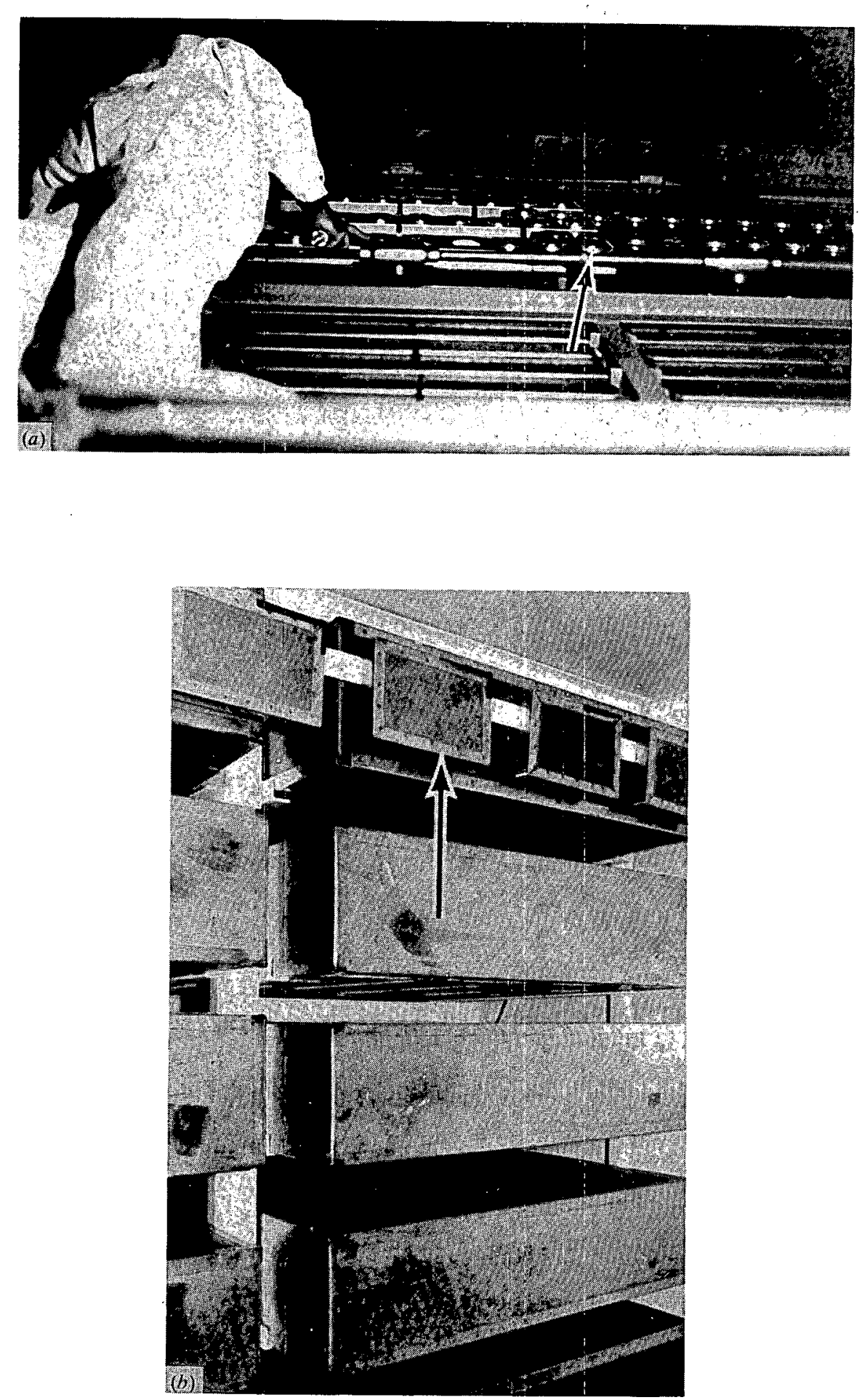

Fig. 8.22. Examples of unacceptable adsorbent sample canister design. Hang-on canisters have no flow through them and do not provide representative samples. (a) Hang-on adsorbent sample canisters suspended upstream of adsorber bank. Note poor access to sample canisters; $(b)$ hang-on adsorbent sample canisters installed upstream of adsorber bank. 


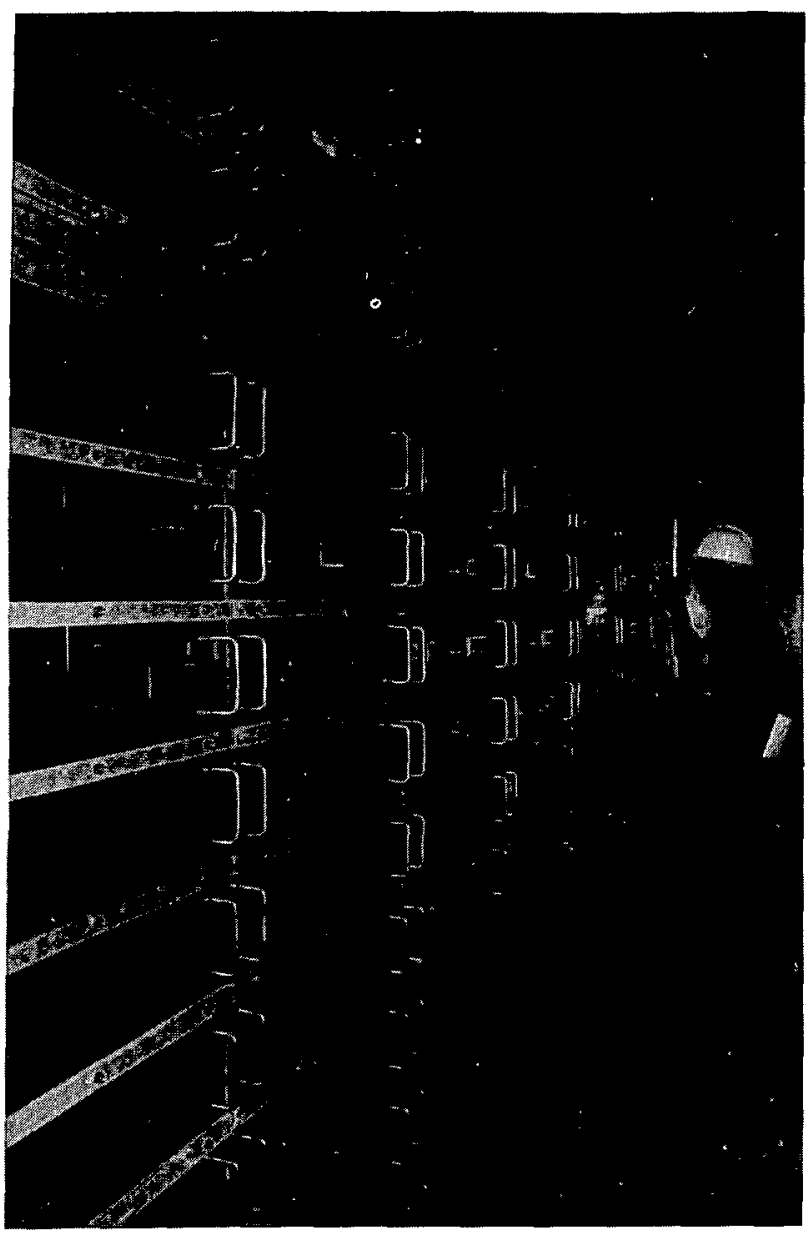

Fig. 8.23. Large bank of tray-type (IES CS-8, type II) adsorber cells. Samples can be taken from installations of this type by removing a cell from the center of the bank. The emptied cell is refilled with new adsorbent and replaced prior to making the inplace test. Note lack of a permanently installed access to upper tiers of cells and structural angles in the floor, which represents a stumbling hazard to service personnel.

\subsection{VISUAL INSPECTION}

Although visual inspection is not an acceptance or surveillance test procedure as such, it is an integral and vital part of every such test. A careful visual examination should be made of each internal and external component prior to installation to verify that the items have been received in satisfactory and serviceable condition. After installation, as part of the acceptance test procedure, the system should be checked to make sure that all required items have been properly installed. A suggested check list of what to look for is given as Appendix A of ANSI N510, Standard for Testing of Nuclear Air Cleaning
Systems. Visual inspection will often reveal deficiencies that could cause test failure or invalidate test results. Visual inspection should be made under a combination of background lighting and supplementary lighting that provides at least $100 \mathrm{ft}-\mathrm{c}$ on the surfaces being examined. Correctable deficiencies should be recorded together with the remedial action taken. Experience has shown that a record of deficiencies and their method of correction is invaluable for future maintenance of the system, and this practice should be followed in both acceptance and surveillance testing. Errors and deficiencies that cannot be corrected immediately, or that require replacement of an item, should be brought immediately to the attention of responsible authorities.

\section{REFERENCES FOR CHAP. 8}

1. E. J. Bauer et al., "Use of Particle Counts for Filter Evaluation," pp. 53-59 in ASHRAE Journal, American Society of Heating, Kefrigerating and Air-Conditioning Engineers, October 1973.

2. ANSI N510, Testing of Nuclear Air-Cleaning Systems, American National Standards Institute, New York, 1975.

3. Regulatory Guide 1.52, Design, Testing, and Maintenance Criteria for Atmospheric Cleanup System Air Filtration and Adsorption Units of Light-Water-Cooled Nuclear Power Plants, U.S. Atomic Energy Commission, Washington, D.C., 1973.

4. High Velocity Duct Construction Standards, Sheet Metal and Air Conditioning Contractors' National Association, Inc., Arlington, Va., 1969.

5. Industrial Ventilation, A Manual of Recommended Practice, 13th ed., American Conference of Governmental Industrial Hygienists, Lansing, Mich., 1974.

6. IES CS-8, Standard for High Efficiency Gas-Phase Adsorber Cells, Institute of Environmental Sciences, Mt. Prospect, 11l., 1975.

7. A disk or baffle centered in the duct having a face area equal to one-half the cross-sectional area of the duct. To reduce pressure drop when not in use, the disk is usually pivoted so that it can be turned parallel to the axis of the duct.

8. A. O'Nan et al., "Reliability and Testing Considerations in the Design of Nuclear Reactor Filtration Systems," Proc. 13th AEC Air Clean. Conf., ERDA Report CONF-700816, May 1975.

9. E. A. Parrish and R. W. Schneider, "Review of Inspection and Testing of Installed High Efficiency Particulate Air Filters at ORNL," Proc. Symp. Treat. Airborne Radioact. Wastes, International Atomic Energy Agency, Vienna, 1968.

I0. J. J. Smith, Inspection Engineering Department, Oak Ridge National Laboratory, personal communication to $C$. A. Burchsted.

11. IES CS-2, Standard for Laminar-Flow Clean Air Devices, Institute of Environmental Sciences, Mt. Prospect, Ill., 1975.

12. K. H. Knuth, "An Evaluation of Two Portable Thermal Aerosol Generators for In-Place Filter Testing," Proc. Ninth AEC Air Clean. Conf., USAEC Report CONF-660904, 1967.

13. Letter, F. T. Williams, Frontier Enterprises, Inc., to C. A. Burchsted, April 16, 1975.

14. F. J. Linck and J. A. Greer, "In-Place Testing of Multiple Stage HEPA Filter Plenums," Proc. 13th AEC Air Clean. Conf., ERDA Report CONF-740807, March 1975. 
15. Monthly Filter Test Report, Oak Ridge National Laboratory, 1970-1975.

16. Operators of ERDA Quality Assurance Stations, Richland, Wash., and Oak Ridge, Tenn., personal communications to C. A. Burchsted.

17. D. R. Muhlbaier, Standardized Nondestructive Test of Carbon Beds for Reactor Confinement Applications, USAEC Report DP-1082, Savannah River Laboratory, July 1967.

18. R. E. Adams and W. E. Browning, Jr., lodine Vapor Adsorption Studies for the NS "Savannah" Project, USAEC Report ORNL-3726, Oak Ridge National Laboratory, February 1965.

19. J. E. Mecca and J. D. Ludwick, In-Place Testing of the Hanford Reactor Charcoal Confinement Filter Systems Using lodine Tagged with lodine-I3I Tracer, USAEC Report DUN-SA105, Douglas United Nuclear, Inc., June 1969.

20. Registered trademark, E. I. du Pont de Nemours and Co.

21. W. L. Anderson, "The Effect of DOP on Activated Carbon," Proc. 13th AEC Air Clean. Conf., ERDA Report CONF-740807, 1975.

22. RDT M16-1, Gas-Phase Adsorbents for Trapping Radioactive lodine and lodine Compounds, Energy Research and Development Administration, current issue.

23. D. A. Collins, L. R. Taylor, and R. Taylor, "The Development of Impregnated Charcoals for Trapping Methyl lodide at High Humidity," Proc. Ninth AEC Air Clean. Conf., USAEC Report CONF-660904, January 1967. 


\section{Special-Application Requirements}

\subsection{INTRODUCTION}

Preceding chapters of this handbook have discussed general requirements of high-efficiency air cleaning systems as they pertain to the more usual applications. This chapter discusses special requirements that may have to be considered for certain applications, including remote handling of filters and/or adsorbers, shielding, design to resist natural phenomena such as a tornado or earthquake, provision for fire protection, high-capacity sand and deep-bed glass fiber filters, ESF systems, and considerations for radiochemical plant ventilation and off-gas systems.

\subsection{REMOTE MAINTENANCE}

In some radiochemical, fuel-reprocessing, and reactor postaccident cleanup applications, radiation levels may be so high that direct access and contact maintenance will be impossible. Therefore, the servicing and replacement of filter and adsorber cells must be accomplished by remote methods. Remotely maintainable systems must achieve the same objectives of high collection efficiency and reliability as other installations, but design and construction are complicated by the necessity for radiation shielding and the need to manipulate clamping devices and handle components indirectly and from a distance. Federal regulations specify a maximum exposure to personnel in restricted areas of 3 rems to the whole body and 18.75 rems to the hands and forearms in any calendar quarter.' If radiation levels in filters or adsorbers approach or could reach these levels, contact maintenance may be prohibited, and consideration must be given to remote procedures.

Radiation exposure can be minimizèd by limiting the time of exposure, by attenuating the radiation by means of shielding, and by reducing the intensity of exposure by keeping a safe distance from the source (intensity follows the inverse square law). A practice in some low to moderate hazard systems has been to limit the time of exposure by sending workmen into contaminated housings in relays. Such procedures run the risk of exhausting the permissible radiation allowance of personnel so that their availability for work in other contaminated areas of the plant is limited. Even in borderline cases, it is advisable to consider remote maintenance.

Specific recommendations on how remote maintenance should be accomplished cannot be made. Only a few truly remote systems have been built to date, and approaches to the problem have varied widely. The installations described below are representative and illustrate some of the problems and factors that must be given consideration in designing such systems.

\subsubsection{General Considerations}

Clamping devices and components (filters, adsorber cells) of remotely maintained systems are handled by special extended-reach tools; electromechanical manipulators; solenoid-, pneumatically-, or hydraulically-actuated devices; cranes; or other indirect means. In some systems (Figs. 9.1 and 9.2 and Figs. 9.5 through 9.14), filters are installed on a removable mounting frame that is replaced as a complete assembly by means of a crane. In three of the systems illustrated, the entire housing is replaced. In most cases, housings will be enclosed in concrete vaults or pits, with heavy concrete plugs to seal access ports. Designers must recognize that workmen do not have the close control over movement of tools, equipment, and components that they do in directaccess contact-maintenance systems. Careful attention must be paid to filter (adsorber) withdrawal and handling space, and, if alignment guides are not provided, access ports must be generously sized to permit the easy passage of components when handled by crane or manipulator. When filters and adsorbers are installed on a removable frame which is, in turn, sealed into a housing, heavier construction is needed 


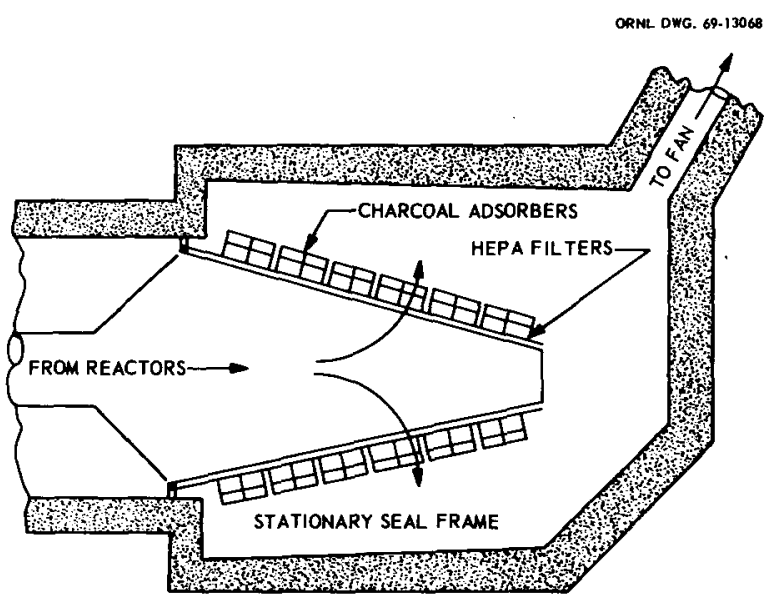

Fig. 9.1. Plan view of the bypass filter pit at Brookhaven National Laboratory. Each stack of eight HEPA filters and charcoal adsorbers is clamped to a removable mounting frame that in turn is clamped to the stationary seal frame, as shown in Fig. 9.2.

to prevent damage to the frame and to the sealing surfaces of the housing from inadvertent bumping of the removable frame against the stationary frame or the sides of the access port. Recommendations for the design and construction of concrete vaults and pits are given in ANSI N101.6, Concrete Radiation Shields. ${ }^{2}$ This standard includes recommendations for roof and penetration plug design, clearances, and tolerances.

In some systems, the contaminated filter or assembly of filters is withdrawn into a special cask or enclosure to permit safe transport through occupied areas of the building or plant to a disposal area. Building openings, areaways between buildings, and ground clearances for power lines and other utilities must be adequate to permit easy passage of the heavy shielded cask and the truck or trailer on which it is hauled. Underground pipelines along the route may have to be reinforced to prevent crushing under the load.

For hot cells, caves, and canyons, it is recommended that first-stage HEPA filters be installed at the duct opening and in a manner whereby they can be replaced by withdrawal into the contained space. In most cases this will permit contact maintenance on the second-stage filters installed downstream in the duct. Provision must be made for access to the firststage filters and for withdrawing them into the cell without interfering with process or experimental equipment in the cell.

It is often possible to design systems for both contact and remote maintenance, that is, to provide for contact maintenance when radiation levels are low and for remote access when radiation levels become high. This approach is particularly appropriate for reactor postaccident cleanup systems where radiation levels during normal operating conditions are well within personnel tolerances, but may be prohibitively high following an accident. The filters of such a semiremote system are held to the mounting frame in the conventional manner, but the mounting frame can be removed as a whole or as a segment, if necessary. Construction of the removable mounting frame and the stationary frame to which it seals must be precise to ensure a reliable and leaktight seal, and construction of both must be heavier than required for contact maintenance systems to withstand the rough treatment that they might receive during a remote filter or adsorber change.

Each step of a remote filter (adsorber) change, from initial dressing up of personnel in protective clothing to final disposal of contaminated components and decontamination of equipment and the area, must be carefully planned before system design is frozen. Overlooking any detail may complicate operations in the field and result in unduly high labor costs, spread of contamination, injury to personnel, or overexposure. Clearances, temporary storage of new and dirty components, equipment space, access to and from the area, decontamination procedures, radiation monitoring, utilities, and handling facilities must all be carefully examined. It is often desirable to build a model or full-size mock-up of the proposed installation to ensure that all factors have been considered. The mock-up or model can later be used for crew training.

\subsubsection{Brookhaven Reactor Bypass Filter System}

This system ${ }^{3}$ is installed in an unlined concrete pit (Fig. 9.1) which has removable concrete shielding blocks in the ceiling to provide access to the filters and adsorber cells. These components are installed on 12 removable multifilter mounting frames which, in turn, are sealed to stationary frames in the pit, as shown in Fig. 9.2. To change filters or adsorbers remotely, the shielding blocks over the stationary sealing frames are removed, the latches that clamp the removable frames to the stationary frames are released from above by means of extended-reach tools, and the removable frames are hoisted out by crane.

Radiation levels are low enough under normal operating conditions to permit direct access for filter 


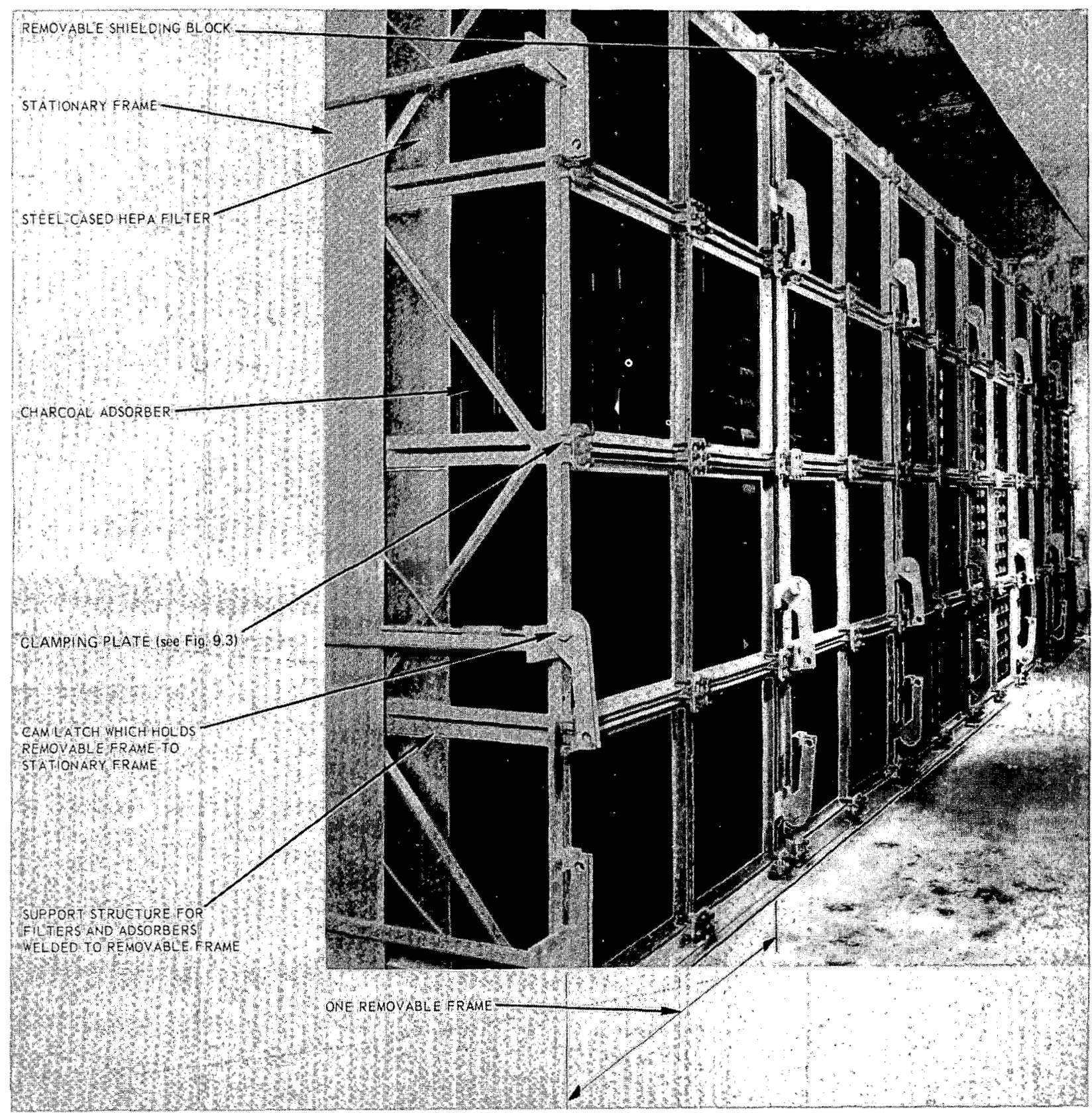

Fig. 9.2. General view of filter bank, Brookhaven bypass filter pit. Cam latches can be released from outside the pit by means of extended-reach tools, enabling the frame assembly to be hoisted out by crane through an opening in the ceiling. Note undesirable back-toback installation of filters and type I adsorber cells. Courtesy Brookhaven National Laboratory.

and adsorber replacement. Figure 9.3 illustrates one of the clamping-plate assemblies that hold these components to the removable mounting frames. The plate is bolted to the frame after the filters and adsorbers have been positioned in the support structures; the clamping screws are then tightened on the pressure-distribution rings. This type of clamping permits the readjustment of individual filters or adsorbers after installation, but does not permit replacement without upsetting the seals of surrounding components. The practice of clamping adsorber cells directly to the faces of the HEPA filters is generally not recommended; however, it does represent one of the compromises sometimes made in a remotely maintainable system. The coupling of filters and adsorbers complicates contact maintenance 


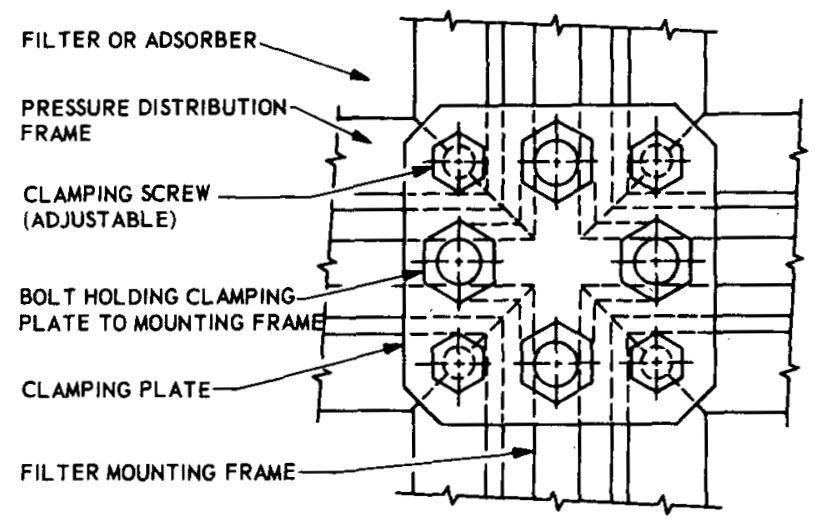

Fig. 9.3. Clamping plate assembly, Brookhaven bypass filter pit. Clamping pressure on individual filters is adjustable, but entire assembly must be removed to replace a filter.

because it is necessary to replace both filters and adsorbers at the same time and to remove the adsorber cells to get to the filters.

\subsubsection{Hanford Reactor Filter System}

The Hanford reactor air cleaning filters ${ }^{4}$ are installed in underground pits having the configuration shown in Fig. 9.4. Each compartment contains 36 moisture separators, 36 HEPA filters, and 36 pleatedbed adsorber cells installed on removable frames, as shown in Fig. 9.5. These components are changed by replacing the removable frames, as shown in Figs. 9.5

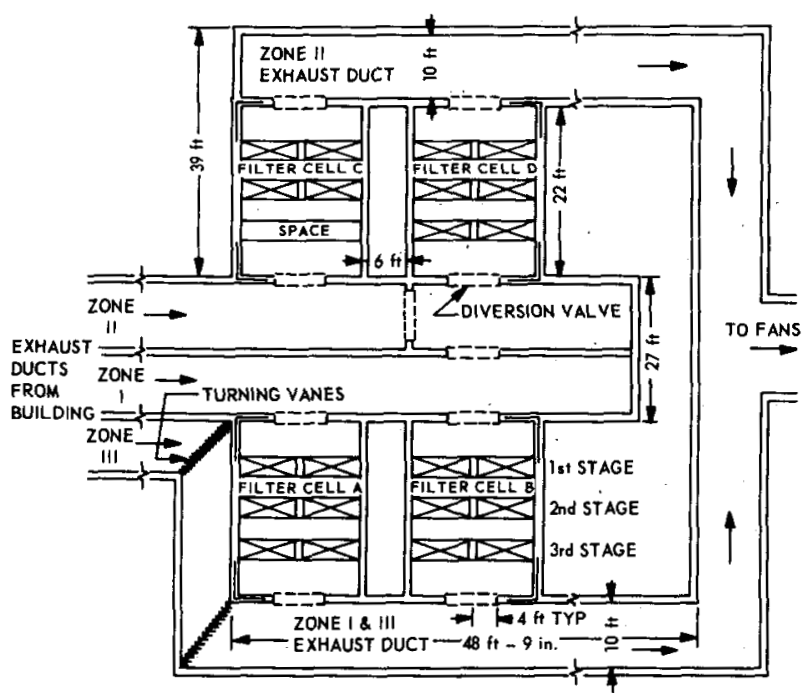

Fig. 9.4. Plan view of Hanford reactor filter system. First stage contains moisture separators, second stage contains HEPA filters, third stage (cells A, B, and D) contains pleated-bed charcoal adsorbers. Cells $A, B$, and $C$ are on-line, cell $D$ is normally held in standby. Courtesy ERDA, Richland Operations Office. through 9.14, which illustrate some of the problems of handling, space, and contamination control inherent in remotely maintainable systems. Radiation levels during the HEPA-filter change shown were not high enough to prevent direct access or to require burial of the contaminated mounting frame and its parts. The operation was constantly monitored (Fig. 9.13), and contaminated items were protected with plastic bags (Figs. 9.9 and 9.10) to minimize the spread of radioactive dust that might fall from the contaminated filters or frame during handling and storage. If this operation had been done after a major reactor accident, personnel would not have been allowed so close to the contaminated housing or filters, and the entire frame assembly, including filters, might have had to be disposed of as radioactive solid waste. The size of the mounting frame, approximately $22 \times 9 \times 3 \mathrm{ft}$, is indicative of the disposal problems that could be encountered.

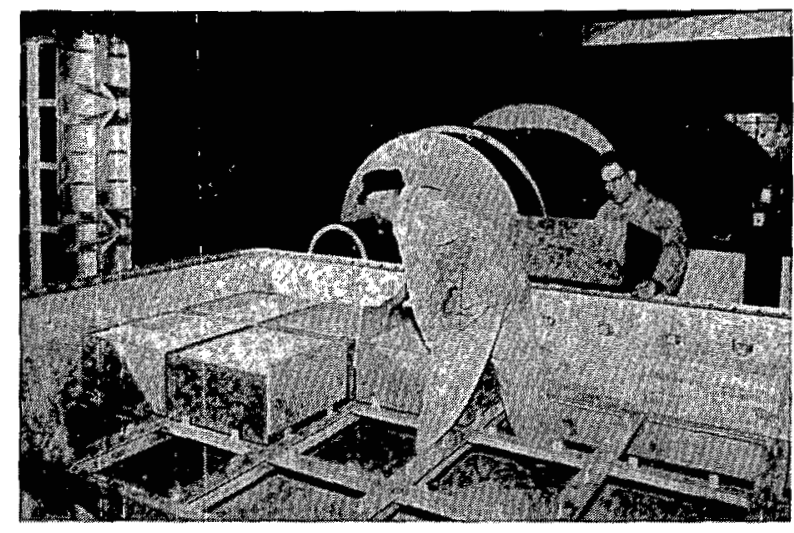

Fig. 9.5. Remote filter change, Hanford production reactors. Loading new filters in removable mounting frame. Courtesy ERDA, Richland Operations Office.

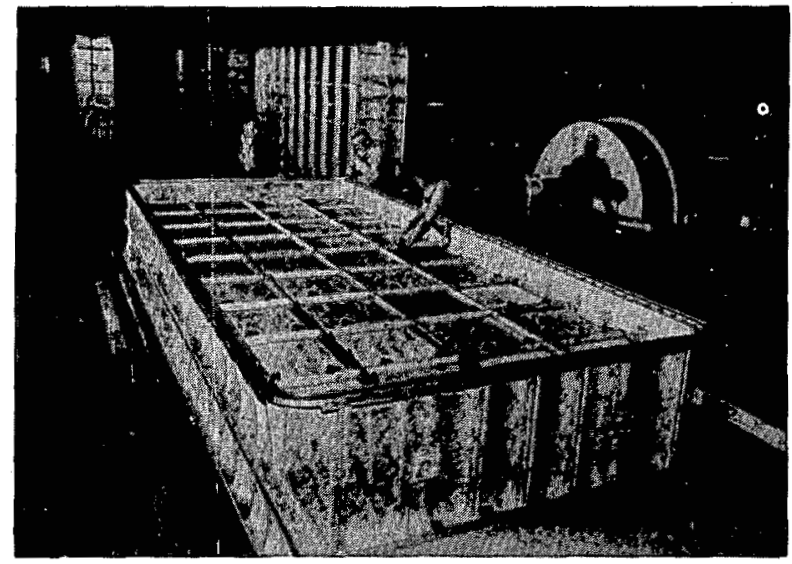

Fig. 9.6. Remote filter change, Hanford production reactors. Filter installation complete. Courtesy ERDA, Richland Operations Office. 


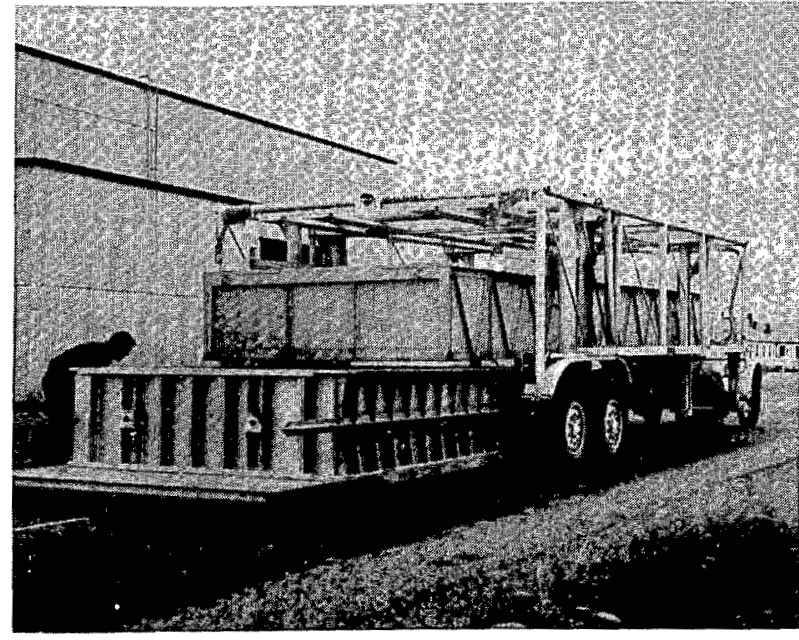

Fig. 9.7. Remote filter change, Hanford production reactors. Delivering new frame assembly to installation site. Note special trailer, protective box, and storage space required. Courtesy ERDA, Richland Operations Office.

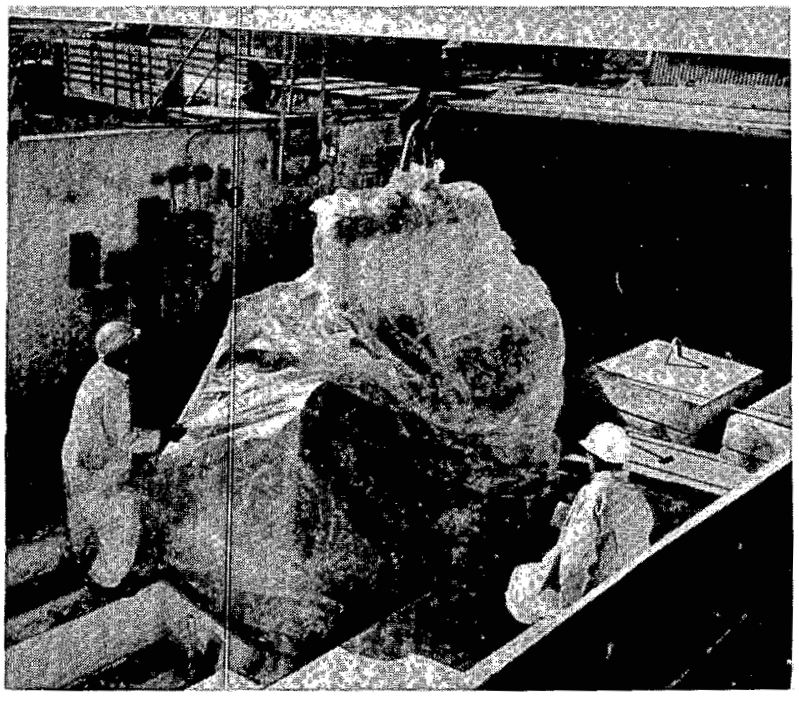

Fig. 9.9. Remote filter change, Hanford production reactors. Withdrawing contaminated frame assembly into plastic contamination shield. Courtesy ERDA, Richland Operations Office.

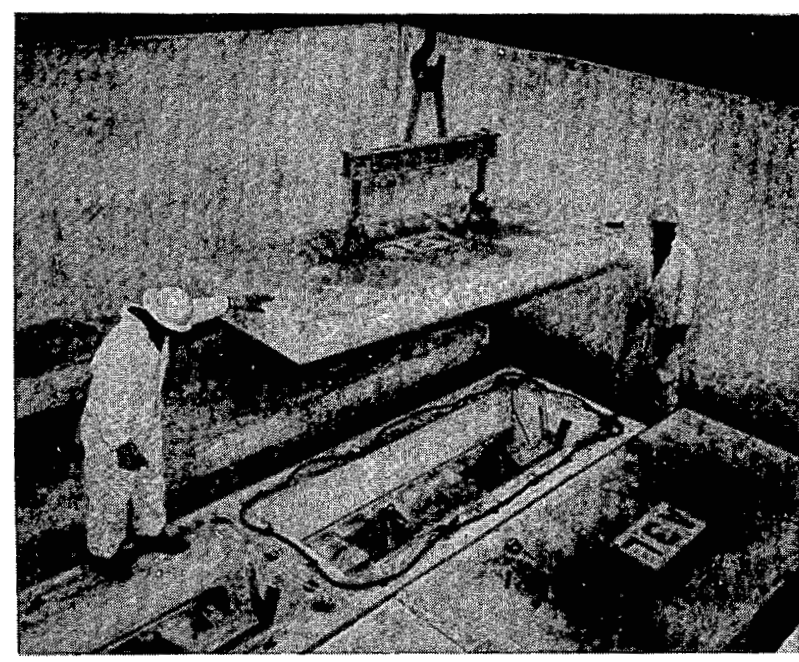

Fig. 9.8. Remote filter change, Hanford production reactors. Removing shielding blocks from filter pit. Note inflatable seal between block and pit. Courtesy ERDA, Richland Operations Office.

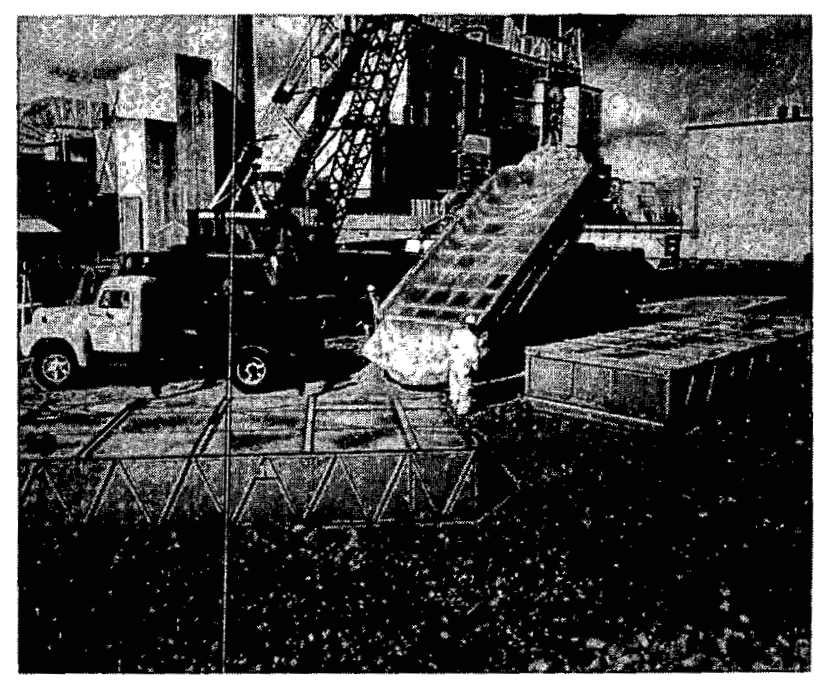

Fig. 9.10. Remote filter change, Hanford production reactors. Temporary storage of contaminated frame assembly. Note space required. Courtesy ERDA, Richland Operations Office. 


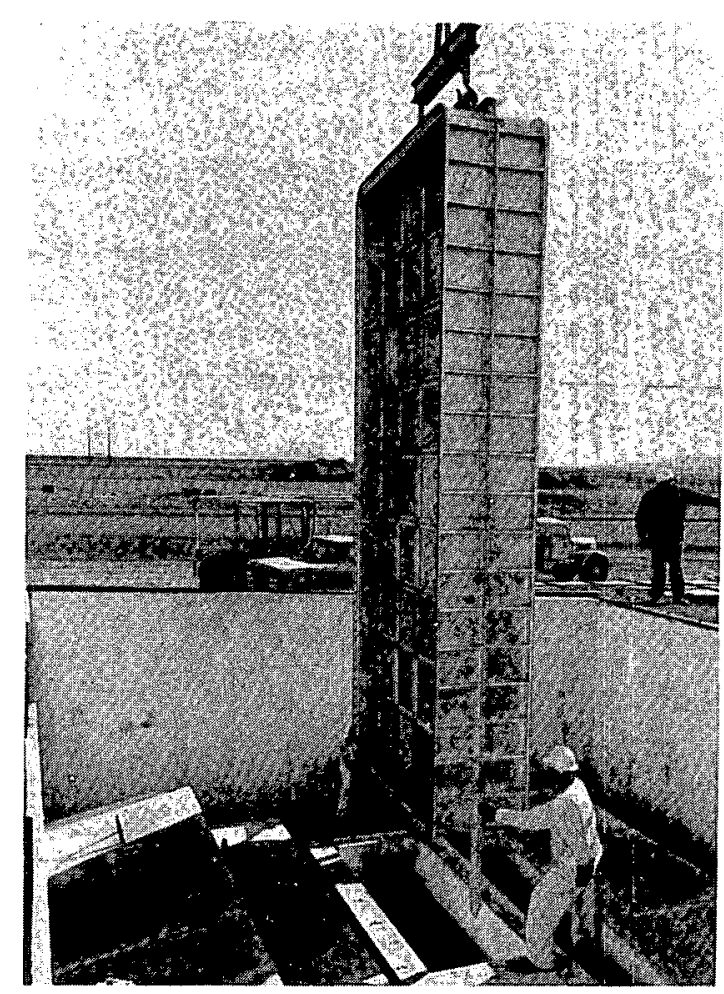

Fig. 9.11. Remote filter change, Hanford production reactor. Positioning new frame assembly over filter pit opening. Note alignment pins. Courtesy ERDA, Richland Operations Office.

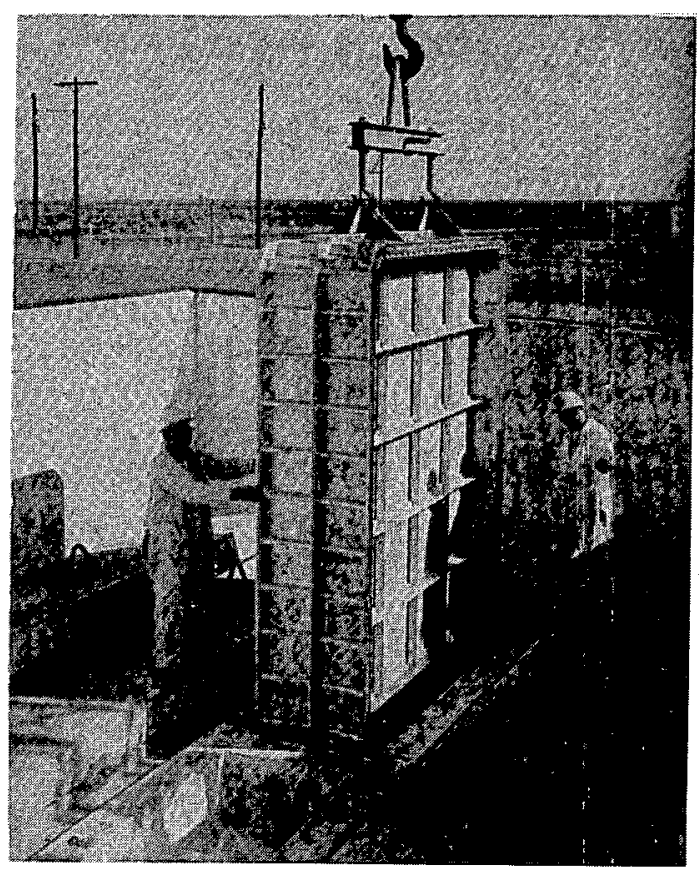

Fig. 9.12. Remote filter change, Hanford production reactor. Lowering new frame assembly into pit. Note lifting assembly. Courtesy ERDA, Richland Operations Office.

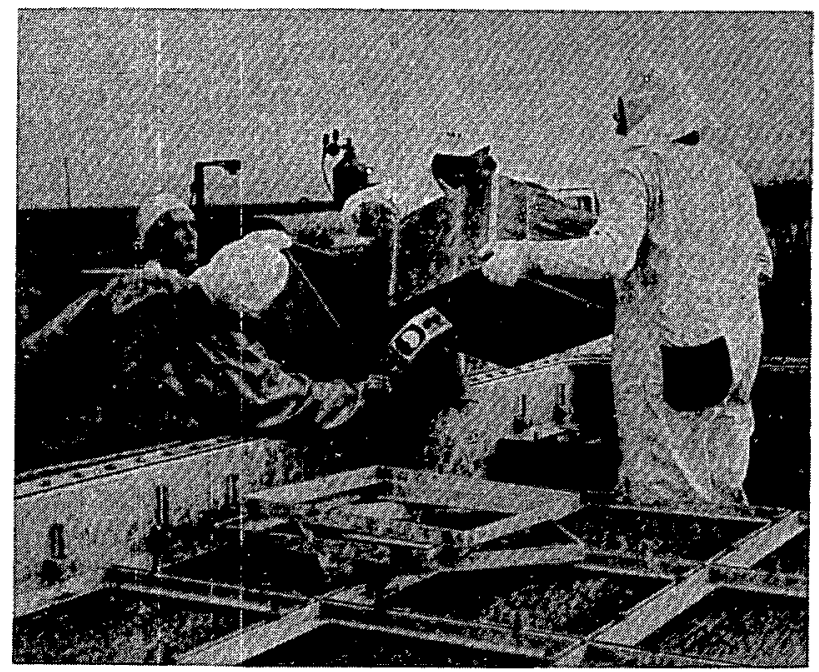

Fig. 9.13. Remote filter change, Hanford production reactor. Disassembling used frame assembly. Note radiation monitoring, protective clothing. Courtesy ERDA, Richland Operations Office.

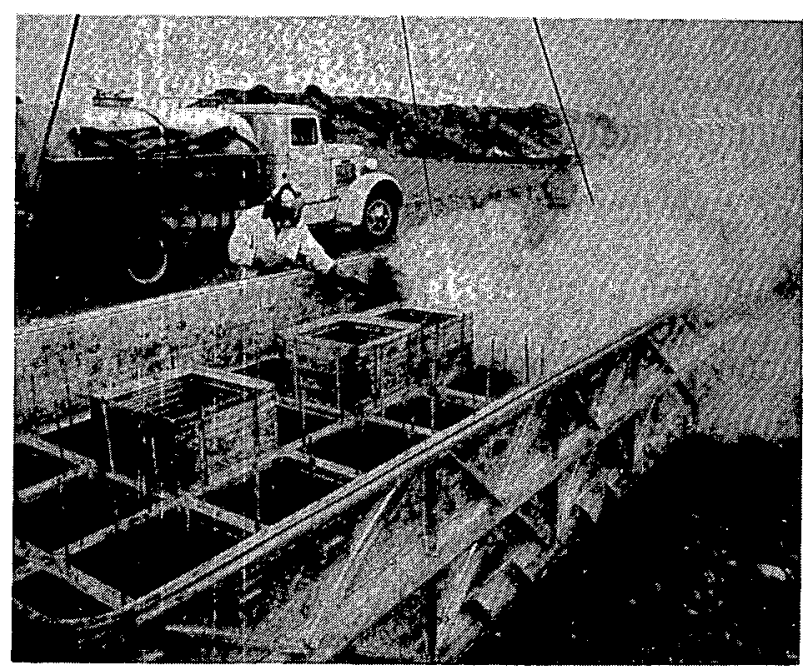

Fig. 9.14. Remote filter change, Hanford production reactor. Cleaning used frame assembly. Note portable steam supply, protective clothing. This type of cleaning is permissible only when contamination levels are very low. Had the frame been badly contaminated, it would probably have had to be buried. Courtesy ERDA, Richland Operations Office. 


\subsubsection{HFIR Filter System}

Figure 9.15 shows the remotely maintained underground air cleaning system of the High Flux Isotope Reactor (HFIR) at Oak Ridge National Laboratory, Oak Ridge, Tennessee. ${ }^{5}$ The small system on the right handles normal off-gas from the reactor and other point sources, and the larger system on the left treats the exhausted air from the continuously vented containment building. Two "filter trains" of each system are on-line at all times, with the third in standby. Each train consists of a prefilter, a HEPA filter, two pleated-bed adsorption stages, and a final HEPA-filter stage. Components in the 1000-cfm nominal capacity off-gas system are individual 1000 cfm units. Components in the building ventilation system are banded together in stacks of three with stainless steel strapping, as shown in Fig. 9.16, using a commercially available banding device. There are four sealing faces in each of the stationary frames in each pit; a stack of filters (adsorber cells) is installed by forcing it against the stationary frame by means of the removable wedge installed between the back side of the filter stack and the stationary wedge. Bottoming lugs on the stationary wedge prevent overstressing the filter cases. The stacks of filters and the removable wedges are installed and removed by means of a crane.
This type of clamping system calls for a high degree of accuracy in installation and adherence to very close fabrication tolerances. The stationary frame is made from square tubing and the wedges from $3 / 8$-in. plate. The flatness and parallelism of the stationary frame and seating surfaces of the wedges must be within $\pm^{1 / 16}$ in. in $6 \mathrm{ft}$ and preferably closer. The spacing between the sealing face of the stationary frame and the stationary wedge is critical and must be maintained within $\pm^{1 / 32}$ in. of specified values. Such tolerances are difficult to maintain during construction, and the mounting frame or wedge can be knocked out of tolerance by careless handling during a filter (adsorber) change.

\subsubsection{Savannah River Reactor Filter System}

This system ${ }^{6}$ differs from the preceding systems in that the entire filter house is removed and disposed of if it becomes contaminated. Each housing contains a bank of 20 moisture separators, 32 HEPA filters, and 32 pleated-bed carbon-filled adsorber cells. The complete system consists of five once-through housings, four normally on-line and one in standby. The housings are mounted on railroad trucks that run on rails to the edge of the roof of the reactor building. Isolation dampers are installed in the building, as shown in Fig. 9.17, and the housing (Fig. 9.18) is

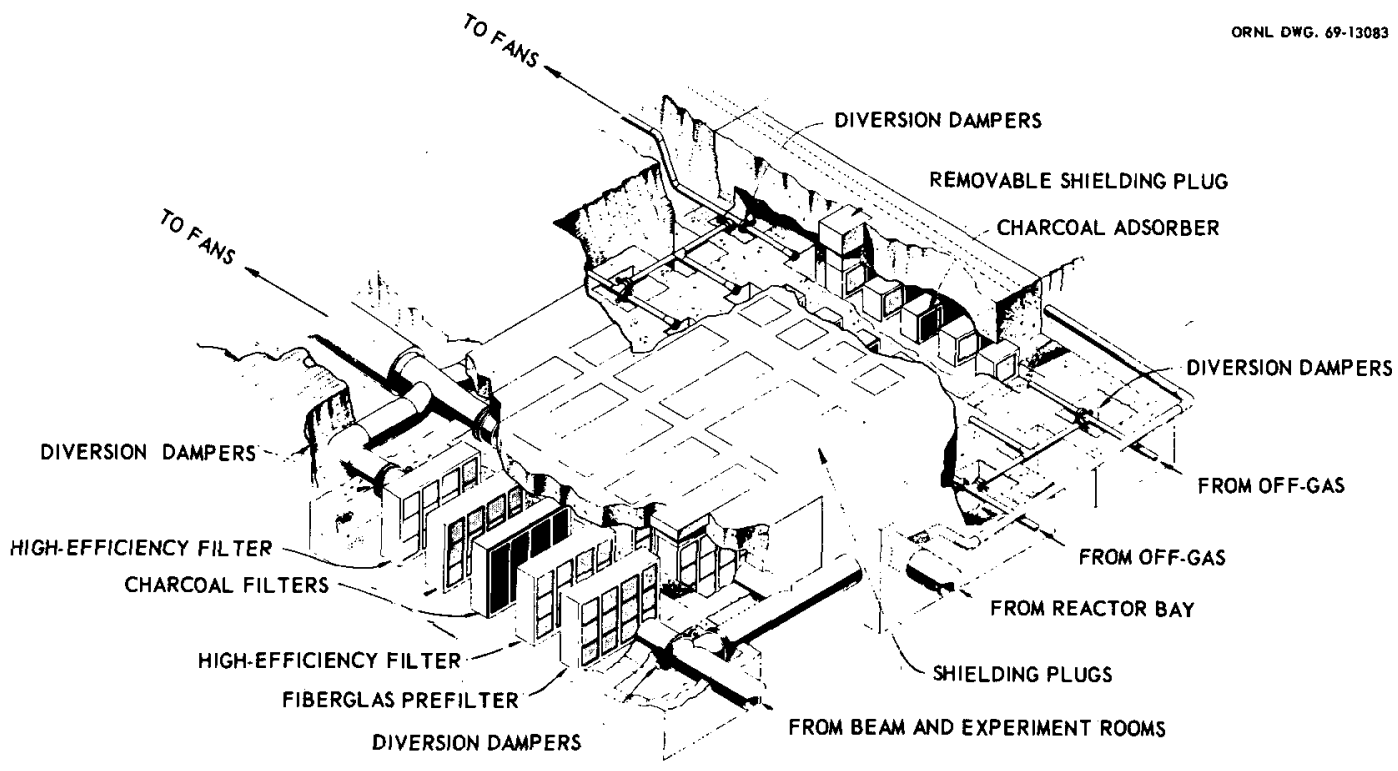

Fig. 9.15. General arrangement of underground filter pits at High Flux Isotope Reactor, Oak Ridge National Laboratory. Demisters (not shown) are located in the ducts leading to the pits. Courtesy Oak Ridge National Laboratory. 


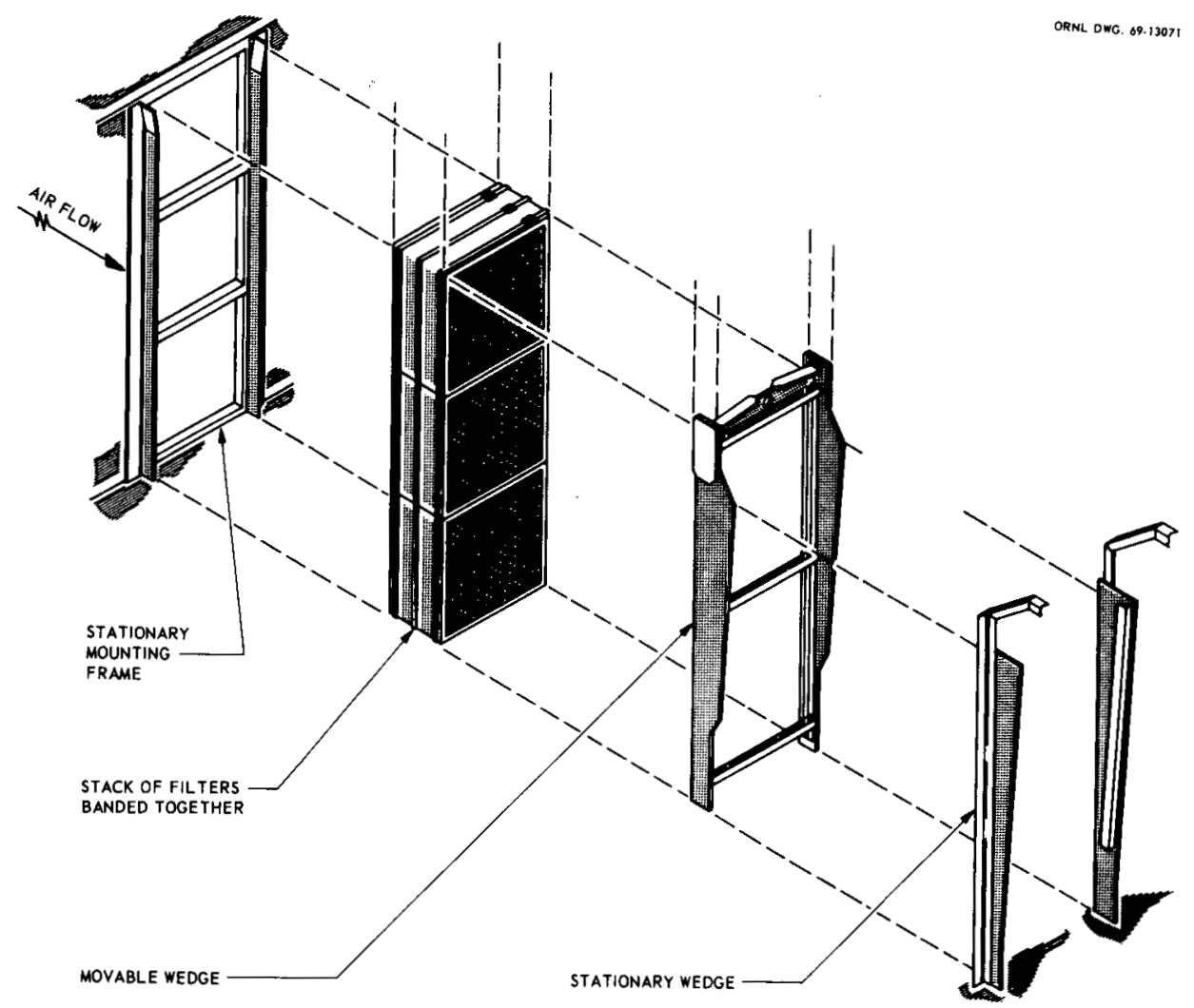

Fig. 9.16. Exploded view of filter clamping method, High Flux Isotope Reactor filter system. Actual distance from face of stationary mounting frame to stationary wedge is approximately 15 in.

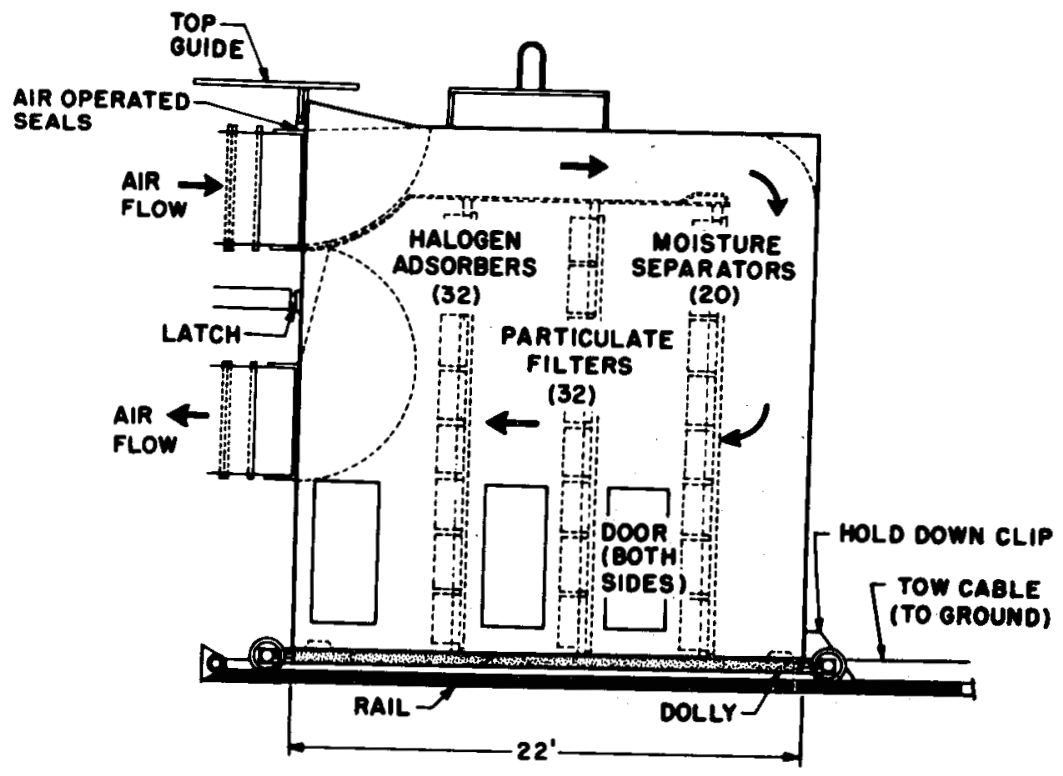

Fig. 9.17. Section through Savannah River Reactor confinement system filter compartment, as installed on roof of reactor building. From J. W. Little, Jr., and J. W. Joseph, Jr., "Confinement of Airborne Activity from Melted Antimony Slugs," Proc. /2th A EC Air Clean. Conf., USAEC Report CONF-720823, January 1973. 
sealed to the building by means of an inflatable pneumatic seal. To remove a housing, it is released and drawn away from the building by means of controls installed outside of the building at ground level. It is then lifted by crane and lowered to a railroad siding or truck trailer on the ground. Isolation valves and clamps holding the housing to the building are controlled either from the reactor control room or from the local station outside of the building.

Radiation levels are low enough under normal operating conditions to permit contact maintenance. Access doors in the opposite side of the housing shown in Fig. 9.18 permit entry to each chamber. Components are clamped to the mounting frames by a conventional nut-and-bolt arrangement. In November 1970, when an irradiated antimony source rod overheated while suspended in air in one of the reactors, about $6250 \mathrm{Ci}$ was carried by the building ventilation air to the confinement filters. Of this amount, only $3 \mathrm{mCi}$ escaped from the building exhaust stack, indicating a $99.999 \%$ capture efficiency for the filters. ${ }^{7}$ The four units (housings) on-line at the time of the accident were continued in service

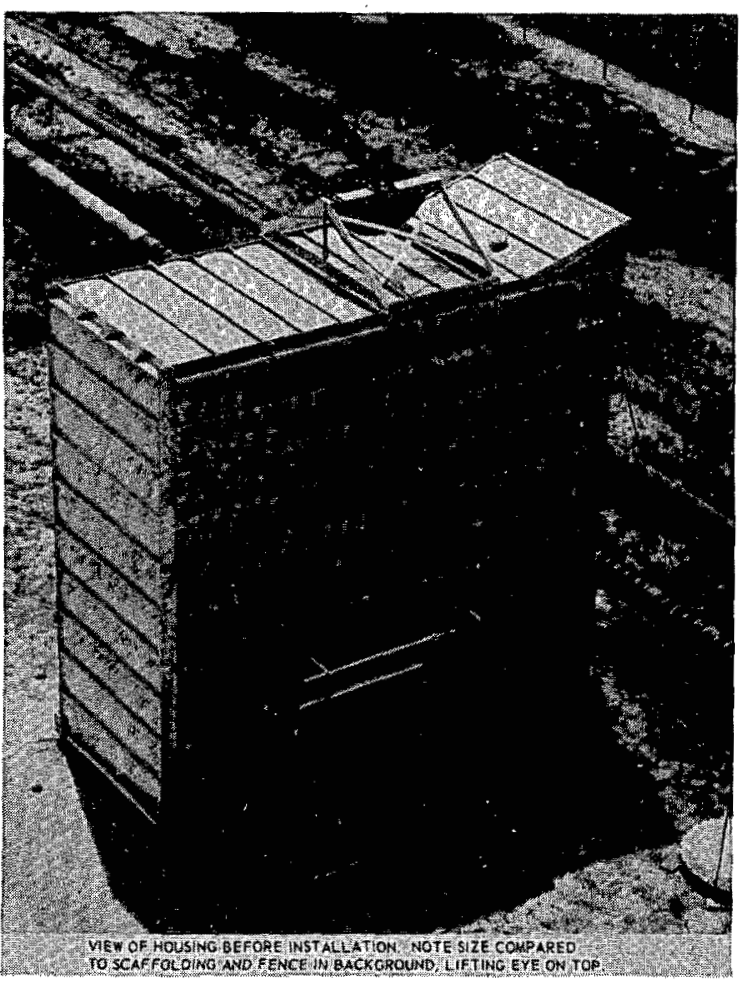

Fig. 9.18. Photo of Savannah River Reactor confinement system filter compartment. Note size relative to fence and scaffolding. during the next three months while the reactor bay was held for fission-product decay and decontamination. The units were then removed from the building by means of remote handling procedures, lowered to a modified railroad car, and moved to an aboveground storage area where they remain. Before removal from the building, the compartments were filled with expanding urethane foam to fix any loose contamination and to provide a seal if the compartment flapper doors failed to seal. The compartments were removed from the roof by a 7-ton motor crane equipped with a shielded cab, a 120-ft boom, and a special remote-handling hook with long tag lines. The most critical crane operation was lifting the compartment straight up off the roof; the crane operator, located at the base of the building, could not see the load and had to depend on two spotters with binoculars and short-wave radios for directions. One spotter was located on an adjacent roof and the other in a cherry picker attached to the crane. The maximum exposure of the crane operator was 200 $\mathrm{mR} / \mathrm{hr}$. The train that moved the compartments to the storage area was made up of the loaded car, three spacer cars, and a locomotive. The train was preceded by a track motorcar occupied by personnel who visually inspected the track, positioned the switches, and opened security-fence gates. A second track motorcar followed the train to restore switches to the normal position and to carry a health physicist who surveyed the track to verify that no contamination was released. The maximum exposure to the train crew was $30 \mathrm{mR} / \mathrm{hr}$. The contaminated compartments were removed from the train by a 100 -ton crawler crane with a 65 -ft boom and a special remotehandling hook. Exposure to the crane operator was less than $400 \mathrm{mR} / \mathrm{hr}$. The movement of each load to the storage area took $1 \mathrm{hr}$, and removal and replacement of the contaminated compartments took four days. "In the design of containment systems, much thought is given to the cleanup if gross radioactive contamination occurs. Viewed from this aspect, the aftermath of the accident is a success story for a good containment system that was severely tested."

\subsubsection{Remotely Maintainable Fish Filter System ${ }^{8}$}

Figure 9.19 shows a series-segmented, remotely maintainable air cleaning facility intended for installation in a shielded vault or underground pit. The interconnecting ducts have tapered-back flanges and are sealed with $\mathrm{V}$-band or ring-clamp couplings that 


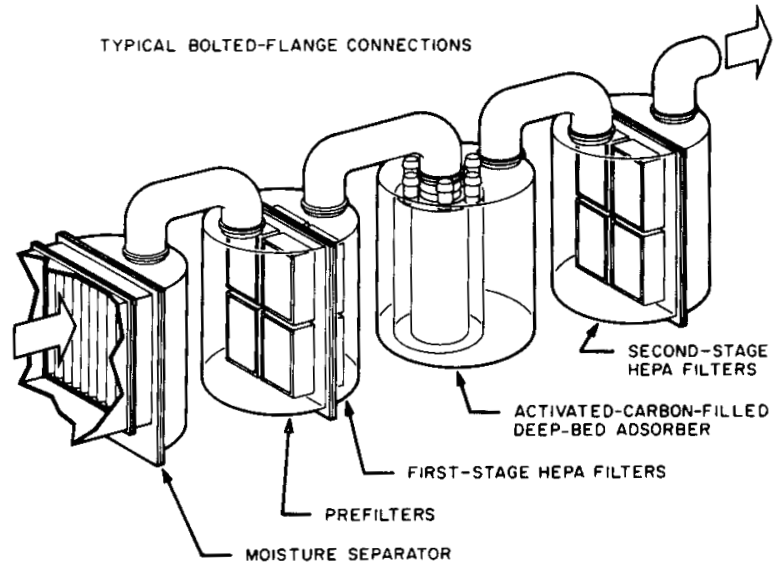

Fig. 9.19. Fish remotely maintainable tub system. Courtesy J. F. Fish, American Air Filter Co.

can be operated by a reach rod or extended-reach tool from outside of the vault or pit. After disconnection, the tubs can be lifted out and replaced by crane, similar to the Savannah River procedure but on a smaller scale. If necessary, contaminated tubs could be withdrawn into a handling cask in the manner described in the next section. Although not shown in Fig. 9.19, the addition of isolation dampers or flapper valves to seal the individual tubs after disconnection would be a simple matter. Fire protection of the carbon-filled deep-bed adsorber is also a simple matter in this design, since it would require no more than filling the tub with water. Being modular, the design offers substantial flexibility and can be adapted to a wide range of application requirements. Individual tubs would be installed in separate concrete shielding vaults and would be easily handled by an overhead crane. The simplicity of the design makes it readily adaptable to remote handling procedures.

\subsubsection{Remotely Maintainable TURF Filter System}

This system is installed in a radiochemical plant, the Thorium-Uranium Recycle Facility (TURF) at Oak Ridge National Laboratory, Oak Ridge, Tennessee. ${ }^{9}$ The filters are installed in a steel enclosure sealed to the building exhaust system ducts by means of the spring-loaded bellows assemblies shown in Fig. 9.20. The bellows are contracted by the hydraulic cylinders to release the enclosure and then returned to the seal position by the springs. The springs also impose a continuous pressure on the gasket of the enclosure while it is in position. An overall view of a similar installation is shown in Fig. 8.9. Each housing contains a bank of three prefilters in series with a bank of three HEPA filters. To replace filters, the isolation valves located in the ductwork are closed, the shielding block is removed, the portable shielded carrier (Fig. 9.21) is moved into position, and the

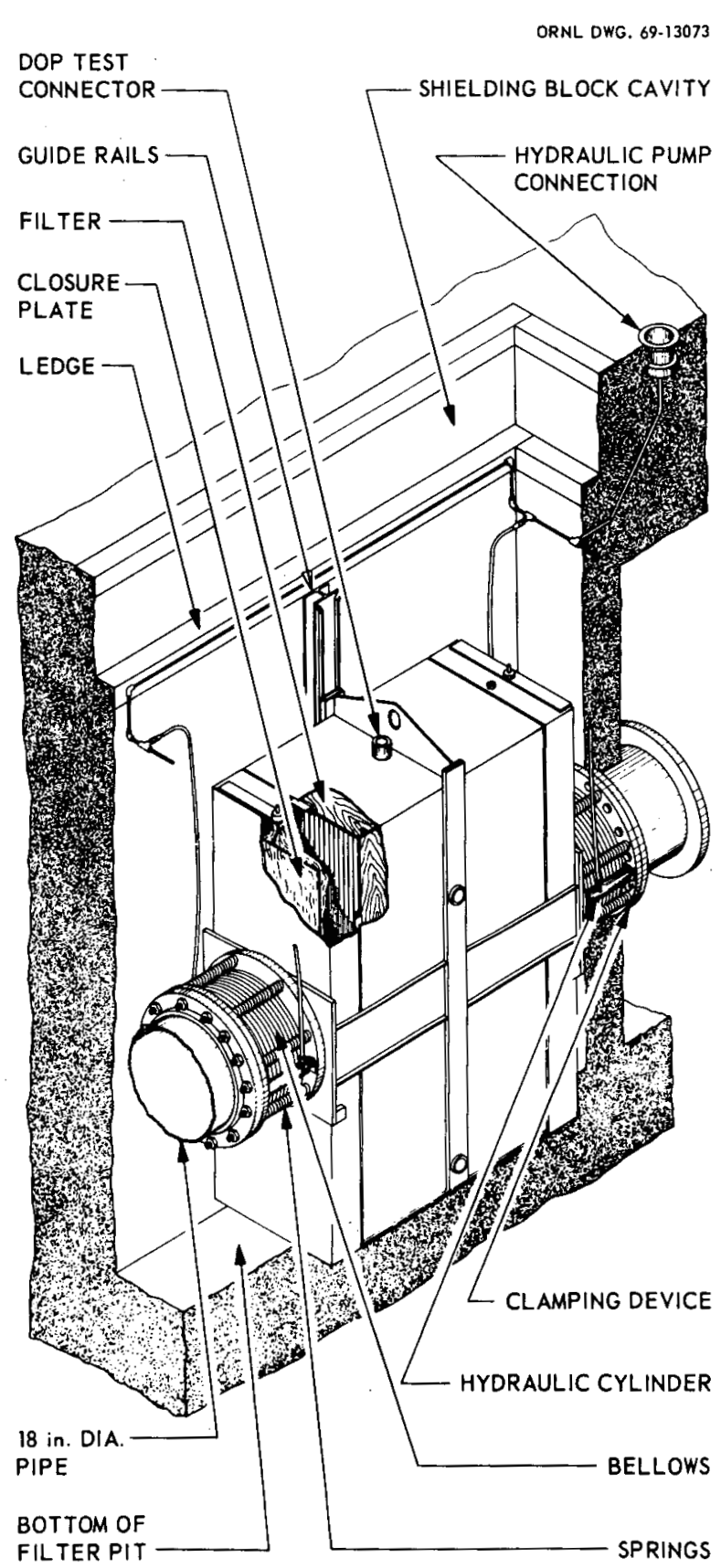

Fig. 9.20. TURF remotely maintainable filter housing, as installed. Filter pit shielding block removed. 


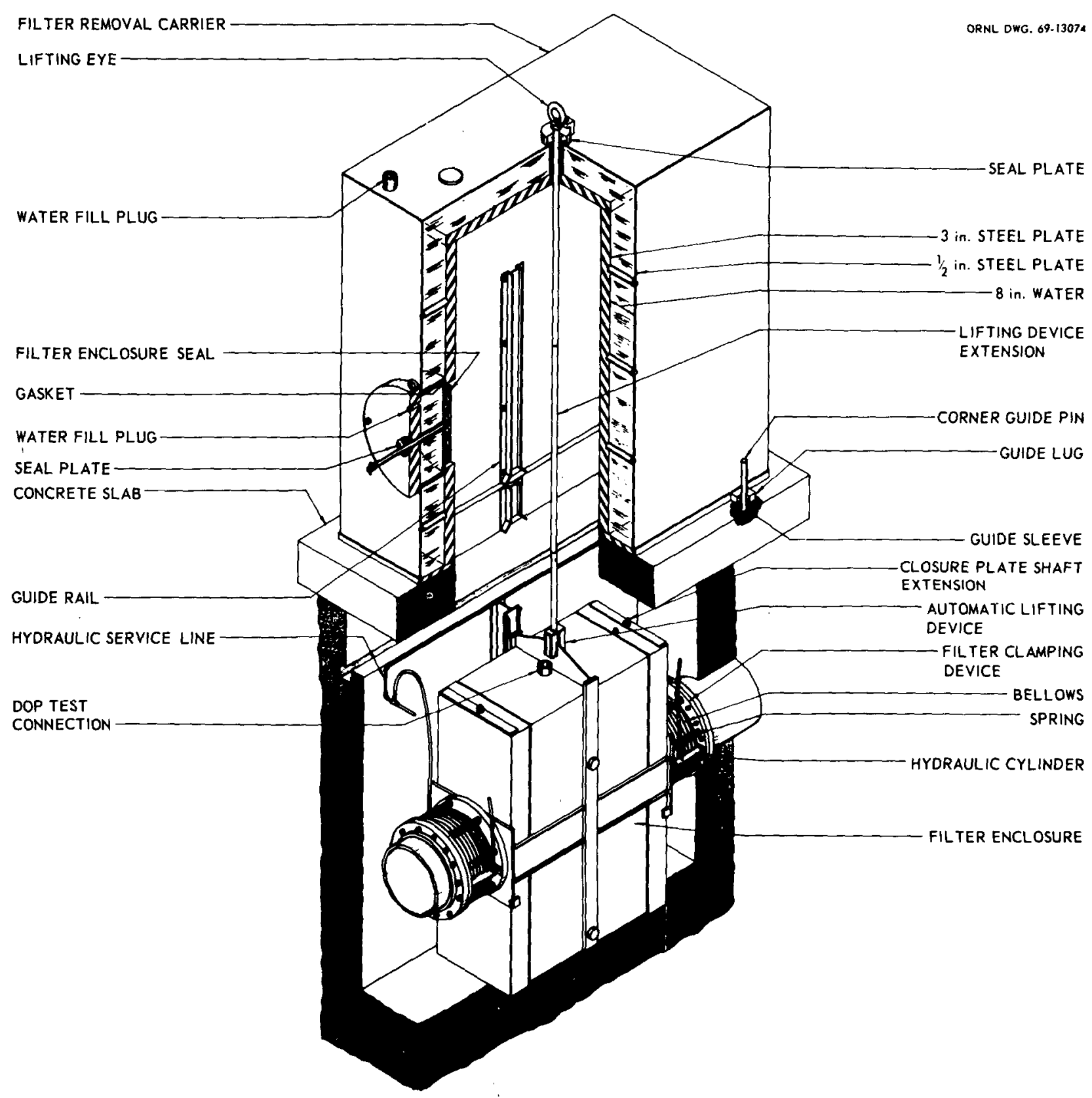

Fig. 9.21. TURF remotely maintainable filter installation with carrier-cask positioned in preparation for removal.

closure plates (Fig. 9.20) are dropped by means of an extended-reach tool. The bellows seals are compressed hydraulically to release the housing, a lifting rod (Fig. 9.21) is hooked into the eye of the housing, and a mobile crane hoists the enclosure into the carrier. After the bottom plate is installed on the carrier, the assembly is lifted to a truck-trailer for removal to the burial ground. The housing is not salvaged.

\subsubsection{Remotely Maintainable HWESF Filter Assembly ${ }^{10}$}

Figure 9.22 shows one of the remotely replaceable filter housings for the Hanford Waste Encapsulation 
and Storage Facility (HWESF) at Richland, Washington. This is one of a pair of redundant type 304-L stainless steel housings, each of which contains a bank of six prefilters and two series banks of six (each) HEPA filters. The housings are approximately $5 \times 7 \times 16 \mathrm{ft}$ long and seal to the ductwork by means of the saddle-and-wedge arrangement shown in Fig. 9.23. Saddle assemblies are seal-welded to each duct opening, and wedge assemblies are bolted to flanges

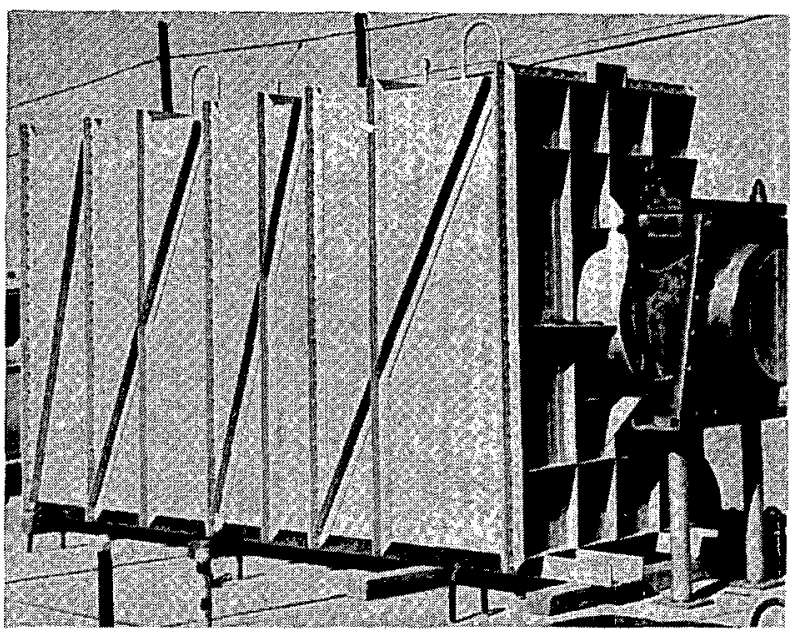

Fig. 9.22. Housing of HWESF remotely maintainable filter installation, Richland, Washington. Note wedge-and-saddle duct connector at right. at each end of the housing. To install a housing, a gasket is dropped into each saddle and the housing is lowered so that the wedges engage the saddles. Captive nuts on the wedges engage bolts on the top surfaces of the saddles and are tightened to force the wedges down and compress the gaskets. A breakaway bolt, engaging a fixed nut on the upper surface of the wedge (Fig. 9.23b), is provided to release the wedge when the housing is to be removed. If heavily contaminated (loadings up to $30 \mathrm{MCi} \mathrm{Sr}-90$ and 30 $\mathrm{MCi}$ Cs-137 may be expected on the first-stage HEPA filters), the entire housing can be discarded. The system is designed to seismic category I.

\subsubsection{Hot-Cell Filter Systems}

First-stage exhaust filters that are installed from inside a hot cell require no special shielding; however, careful planning of filter-change procedures is required to avoid disruption of operations and interference with equipment installed in the cell. Figure 9.24 shows a typical prefilter-HEPA filter installation. The filters are clamped in place with special wing nuts to facilitate handling by the electromechanical manipulators. To remove contaminated filters, the wing nuts are removed and the filters are picked up by the manipulator, placed in a plastic bag or shielded container, and positioned beneath the cell access port preparatory to removal
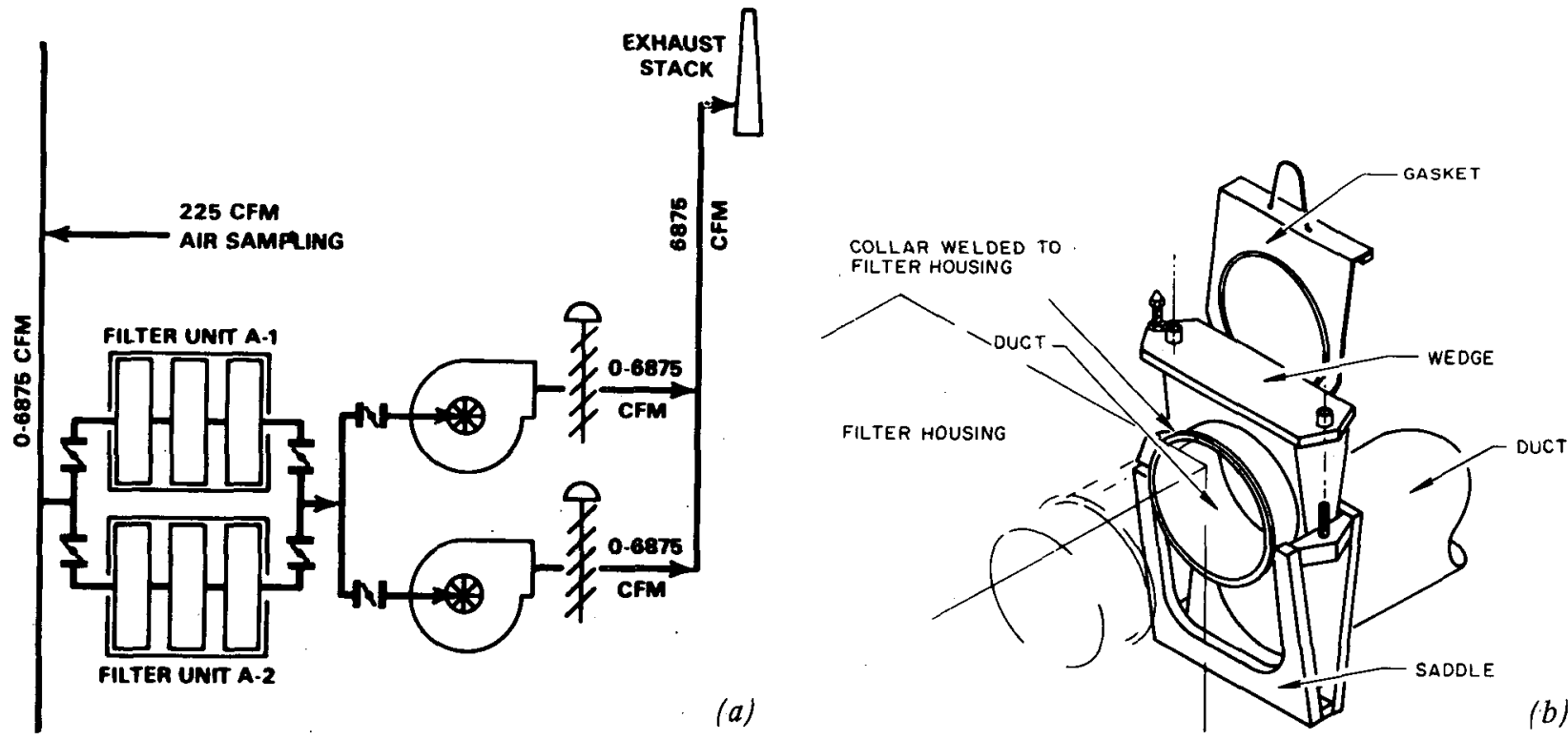

Fig. 9.23. General arrangement and duct-connector details, HWESF remotely maintainable filter system. (a) Schematic of HWESF remotely maintainable filter system. $(b)$ Wedge-and-saddle duct connector. Wedge assembly bolted to flange of housing, saddle assembly seal-welded into duct. This detail is reversed from the view shown in Fig. 9.22. From E. D. Rice and C. G. Caldwell, "Waste Encapsulation and Storage Facility Ventilation Facility," Proc. 12th AEC Air Clean. Conf., USAEC Report CONF-720823, January 1973. 


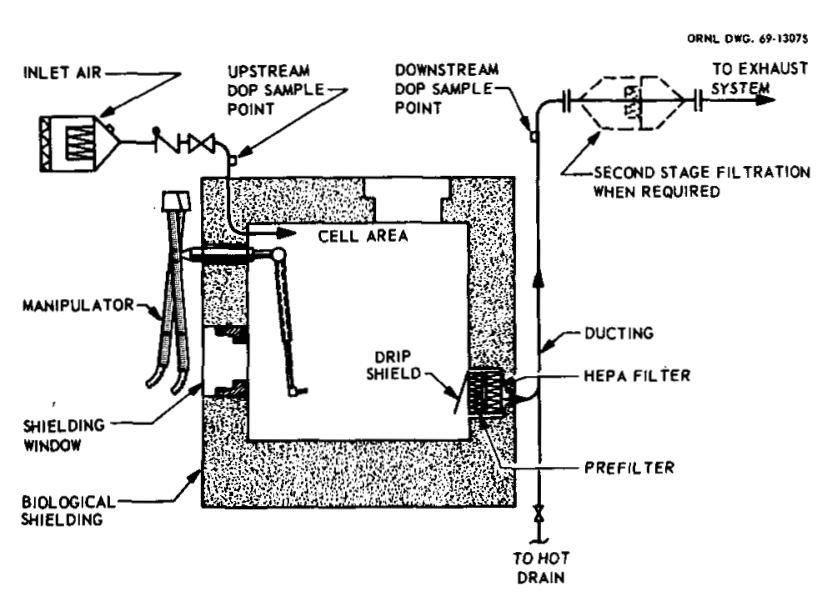

Fig. 9.24. Hot-cell filter installation. First-stage filters replaced remotely from inside of cell, using manipulators. Second-stage filters replaced by contact service techniques.

by means of a hoist or extended-reach tool. Filter installation design requirements for this type of installation are discussed in Chap. 6. Second-stage filters are installed outside of the cell, often in a caisson-type enclosure for bag in, bag out maintenance.

Figure 9.25 shows a hot-cell first-stage HEPA filter that is changed from outside the cell. This is an "incessant" filter installation in which the old filter is pushed out of position by the new filter as it is slid into place, thereby keeping the duct opening essentially closed at all times during a filter change. Contamination levels in this installation are high, and the assembly is heavily shielded with lead. A filter is replaced by positioning the lead-shielded carrier (Fig. 9.26) at the discharge end of the housing, removing the shielding doors, and pushing in a new filter. When the contaminated filter is completely inside the disposal can (Fig. 9.26), the door of the carrier is closed and the doors of the housing are replaced. The filters have gaskets on both faces and seal in place by the interference fit between the gaskets and the mating sealing surfaces inside the housing. This is not a highly reliable method of sealing HEPA filters. The installation is costly and requires considerable manpower (three to five man-days) to effect the change of a single $1000-\mathrm{cfm}$ filter. The mechanical features of filter changers of this general type have presented considerable problems at some sites, with the result that the changers are often operated by opening both ends of the housing, removing the old filter by hand, and pushing the new filter in by hand. Incessant filter installations are much overrated.

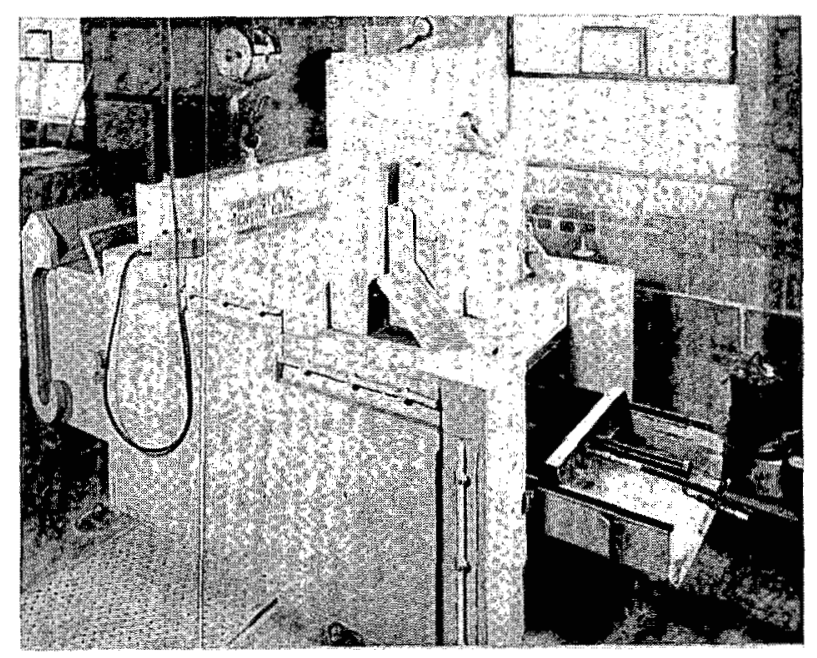

Fig. 9.25. Hot-cell first-stage filter housing designed for semiremote maintenance.

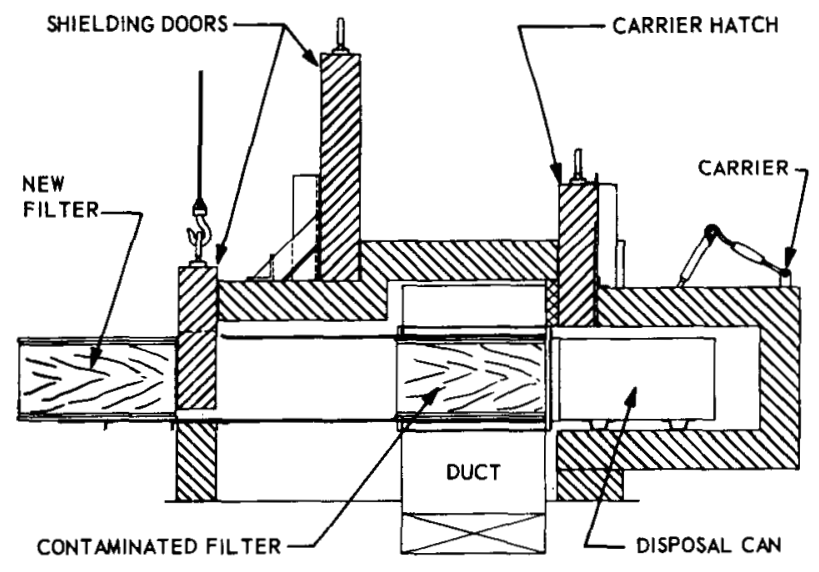

Fig. 9.26. Section through Argonne "incessant" filter changer showing method of operation. When new filter is pushed into position, the old filter is pushed into disposal can in carrier.

\subsection{SHIELDING}

Ducts and housings may have to be shielded when gamma radiation "shine" exceeds exposure limits specified by federal regulations. ${ }^{1}$ Levels as high as $1000 \mathrm{rems} / \mathrm{hr}$ may be expected at first-stage filters serving fuel reprocessing or radiochemical operations. Radiation must be reduced to tolerable levels if personnel are to occupy, even occasionally, adjacent areas of the building. Exhaust ducts and housings may have essentially the same hazard classification (Sect. 2.2.1) as the contained space (i.e., glove box, hot cell, building space, containment) they evacuate, and should, therefore, be installed inside building spaces that provide some degree of secon- 
dary containment. When such building spaces are occupied, even infrequently, shielding must be provided if gamma radiation is, or could be, a problem. Requirements for and the design of shielding are described in several references, including:

Reactor Shielding for Nuclear Engineers, USAEC Report TID-25951, 1973 (available from National Technical Information Service, Springfield, Va.).

Engineering Compendium of Radiation Shielding, International Atomic Energy Agency, SpringerVerlag, New York, Berlin, vol. 1, Shielding Fundamentals and Methods, 1968; vol. 2, Materials, 1975; vol. 3, Shield Design and Engineering, 1970.

Current information on shielding, including computer codes, can be obtained from the Radiation Shielding Information Center, Oak Ridge National Laboratory, Oak Ridge, Tennessee. Recommendations on the construction of concrete radiation shields can be found in ANSI N101.6. ${ }^{2}$

\subsection{NATURAL PHENOMENA}

The ability of a system to survive and function during and/or following an earthquake or tornado must be taken into consideration in the design of ESF air cleaning systems. Such systems, by definition (Chap. 1), must be capable of withstanding the effects of a design basis earthquake or tornado and still remain operable and capable of performing their design functions.

\subsubsection{Earthquake}

The problem of earthquake arises from the possibility of malfunction of fans, dampers, filters, or other functional components of the system, or rupture or structural damage of pressure-boundary components (ducts, housings, fan or damper casings) when the system is subjected to rapid, violent, repetitive shaking or dislocations, either as a lumped mass or as parts of the assembly are dislocated independently relative to one another. Fortunately, the physical masses of air cleaning system components are generally small relative to the massive concrete building elements to which they are anchored; if natural frequencies are greater than about $30 \mathrm{~Hz}$ and the parts of any single air cleaning unit (as defined in Appendix D) are anchored to the same building element, a satisfactory earthquakeresistant air cleaning system can be achieved fairly easily. Problems arise when portions of the same air cleaning unit (e.g., different segments of the ductwork) are anchored to different building elements that can vibrate independently. An approach to the design and to design qualification of earthquake-resistant air cleaning systems is suggested in Appendix D.

\subsubsection{Tornado}

The effects of a tornado manifested in structural damage may arise from missiles, wind, or atmospheric pressure changes that occur when the funnel cloud passes over the building. Assuming that the building is of tornado-resistant construction, damage to the air cleaning system will result mainly from pressure changes that occur in the stack, ducts, and building spaces surrounding the ducts. The current design basis tornado (DBT) hypothesizes that pressure on the building will decrease by as much as 3 psi over a 2-sec period, remain at the depressed level for $3 \mathrm{sec}$, then return to normal. ${ }^{11}$ Because operation of a ventilation system is highly dependent on stable atmospheric conditions to maintain pressure differentials between containment zones of a building and to prevent the release of contaminants, it is likely that system upset, overrunning or reversal of fans, or even reverse flow could occur due to the atmospheric depressurization; failure of dampers could accentuate the condition. ${ }^{12}$ On the other hand, stack, ducts, and fans would attenuate the depressurization, and it is unlikely that filters in the exhaust system would experience pressure differentials hypothesized by the Regulatory Guide. ${ }^{1}$ The studies of Anderson and Anderson ${ }^{13}$ and W. S. Gregory ${ }^{14}$ indicate that HEPA filters that meet requirements for nuclear service, unless they have seriously deteriorated, are capable of withstanding any pressure differential they are likely to experience under tornado conditions. The effects of high airflow rates, large pressure differentials, and sustained pressurization or depressurization on air cleaning systems and components are relatively unknown. A study is under way to mathematically model the dynamic effects of tornados and pressure transients on air cleaning and ventilation systems, and to develop methods for describing, analyzing, and calculating the forces to which these systems would be subjected, along with their response to these forces. ${ }^{12}$

Still lacking, however, are solid data on the characteristics of tornados. Investigations indicate that the design basis characteristics presently specified ${ }^{11}$ may be too severe. Studies indicate that 
the maximum wind speed of a tornado probably does not exceed $225 \mathrm{mph}$, and that the maximum depressurization may be not more than $10 \%$ of an atmosphere $(\sim 1.5 \mathrm{psi}){ }^{15,16}$ These pressure deficiency values are consistent with preliminary results of a study being conducted for ERDA's Oak Ridge, Tennessee, facilities. ${ }^{17}$ There is an indication that the most destructive tornados are the more rapidly moving ones, which implies that the 5-mph translational speed (which controls the period over which the maximum pressure deficiency is applied) of Regulatory Guide $1.76^{11}$ is probably too conservative. ${ }^{18}$ Preliminary results of the Oak Ridge study confirm this and indicate a maximum depressurization rate of only $0.5 \mathrm{psi} / \mathrm{sec}$ as compared with $2.0 \mathrm{psi} / \mathrm{sec}$ in Regulatory Guide $1.76 .{ }^{17}$ The Regulatory Guide recognizes that the statistical frequency and severity of tornados vary from one part of the country to another and provides guidance for the application of wind speed and pressure values in particular locations. ${ }^{11}$

\subsection{FIRE AND SMOKE PROTECTION}

\subsubsection{Operating Procedures}

Operating procedures are the first line of defense against filter failure due to fire. The possibility of fire is sometimes overlooked by the designer because it is assumed that, with fire-resistant filters and steel ducts, there is nothing to burn. This is a misconception. The dust accumulated in ducts and collected in the filters is often highly flammable, and even a fireresistant filter can be destroyed by sparks, by flaming trash carried into the housing, or by burning dust. Heavy smoke accumulations can cause plugging and subsequent rupture of both prefilters and final HEPA filters when, as is often the case, fans must be kept running to maintain a safe environment. All of these conditions can result from a fire in the contained space. Duct and filter fires may also be caused by transmission from fires in surrounding areas or adjacent equipment, from welding and burning operations conducted within the duct or housing or in adjacent building spaces, or from static discharges within the duct or housing.

Solvent fires present one of the most serious hazards. Not only is a heavy viscous smoke often generated (Table 9.1), but duct temperatures can rapidly build to $1000^{\circ} \mathrm{C}$ or higher, particularly when the fire occurs right at the duct entrance as in the case of a chemical fume hood, hot cell, or glove box. It is imperative that the quantities of solvents and other

\begin{tabular}{|c|c|c|c|}
\hline \multirow{2}{*}{$\begin{array}{c}\text { Material } \\
\text { burned }\end{array}$} & \multicolumn{2}{|c|}{$\begin{array}{l}\text { Quantity bumed to } \\
\text { produce plugging in- }\end{array}$} & \multirow{2}{*}{$\begin{array}{l}\text { Quantity } \\
\text { index }\end{array}$} \\
\hline & $\begin{array}{l}50 \text {-cfm filter } \\
\text { (g) }\end{array}$ & $\begin{array}{l}1000-\mathrm{cfm} \text { filter } \\
(\mathrm{kg})\end{array}$ & \\
\hline Cotton gauze wipes & 650 & 15 & 13 \\
\hline Cellulose paper wipes $^{b}$ & 600 & 13.75 & 12 \\
\hline Polyethylene film ${ }^{c}$ & 230 & 5.3 & 4.6 \\
\hline Polyurethane-rubber film ${ }^{r}$ & 110 & 2.53 & 2.5 \\
\hline Polyvinyl chloride film ${ }^{c}$ & 60 & 1.37 & 1.2 \\
\hline Amsco solvent ${ }^{c}$ & 50 & 1.15 & 1 \\
\hline D2EHPA solvent ${ }^{c}$ & 50 & 1.15 & 1 \\
\hline
\end{tabular}

"Filters operated at nominal airflow capacity; $100 \%$ of burning residue reached filters.

"Smoke from cellulosic material consisted of large hair-like particles that collected as a coarse, matted, porous layer on the surface, sometimes bridging the space between pleats.

'Smoke from plastics and solvents consisted of very small, sticky particles that coated the surface fibers and clogged the interstices between fibers with an impermeable layer.

Note: Flanders Separatorless filter will accommodate approximately 37 to $47 \%$ more smoke before plugging than conventional construction.

flammable fluids permitted in a filtered enclosure or room be severely limited, and that those that are allowed be stored in Underwriters' Laboratories- or Factory Mutual-approved safety containers. Adequate ventilation rates must be maintained under normal operating conditions to keep combustible vapor concentrations within safe limits. When fire results from the ignition of flammable gases that are already at high temperature, such as the off-gas from an incinerator or furnace, duct temperatures may reach $2000^{\circ} \mathrm{C}$ or higher. ${ }^{19}$

Pyrophoric metal dusts create special hazards. Pyrophoric dust fires in glove boxes, fume hoods, machine-tool hoods, and other small enclosures occur close to the duct entrance and may give rise to duct temperatures on the order of $1800^{\circ} \mathrm{C}$ or higher. Burning metal fragments may be given off, then captured by the ventilation system and conveyed to the ducts and filters. Since the presence of combustible material cannot be eliminated in this instance, and fire extinguishment by water spray may intensify the fire, recourse often is taken to inert or exclude oxygen from the work environment under normal operating conditions. Argon, helium, nitrogen, and carbon dioxide have all been used as cover gases for this purpose (carbon dioxide freezes moisture in the air and could cause filter plugging by ice crystals). Because pyrophoric metal operations may require oxygen concentrations to be reduced to $1 \%$ or less, severe limitations are placed on duct and housing design to avoid air infiltration. 
Regular and thorough housekeeping will prevent the accumulation of trash and potentially flammable dust in operating areas. Not so obvious, and therefore generally overlooked, is the use of low-lint clothing by operating and maintenance personnel and the provision of at least moderately efficient filters in the building supply air system and in intakes to contained operating spaces of the building (at least $30 \%$ ASHRAE dust-spot efficiency). The major source of lint, one of the most flammable constituents of common dust, is the fretting of clothing as personnel move about in the building. Another major source of dust in exhaust filters is the atmospheric dust brought in with building supply air. The control of such dusts at the source reduces dust loading on exhaust filters and thereby reduces the potential fire hazard in those components. Dust control can also reduce system operating costs; it is much less expensive to change filters in the uncontaminated supply system than in the contaminated exhaust system.

Heat, the third element of fire generation, can be controlled by maintaining adequate ventilation rates for cooling and by excluding, isolating, or shielding items of equipment that produce heat, sparks, or flame. The use of explosion-proof motors and switches in contained spaces should be mandatory, and low-total-heat-output devices such as induction coils and furnaces should be employed instead of Bunsen burners and gas furnaces. One source of heat that may be overlooked is welding and burning within or adjacent to a duct or filter housing. Welding in an adjacent building space has been the cause of at least one serious filter fire.

Maintenance operations often introduce a particular hazard from the standpoint of fire. Operating personnel are generally well indoctrinated in fire prevention and control in their particular areas. Maintenance personnel, however, are not only less familiar with requirements for the particular area, but oftentimes bring into it solvents, paints, lubricants, and other flammable materials in quantities considerably greater than normal operating procedures might permit. Preplanning maintenance procedures may be just as important as preplanning normal operational procedures. Maintenance should be performed in accordance with work-permit procedures that have been reviewed and approved by the plant fire-protection department whenever solvents and other flammable materials are involved.

\subsubsection{System Design}

The second line of defense against filter and duct fires is the design of the ventilation and air cleaning system. Because the loss of filters may be the most serious consequence of a fire, the first decision must be to use fire-resistant filters, that is, HEPA filters that meet the requirements of UL-586 and prefilters that meet the requirements of UL-900. ${ }^{20,21}$ The fireresistant HEPA filter, in both steel- and wood-cased construction, is designed to withstand air temperatures of 700 to $750^{\circ} \mathrm{F}$ for at least 10 to $15 \mathrm{~min}$ without serious degradation of function, so long as airflow is continued and they do not become plugged. There is, however, a rapid decrease in the tensile strength of the medium at about $450^{\circ} \mathrm{F}$; at temperatures above $800^{\circ} \mathrm{F}$ the fibers begin to break, curl up, and "pill," leaving pinholes in the medium. ${ }^{22}$ Extended exposure to temperatures above $800^{\circ} \mathrm{F}$ will cause destruction of the case of wood-càsed filters and warping of the case of steel-cased filters, resulting in bypassing of unfiltered air. Rapid deterioration of all but ceramic-sealed filters can be expected at temperatures above $1200^{\circ} \mathrm{F}$. The medium of HEPA filters is thin $(0.015$ in.) and can be destroyed by incandescent sparks, flaming trash, or burning dust on its surface. The filter can also be plugged rapidly by heavy smoke concentrations, particularly those from burning plastics or solvents, and this can lead to rupture of the medium if the fans have sufficient suction and are kept running. It is essential, therefore, to locate the final HEPA filters where they will be least exposed to the hostile environment of a fire and to protect them with prefilters from sparks, fragments of burning material, smoke, and heat generated by a fire.

Duct-Entrance Filters. Duct fires are serious because they occur in the main conduit leading directly to the filters. Primary protection against duct fires can be obtained by installing at least moderately efficient filters (30 to 45\% ASHRAE dust-spot efficiency) ${ }^{23}$ at duct entrances to prevent the accumulation of flammable dust inside the ducts. These prefilters provide some protection for the HEPA filters downstream from smoke generated in a fire in the contained space served by the exhaust system. They also provide a sacrificial barrier between that space and the HEPA filters to at least delay the spread of a fire to the HEPA filters. The type of filter used is important, as can be seen from a comparison 
of Figs. 9.27 and 9.28; the panel or furnace-type prefilter in Fig. 9.27 ruptured after a rapid increase in pressure drop caused by the collection of neoprene smoke and offered only minimal protection to the downstream HEPA filter, even at the beginning of the fire. In the second case, the high efficiency $(\sim 85 \%$ ASHRAE dust-spot) filter protected the HEPA filter throughout the fire.

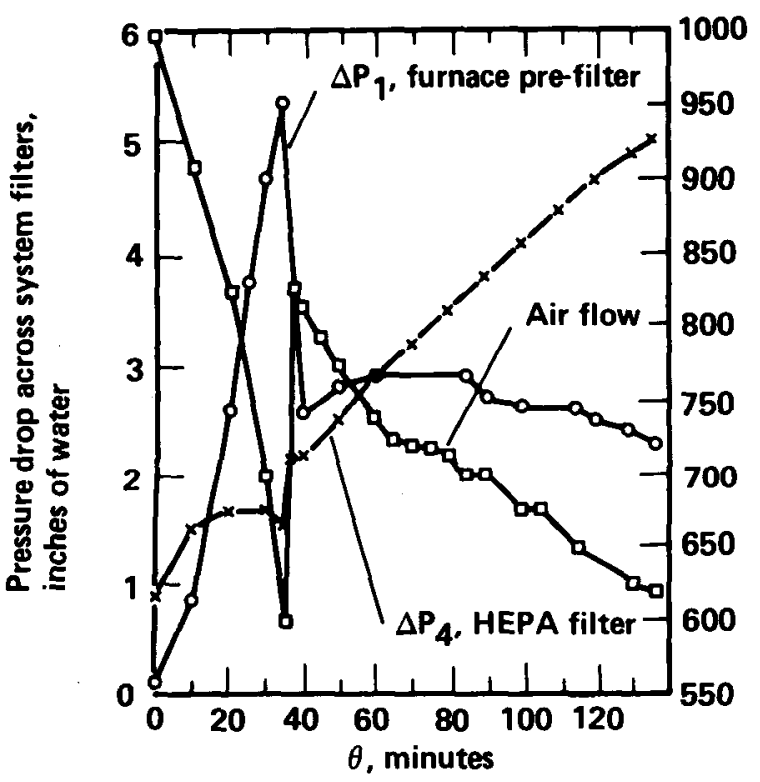

Fig. 9.27. Plot of airflow and resistances of HEPA and furnacetype prefilters against duration of fire in which smoke from burning neoprene is generated. Note rupture of prefilter at about $35 \mathrm{~min}$, the effect of prefilter on prerupture airflow, and poor protection of the HEPA filter, even at early stage of fire. Courtesy Lawrence Livermore Laboratory.

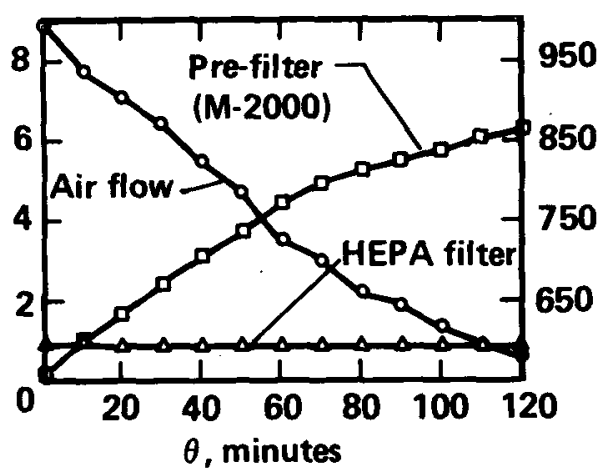

Fig. 9.28. Plot of airflow and resistances of HEPA and $85 \%$ efficiency prefilter against duration of fire in which neoprene smoke is generated. Note continued good protection of HEPA filter throughout fire but steady reduction of airflow as prefilter is plugged. Courtesy Lawrence Livermore Laboratory.
Because the duct-entrance filter is the major dust collector, it is also the primary component in which a fire could occur. Protection of the HEPA filter downstream from sparks and burning fragments from the duct-entrance filter may be needed if the distance between them is not great. If it is less than 20 to $30 \mathrm{ft}$, a fine ( 20 to 30 mesh) screen may be installed downstream of the duct-entrance filter; such screens must be located where they are convenient for periodic cleaning. Because lint tends to bridge the openings, screens, and coarse filters (e.g., furnace filters), the installation of fine-mesh screens on the face of the duct-entrance filter is not recommended; however, this does not preclude the installation of a 4mesh screen for physical protection of the filter. For glove-box and hot-cell applications, the ductentrance filter should be designed for withd rawal into and replacement from the contained space. The filter should also be afforded maximum protection against the effects of or ignition by a fire in the contained space.

Prefilters. Prefilters are usually provided in the central filter house in addition to or in lieu of ductentrance filters. Again, fire is more likely to occur in the prefilter than in the HEPA filter downstream. Prefilters should never be mounted directly on the face of the HEPA filter or on the opposite side of a common mounting frame with the HEPA (i.e., backto-back). A spacing of at least $36 \mathrm{in.} \mathrm{between} \mathrm{the}$ downstream face of the prefilter and the upstream face of the HEPA is recommended, not only for maintainability (Fig. 4.26) but to provide space in which burning fragments and sparks can burn out or settle to the floor of the filter house.

Duct Runs. High temperatures in the central exhaust filter house can be minimized by long runs of duct preceding the housing, by intake of dilution air from streams from other contained or occupied spaces of the building, or by cooling the outside of the duct with water sprays. Cooling by water sprays installed inside the duct has also been employed in some applications. The cooling effect of long runs of duct is illustrated in Fig. 9.29. Without the contribution of dilution air from side streams, at least $100 \mathrm{ft}$ of duct is needed to obtain a $50 \%$ reduction of air temperature in a fire of short duration; this approach, therefore, is not always viable.

Flame Arresters. Metal-mesh flame arresters and gas coolers are of limited value for protecting HEPA filters unless they are located well upstream of the filter. Tests by the AEC showed that no commercially 


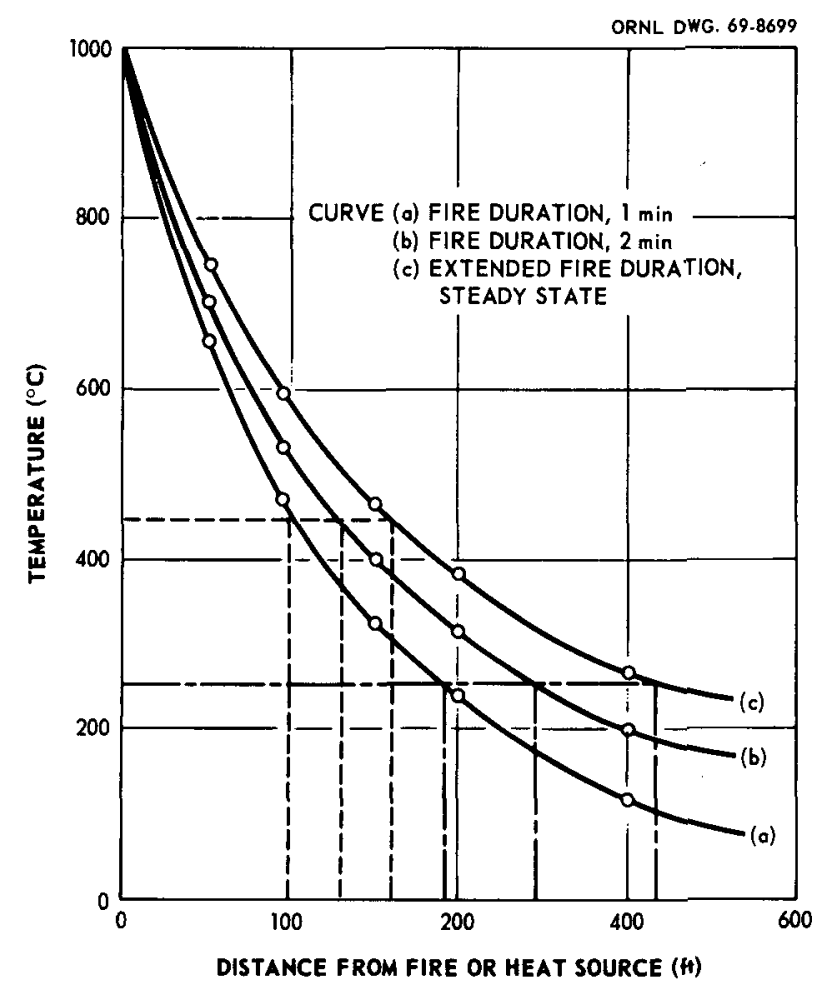

Fig. 9.29. Cooling rate of air in a 12-in.-diam uninsulated duct carrying $1000 \mathrm{cfm}$ of air. Dashed lines show the length required to cool air from initial temperature of $1000^{\circ} \mathrm{C}\left(1832^{\circ} \mathrm{F}\right)$ to $450^{\circ} \mathrm{C}$ $\left(842^{\circ} \mathrm{F}\right)$ and $250^{\circ} \mathrm{C}\left(482^{\circ} \mathrm{F}\right)$ for various fire conditions. From S. E. Smith et al., Protection Against Fire Hazards in the Design of Filtered Ventilation Systems of Radioactive and Toxic Gas Process Buildings, UKAEA Report AWRE 0-24/65. Atomic Weapons Research Establishment, Great Britain, July 1965.

available flame arrester prevented failure of the HEPA filters when installed close to the filter. To be effective, such devices must have considerable heat capacity and must be located at least 4 to $5 \mathrm{ft}$ ahead of the HEPA filters.

Redundant and Standby Units. Where continued operation of a ventilation system is necessary in the event of fire, system upset, or other emergency, a redundant standby air cleaning unit and fan is essential. The standby unit provides not only the capability of switching to an unimpaired unit in the event of damage to the on-line unit, but also permits isolation of the on-line unit for fire fighting (if the fire is in the filter housing) or repairs, with only minimal interruption of building airflow. A stand by unit also permifts routine testing and maintenance of the system without interrupting facility operations. This advantage can repay the cost of the extra equipment in only a few years when downtime is a significant expense factor. Manual activation of such standby units is often recommended if there are attendants on duty full-time. Manual changeover gives the time delay needed for personnel to make judgments concerning preplanned emergency procedures, where regard for control of differential pressures between hazard zones of the facility must be considered. A factor sometimes overlooked in such procedures is that switching from a high-resistance bank of used filters to a low-resistance bank of clean filters may also upset the pressure differentials on which proper airflows in the facility depend. Compensation for such pressure differentials is generally provided by control dampers or periodically adjusted balancing dampers in the duct system.

Smoke Venting. A factor sometimes overlooked when supply and exhaust fans can be shut down to fight a fire in a contained or occupied space of the building is that the building intake and exhaust systems, unless properly fire- and smoke-dampered, may serve as flues to vent the building. Venting through the supply air system, unless it is fitted with HEPA filters, will result in the escape of potentially contaminated smoke and gases. On the other hand, venting through the exhaust air cleaning system may plug the duct-entrance filters and/or prefilters and possibly the final HEPA filters, making replacement necessary before post-fire cleanup can begin. Contaminated smoke may also escape through inadvertent building openings such as cracks, thereby bypassing the air cleaning system. This possibility points up the advantage of procedures that assume continued operation of the exhaust system (but shutdown of the supply fans). As for any building construction, adequately designed fire dampers are essential in at least the nonradioactive systems of the building (supply-air and Zone I ventilation systems) and should be considered in the radioactive systems (exhaust, recirculating-cleanup). Some years ago, a study by SMACNA and Underwriters' Laboratories demonstrated that many commercially available fire dampers will warp or fail structurally under fire conditions. It is necessary, therefore, to carefully evaluate such items before installation in supply or exhaust ducts of a nuclear containment system.

Soldered Joints and Connections. Another factor sometimes overlooked is the construction of pneumatic lines for damper and fan control and instrument sensing lines. The use of soft-soldered or plastic tubing in a system where the hazard of fire exists is obviously inappropriate. The failure of such lines under fire conditions could render the entire air 
cleaning and ventilation system inoperative at the time is most needed.

\subsubsection{Fire Detection}

The first requisite for effective fire control in a ventilation or air cleaning system, or in a building space that can affect the air handling system, is a sensitive fire detection system. If protective action can be started soon enough, fire in operating areas, ducts, and filter housings may be controllable with a minimum of filter damage and escape of contamination. If a fire gets a good start, however, the filters will probably be lost, and contaminated smoke will be released to the environment or occupied areas of the building. All that can be accomplished in that instance is minimization of casualty losses to the filter housing and mounting frames, or to the building. Assuming proper attention to operational procedures and air handling system design, the likelihood of a fire in the final filters is remote. However, it can never be discounted completely. The same can be said for fires in adsorber systems where continuous airflow must be maintained to remove fissionproduct-decay heat.

There are three basic types of fire detectors: flameactuated, smoke-actuated, and heat-actuated. Since airflow carries flame through a filter, preventing its appearance on the upstream face and delaying its appearance on the downstream face until extensive damage has already been done, flame-actuated devices are not suitable. Because the first indication of filter fire is smoke from burning dust or from the volatilized organic binder of the filter paper, smokeactuated detectors give the most rapid response to a fire. However, the ionization gages used in most smoke detectors may give false indications in radiation fields, and therefore they usually require complex redundant-coincident signaling systems for operational reliability. The products-of-combustion smoke detector can be useful and is discussed below.

Heat-Actuated Detectors. Although slower responding than the smoke detector, certain types of heat-actuated detectors are satisfactory for many duct and filter applications. The rate-compensated detectors and continuous fire detectors (CFD) that have found widespread use in aircraft applications are particularly attractive. The CFD, which consists of a coaxial cable filled with a eutectic salt, is useful in the protection of ducts and large filter banks. The eutectic salt is nonconducting when solid but conducting when melted by the heat given off by a fire in any part of the area "covered" by the cable. When the salt melts, the cable is shorted and signals an alarm or fireprotective device. When the heat is taken away, that is, when the fire is out, the salt freezes and the alarm circuit is opened. However, the detector remains operational and can signal an alarm again should the fire rekindle. To ensure positive personnel response to a fire alarm, true or false, a manual reset is recommended for the audible and visual alarms activated by the detector. Audible and visual alarms should be provided locally and at a central control panel.

The CFD is simple and reliable and requires a minimum of maintenance. ${ }^{24}$ Control circuits are simple, and redundancy or coincidency is not required for operational reliability. A wide range of signaling temperatures is available by selection of the appropriate eutectic salt combination, and the system can be tailored to the requirements of the application. CFD systems can be zoned to indicate the general area in which a fire or temperature rise occurs; that is, the system can be segmented, with each segment triggering a separate alarm. When double action such as an alarm on temperature rise to some predetermined level plus activation of fire extinguishing devices at a higher temperature is desired, two cables with different salt combinations can be installed in parallel. Because of its low maintenance requirements, this type of detector is particularly suitable for ducts and other potentially radioactive areas where access is limited.

Products-of-Combustion Detectors. A productsof-combustion detector is probably the most suitable type for carbon-filled adsorbers. Because fires generated by fission-product-decay heat are likely to occur in isolated spots deep within the bed of the adsorber, and because the carbon bed provides excellent heat insulation, flame-actuated (infrared) and heat-actuated detectors are of limited value. However, carbon gives off $\mathrm{CO}$ and $\mathrm{CO}_{2}$ at oxidation temperatures substantially below the flame stage, and the products-of-combustion smoke-actuated detector can, if properly located, sense the incipient fire before it gets a good start. Detectors are needed both upstream and downstream of the adsorbers to eliminate the possibility of falsely indicating fire in the adsorbers when the fire is actually in an operating area of the facility. Whereas HEPA filters can tolerate some modest degree of wetting without significant inpairment, ${ }^{24}$ the discharge of deluge sprays to a carbon-filled adsorption system may 
render the carbon ineffective and also initiate serious corrosion of the stainless steel containers. The downstream detector must be located where excellent mixing of the air coming through all of the beds in the stage has occurred. It must also be very sensitive to offset the effect of dilution of the products of combustion with clean air. Detectors capable of accurately detecting $\mathrm{CO}$ and $\mathrm{CO}_{2}$ at levels of 0.005 ppm above normal background are available. An upstream detector is necessary to discriminate between products of combustion produced in the adsorbers and those produced from fire in an upstream operating area.

\subsubsection{Fire Control}

Because the possibility of a fire that can affect the final filters cannot be eliminated entirely, some provision for fire fighting is necessary. The minimum protection required in any air cleaning system is the provision for introducing hoses into strategic locations or openings in the duct and filter house. This requires that a water hydrant and hose station be located within a reasonable distance and that provision be made for draining the duct and/or housing during and/or after the application of water. Hoses should be equipped with fog nozzles to provide maximum cooling with minimum application of water. The use of a hose can only be considered a lastditch solution and cannot be expected to save the filters or prevent the spread of contamination. At best, a hose can serve only to prevent further damage to the filter mounting frames and housing, the duct, or the building; similar observations can be made for the common types of sprinkler systems, both automatic and manually actuated, ${ }^{25}$ if installed inside the filter house.

Sprinklers. Common types of sprinklers are useful when installed above ducts to provide cooling or to extinguish fires in adjacent areas. Where the potential of high duct temperatures exists and continued operation of the air cleaning system is essential in the event of fire oxplosion in operating areas of the building, the installation of deluge-type sprinklers above the duct is recommended. These sprinklers should be controlled from a CFD installed directly to the underside of the duct and containing a eutectic salt combination commensurate with the desired limiting duct-wall temperature. For large ducts, additional automatic sprinklers, individually equipped with fusible elements, should be installed under the duct for protection against fires in the space below the duct.
The provision of sprinklers within ducts or filter housings is the exception rather than the rule, except for the deluge systems provided on carbon-filled adsorption systems in nuclear power reactors. Fog nozzles, with as fine a droplet-size distribution as possible, are recommended for maximum cooling and smoke-particle capture. To limit the volume of water discharged, consideration should be given to an automatic recycling deluge system. Limiting water discharge is desirable not only from the standpoint of limiting the amount that can potentially get to the filters downstream, but also because the water discharged must be collected and treated as contaminated liquid waste. Individual recycling nozzles (controlled by bimetallic valves on the individual sprinkler heads) are not suitable because the source of heat may be remote from the sprinklers. A deluge system is one in which the sprinklers are normally open with water flow controlled by a quick opening valve in the line leading to the sprinkler heads. When this valve is opened, water is discharged from all the open sprinklers at the same time.

Sprinklers, or more accurately deluge sprays, within the filter house have in practically every case been provided for extinguishing possible fires in the carbon-filled adsorbers of power reactors. These are provided primarily to prevent casualty losses from the fire rather than from any loss of containment. Spraying of water on an adsorber fire is, at best, a last-ditch effort since containment of radioiodine would already have been lost, because both trapped radioiodine and the impregnant added to enhance organic iodide capture desorb at a temperature considerably below the ignition temperature of the carbon. Such desorption would constitute loss of containment for radioiodine.

Protection of Carbon-Filled Adsorption Systems. To prevent loss of containment for radioactive iodine and iodine compounds, carbon-bed temperatures must be maintained at a level at which impregnants and trapped radioiodine cannot desorb. As discussed in Sect. 3.4, this requires that the bed(s) be large enough so that specific loadings of iodine cannot exceed $2.5 \mathrm{mg} / \mathrm{g}$ carbon, and that airflow through the bed be maintained at some level in excess of 6 (preferably 10) lin fpm. If bed temperatures can be maintained below that at which desorption of impregnants and trapped radioiodine takes place, there is little likelihood of carbon ignition. If a fire should start, however, there is serious doubt that present-day deluge systems could extinguish it. ${ }^{26}$ Total flooding or dumping the carbon into a 
container of water appears to be the only effective means of extinguishing a carbon-bed fire at this time. Successful tests of fire extinguishment in a proprietary PSU adsorber design were reported by one equipment manufacturer ${ }^{27}$ but apparently required large volumes of water. Carbon dioxide and gaseous nitrogen are ineffective against activated carbon fires because the fire feeds on the oxygen adsorbed in the pores of the carbon, and the quantity of liquid nitrogen that would be required to provide effective cooling would be unavailable in most cases. ${ }^{26}$

Smothering Systems. Inert-gas smothering systems have been used with some success in a number of containment systems, particularly in glove box and hot cell applications. The most common type is $\mathrm{CO}_{2}$ or gaseous nitrogen, with the discharge located within the contained space (glove box, hot cell) rather than in the duct or filter housing. In most cases, supply fans to the contained space must be shut down and exhaust fans must be kept running (at reduced flow, however) to avoid pressurizing the containment either by the discharge of the smothering agent or by the expansion of air and gases due to the fire. Ducts, filter housings, and housing doors must be capable of withstanding the pressures that could prevail in the system under these conditions without leaking. A 34 to $40 \%$ concentration of $\mathrm{CO}_{2}$ or $\mathrm{N}_{2}$ must be maintained for effective control. Argon and helium have also been used for this purpose, but both may be too expensive for consideration in anything but a closed system (i.e., one in which the fans are shut down and the fire location is isolated).

Halon Systems. Carbon dioxide, nitrogen, and argon systems depend on smothering the fire and present an asphyxiation hazard when employed in occupied or potentially occupied areas. Carbon dioxide systems freeze moisture in the air, creating a plugging potential to filters (if airflow is continued during the fire) and obscuring the vision of personnel fighting the fire or who might be trapped in the treated space. Halon-1301, a chlorinated fluorocarbon, overcomes both of these problems. This agent functions on the basis of chemically tying up oxygen in the air or by reacting with combustion products produced by the fire (the mechanism is not clearly understood). ${ }^{28}$ In the quantities required, Halon1301 causes no obscuration of the work space and presents a minimal toxic or carcinogenic hazard. As with any inerting agent, Halon-1301 works most effectively in a closed space or in one which has minimum airflow. Halon-1301 has been used for fire protection and explosion suppression in many in- dustrial applications and gives promise of relatively inexpensive protection in glove boxes, hot cells, and other nuclear-containment applications. When released to inert a fire in a contained space (hot cell, glove box), the Halon drawn out through the exhaust duct also serves to prevent the ignition of a fire in the duct or in final filters. A concentration of approximately 6 to $8 \%$ Halon-1301 in air is sufficient to inert the atmosphere for most combustibles, and a concentration as low as 3 to $4 \%$ is sufficient to prevent reignition. This agent is not suitable for metal fires, activated carbon fires, or deep-seated fires. The fact that the gas is nonsuffocating and nonobscuring (in the concentrations required) is a decided advantage for its use in occupied or potentially occupied areas, making the provision of a time delay to evacuate personnel unnecessary. (A time delay is mandatory for smothering agents such as $\mathrm{CO}_{2}$ and nitrogen.)

Halon systems are often compared unfavorably with other types of inerting systems $\left(\mathrm{CO}_{2}\right.$ and nitrogen) and with sprinkler systems because of cost. However, these comparisons are generally based on the unit price of Halon and do not take into consideration the small quantity of agent needed for effective control. When compared with the complexity of a smothering (e.g., $\mathrm{CO}_{2}$ ) system or conventional sprinkler system, the Halon system may offer a decided cost advantage in those applications where it can be used. ${ }^{29}$ Minimum requirements for Halon systems are given in NFPA $12 \mathrm{~A} .{ }^{28} \mathrm{~A}$ system engineered specifically to meet the special needs of a nuclear air cleaning system may incorporate special nozzles, flow rates, methods of application, nozzle placement, pressurization levels, and quantities of agent that may differ from those detailed in NFPA 12A. However, all other requirements of that standard must be observed. Applicability, limitations, and precautions in the use of the agent are also covered by NFPA $12 \mathrm{~A}$.

Metal Fires. Metal fires, particularly fires in waterreactive metals such as sodium, present special problems. Water and inerting agents such as the Halons cannot be used, and inert atmospheres such as nitrogen and $\mathrm{CO}_{2}$ require practically the total exclusion of oxygen to be effective. The fire must be treated in the operating space before it can reach the ducts or final filters, which requires an effective ductentrance filter, preferably one of the HEPA type if the metal dusts are finely divided. However, most of the fire extinguishing agents that are effective against such fires produce copious clouds of dust that, when released, pose a threat of rapidly plugging the duct- 
entrance filter, which, in turn, poses the threat of overpressurizing the glove box or hot cell and causing the blowback of contamination to occupied spaces of the building. The most effective method to date for extinguishing metal fires is flooding with carbon microspheres (CMS) ${ }^{30}$ The CMS method has been shown to be extremely effective against plutonium, sodium, uranium, sodium-potassium ( $\mathrm{NaK})$, magnesium, aluminum, lithium, and other fires producing intense heat. The material can be dispensed automatically or manually and produces essentially no dust when dispensed either way. In addition, it has negligible chloride content (and therefore poses no threat to stainless steel equipment and cells), is very easy to clean up because it does not produce dust or airborne particulates, and is inexpensive $(<10 \mathrm{c}$ per pound) and readily available. ${ }^{3 !}$

\subsection{DEEP-BED SAND FILTERS}

Sand filters have been used in the ventilation and process exhaust systems of radiochemical processing facilities since 1948. The major attractions of sand filters include large dust-holding capacity, low maintenance requirements, inertness to chemical attack, high heat capacity, fire resistance, and the ability to withstand shock loadings and large changes in airstream pressure without becoming inoperative. Removal efficiency approaching that of a single HEPA filter is claimed if the proper sands are used and the contact path is long enough.* The disadvantages of DBS filters include high capital cost; large area; high pressure drop and power cost; uncertainties in selection, availability, grading, and handling of suitable sands; and the disposal of the spent unit.

Sand filters are deep (several feet thick) beds of rock, gravel, and sand, constructed in layers graded with about 2 to 1 variation in granule size from layer to layer. Airflow direction is upward, and granules decrease in size in the direction of airflow. A top layer of moderately coarse sand is generally added to

\footnotetext{
*Efficiency tests of sand filters are made with polydispersed DOP aerosol having an NMD of about $0.7 \mu \mathrm{m}$, and using the procedures of the in-place test described in Chap. 8. True efficiency tests of HEPA filters, on the other hand, are made with monodispersed DOP aerosol having an NMD of $0.3 \mu \mathrm{m}$. In addition, tests of very large units such as sand filters are often made under conditions that sometimes give results that are difficult to interpret. For these reasons, although it can be stated that the efficiency of the sand filter approaches that of the HEPA filter, it cannot be assumed that sand-filter efficiency for submicron particles is actually equivalent to that of the HEPA filter.
}

prevent fluidization of finer sand. The rock, gravel, and sand layers are positioned and sized for structural strength, cleaning ability, dirt-holding capacity, and long life. A cross section of a typical sand filter is shown in Fig. 9.30. Ideally, the layers of larger granules, through which the gas stream passes first, remove most of the larger particles and particulate mass, and the layers of finer sands provide highefficiency removal. Below the fixed bed of sand and gravel is a course of hollow tile which forms the air distribution passages. The filter is enclosed in a concrete-lined pit. The superficial velocity is around 5 $\mathrm{fpm}$, and the pressure drop across seven layers, sized from $3 \frac{1}{2}$ in. to 50 mesh, is from 7 to 11 in.wg. Collection efficiencies up to $99.98 \%$ (by in-place test with polydisperse 0.7-NMD DOP) have been reported. ${ }^{32}$ The approximate capital cost of a sand filter is $\$ 35$ per cfm in 1976 dollars.

Sand filters have received renewed interest in the past few years because of the increased concern for the effects of natural phenomena (earthquake, tornado), fire, and explosion and because procurement and maintenance costs of alternative air cleaning

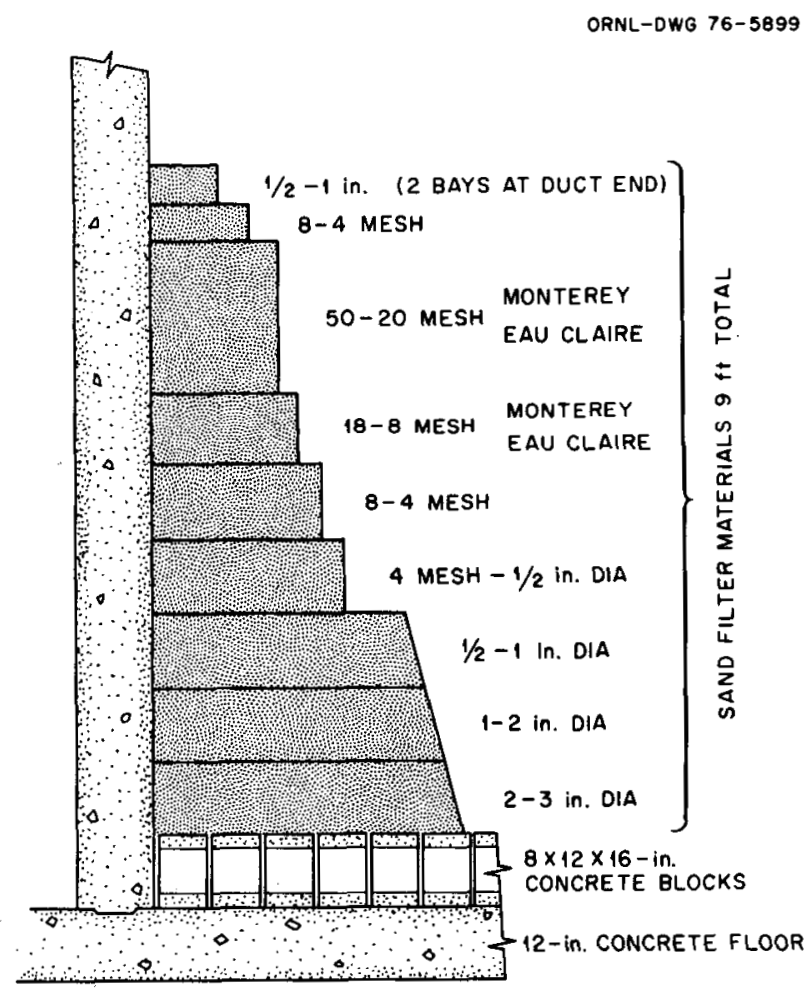

Fig. 9.30. Section through typical sand filter. Table 9.3 lists properties of sand and aggregates used for filtration media. Note course of special hollow concrete block at bottom for distributing inlet air throughout the bed. 
methods have increased substantially. Sand filters are characteristically one-of-a-kind designs. They are literally constructed in the field as the gravel is positioned and the sand is poured in place. A view of a sand filter under construction is shown in Fig. 9.31. No standards exist, so most of the information for new designs must come from reports of previous applications. A bibliography and review of sand filters built prior to 1970 was prepared by Argonne National Laboratory. ${ }^{32}$

Following the initial installation of a sand filter at Hanford, nine others were installed at Hanford, Savannah River, and the Midwest Fuel Recovery Plant at Morris, Illinois. All but one ${ }^{33}$ of these were designed for cleaning ventilation air from fuel reprocessing facilities, and only four (all at Savannah River) are currently used for this purpose. There is a sand filter in the roof of the Zero Power Research Reactor $^{34}$ at Idaho Falls, but it is for emergency exhaust cleanup only and is not operated under normal conditions. Details of existing U.S. sand filters are given in Table 9.2. Properties of sands and aggregates used as the filtration media of these filters are given in Table 9.3.

\subsubsection{Design of Deep-Bed Sand Filters}

A rough approximation of the collection efficiency of sand, on an activity basis, is given by the following equation: ${ }^{35}$

$$
\eta=1-\exp \left(-K L^{1 / 2} V^{-1 / 3} D^{-4 / 3}\right)
$$

where

$\eta=$ fractional collection efficiency on a radioactivity or mass basis;

$L=$ depth of fine sand, $\mathrm{ft}$;

$V=$ superficial gas velocity, fpm;

$D=$ average sand grain diameter, in.;

$K=$ proportionality factor.

(Note: values of $L, V$, and $D$ vary with sands from different sources of the same mesh size and must be determined experimentally for any given sand.)

Values for the proportionality constant, $K$, for several sands tested at Hanford are

\begin{tabular}{lc}
\multicolumn{1}{c}{ Type of sand } & K \\
\hline Hanford & 0.053 \\
AGS flint & 0.045 \\
Rounded grain sand (Ottawa, Eau Claire, Monterey) & 0.035
\end{tabular}

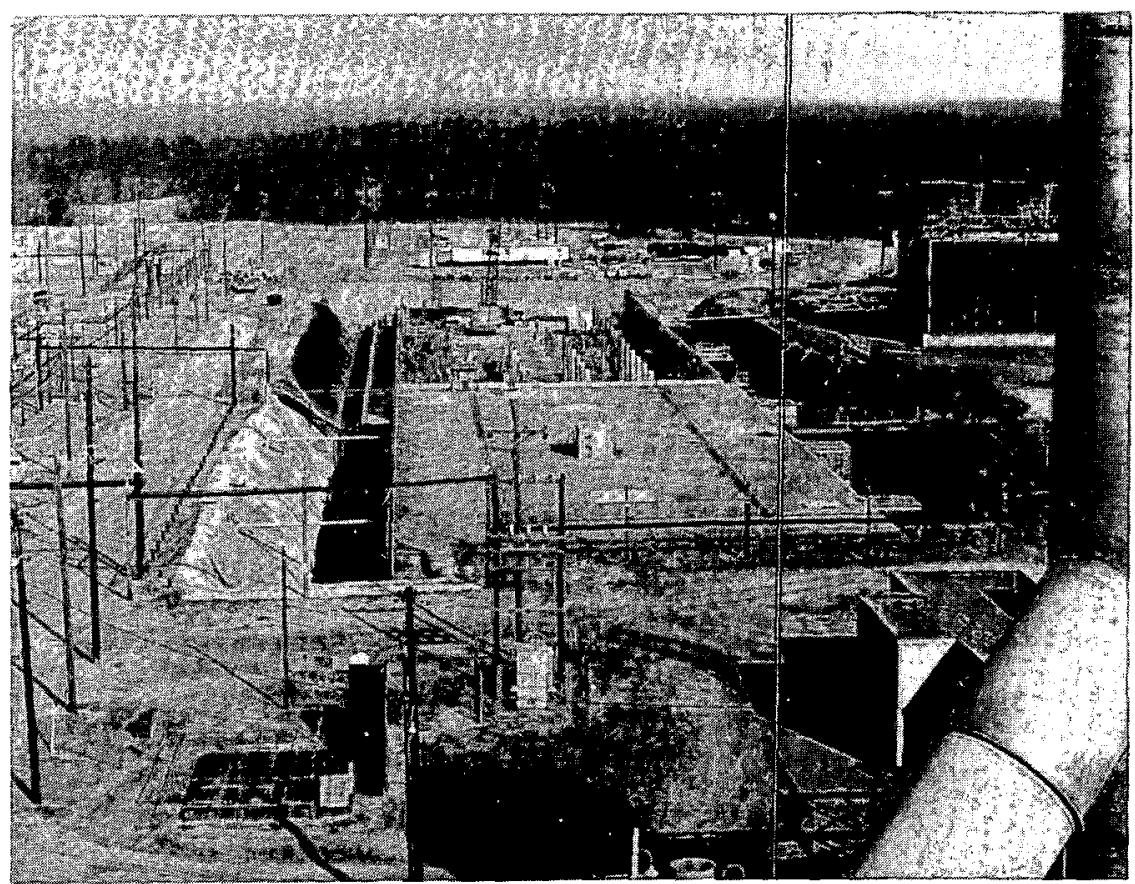

Fig. 9.31. View of sand filters at ERDA Savannah River Laboratory. Existing sand filter in foreground, new filter under construction beyond. 
Collection efficiency on a radioactivity basis gives a higher number than the collection efficiency on a count basis, as reflected by the DOP test, since larger, more easily collected particles may carry more radioactivity and bias the analysis to give more worth to larger particles. The relationship between count and activity collection efficiency cannot be determined without accurate information on aerosol size distribution and the relationship of aerosol size to radioactivity.

The approximate void fraction of a sand bed is generally about 0.4 . Sand permeability tests showed that intense vibration can cause extreme compaction, resulting in near doubling of the pressure drop. ${ }^{36-38}$ Factors that must be considered include the effects of compaction, steam injection, relative humidity, and velocity change on efficiency and pressure drop. Besides permeability and filtration requirements, the sand must be abrasion-and fracture-resistant and must resist corrosion from the fumes likely to be present in the exhaust air stream.
Filter life is determined by the increase in pressure drop and the decrease in gas flow caused by the collection of solids within the sand bed. Filter life can be significantly reduced if solids collection is concentrated in small fractions of the bed or on the finer sand. Uniform concentration of coarse aggregate layers upstream of the fine sand layer tends to maximize filter life.

Clogging of sand filters is aggravated by local decreases in porosity at the interfaces between graded layers. The mixing of aggregates (sand, gravel) at the interfaces usually results in a lower void fraction at the interface than if no mixing is permitted. The extent of reduction in void fraction depends on the characteristics of the aggregates and on the technique used to charge them into the filter bed. The lowest layer may require hand placement for the first few inches so that no rocks fall through the openings in the distribution blocks (Fig. 9.32). Significant improvement in filter life can be obtained by careful attention to loading.

Table 9.2. Dimensions and operating data of existing U.S. sand filters

\begin{tabular}{|c|c|c|c|c|c|c|}
\hline $\begin{array}{l}\text { DBS } \\
\text { filter } \\
\text { No. }{ }^{a}\end{array}$ & $\begin{array}{c}\text { Plan } \\
\text { dimensions } \\
\quad(\mathrm{ft})\end{array}$ & $\begin{array}{l}\text { Design } \\
\text { flow } \\
\text { (cfm) }\end{array}$ & $\begin{array}{l}\text { Design } \\
\text { superficial } \\
\text { velocity } \\
(\mathrm{fpm})\end{array}$ & $\begin{array}{c}\text { Design } \\
\text { pressure } \\
\text { drop } \\
\text { (in.wg) }\end{array}$ & $\begin{array}{c}\text { Date of } \\
\text { initial } \\
\text { operation }\end{array}$ & $\begin{array}{c}\text { Present } \\
\text { status } \\
\text { of DBS }\end{array}$ \\
\hline 1 & $108 \times 46$ & 25,000 & 5.0 & 5.0 & 1948 & Standby \\
\hline 2 & $108 \times 46$ & 25,000 & 5.0 & 7.0 & 1948 & Standby \\
\hline 3 & $96 \times 96$ & 40,000 & 4.3 & 10.0 & 1950 & $c$ \\
\hline 4 & $85 \times 85$ & 40,000 & 5.5 & 12.0 & 1951 & Active \\
\hline 5 & $240 \times 100$ & 115,000 & 4.8 & $\sim 10.0^{\circ}$ & 1954 & Standby \\
\hline 6 & $240 \times 100$ & 115,000 & 4.8 & $9.2^{\prime}$ & 1955 & Standby \\
\hline 7 & $360 \times 100$ & 210,000 & 5.8 & & 1975 & Active \\
\hline 8 & $360 \times 100$ & 210,000 & 5.8 & & 1976 & Active \\
\hline 9 & $140 \times 103$ & 74,000 & 5.1 & & 1974 & Active \\
\hline 10 & $72 \times 78$ & 32,000 & 5.7 & & 1974 & $d$ \\
\hline 11 & 50 to $62.5($ diam $)$ & $e$ & $e$ & & 1968 & Active $^{e}$ \\
\hline
\end{tabular}

${ }^{a}$ Filter identification

1. C Plant, Building 291-T, Hanford West Area, Richland, Wash.

2. B Plant, Building 291-B, Hanford East Area, Richland, Wash.

3. U Plant, Building 291-U, Hanford, Richland, Wash.

4. Redox Facility, Building 291-S, Hanford, Richland, Wash.

5. F Area, Building 294-F (old), Savannah River Plant, Aiken, S. C.

6. H Area, Building 294-H (old), Savannah River Plant, Aiken, S. C.

7. F Area, Building 294-1 F (new), Savannah River Plant, Aiken, S. C.

8. H Area, Building 294-1H (new), Savannah River Plant, Aiken, S. C.

9. SRL, Building 794-A, Savannah River Laboratory, Aiken, S. C.

10. Midwest Fuel Recovery Plant (MFRP), Morris, III.

11. Zero Power Plutonium Reactor Facility, Argonne National Laboratory, Idaho Falls, Idaho. ${ }^{b}$ Inlet side shown first, outlet side italicized.

${ }^{c}$ Unit in service, process operation was discontinued in 1975.

${ }^{d}$ MFRP is not engaged in reprocessing, only storage; sand filter is active.

This is an emergency relief system. 
Table 9.3. Properties of sands and aggregates used in existing U.S. sand filters

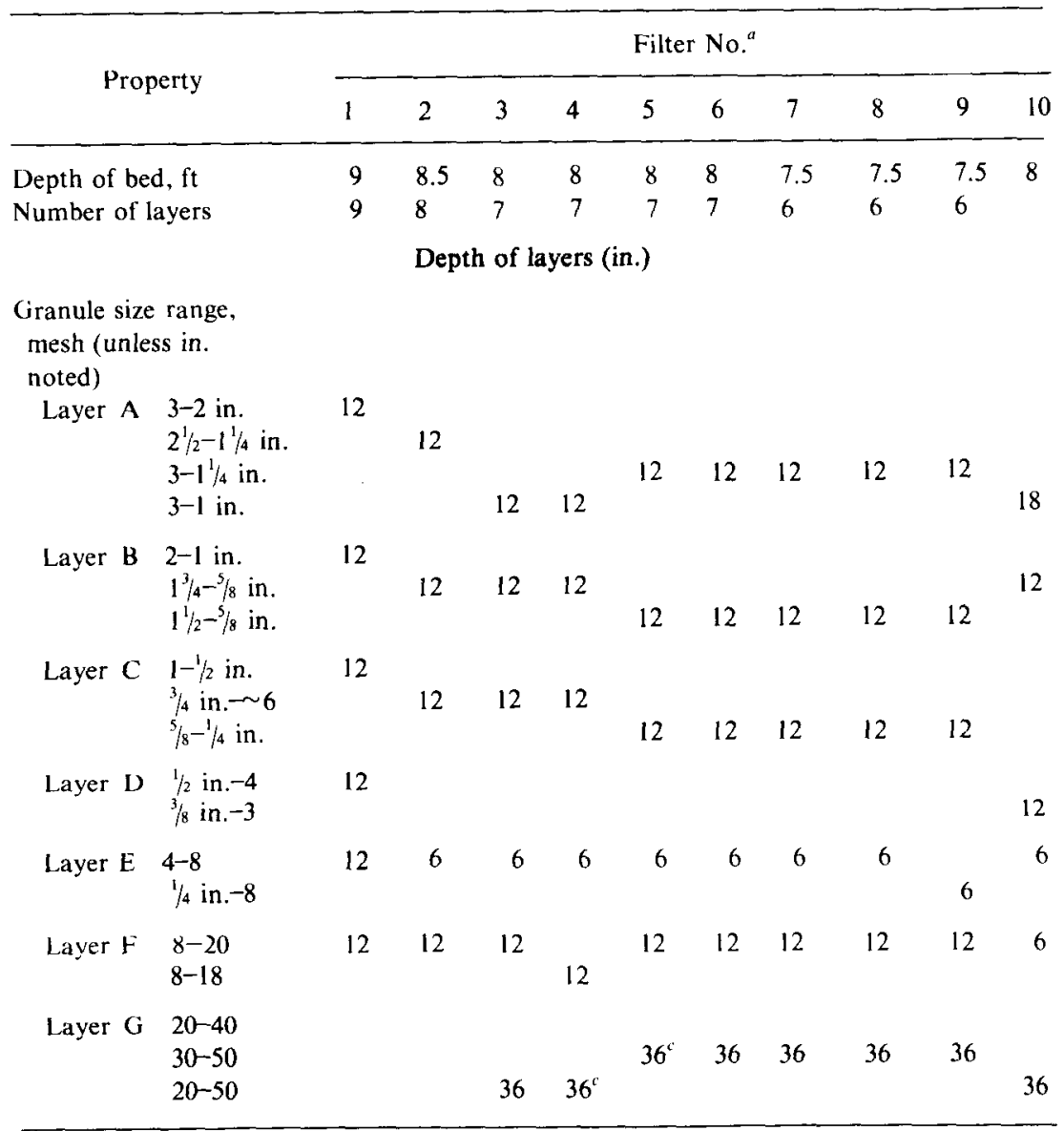

${ }^{a}$ See Table 9.2 for location corresponding to number.

${ }^{b}$ Cable and wire mesh of footnote $a$ catenary cross-section support, deep bed.

'Removed 12 in. from G layer, July 1972, to reduce pressure drop.

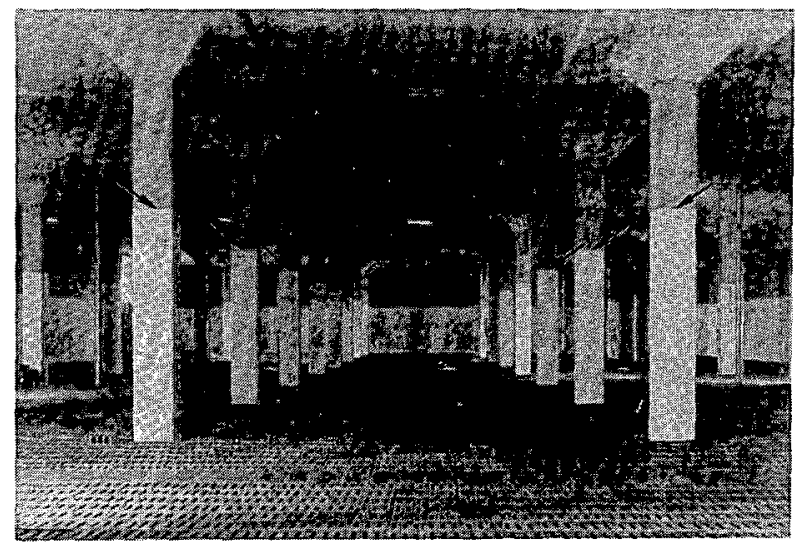

Fig. 9.32. Interior of new sand filter at Savannah River Laboratory before loading of sand and aggregate. Note course of hollow tile for air distribution. When finished, sand will come to the level marked by arrows.
The sand filter housing is a poured concrete structure, partially underground, with walls capable of withstanding the design basis earthquake without cracking and the design basis flood without leaking. The floor has channels for distributing the incoming air and is covered by the special hollow block shown in the view of an empty sand filter (Fig. 9.32). An isometric of this filter is shown in Fig. 9.33. The floor and the distribution system must bear the weight of the sand column above it. With corrosion and aging, withstanding this weight has been a problem in some sand filters. The floor should be sloped to a drain and have a built-in capability for drainage if it becomes necessary. It is often prudent not to connect the drain line, so that a determination of what to do with the drainage can be made after the event if flooding occurs. The filter should be on the suction side of the 


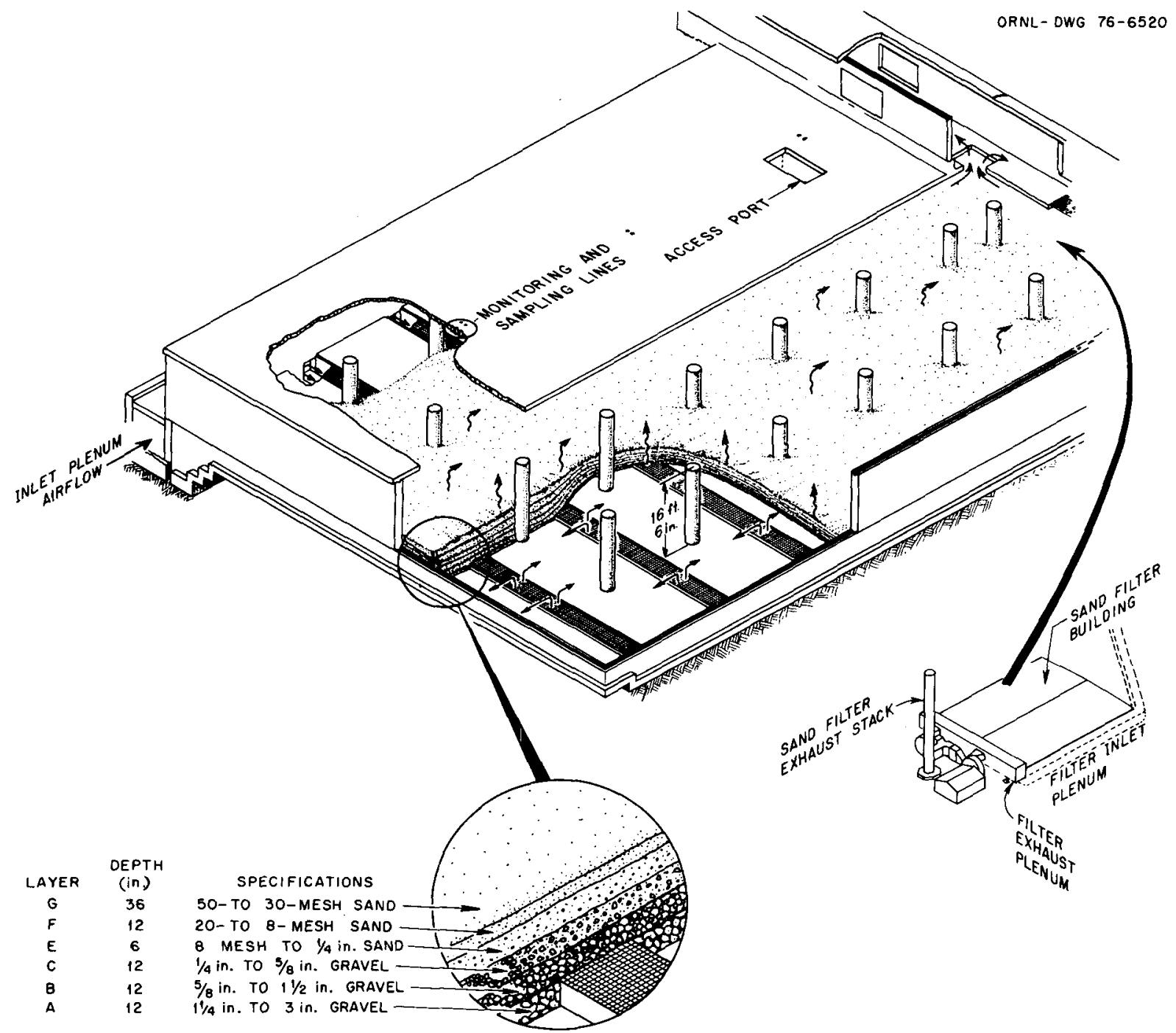

Fig. 9.33. Overall isometric view and details of new sand filter at Savannah River Laboratory.

fan so that it is negative to the atmosphere and all leakage is inward.

When a sand filter is used in series with HEPA filters, it should be upstream of the HEPA filters. In this position, the high dust-holding, fire-resistance, and pressure-surge-attenuating characteristics of the sand filter can protect the HEPA filters that provide the final containment barrier.

\subsubsection{Plugging of Deep-Bed Sand Filters}

Some filters have experienced plugging at low dust loadings. In one case, the plugging was caused by moisture entering through cracks in the concrete sidewalls of the unit. ${ }^{39}$ In another instance, plugging was caused by crystal growth in the filter media fines, probably due to a reaction of nitric acid vapors from the process building with calcite, with dolomite present in the original sand, and with cement dust generated by severe erosion and acid attack on the concrete entry ducts and support structures.

\subsubsection{Disposal of Spent Media}

Deactivation of existing filters is generally accomplished by sealing and abandoning the filter. Spent media are stored in place within the unit. The total unit is replaced by a new filter located close by. Present government regulations for radioactive solid waste, though unclear, may rule out such in-place 
disposal in the future. If the material is handled as high-level radioactive waste, each 1000 -cfm capacity of filter would require about two hundred 55-gal drums for disposal.

\subsection{DEEP-BED GLASS-FIBER FILTERS}

\subsubsection{Introduction}

Another type of large-capacity long-lived filter that has been used successfully for the prefiltration of ventilation and process air in a number of AEC/ERDA radiochemical and fuel reprocessing operations is the deep-bed glass-fiber (DBGF) filter. Developed as an alternative to the more costly sand filter, the DBGF filter employs a medium that has more controllable physical features and more assured availability than filter-grade sands, permits a larger airflow per unit of volume at lower pressure drop, has lower operating costs, and potentially lower spentunit disposal cost than a sand filter of equivalent airflow capacity. On the negative side, the DBGF filter does not have nearly the particle-collection efficiency of the sand filter; has less corrosion resistance, particularly from hydrogen fluoride, and less fire resistance; and lacks the heat-sink and selfrepair properties and capability of the sand filter to snub shocks and high-pressure transients.

DBGF filters are deep ( 8 to 84 in.) beds of compacted fiberglass insulating wool contained in stainless steel boxes (trays) having opaque sides and perforated screens at the top and bottom. Units as small as 200 -cfm airflow capacity, or smaller, have been employed in process off-gas systems, and units as large as 150,000 -cfm airflow capacity have been used in building and cell-exhaust installations. Figure 9.34 shows a view of the newly completed DBGF filter at the Idaho National Engineering Laboratory (INEL) and illustrates the typical construction details of a large unit. The first use of a DBGF filter was a 250-cfm unit in a dissolver cell exhaust at Hanford in $1950 .{ }^{40}$ By 1953,11 units with airflow capacities from 200 to $20,000 \mathrm{cfm}$ had been installed. ${ }^{41,42}$ A 126,000$\mathrm{cfm}$ unit was installed in the canyon exhaust of the Purex plant at Hanford in $1955,{ }^{43}$ and a 150,000 -cfm unit has just been completed for the Idaho Chemical Plant at INEL. DBGF filters have also been used successfully in a number of hot-cell applications for process off-gas cleanup.

\subsubsection{Design and Operation}

The INEL unit shown in Fig. 9.34 is typical of current large-scale DBGF filter design. The filter is housed in a $40 \times 80 \times 14$ - $\mathrm{ft}$-high concrete-lined pit and is divided into four individually isolable bays. Each bay contains four stacks of filter trays arranged in parallel, and each stack contains five trays in series. The trays are supported by and seal to steel embedments in the concrete. Tray (bed) depths and the packing densities of the fiberglass wool in the trays are given in Table 9.4. Airflow is upward and, at the design airflow of $50 \mathrm{fpm}$, initial (clean filter) pressure drop is about $1.5 \mathrm{in.wg}$. The final pressure drop, after a total dust loading estimated to be 10,500 $\mathrm{lb}$, is $8 \mathrm{in}$.wg. In-place tests of the filter, using $0.7-\mu \mathrm{m}$ NMD DOP aerosol, indicated an efficiency of about

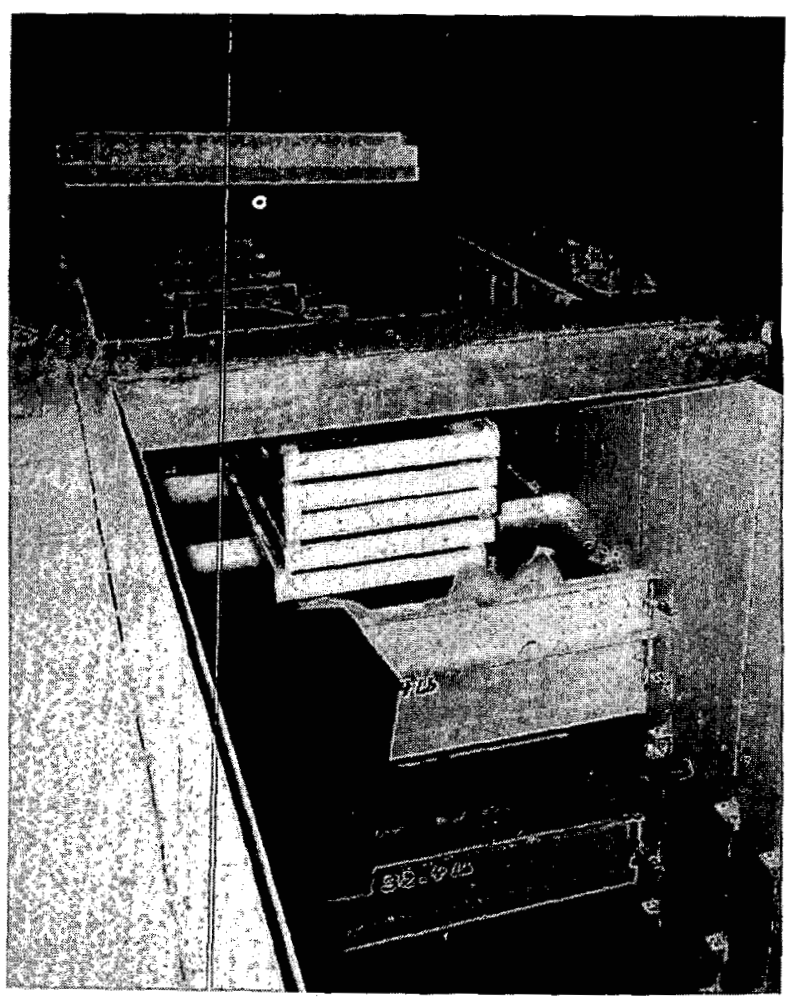

Fig. 9.34. View of the DBGF filter at the Chemical Processing Plant, Idaho National Engineering Laboratory. Courtesy Allied Chemical Co.

Table 9.4. Depth and media-packing densities of an Idaho chemical processing plant DBGF filter ${ }^{a}$

\begin{tabular}{ccc}
\hline Stage & $\begin{array}{c}\text { Bed depth } \\
\text { (in.) }\end{array}$ & $\begin{array}{c}\text { Packing density } \\
\left(\mathrm{Jb} / \mathrm{ft}^{3}\right)\end{array}$ \\
\hline 5 & 18 & 3.0 \\
4 & 18 & 1.5 \\
3 & 18 & 1.5 \\
2 & 15 & 0.7 \\
1 & 15 & 0.7 \\
\hline
\end{tabular}

${ }^{a}$ Airflow direction is upward. 
$80 \%$, but there is some question as to the accuracy of this value because of difficulties of introducing the DOP and obtaining a meaningful upstream sample. The calculated mass efficiency or arrestance is 99.95\% (i.e., a mass decontamination factor of 2000).

Early predictions of achievable DBGF filter efficiencies were overly optimistic. Although some eight fiber types were tested and gave promise of efficiencies approaching that of a HEPA filter, only one, the Owens Corning Fiberglas type 115K, was found to be satisfactory under field conditions. ${ }^{43}$ The key to $115 \mathrm{~K}$ fiber performance is that it has a permanent curl that, when the fiberglass wool is packed into trays at packing densities of from 0.7 to 9 $\mathrm{lb} / \mathrm{ft}^{3}$, resists matting; the other fibers, being straight, tended to pack down under operating conditions, resulting in extremely high pressure drop at even low airflow velocity. ${ }^{44}$ The satisfactory operation of a DBGF filter is a function of collection efficiency, airflow and pressure-drop characteristics, and filter life, and depends on maintainability, testability, and details such as tray, joint, and overall filter design, flow and service connections, instrumentation, and disposability of spent media. Airflow capacity is a function of filter size. The mass collection efficiency and pressure drop at design airflow velocity can be determined from the following equations: ${ }^{43,45}$

$$
\begin{aligned}
& \log \mathrm{DF}_{r}=C L^{a} P^{h} V^{c}, \\
& \eta_{r}=1-\frac{1}{D F}, \\
& R=K L^{x} P^{y} V^{z},
\end{aligned}
$$

where

$$
\begin{aligned}
\mathrm{DF}_{r}= & \text { decontamination factor, based } \\
& \text { radioactivity (dimensionless); } \\
C & =\text { constant (from Table } 9.5) ; \\
L & =\text { bed depth, in.; } \\
P & =\text { fiber packing density, } \mathrm{lb} / \mathrm{ft}^{3} ; \\
V & =\text { airflow velocity, fpm; }
\end{aligned}
$$

$$
\begin{aligned}
\eta_{r}= & \text { collection efficiency based on radioac- } \\
& \text { tivity (decimal); } \\
R= & \text { resistance, in.wg; } \\
K & =\text { constant, from Table } 9.5 ; \\
a, b, c, & =\text { empirical constants, from Table } 9.5 . \\
x, y, z & \text { em }
\end{aligned}
$$

Values of the constants and exponents determined for several of the fibers tested at Hanford ${ }^{43}$ including fiber $115 \mathrm{~K}$, are given in Table 9.5. The DF and pressure drop of a multibed (series) filter are the sums of the DFs and pressure drops, respectively, of the individual beds. From these equations, a filter filled with $115 \mathrm{~K}$ fiber and operated at an airflow velocity of $20 \mathrm{fpm}$ would require $40.62 \mathrm{in}$. of bed depth to produce a $\mathrm{DF}_{r}$ of $2000 . \mathrm{DF}_{r}$ is more closely related to mass DF, or arrestance, than it is to number DF, the value used for the HEPA filter.

As with the sand filter, the collection efficiency of the DBGF filter increases with increasing velocity through the bed; however, collection efficiency for small (submicron) particles drops off with increasing velocity, because the effectiveness of the diffusion mechanism (on which trapping of submicron-sized particles is dependent) decreases (Figs. 2.8 and 2.17). By careful selection of packing density, bed depth, and airflow velocity, collection efficiency equivalent to that of a group II to group III ventilation air filter (Sect. 3.3) can be achieved. The other fibers tested, although found to be unsatisfactory for the DBGF unit, have been used in the media

\begin{tabular}{|c|c|c|c|c|c|c|c|c|c|c|}
\hline \multirow{2}{*}{ Type of fiber } & \multirow{2}{*}{ Type glass } & \multirow{2}{*}{$\begin{array}{c}\text { Fiber diameter } \\
\qquad(\mu \mathrm{m})\end{array}$} & \multicolumn{8}{|c|}{ Constants to be used in Eqs. (9.2) and (9.4) } \\
\hline & & & C & $a$ & $b$ & $c$ & $K$ & $x$ & $y$ & $z$ \\
\hline $\mathrm{AA}$ & E & 1.3 & 4.6 & 0.8 & 1.0 & -0.2 & 0.082 & 1.0 & 1.5 & 1.0 \\
\hline $\mathbf{B}$ & $\mathrm{E}$ & 2.5 & & & & -0.25 & & & & 1.0 \\
\hline $115 \mathrm{~K}$ & $\mathrm{C}$ & 30 & 0.054 & 0.9 & 0.9 & -0.4 & 0.00020 & 1.0 & 1.5 & 1.0 \\
\hline
\end{tabular}
of replaceable medium filters (Fig. 3.7) installed in series with and downstream of the DBGF filter. Equations (9.2) through (9.4) can also be used for determining the $D F_{r}$, efficiency, and pressure drop of these unitized modular filters. Some of the earlier Hanford canyon-exhaust installations employed a DBGF forefilter followed by a bank of afterfilters (Fig. 3.7) filled with AA-fiber medium.

Although there is an economic incentive to increase velocity through the DBGF filter (bed area is inversely proportional to volumetric flow rate, and

Table 9.5. Fiber characteristics, constants; and exponents for calculating decontamination factors and pressure drops of DBGF filters [Eqs. (9.2) and (9.4)] 
capital cost is directly proportional to bed area), experience shows that a maximum velocity of about $50 \mathrm{fpm}$ is desirable. Airflow in units larger than 10,000 -cfm airflow capacity is usually upward, with filter beds installed horizontally, as shown in Figs. 9.34 through 9.37. Although there are currently no standards for DBGF filters, it is likely that future regulations will require that they be capable of withstanding natural phenomenon incidents, as dis- cussed in Sect. 9.4. Large units are in most cases installed underground or partially underground, with earth cover, for the attenuation of collected radioactivity. If moisture is likely to be present, stainless steel cladding of the concrete pit is recommended (Sect. 9, ANSI N 101.6) ${ }^{46}$ To avoid plugging and premature failure or excessive resistance, provision for backflushing is sometimes made. Backflushing sprays are generally necessary only for the first one or

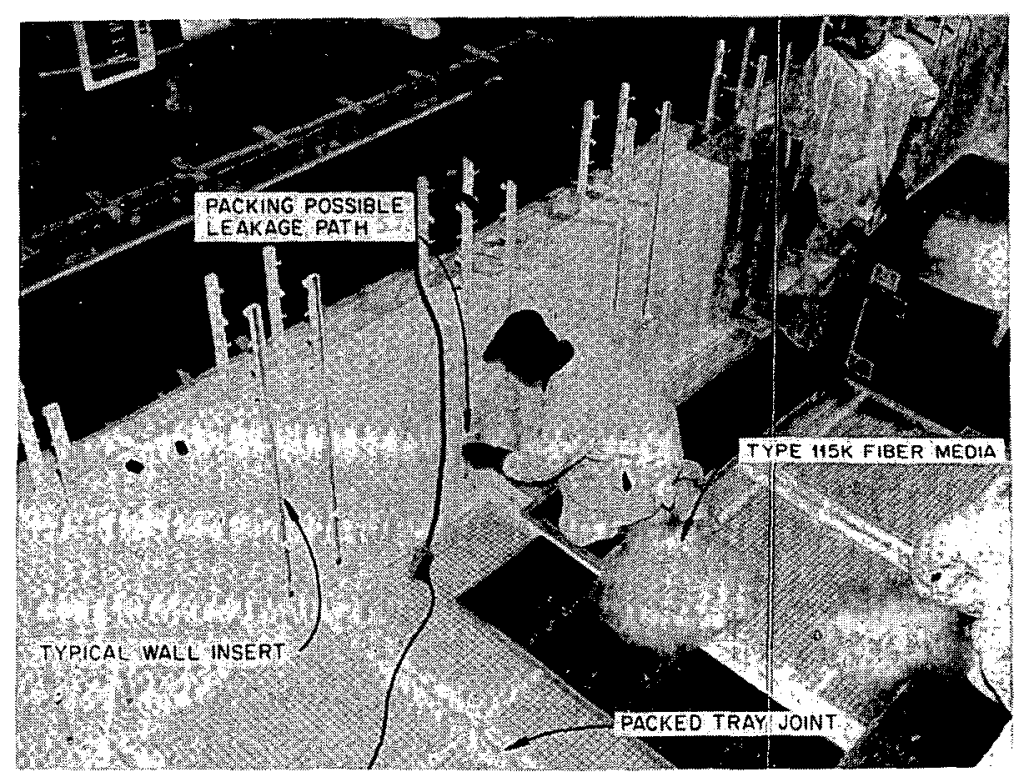

Fig. 9.35. Joints between walls and trays of 291-A-2 DBGF filter, Purex plant, being packed with loose fiber to prevent bypassing. Courtesy Atlantic Richfield Hanford Co.

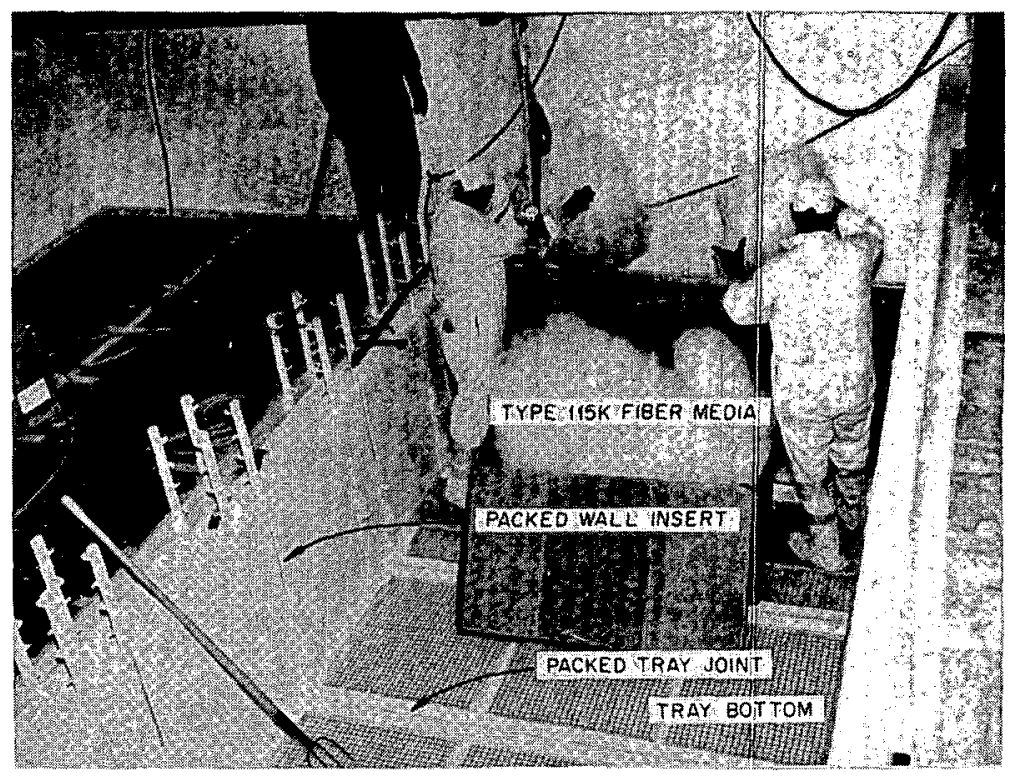

Fig. 9.36. Packing media into trays of a 291-A-2 DBGF filter under construction at the Purex plant. Note packed wall joints, embedments in walls. Courtesy Atlantic Richfield Hanford Co. 


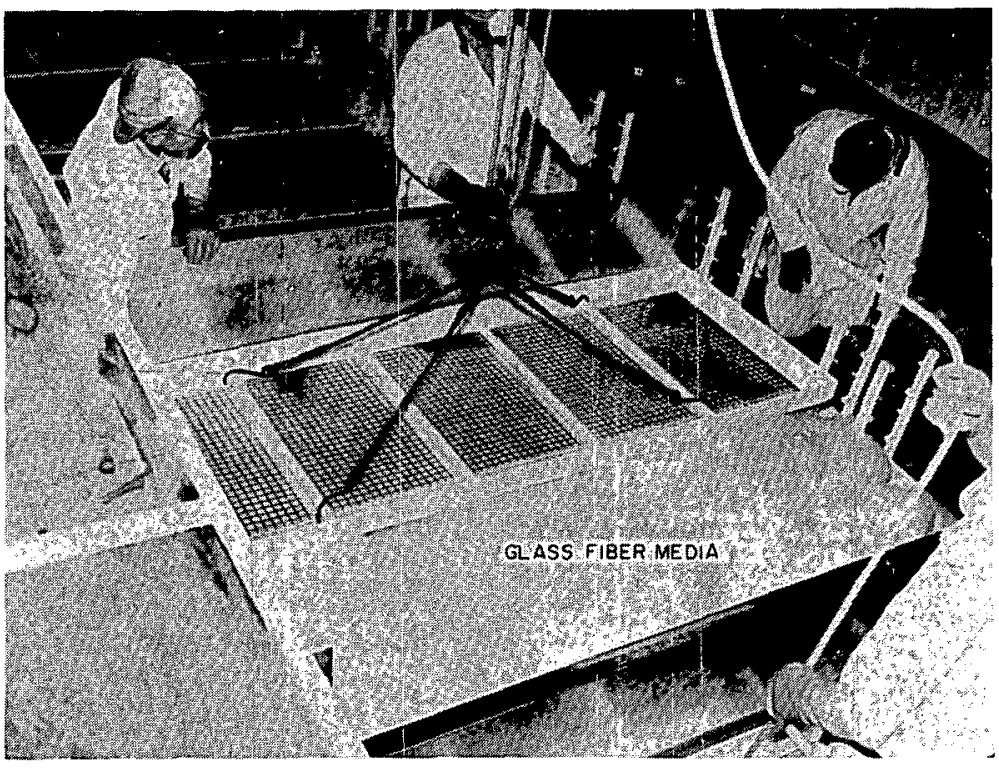

Fig. 9.37. Installing top screen on fiber-backed tray of a 291-A-2 DBGF filter under construction at the Purex plant. Courtesy Atlantic Richfield Hanford Co.

two stages of the filter. Large DBGF filters should be segmented (Sect. 2.4.12), with each section contained in an isolable, individual vault, as in the INEL filter, to provide flexibility for maintenance and testing. Provisions for in-place testing with DOP should be built into the filter, with particular attention given to the ability to achieve a satisfactory air-aerosol mixture entering the filters and the ability to take reliable samples immediately upstream and at a point downstream, where there is good mixing of the filtered air and penetrating contaminants.

Instrumentation to establish the dew point of the inlet air, the flow rate, the pressure drop across individual trays (not stages), and the inlet air temperature is recommended. The capability of monitoring the radiation level in various parts of the filter and the provision for removing strategically located samples of media from time to time are desirable.

In large filters, the medium is contained in modular, interchangeable trays. Figure 9.38 shows the bottom screen assembly of a typical tray. Besides holding the filter medium in place, the trays are also designed, and must be structurally reinforced, to apply the compressive force required to give the desired packing density. The compressive pressure necessary to produce the required packing density of $115 \mathrm{~K}$ fibers, over the range of from 0.7 to $9 \mathrm{lb} / \mathrm{ft}^{3}$, can be found from the following equation:

$$
P_{c}=2.1(P-0.6)^{2},
$$

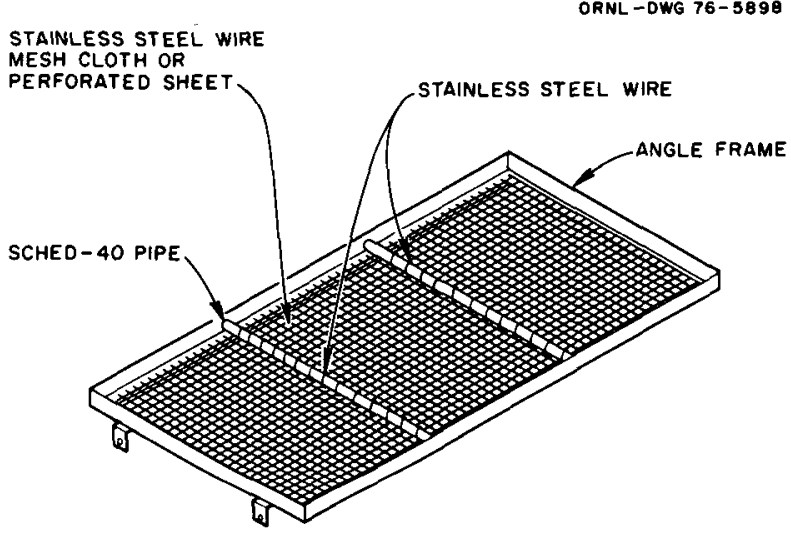

Fig. 9.38. Typical top or bottom screen for DBGF filter trays.

where

$$
\begin{aligned}
P_{c} & =\text { compressive pressure, } \mathrm{lb} / \mathrm{ft}^{2} ; \\
P & =\text { packing density, } \mathrm{lb} / \mathrm{ft}^{3} .
\end{aligned}
$$

Compressing type $115 \mathrm{~K}$-fiber insulating wool to a density of $9 \mathrm{lb} / \mathrm{ft}^{3}$ requires a compressive pressure of about $150 \mathrm{lb} / \mathrm{ft}^{2}$.

\subsubsection{Wet Operation}

Although reports have indicated that a DBGF filter can provide satisfactory service when operated so that water continuously condenses in the beds, ${ }^{47}$ wet operation is not recommended. Downstream filters, whether HEPA or the extended-medium type shown in Fig. 3.7, will not tolerate high moisture 
loadings and lose performance, or deteriorate, when operated continuously wet, ${ }^{43,45}$

\subsubsection{Plugging}

The high dust-holding capacity of the DBGF filter should not induce the operator to totally forego the pretreatment of air discharged to it. Some contaminants must be removed from the process exhaust streams, and the operator must be as sensitive to the limitations of the DBGF filter as he is to the limitations of the HEPA filter. The first large DBGF filter at the Hanford Purex plant was originally operated in a downflow condition. The gradual buildup of ammonium nitrate on the upper fiberglass layers finally increased the pressure drop, over a period of nine years, to the point that the system could no longer meet canyon ventilation airflow requirements. ${ }^{48}$ The effect of plugging is shown in Fig. 9.39, which illustrates the effect of loading a 2-in.thick bed of $115 \mathrm{~K}$-fiber medium, packed to a density of $6 \mathrm{lb} / \mathrm{ft}^{3}$ at $25 \mathrm{fpm}$ airflow velocity, with methylene blue. As the load increased from 0 to 200 grains of methylene blue per square foot, the pressure drop across the bed increased by only $0.5 \mathrm{in.wg}$. With an additional 60 grains $/ \mathrm{ft}^{2}$, however, the pressure drop increased to $5.5 \mathrm{in.wg}$, or ten times that at the start of this additional loading. This increase suggests possible difficulties in the operation of the filter. If particulate concentrations in the exhaust stream were constant over the life of the unit, the timing of the sharp knee of the load vs pressure-drop curve could

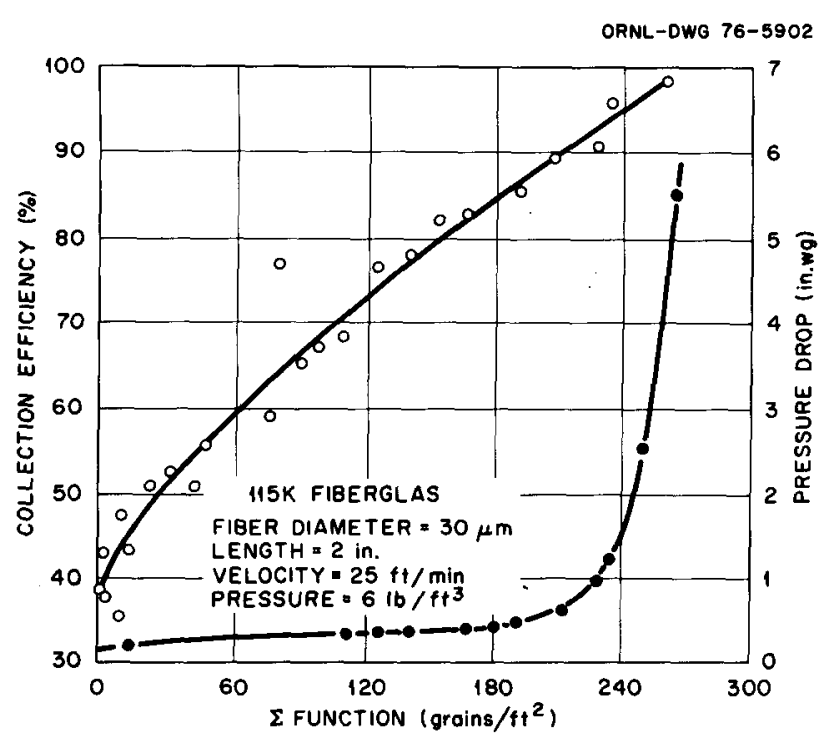

Fig. 9.39. Characteristic collection efficiency and loading curves for DBGF filter. be predicted. In actual operation, however, particulate concentrations vary substantially from time period to time period, depending on what is occurring at the source. A predetermined pressure drop is generally established as the indicator that a rapid pressure rise is imminent; if the critical loading that determines this guide is located to the right of the knee of the curve, plugging could occur before preplanned preventive action can be taken. If the set point precedes the knee, a small difference in pressure drop can make a large difference in the time interval between when the preventive action is taken and when it is needed.

\subsubsection{Disposal of Spent Filters}

Because low maintenance requirements are characteristic of large DBGF filters, little consideration was given to the disposal of spent media in early DBGF filter designs. In small units, the entire unit, including container, can be replaced, and the spent unit can be disposed of as solid waste. For large units, as with sand filters, it was considered sufficient that the inlet and exit ducts of the filter could be sealed and the contaminated media left in place. Present requirements for solid-waste handling, though clouded, may rule out such in-place disposal. If new requirements indicate that alpha-bearing material, such as DBGF filter media, must be sealed and safely removed to a site yet to be designated for permanent storage, it may be necessary to design future DBGF filters so that trays or media can be replaced. Media replaceability should not prove too difficult if it is considered in the original filter design; however, trayremoval capability adds substantially to capital costs. Tray-removal capability requires an alternate airflow path during the change-out operation (this can be provided with proper segmentation and the provision of isolation valves), the ability to decontaminate or shield the tray being removed, and an alpha-sealed, and probably remote, handling system. The flexibility gained by the ability to replace trays and media may be advantageous, however, because exact knowledge of future exhaust-air particulate loadings, after the filter goes into operation, is lacking and difficult to estimate before startup.

\subsection{REACTOR ENGINEERED-SAFETY- FEATURE AIR CLEANING SYSTEMS}

\subsubsection{Introduction}

Probably in no other field of human endeavor have such extensive, careful, and costly efforts been taken 
to prevent the occurrence of a major accident as those that have been taken in the design of nuclear reactors. As a result of these efforts, the likelihood of a major accident is considered to be extremely remote. ${ }^{49}$ No matter how remote the possibility, however, such accidents must be planned for, and ESF systems must be provided to mitigate the possible consequences. Major ESF systems include the containment postaccident air cleaning system and the control room protection air cleaning system, both of which must be designed to survive the structural and environmental effects of the worst combination of core disruptive accident and natural phenomenon (e.g., earthquake) that can be reasonably postulated for the reactor and yet remain operable during, or be capable of startup and operation following, that incident. Other ESF air cleaning systems include those serving ESF equipment areas and fuel storage areas of the plant. These latter systems are remote from the reactor and would be subjected to a lesser DBA; on the other hand, they must be capable of withstanding a safe shutdown earthquake (SSE), a tornado, or other natural phenomena postulated for the site.

\subsubsection{Reactor Containment}

Requirements of the containment postaccident air cleaning system depend, in large measure, upon the type of containment employed. ${ }^{50}$ There are five basic types of reactor containment: single-pressure containment, double-pressure containment, pressure containment with shield building, vented confinement, and containment/confinement.

In pressure containment (Fig. 9.40), the reactor vessel head space (or head space vault) and reactor bay are enclosed by a large $\left(2 \times 10^{6} \mathrm{ft}^{3}\right.$ or larger volume), ASME Code-constructed, ${ }^{51}$ leaktight (0.1 vol $/ 24-\mathrm{hr}$ maximum permissible leak), steel or steel and concrete vessel designed to withstand the maximum temperatures and pressures developed in the DBA and SSE. A recirculating air cleaning system is provided to minimize the airborne radioactive material that escapes from the pressure containment. Pressure containment with internal recirculating (or kidney) air cleaning facilities was used for several early pressurized-water reactors (PWR) and has been proposed for liquid-metal fast breeder reactors (LMFBR). Because of the extremely severe postaccident service environment (temperatures to $275^{\circ} \mathrm{F}$; pressures to as high as 65 psia over the $1-$ to 10-sec period following a core disruptive accident; relative humidity of $100 \%$; air densities of two to three times normal; and, if containment sprays are used to reduce pressure, sensible moisture in a heavyrain condition in a PWR), the possible low reliability of filters and adsorbers under this environment, and the inability to repair or replace air cleaning system components following an accident, the internal recirculating or kidney system concept has apparently been abandoned in modern power plant practice, at least for light water reactors (LWR).

An alternative to the internal kidney system is the external recirculating system shown in Fig. 9.41. In this design, air cleaning components are located in pits outside of the containment structure. Isolation dampers in the ducts leading from and to the containment structure permit isolation of the air cleaning system components until the initial pressure transient has passed and pressure across the system

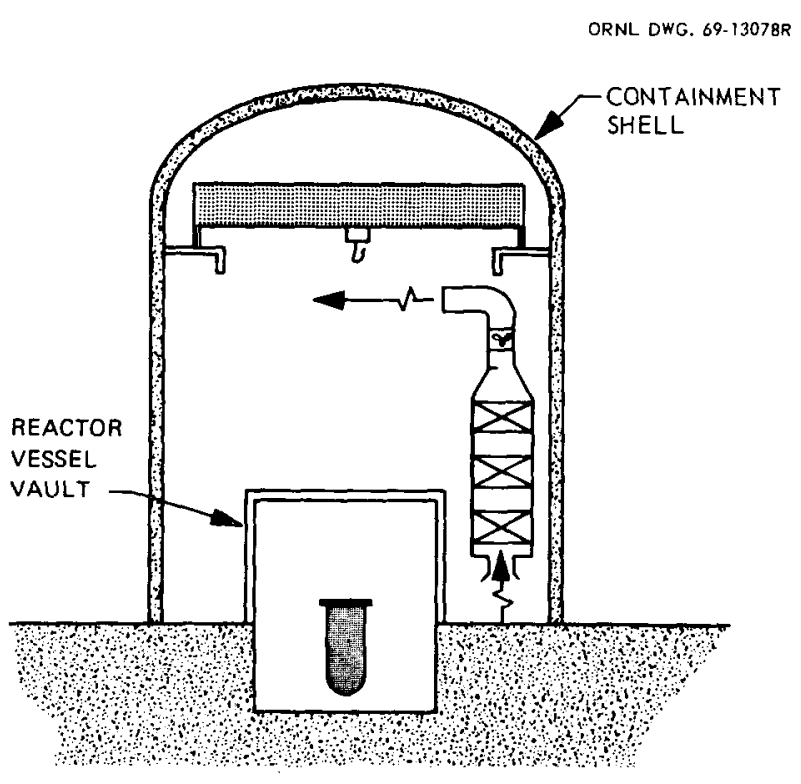

Fig. 9.40. Pressure containment with internal recirculating or kidney ESF postaccident air cleaning system.

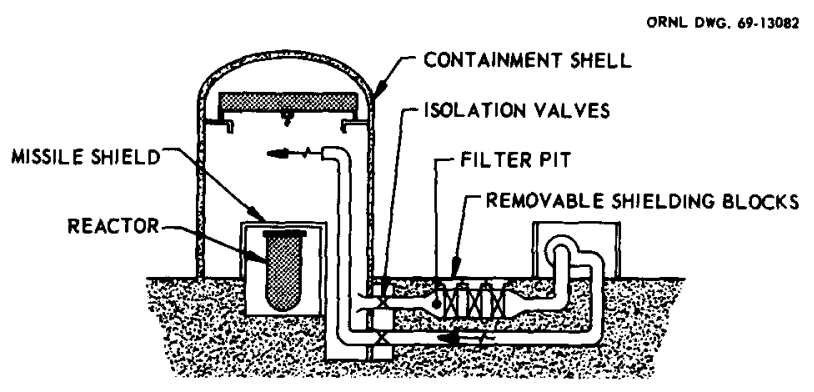

Fig. 9.41. Pressure containment with external recirculating postaccident air cleaning system. In this concept, note capability to repair or replace air cleaning components after an accident. 
can be equalized. System components are protected from missiles in the containment, and, with redundant systems, can be repaired or replaced remotely (Sect. 9.2) if necessary. Bypass dampers can be provided to permit operation of the system in the once-through mode for purging the containment. This concept, first proposed in the earlier edition of this handbook, ${ }^{52}$ has been considered for power reactors. ${ }^{53}$

Double containment (Fig. 9.42) has been proposed for the $\mathrm{LMFBR}^{54}$ In this concept, a pressure containment similar to that discussed above surrounds an inner ASME Code-constructed pressure containment surrounding only the reactor vessel head space. The inner vessel, which must be removable to permit access to the reactor core, has a permissible leak rate of 1 vol per $24 \mathrm{hr}$ but is designed to withstand the maximum pressures and temperatures of a DBA. Kidney-type ESF containment postaccident air cleaning facilities are provided in the outer containment space. Since these facilities "see" only the radioactive material that leaks from the inner containment, and most of the particulate matter emitted in a core disruptive accident would settle or plate out in the inner containment, the loading and environmental conditions to which they are subjected are substantially less than in the case of single containment.

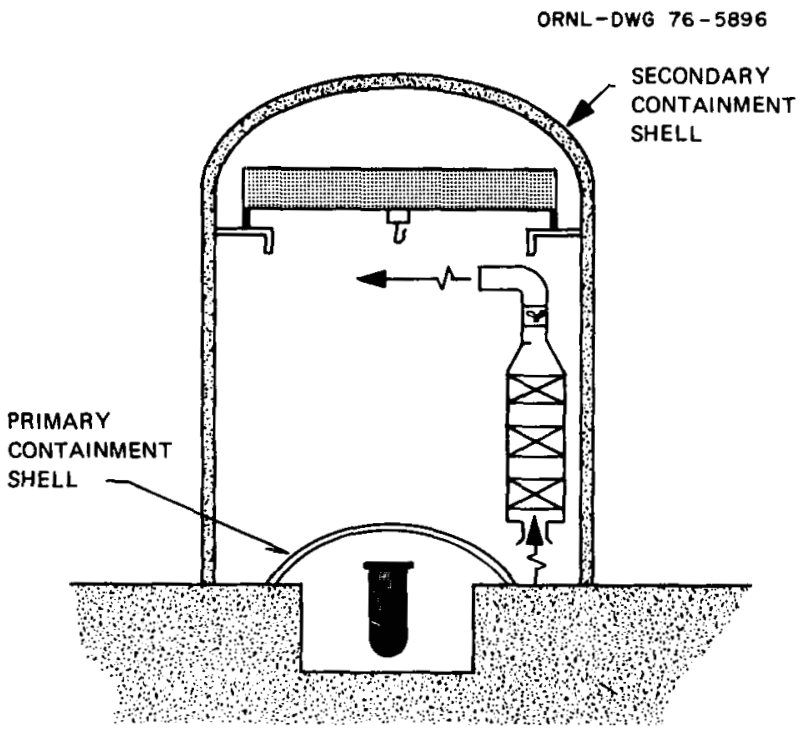

Fig. 9.42. Double containment as proposed for a commercial scale LMFBR. Both containments designed to ASME Code, primary containment shell removable for access to reactor vessel head space. Internal recirculating (kidney) postaccident air cleaning system, no provision for postaccident air cleaning in primary.
Pressure containment with shield building (Fig. 9.43) has been employed in most recent PWRs and in some recent boiling-water reactors (BWR). In this concept, an annular shield building of essentially conventional construction surrounds the pressure containment structure. Any leakage from the primary containment is to the shield space. ESF air cleaning facilities are provided in or adjacent to the shield space. These may be once-through, discharging to the atmosphere, once-through and discharging back to the primary containment (pump-back), or recirculating within the shield space. In most cases, shield space is maintained at a lower pressure than either the primary containment or the atmosphere. Shield-building ESF air cleaning facilities are small (4000- to $6000-\mathrm{cfm}$ installed capacity in the basic system, with $100 \%$ redundancy) compared with incontainment kidney systems (as large as 100,000-cfm installed capacity in the basic system, usually with $100 \%$ redundancy; a 200,000-cfm ESF kidney system with 100\% redundancy was proposed for the LMFBR). As the components are protected from the severe postaccident environment of the primary containment, shield-building systems are obviously much more reliable. A variant of the shield-building concept is the penetration vault that has been used in some reactors. In this design (Fig. 9.44), the shield volume surrounds only the area of the containment structure at which steam lines, piping, electrical conduits, and other penetrations occur.

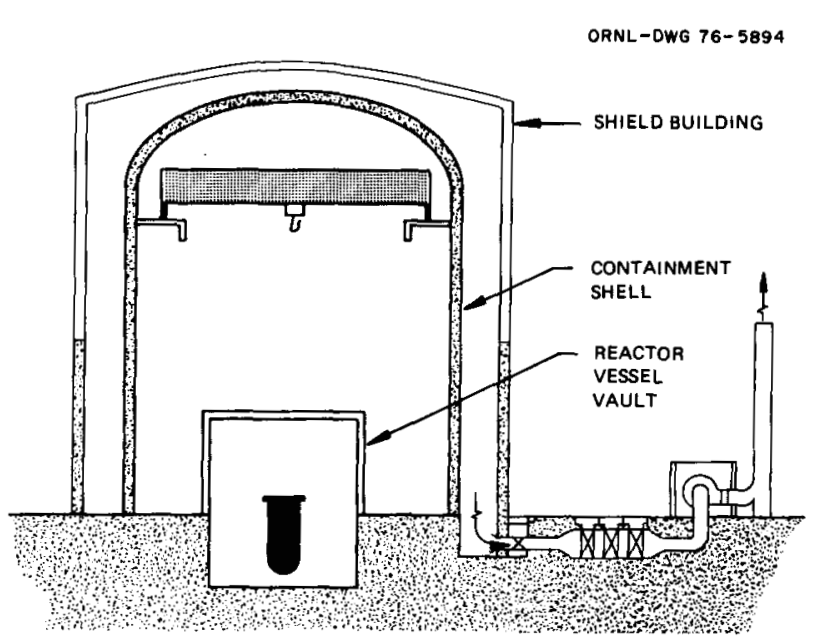

Fig. 9.43. Pressure containment with shield building. Oncethrough ESF air cleaning system vents shield space to remove contaminants leaked to shield space from containment. External air cleaning facility permits repair or replacement of components following accident. 
Vented confinement (Fig. 9.45) is employed in ERDA production reactors and most research reactors. In this design, the reactor vessel head space (or head space vault) and reactor bay are enclosed in a low-leakage building of special, but essentially conventional design. ${ }^{55}$ The containment structure is not an ASME Code vessel. ESF air cleaning facilities may be either recirculating, as in the Savannah River reactors, or once-through, discharging to the atmosphere through a high stack (elevated release may reduce offsite doses by an order of magnitude or more, as compared with ground-level release). The confinement building is maintained at negative pressure relative to ambient by means of the exhaust system. In most ERDA and research reactors, the same set of air cleaning facilities serves both the normal operational and postaccident functions. If vented confinement should be used for commercial

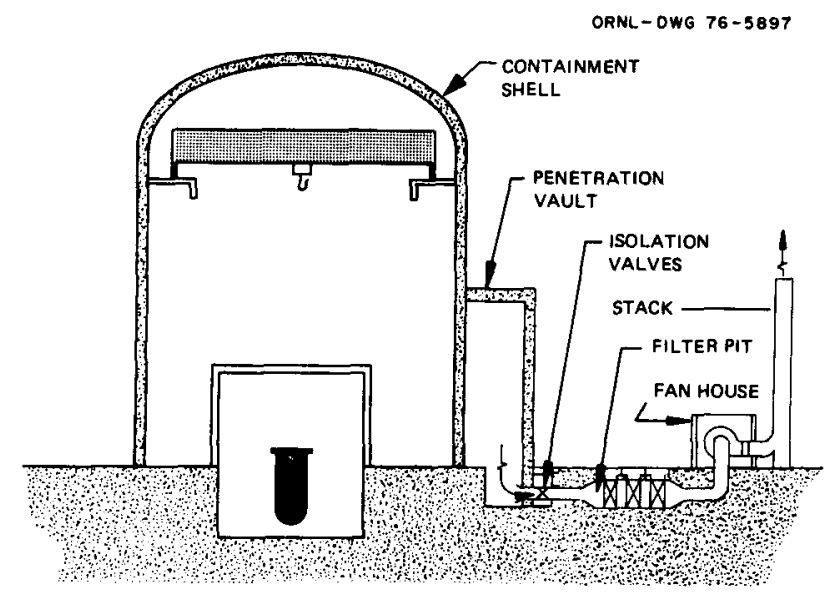

Fig. 9.44. Pressure containment with vented penetration vault.

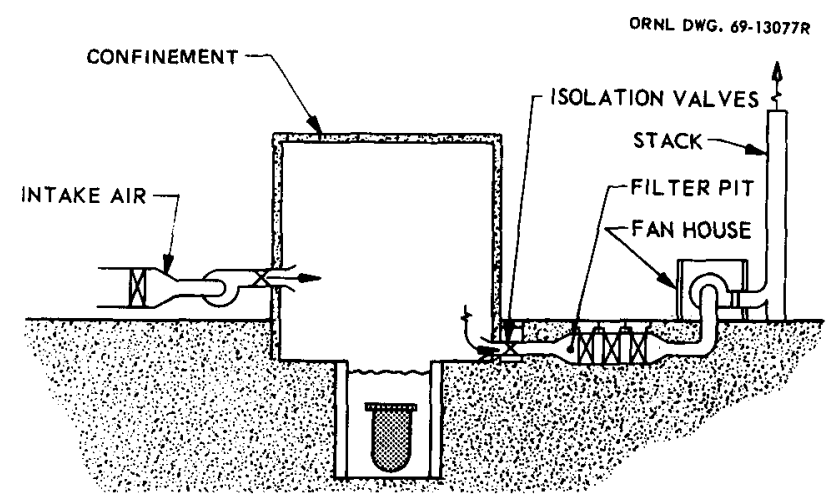

Fig. 9.45. Vented confinement as used in production and research reactors. Once-through air cleaning facilities are on-line at all times during both normal and reactor upset conditions. reactors, it is likely that separate ESF and normal operational systems would be required. The systems are very large (for example, a 128,000-cfm basicsystem installed capacity, plus $25 \%$ redundancy, in the Savannah River reactors).

Containment/confinement (Fig. 9.46) is employed in most $\mathrm{BWRs}$ and is one of the concepts proposed for commercial scale LMFBRs. The reactor vessel, or reactor vessel head space, is enclosed in an ASME Code-constructed containment vessel which, in turn, is surrounded by a confinement building similar to that used for vented confinement. Unlike vented confinement, air cleaning system components are not exposed to the severe postaccident environment of a $\mathrm{DBA}$ and are required to remove only the small quantity of material that leaks from the containment vessel or around containment penetrations. Therefore, ESF air cleaning facilities are small, ranging from as low as 4000 -cfm basic-system airflow to as much as $16,000 \mathrm{cfm}$ for BWR standby gas treatment systems (SGTS), depending on the size of the confinement building. In all cases, $100 \%$ redundancy of ESF air cleaning facilities is required. A basic-system airflow of $15,000 \mathrm{cfm}$ has been proposed for a $1000-\mathrm{MW}$ (e) LMFBR employing the containment/confinement design. ${ }^{54}$

If air cleaning equipment is located high in the containment or confinement building, as has been done in some reactors to conserve space on the reactor or fuel-loading floor, ${ }^{56}$ protection against extreme shaking in the event of an earthquake is needed (the amplification of ground-level earthquake

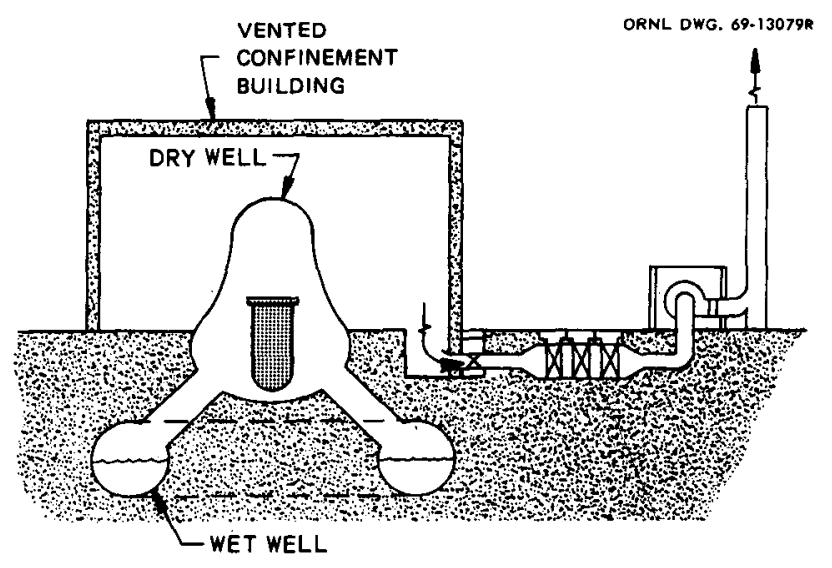

Fig. 9.46. Containment/confinement for BWR with oncethrough, external-standby gas-treatment system. In event of accident, steam and gases released in dry well expand into wet well where they come in contact with water to reduce pressure and capture some particulate matter and iodine. 
acceleration may be as great as 30 to 150 , depending on the height of the equipment above ground level)..$^{57}$

In all containment concepts, non-ESF air cleaning facilities are usually provided in the containment or confinement building for air cleaning under normal operating, maintenance, or shutdown conditions, and for containment purge. Non-ESF systems are generally much smaller than in-containment postaccident air cleaning systems and are not required to be redundant. In commercial power reactors non-ESF systems are independent of the ESF systems and must be shut down in the event of an accident. Although non-ESF systems do not have to meet the postaccident and earthquake survival requirements of the ESF system, if located in areas where ESF facilities of any type exist, they must be at least designed to resist falling or tearing loose during a core disruptive accident or SSE.

\subsubsection{Light Water Reactors}

Guidelines for the design of light water reactor ESF air cleaning facilities are given in Regulatory Guide $1.52,{ }^{58}$ which recommends a sequence of air cleaning components consisting of demister, prefilter, HEPA filter, adsorber, and final HEPAfilter stage. A heater is also recommended upstream of the adsorbers to maintain the relative humidity of the air entering the adsorbers at approximately $70 \%$ following a loss-of-coolant accident (LOCA). Because radioiodine is the contaminant of major concern in the event of a light water reactor LOCA, the need for two stages of HEPA filters is often questioned, particularly in that prefilters are also required upstream of the first-stage HEPA filters. The first-stage HEPA filters have two functions: (1) protection of the adsorbers from particulates which could "blind" the adsorbent granules, and (2) holdup of iodine-bearing particles. Without the first-stage HEPA filters, these particles could penetrate the adsorber beds and be caught on the downstream HEPA filters, and the iodine adsorbed on them [which accounts for $5 \%$ of the postulated iodine load (Sect. 3.4)] would desorb to the air being discharged from the system. The second-stage HEPA filters prevent the loss of iodine-bearing adsorbent fines and also provide backup protection in the event of failure of or damage to the first-stage HEPA filters.

Components of the ESF air cleaning system must be designed, constructed, tested, and maintained to ensure effective and reliable operation when subjected to the postulated environment and service conditions of the DBA. The least-stringent postaccident environment and service conditions would be encountered in the confinement building of a containment/confinement system or in the shield building of a pressure-containment/shield-building system. Although the ESF air cleaning facilities would have to withstand the SSE and humidity of $100 \%$, pressure and temperature transients would be nil, and particulate concentrations and radioactivity levels would be relatively low, compared with ventedcontainment or pressure-containment systems. Other conditions that have to be considered are pressure transients due to a design basis tornado or to an inadvertently opened or closed damper and shock, vibration, physical displacement resulting from a postulated simultaneous SSE.

Simple vented confinement, because there is no inner containment, is subject to much more stringent postaccident service conditions than the containment/confinement or pressure-containment/shield-building designs. Postaccident service conditions may include radiation levels in air cleaning components of $10^{7}$ to $10^{8} \mathrm{rads}$, temperatures as high as $275^{\circ} \mathrm{F}$, and pressures of 2 to 3 psi above atmospheric, in addition to large volumes of condensing steam and, perhaps, sensible moisture. In addition, the system must be designed to withstand the design basis earthquake and tornado. The most severe postaccident conditions would be encountered in a pressure containment with an ESF kidney air cleaning facility. In addition to high radiation levels $\left(10^{7}\right.$ to $10^{8}$ rads), high temperatures (up to $275^{\circ} \mathrm{F}$ ), and large volumes of condensing steam and sensible moisture, ducts and equipment housings may be subjected to high collapsing pressures, as great as 40 to 45 psig during the first few minutes following the core disruption due to the lag of pressure rise in the duct relative to pressure rise in the containment, unless pressure-relief dampers are provided. Fans and motors are required to operate at very high temperatures in saturated air and in air densities of 2 to $3 \mathrm{~atm}$. If chemical sprays are discharged in the containment (to reduce pressure and react with airborne iodine), corrosion of metal parts and chemical attack of filter and adsorber media may also take place.

In all reactor postaccident situations, fissionproduct-decay heating of carbon in the adsorbers must be considered. With insufficient provision for cooling in the event of main-fan failure, heating of the carbon may result in desorption of the already 
trapped radioiodine (which would constitute failure of the system) or of the impregnants on which radioiodine trapping depends, or even in ignition of the carbon. Tests have shown that deluge water sprays, often provided for extinguishing carbon fires, are of limited value ${ }^{59}$ in addition, the water washes out both the impregnant and any trapped radioiodine, ${ }^{60}$ thus causing further loss of iodine containment and creating a substantial liquid waste problem.

Demisters are required in all systems because of high sensible moisture and possible steam loadings which can plug HEPA filters and degrade the effectiveness of activated carbons for organic iodine compounds. Demisters require adequate drains to carry the collected water to the liquid waste system. If drains are not properly designed and maintained, a bypass of the HEPA filters and adsorbers may be created (through the drain system), which would result in failure or degradation of the air cleaning function. Controls, instruments, sensing and air lines, electrical equipment, and electrical wiring serving the air cleaning system must also be designed to withstand the postulated postaccident environment and conditions without failure. Redundant-unit ductwork and equipment must be geographically isolated, shielded, or installed in individual vaults to protect against single failure from missiles resulting from burst piping or failed equipment and from falling pipes, equipment, and ducts. Redundant units are always required to provide backup air cleaning capacity in the event of on-line unit failure. Provision for remote maintenance, though rarely considered, is desirable to permit the reactivation of failed units or the replacement of damaged or failed components.

\subsubsection{High-Temperature Gas-Cooled Reactors}

Single pressure containment has been proposed for the commercial high-temperature gas-cooled reactor (HTGR). ${ }^{61}$ However, because of the low specific heat of the gas and certain features of the concrete reinforced pressure vessel employed, the postaccident containment environment and service conditions will not be as severe as in the LWR single containment. Although temperature and pressure may reach $700^{\circ} \mathrm{F}$ and $40 \mathrm{psig}$, respectively, the duration of these transients will be brief, and startup of the ESF air cleaning facilities can be delayed until the worst of their adverse effects have passed. A typical 1000MW(e) HTGR employs a recirculating containment postaccident air cleanup system consisting of four 18,000-cfm (installed capacity) units located $90^{\circ}$ apart within the containment structure. ${ }^{62}$ The basic system installed capacity is $36,000 \mathrm{cfm}$, with $100 \%$ redundancy. During normal and maintenance operations, the containment is held at a slight negative pressure by a $20,000-\mathrm{cfm}$ combination recirculating and once-through purge and containment cooling system operating in a "feed and bleed" mode. If an accident occurs, this system is shut down, and the ESF units are brought on line following a 2sec to 15-min delay to permit decay of initially high postaccident temperature and pressure. Although there are no regulatory guides governing hightemperature gas-cooled reactor ESF air cleaning facilities, it is likely that an air cleaning unit configuration similar to that recommended in Regulatory Guide $1.52^{58}$ for LWRs would be advised, that is, a train consisting of demisters, prefilters, firststage HEPA filters, adsorbers, and second-stage HEPA filters. Although the reactor coolant is helium gas, free water or steam could be present in the containment due to the rupture of feedwater or secondary-coolant piping; therefore, demisters would be required. All requirements of the LWR for protection of ESF air cleaning facilities from tornados, earthquakes, pressure transients, missiles, and other postaccident environmental and service conditions would apply equally to the HTGR.

\subsubsection{Liquid-Metal Fast-Breeder Reactors}

Two features influence the design of ESF air cleaning facilities for LMFBRs. First, the large plutonium inventory of the fuel makes inhalation of plutonium-bearing aerosols the major concern rather than inhalation of radioiodine, as is the case in LWRs and HTGRs. Second, there is potential for a sodium fire which can produce large quantities of thick, viscous fumes (sodium hydroxide and oxides) that can rapidly plug many air cleaning devices, especially HEPA filters. Although one may not normally associate water with an LMFBR, burning sodium in contact with concrete can release substantial quantities of water (hydration from the concrete), creating a relative humidity in the contained space approaching $100 \%$.

Three containment concepts have been considered for the commercial-scale [1000-MW(e)] LMFBR: single containment, double containment, and containment/confinement. For the singlecontainment case, a 200,000-cfm basic-system installed capacity (plus $100 \%$ redundancy) postaccident air cleanup system was proposed, ${ }^{54}$ consisting of 
low-efficiency ( $\sim 50 \%$ ASHRAE dust spot) prefilters and one bank of HEPA filters. Radioiodine adsorbers were not considered necessary because of the ready reaction of iodine with sodium and sodium fumes, and because the large installed capacity was designed to accommodate the large quantities of sodium fumes that would evolve. ${ }^{63}$ As with the PWR, the severe postaccident environment in the containment space makes serious consideration of this type of system unlikely. Postaccident environm ntal conditions include a gas temperature of $300^{\circ} \mathrm{F}$, maximum containment pressure of $10 \mathrm{psig}$, maximum $\mathrm{RH}$ of $100 \%$, and $0.7 \mathrm{~g} / \mathrm{cm}^{3}$ particulate concentration. In addition, the probable poor reliability of HEPA and other fiberglass-medium filters in a caustic environment and the probable poor performance of any filter against very sticky particles, as would exist with moist sodium fumes, would further legislate against this choice of system.

Double containment with ESF air cleaning facilities in the secondary containment space has a much greater chance of successful and reliable operation under accident conditions. A much smaller system (72,000- to 75,000-cfm basic-system installed capacity, plus $100 \%$ redundancy), again consisting of prefilters and HEPA filters only, is required since the secondary containment "sees" only leakage from the primary (the primary containment vessel is assumed to leak a maximum of 1 vol per $24 \mathrm{hr}$ ), and the possibility of a sodium fire in the secondary containment space is eliminated by inerting that space with nitrogen. Again, radioiodine is contained in the primary containment space due to the ready reaction of iodine with sodium and sodium fume and the plate-out and settling of the fume particles within the primary space. Although proposed for a 1000-MW(e) plant, ${ }^{64}$ double containment has the drawbacks of high temperature (up to $300^{\circ} \mathrm{F}$ over a $60-\mathrm{hr}$ period) and humidity (up to $100 \% \mathrm{RH}$ ) in the secondary containment space, which may make for low longterm reliability of filters and the inability to replace or repair components following an accident. This inability can be offset by providing an external recirculating loop, as shown in Fig. 9.41.

Containment/confinement with ESF air cleaning facilities in the confinement space appears to be a promising system for commercial LMFBRs. The primary containment is identical to that proposed for the double-containment case and; as in the doublecontainment case, the ESF air. cleaning facilities "see" only the material that leaks from the primary containment. The confinement building is con- tinuously vented by a non-ESF purge and cooling system during normal and maintenance operations and by the ESF system in the event of an accident. Therefore, the temperature in the confinement space is no more than slightly above a mbient $\left(110^{\circ} \mathrm{F}\right)$ unless there is a sodium fire, in which case the temperature might reach 200 to $300^{\circ} \mathrm{F}$ during the period of the fire. A sodium fire in the reactor bay is a major consideration of this concept, and a very large prefilter stage would be required to accommodate the fume arising from a burning $1000-\mathrm{ft}^{2}$ sodium spill. Furthermore, because the fire might release water of hydration from the concrete (the water content of concrete averages $\left.9 \mathrm{lb} / \mathrm{ft}^{3}\right)$, ${ }^{65}$ the fume would be sticky and would tend to clog air cleaning devices. Although airflow requirements of the system are small $(15,000$ $\mathrm{cfm}$ ), the basic-system installed capacity of the prefilter and the first-stage HEPA filter stages would have to be about $480,000 \mathrm{cfm}$ to accommodate the large volume of fume resulting from a sodium fire in the confinement space. $^{63}$ Because venting would continuously remove sodium fume in the containment atmosphere, no reaction between iodine and sodium in the secondary space can be assumed, and iodine adsorbers would be required. Extrapolating recommendations from Regulatory Guide $1.52,{ }^{58} \mathrm{a}$ second stage of HEPA filters is necessary. The installed capacity of the adsorbers and second-stage HEPA filters would be $15,000 \mathrm{cfm}$, and $100 \%$ redundancy of all stages, including the very large prefilter and first-stage HEPA filter stages, would be necessary. An alternative to the very large $(960,000$ cfm total installed capacity) prefilter and first-stage HEPA filter stages might be a 480,000 -cfm-capacity sand filter. As noted in Sect. 9.6, sand filters can be designed to approach the efficiency of a HEPA filter; therefore, first-stage HEPA filters could probably be eliminated. The sand filter has the advantages of providing an excellent heat sink and shock snubber, and because redundancy would not be required, might be cost-competitive with the large prefilter and HEPA-filter stages.

\subsubsection{Control Room Protection Air Cleaning Systems}

Control room protection air cleaning systems are ESF systems that must meet the requirements of Regulatory Guide $1.52 .{ }^{58}$ Unless the internal components(filters, adsorbers) are located at the wall penetration of or within the controlled space, the system is generally of forced-flow configuration and operates in a recirculating mode. In most cases, 
the air cleaning facilities are external to the control room (controlled space). Positive pressure in the housings and ducts downstream of the fan minimizes inleakage of potentially contaminated air from building spaces surrounding the control room. Most systems have provisions for makeup air from outside of the building, with isolation dampers to cut off makeup airflow if necessary. The proposed control room for a 1000-MW(e) HTGR has two makeup air ducts that can draw air from two geographically isolated areas outside of the reactor site boundary, permitting a constant supply of makeup air even in the event of an accident. ${ }^{61}$ The location of control room protection system components within the control room has the advantage of maintainability under accident conditions; however, it also has the disadvantage that maintenance operations must be conducted within the control room, an activity that may be untenable to some operators.

The component train of a control room protection system should include prefilter, HEPA filter, adsorber, and second-stage HEPA filter. Prefilters are recommended even though the system recirculates very clean air, because the lint generated by personnel moving about in occupied spaces can bridge the pleats of HEPA filters, reducing their capacity. Makeup ducts should be fitted with prefilters and one stage of HEPA filters, and should have a high-quality isolation damper to cut off the makeup air in the event of a release of toxic or debilitating industrial gases (e.g., chlorine) in the area of the makeup intake. Redundancy is necessary and is usually provided by two or more totally independent and geographically isolated systems, each capable of furnishing the needs of the control room.

\subsection{FUEL REPROCESSING PLANT AIR CLEANING}

\subsubsection{Introduction}

The requirements for air cleaning in fuel reprocessing facilities differ greatly from those for a power reactor. Basically, the difference stems from the fact that day-to-day operations in a reactor are clean, whereas day-to-day operations in a reprocessing facility are inherently dirty. In a reactor, air cleaning facilities are designed to accommodate a large radioactivity release under accident condition, whereas the fuel reprocessing facility must accommodate the potential for smaller, but still substantial, releases under normal operating conditions. Effluent air and gases from reprocessing operations are likely to contain substantial quantities of acid or caustic that must be removed before getting to final air cleaning facilities. In a reactor there are several lines of containment for fissile material and fission products, including the fuel cladding, the reactor vessel, and the containment structures; in the fuel reprocessing plant these lines are all lacking, and, although the fuel is handled one rod at a time, the cladding is purposely removed to release the fissile and radioactive materials (under controlled conditions) for processing. In a reactor, fuel is always in an essentially static condition except when it is being loaded into or unloaded from the reactor vessel or being moved to or from the storage pool. On the other hand, in a reprocessing plant, the fuel and its subsequent byproducts are constantly in an active condition, being chopped, dissolved, leached, or otherwise acted upon. The potential for a release of radioactive material or nuclear criticality incident in the fuel reprocessing facility, therefore, is an ever-present condition.

The requirements for the design, construction, testing, and maintainability of air cleaning systems for the fuel reprocessing and radiochemical facility differ little from those for reactors. That is, generally the same components (demisters, prefilters, HEPA filters, ducts, fans, dampers, and housings) are employed, and differences are in the details of application rather than in the basic principles of application. Basically, the design and installation of air cleaning components and equipment should follow the guides in this handbook. Other guides and standards of particular interest in fuel reprocessing and radiochemical applications include:

Regulatory Guide 3.12, General Design Guide for Ventilation Systems of Plutonium Processing and Fuel Fabrication Plants.

Regulatory Guide 3.14, Seismic Design Classification for Plutonium Processing and. Fuel Fabrication Plants.

Regulatory Guide 3.18, Confinement Barriers and Systems for Fuel Reprocessing Plants.

Regulatory Guide 3.20, Process Offgas Systems for Fuel Reprocessing Plants.

Regulatory Guide 3.24, Guidance on the License Application, Siting, Design, and Plant Protection for an Independent Spent Fuel Storage Installation. 
Code of Federal Regulations, Title 10, Part 20, Standards for Protection Against Radiation.

Code of Federal Regulations, Title 10, Part 50, Appendix P, General Design Criteria for Fuel Reprocessing Plants.

ANSI N101.3, Guide to Principal Design Criteria for Nuclear Fuel Reprocessing Facilities.

ANSI N303, General Requirements for Control of Gaseous Effluents Containing Radioactive Material at Nuclear Fuel Reprocessing Facilities.

Air and gas cleaning systems fall in one or the other of two broad categories, ventilation or off-gas. Ventilation air cleaning systems are often very large, as much as 250,000 to $300,000 \mathrm{cfm}$, although the trend appears to be toward smaller once-through systems. These systems are fed from a number of small branch lines, each of which is generally equipped with at least a HEPA filter at the duct entrance. The central exhaust air cleaning system generally consists of a bank of prefilters and a bank of HEPA filters, although a DBGF prefilter followed by one stage of HEPA filter, or a sand filter alone with no HEPA filters at the central-exhaust plenum, is used in some ERDA installations. Normal off-gas systems are generally small, seldom more than $1000-\mathrm{cfm}$ and often 100-cfm airflow. Gases evolved in chemical operations are pretreated by condensation, scrubbing, or other chemical engineering techniques to remove acids, caustics, excess moisture, and other materials that could harm filters or adsorbents. In some plants, off-gas exhausts directly to a high stack; in others it is discharged to the central buildingexhaust air cleaning system to provide series redundancy of the final filtration step.

\subsubsection{Light Water Reactor Spent Fuel Reprocessing}

The Barnwell Nuclear Fuel Plant located near Barnwell and Aiken, South Carolina, represents the present-day design of equipment and systems and the current state-of-the-art techniques for ensuring that any release of radioactive material to the environment, under both normal and system upset conditions, is maintained at levels that meet current ALARA criteria. ${ }^{66}$ The Barnwell plant has not gone into operation as of June 1976.

Air in operating cells and galleries is maintained at less than atmospheric pressure so it will flow from areas of no contamination toward areas of increasing contamination potential. Exhaust air from sources of potential contamination is passed through a ductentrance filter near the source, then to the main building or laboratory ventilation system. Air pressure in occupied areas and aisles is maintained at slightly higher than atmospheric. All ventilation air is exhausted through one of two ventilation air cleaning systems having a single bank of HEPA filters, then to a 100-m stack. Most first-stage duct-entrance HEPA filters will be changed remotely, but radioactivity levels at certain low-activity cells and at the central building-exhaust plenum are expected to be low enough to permit contact maintenance. The quantities of radioisotopes in the ventilation air streams will be relatively insignificant under normal operating conditions. Ventilation air for fuel receiving and storage areas is independently supplied and exhausted directly to the atmosphere or recirculated (through roughing filters only) without additional treatment since no contamination is expected at these points.

Off-gas from the shear and dissolver is passed through a dust screen to remove large particles, through a condenser to remove most of the water and soluble contaminants, through a mercuric nitrate-nitric acid scrubber to remove noncondensible iodine, through a vapor-liquid phase separator, and through an absorption column where nitrogen and nitrogen oxides will be oxidized with air and absorbed in water. This dissolver off-gas stream is then discharged to the main process vessel off-gas (VOG) system. The nitric acid and iodine content of gases entering the VOG system should be quite low; however, an additional iodine scrubber is provided. The VOG system, consisting of a condenser, a vapor-liquid phase separator, a second iodine scrubber, and a gas heater, then exhausts to the stack through an air cleaning unit having HEPA filters (two stages) and zeolite-filled adsorbers. No provision is made for trapping or removing the noble gases. It has been proposed that the VOG stream, after pretreatment and one stage of HEPA filters and adsorbers, be discharged to the building-exhaust air cleaning system. For reasons given earlier, a second stage of HEPA filters should be provided downstream of the adsorbers.

\subsubsection{LMFBR Spent Fuel Reprocessing}

Plutonium produced in the operation of LMFBRs must be separated from other components of the 
spent fuel, fabricated into new fuel assemblies, and then returned to the reactor in as short a time as practical in order to benefit from the LMFBR's more efficient use of uranium. Air and gas cleaning techniques employed in reprocessing fuel from LWRs (Sect. 9.9.1) are similar in many ways to those which will be used for processing LMFBR fuel. However, there are special problems that must be dealt with, including the handling of thermally hot, sodium-contaminated fuel assemblies, the design, construction, and operation of very low leakage cells for reprocessing activities, the recirculation and cleanup of argon or nitrogen cell atmospheres, and the application of the near-zero release concept (Sect. 9.9.4) to provide a high degree of containment of not only plutonium, uranium, and iodine, but also tritium, krypton, xenon, and possibly ruthenium.

One concept of an air-gas cleaning system for an LMFBR fuel storage and reprocessing facility utilizes at least three stages of HEPA filtration, a 300-m offgas stack, a sand filter for natural or DBA emergency protection, iodine removal, and provisions for the future retention of tritium, krypton, xenon, and ruthenium. Under this concept, cells are designed for very low air inleakage, on the order of $0.01 \mathrm{cfm} /$ cell under sealed test conditions. This and other Zone I (Sect. 2.2.1) exhaust rates are limited to a total of 100 $\mathrm{cfm}$. Recirculation of the cell inert atmosphere (nitrogen) is a requirement. In cells where sodium is present, both the oxygen and moisture content must be controlled to a maximum of $50 \mathrm{ppm}$ by volume. The regulation of pressure and temperature is necessary, and redundant air moving and air cleaning equipment must be provided. The removal of sodium fumes and organic vapors is also necessary in recirculating streams where these contaminants are likely to occur. Dual silver-zeolite adsorbers provide iodine retention, with provisions being made for obtaining higher retention factors for iodine $\left(10^{7}-10^{9}\right)$ by combining nitric acid scrubbing (Iodox process, under development) and solid adsorbents. Problem areas associated with LMFBR fuel reprocessing, including disassembly, fuel rod shearing voloxidation, dissolution, and solvent extraction, are under investigation, particularly in the areas of retention of the volatile fission products-tritium, $\mathrm{Kr}$ $85, \mathrm{I}-129$, and I-131. It appears that tritium can be removed by exposing the fuel to high temperatures (voloxidation); the released tritium is condensed and stored as tritiated water. Krypton can be removed by fluorocarbon selective absorption. Iodine removal is accomplished by acid scrubbing followed by adsorp- tion on silver-zeolite. Plutonium, uranium, and other radioactive particulates are controllable by existing filtration technology, as previously discussed in this handbook.

Key concepts for LMFBR spent fuel reprocessing facility design include the following:

- Confine radioactive gases and particulates within low-leakage process equipment enclosures by means of HEPA filters. Exhaust and recirculated gas streams from these enclosures should be filtered as close to the contaminant source as practicable. The need for radioactive gas retention facilities downstream from the HEPA filters is yet to be determined.

- Limit the total air-gas exhaust from the cells and other Zone I areas to as low as practical, possibly a maximum of $100 \mathrm{cfm}$. This demands that the hot-cell work areas have very low air inleakage.

- Perform cleaning operations on the cell exhaust stream as necessary to result in a near-zero release level. Cleaning processes should include the removal and retention of essentially all of the plutonium, uranium, iodine, krypton, xenon, tritium, and ruthenium.

- All plutonium-contaminated streams should undergo at least three stages of HEPA filtration before discharge to the plant atmosphere.

- A deep-bed sand filter or equivalent should be provided for fire, shock, and explosion protection, followed by a HEPA filter stage. The final HEPA filter bank must be operable under all possible DBA, earthquake, tornado, and other postulated disaster and emergency conditions.

- Where sodium is encountered, the use of either a nitrogen or argon cell atmosphere is necessary. The cell atmosphere must be recirculated through HEPA filters and gas purifiers for particulate removal, maintenance of gas purity, and cell temperature and pressure control.

- Provide monitors with alarms, redundant air moving and air cleaning equipment, and central control room operation for maximum reliability.

\subsubsection{Near-Zero Release Concept}

In the past, radioactive discharges have been limited to quantities that would yield concentrations of radioactive contaminants at site boundaries well below levels set by national and international agen- 
cies for continuous intake by the public. ${ }^{67}$ The present emphasis is to ensure that releases of radioactive material are, in addition, kept to as low a value as reasonably achievable. It is believed that reductions in effluent activities and volumes to levels approaching near zero can be achieved in future facilities. The near-zero confinement objective can be realized by a reasonable projection of the technology currently in development. Although the related process development work is not complete, it appears that the following retention factors can be attained: iodine, $10^{10}$; noble gases and tritium, 10 ; and particulates, $10^{16} .{ }^{16}$

As LMFBRs (with their higher burnup levels, higher specific power, and the economic incentive to reduce spent-fuel preprocessing decay time) assume their projected role in the power economy of the future, the input level of fission products to reprocessing plants will increase significantly. This higher input level of activity, coupled with possible reductions in the permissible release of activity to the environment, will place very stringent demands on effluent control systems and require advanced processes for the control and removal of the volatile fission products from effluent streams. The current practice of using once-through ventilation for cell enclosures at rates in the $100,000-\mathrm{cfm}$ range is not compatible with the near-zero release concept of activity from the plant. The removal of trace concentrations of tritium, krypton, and iodine from very large air and gas flows is economically infeasible as well as technically unsound.

Key factors in reducing the quantity of radioactivity released to the environment to near zero include a reduction in the volume of effluents, low air inleakage into cells, and avoidance of bypassing of conta minant trapping systems. The practical extent of the treatment of an effluent is determined in large measure by the volume of the effluent to be treated. A large shielded fuel examination facility (the High-Level Fuel Examination Facility at the National Reactor Testing Station, Arco, Idaho) is operating with an air infiltration rate of $0.004 \mathrm{cfm}$. It is believed that a practical infiltration rate for a 5-tonne/day reprocessing facility, designed for near-zero radioactivity release, is $100 \mathrm{cfm}$ or less. To meet these objectives, a high degree of overall containment must be maintained during all phases of plant life, including routine operation, maintenance, and decommissioning at the end of the plant's useful life.

\subsubsection{HTGR Spent Fuel Reprocessing Air Cleaning Systems}

Although no HTGR fuel processing plant is yet in the design stage, it is likely that air and gas cleaning facilities for such a plant would be similar in many respects to those of an LWR spent fuel reprocessing plant. Building design and containment philosophy are similar except that Zone III areas of the plant (operating areas and aisles) would probably be maintained at a slight negative pressure relative to the out-of-doors, and galleries and cells would be maintained at progressively lower pressures in order to maintain airflow in the building from areas of low hazard to areas of progressively higher hazard.

The total projected off-gas volume from the headend processes and dissolver is $380 \mathrm{scfm}$. The nitrogen oxides in the dissolver off-gas stream are catalytically decomposed to $\mathrm{N}_{2}$ and $\mathrm{H}_{2} \mathrm{O}$, using ammonia. The head-end and dissolver off-gas streams are then mixed at subatmospheric pressure and compressed. Excessive $\mathrm{CO}$ and/or $\mathrm{H}_{2}$ are removed in a hightemperature oxidizer by conversion to $\mathrm{CO}_{2}$ and tritiated water. Iodine is removed by adsorption on lead-zeolite and silver-zeolite beds in series. Radon is held up on a molecular-sieve bed for decay to stable lead, and tritiated water vapor is removed by adsorption in a molecular-sieve bed (the tritiated water is fixed in concrete for disposal). The gases are then passed through a HEPA filter, compressed to a pressure in excess of $20 \mathrm{~atm}$, cooled to $-35^{\circ} \mathrm{C}$, and treated to remove krypton. Krypton is removed by absorption in liquid $\mathrm{CO}_{2}$, and the decontaminated gases are vented to the main ventilation exhaust system. Krypton is then stripped from the liquid $\mathrm{CO}_{2}$ by fractional vaporization and desublimization to freeze out the remaining $\mathrm{CO}_{2}$, after which it is compressed and bottled for indefinite storage. A final system design description for off-gas treatment had not been issued at the time this handbook was published.

\subsubsection{Air Cleaning System Costs, Fuel Reprocessing}

Comparisons of construction costs of cell ventilation exhaust air cleaning systems for reprocessing plants can be misleading because of the difficulty in establishing valid equivalency bases. The extent of the construction costs included in the figures reported, the type of construction contract, regional cost differentials, differences in the terrain on which 
the facility is built, and cost escalation are some of the factors that make such comparisons misleading. With these words of caution, a construction cost comparison of fuel reprocessing and radiochemical plant exhaust air cleaning systems is given in Tables 9.6 and 9.7 .

Table 9.6. Estimated unit cost (dollars per cfm of installed capacity) of various fuel reprocessing plant exhaust air cleaning systems (based on data in Table 9.7)

\begin{tabular}{lcc}
\hline \multicolumn{1}{c}{ Type of system } & $\begin{array}{c}\text { Number of } \\
\text { systems }\end{array}$ & $\begin{array}{c}\text { Cost in } \\
\text { 1975 dollars }\end{array}$ \\
\hline Sand filter & 7 & 25.76 \\
UBGiF filter & 2 & 9.21 \\
UBGF, one HEPA filter & 1 & 16.67 \\
One prefilter, two HEPA filters & 3 & 4.08 \\
Two prefilters, three HEPA filters & 2 & 11.76 \\
\hline
\end{tabular}

\section{REFERENCES FOR CHAP. 9}

1. "Standards for Protection Against Radiation," Code of Federal Regulations, Title 10, Part 20 (10 CFR 20), Sect. 20.101 (b), 1974.

2. ANSI N101.6, Concrete Radiation Shields, American National Standards Institute, New York, 1972.

3. K. O. McClintock, "The Design, Test, and Use of the Brookhaven National Laboratory Reactor Bypass Filter Facility," Proc. Ninth AEC Air Clean. Conf., USAEC Report CONF660904, January 1967.

4. W. Green et al., "Hanford Experience with Reactor Confinement," Proc. Eighth AEC Air Clean. Conf., USAEC Report TID$7677,1963$.

5. F. T, Binford and E. N. Cramer(eds.), The High Flux Isotope Reactor, USAEC Report ORNL-3572, vol. 1, Oak Ridge National Laboratory, May 1964.

6. W. S. Durant et al., Activity Confinement System of the Savannah River Plant Reactors, USAEC Report DP-1071, Savannah River Laboratory, August 1966.

7. J. W. Joseph, Jr., and J. W. Little, Jr., “Activity Confinement and Decontamination After Failure of an Sb-Be Source Rod," Nucl. Saf. 14(4), 362-72 (July-August 1973).

Table 9.7. Construction cost comparison, reprocessing plant cell and building ventilation-exhaust air cleaning systems ${ }^{a}$

\begin{tabular}{|c|c|c|c|c|c|c|c|}
\hline Location & Facility No. & Type & $\begin{array}{c}\text { Installed } \\
\text { capacity } \\
\left(\operatorname{scfm} \times 10^{3}\right)\end{array}$ & $\begin{array}{c}\text { First } \\
\text { cost } \\
\left(\$ \times 10^{6}\right)\end{array}$ & $\begin{array}{l}\text { Completion } \\
\text { date }\end{array}$ & $\begin{array}{c}\text { Unit } \\
\text { cost } \\
\text { in year } \\
\text { of } \\
\text { construction } \\
(\$ / \mathrm{cfm})\end{array}$ & $\begin{array}{l}\text { Unit } \\
\text { cost } \\
\text { related } \\
\text { to } 1975 \\
\text { dollars } \\
(\$ / \mathrm{cfm})\end{array}$ \\
\hline Hanford & $291-B$ & Sand & 26 & 0.311 & 1948 & 11.96 & 30.98 \\
\hline Hanford & $291-\mathrm{T}$ & Sand & 25 & & 1948 & & \\
\hline Hanford & $291-U$ & Sand & 44 & & 1950 & & \\
\hline Hanford & $291-S$ & Sand & 40 & & 1951 & & \\
\hline $\mathrm{SRP}^{x}$ & 294-F & Sand & 115 & 1.51 & 1954 & 13.13 & 27.70 \\
\hline $\mathrm{SRP}$ & $294-\mathrm{H}$ & Sand & 115 & 1.25 & 1955 & 10.87 & 22.39 \\
\hline Hanford & 291-A-1 & DBGF & 126 & 0.556 & 1955 & 4.41 & 9.08 \\
\hline Hanford & 291-A-2 & DBGF & 126 & 0.671 & 1965 & 5.33 & 9.33 \\
\hline Hanford & $291-B a$ & 1 pre., 2 HEPA & 75 & & 1967 & 2.33 & 3.87 \\
\hline Hanford & 291-Bb & 1 pre., 2 HEPA & 75 & $0.350^{\circ}$ & 1967 & 2.33 & 3.87 \\
\hline Hanford & $291-B c$ & 1 pre., 2 HEPA & 75 & 0.254 & 1972 & 3.39 & 4.51 \\
\hline $\mathrm{SRL}^{e}$ & $794-A$ & Sand & 74 & $1.94^{\prime}$ & 1974 & 26.22 & 28.84 \\
\hline Morris, Ill. & MFRP & Sand & 32 & 0.400 & 1974 & 12.50 & 13.75 \\
\hline Hanford & 291-Bd & 2 pre., 3 HEPA & 75 & 0.900 & 1975 & 12.00 & $\mathrm{I} 2.00$ \\
\hline SRP & $294-1 F$ & Sand & 210 & 5.60 & 1975 & 26.67 & 26.67 \\
\hline Idaho Falls & 756 & DBGF, HEPA & 150 & 2.50 & 1976 & 16.67 & 16.67 \\
\hline SRP & 294-1 H & Sand & 210 & 6.30 & 1976 & 30.00 & 30.00 \\
\hline Hanford & 291-A-3 & 2. pre., 3 HEPA & 126 & 1.45 & 1977 & 11.51 & 11.51 \\
\hline
\end{tabular}

${ }^{a}$ Most of the information in this table is based on data from H. A. Lee, Engineering Study-B Plant, Fifth Filter Cell, USAEC Report ARH-CD-447, Atlantic Richfield Hanford Co., September 1975.

${ }^{h}$ Costs adjusted to 1975 dollars on basis of Chemical Engineering Cost Index, McGraw-Hill, New York, 1976.

'Savannah River Plant.

"Total cost of 291-Ba and 291-Bb was $\$ 350,000$.

'Savannah River Laboratory.

${ }^{\prime}$ D. Zippler, Savannah River Plant, personal communication to J. E. Kahn. 
8. U.S. Patent No. 3,847,574, J. F. Fish, Charcoal Filter Arrangement (assigned to American Air Filter Co., Louisville, Ky.).

y. C. A. Hahs, "Developments in Contaminated Filter Removal Equipment," Proc. Ninth AEC Air Clean. Conf., USAEC Report CONF-660904, January 1967.

10. E. D. Rice and C. G. Caldwell, "Waste Encapsulation and Storage Facility Ventilation System," Proc. 12th AEC Air Clean. Cont., USAEC Report CONF-720823, January 1973.

11. Regulatory Guide 1.76, Design Basis Tornado for Nuclear Power Plants, U.S. Atomic Energy Commission, Washington, D.C., April 1974.

12. W. S. Gregory and G. A. Bennett, Ventilation Systems Analysis During Tornado Conditions, ERDA Report LA-5894PR, Los Alamos Scientific Laboratory, March 1975.

13. W. L. Anderson and T. Anderson, "Effect of Shock Overpressure on High-Efficiency Filter Units," Proc. Ninth AEC Air Clean. Conf., USAEC Report CONF-660904, January 1967.

14. W. S. Gregory, HEPA Filter Effectiveness During Tornado Conditions, ERDA Report LA-5352-MS, Los Alamos Scientific Laboratory, 1973.

15. P. Dergarabedian and F. Fendell, "A Method for Rapid Estimation of Maximum Tangential Wind Speed in Tornados," Mon. Weather Rev. 99(2) 143-45 (February 1971).

16. P. Dergarabedian and F. Fendell, "One- and Two-Cell Tornado Structure and Funnel-Cloud Shape,"J. Astronaut. Sci. 21(1) 26-31 (July-August 1973).

17. J. E. Beavers, Oak Ridge Gaseous Diffusion Plant, personal communication to C. A. Burchsted.

18. F. Fendell, TRW Systems, Inc., personal communication to C. A. Burchsted.

19. S. E. Smith et al., Protection Against Fire Hazards in the Design of Filtered Ventilation Systems of Radioactive and Toxic Gas Process Buildings, UK AEA Report AWRE O-24/65, Atomic Weapons Research Establishment, Great Britain, July 1965.

20. UL-586, Safety Standard for Figh Efficiency Air Filter Units, Underwriters' Laboratories (also ANSI B132.1), current issue.

21. UL-900, Safety Standard for Air Filter Units, Underwriters Laboratories (also ANSI B124.1), current issue.

22. C. A. Burchsted, "Environmental Properties and Installation Requirements of HEPA Filters," Proc. Symp. Treat. Airborne Radioact. Wastes, International Atomic Energy Agency, Vienna, 1968.

23. ASHRAE 52-68, Method of Testing Air Cleaning Devices Used in General Ventilation for Removing Particulate Matter, American Society of Heating, Refrigerating and AirConditioning Engineers, 1968.

24. F. J. Linck, Dow Chemical Co., Rocky Flats Division, personal communication to $\mathrm{C}$. A. Burchsted.

25. D. J. Keigher, fire protection engineer, Los Alamos Scientific Laboratory, personal communication to C. A. Burchsted.

26. J. L. Murrow, "Carbon Adsorber Fire Extinguishment Tests," Proc. I/th AEC Air Clean. Conf., USAEC Report CONF700816 , December 1970.

27. A Report on the Extinguishment of Activated Carbon Fires in $\mathrm{CVI} \mathrm{HECA}{ }^{\text {tm }}$ Adsorber Beds, Grinnell Fire Protection Systems Co. and CVI Corp., Feb. 6, 1975.

28. NFPA 12A, Standard on Halogenated Fire Extinguishing Agent Systems--Halon 1301, National Fire Codes, vol. 1, National Fire Protection Association, 1975.
29. A. H. Hill, Savannah River Laboratory, personal communication to $C$. A. Burchsted.

30. C. R. Schmitt, "Carbon Microspheres as Extinguishing Agents for Metal Fires," J. Fire Flammability 5 (July 1974).

31. Letter, J. M. Case, plant manager, Oak Ridge Y-12 Plant, Union Carbide Corp. Nuclear Division, to ERDA Oak Ridge Uperations Office, May 22, 1975.

32. R. A. Juvinall, R. W. Kessie, and M. J. Steindler, Sand-Bed Filtration of Aerosols: A Review of Published Information on Their Use in Industrial and Atomic Energy Facilities, USAEC Report ANL-7683, Argonne National Laboratory, June 1970.

33. R. A. Moyer, J. H. Crawford, and R. E. Tatum, "Deep-Bed Sand Filter at Savannah River Laboratory," Proc, 13th AEC Air Clean. Conf., USAEC Report CONF-740807, 1975.

34. H. Lawroski, "Zero Power Plutonium Reactor Facility," Nucl. New:s 11(47) (February 1968).

35. C. E. Lapple, Interim Report-200 Area Stack Contamination, USAEC Report HDC-743, General Electric Co. Oct. II, 1948.

36. J. B. Work, Decontamination of Separation Plant Ventilation Air, USAEC Report HW-11529, General Electric Co.. Richland, Wash., Nov. 10, 1948.

37. C. E. Lapple, 200 Area Stack Contamination, Interim Report, USAEC Report HDC-978, General Electric Co. Kichland, Wash., Jan. 24, 1949.

38. (i. A. Schurr, D. B. Zippler, and D. C. Guyton, "Deep-Bed Filter Tests," Proc. 12th AEC Air Clean. Conf., USAEC Report CONF-720828, January 1973.

39. (i. H. Sukes and J. A. Harper, "Design and Operation of a Large Sand Bed for Air Filtration," Proc. Symp. Treat. Airhorne Radioact. Wastes, International Atomic Energy Agency, Vienna. 1968

40. A. (i. Blasewitz, "Dissolver Off-Gas Filtration," Proc" Second AEC Air Clean. Semin., USAEC Report WASH-149, 1954.

41. A. G. Blasewitz, "Hanford Air Cleaning Operations," Proc'. Third AEC Air Clean. Conf., USAEC Report WASH-170, 1954.

42. A. G. Blasewitz and B. F. Judson, "Filtration of Radioactive Aerosols by Glass Fibers," Chem. Eng. Prog. 51, 47 (June 1955)

43. A. G. Blasewitz et al., Filtration of Radioactive Aerosols hy Glass Fihers, USAEC Report HW-20847, General Electric Co., Apr. 16, 1951.

44. H. Gilbert, consultant to ERDA, personal communication to C. A. Burchsted.

45. W. C. Schmidt, Treatment of Gaseous Effluents, USAEC Report HW-49549A, General Electric Co., Richland, Wash.

46. ANS1 N101.6, Concrete Radiation Shields, American National Standards Institute, New York, current issue.

47. L. L. Zahn, Jr., Comparative Study of Alternative Fibrous Gilass and Sand Exhaust Ventilation Air Filter Installation for Purex, USAEC Report HW-30142, General Electric Co., 1953.

48. E. F. Curren and W. H. Koontz, Investigation of 29/Building Filters for Evidence of Failure or Incipient Failure, USAEC Report ARH-1454, Atlantic Richfield Hanford Co., Necember 1969.

49. An Assessment of Accident Risks in U.S. Commercial Nuclear Power Plants, NRC Report WASH-1400, Nuclear Kegulatory Commission, 1975.

50. W. B. Cottrell and A. W. Savolainen (eds.) U.S. Reactor Containment Technology, USAEC Report ORNL/NSIC-5, Oak, Ridge National Laboratory, 1965. 
51. ASME Boiler and Pressure Vessel Code, Sect. III, "Nuclear Power Plant Components," Subsection NE, "Class MC Components," American Society of Mechanical Engineers, current issue.

52. C. A. Burchsted and A. B. Fuller, Design, Construction, and Testing of High Efficiency Air Filtration Systems for Nuclear Application, USAEC Report ORNL/NSIC-65, Oak Ridge National Laboratory, 1970.

53. Dwight Coddington, Bechtel Corp., personal communication to $\mathrm{C}$. A. Burchsted.

54. K. K. Hilliard, Hanford Engineering Development Laboratory, personal communication to C. A. Burchsted.

55. K. L. Koontz et al., Low Pressure Containment Buildings, USAEC Report NAA SR-7234, Atomics International, Mar. 15, 1963.

56. Г. Moffette, Gulf General Atomic Co., personal communication to $\mathrm{C}$. A. Burchsted.

57. C. G. Bell, Oak Ridge National Laboratory, personal communication to C. A. Burchsted.

58. Regulatory Guide 1.52, Design. Testing, and Maintenance Criteria for Atmospheric Cleanup System Air Filtration and Adsorption Units of Light Water Cooled Nuclear Power Plants, U.S. Atomic Energy Commission, Washington, D.C., 1973.

59. J. L. Murrow, "Carbon Adsorber Fire Extinguishment Tests," Proc. IIth AEC Air Clean. Conf., USAEC Report CONF700816, December 1970.
60. V. Deitz, Naval Research Laboratory, personal communication of unreported test results from domestic carbon survey to C. A. Burchsted.

61. C. Kang, United Engineers and Constructors, personal communication to C. A. Burchsted.

62. Preliminary Safety Analysis Report, Summit Power Station, Delmarva Power and Light Co., 1975.

63. J. D. McCormack, Hanford Engineering Development Laboratory, personal communication to C. A. Burchsted.

64. C. Newton, "Status of Safety Technology for Radiological Consequence Assessment of Postulated Accidents in LMFBR's," in Program Planning for Development of Radiological Source Terms for Postulated LMFBR Accidents, ERDA Report ERDA56, July 1975.

65. H. Hilsdorf, The Water Content of Hardened Concrete, DASA-1875, University of llinois, Urbana, February 1967.

66. Environmental Report, Barnwell Nuclear Fuel Plant, Docket No. 50-332, Construction Permit No. CPCSF-4, Nuclear Regulatory Commission, 1975.

67. "Standards for Protection Against Radiation," Code of Federal Regulations, Title 10, Part 20 (10 CFR 20).

68. O. O. Yarbro et al., Effluent Control in Fuel Reprocessing Plants, USAEC Report ORNL-TM-3899, Oak Ridge National Laboratory, March 1974. 


\section{Appendix A. Sample Air Cleaning Equipment Specifications}

\section{EQUIPMENT SPECIFICATION ES-1}

HEPA FILTER

1. SCOPE

This specification covers basic requirements for HEPA (high efficiency particulate air) filters for use in supply-air, contaminated exhaust, contaminated air-cleanup, and other nuclear and hazardous air-cleaning applications requiring extremely high arresting efficiency for all sizes, including submicron, of particulate matter.

\section{CLASSIFICATION}

HEPA filters are classified by size and construction in accordance with Tables 1 and 2 .

Table 1. Standard Filter Sizes

\begin{tabular}{lccc}
\hline Size & $\begin{array}{c}\text { Nominal Airflow } \\
\text { Capacity } \\
\text { (scfm) }\end{array}$ & $\begin{array}{c}\text { Face } \\
\text { Dimensions } \\
\text { (in.) }\end{array}$ & $\begin{array}{c}\text { Depth } \\
\text { (in.) }\end{array}$ \\
\hline 1 & 25 & $8 \times 8$ & $3-1 / 16$ \\
2 & 50 & $8 \times 8$ & $5-7 / 8$ \\
3 & 125 & $12 \times 12$ & $5-7 / 8$ \\
4 & 500 & $24 \times 24$ & $5-7 / 8$ \\
5 & 1000 & $24 \times 24$ & $11-1 / 2$ \\
\hline
\end{tabular}

Table 2. Standard Filter Constructions

\begin{tabular}{|c|c|c|c|}
\hline Group & Type & Frame Material & Separator Material \\
\hline 1 & $\begin{array}{l}I-A \\
I-B \\
I-C \\
I-D \\
I I-A \\
I I-B \\
I I-C \\
I I-D\end{array}$ & $\begin{array}{l}\text { Cadmium-plated carbon steel } \\
\text { Cadmium-plated carbon steel } \\
\text { Cadmium-plated carbon steel } \\
\text { Cadmium-plated carbon steel } \\
\text { Chromized steel } \\
\text { Chromized steel } \\
\text { Chromized steel } \\
\text { Chromized steel }\end{array}$ & $\begin{array}{l}\text { Made without separators } \\
\text { Aluminum } \\
\text { Water-proof asbestos } \\
\text { Corrosion-resistant } \\
\text { Made without separators } \\
\text { Aluminum } \\
\text { Water-proof asbestos } \\
\text { Corrosion-resistant }\end{array}$ \\
\hline 2 & $\begin{array}{r}\text { III-A } \\
\text { III-B } \\
\text { III-C } \\
\text { III-D } \\
\text { IV-A } \\
\text { IV-B } \\
\text { IV-C } \\
\text { IV-D }\end{array}$ & $\begin{array}{l}\text { Plywood, Exterior Grade } \\
\text { Plywood, Exterior Grade } \\
\text { Plywood, Exterior Grade } \\
\text { Plywood, Exterior Grade } \\
\text { Wood Ptcl. Board, Ext. Gr. } \\
\text { Wood Ptc1. Board, Ext. Gr. } \\
\text { Wood Ptc1. Board, Ext. Gr. } \\
\text { Wood Ptc1. Board, Ext. Gr. }\end{array}$ & $\begin{array}{l}\text { Made without separators } \\
\text { Aluminum } \\
\text { Water-proof asbestos } \\
\text { Corrosion-resistant } \\
\text { Made without separators } \\
\text { Aluminum } \\
\text { Water-proof asbestos } \\
\text { Corrosion-resistant }\end{array}$ \\
\hline
\end{tabular}


ES-1

Page 2

\section{REFERENCE DOCUMENTS}

The following documents are a part of this specification to the extent specified in the text. The issue of one of these documents in effect on the date of the invitation to bid, including any amendments or other published changes in effect on that date, shall apply. If the specification appears to conflict with the requirements of a referenced document, such conflict shall be brought to the attention of the purchaser for resolution.

\subsection{Military Specifications and Standards.}

MIL-S-46055 Steel, Sheet and Strip, Low Carbon, Chromized

MIL-F-51068 Filter, Particulate, High-Efficiency, Fire-Resistant

MIL-F-51079 Fizter Medium, Fire-Resistant, High-Efficiency

MIL-STD-282 Filter Units, Protective Clothing, Gas-Mask Components, and Related Products, Performance Test Methods

3.2 ERDA Health \& Safety Bulletins (Energy Research and Development Administration).

Filter Unit Inspection and Testing Service (issued annually, number changes with each issue).

\subsection{ASTM Standards (American Society for Testing and Materials).}

ASTM A $165 \quad$ Electrodeposited Coatings of Cadmium on Steel

ASTM A 366 Cold-Rolied Carbon Steel Sheets, Commercial Quality

ASTM D $905 \quad$ Strength Properties of Adhesive Bonds in Shear by Compressive Loading

ASTM D 1056 Sponge and Expanded CeZZuZar Fubber Products

ASTM E 84 Surface Eurning Characteristics of Building Materials

3.4 TAPPI Standards (Technical Association of the Pulp and Paper Industry)

TAPPI T404 Tensile Breaking Strength of Paper and Paperboard

TAPPI T411 Test for Thickness and Density of Paper

TAPPI T413 Ash in Paper 
ES-1

Page 3

\subsection{NBS Standards (National Bureau of Standards).}

NBS PS-1 Product Standard for Softwood Plywood, Commercial and Industrial

NBS CS-236 Commercial Standard for Mat-Formed Wood Particleboard

3.6 UL Standards (Underwriters' Laboratories)

UL-586 Safety Standard for High-Efficiency Air Fizter Units

UL-900 Safety Standards for Air Fizter Units

3.7 IES Standards (Institute of Environmental Sciences, formerly American Association for Contamination Contro1).

CS-1 Standard for HEPA Filters

4. TECHNICAL REQUTREMENTS

4.1 Performance. Filters shall have a maximum penetration of $0.03 \%$ (i.e., a minimum efficiency of $99.97 \%$ ) when tested with monodisperse dioctylphthalate (DOP) smoke having a light-scattering mean droplet diameter of 0.3 micron when operated at rated airflow capacity and at $20 \%$ of rated airflow capacity. Maximum clean-filter pressure drop (resistance) across the filter when operated at rated airflow capacity shall be 1.0 inch of water.

\subsection{Materials}

4.2.1 Filter Medium: glass or glass and asbestos fiber paper having the following properties:

a. DOP Penetration: $0.03 \%$ maximum when tested in accordance with Method 502.1 .1 of MIL-STD-282.

b. Airflow Resistance: $40 \mathrm{~mm}$ water max., when tested at 32 1iters/min./ sq.ft. of medium, in accordance with Method 402.1.1 of MIL-STD-282.

c. Thickness: 0.15 inch minimum, when tested in accordance with TAPPI T411.

d. Basis Weight (density): $48 \mathrm{lbs}$ per $3000 \mathrm{sq}$. ft. when tested in accordance with TAPPI T411.

e. Tensile Strength: $2.5 \mathrm{lb} /$ inch width, minimum, in both machine and cross-machine direction, when tested in accordance with TAPPI T404.

f. Elongation: $1.0 \%$ minimum at rupture in both machine and crossmachine direction, when tested in accordance with TAPPI T404. 
ES-1

Page 4

g. Wet Tensile Strength: $1.25 \mathrm{lb} /$ inch width, minimum, when tested in accordance with TAPPI T404 after soaking in water for 15 minutes.

h. Water repellency: 0 penetration of medium under 30-inch head of water when tested in accordance with Method 603.1 of MIL-STD-282.

i. Folding Characteristics: medium shall show no tears, breaks, or cracks when a specimen, cut in the machine direction, is bent $180^{\circ}$ around a 1/16-in. diameter mandrel. Folded tensile $=50 \%$ original tensile, minimum.

j. Combustible Content: $5.0 \%$ maximum when tested in accordance with TAPPI T413.

k. Tensile Strength After Irradiation: not less than $1.01 \mathrm{~b}$ per in. of width in both the machine and cross direction when tested in accordance with e., above, after exposure to an integrated gamma radiation dose of $6.4 \times 10^{7} \mathrm{rad}$.

1. Water Repellency After Irradiation: 0 penetration of medium under $6 \mathrm{in}$. head of water when tested in accordance with h., above, after exposure to an integrated gamma radiation dose of $6.4 \times 10^{7} \mathrm{rad}$.

\subsubsection{Case Materials:}

a. Type I case: No. 14 US gage carbon steel, ASTM A366.

b. Type II case: No. 14 US gage chromized steel, MIL-S-46055, Type I.

c. Type III case: 3/4-inch fire-retardant treated Grade A-A exterior plywood in accordance with PS-1, having a flame-spread classification of 25 or less when tested in accordance with ASTM E84.

d. Type IV case: 3/4-inch phenolic-resin-bonded exterior wood particle board, batch-treated for fire retardance before forming, in accordance with CS-236 except minimum density shall be $45 \mathrm{lb} / \mathrm{cu} \mathrm{ft}$; filled both sides, flame-spread classification of 25 or less when tested in accordance with ASTM E84.

\subsubsection{Separator Materials shall meet the following requirements.}

a. Type B Separators: aluminum alloy 3003-H19 or 5052-H39, 0.0015 inch thick minimum.

b. Type C Separators: asbestos paper, $4 \mathrm{lb} / 100 \mathrm{sq} f t$ minimum density, containing no more than $5 \%$ combustible materials, qualified in accordance with Appendix A.

c. Type D Separators: asbestos paper, same as Type C, TFE-plastic coated aluminum, or other non-flammable material, qualified in accordance with Appendix B. 
4.2.4 Sealants and Adhesives. Sealants used to seal the filter pack into the frame shall have a minimum shear value of $100 \mathrm{psi}$ at $225^{\circ} \mathrm{F}$ wood (maple or birch) to wood when tested in accordance with ASTM D 905 under a compression loading rate of 0.1 to 0.2 inch per minute. Adhesives used to glue the gaskets to the frame shall have a minimum peel strength of $10 \mathrm{lb}$ per inch at room temperature, metal surface to neoprene surface. Al1 sealants and adhesives shäll be oil resistant and self-extinguishing after exposure to open flame.

4.2.5 Gasket Material shall be oil-resistant, ozone resistant, closed cell neoprene sponge, ASTM D 1056, Grade SCE-43, $1 / 4$ inch thick by $5 / 8$ to $3 / 4$ inch wide ( $+1 / 8$ inch, -0 inch), with cut surfaces on both faces.

4.2.6 Face Guards, when specified, shall be $4 \times 4$ mesh galvanized hardware cloth, expanded-and-flattened expanded metal with maximum openings of $1 \times 1 / 2$ inch, or perforated metal having maximum openings of $1 / 2 \times 1 / 2$ inch. Pressure drop of filters with face guards shall not exceed the requirements of 4.1 .

\subsection{Construction.}

4.3.1 Filters shall be Grade 1 (fire resistant) construction in accordance with Section 6 of IES CS-1, and shall meet the construction requirements of Section 8 of that standard. Case and separator materials shall be as specified by the purchaser; separatorless construction shall be furnished only when specified by the purchaser.

4.3.2 The filter pack of a size 1 or 2 filter shall contain no splices. The filter pack of a size 3 filter may contain one splice. The filter pack of a size 4 or 5 filter shall contain no more than three splices. Splicing shall be in accordance with Section 8.3(1) of AACC CS-1.

4.3.3 Filter media shall not be spot-patched to repair holes or tears, but may be repaired in accordance with Section 8 .e.(3) of AACC CS-1.

4.3.4 Edges of separators (except Type A construction) shall extend at least $1 / 8$ inch beyond the pleats and no closer than $3 / 16$ inch to the plane of the face of the frame. Pleats and separators shall not be kinked more than $1 / 4$ inch from a straight line drawn from end to end of the pleat, and shall be perpendicular to the frame with $\pm 3 / 8$ inch as measured from a perpendicular to the frame drawn from the opposite end of the pleat. The filter core shall be tight when tested as specified in Section 5.3.2.

\subsubsection{Group 1 (metal cased) Filters:}

a. At least the top and bottom case panels of each filter shall have a $3 / 8-$ inch to $1 / 2$-inch flange on the inside, front and back, to restrain movement of the filter pack after assembly of the filter. Case shall have a double-turned $3 / 4$-inch-wide flange on each face. (See Fig. 1.) 
ES-1

Page 6

b. Individual case panels for Type I construction shall be cadmium plated after completion of all shearing, forming, and spot-welding operations, but before assembly into a complete case. Plating shall be Type oS or heavier, in accordance with ASTM Al65.

c. Cases may be assembled with stainless steel, cadmium plated, nickel plated, or chromium plated rivets, bolts, screws, or drive screws. Joint surfaces of individual panels shall be thoroughly coated with adhesive before assembly into the case to ensure a leak-tight corner joint, and assembled case shall meet the dimensional tolerances of 8.b and 8.c of AACC CS-1. Contact areas between the case and the filter pack shall be thoroughly coated with adhesive (sealant) before assembly to ensure a leak-tight assembly.

\subsubsection{Group 2 (wood cased) Filters:}

a. Cases shall have single-rabbeted corners as shown in Fig. 2 and shall be assembled by double-nailing or double screwing with coated box nails or galvanized flat-head wood screws. Points of fasteners shall not penetrate any surface of the case. Joint surfaces shall be thoroughly coated with adhesive before assembly to ensure a leaktight joint. Contact areas with the top, sides, and bottom of the filter core shall be coated with adhesive to minimize any possibility of edge-to-edge leakage through the frame.

b. Cases of Type III (plywood case) filters shall be assembled with the Grade A surface of the plywood on the inside, adjacent to the filter core. Any defects in the outer (Grade B) surface shall be coated with adhesive to prevent leakage through the panel.

\subsubsection{Dimensional and Assembly Tolerances:}

a. Face Dimensions: Less than 24 inches: $+0,-1 / 16$ in. 24 inches and over: $+0,-1 / 8$ in.

b. Depth: $+1 / 16$ in., -0 .

c. Squareness: Face diagonals of both faces of Sizes 1, 2, and 3 filters shal1 be equal within $1 / 16$ inch total allowance; face diagonals of both faces of larger filters shall be equal within $1 / 8$ inch total allowance,

d. Faces: Gasket seating surfaces shall be square with the sides of the frame within +3 degrees, and shall be flat and parallel within $1 / 16$ inch total allowance when measured with one face of the filter resting on a surface plate. Sides of the assembled case shall not bow inward or outward more than $1 / 16$ in. in 12 inches, measured on the outside of the case. 
4.4 Gaskets. Filters shall have a gasket on one face only unless otherwise specified by the purchaser. Gaskets shall be made from strips of neoprene cemented evenly to the flange surface with a rubber-base adhesive; the surface of the flange surface shall be cleaned with a degreasing solvent (fluorocarbon, trichloroethylene, acetone, etc.) before cementing the gasket. Gasket strips shall have keyhole or dovetall joints at the corners and shall not be spliced between the corners; edges of the joint surfaces shall be coated with adhesive during assembly to ensure a leak-tight joint. There shall be no evidence of adhesive, cohesive, or contact failure in the joint between the gasket and the flange face. After curing of the adhesive gaskets shall have a minimum peeling strength of $10 \mathrm{lb}$ per Inch of width.

4.5 Face Guards. Size 4 and 5 filters shall have face guards installed on both faces unless otherwise specified by the purchaser. Face guards shall be firmly embedded all around, with no projecting wires or edges that might penetrate the filter medium or be safety hazards to personnel handling the filters.

4.6 Marking. Each filter shall be marked on the top panel with the following information; marking shall be oriented to read from the downstream side, relative to the position of the filter during test (Section 4). Marking sha11 be by indentation-stamping or indelible ink; ink characters shall be at least $1 / 4$ inch high and shall be clear and distinct; variable data (penetration and pressure drop values) may be handwritten; serial number shall be printed. Marking shall not be furnished on a paper label. Marking shall include the following information:

Manufacturer's name or symbol

Percent penetration, actual, at rated capacity (al1 sizes)

Percent penetration, actual, at $20 \%$ of rated capacity (Sizes 4 and 5 only)
Filter serial number Airflow capacity, cfm

Pressure drop at rated capacity (a11 sizes)

Pressure drop at $20 \%$ of rated capacity (Sizes 4 and 5)

\section{QUALITY ASSURANCE REQUIREMENTS}

5.1 Quality Assurance Plan. Filters shall be manufactured under the surveillance of a formal quality assurance program, in accordance with formalized and documented manufacturing and quality assurance plans. Quality assurance personnel shall not report to the production manage of the manufacturer's plant.

5.2 Qualification. The filter design, construction, and medium shall be qualified in accordance with the following requirements. Qualification tests shall be made by the manufacturer, a qualified testing laboratory mutually acceptable to the manufacturer and the purchaser, or by the $U$. S. Army. Listing of the item in a Military Qualified Products List shall be objective evidence that the product meets the qualification requirements. Once qualified, a given filter design need not be requalified for subsequent shipments to this or other pruchasers unless there has been a change in design, method of manufacturer, or materials, except each manufacturing lot of filter medium shall be tested as specifled below. 
ES-1

Page 8

5.2.1 Filter Medium. Each manufacturing lot of filter medium shall be tested in accordance with the requirements of MIL-F-51079 except with the following modifications: (NOTE: paragraph numbers in text of requirements below refer to the paragraph numbers of MIL-F-51079):

a. Tensile Strength and Elongation. Twenty machine-die-cut specimens, each 1 inch $\times 6$ inches in length, 10 having the long dimension parallel to the machine direction and 10 having the long dimension parallel to the cross direction, shall be conditioned and tested for tensile strength and elongation in accordance with TAPPI T404, using a motorized Instron University Testing Machine at a loading rate of 0.5 inch per minute, or at a loading rate which will complete the test in $10 \pm 2$ seconds, whichever is greater.

b. Tensile Strength After Heated Air. Delete in its entirety.

c. Wet Tensile Strength. Ten test specimens, each 1 inch $\times 6$ inches, five having the long dimension parallel to the machine direction and five having the long dimension parallel to the cross direction, shall be submerged in water at a depth of at least 10 inches for at least 15 minutes, and then tested for wet tensil strength as specified in 4.2.4.2(a). The average of the test results shall be considered the wet tensile strength of the medium.

d. Water Repellency. Three test specimens, each 2-3/4 inches $\times 5-1 / 2$ inches, shall be tested for water repellency using the Q101 Water Repellency Indicator (DLB-125-8-1). Each specimen shall be cut into two equal size squares. The felt (top) side of one square and the wire (bottom) side of the other square shall be tested; the lesser of the two results sha11 be considered the water repellency of the specimen. The minimum water repellency of the three specimens shall be considered the water repellency of the medium.

e. Mildew Resistance. When specified in the Ordering Data, mildew resistance tests of the medium shall be conducted in accordance with method 5750 of FED TEST METHOD 191.

f. Acidity. Delete in its entirety.

g. Thickness. The thickness of the medium shall be determined in accordance with TAPPI standard T411, using a TMI No. 549-M Thickness Tester with $5 / 8$ inch diameter anvils.

h. Combustibles Content. The dried weight of the sample shall be recorded. The ash welght shall be determined in accordance with TAPPI standard T413, except the sample shall be either heated for 2 hours at $800^{\circ} \mathrm{C}$ or for minutes at $1000^{\circ} \mathrm{C}$. Percent combustible content shall be determined from the equation:

$$
\text { Percent Combustible }=\frac{\text { Sample weight }- \text { Ash weight }}{\text { Sample weight }} \times 100
$$


i. Radiation Resistance. Radiation resistance of the medium shall be determined as follows: Twenty 1 -inch $\times$ 6-inch tensile-test specimens (10 with the long dimension parallel to the machine direction and 10 with the long dimension parallel to the cross direction) and three $23 / 4 \times 51 / 2$ inch water repellency specimens shall be subjected to gamma radiation. Irradiated tensile test specimens shall be tested in accordance with 4.2.4.2(a). Irradiated water repellency specimens shall be tested in accordance with $4 \cdot 2.4 .3$.

5.2.2 Filter Design and Materials. Filter design and materials of construction shall be tested for qualification as follows:

a. Resistance to Fire and Hot Air. Each filter design and combination of materials of construction shall be tested for qualification in accordance with UL-586 or the requirements for heated-air and spotflame resistance of MIL-F-51068. Presence of the Underwriters' Laboratories label certifying compliance with UL-586, or listing of the design in a military Qualified Products List, shall be satisfactory evidence of compliance with this requirement.

b. Resistance to Moisture and Overpressure. Each filter design and combination of materials of construction shall be tested for qualification as follows:

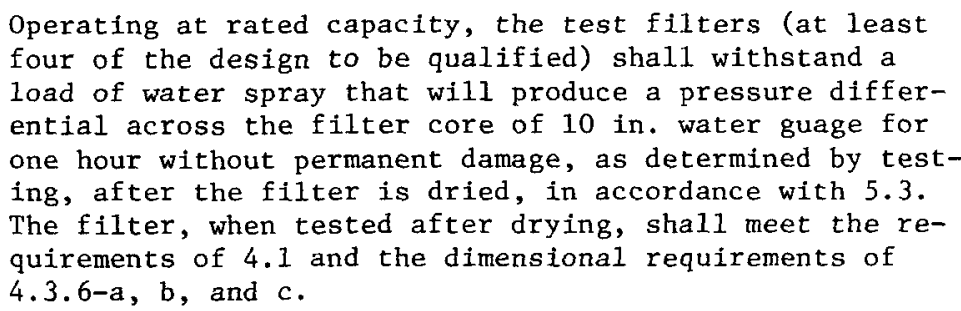
ential across the filter core of 10 in. water guage for one hour without permanent damage, as determined by testing, after the filter is dried, in accordance with 5.3 . The filter, when tested after drying, shall meet the requirements of 4.1 and the dimensional requirements of 4.3.6-a, b, and $c$.

Listing of the design in a military Qualified Products List shall be satisfactory evidence of compliance with this requirement.

c. Resistance to Rough Handling. Each filter design and combination of materials of construction shall be tested for qualification as follows :

The test filters (at least two of the size and design to be qualified) shall withstand rough handling in accordance with Method 105.9 of MIL-STD-282 for $15 \mathrm{~min}$. at $3 / 4 \mathrm{in}$. total amplitude and a frequency of $200 \mathrm{~Hz}$, with pleats and filter faces in a vertical orientation, without visible damage or loss of filtration efficiency, as determined by testing in accordance with 4.3.1. After rough handling, the filters shall meet the requirements of 4.1 and 4.3.6-a, b, and $c$. 
ES-1

Page 10

Listing of the design in a military Qualified Products List shall be satisfactory evidence of compliance with this requirement.

5.2.3 Separator Materials. Asbestos separator material shall be qualified in accordance with Appendix $B$ of this specification. Corrosionresistant separator material shall be qualified in accordance with Appendix $C$ of this specification. Test results of qualification tests shall be made available to the purchaser on request.

\subsection{Production Lot Testing.}

5.3.1 Performance Test. Each filter element shall be individually tested for DOP penetration and airflow resistance in accordance with Method 102.9.1 of MIL-STD-282 except: (1) the Q-107 or Q-76 filter testing penetrometer sha11 be modified to permit the complete filter element to be encapsulated during test in order to establish the presence of frame leaks; (2) tests shall be conducted with the filter element encapsulated; (3) tests shall be made and reported at $100 \%$ of rated capacity and also, for filters larger than 125 cfm rated capacity, at $20 \%$ of rated capacity. The results of tests shall be marked on the case of the filter element; test reports are not required unless expressly specified by the purchaser, and shall comply with the requirements of 4.1. Filters which fail this test shall be rejected.

5.3.2 Core-Tightness Test. A test sha11 be made of the tightness of the filter core. The test may be made on a number of filters corresponding to at 1east $5 \%$ of the number of filters in the shipment, but no fewer than 5 filter elements. The test is made as follows:

Lay filter face down on a flat surface. Place a 4-x-6-inch block of wood, covered with a neoprene pad glued to one surface, at the center of the filter face. Place a 4-1b weight on the block. A force of $4 \mathrm{lbs}$ exerted on the block in a direction parallel to the face of the filter and perpendicular to the pleats shall cause the block to move no more than $1 / 16$ inch from its original position in either direction.

Failure to meet the requirements of this test shall be cause for rejection of the shipment.

5.4 Tests By ERDA Quality Assurance Station. When specified by the purchaser, filters shall be shipped to one of the ERDA Quality Assurance Stations in accordance with instructions contained in the current issue of the ERDA Health and Safety Bulletin on Filter Unit Inspection and Testing. The Quality Assurance Station shall inspect each filter to verify that it complies with this specification and that it is free of visible damage, evidence of poor workmanship, and errors in manufacture, and shall test the filters in accordance with 5.3 above. The Quality Assurance Station sha11 then repack the filters in the manner in which they were shipped, to them, and ship them to the specified delivery site in accordance with the purchaser's instructions. Acceptance of filters shall be at the Quality Assurance Station upon successful completion of the requirements of this section. 


\section{PREPARATION FOR DELIVERY}

6.1 Filter Packaging. Filters shall be individually packaged or packed with individual corrugated paperboard separators in wood or corrugated paperboard cartons having corner braces, inserts, or other means of shock prevention to protect the filters from damage during handing, shipping, and storage. Size 4 and 5 filters shall be enclosed within a corrugated paperboard sleeve having Integral spacers to hold the filter, while in the carton, at least 1 inch from all walls of the carton (separate spacers may be used at the top and bottom of the filters), or be protected with plywood or heavy-paperboard on each face, with spacers to hold the filter, while in the carton, at least 1 inch from all walls of the carton.

6.1.1 Filters shall be placed in the carton so that the faces and the pleats are vertical when the carton is in its normal shipping orientation. Cartons shall be marked FRAGILE - HANDLE WITH CARE and THIS SIDE UP, or similar legends, in black or red heavy block characters at: least $3 / 4$ inch high.

6.1.2 Packing and packaging shall be of a type, weight, thickness and material which is acceptable to the public carrier in compliance with Uniform Freight Classification Rules, the National Motor Freight Classification Rules, or other applicable freight classification rules.

\subsection{Shipping.}

6.2.1 Loading in Public Carriers. Cartons shall be banded to skids so that the specified filter orientation is maintained during shipment, or packed in the carrier's truck or railroad car so that the specified filter orientation is maintained. Cartons shall not be stacked more than 6-1/2 ft high unless a platform is provided to support the tiers of cartons above the 6-1/2 ft height, and other materials shall not be stacked on top of the filter cartons during shipment. A packing list shall be affixed to the outside of one carton of each shipping unit (one skid or pallet load comprises a shipping unit) giving the name of the manufacturer, description of contents, quantity in the order, quality in the shipping unit, indication that the shipment is partial or complete, name of purchaser, and purchaser's order number.

6.2.2 Transhipment. If possible, shipment shall be arranged so that the filters are not disturbed after they leave the manufacturer's plant until they are received at the USERDA Quality Assurance Station, and, after leaving the Quality Assurance Station, until they are received at the delivery site. The common carrier should be instructed that Quality Assurance Station and Purchaser's personnel, respectively, shall be responsible for unloading the truck or railroad car, Railroad cars shall be marked, DO NOT HUMP. The carrier shall report all transshipments to the purchaser.

6.2.3 Sealed Trailer. When specified by the purchaser, filter shall be transported to the delivery site (and to and from the ERDA Quality Assurance Station, when specified) in a sealed trailer or containerized-freight unit. Filters shall be packed and oriented in the trailer or containerized-freight unit as specified in 6.2 .1 above. 
ES-1

Page 12

7. PURCHASE DOCUMENT

Purchase documents for filters bought in accordance with this specification shall (1) cite the number and title of this specification; (2) show the size (see Table 1) and type of construction required (see Table 2);

(3) specify gaskets on both faces, if required (4.4); (4) specify face guards, if required (4.5); (5) spectfy shipment to and testing by an ERDA Quality Assurance Station, with address of station, if required (5.4); (6) specify any special packing or packaging requirements $(6.1)$; (7) method of shipment (6.2); and (8) specify any exceptions to the specification.

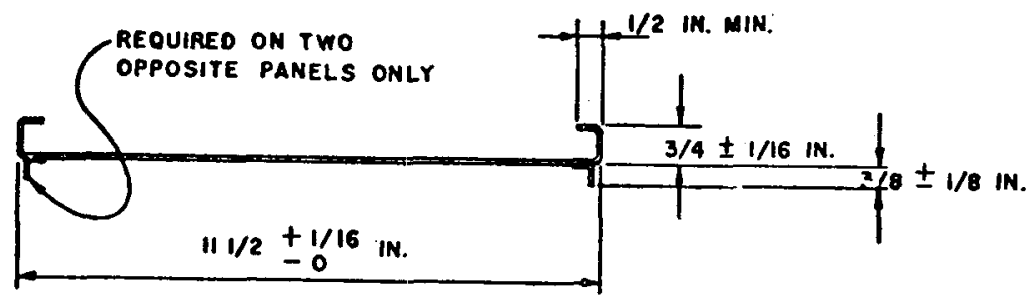

Fig. 1. Section through case panel, metal-cased HEPA filter. 


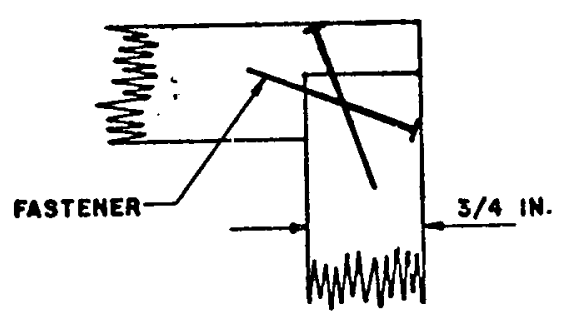

F1g. 2 Corner detail, wood-cased HEPA filter.

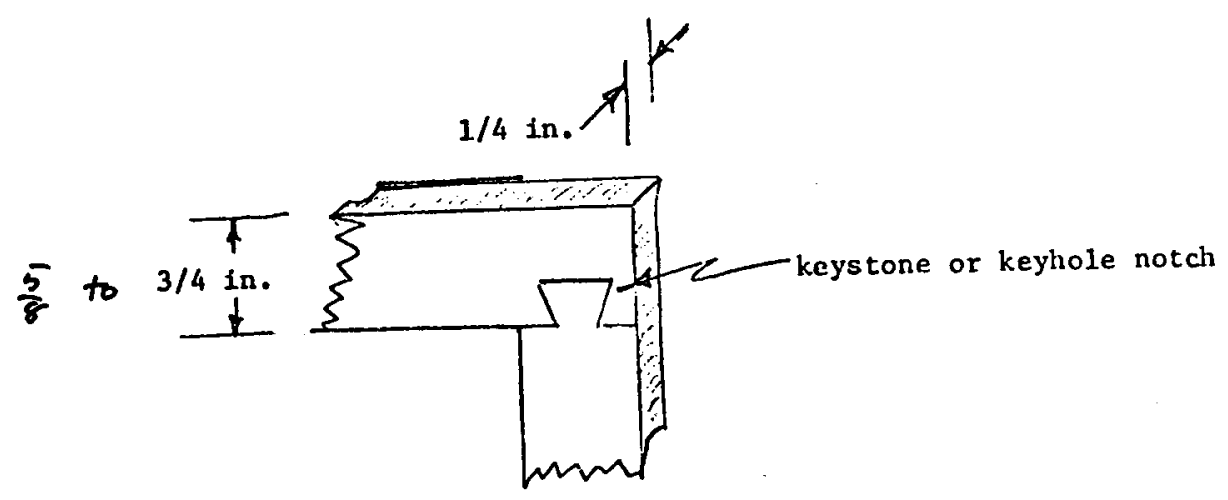

Fig. 3 Corner detall, flat neoprene gasket. 
ES-1

Page 14

A P P E N D I C E S

A. QUALIFICATION TEST FOR MOISTURE RESISTANCE OF SEPARATOR MATERIALS

Ten sections of corrugated separator, each 4 inches long and containing 5 corrugations, shall be immersed in tap water containing 2 to 3 percent by weight trisodium phosphate base detergent for one hour. After soaking, each section, in turn, shall be laid flat and a load of $1 / 2 \mathrm{lb}$ even1y distributed over the tops of the corrugations, shall be placed on the sample. None of the sample shall flatten, and corrugations shall not decrease in height by more than 50 percent.

B. QUALIFICATION TEST FOR CORROSION RESISTANCE OF SEPARATOR MATERIALS Shear.

1. Equipment Requirements. Taber V-5 Stiffness Tester and Taber Specimen

2. Preparation of Test Specimens. Cut 33 test specimens from flat stock, using specimen shear, with long dimension of specimen parallel to machine direction of stock. Divide specimens into eleven sets of two specimens per set, and number each specimen with a set identification. Measure the thickness of each specimen at each end and at the center in accordance with TAPPI T411. Record average weight of each specimen.

3. Conditioning. Condition each set of specimens as follows:

Set 非 1 Control sample; do not condition.

Set \# 2 Soak in $25^{\circ} \mathrm{C}$ water for 1 hour.

Set \# 3 Immerse in $6 \mathrm{M} \mathrm{HCl}$ for 2 minutes, blot, and soak in $25^{\circ} \mathrm{C}$ water for 1 hours.

Set \# 4 Immerse in $10 \%$ HF solution for 1 minute, blot, and soak in $25^{\circ} \mathrm{C}$ water for 1 hour.

Set \# 5 Immerse in $6 \mathrm{M} \mathrm{HClO}_{4}$ for 2 minutes, blot, and soak in $25^{\circ} \mathrm{C}$ water for 1 hour.

Set \# 6 Immerse in $9 \mathrm{M} \mathrm{H}_{2} \mathrm{SO}_{4}$ for 2 minutes, blot, and soak in $25^{\circ} \mathrm{C}$ water for $2^{\mathrm{S}}$ hour.

Set \# 7 Immerse in 7.5M HNO 3 for 2 minutes, blot, and soak in $25^{\circ} \mathrm{C}$ water for 1 hour.

Set \# 8 Immerse in $50 \% \mathrm{NaOH}$ solution for 2 minutes, blot, and soak in $25^{\circ} \mathrm{C}$ water for 1 hour. 
Set \# 9 Steam for 6 hours at approximately atmospheric pressure, blot and soak in $25^{\circ} \mathrm{C}$ water for 1 hours.

Set \#10 Steam for 3 hours at approximately 25 psig, blot, and soak in $25^{\circ} \mathrm{C}$ water for 1 hour.

Set \#11 Heat in furnace at $850^{\circ} \mathrm{F}$ for 5 minutes cool for 5 minutes, and soak in water for $I$ hour.

4. Stiffness Determination. Blot specimen, determine stiffness with Taber V-5 Stiffness Tester while still wet; record stiffner value. Dry specimen and repeat. Average stiffness values for each set; report test values and average-wet-and-dry-stiffness values for each set.

5. Acceptance. Minimum average wet stiffness of any set shal1 be 10 Taber Stiffness Units. Minimum average dry stiffness of any set shall be 12 Taber Stiffness Units. 


\section{Appendix B. Estimating Forms}

The attached forms summarize the information needed to make proper comparisons between candidate filter systems. Labor costs for the first form are derived from the second form. The second form includes the major items involved in replacing filters in a contaminated exhaust system-additional factors may have to be added for a particular system. A separate cost analysis (first form) must be made for each bank of components for each combination to be prepared. The total system cost is the sum of the costs of the individual banks.

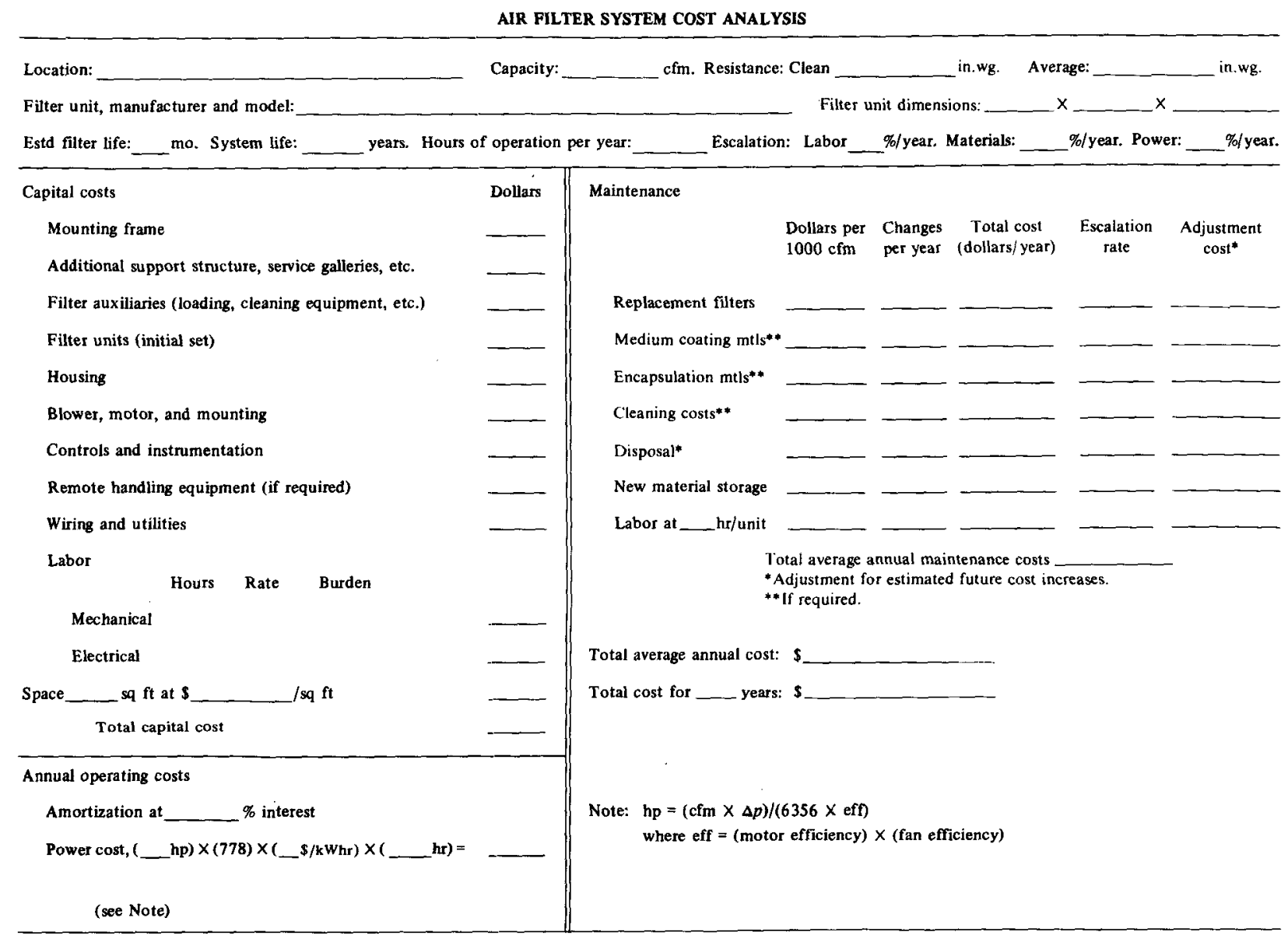


LABOR COST ESTIMATING FORM FOR INTERNAL COMPONENTS OF RADIOACTIVE EXHAUST SYSTEM

\begin{tabular}{|c|c|c|c|c|}
\hline & \multicolumn{2}{|c|}{ Personnel } & \multicolumn{2}{|r|}{ Time } \\
\hline & Type & $\begin{array}{c}\text { Number } \\
\text { of men }\end{array}$ & $x$ hours & $\times$ man-hours \\
\hline \multirow{2}{*}{\multicolumn{5}{|c|}{$\frac{\text { Make ready }}{\text { Planning }}$}} \\
\hline & & & & \\
\hline \multicolumn{5}{|l|}{ Move new components to installation site } \\
\hline Inspect & \multirow{2}{*}{ Technician } & & & \\
\hline Prepare area: place barriers, floor coverings, etc. & & & & \\
\hline $\begin{array}{r}\text { Dress mechanics: coveralls } \square \text { shoe covers } \square \\
\text { respirators } \square \text { tape clothing } \square\end{array}$ & \multirow[t]{2}{*}{ Mechanic } & & & \\
\hline Other & & & & \\
\hline \multirow{2}{*}{\multicolumn{5}{|c|}{$\frac{\text { Replace prefilters, demisters, HEPA filters, adsorber cells }}{\text { Demisters: quantity }}$}} \\
\hline & & & & \\
\hline \multicolumn{5}{|l|}{ Prefilters: quantity } \\
\hline \multirow{2}{*}{\multicolumn{5}{|c|}{$\begin{array}{l}\text { HEPA filters, first stage: quantity } \\
\text { Adsorber cells: quantity }\end{array}$}} \\
\hline & & & & \\
\hline \multicolumn{5}{|l|}{ HEPA filters, second stage: quantity } \\
\hline \multirow{2}{*}{\multicolumn{5}{|c|}{$\begin{array}{l}\text { Bag and or box prefilters, demisters, HEPA filters, adsorber cells } \\
\text { Other }\end{array}$}} \\
\hline & & & & \\
\hline \multicolumn{5}{|l|}{ Cleanup } \\
\hline \multirow{2}{*}{\multicolumn{5}{|c|}{$\begin{array}{l}\text { Remove used components to temporary storage area } \\
\text { Bag and remove tools, etc.; take to decontamination area }\end{array}$}} \\
\hline & & & & \\
\hline \multicolumn{5}{|l|}{ Bag and remove trash } \\
\hline \multicolumn{5}{|l|}{ Clean up area } \\
\hline \multirow{2}{*}{\multicolumn{5}{|c|}{ Health physics survey }} \\
\hline \multirow{2}{*}{\multicolumn{5}{|c|}{$\begin{array}{l}\text { Undress mechanics } \\
\text { Leak test HEPA filter and adsorber banks }\end{array}$}} \\
\hline & & & & \\
\hline \multicolumn{5}{|l|}{ Disposal or shipment to disposal site } \\
\hline \multicolumn{5}{|l|}{ Decontaminate } \\
\hline \multicolumn{5}{|l|}{ Rebalance system } \\
\hline Other & & & & \\
\hline
\end{tabular}




\title{
Appendix C. Care and Handling of HEPA Filters ${ }^{1}$
}

\author{
H. Gilbert and J. H. Palmer
}

High reliance can be placed on the HEPA filter if precautions are taken in handling, storage, and installation. Inspection upon delivery, upon withdrawal from stock, and before and afterinstallation is important. A filter unit should be inspected each time it is handled to guard against installation of a damaged item.

The precautions and recommendations in this handbook are based upon field experience and current development.

\section{C.1 PACKAGING AND SHIPPING}

Packaging practice varies among the filter unit manufacturers. Normally, units are packaged in cardboard cartons with various means of providing internal strengthening and impact resistance of the carton. A carton will usually contain one of the larger units, such as the $1000-\mathrm{cfm}, 24 \times 24 \times 11^{1 / 2}$ in. unit; or it may have two $500-\mathrm{cfm}, 24 \times 24 \times 5 \frac{7}{8}$ in. units. The smaller sizes, the $50-\mathrm{cfm}, 8 \times 8 \times 5 \%$ in., and the $25-$ $\mathrm{cfm}, 8 \times 8 \times 3 \frac{1}{16}$ in. units, frequently are packaged in multiple.

When a filter is placed in the carton, it is inserted so that the pleated folds are vertical to prevent damage in shipment. To prevent sagging of the pleats, vertical positioning of the pleats must be maintained during subsequent handling and storage. Moreover, filter units should also be installed vertically for operation.

The shipping carton is marked with a vertical arrow and the notation "this side up" to indicate positioning of the carton in the transport vehicle. Other markings, "handle with care," "use no hooks," etc., may be found on some containers.

When a filter unit is shipped with pleats in the horizontal position, the vibration that occurs during transportation and the jarring that usually accompanies handling often cause the filter medium to split

1. Updated from H. Gilbert and J. H. Palmer, High Efficiency Particulate Air Filter Units, USAEC Report TID-7023, August 1961. or to break at the adhesive line, which will appear as a hairline crack.

Occasionally a filter unit is positioned improperly in the container by the manufacturer. Cartons frequently are not placed in railroad cars or trucks according to the vertical arrow, and they are not handled consistently with the care designated. Consequently, inspection to verify that filters have been packed properly is necessary upon delivery at destination.

\section{2 INSPECTION AND TEST}

Inspection starts when a delivery of filter units reaches the purchaser, even while the load is still aboard the carrier. As the shipment is being unloaded, each carton should be inspected for external damage and improper positioning in the cargo space (carton placed with arrow directed horizontally). Damaged cartons, including those with corners dented and those improperly oriented in the truck, should be set aside for particularly careful inspection of their contents. Damage will be more prevalent when filter units are loaded with mixed cargoes or are shipped in a partially loaded carrier.

The filter unit must be removed carefully from its carton. The acceptable method for removal is to open the top flaps of the container after removing the sealing tape. With flaps folded back, the carton should be inverted or upended gently to place the exposed end of the filter unit on a flat surface, preferably the floor. The surface must be clear of nuts, bolts, and similar protrusions. Then withdraw the carton from the filter unit. Attempts to remove the filter unit from the carton by grasping below the exposed filter case can result in irreparable damage if fingers puncture the delicate filter medium attached immediately below the case.

When visual inspection is made, a strong lamp should be used to examine the exposed areas of both faces to ensure that no breaks, cracks, or pinholes are evident. In addition, a less intense light, such as a 
flashlight, can be used in a darkened room. The inspector should look for visible defects with the light projected along the full length of each channel created by the separators.

Translucent spots will likely prove to be variations in thickness of the filter medium which occur during manufacture. Breaks or cracks in the medium usually show up on the surface edges of the filter pleats but often are not readily detected. Minor cracks can be of major importance. If the filter unit is installed with this pleat-edge damage, the cracks can be extended by air movement through the unit. After examining each channel, the inspector should examine the adhesive seal around the filter unit face to be sure that the seal is complete and unbroken. When one face of the filter unit has been inspected, the other face should be examined in the same manner and with the same care.

After the inspector has completed a thorough scrutiny of both faces, he should check the corner joints of the frame for adhesive sealing and tightness. Gasketing about the edge of the frame should be inspected for tight mating of gasket strips and good physical condition. Gasket strips should also be examined for full adhesion to the frame.

Cartons showing damage or dented corners and those that are found loaded in improper position upon delivery and that were set aside after being unloaded from the carrier, require careful inspection. The filter unit should be examined at all corners and particularly at the point of carton impact for damage to separators and medium. Exterior damage to several protruding separator edges in a small area will not influence filter unit efficiency if the medium is not mashed, punctured, or broken. Even though the medium may not be broken on one face, damage may occur at the opposite edge of the pleat on the other face. Large areas of mashed separator edges, even though the medium is not damaged, will obstruct the passage of air through the filter unit and thus reduce its life. Improperly stowed filter units should be inspected particularly for cracks alongside the adhesive seal, for extreme sags in pleats and separators, and for slits or breaks in the medium. The procedures outlined above, including examination with lamp and flashlight, should be used for routine inspections.

Repair of a damaged filter unit, particularly the medium, should not be attempted by the user. Any repaired unit must be retested by DOP penetrometer to ensure that hidden damage does not exist which will reduce filtering efficiency. Repair and retest thus become uneconomical for most users.
Materials used in construction of the filter unit must comply with the purchase specification, if any. Compliance, so far as practicable, should be determined at the time of inspection. Filter units that have been inspected and found damaged, defective, or not in conformance with the purchase order should be separated from acceptable units; identified; and, accompanied by necessary records, referred to the purchasing, receiving, or other appropriate department for proper disposal.

Visual inspection of the filter unit to detect physical damage is necessary. Inspection, however, is not a substitute for DOP testing with a penetrometer.

Prior to delivery, efficiency testing of the filter unit by an ERDA Quality Assurance Station is advisable. Such testing will readily disclose a defective filter unit, even when faults in the unit cannot be found by visual inspection. High penetration due to faults results in an excessive release of particles to the atmosphere. The penetrometer also measures the pressure drop, or resistance of the filter unit to the rated airstream. Excessive resistance will shorten the period that the filter unit can be used. Resistance, like penetration, must not exceed a predetermined level.

Standard practice for manufacture of HEPA filters requires that the manufacturer's test results of airflow, penetration, and resistance be marked on the case of the filter unit. These will be found in a stamp that bears the manufacturer's name, or vendor's name, together with the model number and serial number of the filter unit. Penetration and resistance should not be greater than specified by the purchase order. If not specified, penetration should not exceed $0.03 \%$ and resistance should not be more than 1.0 in.wg at rated airflow.

\section{C.3 SHIPPING}

HEPA filters should be shipped under controlled conditions insofar as practicable. Too often, after the cartons have been carefully arranged in a trucktrailer body, the shipper removes them at an interchange station, stacks them temporarily in the terminal (under completely uncontrolled conditions), and then stacks them into another truck-trailer. Handling under such conditions is usually careless, and attention to proper orientation of the cartons may be nonexistent. As a minimum, it is recommended that cartons be steel-banded to a skid or pallet, no more than $6 \frac{1}{2} \mathrm{ft}$ high, in the specified vertical orientation. Skids (pallets) must not be stacked one above the other unless bracing is provided in the truck-trailer body or railroad car to 
prevent the weight of the upper load from resting on the lower. This will force the shipper to keep the cartons in their proper orientation and prevent him from throwing or dropping them indiscriminately.

Another control is to require that the filters be packed properly in a sealed truck-trailer body or in a sealed containerized-freight unit, not to be opened until arrival at the specified delivery point. The trailer or containerized-freight unit should be unloaded by personnel employed at the delivery site who have been thoroughly instructed in the proper care and handling of HEPA filters. Mixed-load shipments should be avoided.

\section{C.4 STORAGE}

Following receipt and inspection, the filter unit should be repacked carefully in the carton in which it was shipped and received. All packing material for internal strengthening of the carton and for protection of the filter unit should be replaced properly. Pleats of the filter unit should be positioned to conform to the orientation marking on the carton; this should be done routinely whether the filter unit will be installed at an early date or whether it will be stored.

Cartons of filter units should be positioned in storage to conform to the vertical arrow, and manufacturer's recommendations for storage heights should be followed. When recommendations are not available, filter units $24 \times 24 \times 11^{1 / 2}$ in. and $24 \times 24 \times$ $57 / 8$ in. should be stacked not more than three filter units high.

Mixing other items and materials with filter units in storage should be avoided to prevent damage to the filter units. Recommended aisle widths consistent with good warehousing practice should be provided to reduce damage of filter units from materialshandling equipment and other traffic. Filter units should not be stored in locations where they will be exposed to dampness, excessive heat or cold, or rapidly changing temperatures.

\section{C.5 HANDLING}

Mechanical warehousing equipment is recommended for handling large quantities of filter units. Skids and pallets should be used to provide a flat bed for movement of the units. Chains, slings, and hooks obviously must not be used. The cartons should be placed on the pallet so that the arrow on the carton points vertically.
In physically handling a packaged filter unit, a person must make certain that the carton is picked up at opposite corners and deposited carefully on the floor or other surface. The carton should not be dropped or jarred. Any filter unit dropped, whether or not in the carton, should be reexamined for damage as prescribed in Sect. C. 2 .

When a filter unit is lifted, it must be grasped only along the outer surface of the case. Even slight contact of fingers at almost any point within the case can puncture the filter medium.

A handle or grip is sometimes attached permanently to the wood filter frame for ease of installation and removal of the filter unit. In such instances, care must be taken in attaching the handle. Screws should not be pounded for starting, and nails should never be used. The recommended method is to drill starting screw holes, making certain that the drill and the length of screws do not penetrate through the frame and pierce the filter medium attached (screws must not be longer than $3 / 4$ in.). Pounding may crack the filter medium and possibly loosen the adhesive seal that bonds the filter pack within the frame. Attachment of a handle to a metal-frame filter unit is not recommended.

Filter units should be kept in shipping cartons when moved from one location to another. When transferred for installation, the units should be unloaded at a point which, so far as practicable, will reduce physical handling. Filter units should remain in cartons until ready for installation and then should be unpacked as prescribed in Sect. C.2.

If for any reason an unpackaged filter unit must be placed with its face on the floor or other surface, the surface must be cleared of every object or irregularity that might damage the filter pack.

\section{C.6 INSTALLATION}

Craftsmen responsible for installation of the filter unit must be carefully instructed in proper handling technique. They should know that the filter pack within the frame is delicate and must not be damaged during installation. Equally important is that the filter unit must be installed so that unfiltered air will not leak past the unit. The following installation procedure should be used:

1. Carefully remove filter unit from shipping carton, following the procedure described under Sect. C.2. 
2. Carefully inspect both faces of the filter unit for cracks in the filter medium, for damage of separators, and for separation of the filter pack at the frame.

3. Ensure that the gasket is cemented firmly to the frame and that the gasket pieces are butted or mated at the joints.
4. The gasket must be compressed firmly. Compression should be applied evenly and equally at all points in increments of $5 \mathrm{ft}-1 \mathrm{~b}$ or less, with the filter frame completely covering the opening.

5. Install the filter with pleats and separators in the vertical position. This will eliminate sagging of pleats from accumulated weight of materials stopped by the filter unit. 


\section{Appendix D. Seismic Design and Qualification of ESF Air Cleaning Systems ${ }^{1}$}

\section{D.1 INTRODUCTION}

External components of the system (e.g., ducts, housings, and fans), insofar as practicable, should be rigidly anchored to major building elements (walls, floors, partitions). These building elements have sufficient stiffness that it can be assumed that the interaction of the air cleaning system on the building is negligible, and that the motion of the building element can be considered as the only input to the system. Where this type of anchoring is not practicable (e.g., as with ductwork), lateral bracing or other means to minimize movement of the external component must be provided. External components of the same system should be anchored to the same building element. Where this is not possible, the motion produced in the building element experiencing the greatest motion under the influence of an earthquake should be used to determine the accelerations of all segments of the system or subsystem. When parts of the system are anchored to more than one building element, displacements of the anchor points of different parts of the system should be considered as $180^{\circ}$ out of phase and must be added to establish the maximum stresses in connections and other parts of the system that could be affected by the differential loading. Connections and anchors must be designed to accommodate simultaneously the combined horizontal, vertical, twisting, and bending motions. Expansion joints, expansion loops, or other means of providing flexibility while preserving the leak integrity of the system, may be used where necessary.

Anchors, attachments, and connections between runs of duct, dampers (valves), and fans (including motor and motor mount), must be designed to transmit the forces associated with the accelerations induced in the air cleaning system and the relative distortions of the building elements to which the external components of the system are anchored.

1. N. J. Mason and P. J. Lama, "Seismic Control for Floor Mounted Equipment," Heat. Piping Air Cond. 48(3), 97-104 (1976).
Ducts and housings (including their pressure boundary welds and flanged connections), and filter mounting frames and doors (including door frames) of housings, should be designed to withstand, without buckling or rupture, the forces associated with equipment accelerations, relative distortions of connected parts, and relative distortions of building elements to which they are anchored.

\section{D.2 COMPONENT QUALIFICATION}

Maximum accelerations, displacements, and relative-velocity changes that can be tolerated by manufactured components (fans, filters, adsorbers, dampers, etc.) without damage or failure of function should be determined as a function of frequency by the manufacturer or the system designer. Qualification may be either by testing in accordance with Sect. D.4 or mathematical analysis in accordance with Sect. D.5 if possible. A component vulnerability spectrum should be prepared from the test or analytical data to show the maximum displacements and accelerations that the equipment can tolerate, as a function of frequency, without damage or loss of function. Qualification of the equipment may be based on either (1) comparison of the component vulnerability spectrum with the response spectrum of the building element to which the component is anchored or (2) demonstration of the operability of the equipment following the test. Natural frequencies of the equipment, installed and operated as it will be in service, should be determined if possible, but may be ignored if proven to be less than $0.3 \mathrm{~Hz}$ or greater than $30 \mathrm{~Hz}$.

\section{D.3 BUILDING-ELEMENT RESPONSE SPECTRUM}

The designer should be furnished with or should compute, for each building element of interest, the maximum accelerations, displacements, and relative velocity changes, as a function of frequency, that can be expected in the building element as a result of the design basis earthquake. 


\section{D.4 TESTING}

Components or the complete system may be qualified by testing under simulated earthquake conditions. The item to be tested is mounted on a biaxial or triaxial vibration generator in a manner that simulates the intended service mounting, and vibratory motion is applied independently to each of the perpendicular axes. Displacement induced in the vertical axis should be considered to be at least 0.67 times the displacement in the major horizontal axis. The magnitudes of horizontal acceleration and displacement are those magnitudes for which the item is to be qualified. Where practicable, it is recommended that the accelerations, displacements, and relative velocity changes be the maximums which the equipment can tolerate without loss of function. For fans, motors, dampers, and other operating devices, sufficient monitoring devices must be used to thoroughly evaluate performance during and after testing; monitoring devices must be located on the equipment or test assembly so that the maximum response is always obtained. Test are made at several sinusoidal frequency steps representative of the range of frequencies for which the item is to be qualified, at the natural frequency, or at a number of predetermined frequencies as outlined below.

\section{D.4.1 Exploratory Vibration Test}

An exploratory test should first be made, using a sinusoidal steady-state input of low magnitude, to determine the presence and location of any natural frequencies within the range of 0.1 to $60 \mathrm{~Hz}$, or the frequency range stated in the project specification. The test should include a minimum of two sweeps at a maximum sweep rate of $2 \mathrm{~Hz}$ and a minimum acceleration of $0.1 \mathrm{~g}$, with dwell at resonance for at least $30 \mathrm{sec}$ and dwell for $1 \mathrm{~min}$ at each natural frequency. If no resonating frequencies are found, the item may be analyzed statically or may be tested (1) by a continuous test (Sect. D.4.2), (2) by sine-beat test (Sect. D.4.3), or (3) by a multiple-frequency test (Sect. D.4.4).

If one or more resonant frequencies are found in the exploratory test, the design of the component should, if possible, be modified to move the resonating frequencies above $30 \mathrm{~Hz}$ or the maximum frequency for which the item is to be qualified, then tested in accordance with Sect. D.4.2. If the item cannot be readily modified, a performance test should be made at the resonant frequency at an amplitude of at least the corresponding value for that frequency from the response spectrum for the building element of interest.

Items having resonance frequencies below $5 \mathrm{~Hz}$ require special consideration because available vibration generators may not be adequate to produce the required dynamic forces. The item may be modified to increase the resonating frequency to a level where testing by the sine-beat method is possible; otherwise, sufficient testing and analysis must be carried out to verify the structural and functional integrity of the item.

\section{D.4.2 Continuous Test}

A continuous sinusoidal motion corresponding to the maximum acceleration to which the item is to be qualified, at the frequency for which the item is to be qualified, is imposed for a length of time conservatively consistent for the service for which the item will be used, during which the item is operated to demonstrate its ability to perform its function. The duration of test is specified in a detailed test procedure. The item is mounted on the vibration generator in a manner that is representative of its installation under service conditions. The vibratory forces are applied to each of the three major. perpendicular axes independently unless symmetry justifies otherwise. Sufficient monitoring equipment must be used to accurately evaluate performance before, during, or after the test, depending on the nature of the item to be tested.

\section{D.4.3 Sine-Beat Test}

The test is made by inducing sine beats of peak acceleration corresponding to that for which the item is to be qualified, at the frequency and amplitude of interest. The duration and amplitude of the beat for each test frequency must be chosen to produce a magnitude equivalent to that produced by the particular building-element response, with appropriate damping factors. For a test at any given frequency, five beats of ten cycles per beat are normally used, with a pause between the beats so that no significant superposition of motion will result. Mounting of equipment and instrumentation is described in Sect. D.4.2.

\section{D.4.4 Multiple-Frequency Test}

The item is tested over the frequency range of 5 to $60 \mathrm{~Hz}$ at a maximum sweep rate of $2 \mathrm{~Hz}$. The input at any given frequency is equal to at least the corresponding frequency of the response spectrum 
for the building element of interest for the operating basis earthquake (OBE). Section D.4.2 discusses the mounting of equipment and instrumentation.

\section{D.4.5 Documentation}

The test and analytical procedures and any modifications of the item must be documented, together with complete results of operational tests conducted during or after the vibration test, as applicable.

\section{D.5 MATHEMATICAL ANALYSIS}

Components or the complete system may also be qualified by a mathematical analysis. The objective of the analysis is to predict the stresses, displacements, and deflections that will develop in critical parts of the component or system as a result of the specified input or time-history motion applied at the base (anchor points) of the component or system by an earthquake. The problem is defined by the physical properties of the system to be analyzed; its mass, stiffness, and damping characteristics; and the timevarying accelerations, displacements, and relative velocity changes introduced at its foundation (anchor points).

If the mass of the component or system to be analyzed is small relative to the mass of the building element to which it is anchored, the supported component or system may be treated as a lumpedmass, multidegree-of-freedom system, having an input at its foundation (anchor points) equal to the motion of the building element to which it is attached (i.e., no interaction assumed).

If the natural frequency of the item (component or system) is less than $0.2 \mathrm{~Hz}$ or more than $40 \mathrm{~Hz}$, the item may be analyzed statically. The seismic forces on each element of interest of the item are obtained by concentrating its mass at its center of gravity and multiplying it by the appropriate maximum floor acceleration. Operating, live, and dead loadings are added to the seismic loadings in their appropriate directions. Displacements may be the limiting factor and must be accounted for in the design analysis. The following damping values may be used:

$\begin{array}{ccc}\text { Type of system } & \begin{array}{c}\text { For } \\ \text { SSE }\end{array} & \begin{array}{c}\text { For } \\ 1 / 2 \text { SSE }\end{array} \\ \text { Welded } & 5.0 & \\ \text { Bolted } & 7.0 & 2.0 \\ & & 5.0\end{array}$

If the mass of the component or system is large relative to the mass of the building element to which it is attached, or if the item is not anchored rigidly to a building element, the interaction of the system on the building element must be considered; the system is dynamically analyzed as a multidegree-of-freedom mathematical model. The item (component or system) may be modeled as a series of discrete mass points connected by mass-free members, with sufficient mass points to ensure adequate representation of the item as it is supported in the building structure. The resulting system may be analyzed using the response spectra model analysis technique or a timehistory (modal or step-by-step) analysis technique. A stress analysis is then made using the inertial forces or equivalent static loads obtained from the dynamic analysis for each vibration mode. If the response spectrum analysis technique is used, the seismic design stress may usually be obtained by taking the square root of the sum of the squares of the individual modal stresses; the absolute sum of the responses should be taken, however, for closely spaced, inphase vibration modes. In the analysis, each of the two major horizontal directions is considered separately and simultaneously with the vertical direction in the most conservative manner.

The analysis must include an evaluation of the effects of the calculated stresses on mechanical strength, alignment (if critical to proper operation of the air cleaning system), and operational (functional) performance of the components and the system as a whole. Maximum displacements at critical points must be calculated and interference or plastic deformation determined and evaluated.

\section{D.6 DOCUMENTATION}

The selected method or methods of seismic analysis, mathematical models and their natural frequencies, input time-histories and corresponding response spectra, damping values, and allowable stress criteria must be shown in a safety analysis report, together with results of all tests and analyses. The documentation must give details which demonstrate that the item meets specified requirements when subjected to the seismic motion for which it is to be qualified. Analytical procedures should be described in sufficient detail to be readily auditable by persons knowledgeable in such analyses. Analytical and test results should be certified by a licensed professional engineer qualified in the analysis of such systems. 


\section{Bibliography}

\section{REGULATORY GUIDES (Nuclear Regulatory Commission)}

Division 1, Power Reactors

1.29 Seismic Design Classification, August 1973.

1.33 Quality Assurance Program Requirements, November 1972.

1.48 Design Limits and Loadings for Seismic Category I Fluid Systems Components, May 1973.

1.52 Design, Testing and Maintenance Criteria for Atmosphere Cleanup System Air Filtration and Adsorption System Units of Light-Water-Cooled Nuclear Power Plants, June 1973.

1.78 Assumptions for Evaluating the Habitability of a Nuclear Power Plant Control Room During a Postulated Hazardous Chemical Release, June 1974.

1.95 Protection of Nuclear Power Plant Control Room Operators Against an Accidental Chlorine Release, February 1975.

Division 3, Fuels and Materials Facilities

3.9 Concrete Radiation Shields, June 1973.

3.12 General Design Guide for Ventilation Systems of Plutonium Processing and Fuel Fabrication Plants, August 1973.

3.14 Seismic Design Classification for Plutonium Processing and Fuel Fabrication Plants, October 1973.

3.16 General Fire Protection Guide for Plutonium Processing and Fuel Fabrication Plants, January 1974.

3.18 Confinement Barriers and Systems for Fuel Reprocessing Plants, February 1974.

3.20 Process Offgas Systems for Fuel Reprocessing Plants, February 1974.

\section{CODE OF FEDERAL REGULATIONS}

10 CFR $20 \quad$ Standards for Protection Against Radiation

10 CFR $50 \quad$ Licensing of Production and Utilization Facilities

10 CFR $100 \quad$ Reactor Site Criteria

\section{CODES AND STANDARDS}

\section{American National Standards}

ANSI B31 Pressure Piping

ANSI N101.6 Concrete Radiation Shields

ANSI N45.2 Requirements for Quality Assurance Programs for Nuclear Power Plants

ANSI N509 Standard for Nuclear. Power Plant Air Cleaning Units and Components

ANSI N510 Standard for Testing of Nuclear Air Cleaning Systems 
ANSI N512 Protective Coatings (Paints) for the Nuclear Industry

ANSI Z9.2 Fundamentals Governing the Design and Operation of Local Exhaust Systems

American Society for Testing and Materials

ASTM A36 Specification for Structural Steel

ASTM A240 Specification for Corrosion-Resisting Chromium and Chromium-Nickel Steel Plate, Sheet, and Strip

ASTM A245 Specification for Flat-Rolled Carbon Steel Sheets

ASTM A380 Recommended Practice for Cleaning and Descaling Stainless Steel Parts, Equipment, and Systems

ASTM A479 Specification for Stainless and Heat-Resisting Steel Bars and Shapes

ASTM A499 Specification for Hot-Rolled Carbon Steel Bars and Shapes

ASTM A500 Specification for Cold-Formed Welded and Seamless Carbon Steel Structural Tubing in Rounds and Shapes

ASTM D1056 Specification for Sponge and Expanded Cellular Rubber Products

ASTM D3467 Method of Test for Carbon Tetrachloride Activity of Activated Carbon

ASTM D3466 Method of Test for Ignition Temperature of Activated Carbon

ASTM D2652 Definitions of Terms Relating to Activated Carbon

ASTM D2854 Method of Test for Apparent Density of Activated Carbon

ASTM D2862 Method of Test for Particle Size Distribution of Granular Activated Carbon

ASTM D2866 Method of Test for Total Ash Content of Activated Carbon

ASTM D2867 Method of Test for Moisture in Activated Carbon

ASTM Ell Specification for Wire-Cloth Sieves for Testing Purposes

Air Moving and Conditioning Association

AMCA $210 \quad$ Laboratory Methods of Testing Fans for Rating

AMCA $500 \quad$ Test Methods for Louvers, Dampers, and Shutters

AMCA 99 Standards Handbook

American Society of Heating, Refrigerating and Air-Conditioning Engineers

ASHRAE 52-68 Method of Testing Air. Cleaning Devices Used in General Ventilation for Removing Particulate Matter

Institute of Environmental Sciences (formerly American Association for Contamination Control)

AACC CS-1 Standard for HEPA Filters

AACC CS-2 Standard for Laminar Flow Clean Air Devices

AACC CS-6 Testing and Certification of Particulate Clean Rooms

AACC CS-8 Standard for High Efficiency Gas-Phase Adsorber Cells

National Fire Protection Association

NFPA 11A Standard for High Expansion Foam Systems 
NFPA 11B Standard for Synthetic Foam and Combined Systems

NFPA 12A Standard for Halogenated Fire Extinguishing Agent Systems-Halon 1301

NFPA 68 Explosion Venting

NFPA 90A Standard for Air Conditioning and Ventilating Systems

NFPA $91 \quad$ Blower and Exhaust Systems, Dust, Stock and Vapor Removal or Conveying

NFPA 12 Carbon Dioxide Extinguishing Systems

NFPA 13 Installation of Sprinkler Systems

Underwriters' Laboratories

UL-586 Safety Standard for High Efficiency Air Filter Units

UL-900 Safety Standard for Air Filter Units

Sheet Metal and Air Conditioning Contractors' National Association

SMACNA Low Velocity Duct Construction Standards

SMACNA High Velocity Duct Construction Standards

SMACNA Manual for the Adjustment and Balancing of Air Distribution Systems

Military Specifications

MIL-F-51068 Filter, Particulate, High Efficiency, Fire-Resistant

MIL-F-51079 Filter Medium, Fire-Resistant, High Efficiency

ERDA Standards

RDT E9-1 HEPA Filters

RDT M 16-1 Gas-Phase Adsorbents for Trapping Radioactive Iodine and Iodine Compounds

RDT M16-3 HEPA Filter Medium, Glass Fiber

American Society of Mechanical Engineers

ASME Boiler and Pressure Vessel Code, Sect. V, Nondestructive Examination.

ASME Boiler and Pressure Vessel Code, Sect. III, Nuclear Power Plant Components.

ASME Boiler and Pressure Vessel Code, Sect. IX, Welding and Brazing Qualifications.

\section{OTHER DOCUMENTS}

\section{American Conference of Governmental Industrial Hygienists}

Industrial Ventilation, current issue.

TLVs-Threshold Limit Values for Chemical Substances and Physical Agents in the Workroom Environment, current issue.

American Society of Heating, Refrigerating and Air-Conditioning Engineers

ASHRAE Handbook and Product Directory-Equipment, current issue.

ASHRAE Handbook and Product Directory-Handbook of Fundamentals, current issue.

ASHRAE Handbook and Product Directory-Applications, current issue. 
ASHRAE Handbook and Product Directory-Systems, current issue.

Air Moving and Conditioning Association

Fan Application Manual

International Atomic Energy Agency

Proceedings of the Symposium on Airborne Radioactivity, 1966.

Proceedings of the Symposium on Treatment of Airborne Radioactive Wastes, 1968.

Techniquesfor Controlling Air Pollution from the Operation of Nuclear Facilities, Safety Series No. 17, 1966.

Air Filters for Use at Nuclear Facilities, Technical Reports Series No. 122, 1970.

\section{AEC and ERDA Reports}

Proceedings of the biennial AEC/ERDA Air Cleaning Conferences, 1952 to present.

Proceedings of the Rocky Flats Symposium on Safety of Plutonium Handling Facilities, USAEC Report CONF-710401, Dow Chemical Co., April 1971.

R. E. Ackley et al., Aging, Weathering, and Poisoning of Impregnated Charcoals Used for Trapping Radioiodide, USAEC Report ORNL-TM-2860, Oak Ridge National Laboratory, March 1970.

C. J. Barton, A Review of Glove Box Construction and Experimentation, USAEC Report ORNL-3070, Oak Ridge National Laboratory, May 1961.

W. L. Belvin et al., Final Report, Development of Fluoride-Resistant HEPA Filter Medium, ERDA Report TID-26649, August 1975.

E. C. Bennett and D. E. Strege, Evaluation of Weathered Impregnated Charcoals for Retention of lodine and Methyl Iodide, USAEC Report UNI-39, United Nuclear Industries, July 1973.

A. H. Dexter, A. G. Evans, and L. R. Jones, Confinement of Airborne Radioactivity, ERDA Report DP-1390, Savannah River Laboratory, October 1975.

W. C. Durant et al., Activity Confinement System of the Savannah River Plant Reactors, USAEC Report DP1071, Savannah River Laboratory, August 1966.

N. B. Garden (ed.), Report on Glove Boxes and Containment Enclosures, USAEC Report TID-16020, AEC Health and Safety Laboratory, June 1962.

W. S. Gregory, HEPA Filter Effectiveness During Tornado Conditions, USAEC Report LA-5352-MS, Los Alamos Scientific Laboratory, 1973.

W. S. Gregory and G. A. Bennett, Ventilation Systems Analysis During Tornado Conditions, USAEC Report LA-5894-PR, Los Alamos Scientific Laboratory, 1975.

G. H. Griwatz et al., Entrained Moisture Separators for Fine Particle Water-Air-Steam Service; Their Performance, Development, 'and Status, USAEC Report MSAR-71-45, MSA Research Corp., March 1971.

R. A. Juvinall et al., Sand-Bed Filtration of Aerosols: A Review of Published Information on their Use in Industrial and Atomic Energy Facilities, USAEC Report ANL-7683, Argonne National Laboratory, June 1970.

J. E. Mecca and J. D. Ludwick, In-Place Testing of the Hanford Reactor Charcoal Confinement Filter Systems Using Iodine Tagged with Iodine-131 Tracer, USAEC Report DUN-SA-105, Douglas United Nuclear, Inc., June 1969.

D. R. Muhlbaier, Standardized Nondestructive Test of Carbon Beds for Reactor Confinement Applications, USAEC Report DP-1082, Savannah River Laboratory, 1967. 
J. R. Murrow, Plugging of High Efficiency Filters by Water Spray, USAEC Report TID-4500, Lawrence Livermore Laboratory, 1967.

J. B. Owen, Control of Personnel Exposure to External Radiation in a Plutonium Chemical Plant, USAEC Report RFP-1254, Dow Chemical Co., 1968.

A. H. Peters, Application of Demisters and Particulate Filters in Reactor Containment, USAEC Report DP812, Savannah River Laboratory, 1962.

M. A. Thompson, Rocky Flats Glovebox Safety Studies, USAEC Report RFP-1547, Dow Chemical Co., 1971.

R. D. Rivers and J. L. Trinkle, Moisture Separator Study, USAEC Report NYO-3250-6, American Air Filter Co., June 1966.

C. C. Wright et al., The Evaluation of Substitutive Filter Framing Materials in Corrosive Environments, USAEC Report K-TL-81, Union Carbide Corp., 1970.

O. O. Yarbro et al., Effluent Control in Fuel Reprocessing Plants, USAEC Report ORNL-TM-3899, Oak Ridge National Laboratory, March 1974.

\section{Reports of Other Organizations}

W. D. Burch and T. A. Arehart, "Safety Review Procedure for Hot-Cell and Radiochemical Processing Facilities at ORNL," Proc. 15th Conf. Remote Syst. Technol., American Nuclear Society, 1967.

C. A. Burchsted, "Fire Prevention and Control in Nuclear Air Cleaning Systems," Proc. Am. Nucl. Soc. Winter Meet., Washington, D.C., Nov. 1972, American Nuclear Society.

D. A. Collins et al., The Development of Impregnated Charcoals for Trapping Methyl lodide at High Humidity, TRG Report 1300(W), United Kingdom Atomic Energy Authority, London, 1967.

V. R. Deitz and C. A. Burchsted, Survey of Domestic Charcoals for Iodine Retention, NRL Memo Report 2960, Naval Research Laboratory, January 1975.

S. E. Smith et al., Protection Against Fire Hazards in the Design of Filtered Ventilation Systems of Radioactive and Toxic Gas Process Buildings, UKAEA Report AWRE 0-24/65, Atomic Weapons Research Establishment, Aldermaston, Berkshire, England, 1965.

J. W. Thomas, "Particle Loss in Sampling Conduits," Proc. Annu. Conf. Internat. Atomic Energy Agency, Vienna, 1967.

C. J. Trickler, NYB Engineering Letters, New York Blower Co., Chicago.

T. E. Wright et al., High Velocity Filters, USAF Report WADC 55-457, ASTIA Document No. AD-142075, Donaldson Co., Inc., 1957.

Books

Manual of Steel Construction, American Institute of Steel Construction, current issue.

Steel Structures Painting Manual, Steel Structures Painting Council, Pittsburgh, current issue.

System Design Manual, Carrier Corp., 1960.

Welding Handbook, American Welding Society, current issue.

C. N. Davies, Air Filtration, Academic Press, New York, 1973.

C. D. Morgan et al., Human Engineering Guide to Equipment Design, McGraw-Hill, New York, 1963.

T. Rockwell III (ed.), Reactor Shielding Design Manual, Van Nostrand Reinhold Co., Princeton, 1956.

N. I. Sax, Dangerous Properties of Industrial Materials, Van Nostrand Reinhold Co., New York, 4th ed., 1975. 
G. N. Walton et al., Glove Boxes and Shielded Cells, Butterworth and Co., Ltd., London, 1967.

P. A. F. White and S. E. Smith, High Efficiency Air Filtration, Butterworth and Co., Ltd., London, 1964.

P. A. F. White and S. E. Smith, Inert Atmospheres, Butterworth and Co., Ltd., London, 1962.

W. E. Woodson and D. W. Conover, Human Engineering Guide for Equipment Designers, University of California Press, 1966.

\section{Journal Articles}

P. Dergarabedian and F. Fendell, "A Method for Rapid Estimation of Maximum Tangential Wind Speed in Tornados," Mon. Weather Rev. 99, 143-45 (February 1971).

P. Dergarabedian and F. Fendell, "One- and Two-Cell Tornado Structure and Funnel Cloud Shape," $J$. Astronaut. Sci. 21, 26-31 (July-August 1973).

C. R. Schmitt, "Carbon Microspheres as Extinguishing Agent for Metal Fires," J. Fire Flammability 5, 223-34 (July 1974).

N. J. Mason and P. J. Lama, "Seismic Control for Floor Mounted Equipment," Heat. Piping Air Cond. 48(3), 97-104 (1976). 


\section{Index*}

Abbreviations and acronyms

list of, 1.9.1

Acronyms and abbreviations

list of, 1.9.1

Adhesives

specifications for HEPA filter, Appendix A.4.2.4

Adsorber banks

definition of, 2.4 .6

Adsorbers

(see Charcoal adsorbers and Inorganic adsorbers)

Air cleaning stage

definition of, 2.4.7

Air cleaning system

definition of, 2.4.3

Air cleaning systems

(see Emergency air cleaning systems, High-efficiency air cleaning systems, and Portable air cleaning systems)

Air cleaning unit

definition of, 2.4.2

Air sampling

for performance testing of high-efficiency air cleaning systems, 2.7

Air-supply filters

for nuclear facilities, 2.3.3

Bank systems

arrangement of, $4.1,4.4 .5$

floor plan of, 4.4.6

horizontal, size and arrangement of, 4.4.2

size and arrangement of charcoal adsorber, 4.4

size and arrangement of HEPA filter, 4.4

size of, 4.4 .4

vertical, size and arrangement of, 4.4.1

Branched system

definition of, 2.4.14

Buildings

air-supply filters, 2.3.3

Canyons

air cleaning requirements, 2.2.1

\footnotetext{
*Prepared by Theodore F. Davis, ERDA Technical Informa-
} tion Center, Oak Ridge, Tennessee.
Cases

corrosion resistance of HEPA filter, 3.2.6

for HEPA filters, 3.2.2

moisture resistance of HEPA filter, 3.2.6

Caves

air cleaning requirements for, 2.2.1

Clamps

for charcoal adsorbers, 4.3.4

for HEPA filters, 4.3.4

Charcoal

density of adsorber grade, 3.4 .4

hardness of adsorber grade, 3.4 .4

physical properties of adsorber grade, 3.4.4

sources of adsorber grade, 3.4.4

Charcoal adsorbers

airflow capacity of, 3.4 .2

airflow capacity testing of, 8.2.3

airflow distribution testing of, 8.2.4

airflow resistance of, 3.4.2

adsorbents for, sampling and testing of, 8.3.4

aging or weathering of , 3.4.2

bank systems of, size and arrangements of, 4.4

capacity of, 3.4.2

change frequency for, 2.3.2

charcoal sources for, 3.4.4

clamping to mounting frames, 4.3.4

construction of, 3.4.3

design of, $3.4 .3,3.4 .6$

efficiency of, 3.4 .2

fire hazards in, design for protection against, 2.5.2

heat effects on, 2.2.4

ignition temperature of, 3.4.2

in-place testing of, 8.3.2

in-place testing of, design considerations for, 8.2.5

in-place testing of multistage, 8.3.3

installation of, 4.2

moisture effects on, 2.2.3

mounting frames for 4.3

mounting frames for, configuration of, 4.3.2

mounting frames for, fabrication of 4.3 .3

mounting frames for, leak testing of, 8.2.2

mounting frames for, structural requirements of, 4.3.1 
performance of, 3.4.2

sealing to mounting frames, 4.3.4

surveillance testing of, 8.3

testing frequency for, 8.3.5

visual inspection of, 8.4

Compartmented unit

definition of, 2.4 .16

Component

definition of, 2.4.1

Control systems

for glove boxes, 7.5.3

Dampers

acceptance testing of, 5.3.5

classification of, 5.3.1

control system for, 5.3.4, 5.6.2

design of, 5.3.3

fabrication of, 5.3.3

inlet vane, 5.6.3

specifications for, 5.3.1

Deep-bed glass-fiber filters

design and operation of, 9.7.2

disposal of spent, 9.7.5

Deep-bed sand filters

design and operation of, 9.6.1

disposal of spent, 9.6.4

plugging of, 9.6.3

Demisters

airflow distribution testing of, 8.2.4

design of, 3.5.2

installation of, 4.2

knitted fabric type, 3.5.3

mounting frames for, 4.3

mounting frames for, configuration of 4 4.3.2

mounting frames for, fabrication of, 4.3.3

mounting frames for, structural requirements of, 4.3.1

nonwoven fiber mat type, 3.5 .3

packed-fiber type, 3.5.4

perforated-plate type, 3.5.4

performance of, 3.5.3

use in postaccident cleanup systems for reactors, 3.5.2

use with charcoal adsorbers, 3.5

use with HEPA filters, 3.5

wave-plate type, 3.5.3

DOP aerosol generator

for in-place testing of HEPA filters, 8.3.1

Ducts

anchors for, 5.2.6

engineering analysis of, 5.2.3

functional design of, 5.2.1

hangers for, 5.2.6 leak testing of, 8.2.1

leaktightness of, 5.2.8

mechanical design of, 5.2.2

protective coatings for, 5.2.5

protective paints for, 5.2.5

radiation shielding of, 9.3

sound proofing of, 5.2.7

supports for, 5.2.6

Earthquakes

effects on high-efficiency air cleaning systems,

9.4.1, Appendix D

Emergency air cleaning systems

design of, 6.5.3

Entrainment separators

(see Demisters)

Face guards specifications of HEPA filter, Appendix A.4.2.6

Fans

capacity requirements of, 5.4.4

installation of, 5.4.6

installation of multiple systems of, 5.4.3

location of, 5.4.7

maintenance of, 5.4.5

performance requirements of, 5.4.2

reliability of, 5.4 .5

variable speed control, 5.6.4

Filter bank

definition of, 2.4.5

Filter media

corrosion resistance of HEPA, 3.2.6

for HEPA filters, 3.2.2

moisture resistance of HEPA, 3.2 .6

radiation resistance of HEPA, 3.2.6

Frames

for HEPA filters, 3.2.2

Freon

use in testing of charcoal adsorbers, 8.3.2

Fuel reprocessing plants

air cleaning systems for, 9.9

air cleaning system for, cost comparison of, 9.9.6

Barnwell, air cleaning system for, 9.9.2

for HTGR fuels, air cleaning system for, 9.9.5

for LMFBR fuels, air cleaning system for, 9.9.3

Fume hoods

HEPA filters for, 6.5.2

Gaskets

adhesives for HEPA filter, 3.2.2

for HEPA filters, 3.2.2

specifications for HEPA filter, Appendix A.4.2.5

Glossary, 1.9

dictionary of acronyms and initialisms, 1.9.1 
Glove boxes

air cleaning requirements for, 2.2.1

airflow requirements for, 7.2.3

control systems for, 7.5.3

design of, 7.1.1

evolved gases from, dilution of, 7.2.1

exhaust cleanup requirements for, 7.2.7

exhaust filters for, inside location of, 7.3.2

exhaust filters for, outside location of, 7.3.3

exhaust HEPA filters for, 7.3.1

exhaust manifolds for, 7.2.6

exhaust requirements for, 7.2.4

explosion protection of, 7.5.1

filter replacement, 7.4

filter systems for, design of, 7.3

fire protection of, 7.5.1

heat dissipation requirements of, 7.2.2.

HEPA filters for, DOP testing of, 7.5.4

HEPA filters for, selection of, 7.3.5

inert atmospheres for, 7.5.2

inlet HEPA filters for, 7.3.4

instrumentation for, 7.5.3

prefilters for, 7.3.6

pressure-surge relief in, 7.2.5

protective atmospheres for, 7.5.2

radiation shielding for, 7.5.5

vacuum-surge relief in, 7.2.5

ventilation systems for, 7.2

Hanford Waste Encapsulation and Storage Facility

air cleaning system for, description of remotely

maintained, 9.2.8

\section{HEPA filters}

airflow capacity of, 3.3.3

airflow capacity testing of, 8.2.3

airflow distribution testing of, 8.2.4

airflow resistance of, 3.2.1, 3.3.3

bank systems of, arrangement of, 4.1, 4.4

care and handling of, Appendix $\mathrm{C}$

cases for, corrosion resistance of, 3.2.6

cases for, moisture resistance of, 3.2.6

casing materials for, 3.2 .2

change frequency for, 2.3.2

changing of single systems of, bagging method for, 6.2 .3

clamping to mounting frames, 4.3.4

classification of, Appendix A.2

construction of wood-and steel-cased, 3.2.2

construction specifications for, Appendix 4.3

corrosion of, 2.2.5

corrosion resistance of, 3.2.6

cost of, 3.2.7 decontamination factor of multistage, increase of,

2.6.2

definition of, 3.2

design and uses of cylindrical, 6.4

dust-holding capacity of, 3.2.1, 3.3.3

efficiency of, 3.2.1

efficiency of, effect of airflow uniformity on, 2.3.7

efficiency requirements of, 1.3

environmental properties of, 3.2.6

face guards for, specifications for, Appendix A.4.2.6

filter media for, 3.2.2

filter media for, corrosion resistance of, 3.2.6

filter media for, moisture resistance of, 3.2.6

filter media for, radiation resistance of, 3.2.6

fire hazards in, design for protection against,

$$
\text { 2.5.2 }
$$

fire resistance of, 3.2 .5

frame materials for, 3.2 .2

for fume hoods, 6.5.2

gasket adhesives for, 3.2.2, Appendix A.4.2.5

gaskets for, 3.2.2

for glove boxes, 7.3.4, 7.3.5, 7.5.2

handling of, Appendix C.5

heat effects on, 2.2.4

hot air resistance of 3.2 .6

housing for single systems of, 6.2

in-place testing of, 8.3.1

in-place testing of, design considerations for, 8.2.5

in-place testing of multistage, 8.3.3

inspection of, post delivery methods for,

Appendix C.2

inspection of, visual methods for, 8.4

installation of, 4.2, Appendix C. 6

installation of, human factor aspect of, 6.5.1

installation of single systems of, 6.2.1, 6.3

location on mounting frames, upstream vs

downstream, 4.4.3

mechanical properties of, 3.2 .4

moisture effects on, 2.2.3

moisture protection of, demisters for, 3.5

moisture resistance of, 3.2.6

mounting frame materials for, Appendix A.2

mounting frames for, 4.3

mounting frames for, configuration of, 4.3.2

mounting frames for, fabrication of , 4.3.3

mounting frames for, leak testing of, 8.2.2

mounting frames for, structural requirements of,

\subsection{1}

operation to high pressure drop, 2.3.5

overpressure in, resistance to, 3.2.4 
packaging and shipping of, Appendix $\mathrm{C}$ performance characteristics of, 3.2.1 performance requirements of, Appendix A.4 plugging resistance of, 3.2.6 purchase documents for, Appendix A.7 quality assurance requirements for, Appendix A.5 radiation resistance of, 3.2.6 sealants for, 3.2.2, Appendix A.4.2.4 sealants for, hot air resistance of, 3.2.6 sealing to mounting frames, 4.3.4 separators for, 3.2.2 separators for, corrosion resistance of, 3.2.6 separators for, hot air resistance of, 3.2.6 separators for, moisture resistance of, 3.2.6 separators for, qualification test for corrosion resistance of, Appendix A.A separator materials for, Appendix A.2 separator materials for, qualification test for moisture resistance of, Appendix A.A. series redundancy in multistage, 2.6.1 service life of, effect of overrating on, 2.3.6 service life of, effect of prefilters on, 2.3.4 service life of, effect of underrating on, 2.3.6 shipping of, Appendix C.3 shipping of, packaging methods for, Appendix C.1 shipping of, preparation for, Appendix A.6 shock resistance of, 3.2.4

size of, 3.2.1, Appendix A.2 specifications for, Appendix A storage of, Appendix C.4 supports for, 4.3.5 testing frequency for, 8.3.5 testing of, post delivery methods for, Appendix C.2 testing of, surveillance techniques for, 8.3 use in glove box exhaust systems, 7.3.1 weight of, 3.2.3

High-efficiency air cleaning systems acceptance testing of, 8.2

air intakes for, 5.5 component installation for, 4.2 components of, definitions of, 2.4 control systems for, description of automatic, 5.6.5 control systems for, description of central, 5.6.6 cost consideration for, 1.7 cost estimation of, forms for, Appendix B dampers for, 5.3 deep-bed glass-fiber filters, 9.7 deep-bed sand filters, 9.6 demisters for use in, 3.5 design and construction of, coordination of, 1.6 design considerations for, 1.3 design of emergency, 6.5.3 design of fuel reprocessing plant, 9.9

design of portable, 6.5.3

design of remotely maintained, 9.2

ductwork for, 5.2

earthquake protection of, 9.4.1, Appendix D

emergency design considerations for, 2.5

environmental considerations for, 2.2

equipment failure in, design for protection

against, 2.5.3

exhaust stacks for, 5.5

fans for, 5.4

filter change in, frequency of, 2.3.2

filters used in, 1.3

fire control in, 9.5.4

fire detection in, 9.5.3

fire protection of, 9.5

flexibility of, 1.5

flexibility of, 1.5

housings for, 4, 8.2.1, 9.3

instrumentation for, 5.6.7

internal components of, 3

layout considerations for emergency situations,

2.5.4

maintainability of, 2.3.8

maintenance of, 2.3.8

maintenance of, remote methods for, 9.2

mechanical shock in, design for protection against, 2.5.1

mechanical vibrations in, 2.2.6

moisture effects on, 2.2.3

operating modes for, 2.3.1

overpressure in, design for protection against, 2.5.1

performance testing of, air sampling techniques for, 2.7

power outage in, design for protection against, 2.5.3

smoke protection of, 9.5

space considerations for, 1.4

stacks for, 5.5

testability of, 2.3.8

tornado protection of, 9.4 .2

zoning for, 2.2.1

High-efficiency particulate air filters

(see HEPA filters)

Hot cells

air cleaning requirements of, 2.2.1

air cleaning systems for, description of remotely maintained, 9.2.9

Housings

construction of single HEPA filter, 6.2.2

design and layout of, 4

design of masonry and concrete, 4.5.4 
design of steel, 4.5 .3

doors for, 4.5 .7

drains for, 4.5 .8

floor materials for, 4.5.6

installation of single HEPA filter, 6.2.4

leak testing of, 8.2.1

leaktightness of, 4.5.9

paints and coatings for, 4.5.11

radiation shielding of, 9.3

sealing to mounting frames, 4.5.5

for single HEPA filter systems, 6.2

Inert atmospheres

for glove boxes, 7.5.2

Inorganic adsorbers

for radioiodine, 3.4

Installed capacity

definition of, 2.4.8

Instrumentation

for glove boxes, 7.5.3

Isolable unit

definition of, 2.4.15

Measuring units

metric equivalents for, 1.9.2

Mechanical vibrations

effect on high-efficiency air cleaning systems, 2.2 .6

Mist eliminators

(see Demisters)

effect on HEPA filters, 2.2.3, 3.2.6

Moisture separators

(see Demisters)

Mounting frames

clamping to charcoal adsorbers, 4.3.4

clamping to HEPA filters, 4.3.4

configuration of, 4.3.2

fabrication of, 4.3 .3

for charcoal adsorbers, 4.3

for demisters, 4.3

for HEPA filters, 4.3

leak testing of charcoal adsorber, 8.2.2

leak testing of HEPA filter, 8.2.2

materials for, Appendix A.2

sealants for HEPA filter, Appendix A.4.2.4

sealing to charcoal adsorbers, 4.3.4

sealing to HEPA filters, 4.3.4

sealing to housings, 4.5 .5

seals for, 4.3.4

structural requirements for, 4.3.1

Nuclear facilities

air cleaning requirements for different zones of, 2.2.1

air-supply filters for, 2.3.3
Parallel system

definition of, 2.4.11

Particulates and gases

distributionin úrban air, 2.2.2

Penetrometers

for in-place testing of HEPA filters, 8.3.2

Portable air cleaning systems

design of, 6.5.3

Prefilters

airflow capacity of, 3.3.3

airflow capacity testing of, 8.2.3

airflow distribution testing of, 8.2.4

airflow resistance of, 3.3.3

change frequency for, 3.3.6

classification of, 3.3.1

construction of, 3.3.3

corrosion resistance of, 3.3.6

cost of, 3.3.7

design of, 3.3 .3

dust-holding capacity of, 3.3.3

effect on HEPA filter service life, 2.3.4, 3.3.7

efficiency of, 3.3.2

fire resistance of, 3.3.4

for glove boxes, 7.3.5

hot air resistance of, 3.3.5

maintenance considerations for, 3.3.6

moisture effects on, 2.2.3

moisture resistance of, 3.3.6

performance of, 3.3.2

plugging of, 3.3.6

Protective atmospheres

for glove boxes, 7.5.2

Radioiodine

removal from air streams, inorganic adsorbers for, 3.4

removal from gas streams, charcoal adsorbers for, 3.4

use in testing of charcoal adsorbers, 8.3.2

Radioisotopes

hazard classification of, 2.2.1

Reactors

air cleaning systems for, 9.8

control rooms of, air cleaning systems for, 9.8.6

postaccident filter cleanup systems for, use of demisters in, 3.5.2

radioactivity containment, design of air cleaning systems for, 9.8

Reactors (Brookhaven)

air cleaning system for, description of remotely maintained, 9.2.2 
Reactors (Hanford Production)

air cleaning system for, description of remotely maintained, 9.2.3

Reactors (HFIR)

air cleaning system for, description of remotely maintained, 9.2.4

Reactors (high-temperature gas-cooled) postaccident containment of, air cleaning systems for, 9.8.4

Reactors (light water) postaccident containment of, air cleaning systems for, 9.8.3

Reactors (LMFBR)

postaccident containment of, air cleaning system for, 9.8.5

Reactors (Savannah River)

air cleaning system for, description of remotely maintained, 9.2.5

Redundant system

definition of, 2.4.13

Sealants

for charcoal adsorbers, 4.3.4

for HEPA filters, 3.2.2, 4.3.4

for HEPA filters, hot air resistance of, 3.2.6

for HEPA filters, specifications for, Appendix A.4.2.4

Segmented system definition of, 2.4.12

Separators corrosion resistance of HEPA filter, 3.2.6 corrosion resistance of HEPA filter, qualification test for, Appendix A.A

for HEPA filters, 3.2.2, Appendix A.2

hot air resistance of HEPA filter, 3.2.6

moisture resistance of HEPA filter, 3.2.6

Silver nitrate

adsorption and retention of radioiodine, 3.4.5

Silver zeolite adsorption and retention of radioiodine, 3.4.5

Single-component air cleaning unit definition of, 2.4.9

Single-path system definition of, 2.4.10

Smoke protection of high-efficiency air cleaning systems, 9.5

Stacks design of, 5.5.2

Standardized terms and phrases definitions of, 1.9.3

Terms and phrases definitions of, 1.9.3

Thorium-Uranium Recycle Facility air cleaning system for, description of remotely maintained, 9.2.7

Tornados

effects on air cleaning systems, 9.4.2

Units of measure metric equivalents for, 19.2

Ventilation system definition of, 2.4.4 